\title{
Addendum 2: Logs of Monitor Wells Drilled May 1988 through December 1992
}

\author{
November 1993
}

\section{Contributors}

\author{
J. Stout* \\ B. Qualheim \\ R. McPherrin ${ }^{\dagger}$ \\ K. Barber ${ }^{\ddagger}$ \\ R. Hedegaard ${ }^{\S}$ \\ W. McConihe** \\ T. Miller**
}

\begin{abstract}
*Ray Raskin Assoc. Inc., Tempe, Arizona ${ }^{\dagger}$ LLNL Plant Engineering, Livermore, California **Brown and Caldwell, Pleasanton, California ${ }^{\ddagger}$ Allied Signal Technical Services, Livermore, California ${ }^{8}$ ICF Kaiser Engineers, San Francisco, California

Environmental Restoration Division




\title{
Preface
}

The logs in this addendum were plotted in a new format by the same software package (LOGGER by Rockware, Denver, CO) that was used in the original publication. The scale remains the same, 1 inch $=15$ foot.

The header is totally automated with a subheading indexing the well-const uction symbols. Geophysical curves are labeled in their respective channels, and percentage core recovery is plotted in a histogram. Lithologic symbols are plotted to scale in a channel similar to previous logs. The lithologic description also has been automated to assure consistency in terminology. Descriptions are more extensive and are referenced by leader lines to the lithologic symbol.

Figures 1a and 2a are included for this Addendum: Figure 1a is a plot of all the monitoring well locations at the LLNL Main Site. Figure 2a details the gasoline spill area well locations in the vicinity of Building 403.

\section{DISCLAIMER}

\begin{abstract}
This report was prepared as an account of work sponsored by an agency of the United States Government. Neither the United States Government nor any agency thereof, nor any of their employees, makes any warranty, express or implied, or assumes any legal liability or responsibility for the accuracy, completeness, or usefulness of any information, apparatus, product, or process disclosed, or represents that its use would not infringe privately owned rights. Reference herein to any specific commercial product, process, or service by trade name, trademark, manufacturer, or otherwise does not necessarily constitute or imply its endorsement, recommendation, or favoring by the United States Government or any agency thereof. The views and opinions of authors expressed herein do not necessarily state or reflect those of the United States Government or any agency thereof.
\end{abstract}

*This work was performed under the auspices of the U.S. Department of Energy by Lawrence Livermore National Laboratory under contract No. W-7405-Eng-48. 


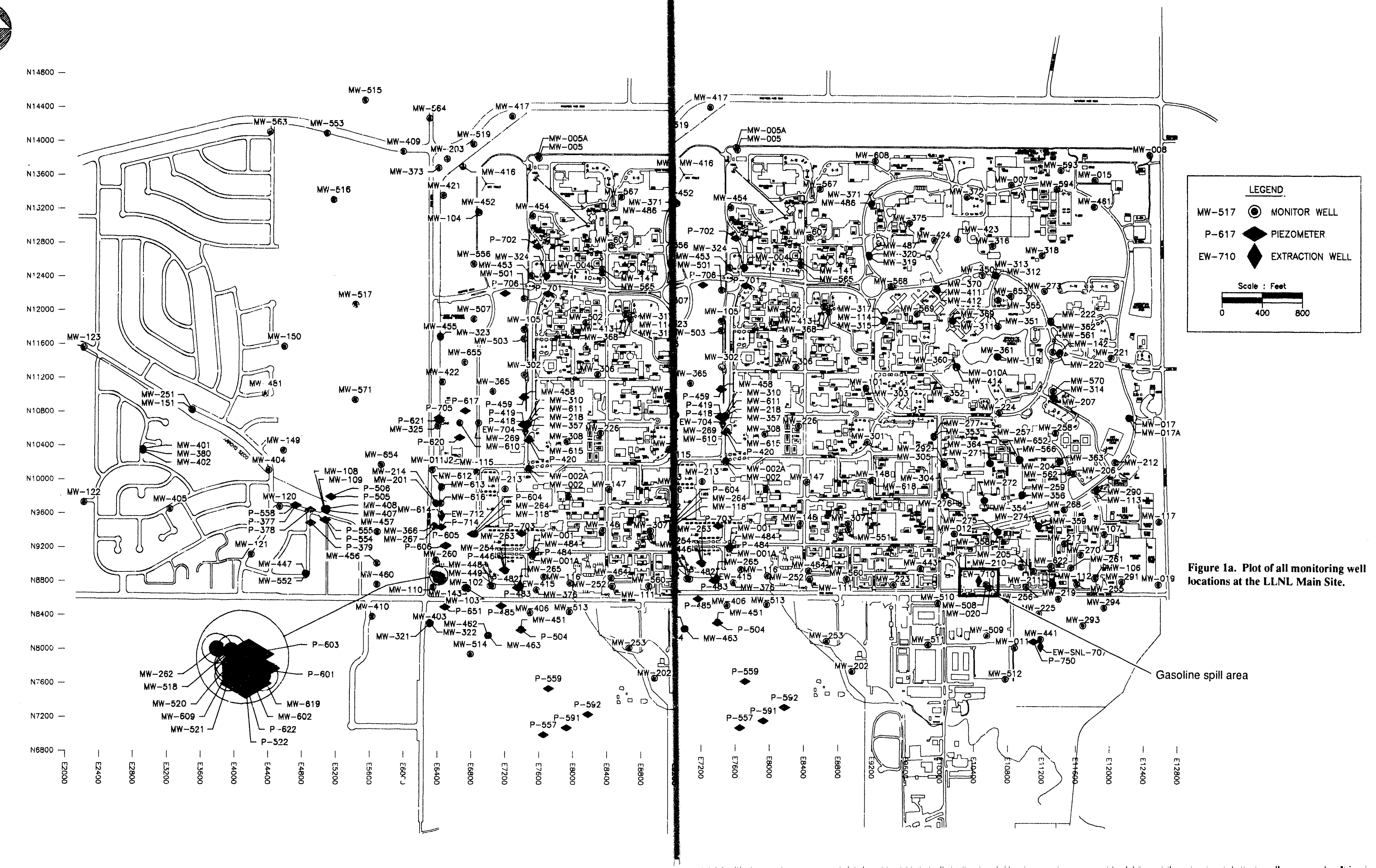




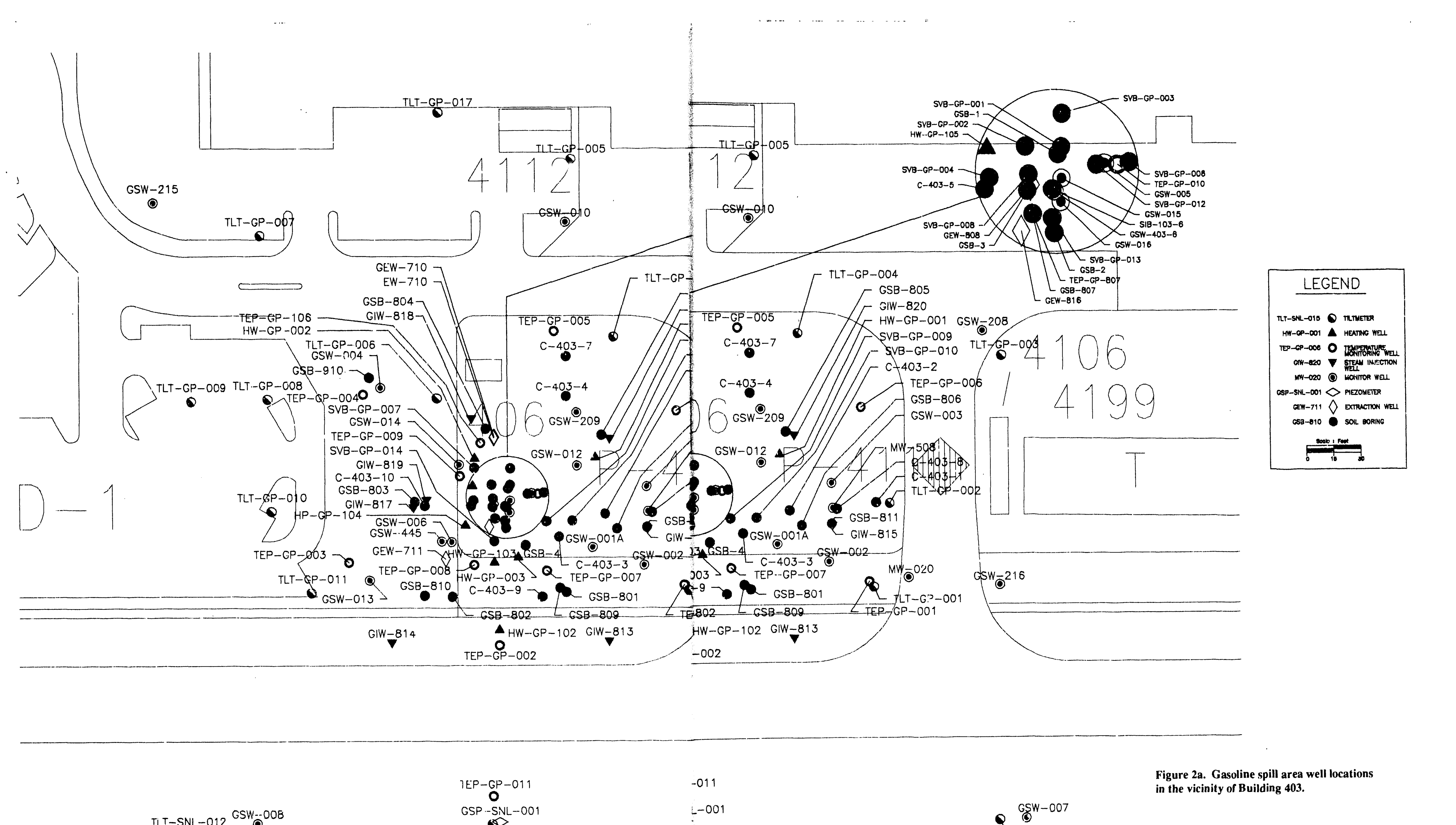

Addendum 2

Logs of Monitor Wells Drilled May 1988 through December 1992 
cember 1992

$\begin{array}{ll}\text { MW-616 } & \text { P-555 } \\ \text { MW-617 } & \text { P-557 } \\ \text { MW-618 } & \text { P-558 } \\ \text { MW-619 } & \text { P-559 } \\ \text { MW-652 } & \text { P-591 } \\ \text { MW-653 } & \text { P-592 } \\ \text { MW-654 } & \text { P-601 } \\ \text { MW-655 } & \text { P-603 } \\ \text { P-418 } & \text { P-604 } \\ \text { P-419 } & \text { P-605 } \\ \text { P-420 } & \text { P-606 } \\ \text { P-459 } & \text { P-620 } \\ \text { P-482 } & \text { P-621 } \\ \text { p-483 } & \text { P-622 } \\ \text { P-484 } & \text { P-651 } \\ \text { P-504 } & \text { P-702 } \\ \text { P-505 } & \text { P-706 } \\ \text { P-506 } & \\ \text { P-522 } & \\ \text { P-554 } & \end{array}$



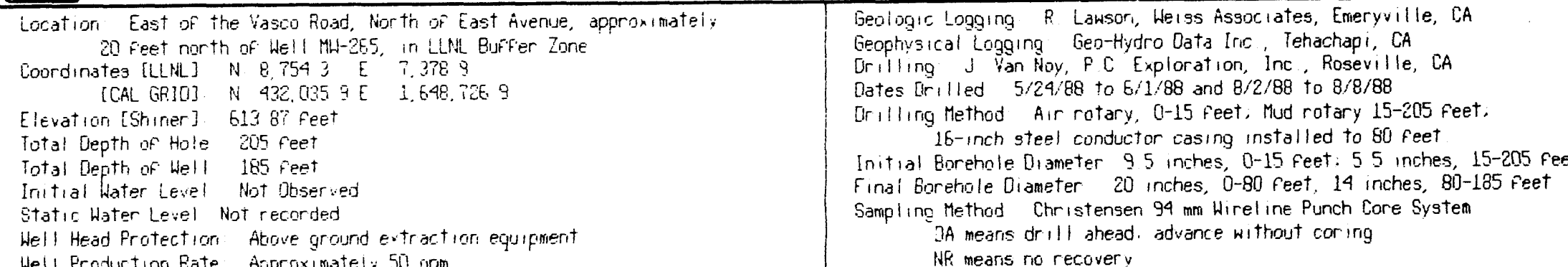


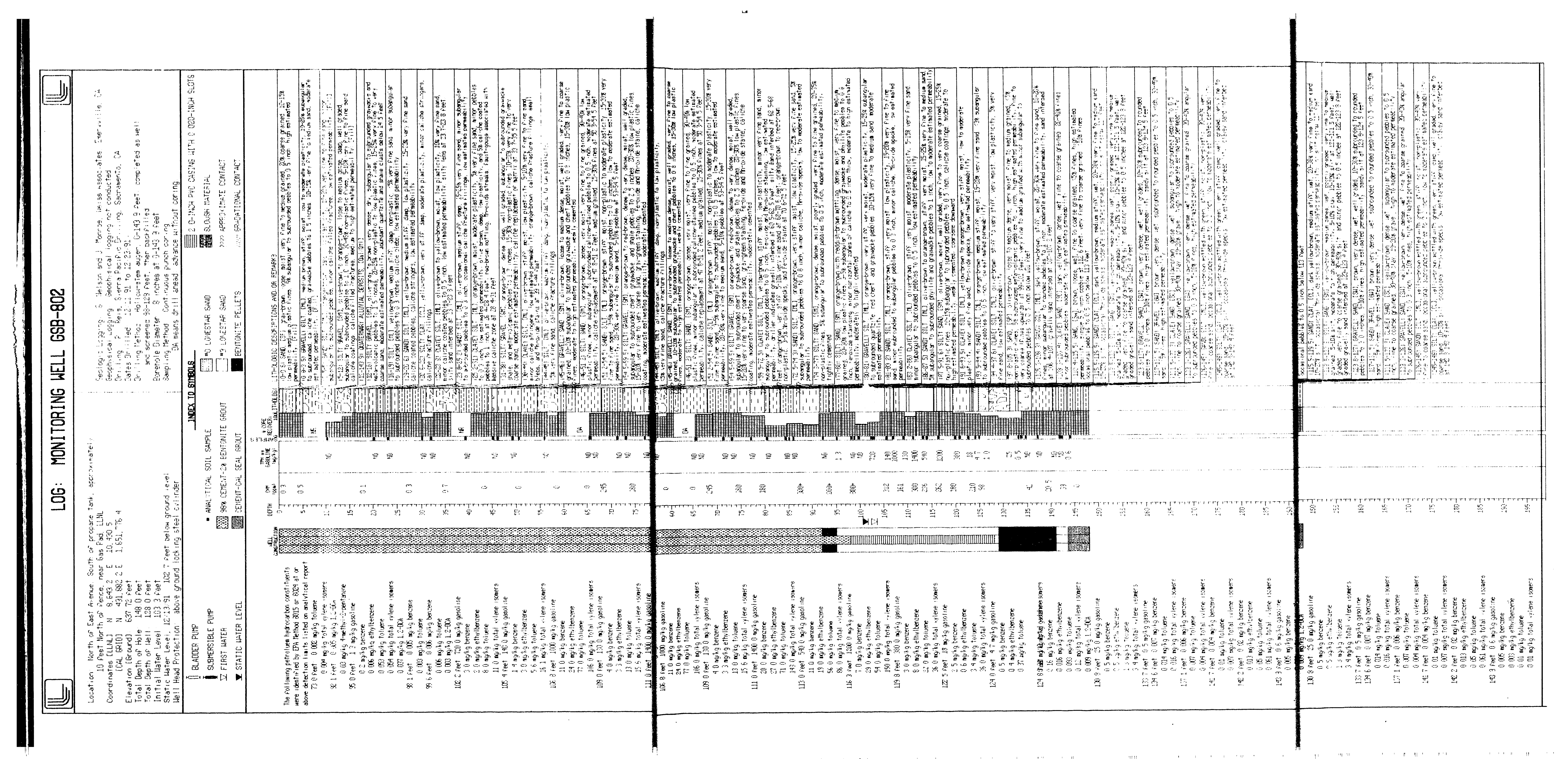



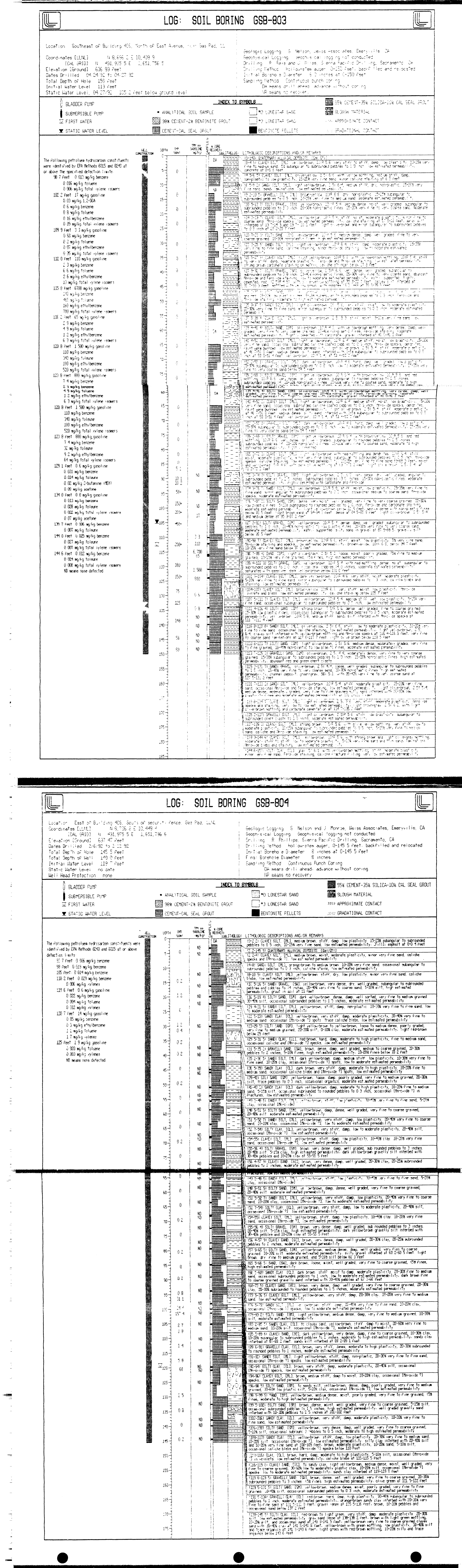


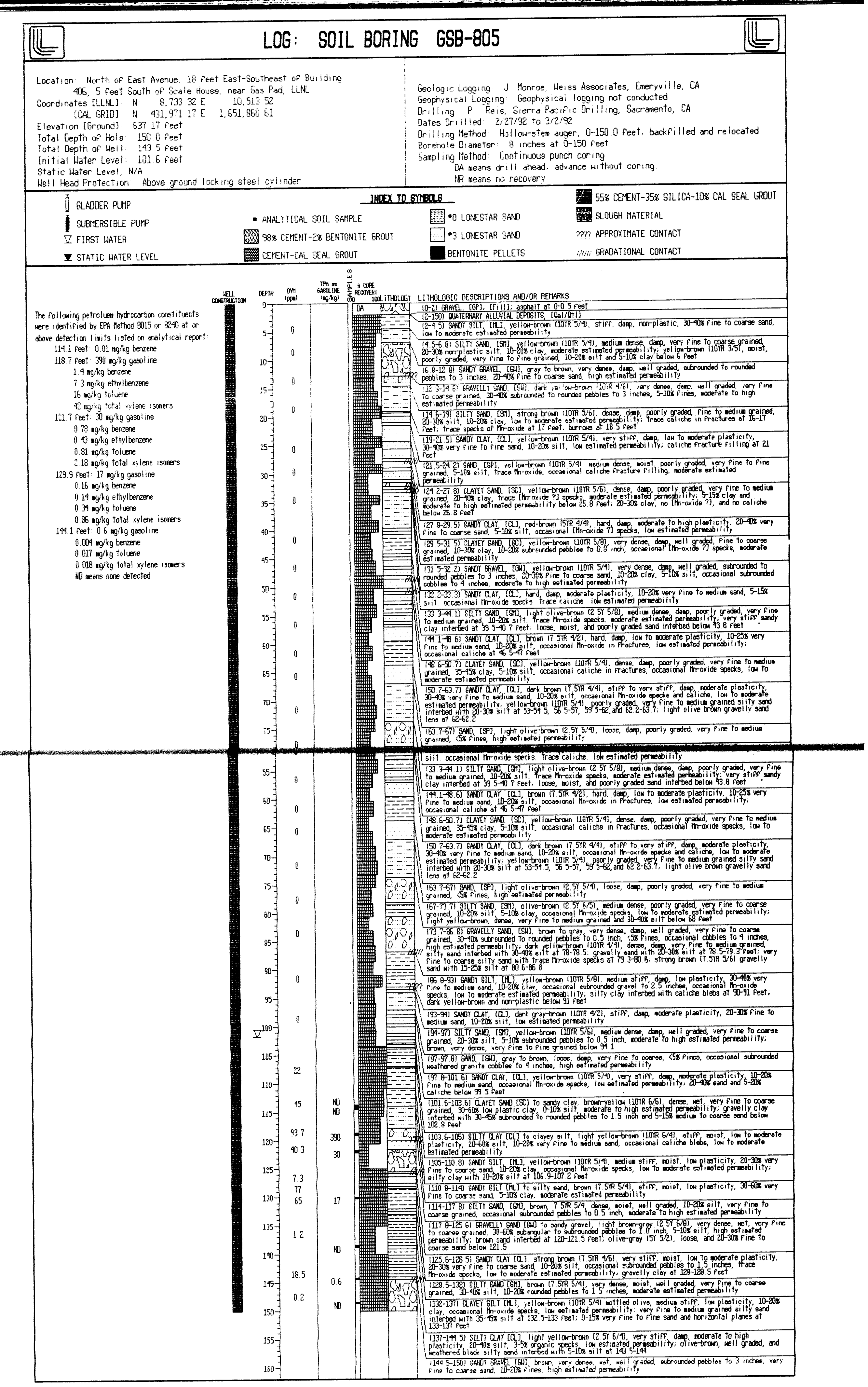

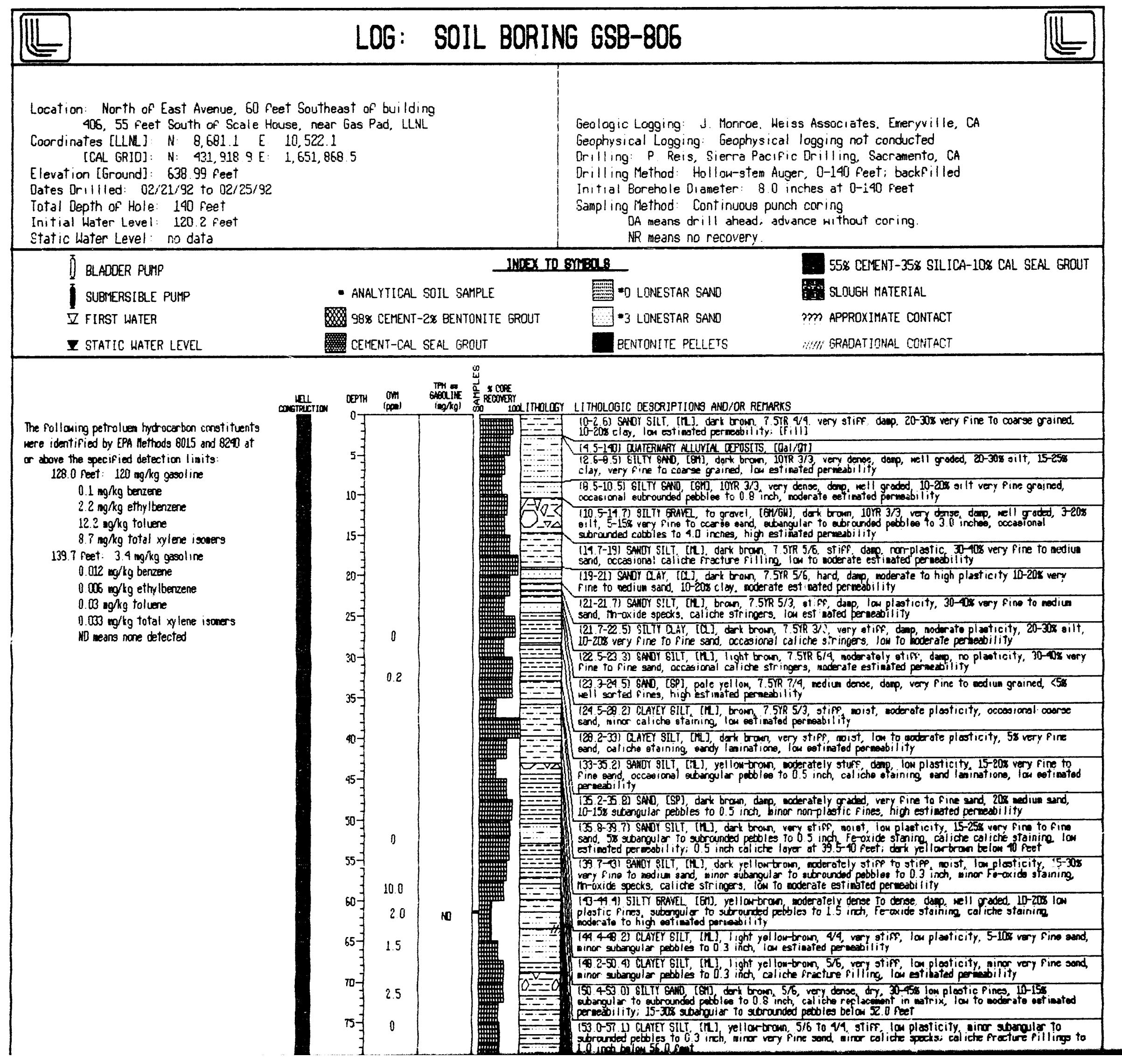





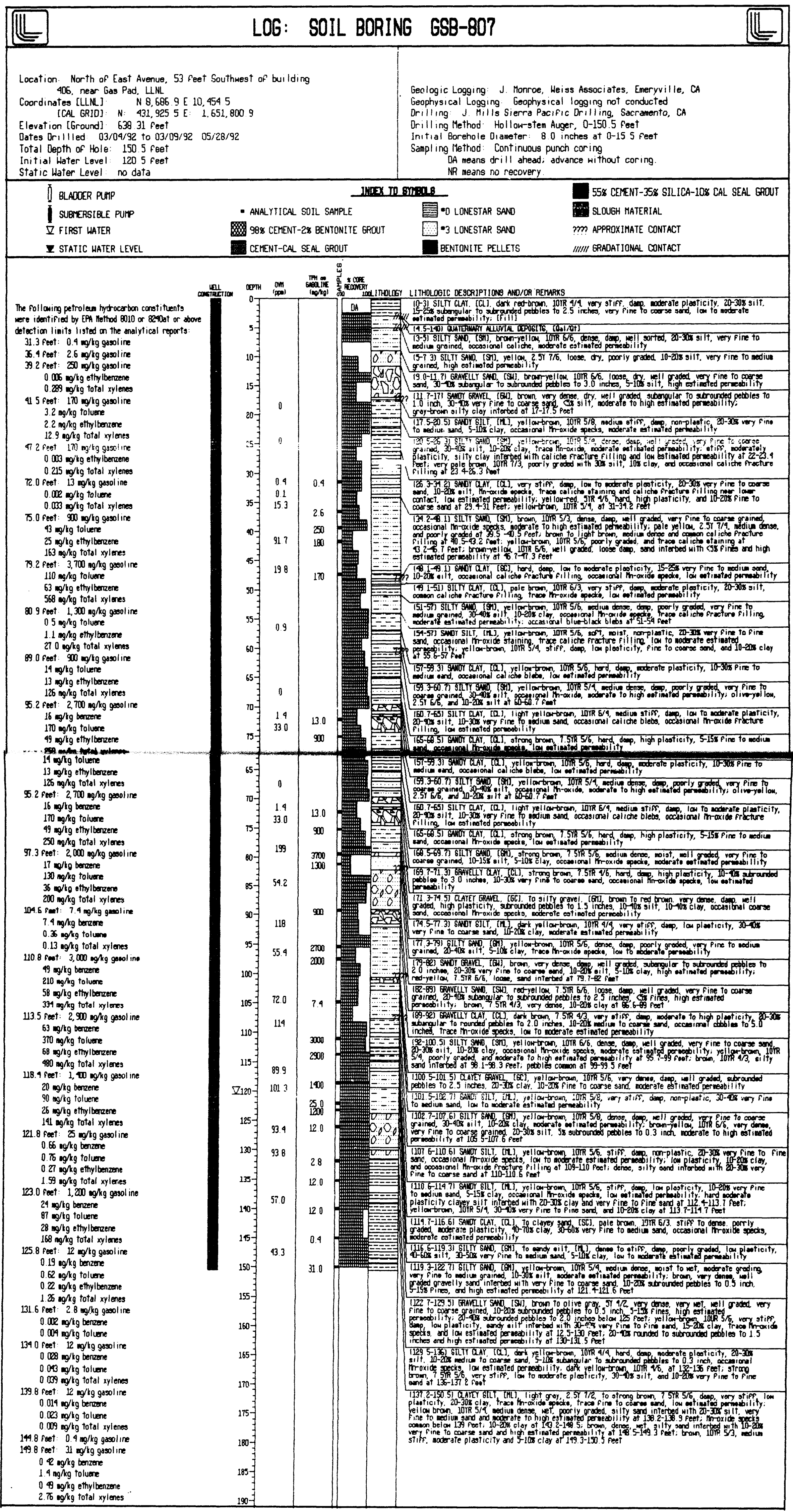



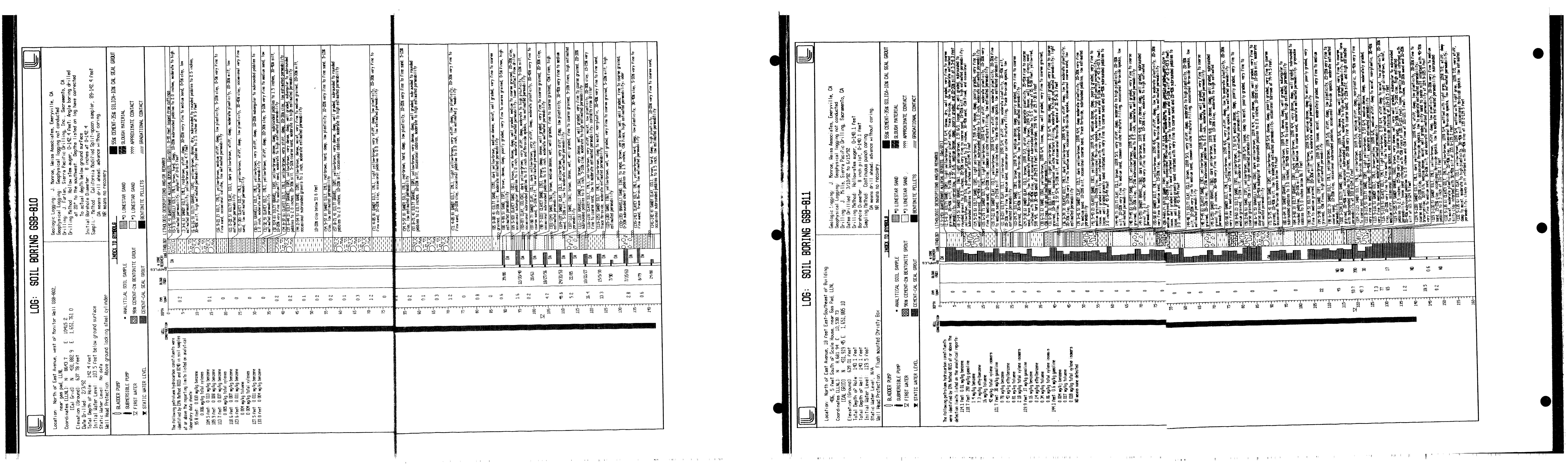


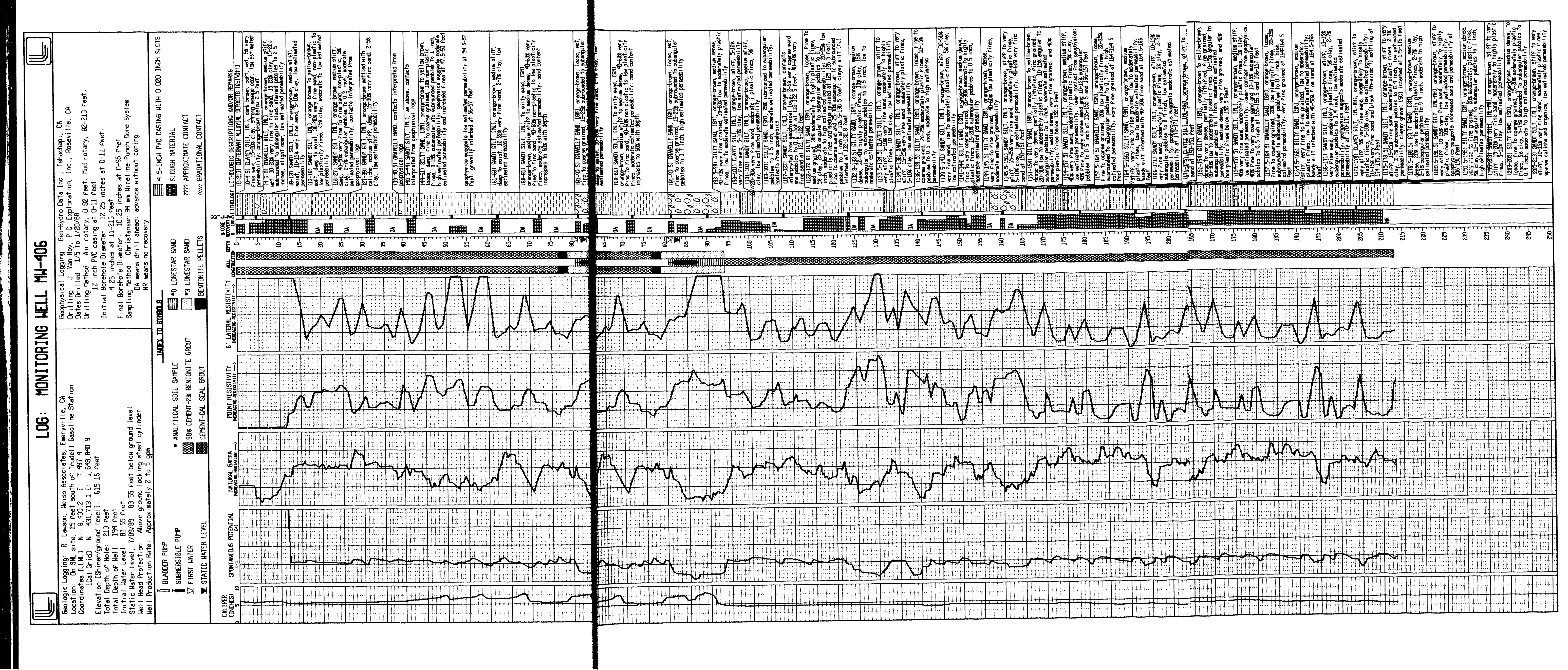




\section{LOG: MONITDRING WELL MW-414}

Location: South of Building 4675, 25 feet southwest of MLL-10A, and 11 feet south of $M-360$, LLNL

Coordinates [LLM]: N: $11,254.3$ E: 10, 179.9

[Col Grid]: $N: 434,496.3 \mathrm{E}: 1,651,562.2$

Elevation [Shiner/ground level]: $612.38 \mathrm{feet}$

Total Depth of Hole: 179 feet

Total Depth of thell: 74 feet

Initial hater Level: 66.3 feet

Static Hater Level, 7/09/89: 68.3 feet below ground leve

Hell Head Protection: Above ground locking steel cylinder
Geologic Logging: R. Lawson, Weiss Associates, Emeryville, CA

Geophysical Logging: Georand, Sacramento, CA

Orilling: Ine Roseville, CA

Dates Drillied: $5 / 11$ to $5 / 20 / 88$

Drilling Method: Air rotary 0-21.5 feet; Mud ratary, 21.5-179 feet

Initial Borehole Diameter: 5.5 inches, 0-179 feet

Final Borehole Diameter: 9 inches, 0-79 feet

Sampling Method: Christensen 99 Wirel ine Punch Core System, below 22 feet 25-inch spl it-tube sampler hydraulically advanced, $5,10,15$, and 20 feet. DA means advance without cor irig Hell Production Rate: Approximately $0.5 \mathrm{gpm}$

BLADOER PUTP
SLBERSIBLE PUMP

4.5-INCH PVC CASING HITH O.020-INCH SLOTS SLOUGH MATERIAL

m? APPROXIMATE CONTACT

IIIII GRADATIONAL CONTACT

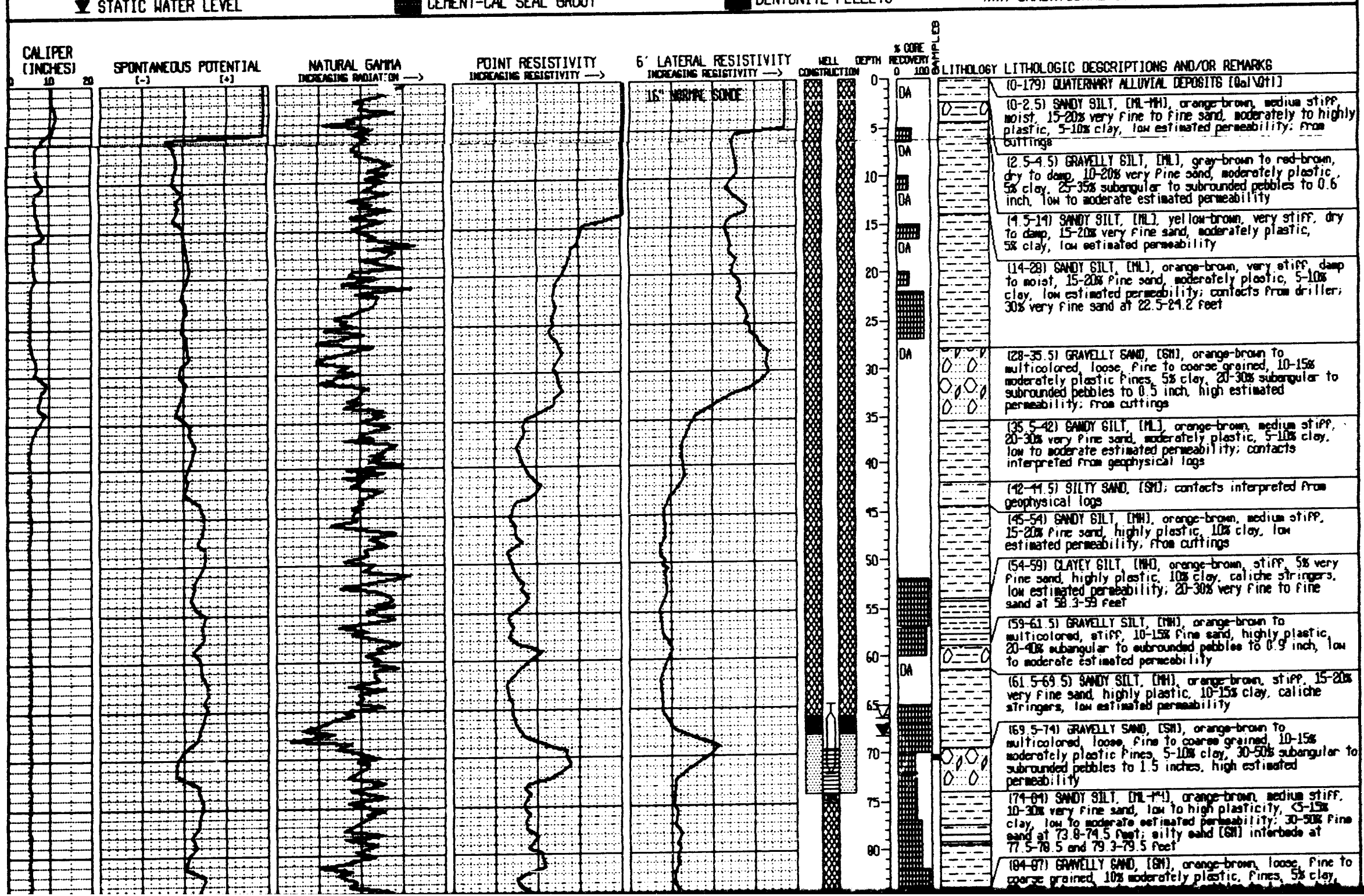




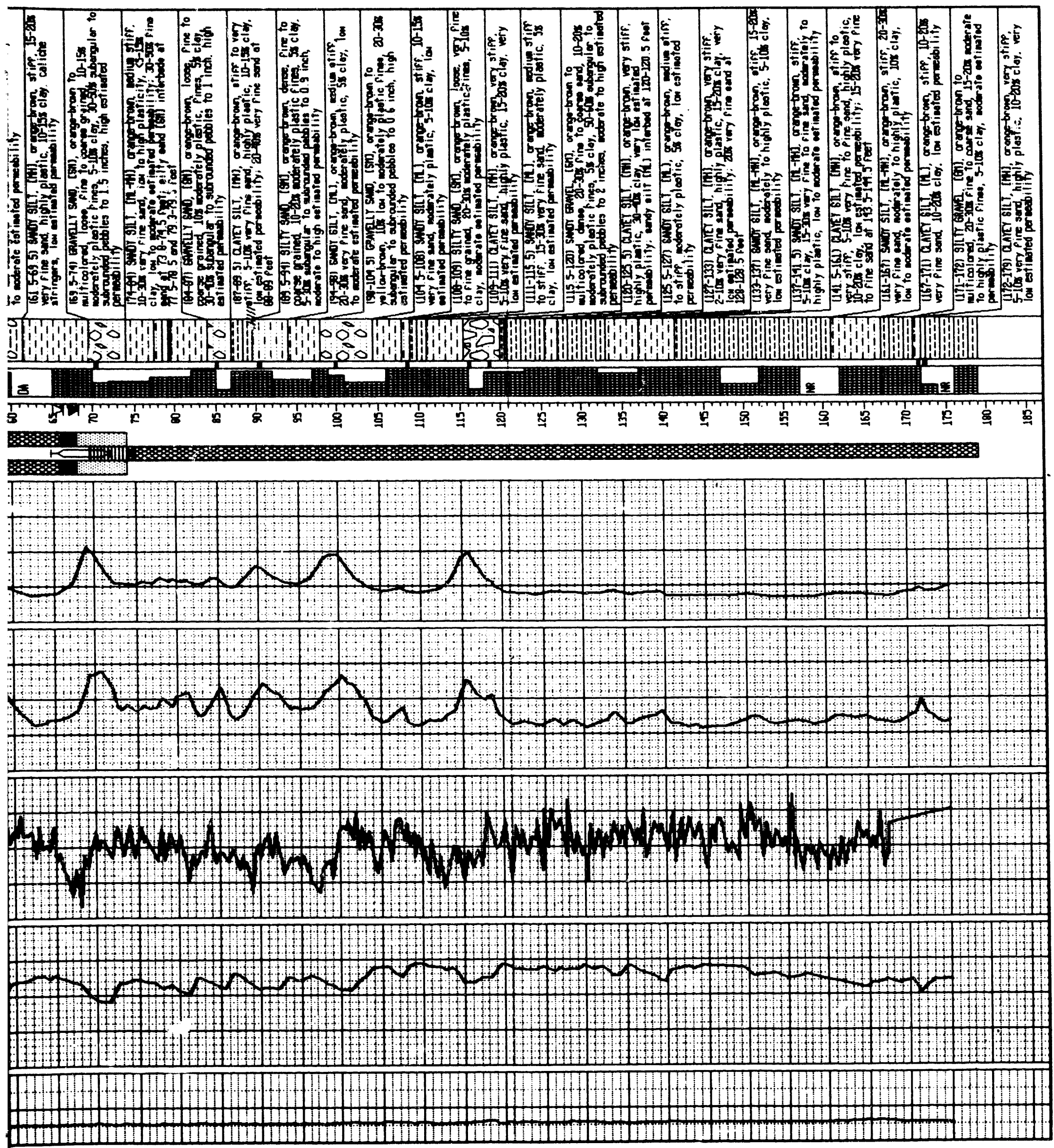




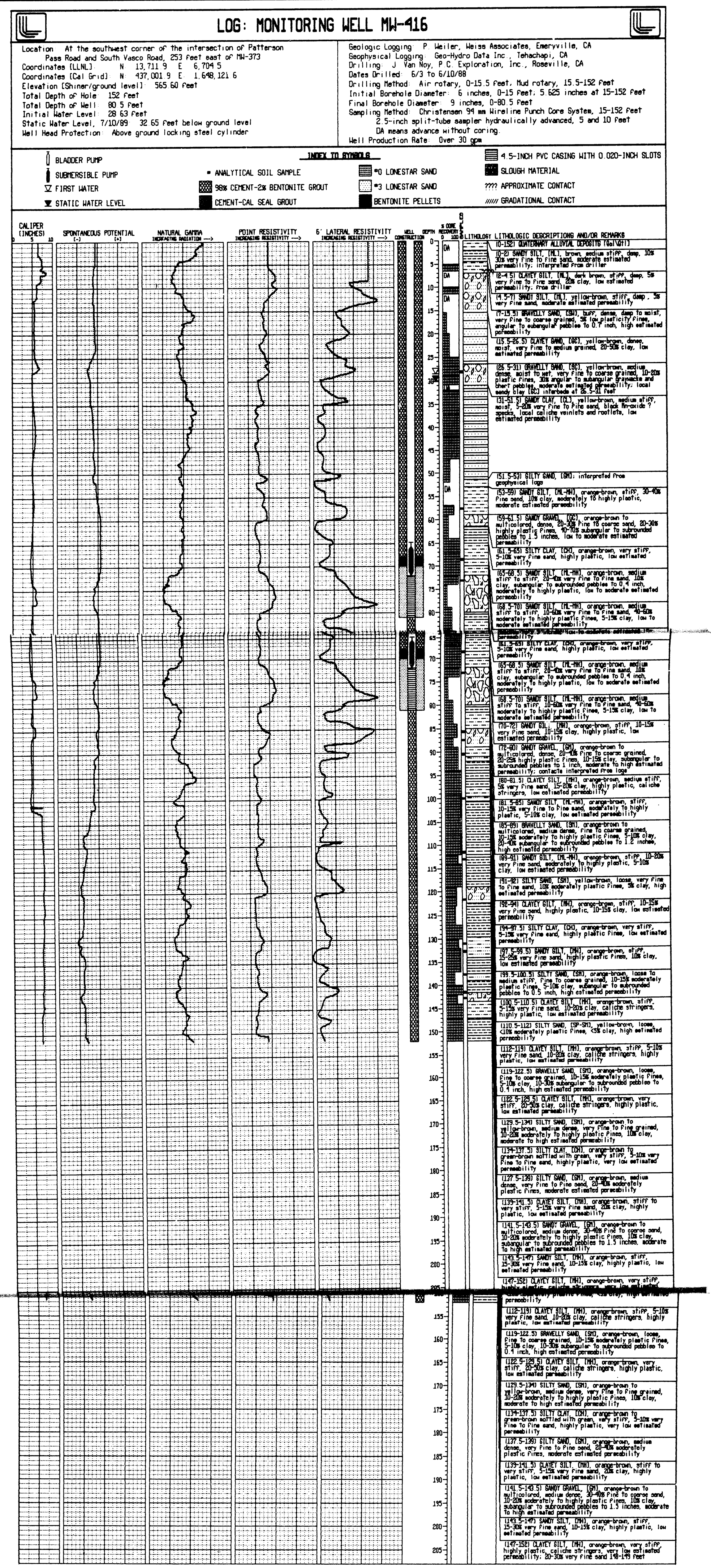




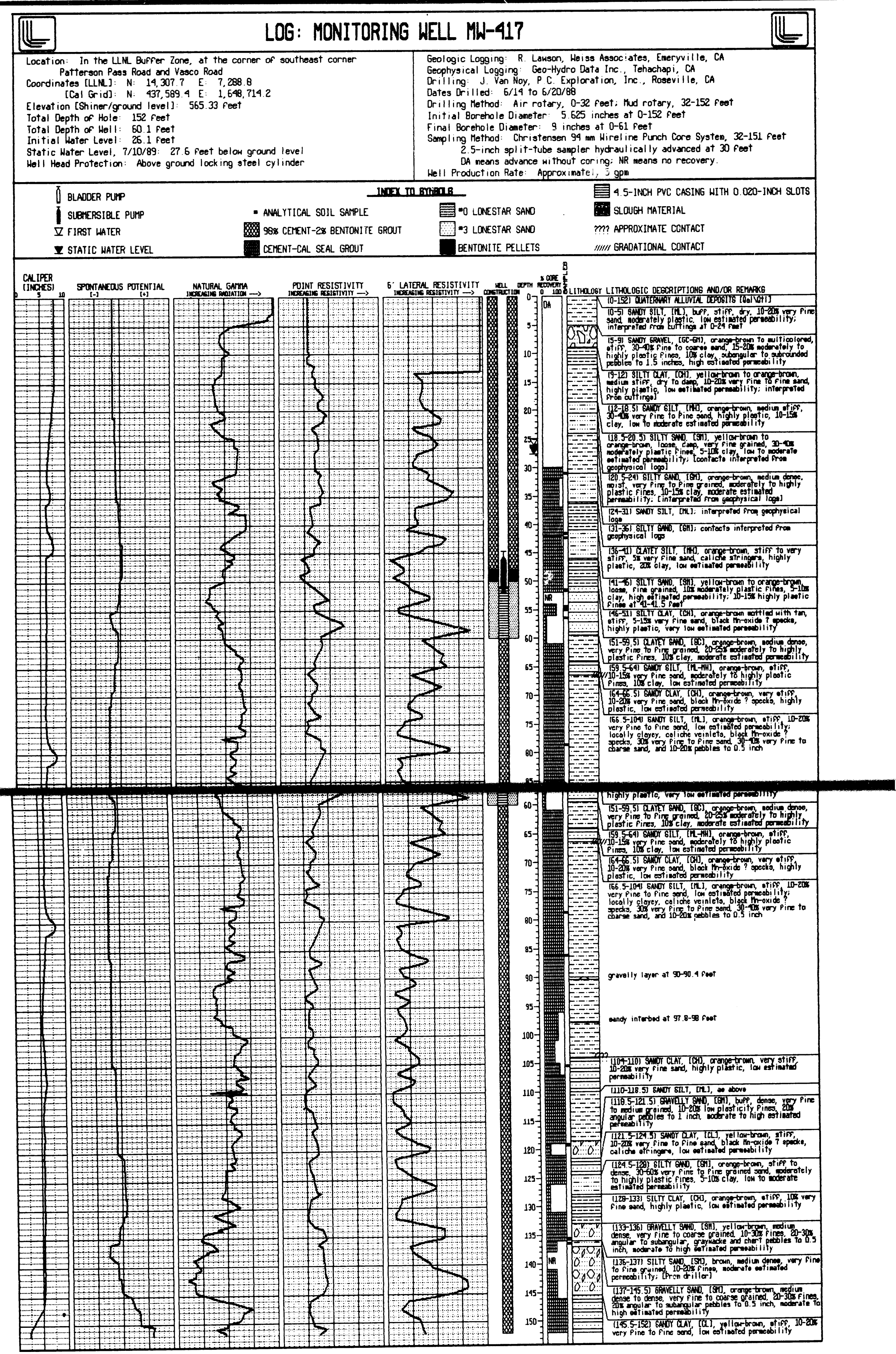




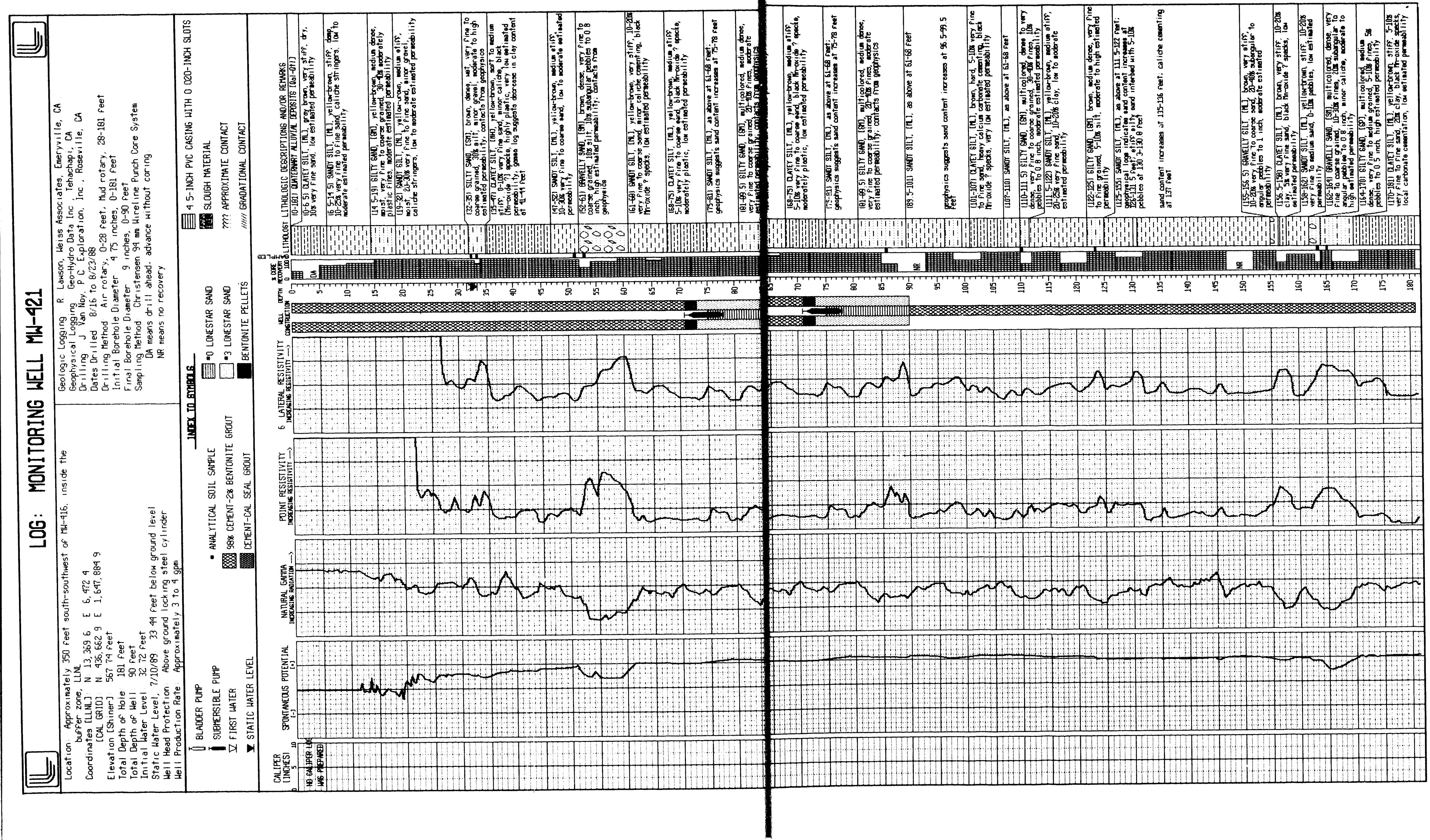


LOG: MONITORING WELL MLL-422

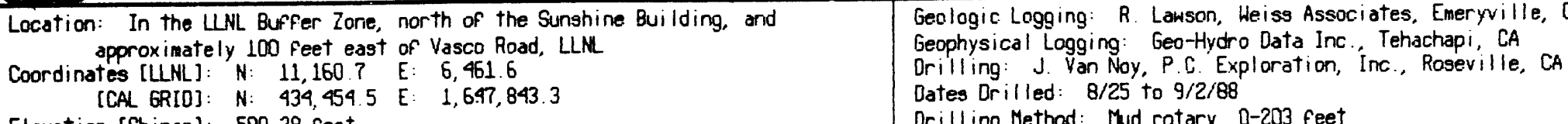

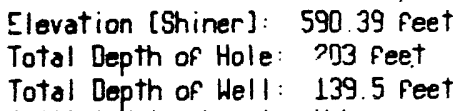

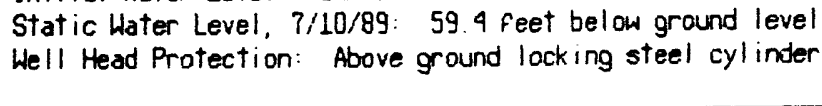

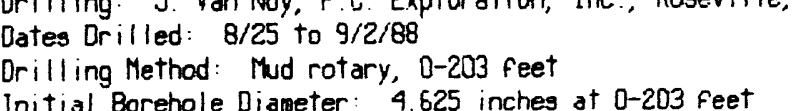

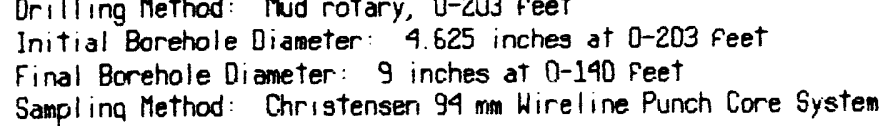

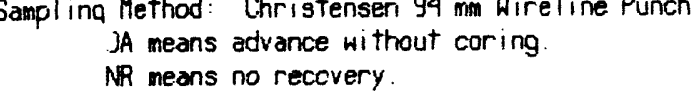

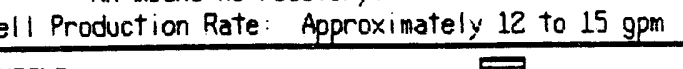

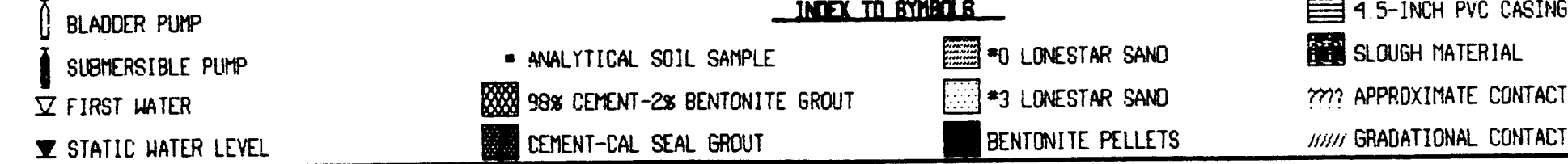

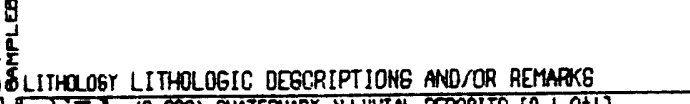

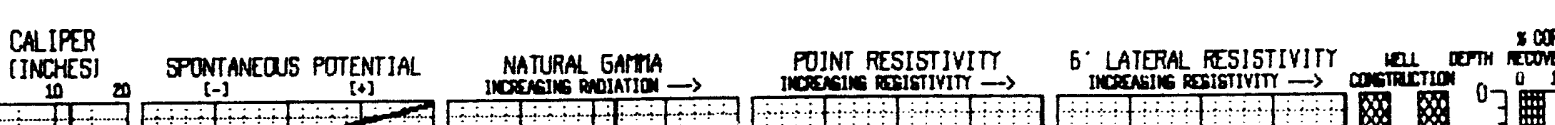

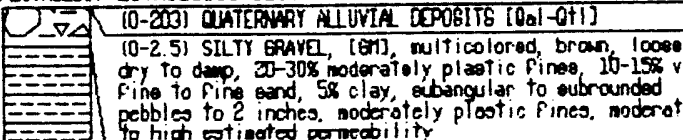

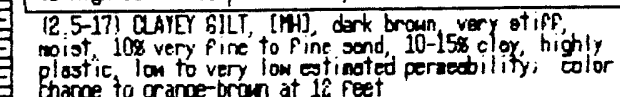
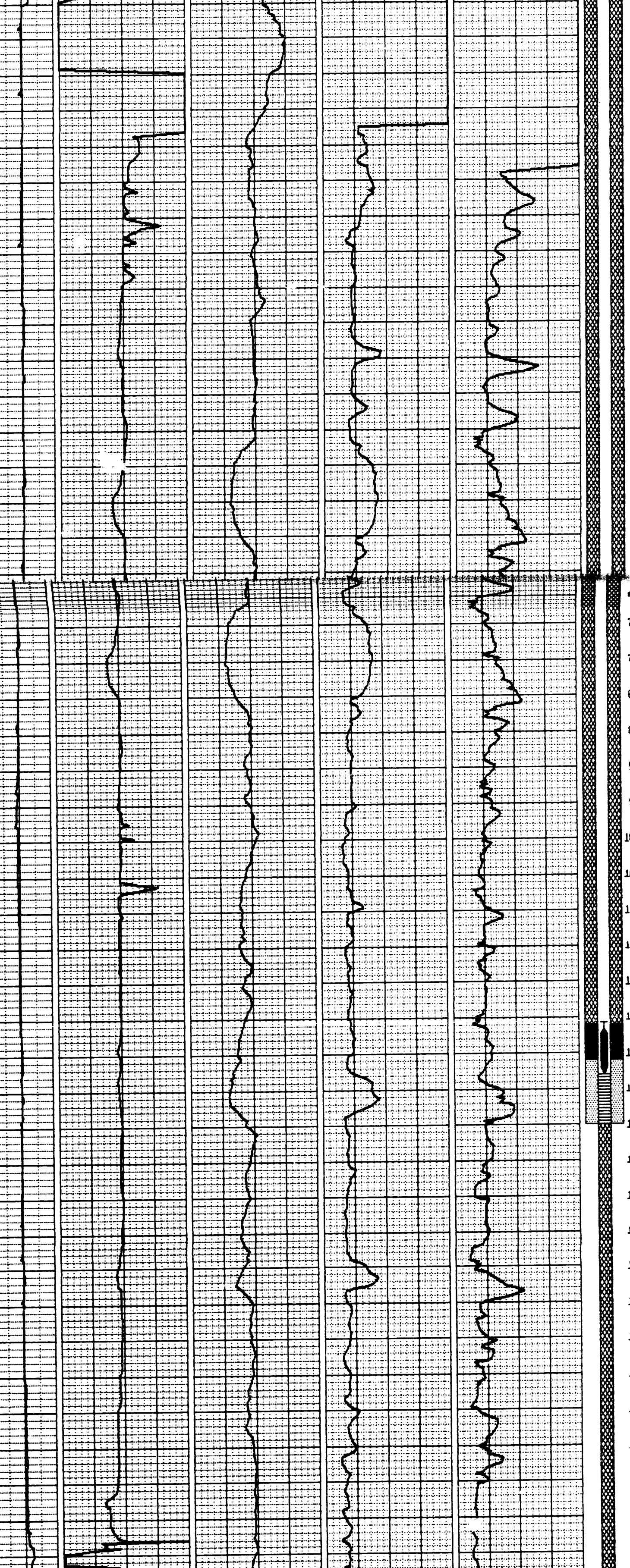

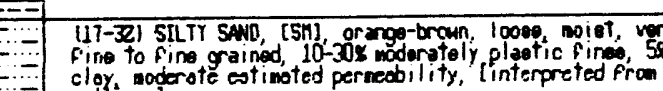

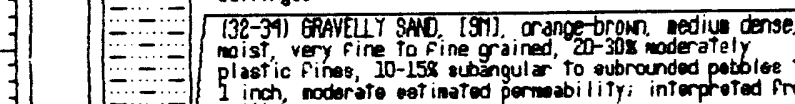

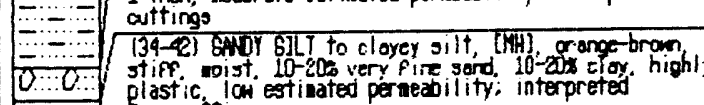

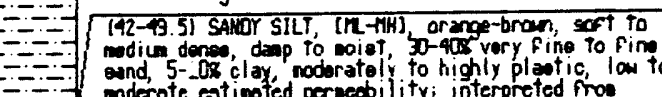

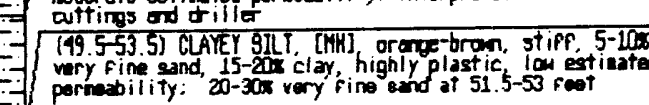

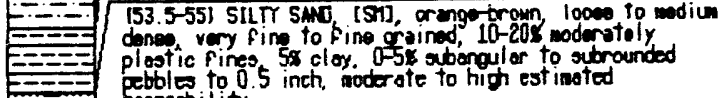

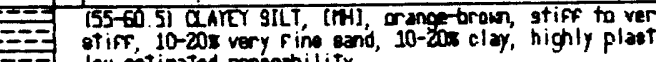

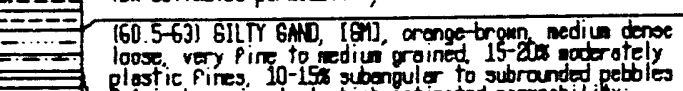

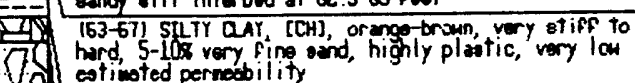

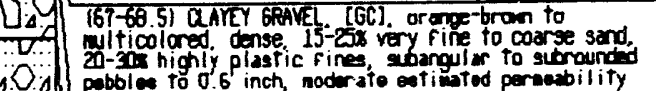

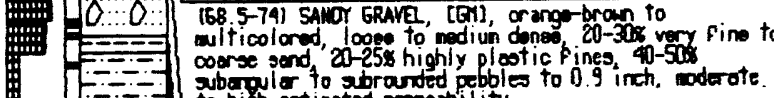
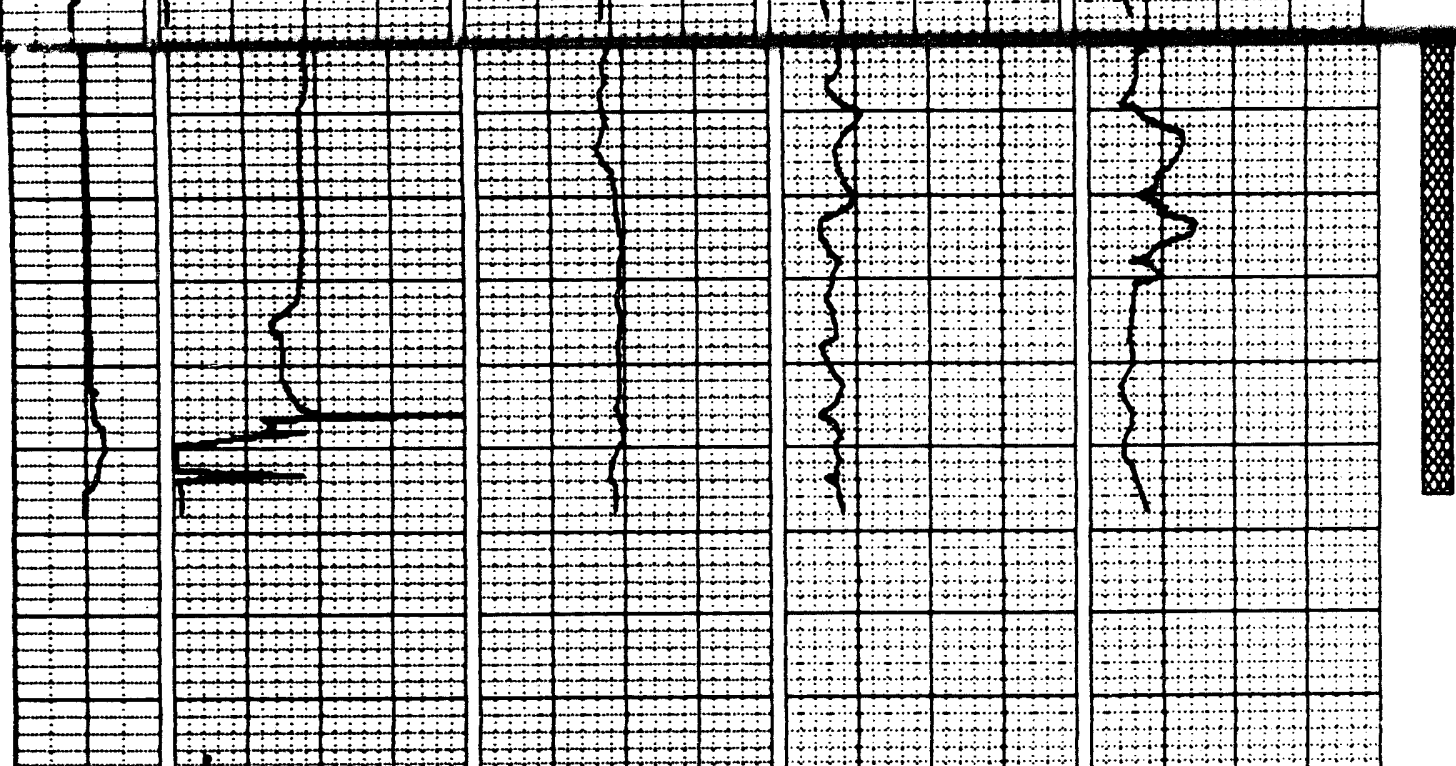

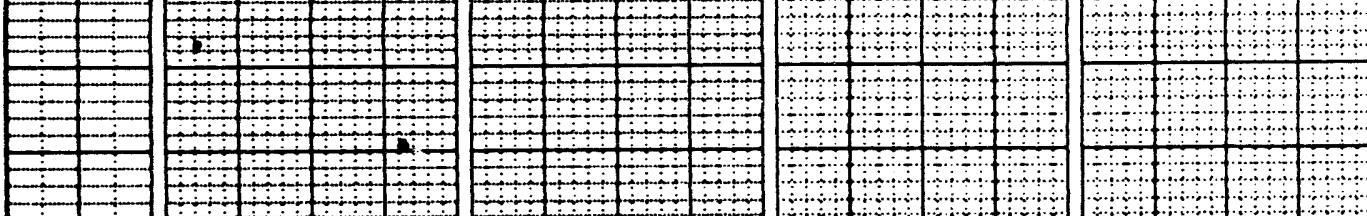

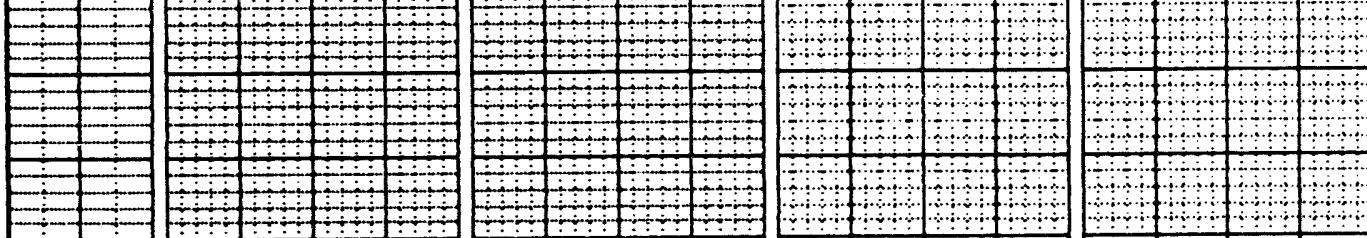

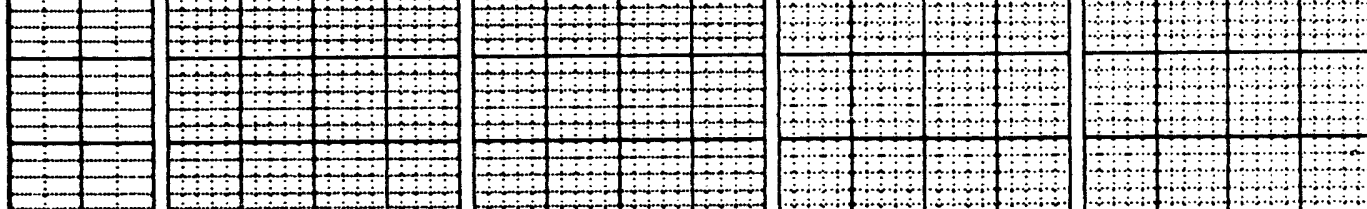

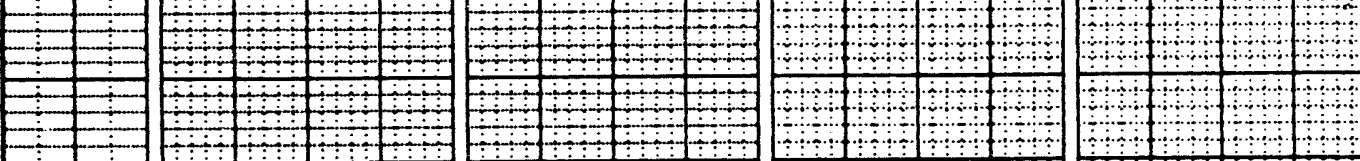

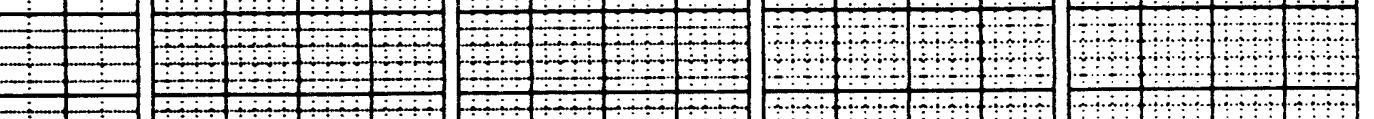

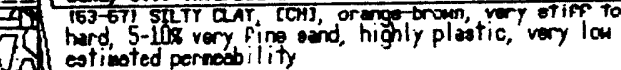

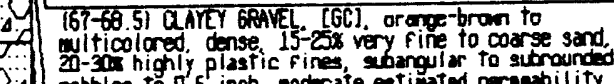

10.0.

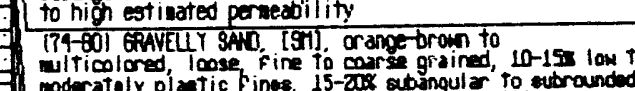

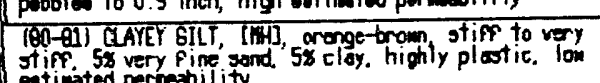

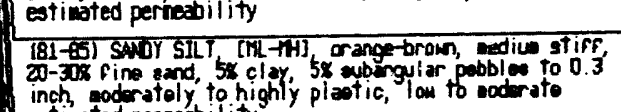

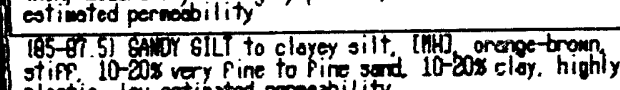

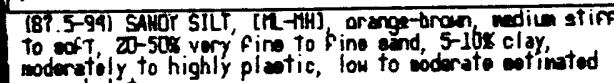

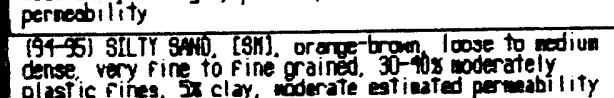

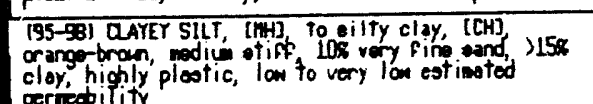

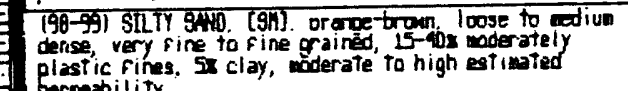

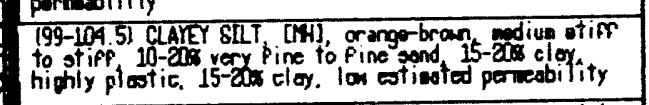

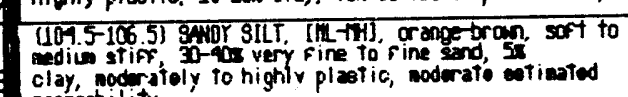

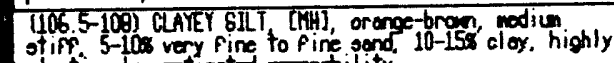

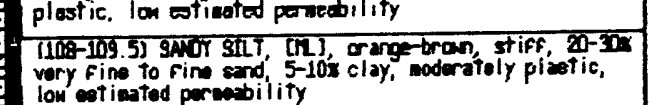

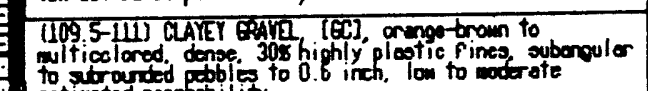

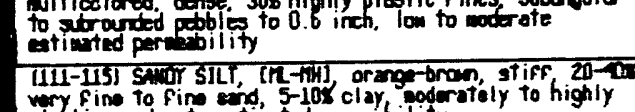

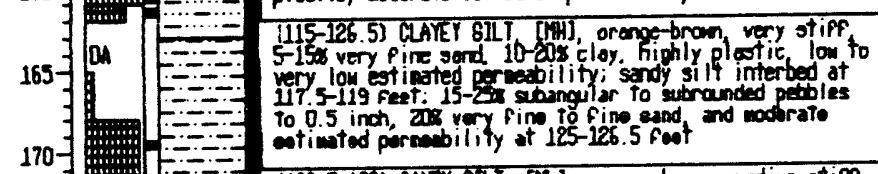

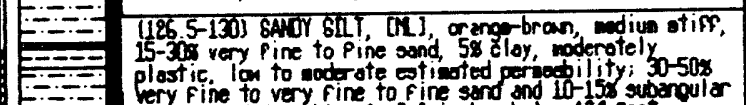

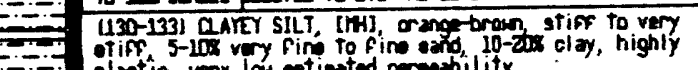

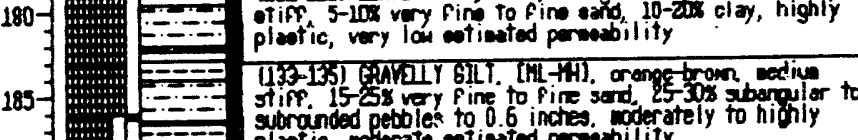

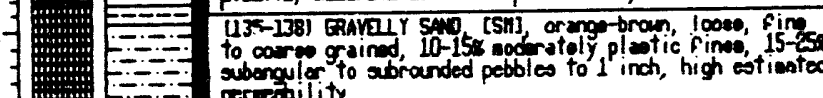

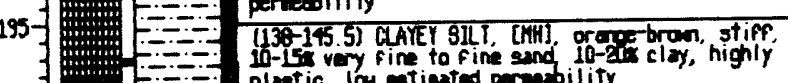

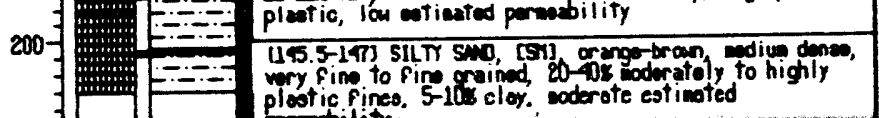

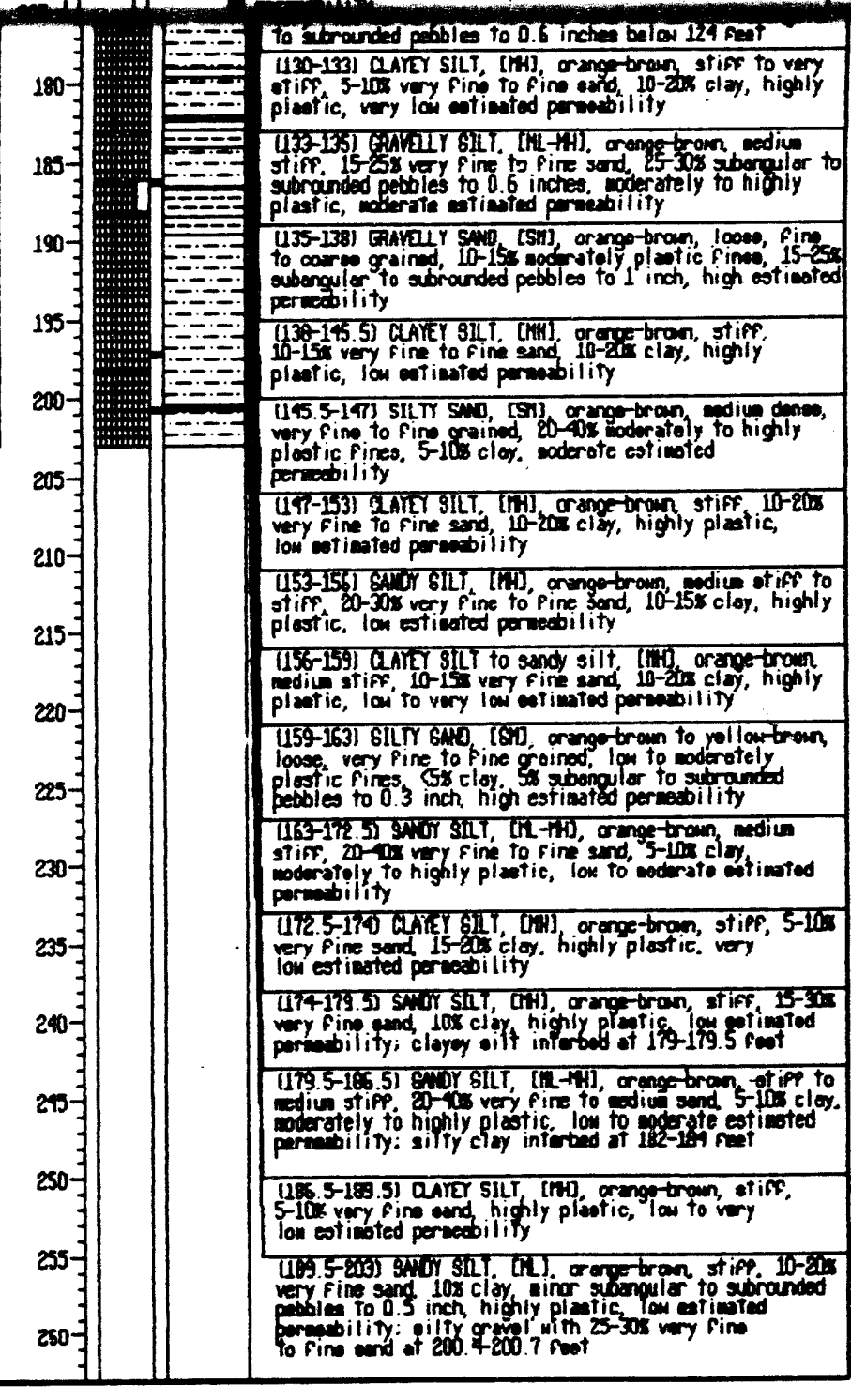




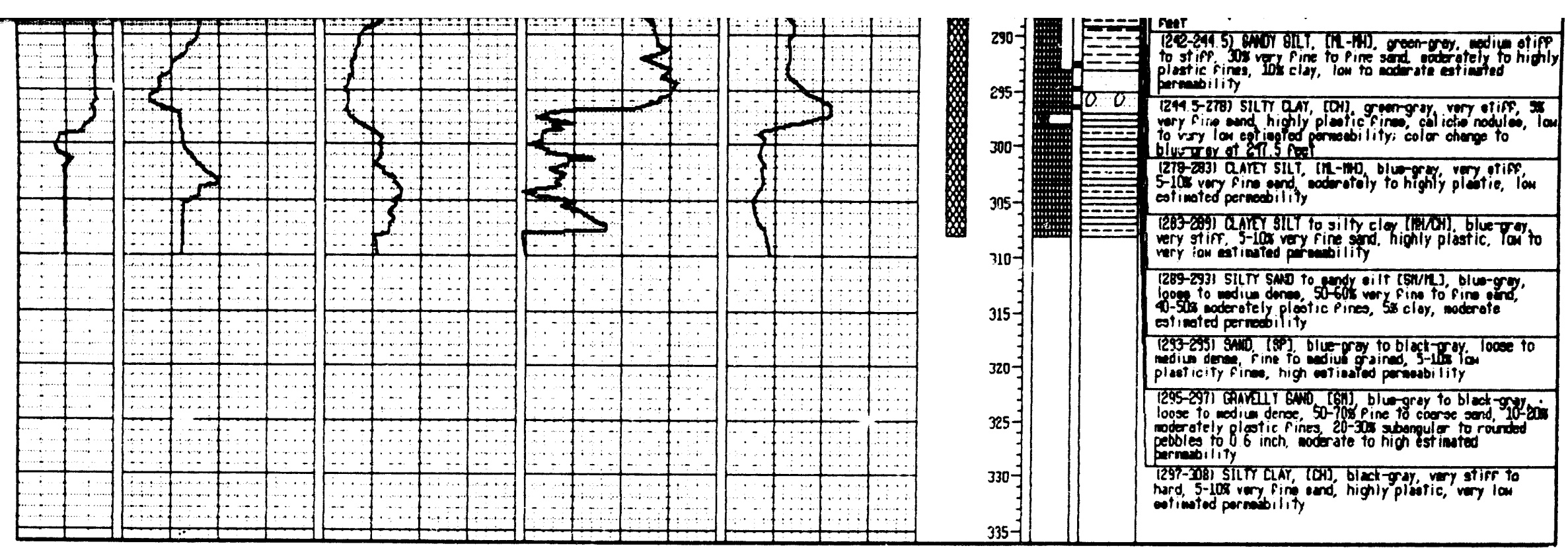




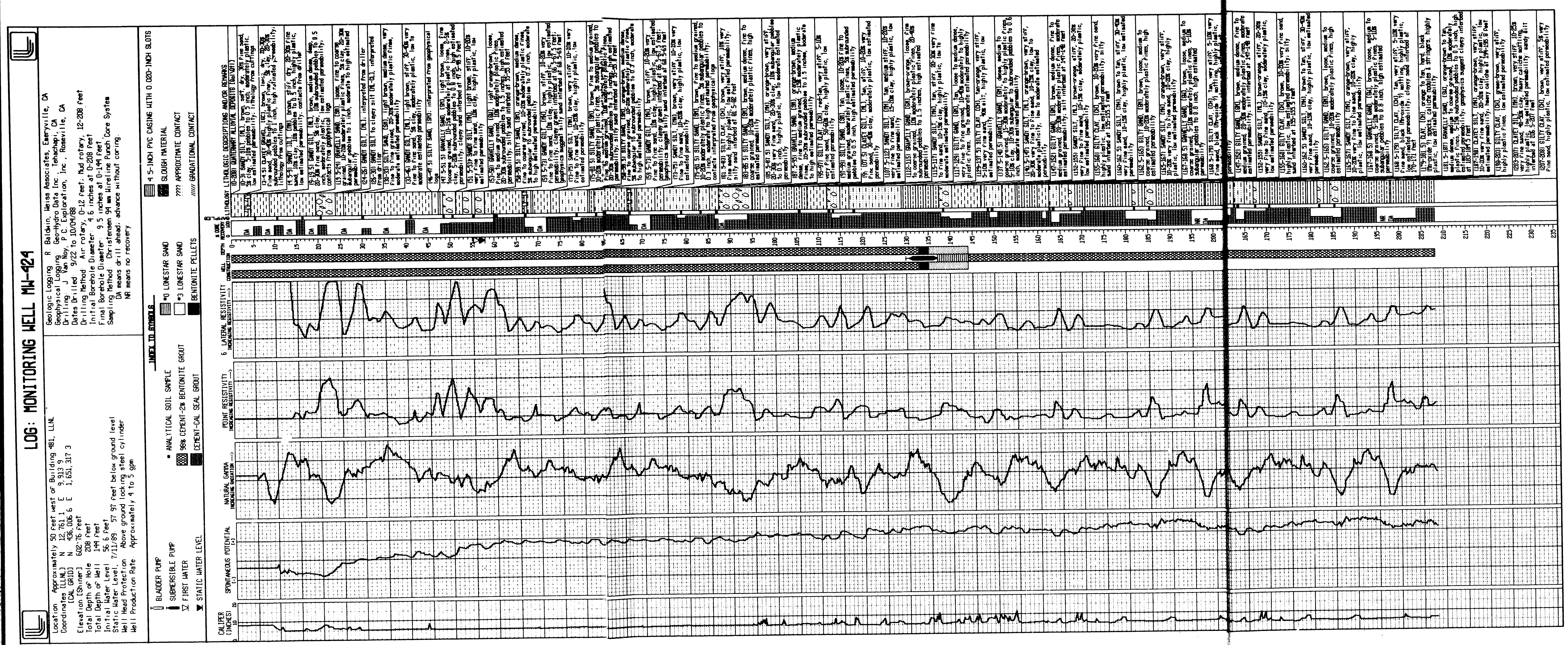




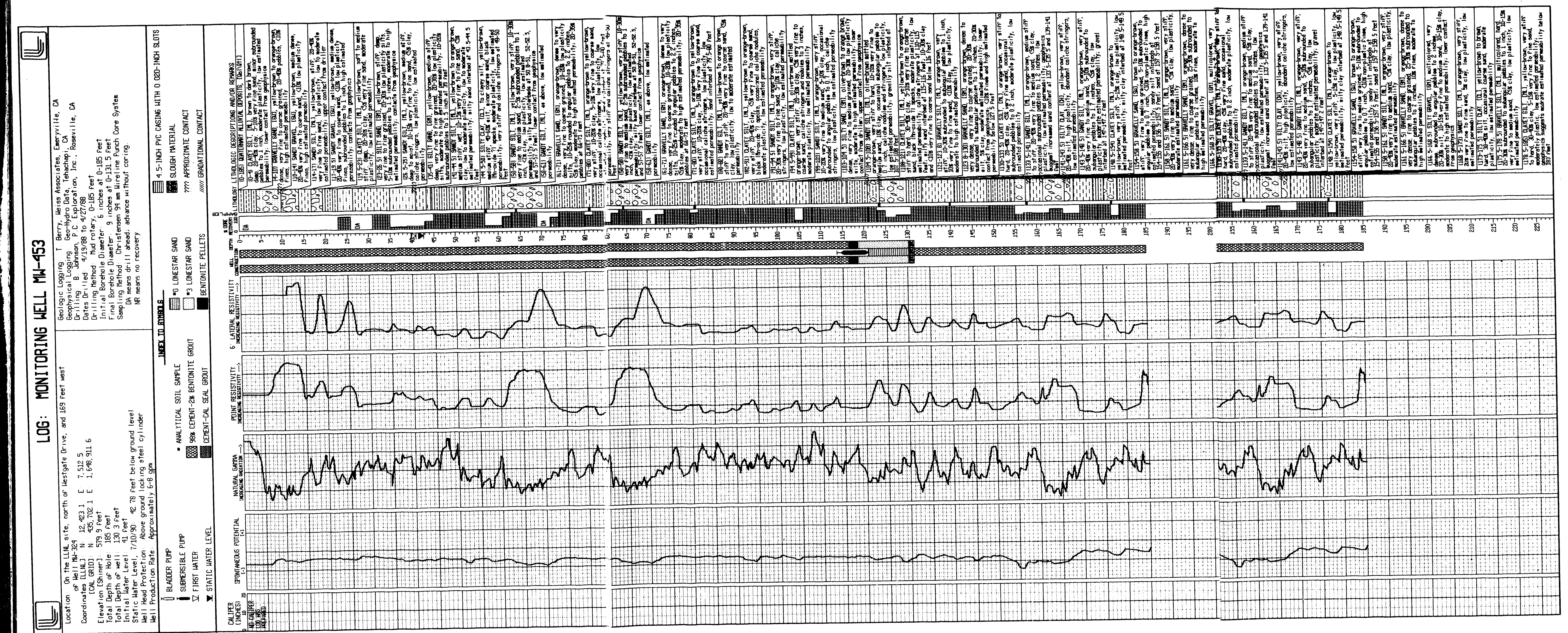




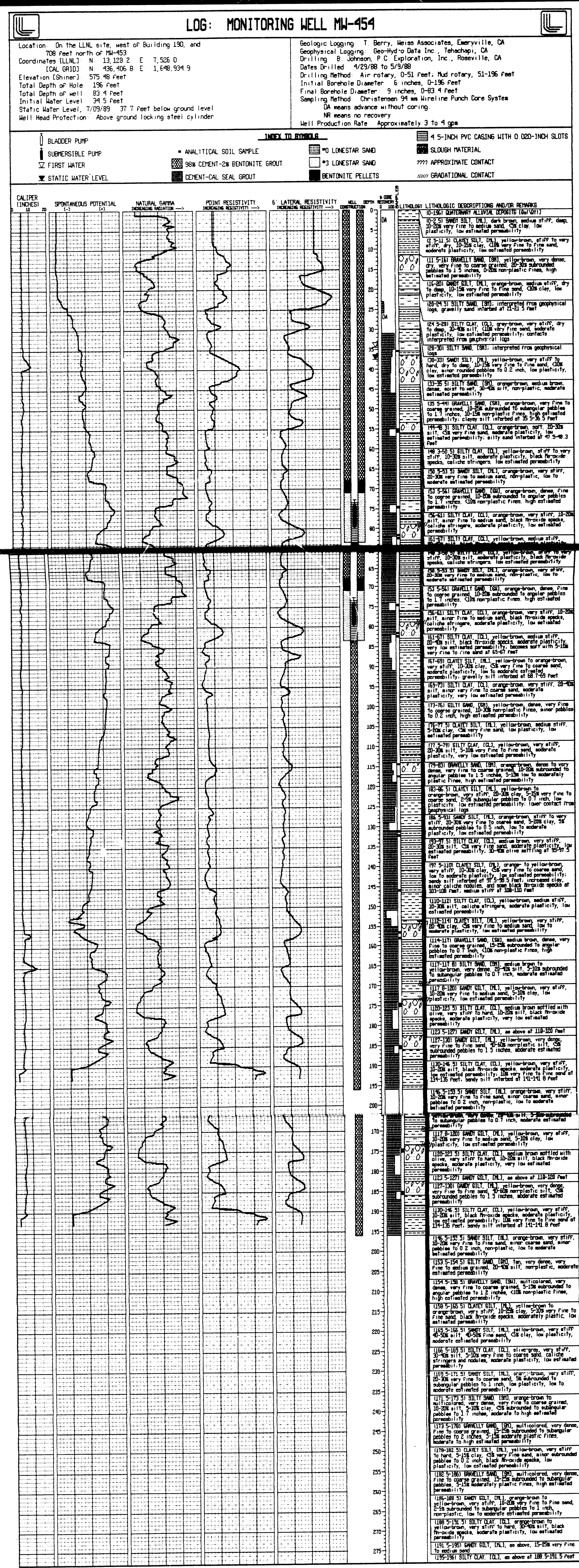




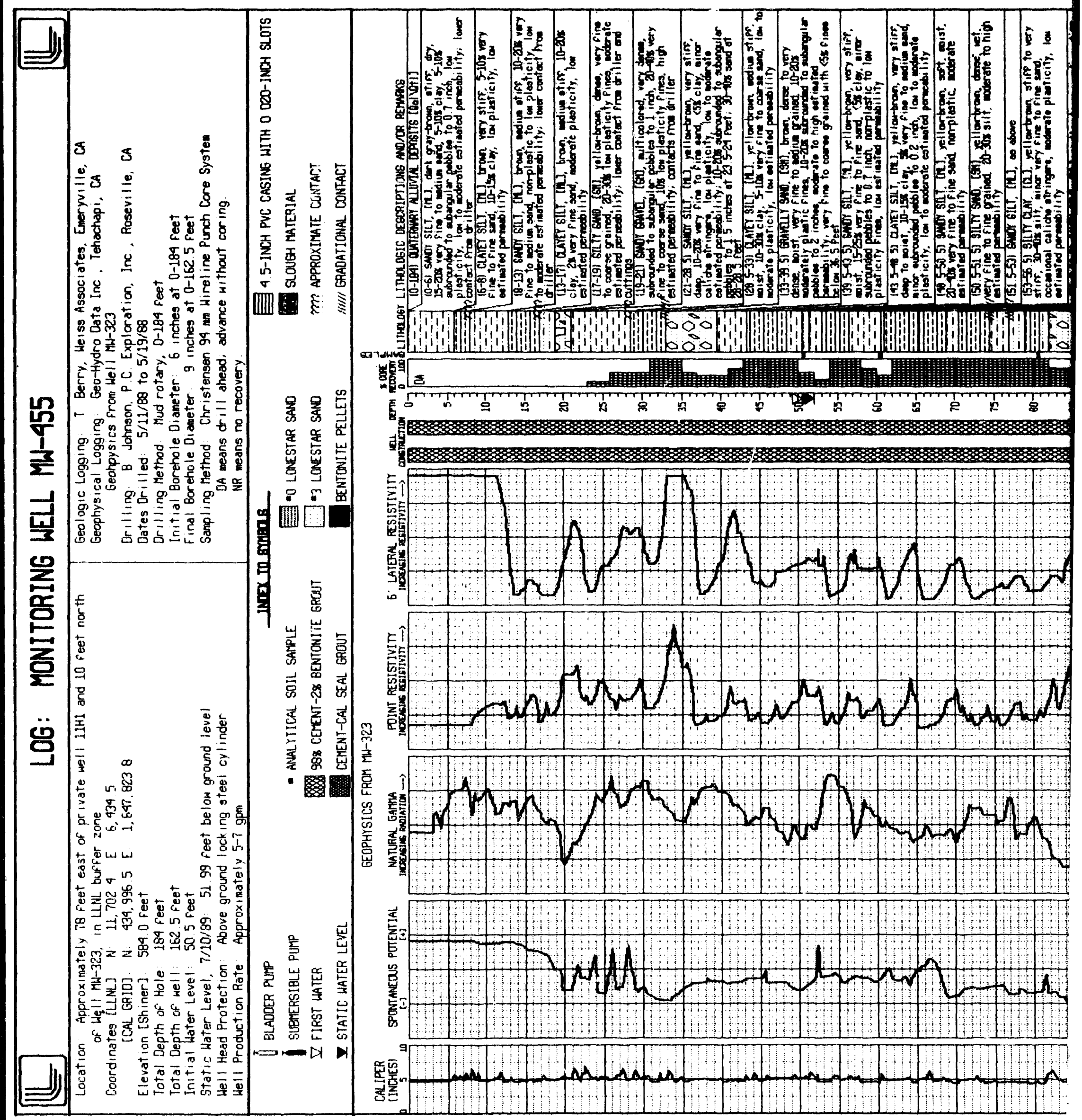




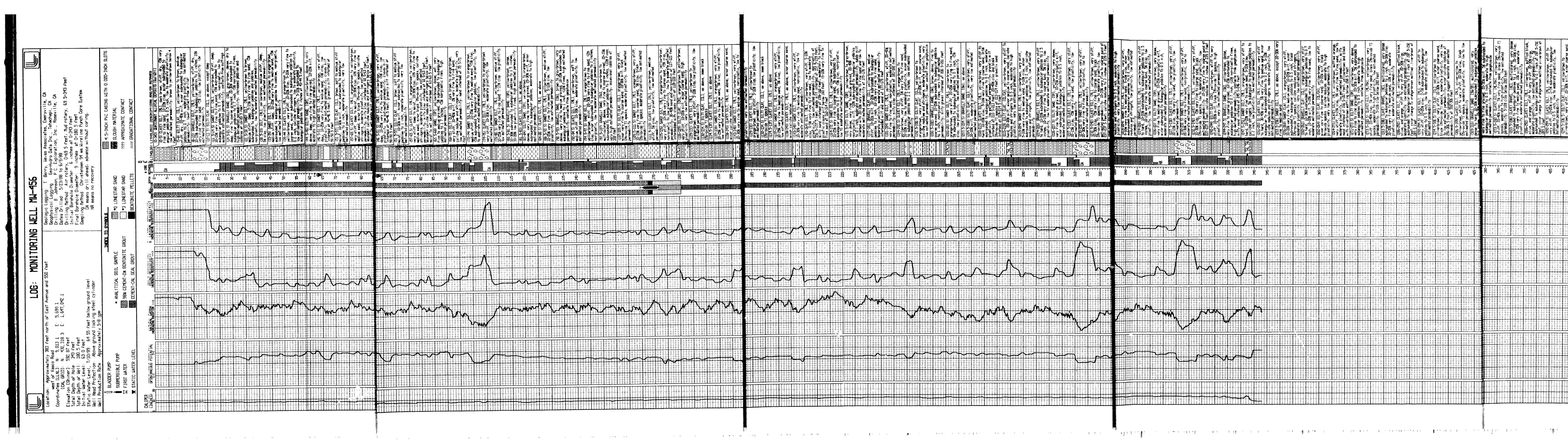




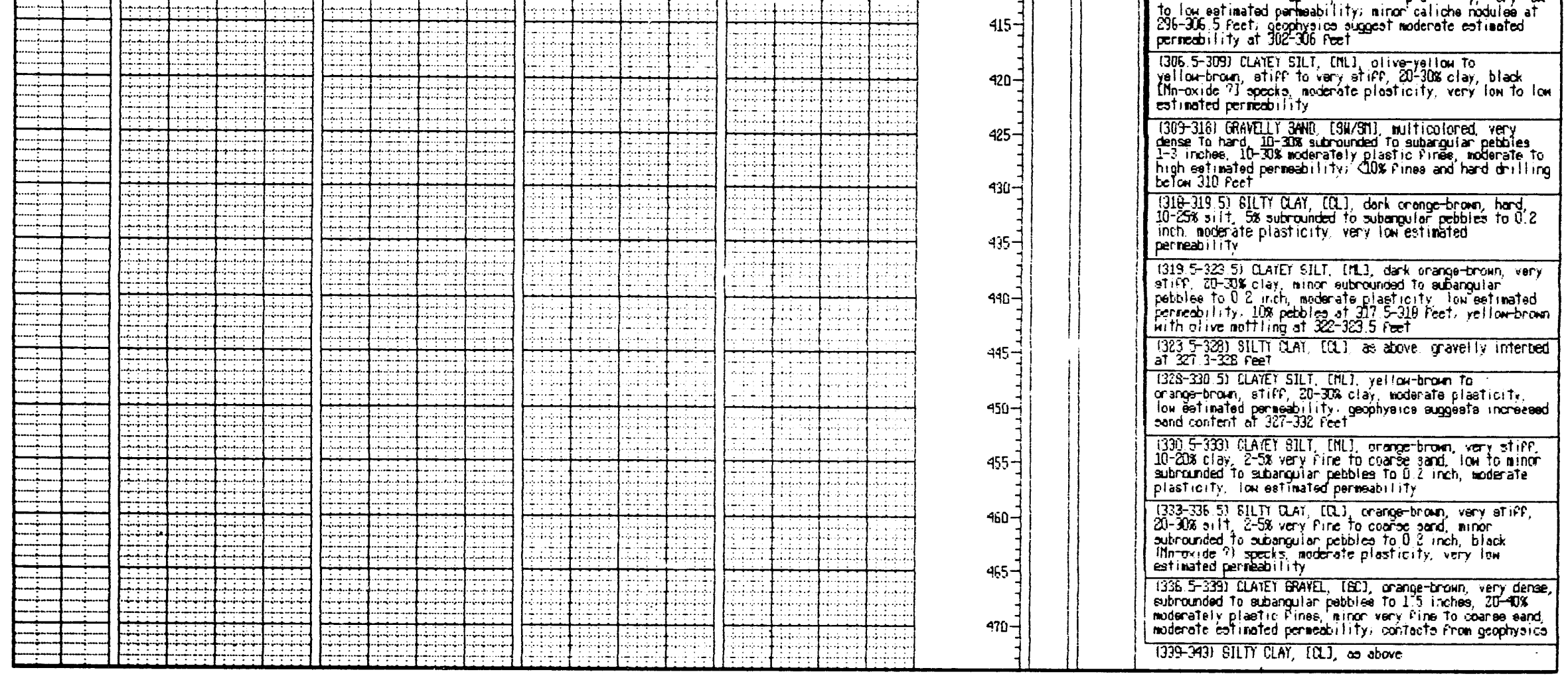




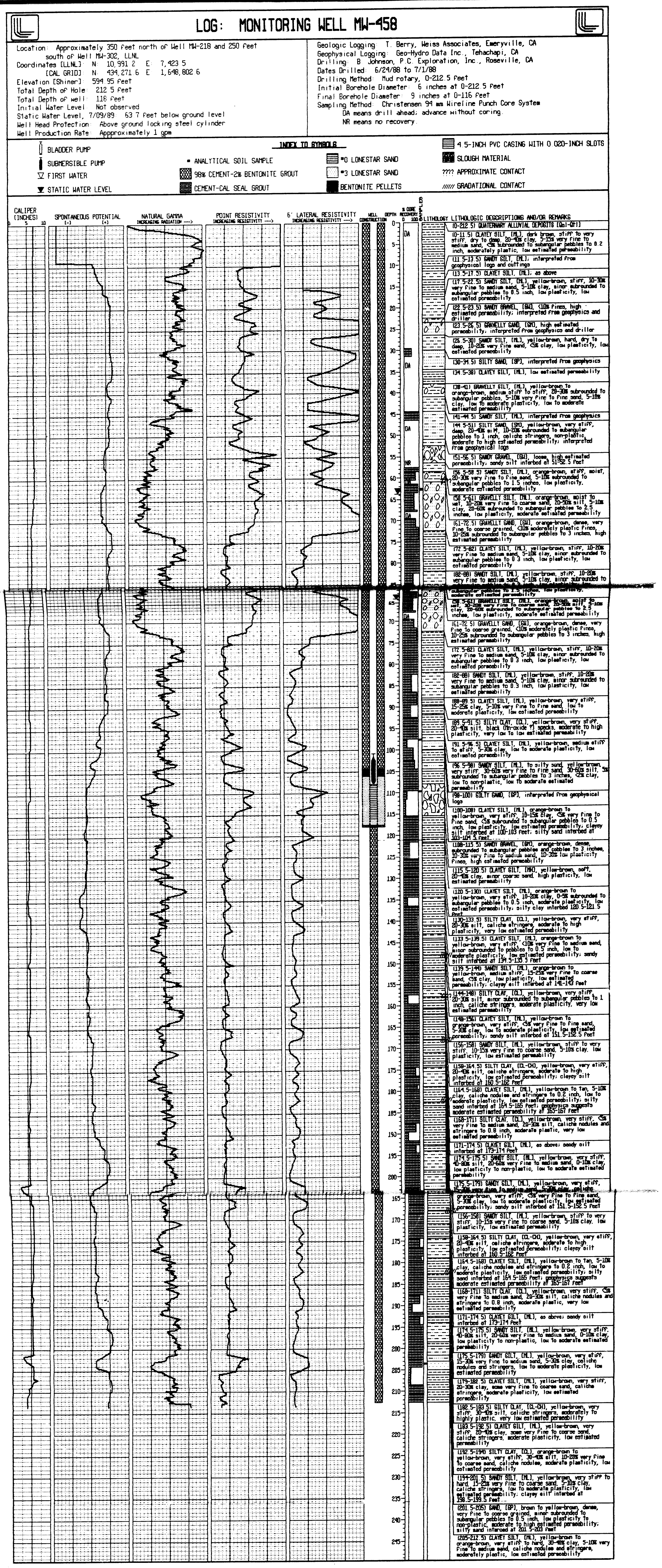




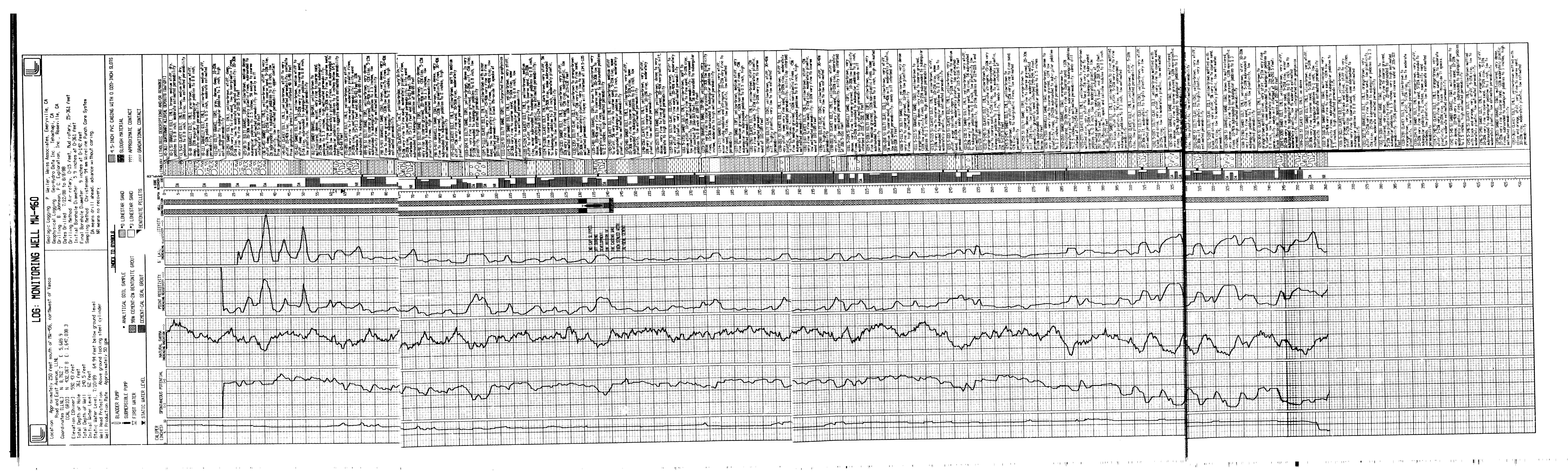




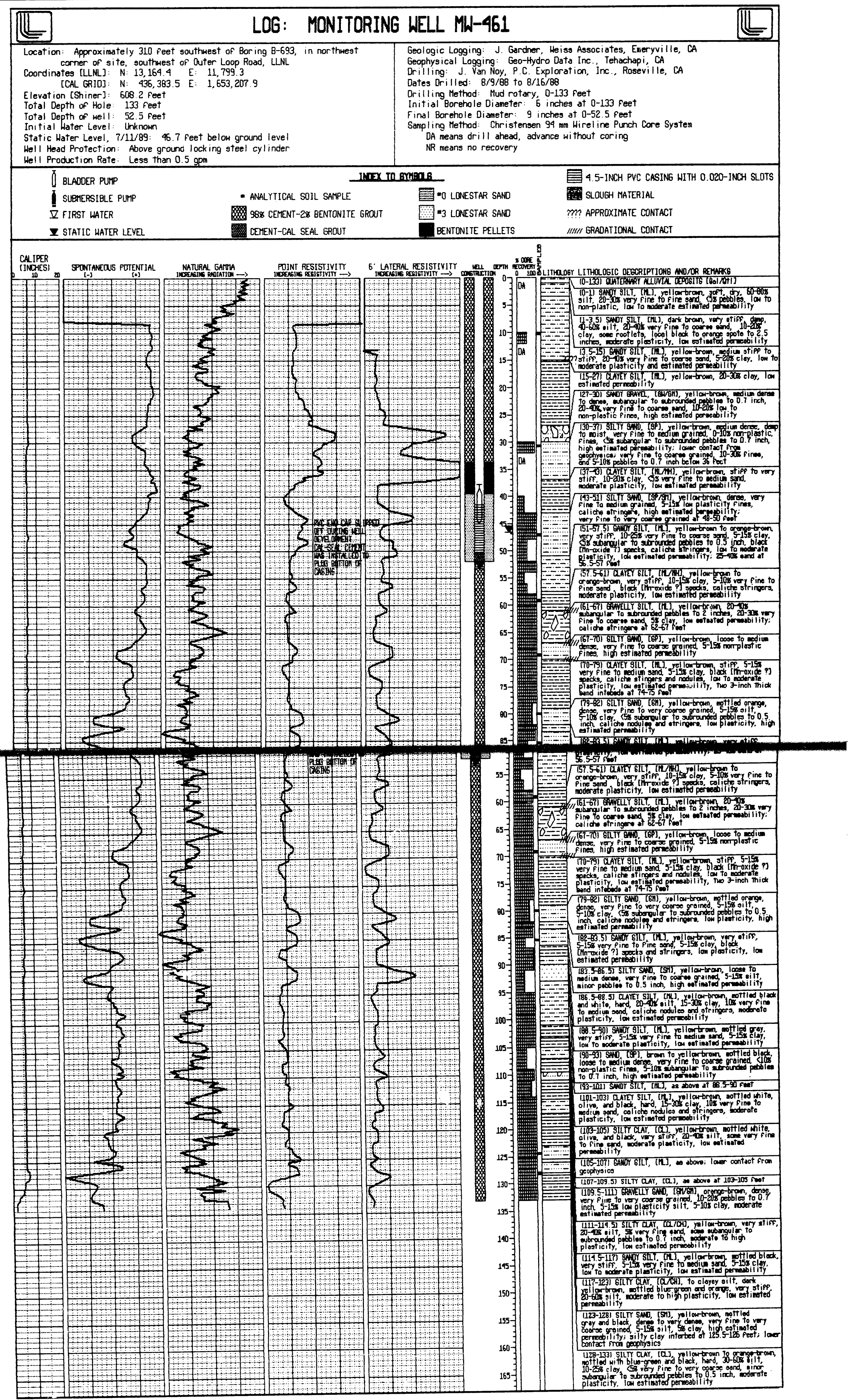




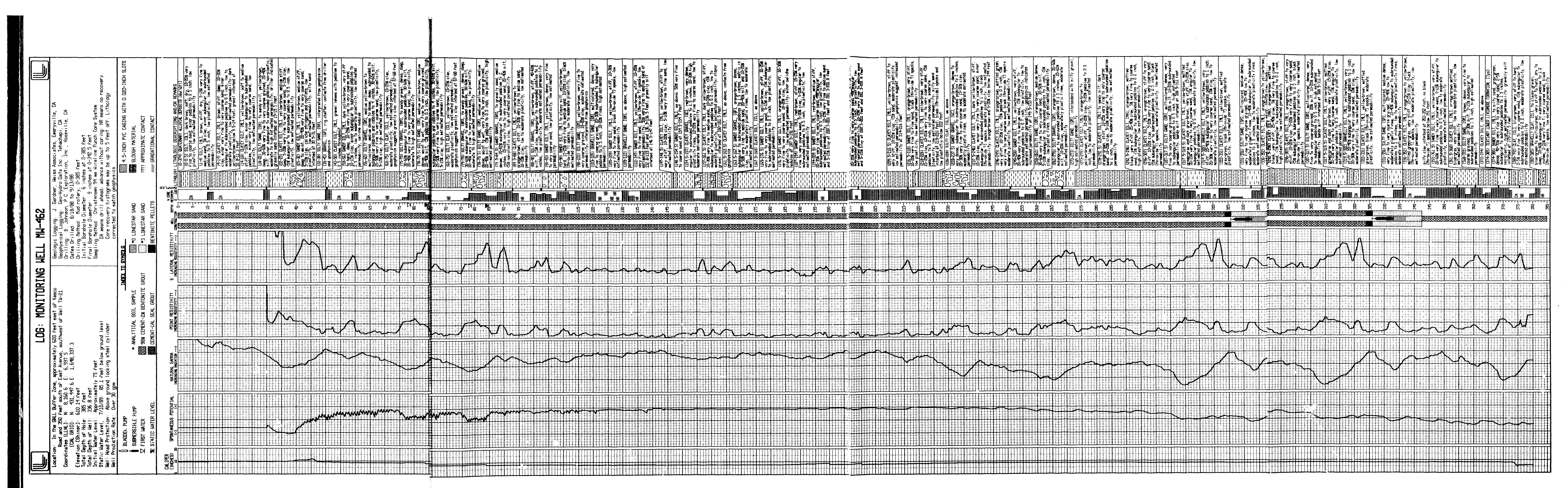




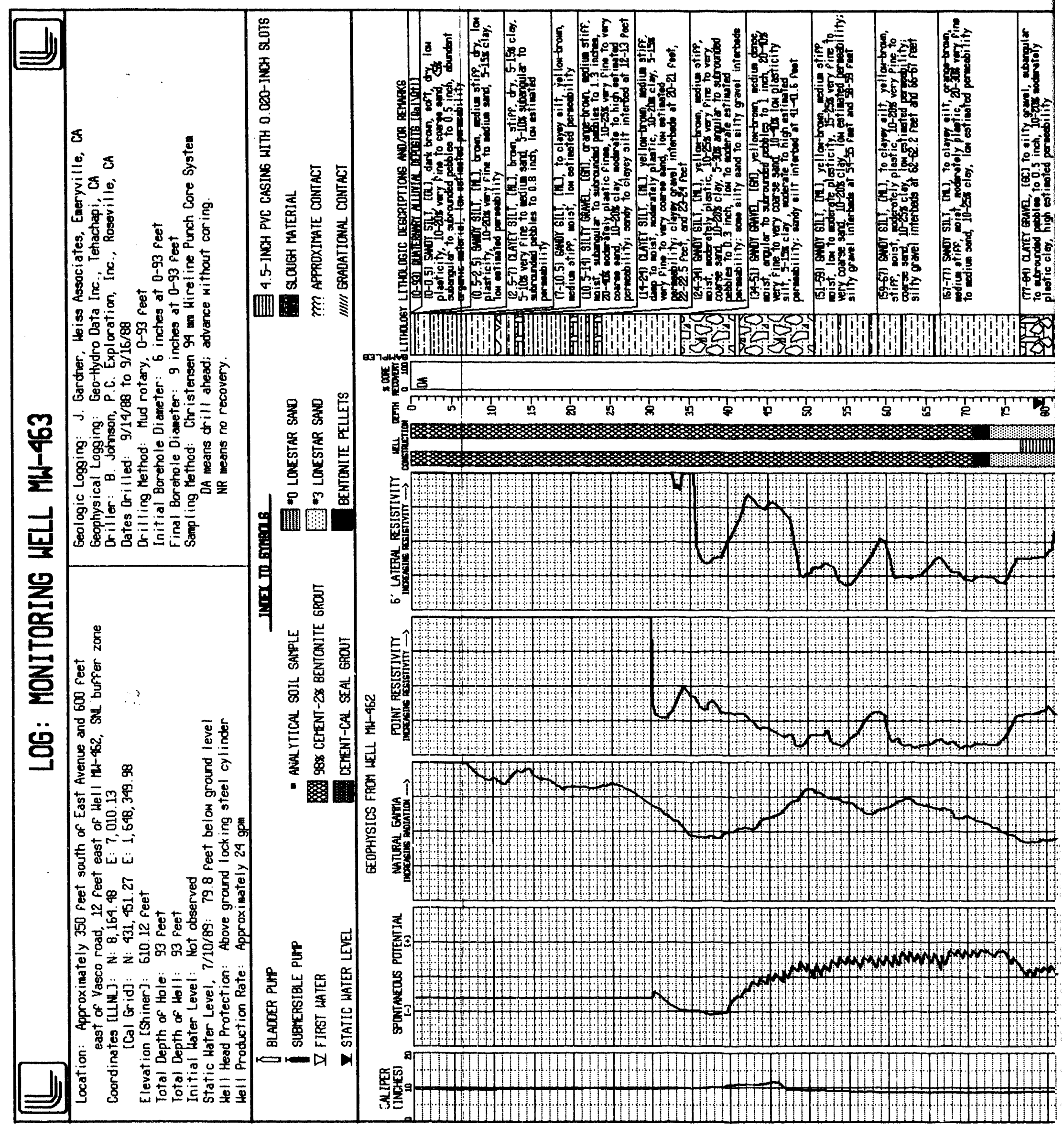




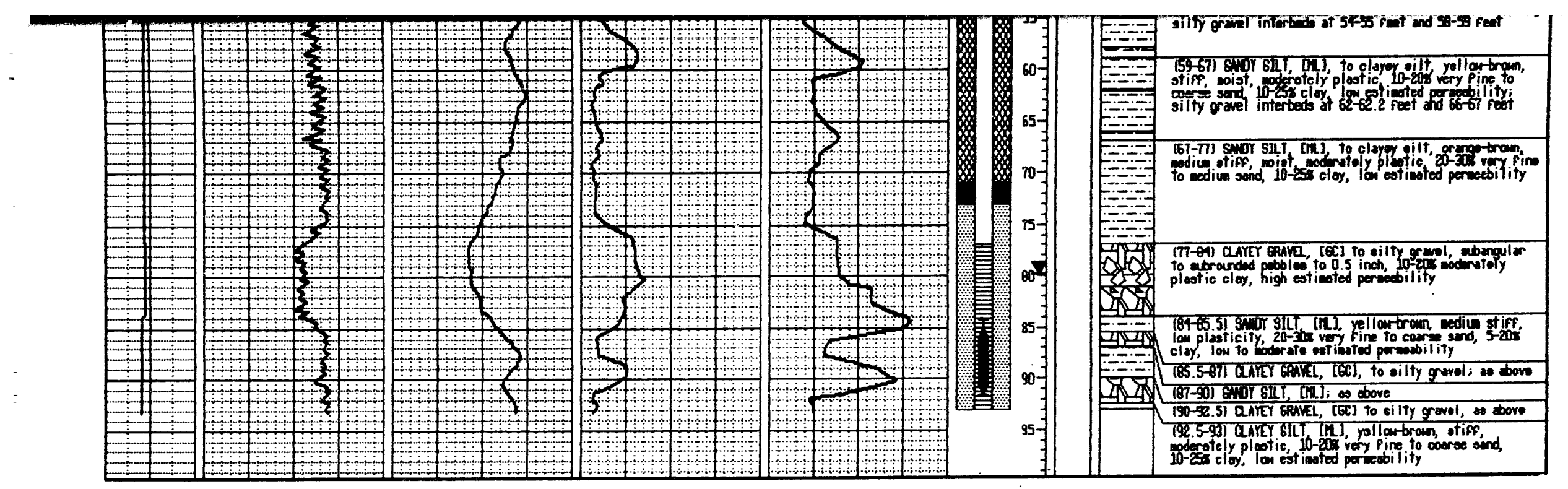




\section{LOG: MONITORING WELL MW-464}

Location: On sidewalk next to South Mall Road and 35 feet east of Avenue B, north of Building 212, LLM

Coordinates [LUN]: N: 8,843.66 $E: 8,452.05$

[Cal Grid]: $N: 432,110.22 \quad E: 1,649,801.09$

Elevation [Shiner]: 623.63 feet

Total Depth of Hole: 253 feet

Total Depth of thal : 104 feet

Initial Later Level: Not observed

Static Hater Level, 7/11/89: 89.1 feet below ground level

Hell Head Protection: Christy Box, ground level traffic grade box

Hell Production Rote: Approximately $9 \mathrm{gpm}$

\begin{tabular}{|c|c|c|}
\hline II BLADOER PUAP & \multicolumn{2}{|c|}{ INIX In mpens } \\
\hline SUREERSIBLE PUIPP & - ANALYTICAL SOIL SAMPLE & =0 LONESTAR SAND \\
\hline Q FIRST WATER & 988 CEMENT-28 BENTONITE GROUT & *3 LONESTAR SAND \\
\hline I STATIC HATER LEVEL & CEMENT-CAL SEAL GROUT & BENTONITE PELLETS \\
\hline
\end{tabular}

Geological Logging: J. Gardner, Weiss Associates, Emeryville, CA

Geophysical Logging: Geo-Hydro Data Inc., Tehachapi, CA

(a) Roseville, CA

Dates Drilled: 9/20/88 to $9 / 30 / 88$

Drilling Method: Mud rotary, 0-253 feet

Initial Borehole Diameter: 6 inches at 0-253 feet

Final Borehole Diameter: 9 inches at 0-107 feet

Sampling Method: Christensen $94 \mathrm{~mm}$ Wirel ine Punch Core System

DA means drill ahead; advance without coring.

NR means no recovery.

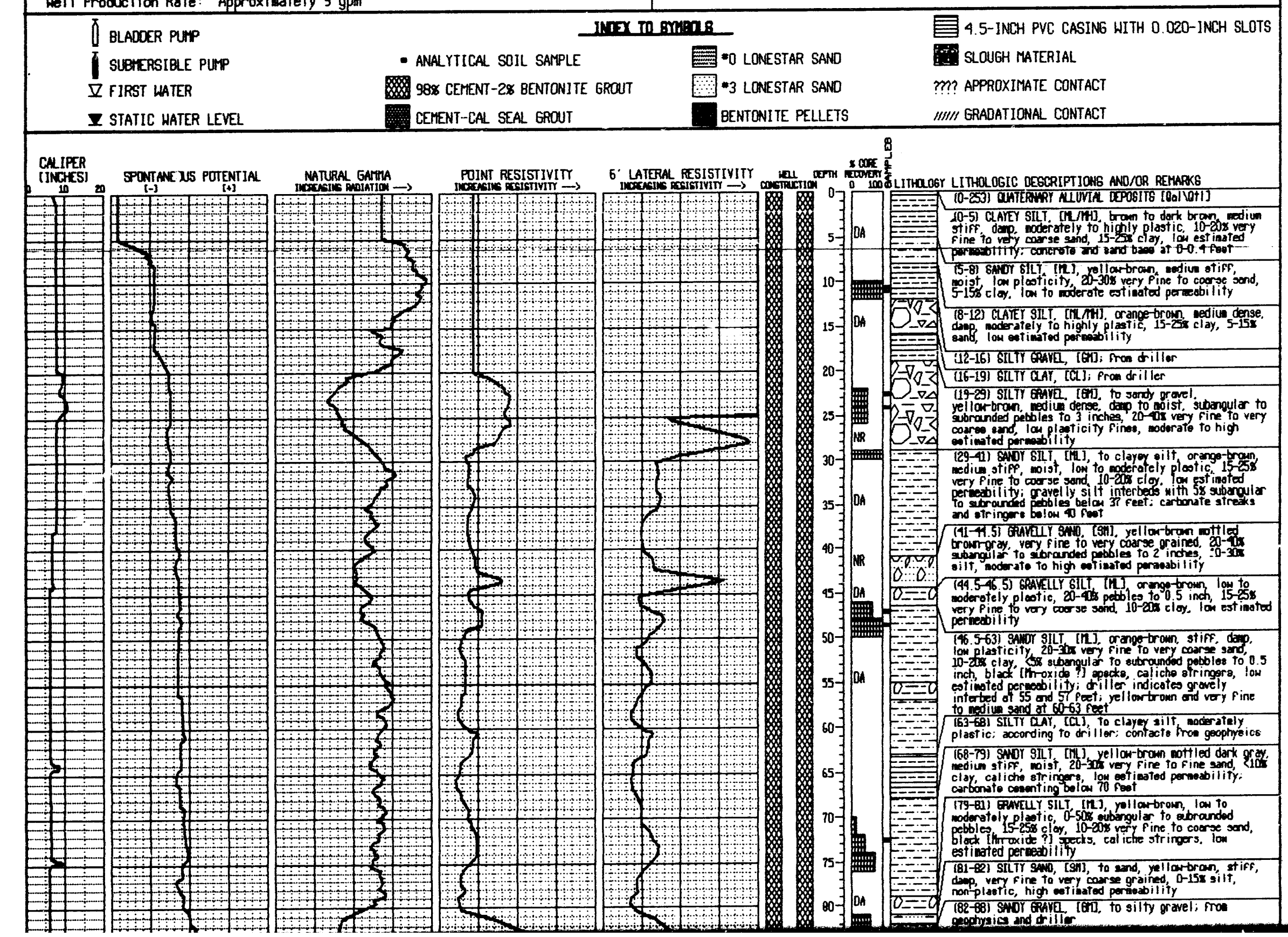




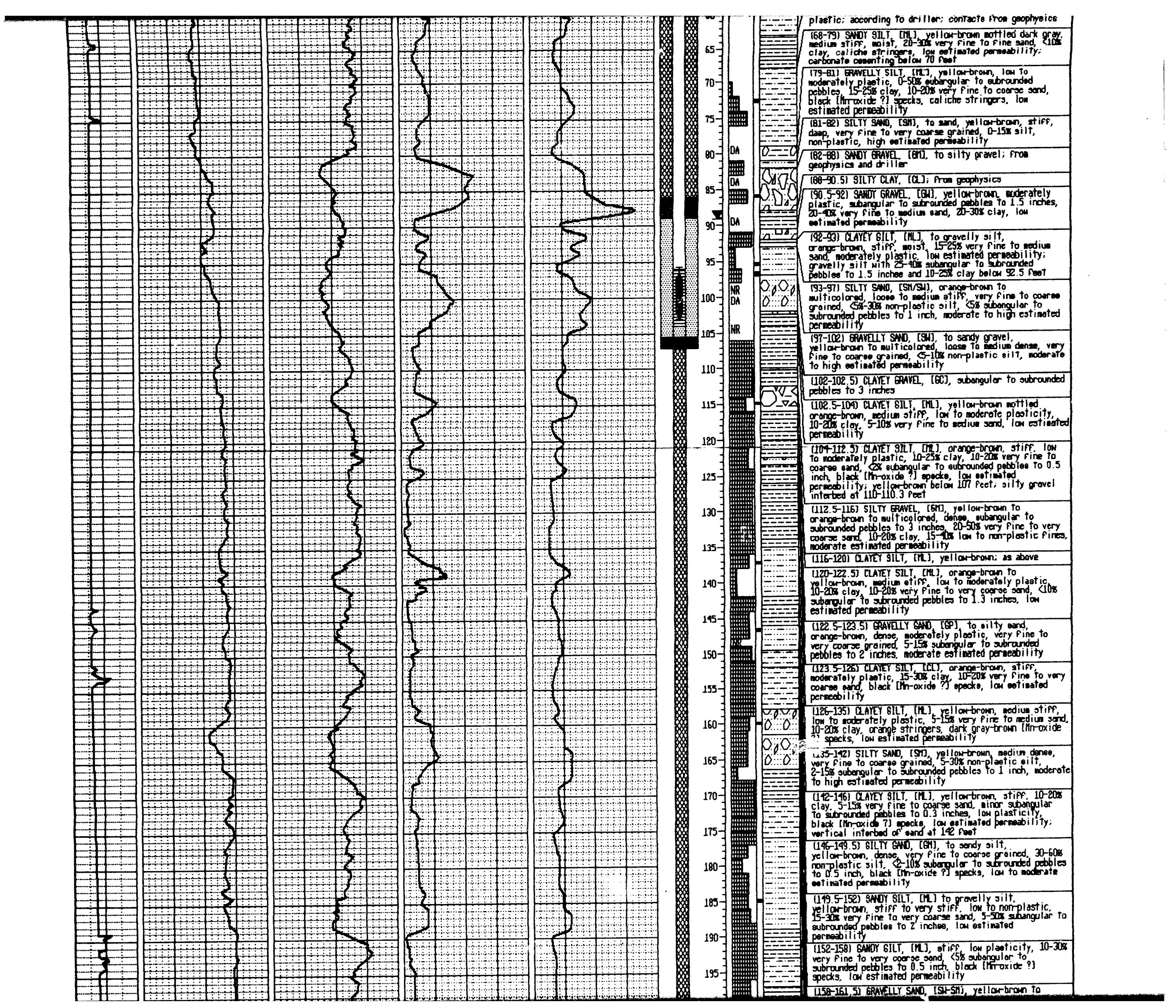




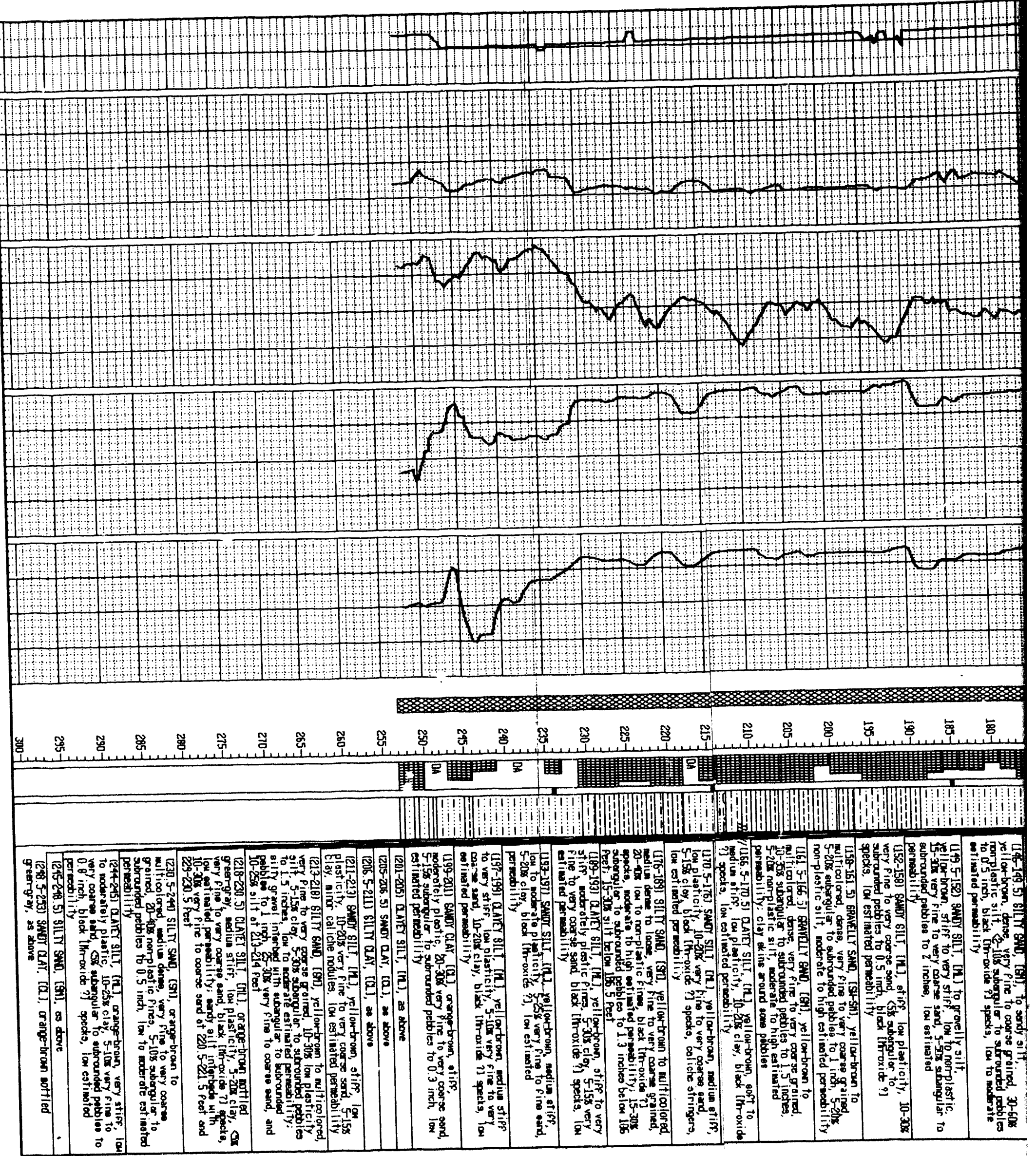




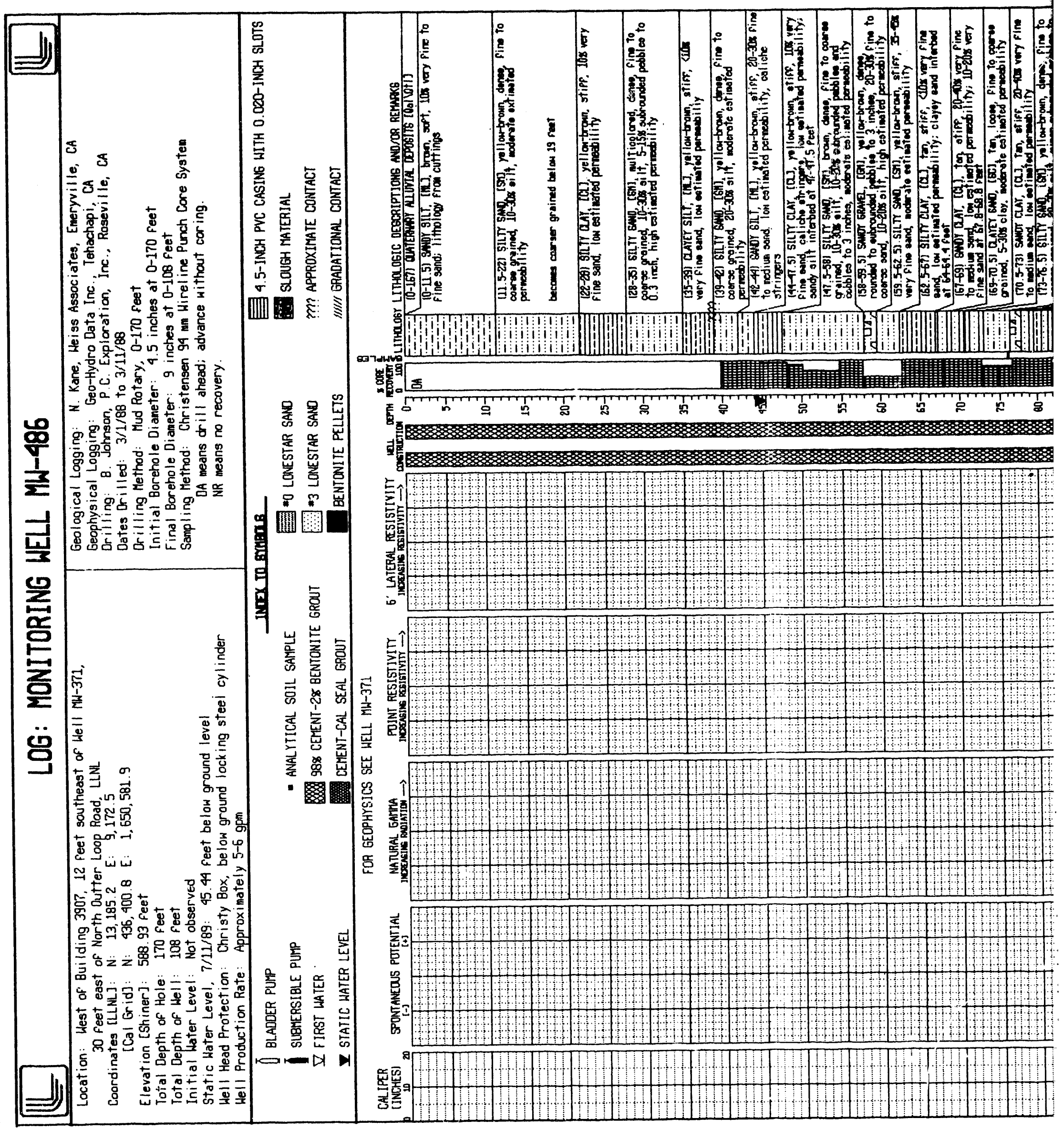




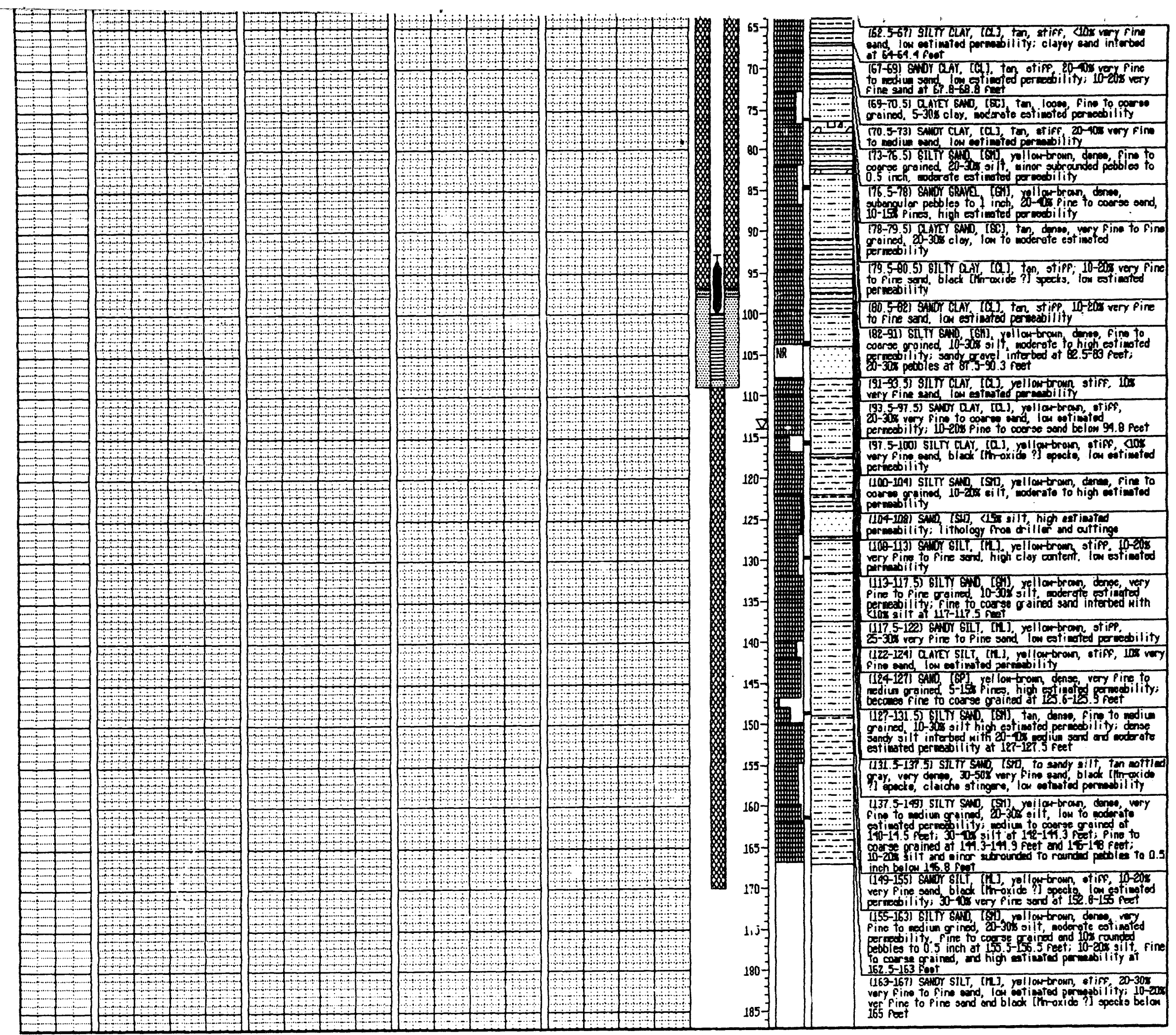




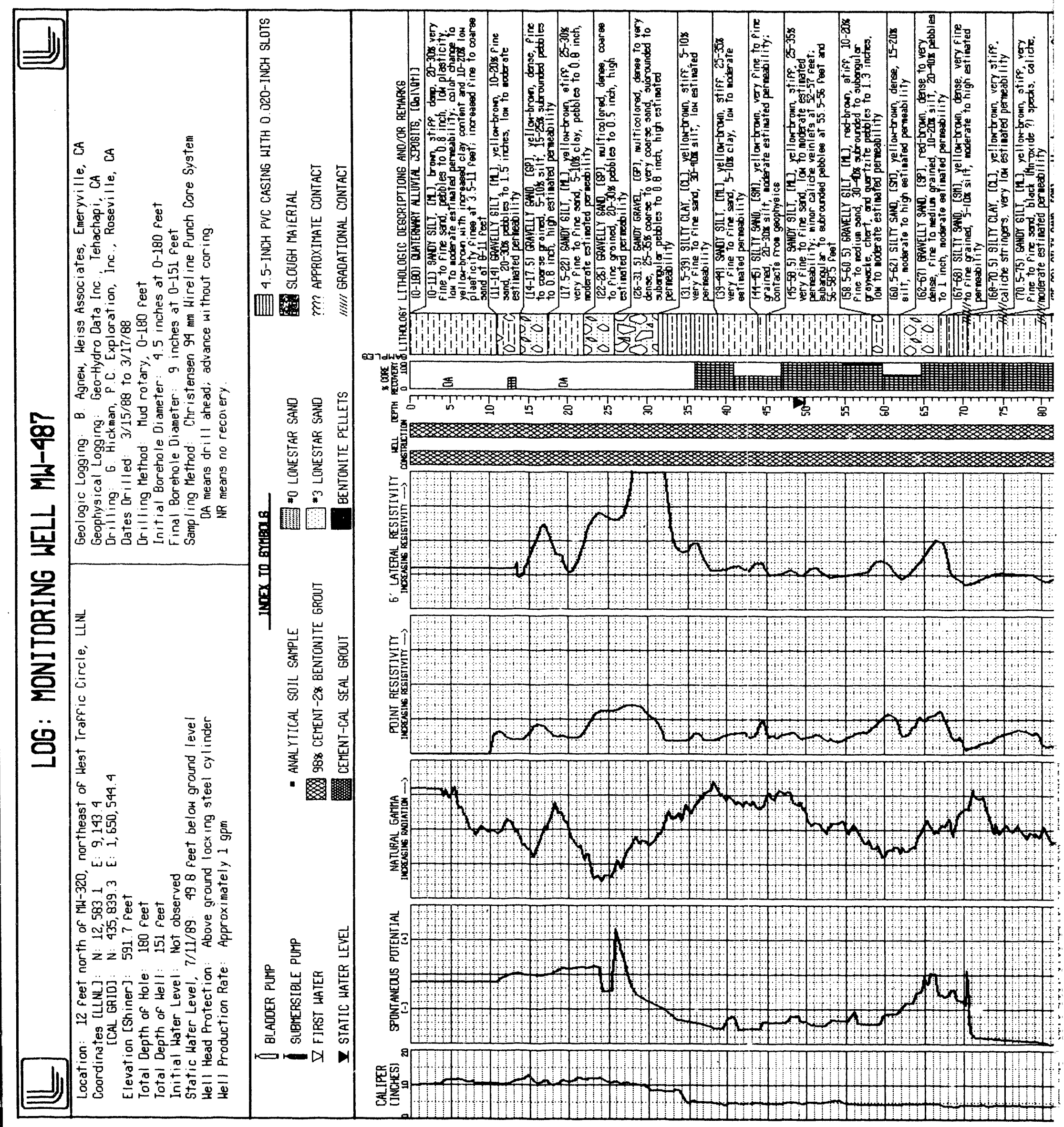




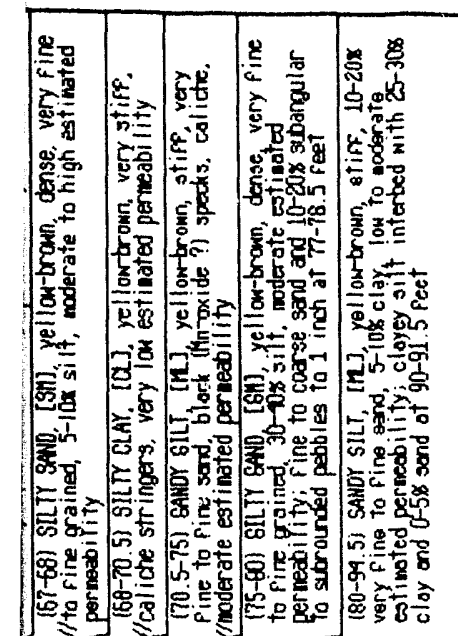

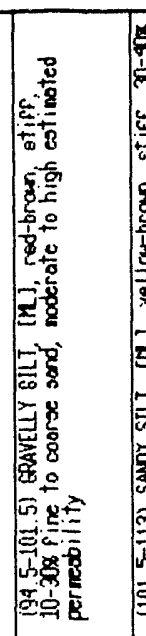

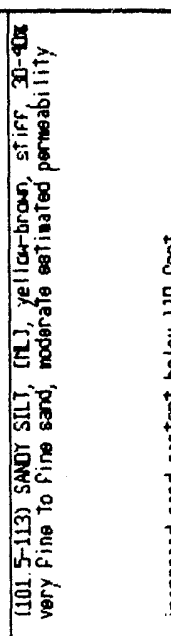

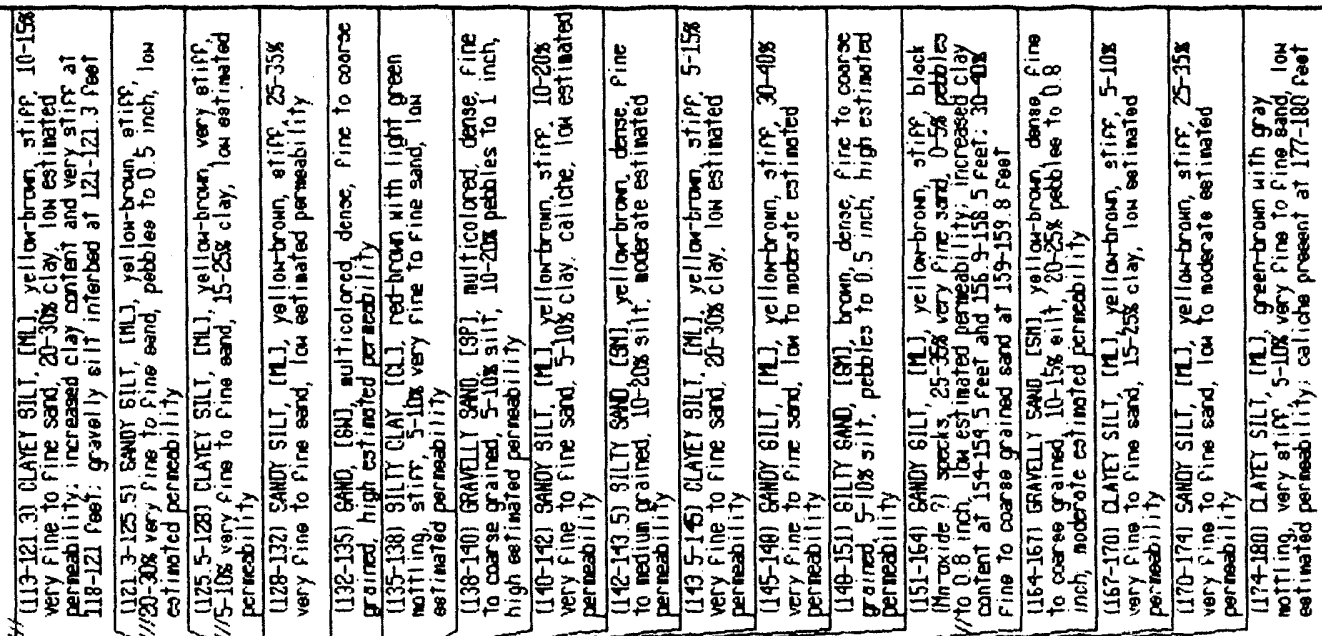
IIfili
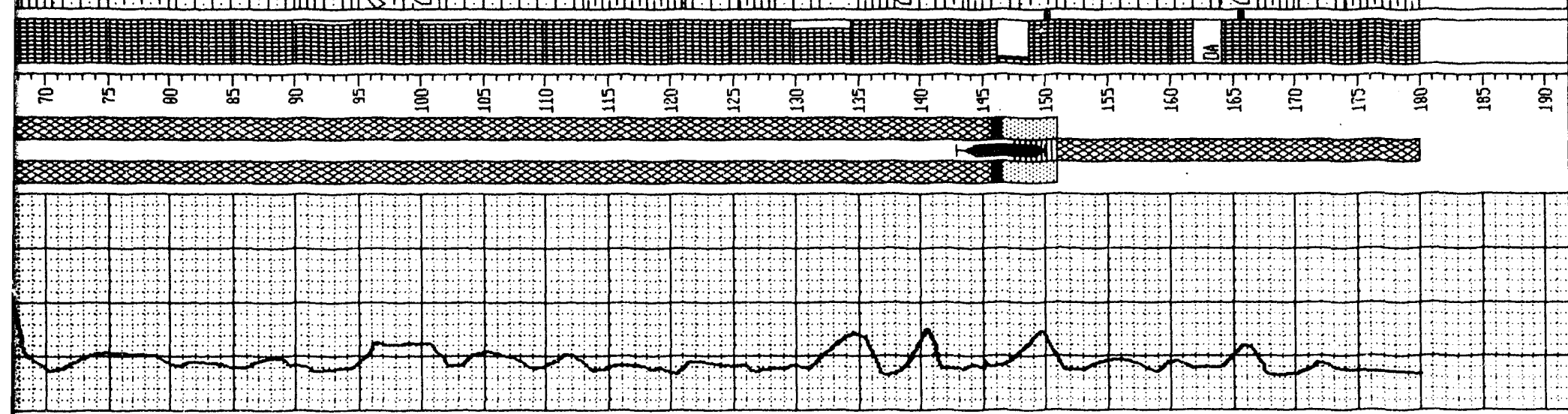

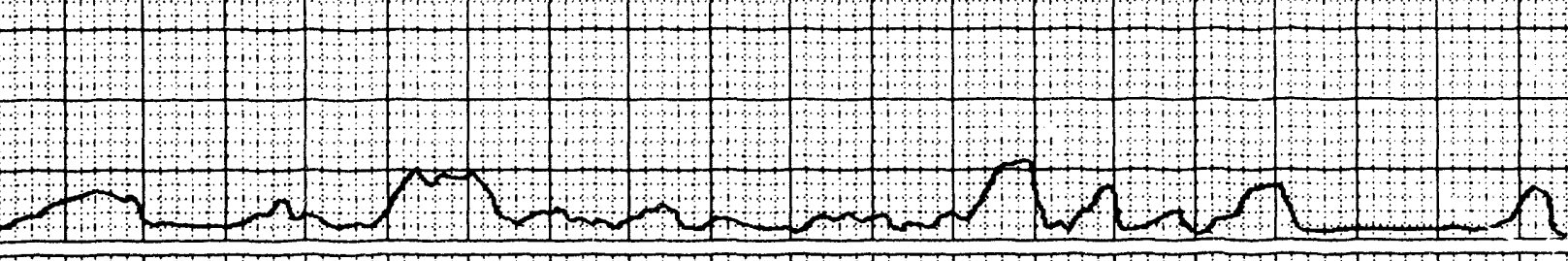

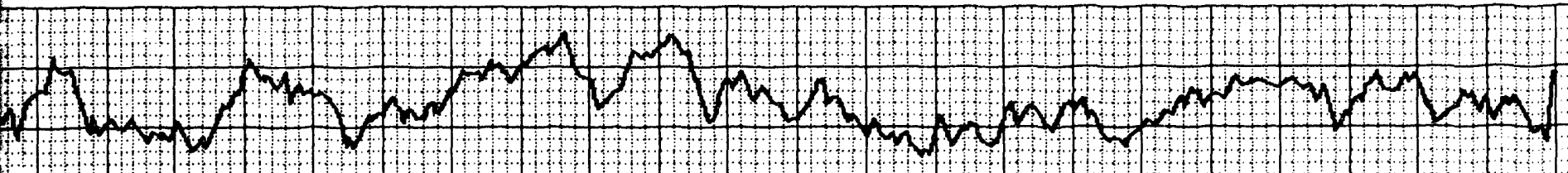




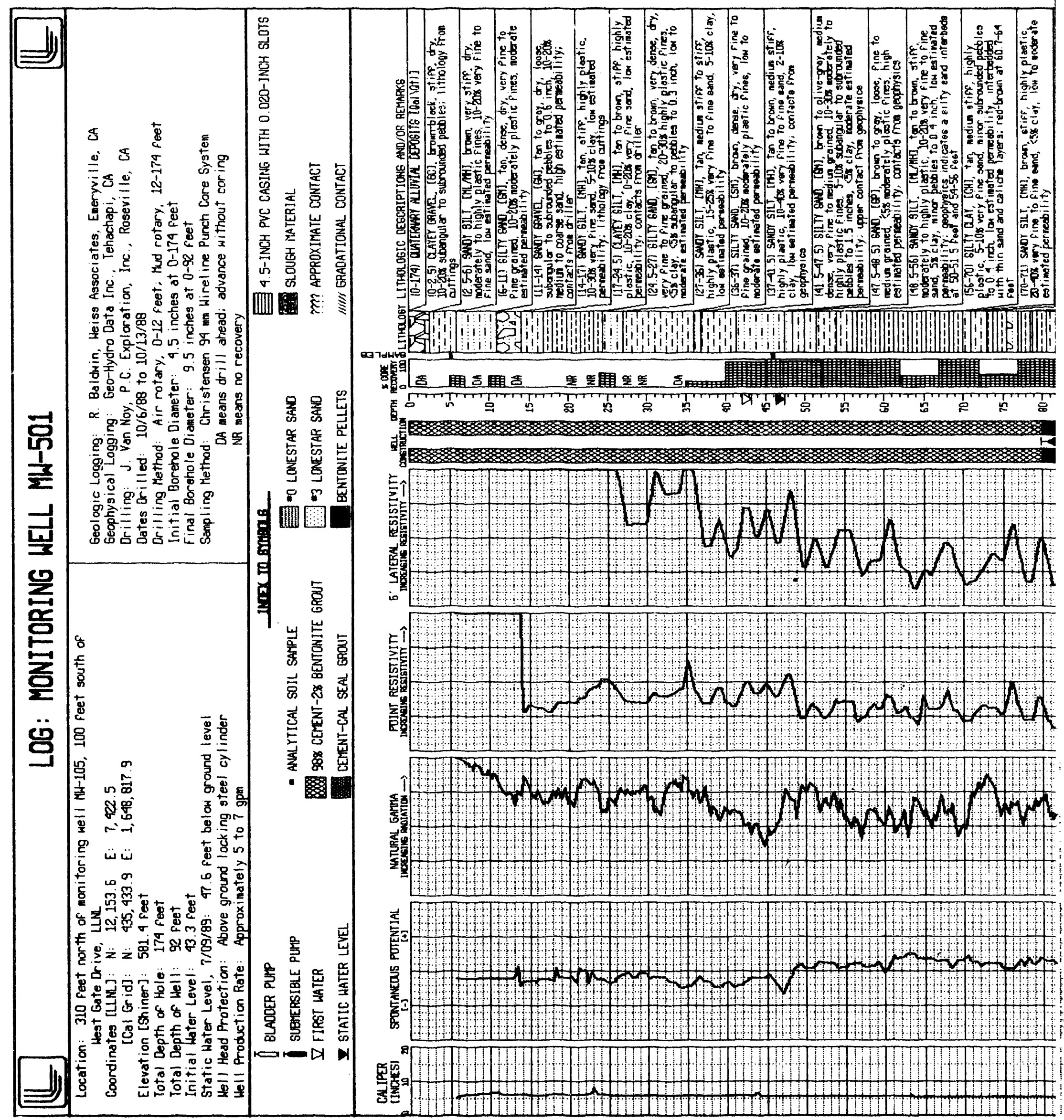




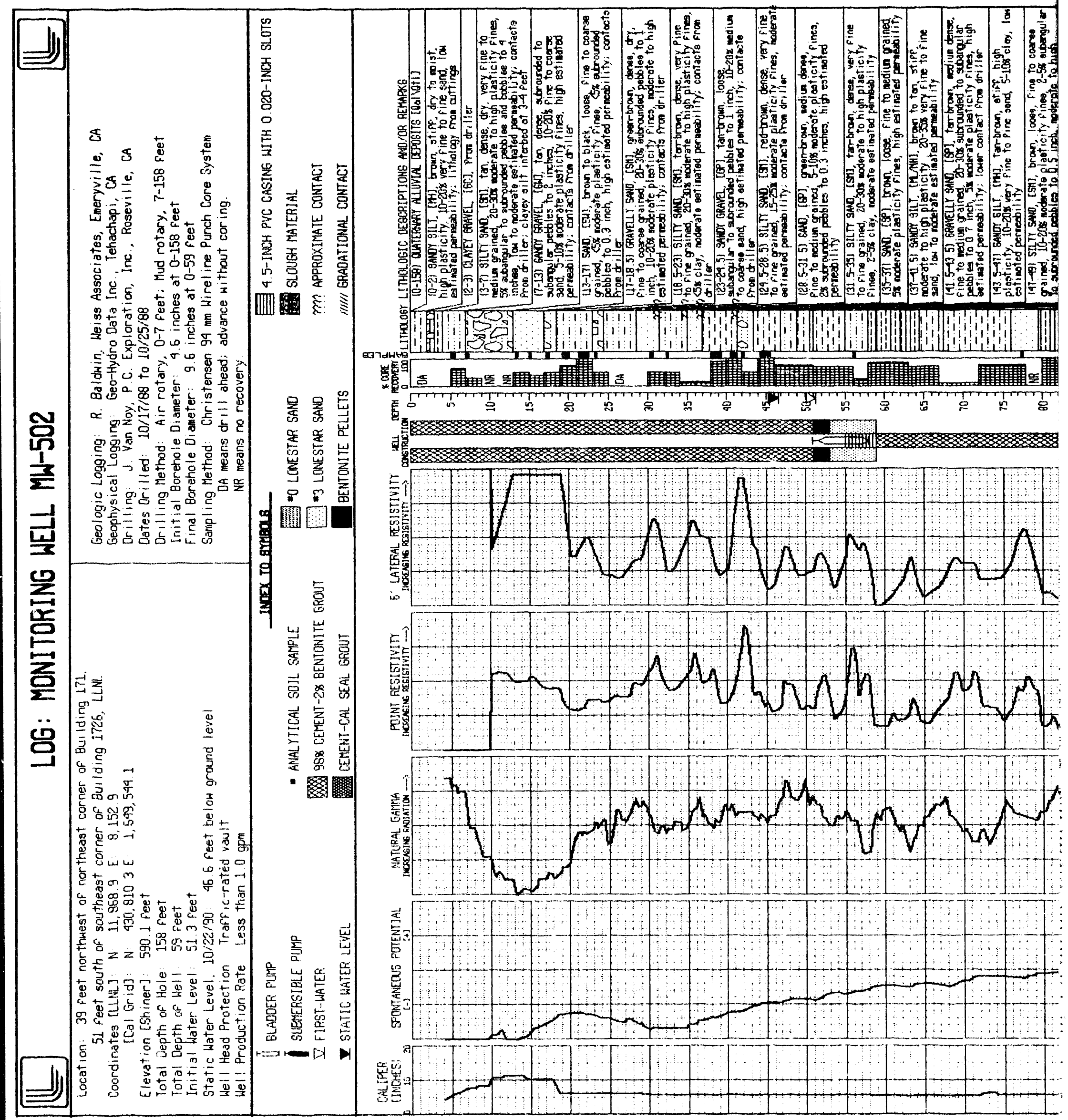




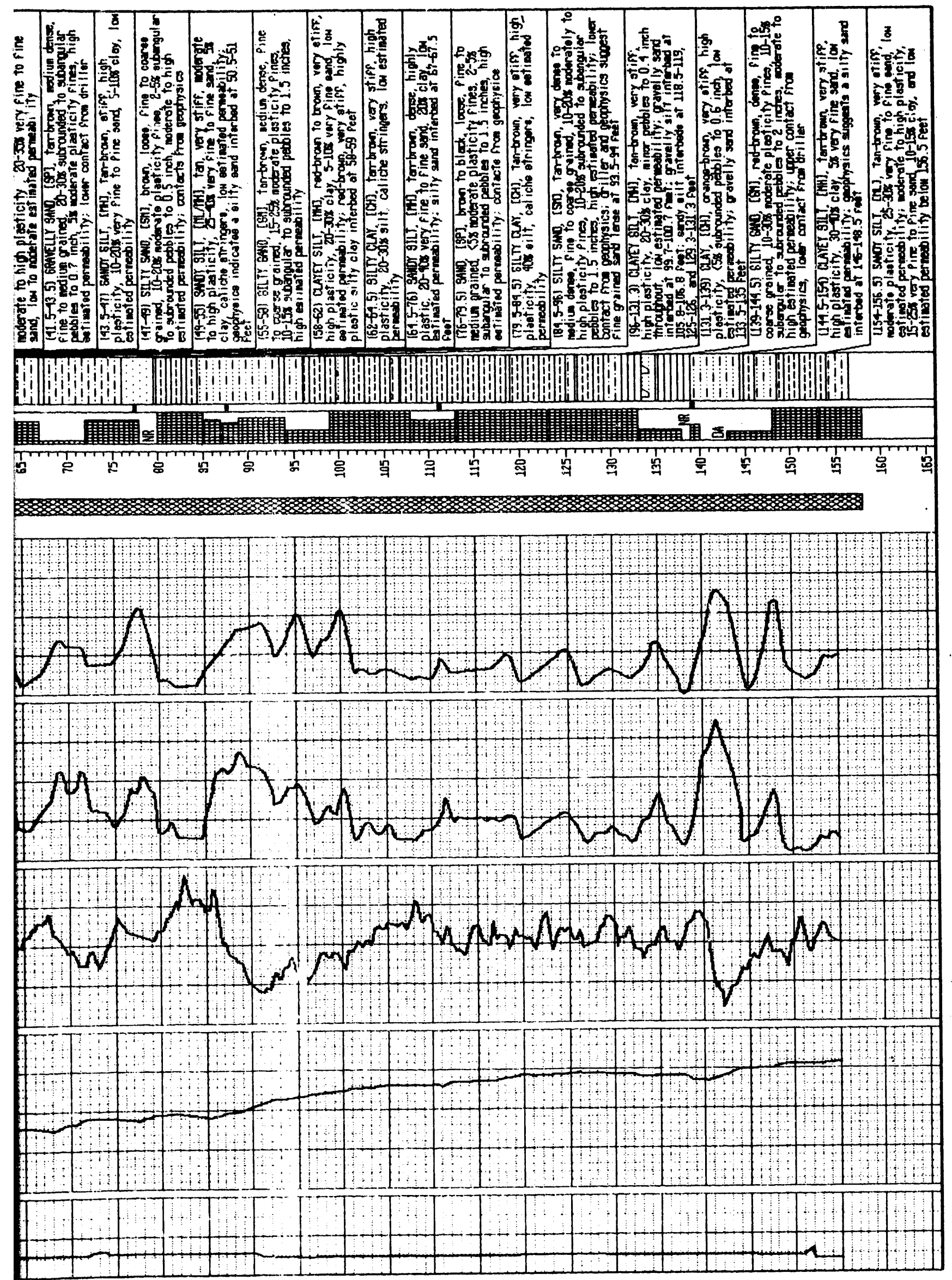




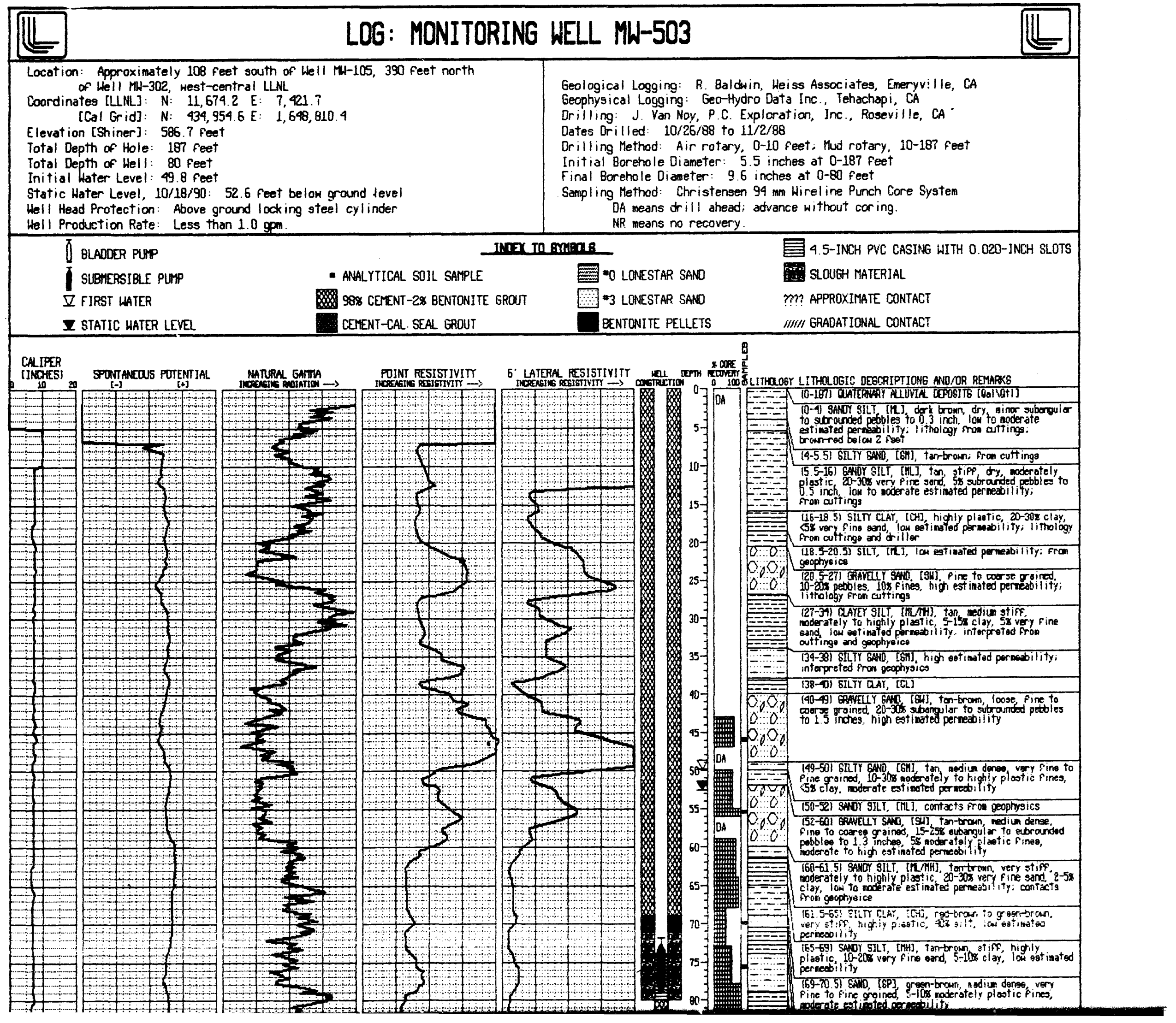




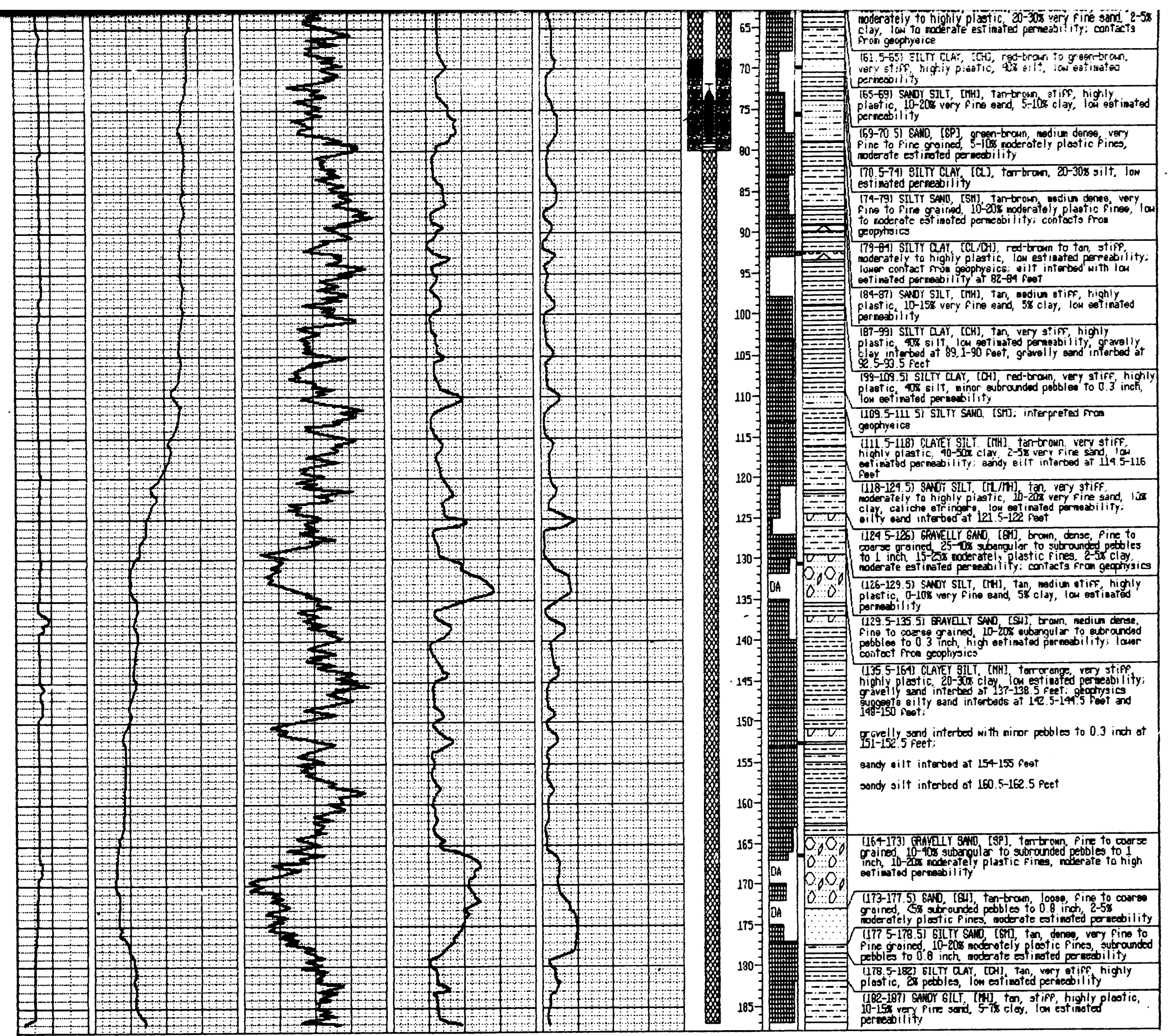




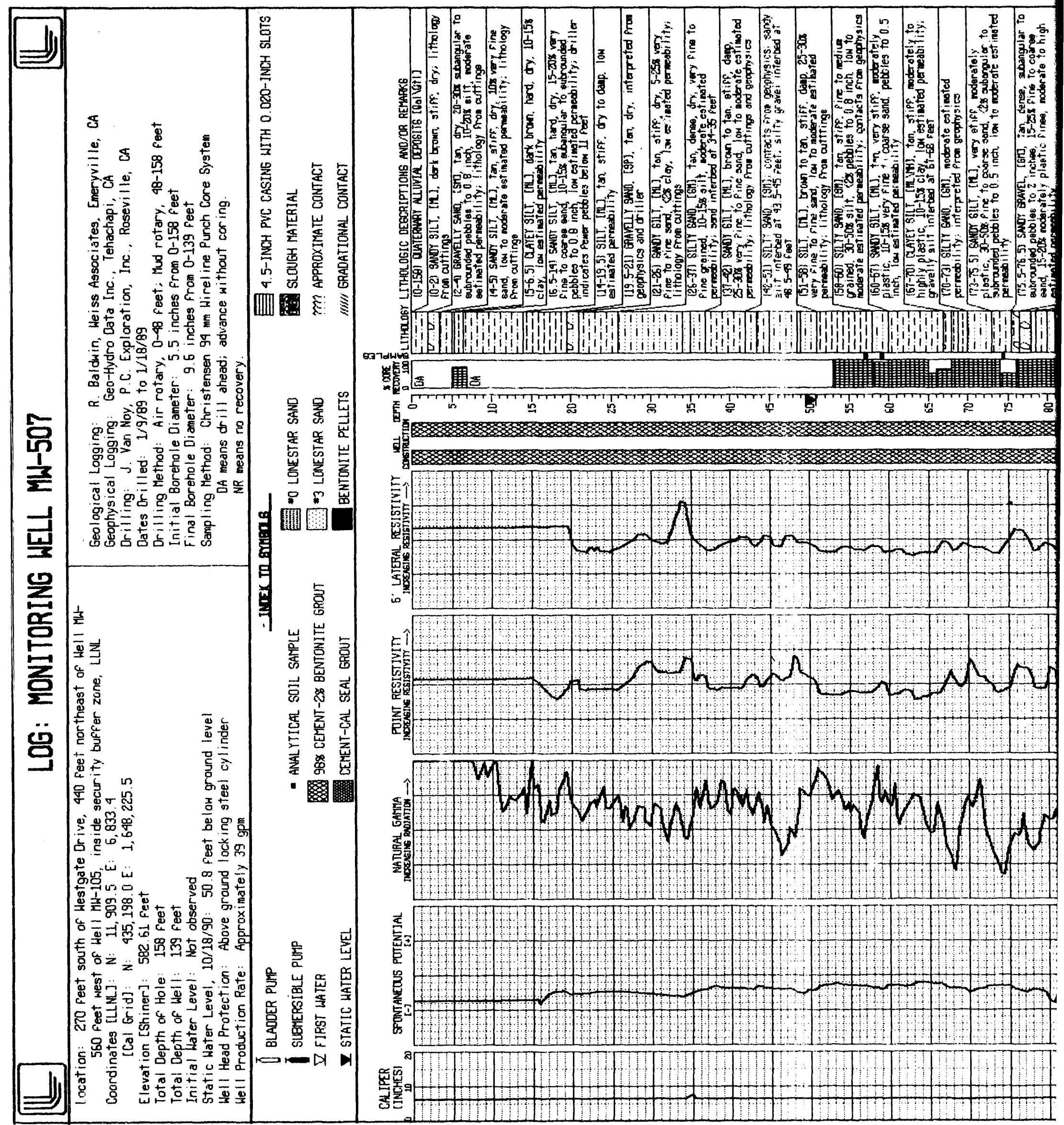




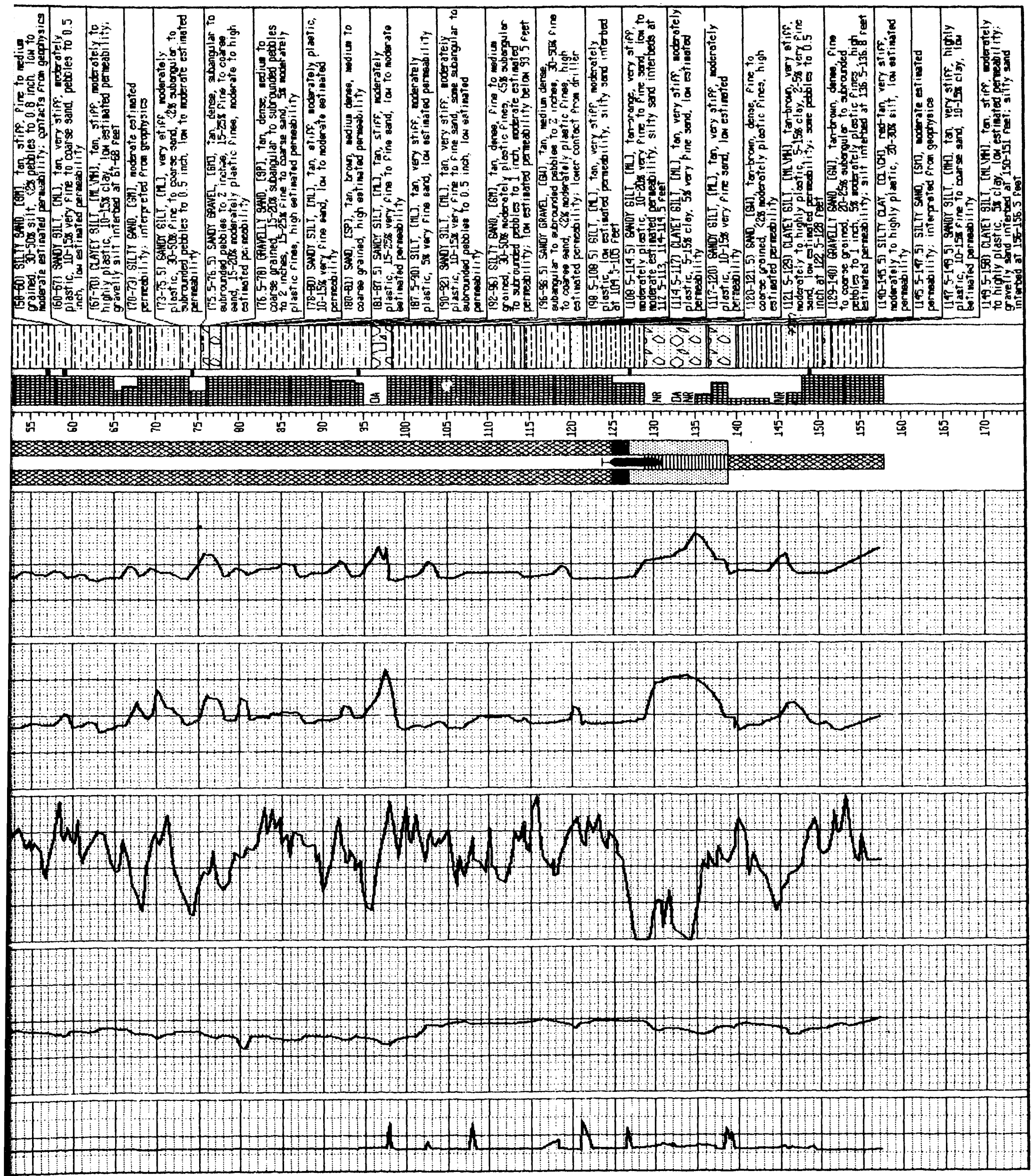




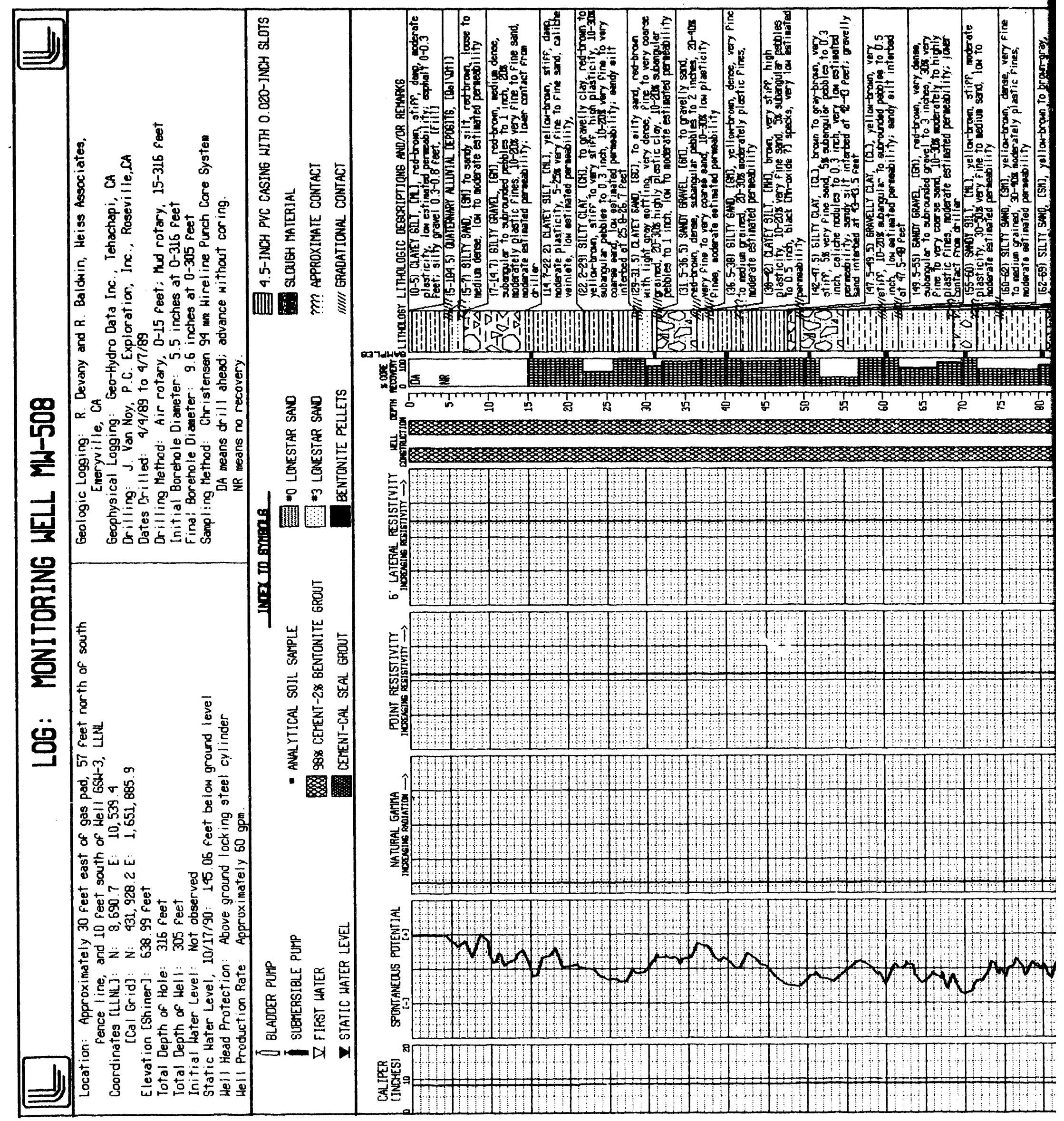




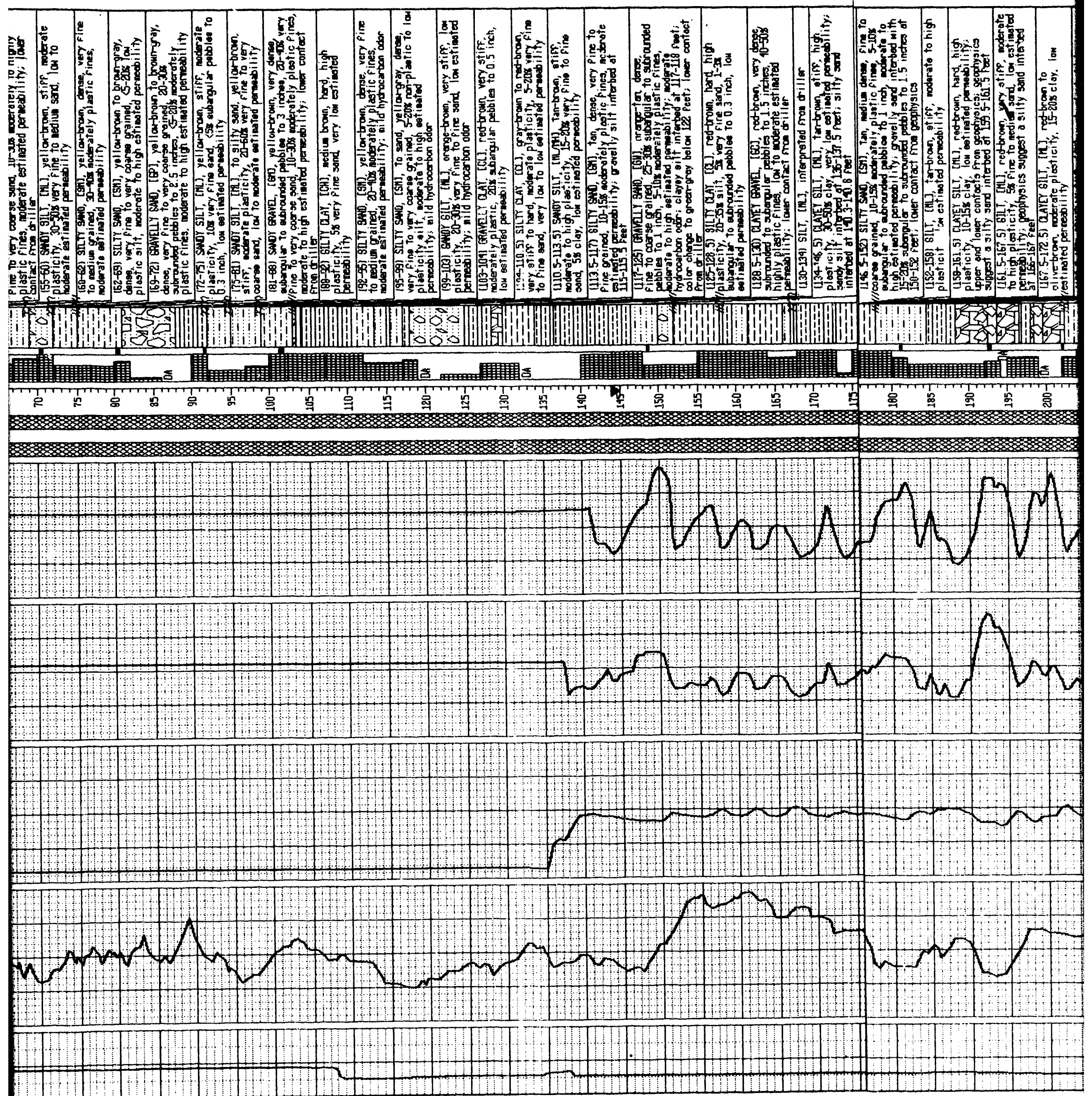




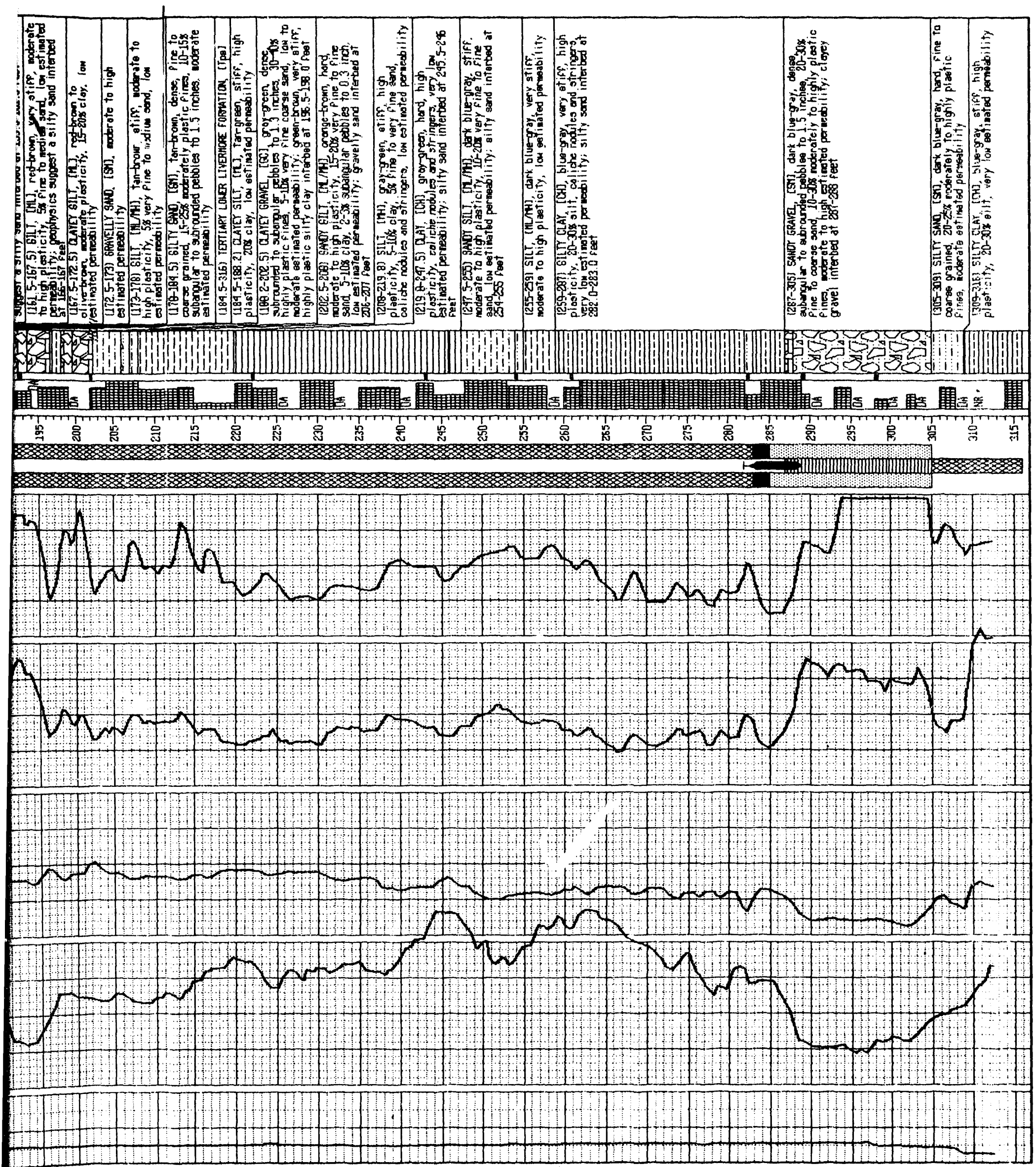




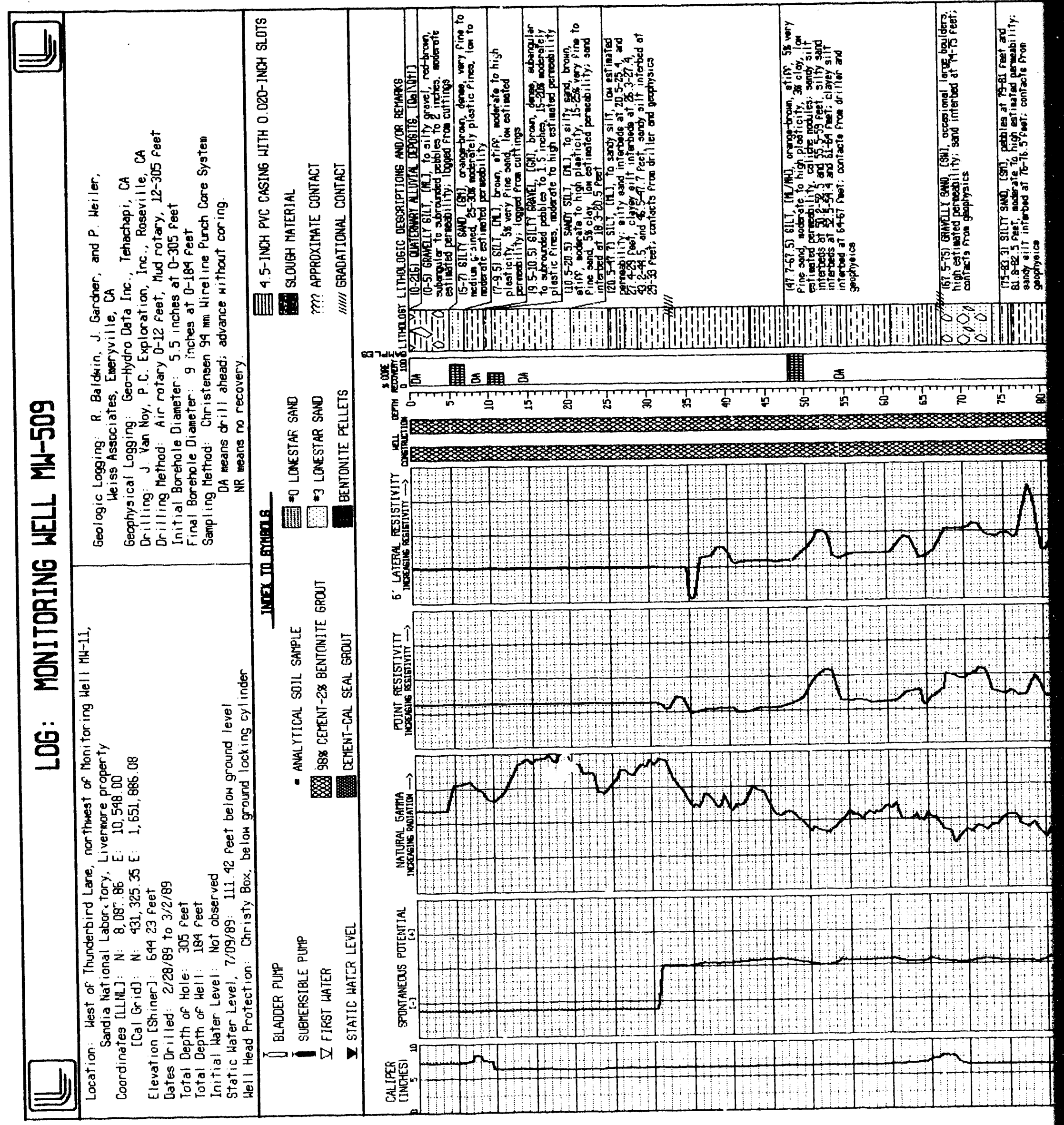




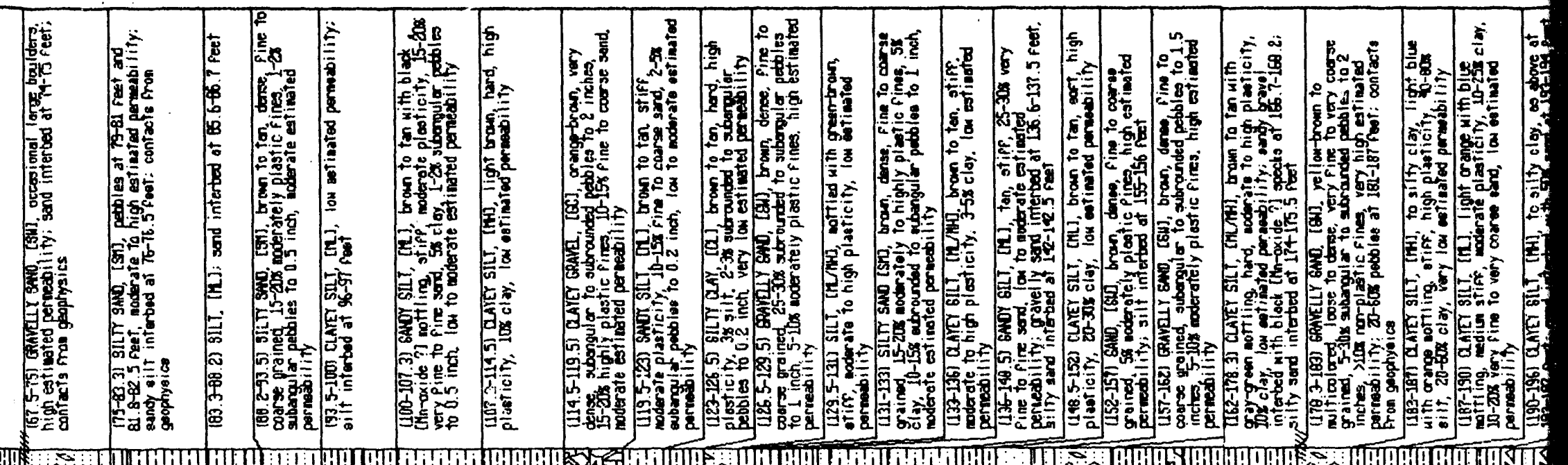

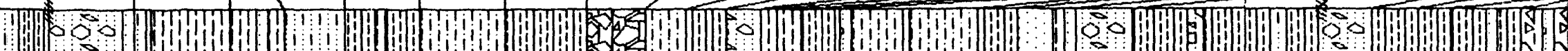
L 工要

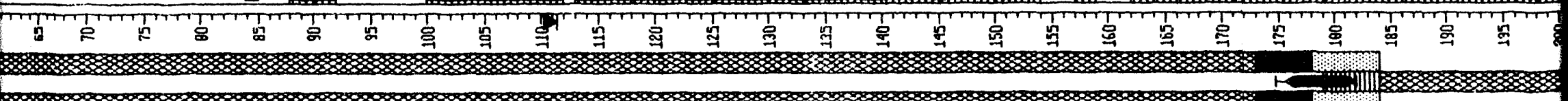

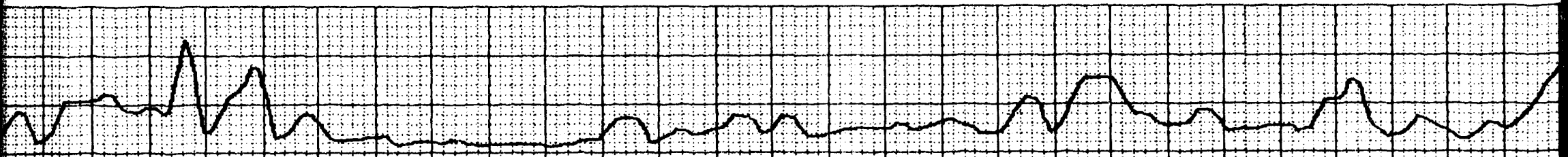

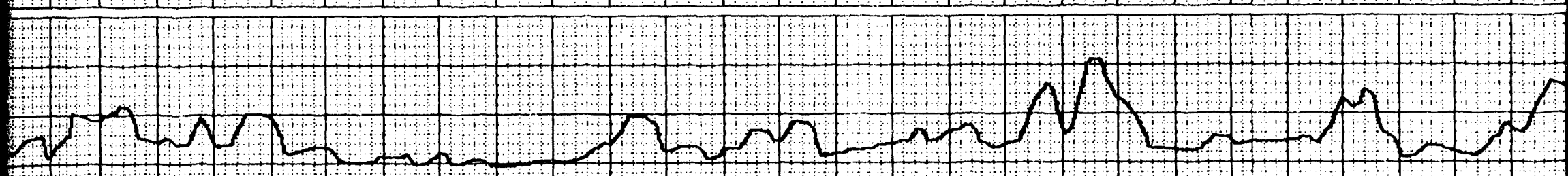

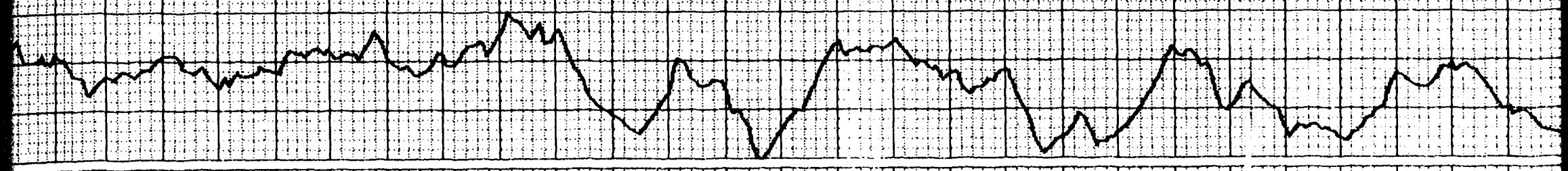




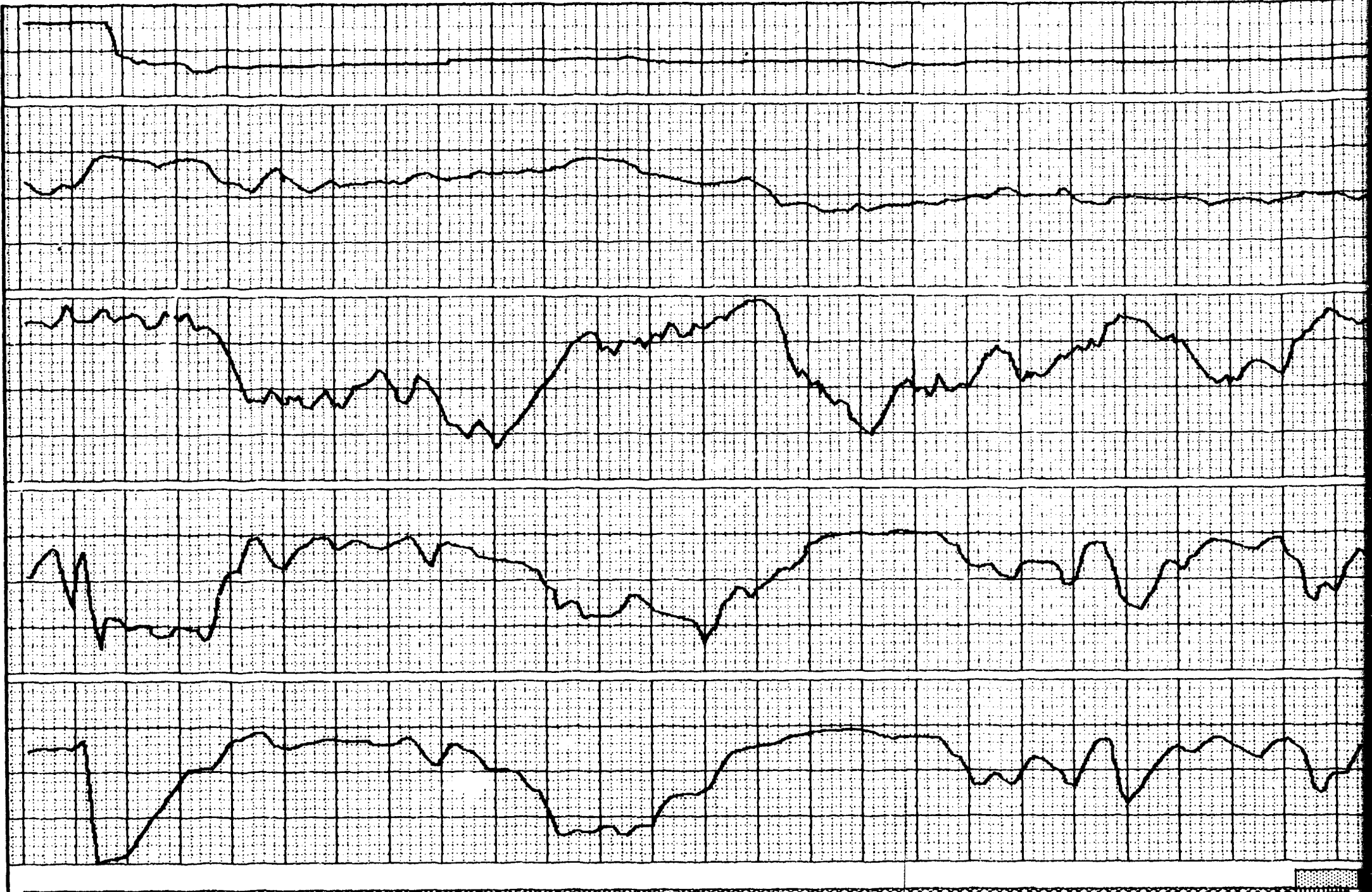

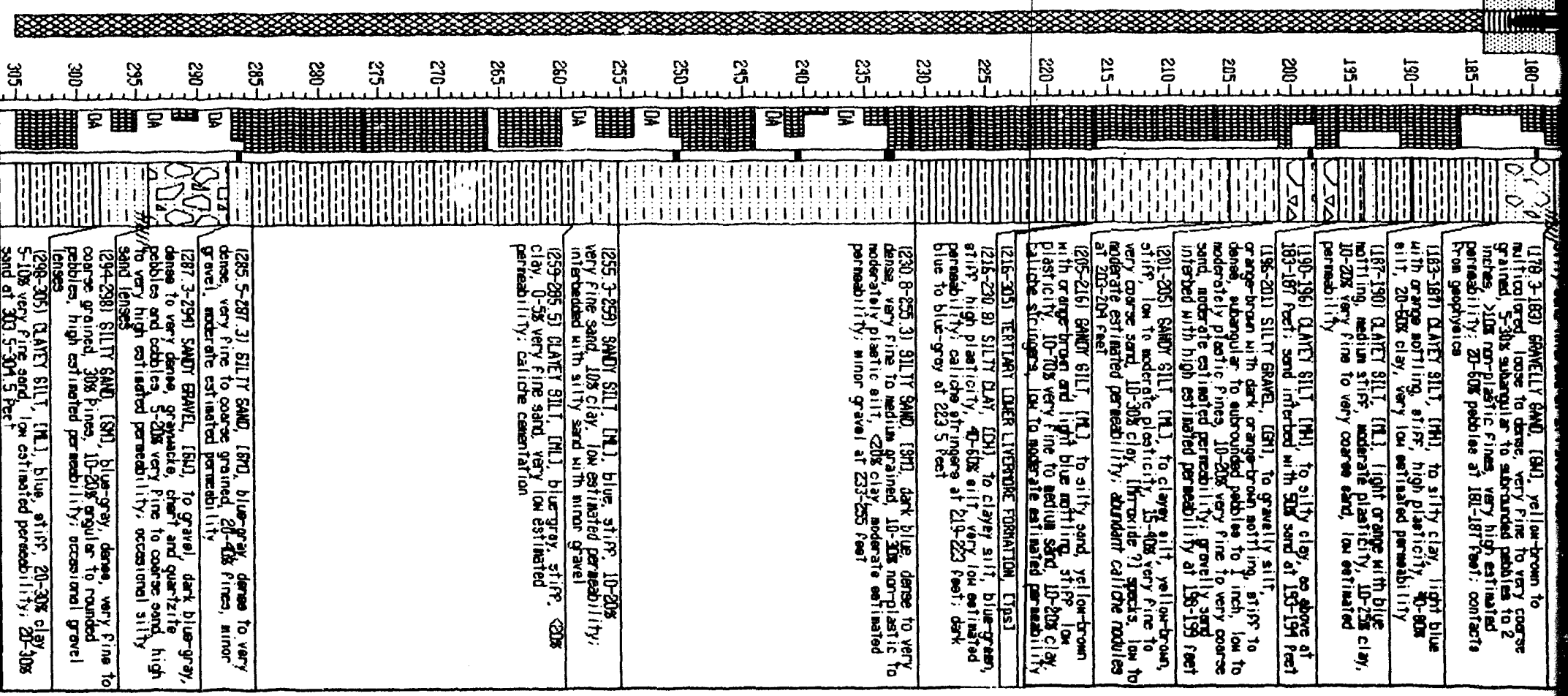




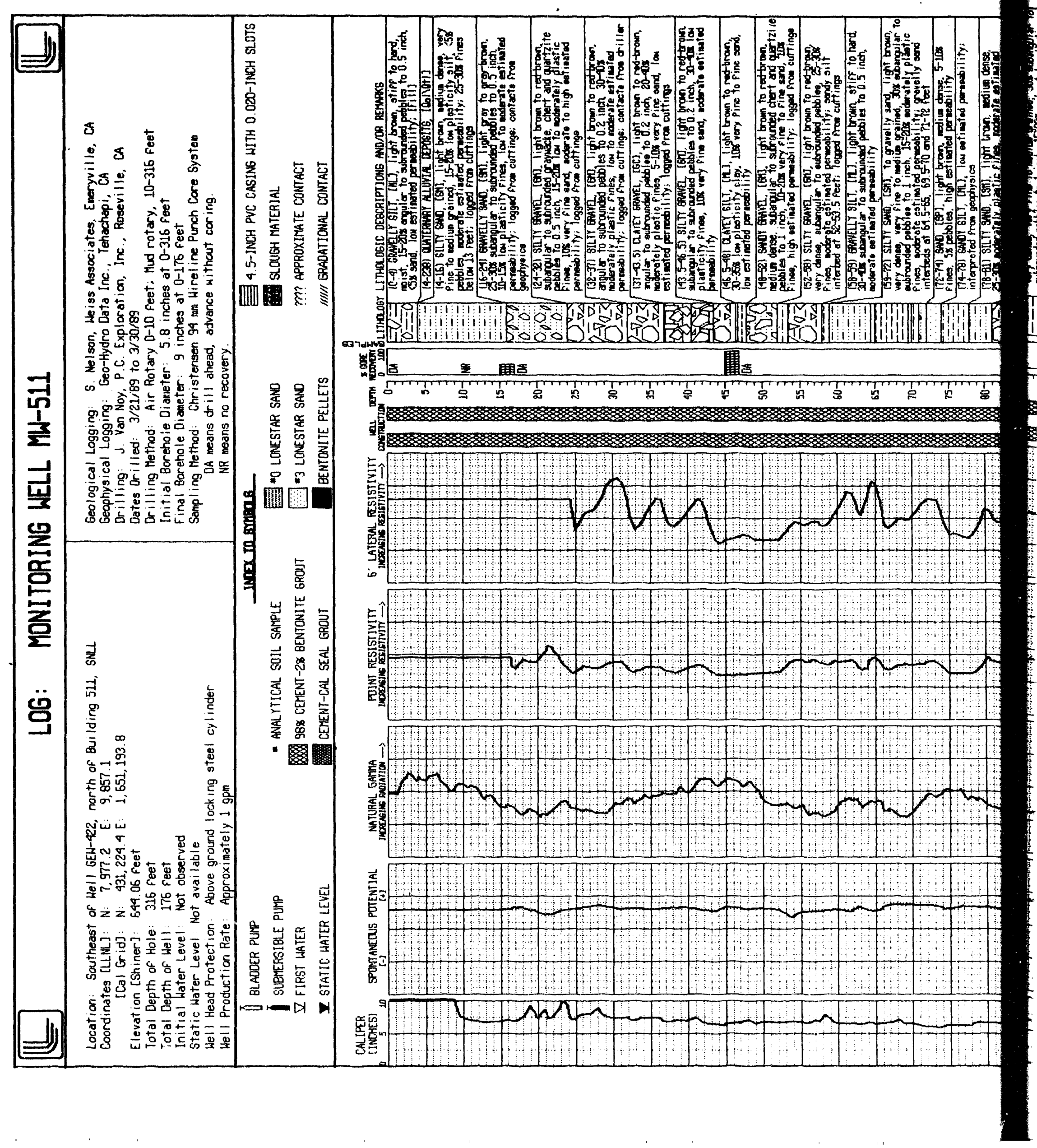




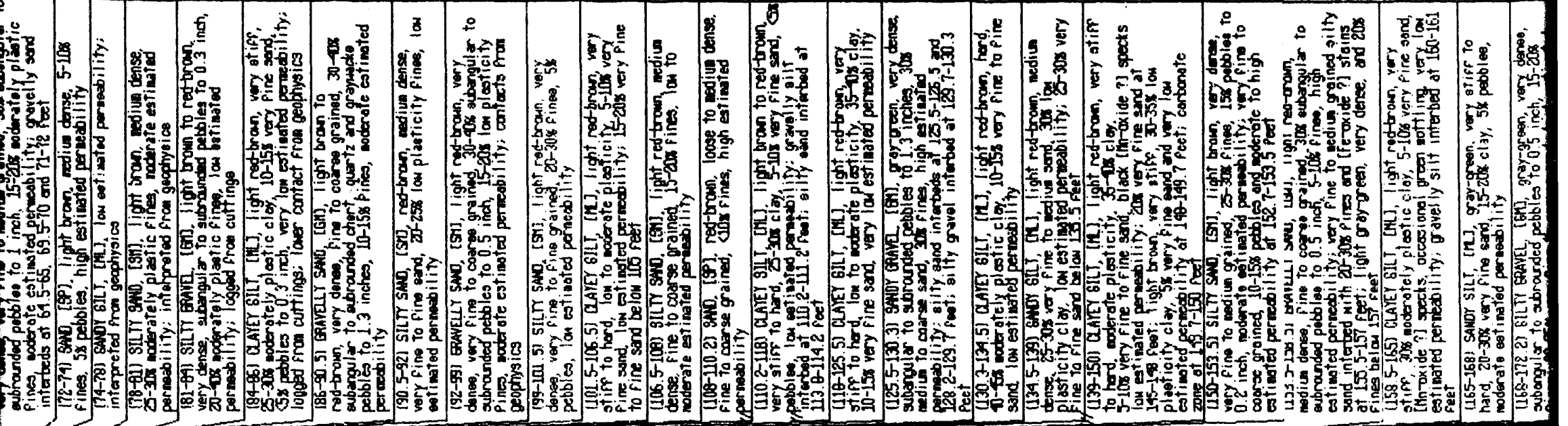
Intand

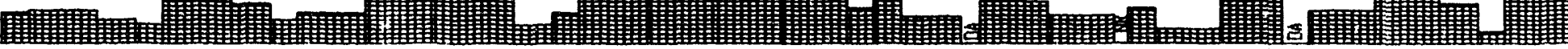

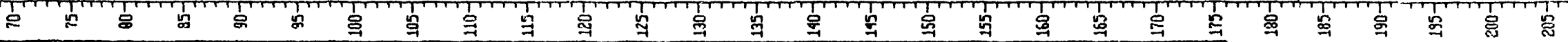

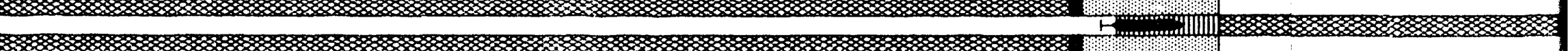

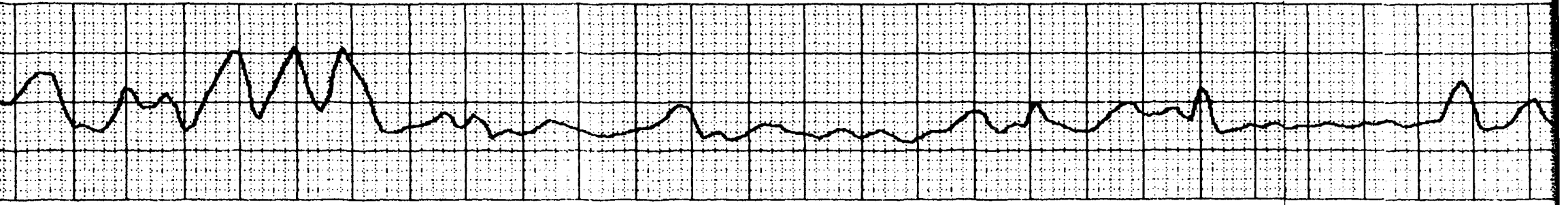

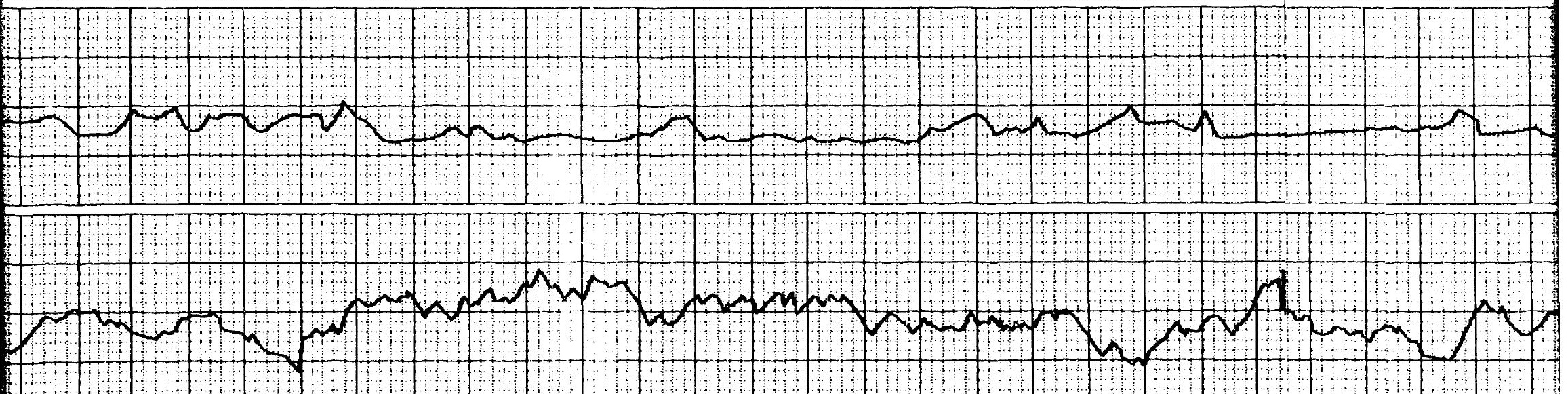

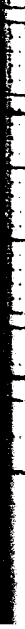




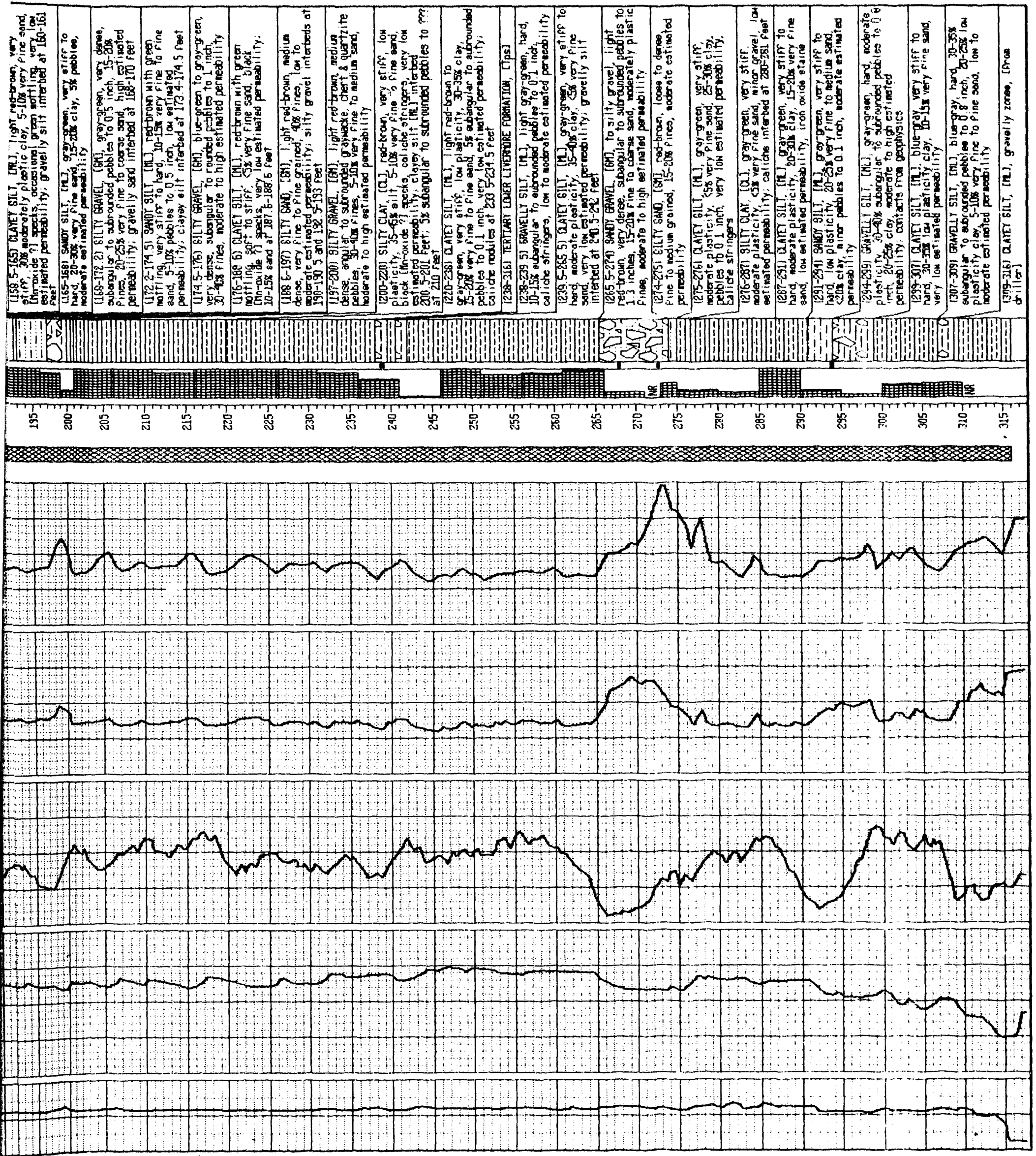




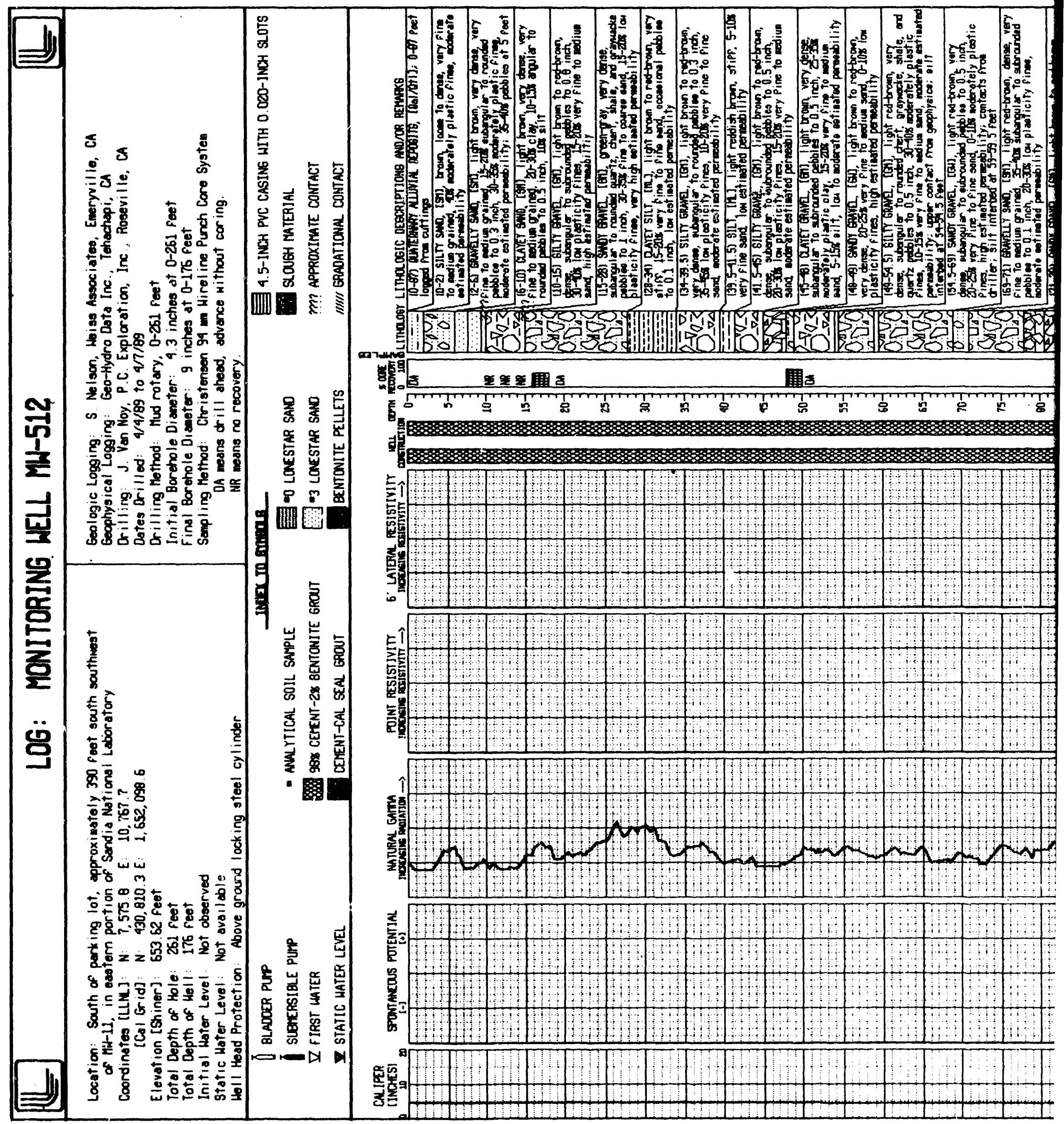




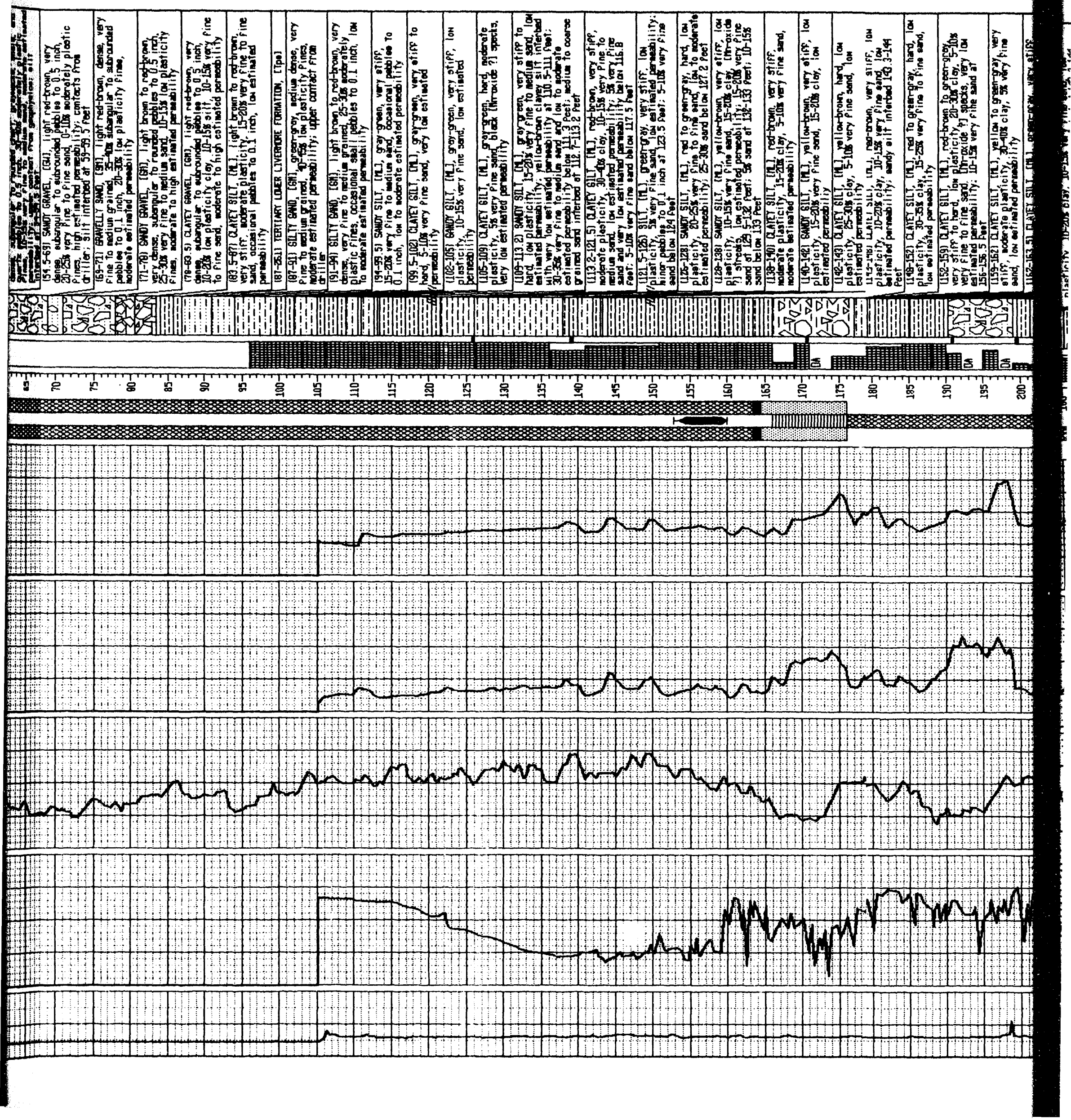




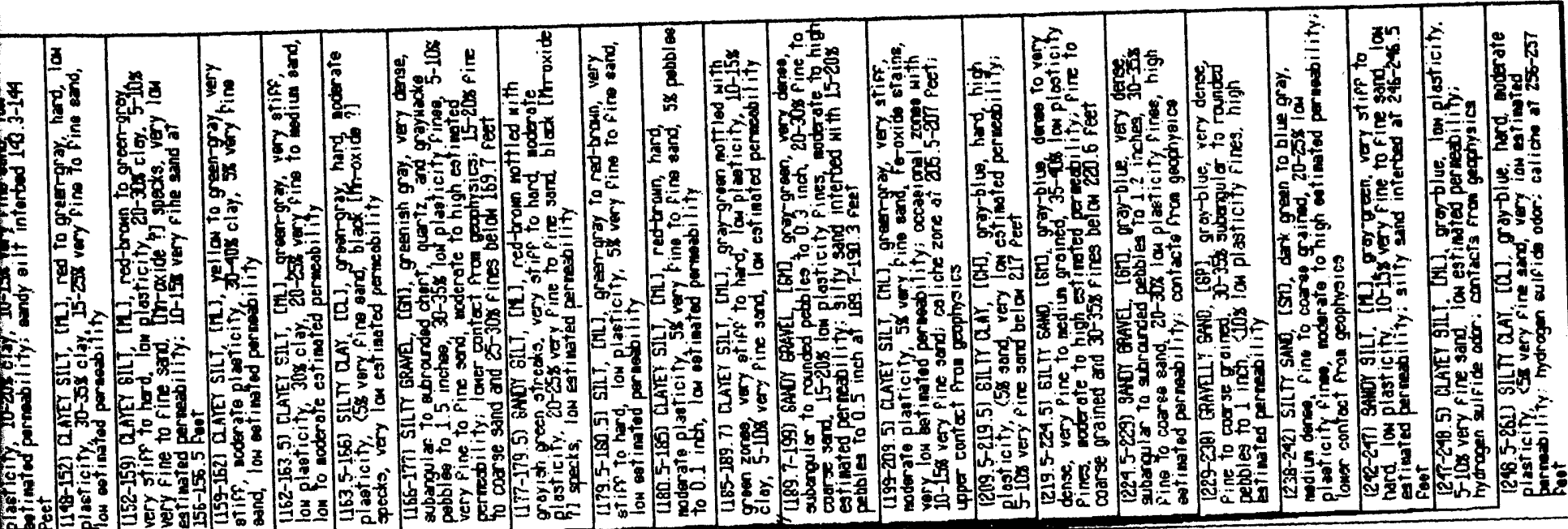

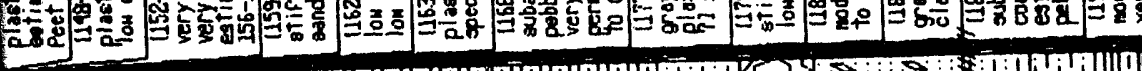

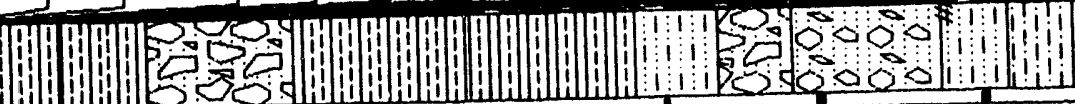

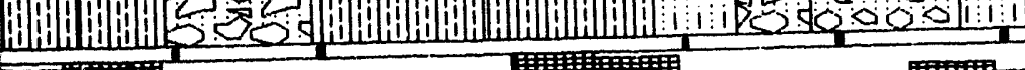

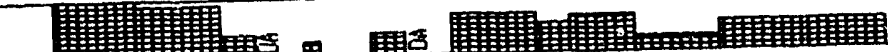

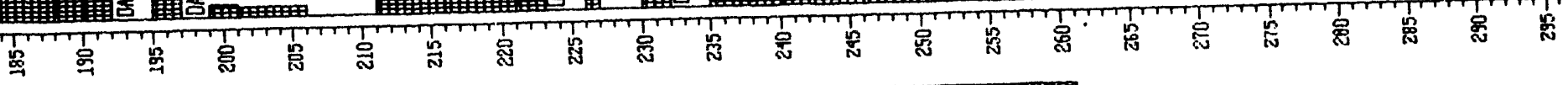

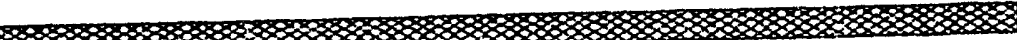

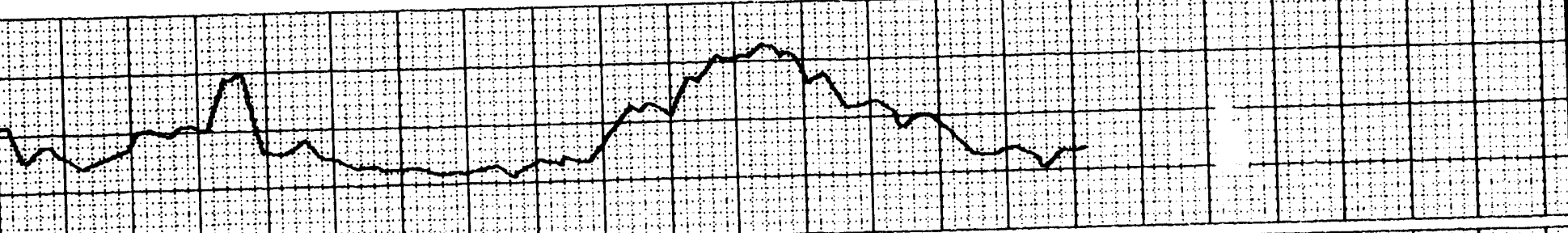

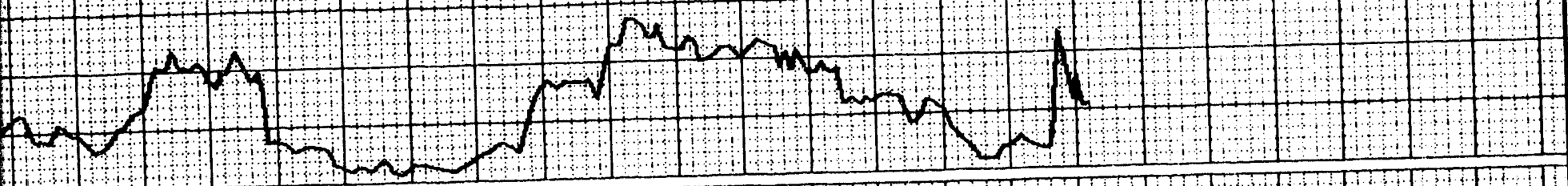

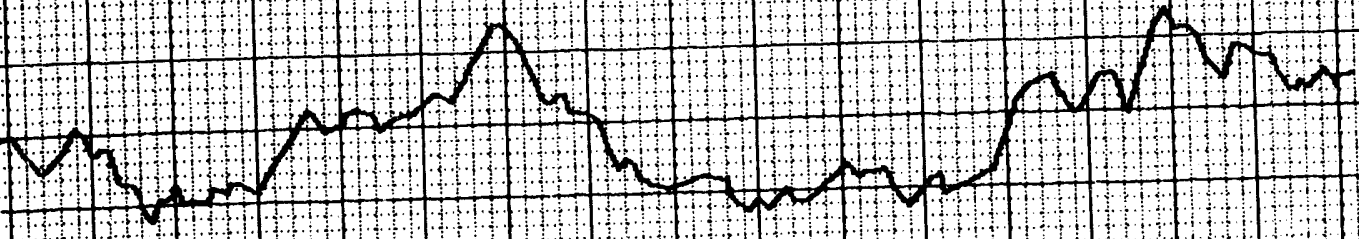

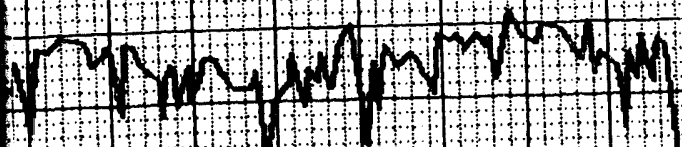

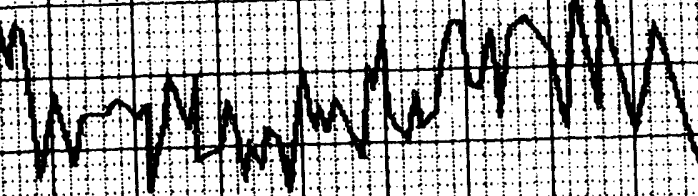




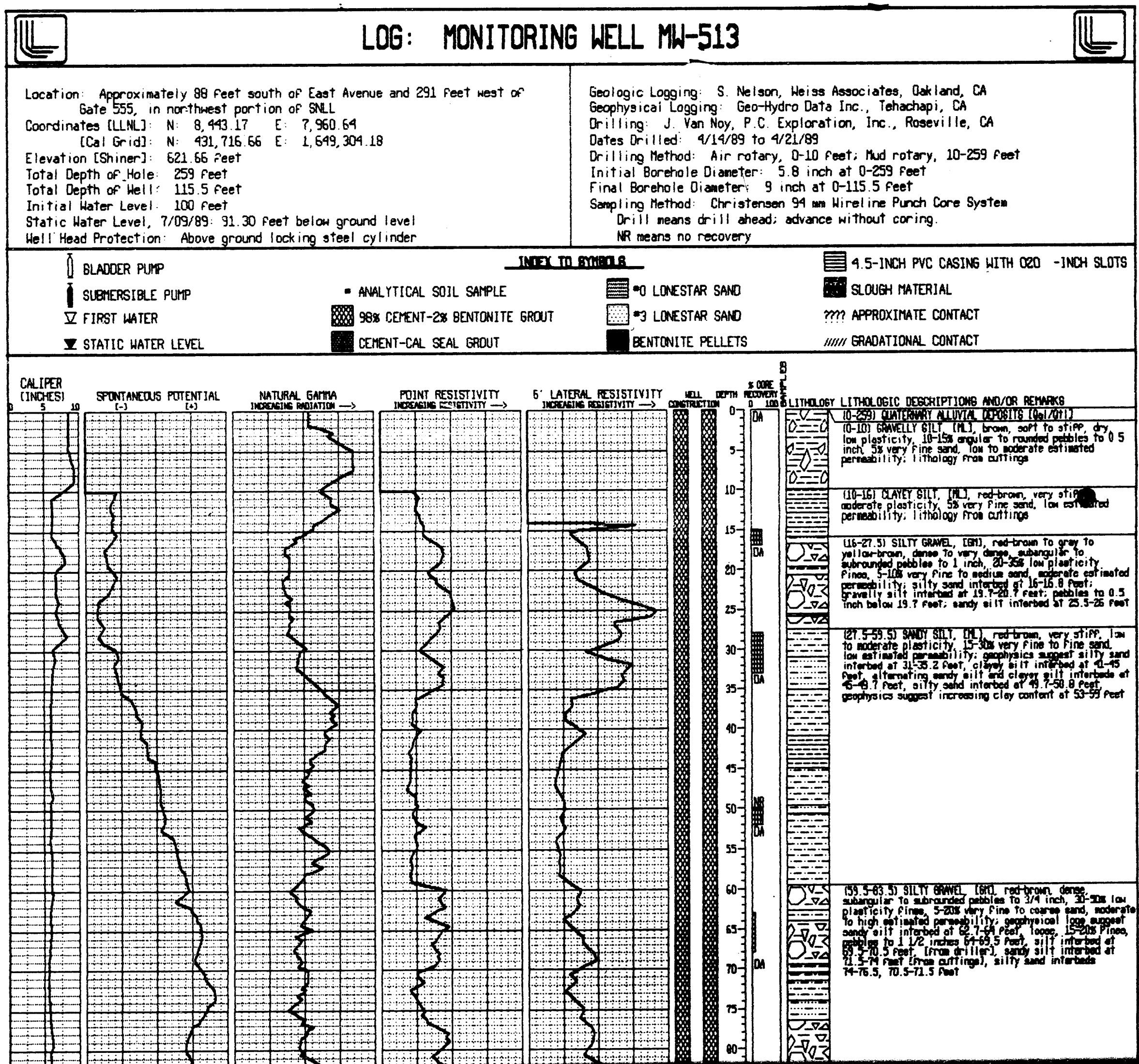




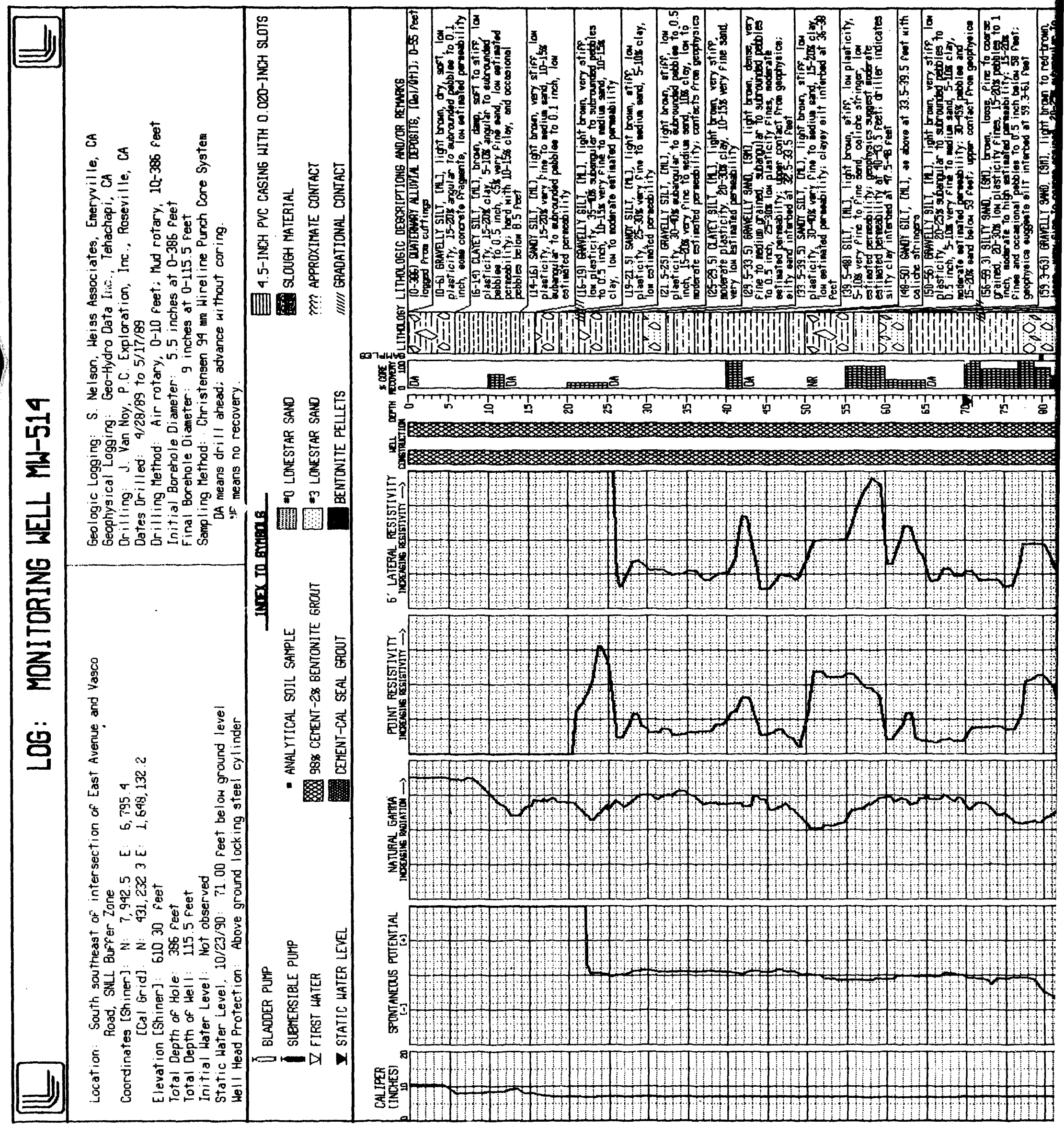




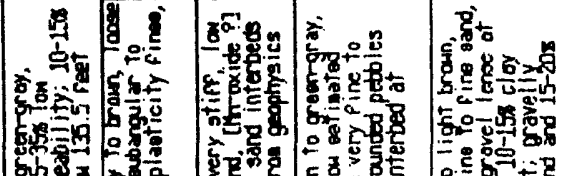

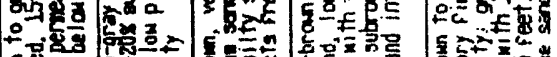

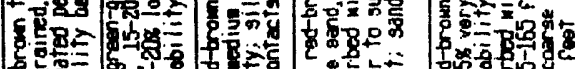

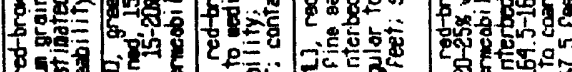
25. 空

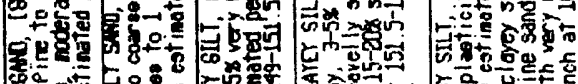

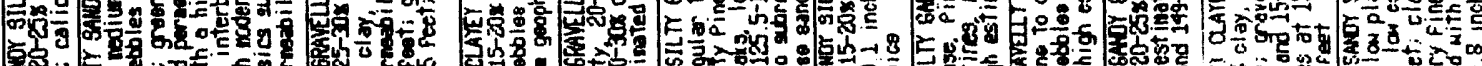

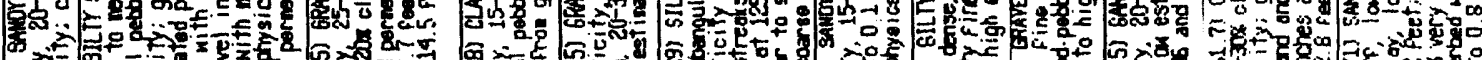
作 tro

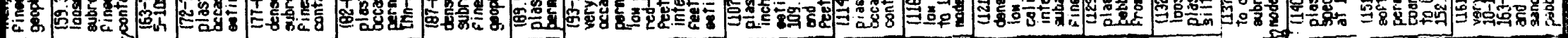
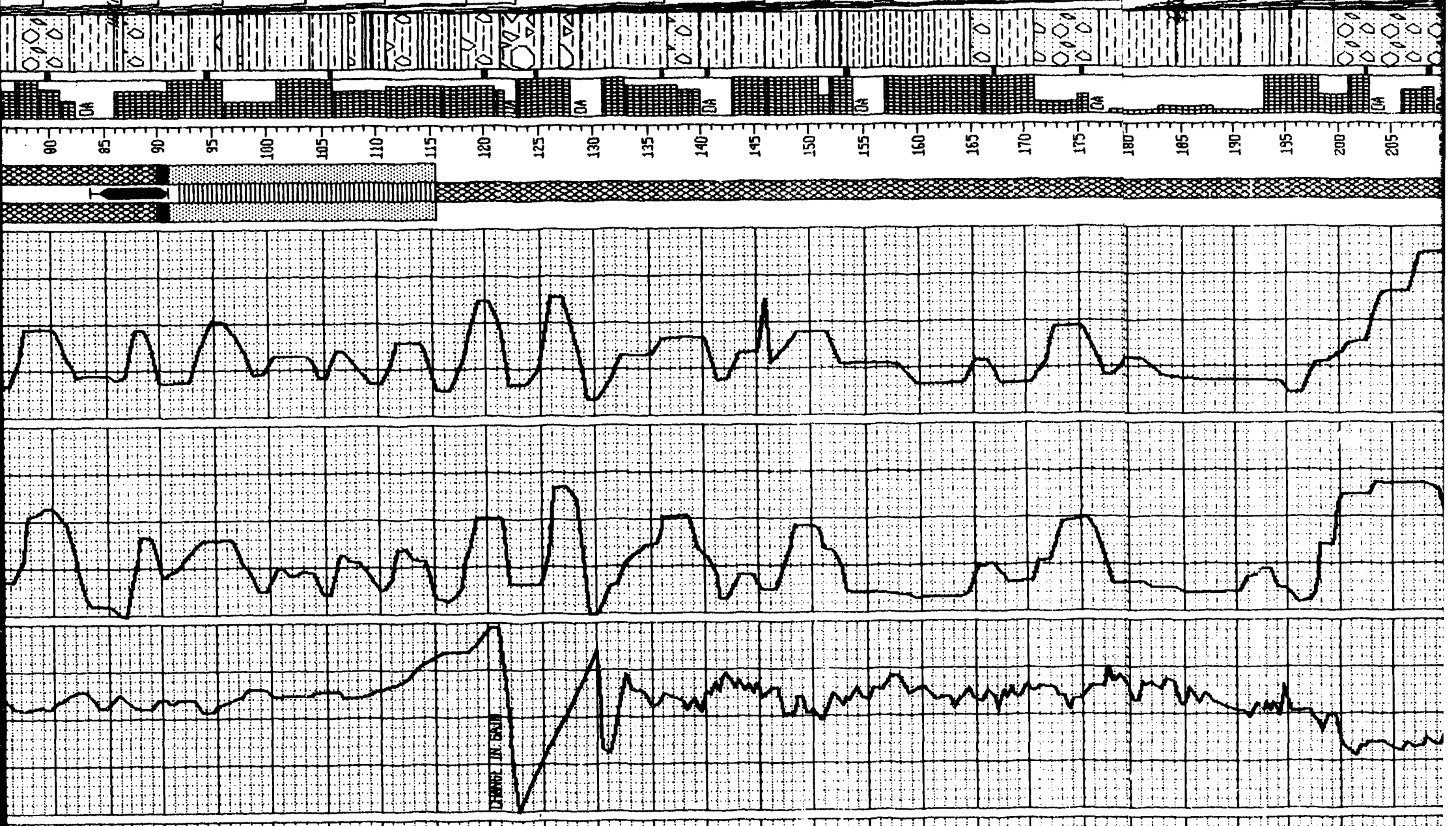

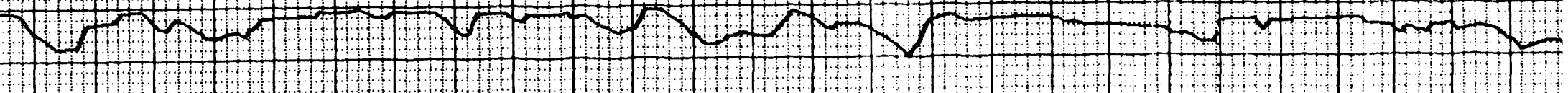



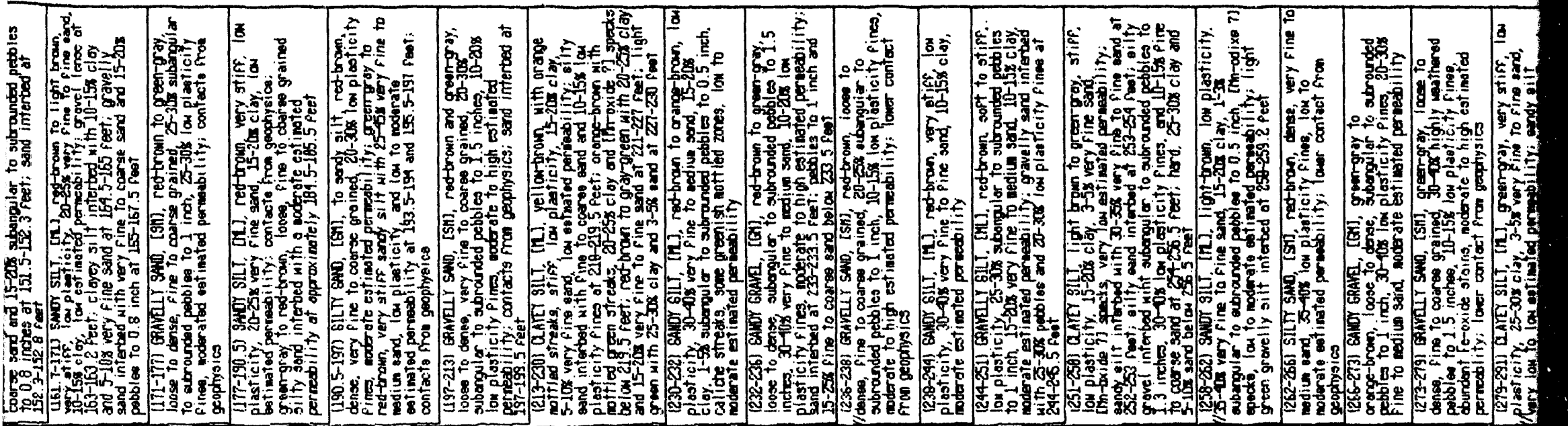

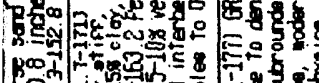

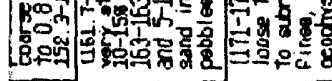

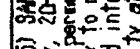

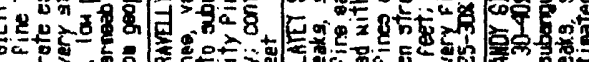

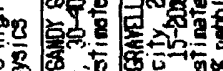

等

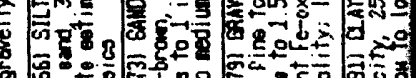

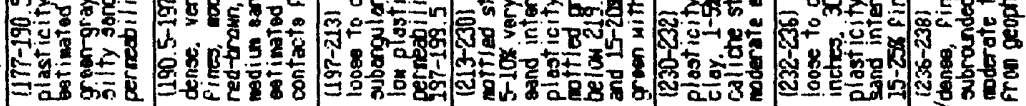

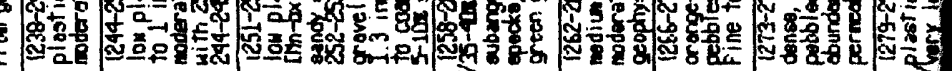

00000 .

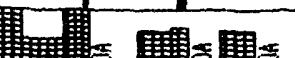

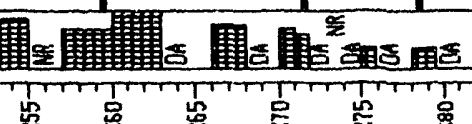

i l 電占

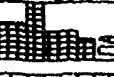
1
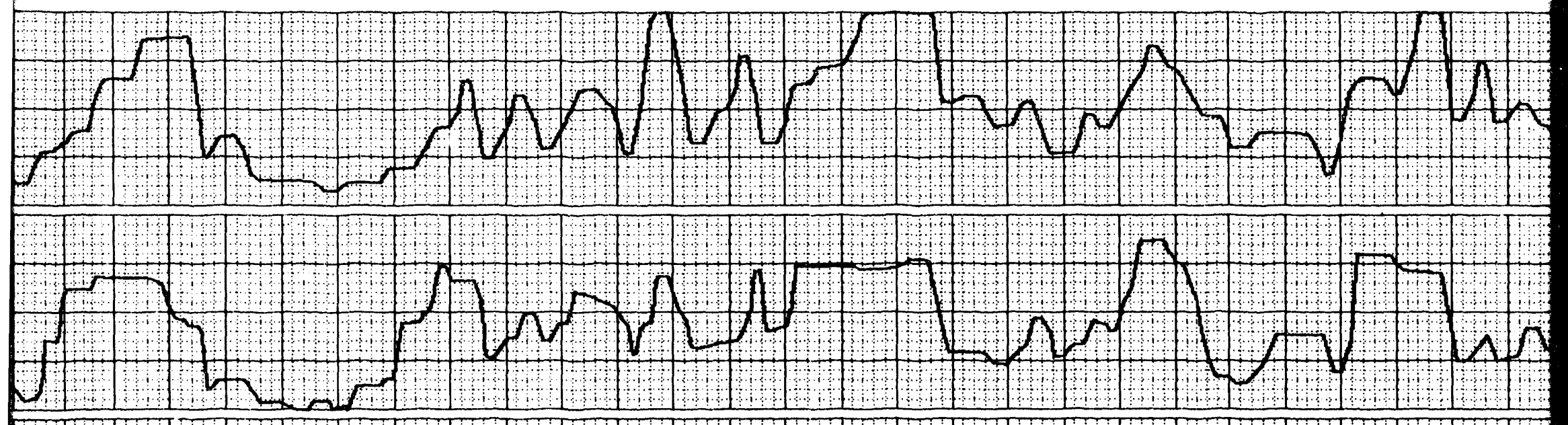

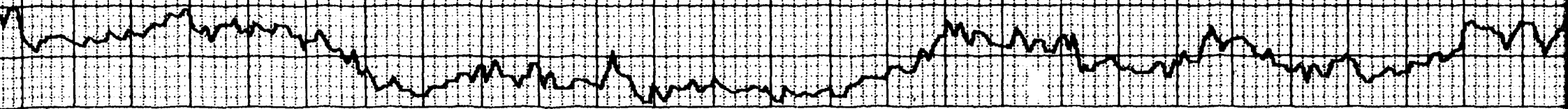




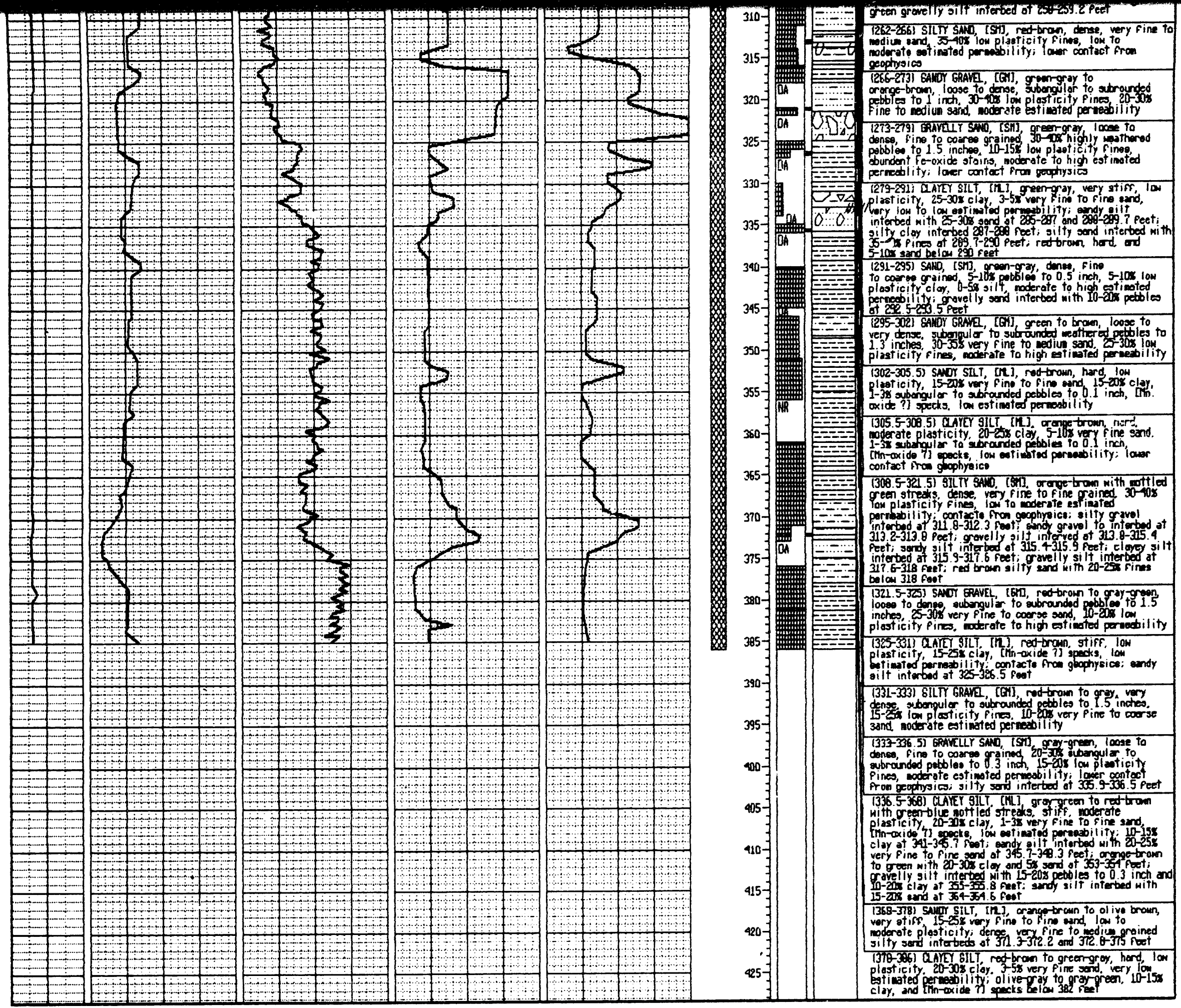




\section{LOG: MONITORING WELL ML-515}

Location: Approximately 900 feet west of Vasco Road, widway between Patterson Pass Road and railiraad tracks, nor thwest of LLNL Coordinates [LIML]: N: $19,492.64$ E: $5,549.66$

[CAL GR]D]: $N: 437,798,54 \mathrm{E}: 1,646,977,99$

Elevation [Shiner]: $555.76 \mathrm{Ft}$; [Ground]: $555.30 \mathrm{ft}$

Total Depth of Hole: 211 feet

Total Depth of Hell: 78 feet

Initial Hater Level: Not observed; possibly near 30 feet

Static Water Level, 5/30/89: less than 58 feet below ground level Well Head Protection: Above ground locking steel cylinder

\section{BLADOER PUTP \\ SLEMERSIBLE PUMP \\ Q FIRST WATER}

I STATIC WATER LEVEL
- analytical soll sample

988 CEMENT-2Х BENTONITE GROUT

CEMENT-CAL SEAL GROUT

Geologic Logging: S. Nelson, Heiss Associates, Emeryvillle, CA

Geophysical logging: Geo-Hydro Data Inc., Tehachapi, CA

Orilling: J. Ven Noy, P.C. Exploration, Inc., Roseville, CA Dates Drilled: 5/22/89 to 5/24/89

Drilling Method: Mud rotary, 0-211 feet

Initial Borehole Diameter: 5.8 inches of $0-211$ feet

Final Borehole Diameter: 9 inches at 0-78 feet

Sampling Method: Christensen 94 mirel ine Punch Coring System DA means or ill ahead; advance without coring. NR means no recovery.

INTXIn mats

" 0 LONESTAR SAND - y LONESTAR SAND

BENTONITE PELLETS
4.5-INCH PVC CASING WITH 0.020-INCH SLOTS SLOUGH MATERJAL

m? APPROXIMATE CONTACT

IIIII GRADATIONAL CONTACT

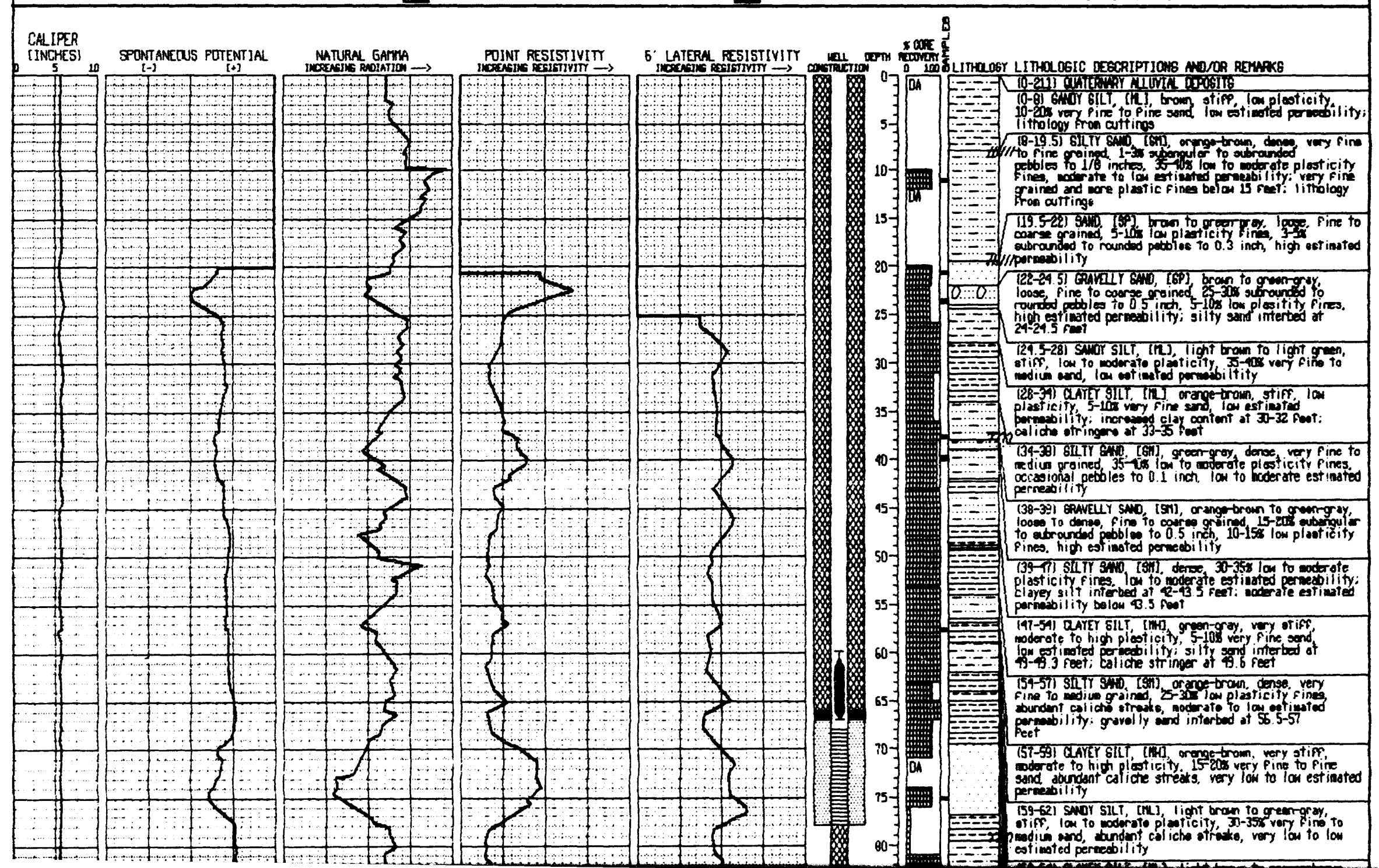




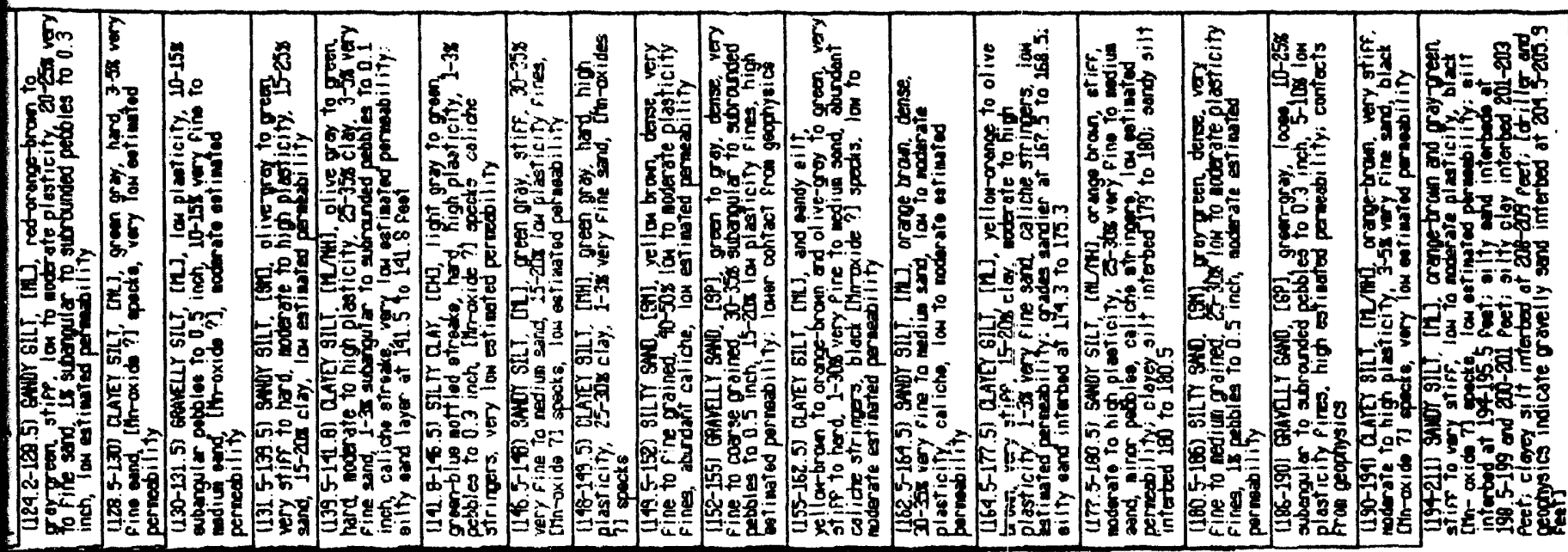

10s

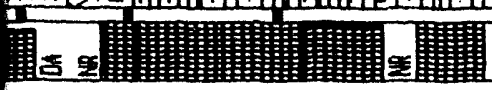

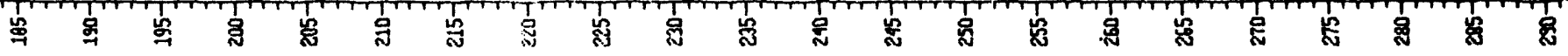
(1)

\begin{tabular}{|l|l|l|l|l|l|l|l|l|l|l|l|l|l|}
\hline \hline \\
\hline
\end{tabular}




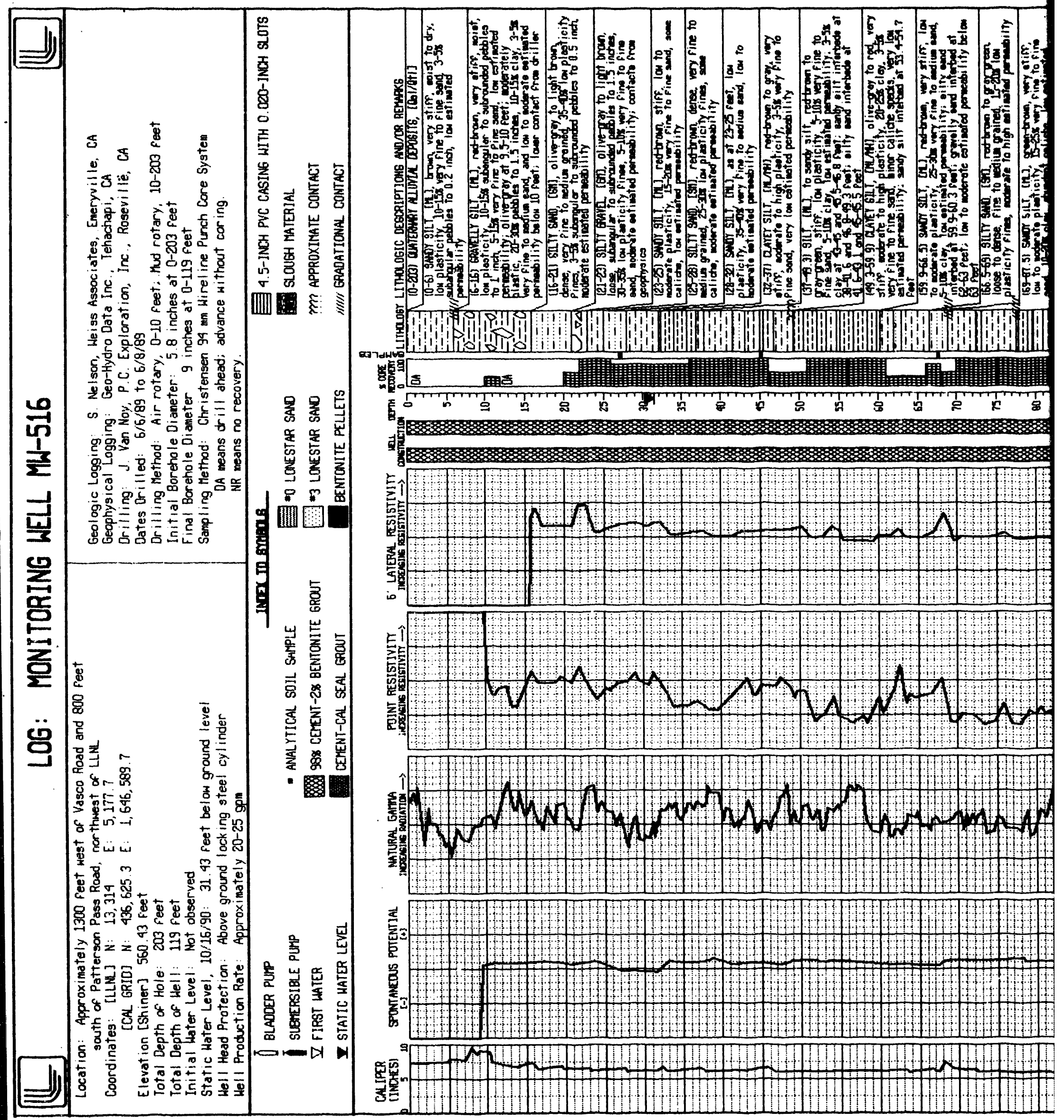




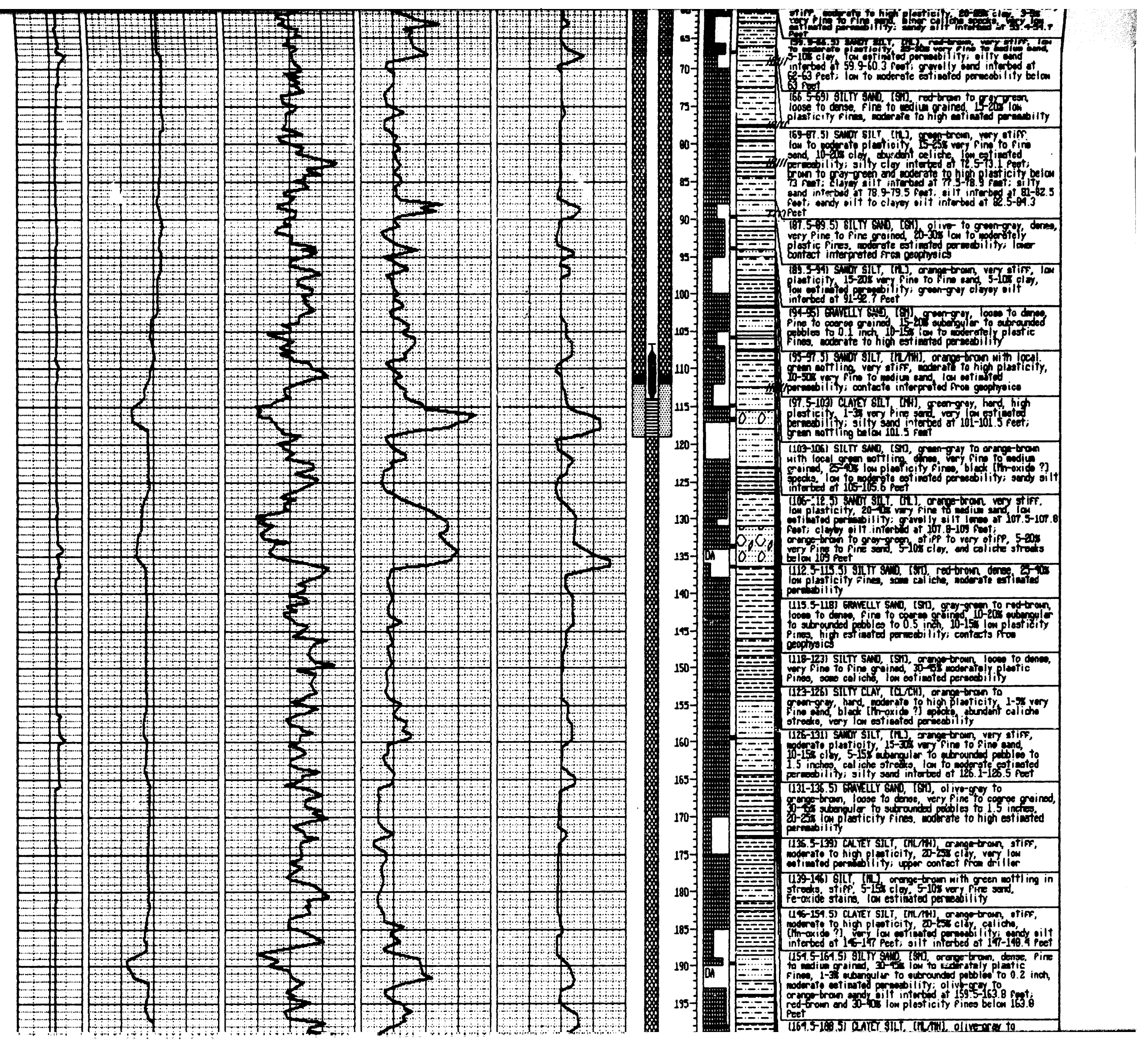




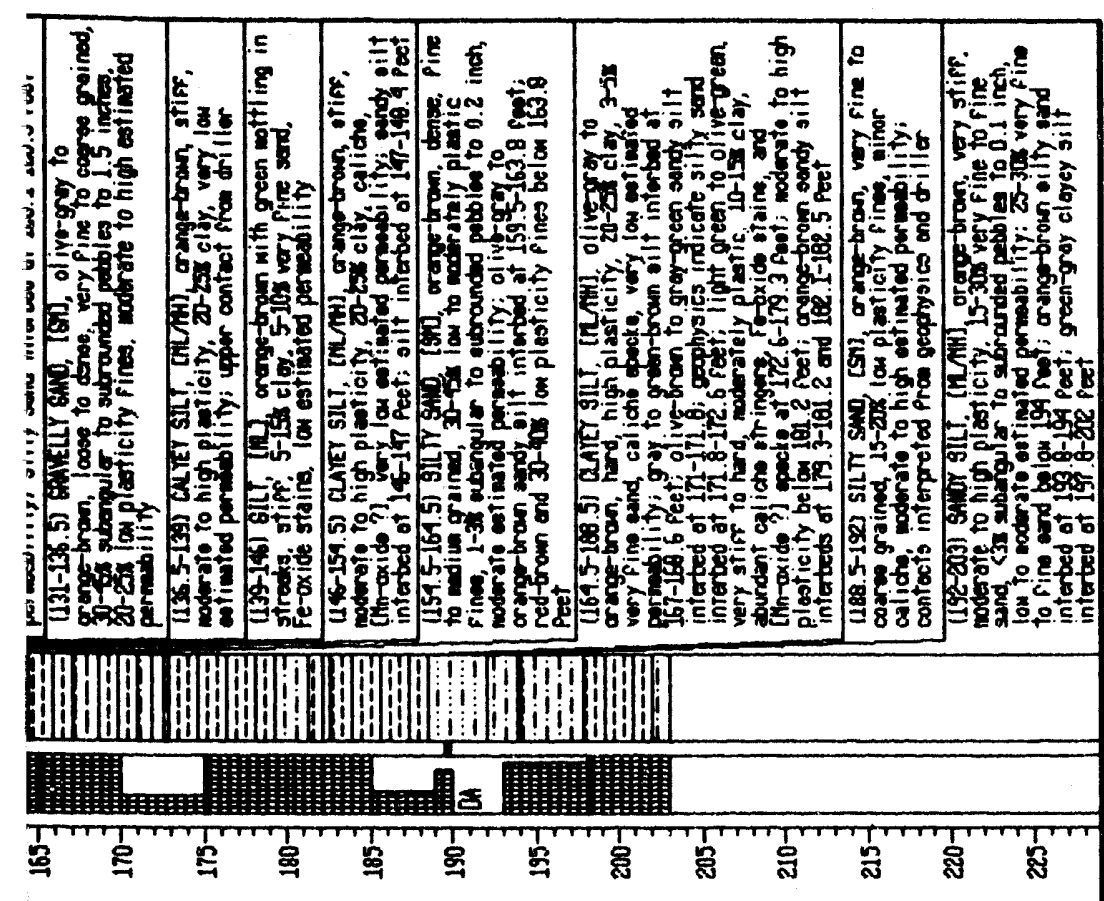
极

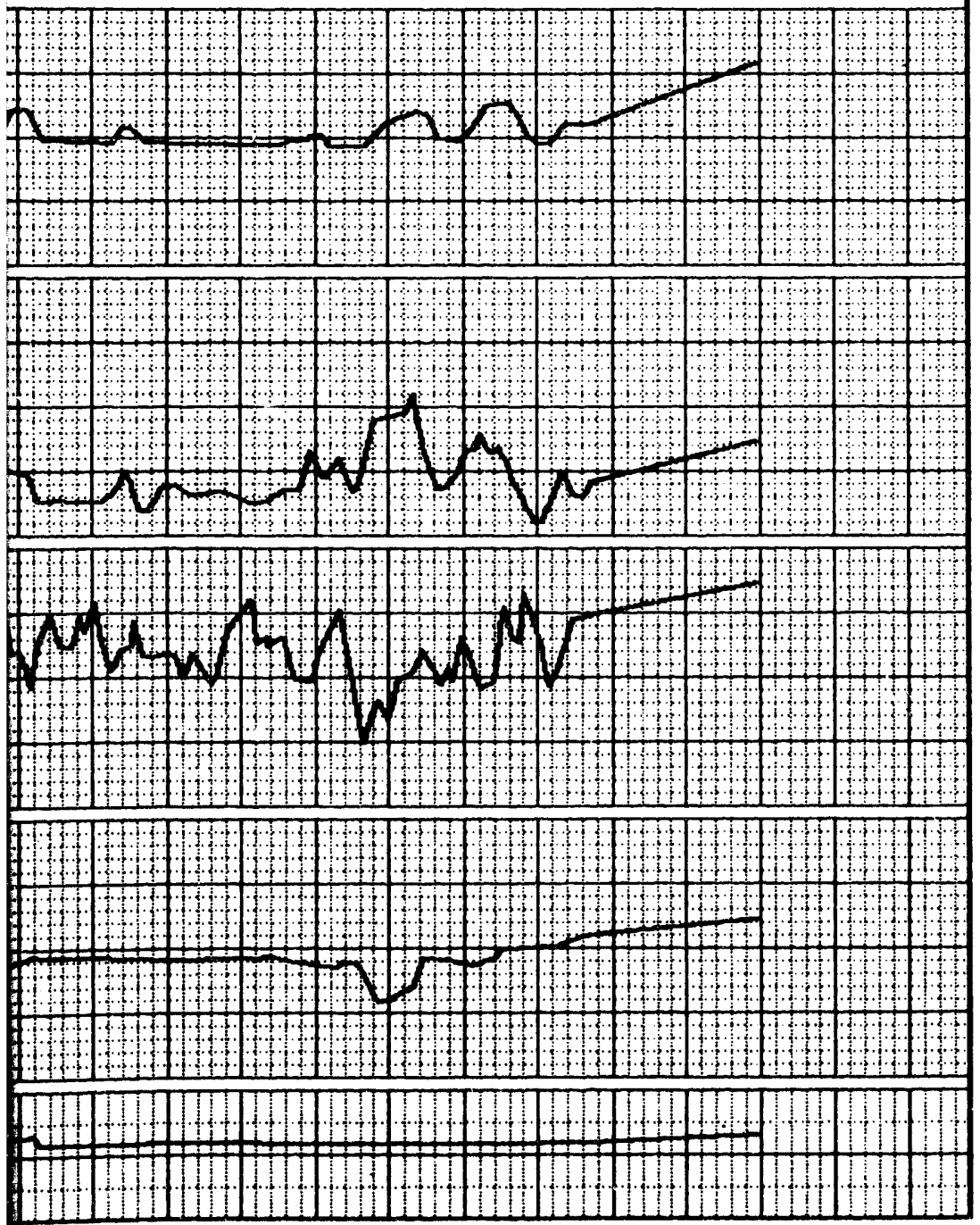




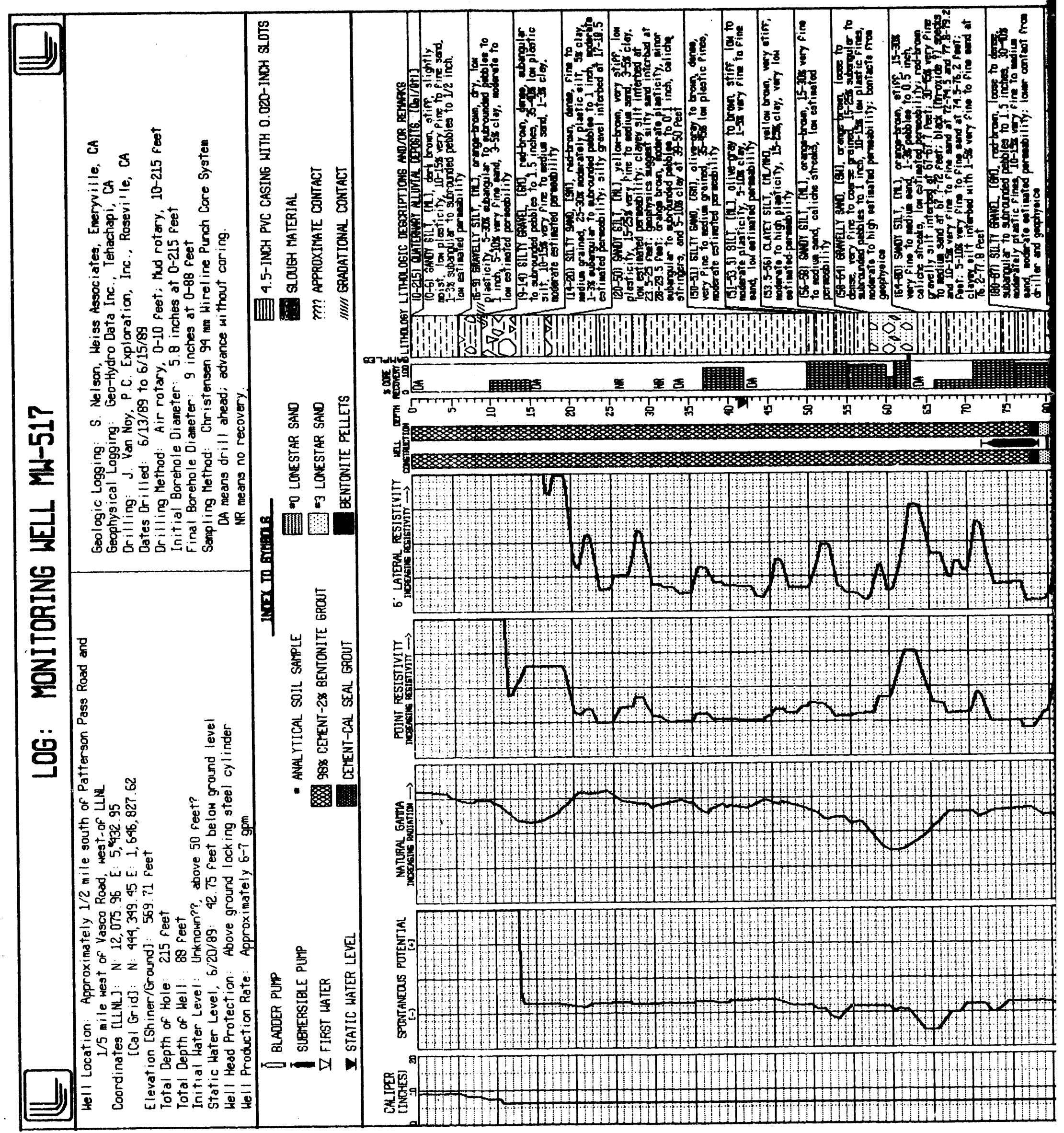




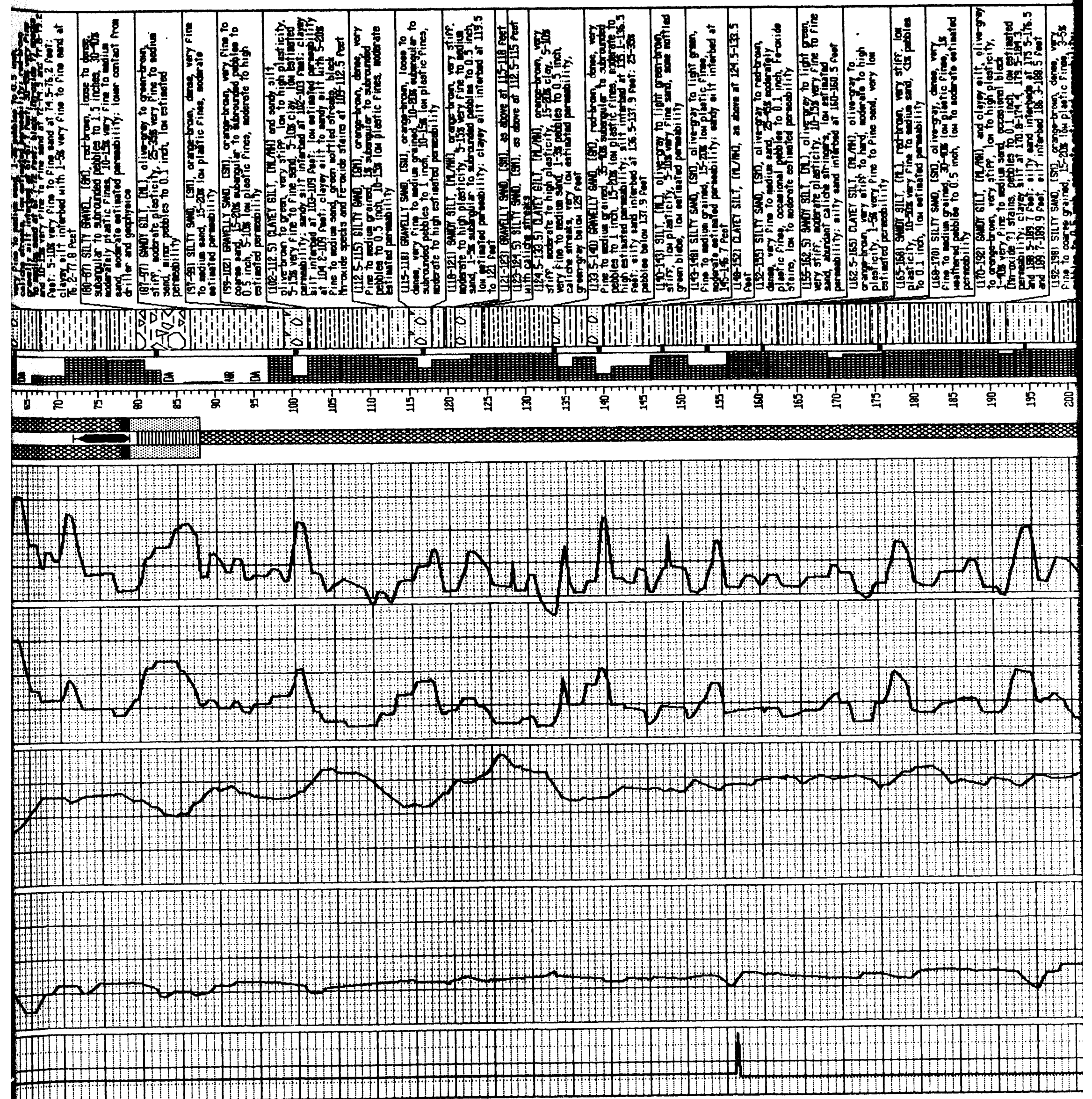




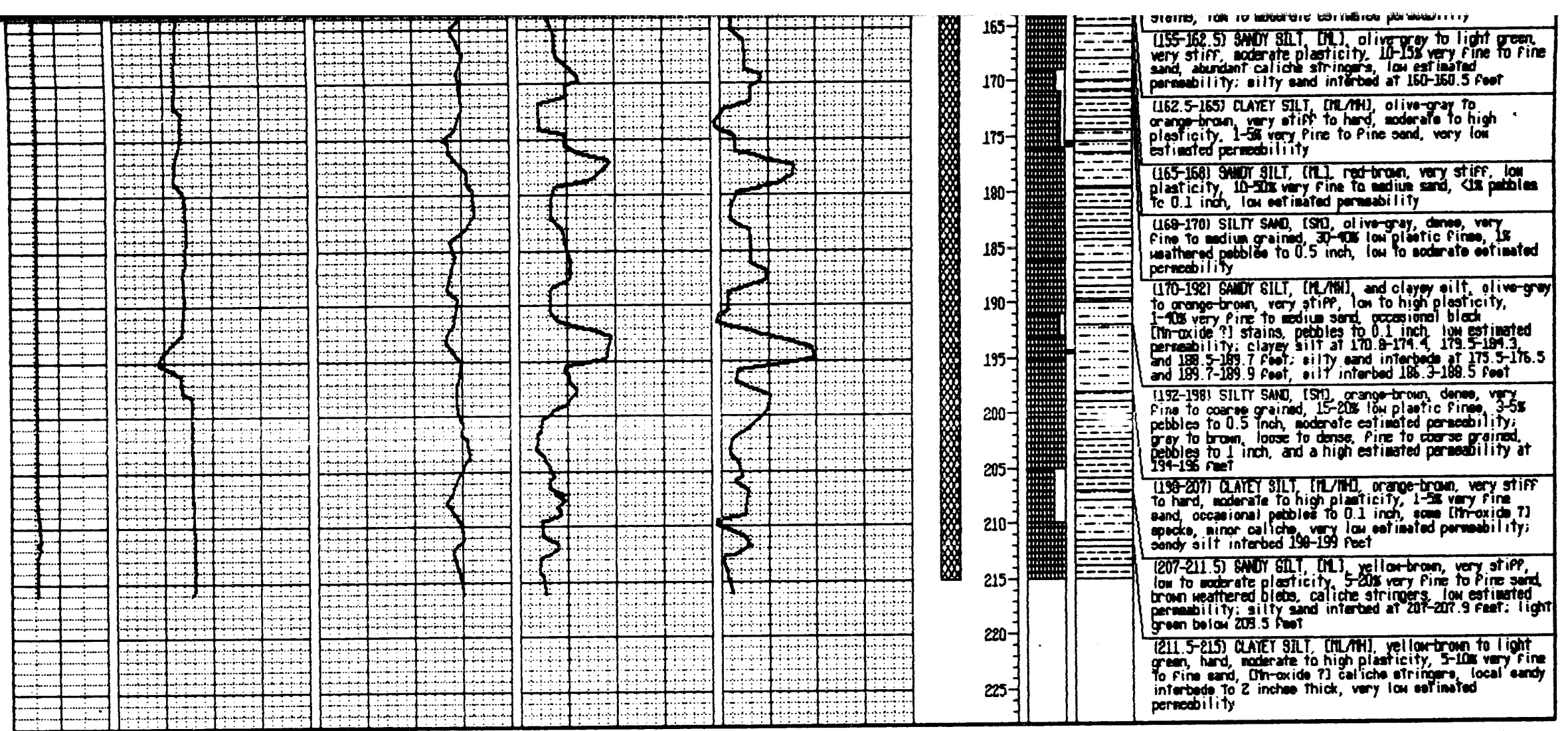




\section{LOG: MONITORING WELL MW-518}

Location: In the LLM Buffer zone, north of East Avenue, east of lasco Road, approximately 30 nest of MH-262

Coordinates [LLML]: $\mathrm{N}: 8,883.9$ E: $6,392.7$

[Cal Grid]: N: 432, 179.1 E: 1,647,742.?

Elevation [Shiner]: 601.38 feet

Total Depth of Hole: 251 feet

Total. Depth of Hell: 139.3 feet

Initial hater Level: 49.5 feet

Static Hater Level: Not avai iable

Well Head Protection: Above ground locking steel cylinder

Well Production Rate: Approximately $2.5 \mathrm{gpm}$

DLADDER PUMP

1 SUBuERSIBLL PUTP

Z FIRST WATER

I STATIC WATER LEVEL

Feologic Logging: S. Nelson, Heiss Associates, Emeryville, CA

Geophysical Logging: Geo-Hydro Data Inc., Tehachapi, CA

Orilling: I Van Nay P.C. Exploration, Inc. Roseville, CA

Dates Drilled: 7/17/89 to 7/27/89

Drilling Method: Mud rotary, 0-251 feet

Initial Borehole Dianeter: 5.8 inches at 0-251 feet

Final Borehole Diameter: 9 inches of 0-139.3 feet

Sampling Method: Christensen 94 mirel ire Punch Core System

DA means of ill ahead, advance without coring. NR means no recovery.

\section{IIT m mmans}

- analytical soil sample

98\% CEMENT-2\% BENTONITE GROUT

CEMENT-CAL SEAL GROUT
-O LONESTAR SANO

- o lonestar sano

BENTONITE PELLETS
4.5-INCH PVC CASING WITH O.O2O-INCH SLOTS

SLOUgh matealal

mI APPROXIMATE CONTACT

IIIII GRADATIONAL CONTACT

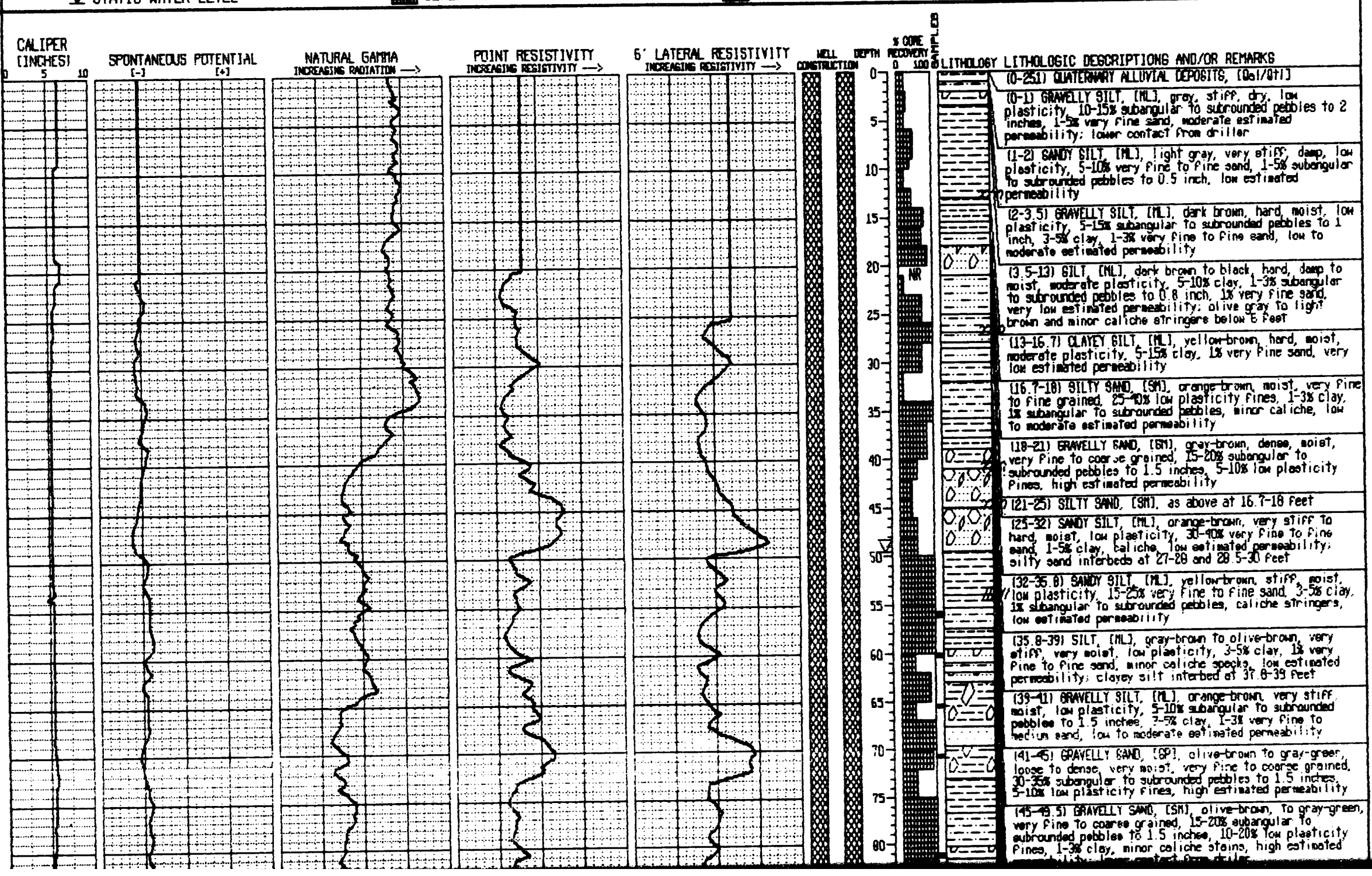




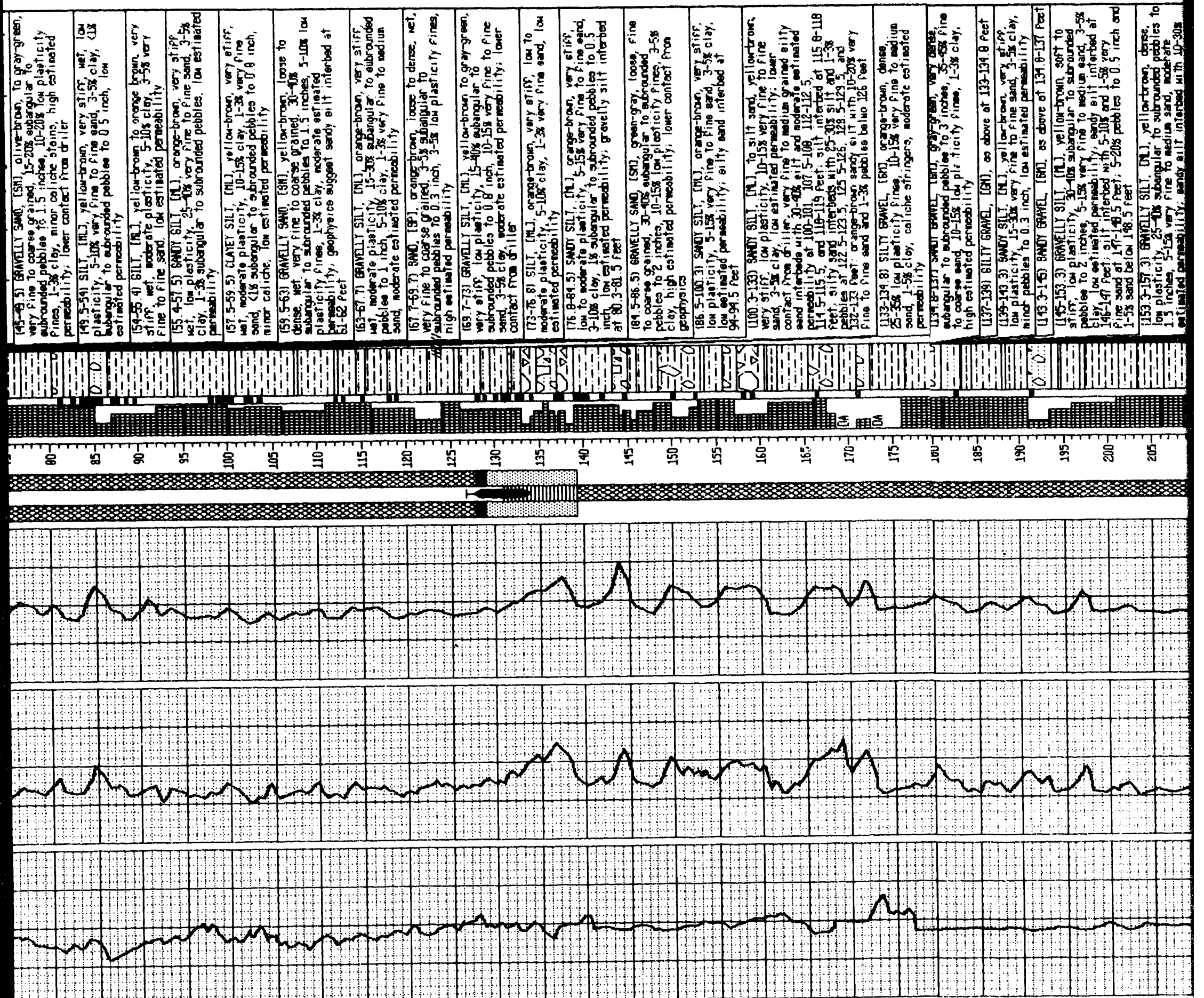




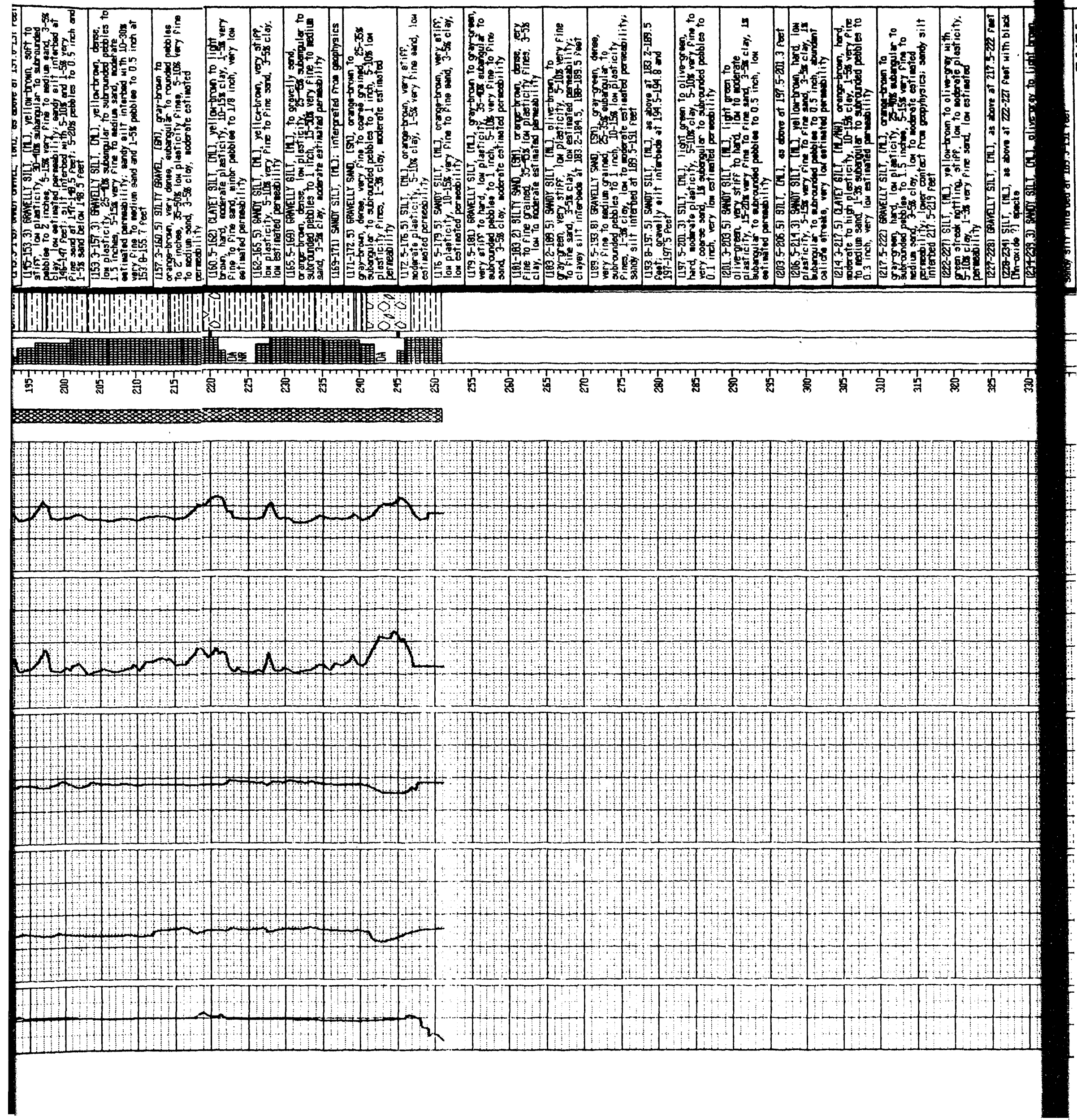



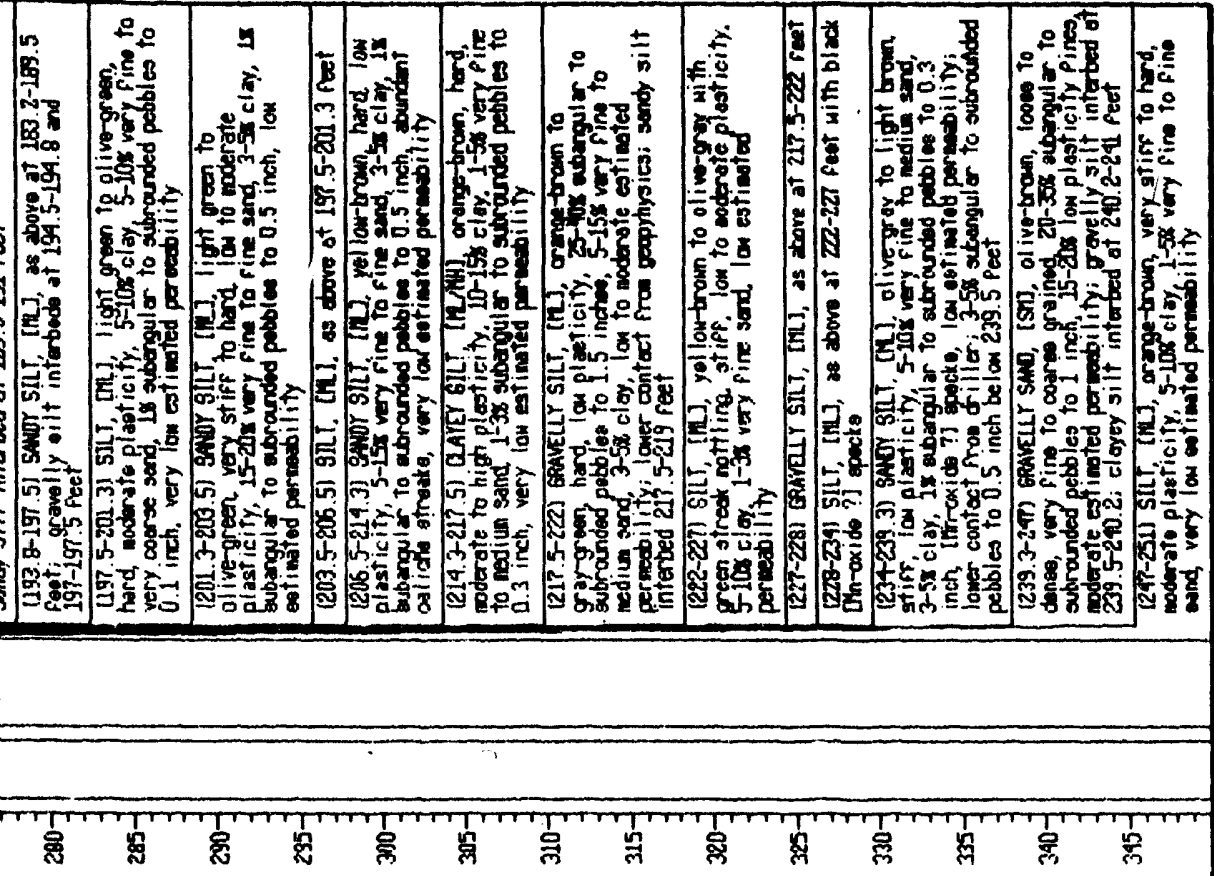

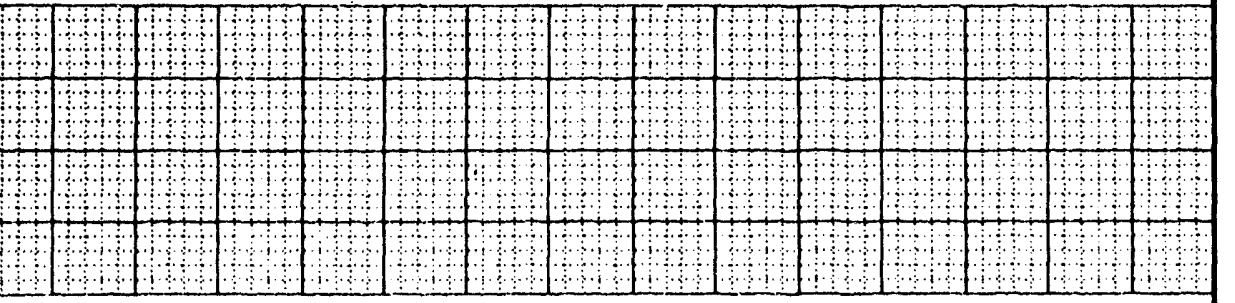

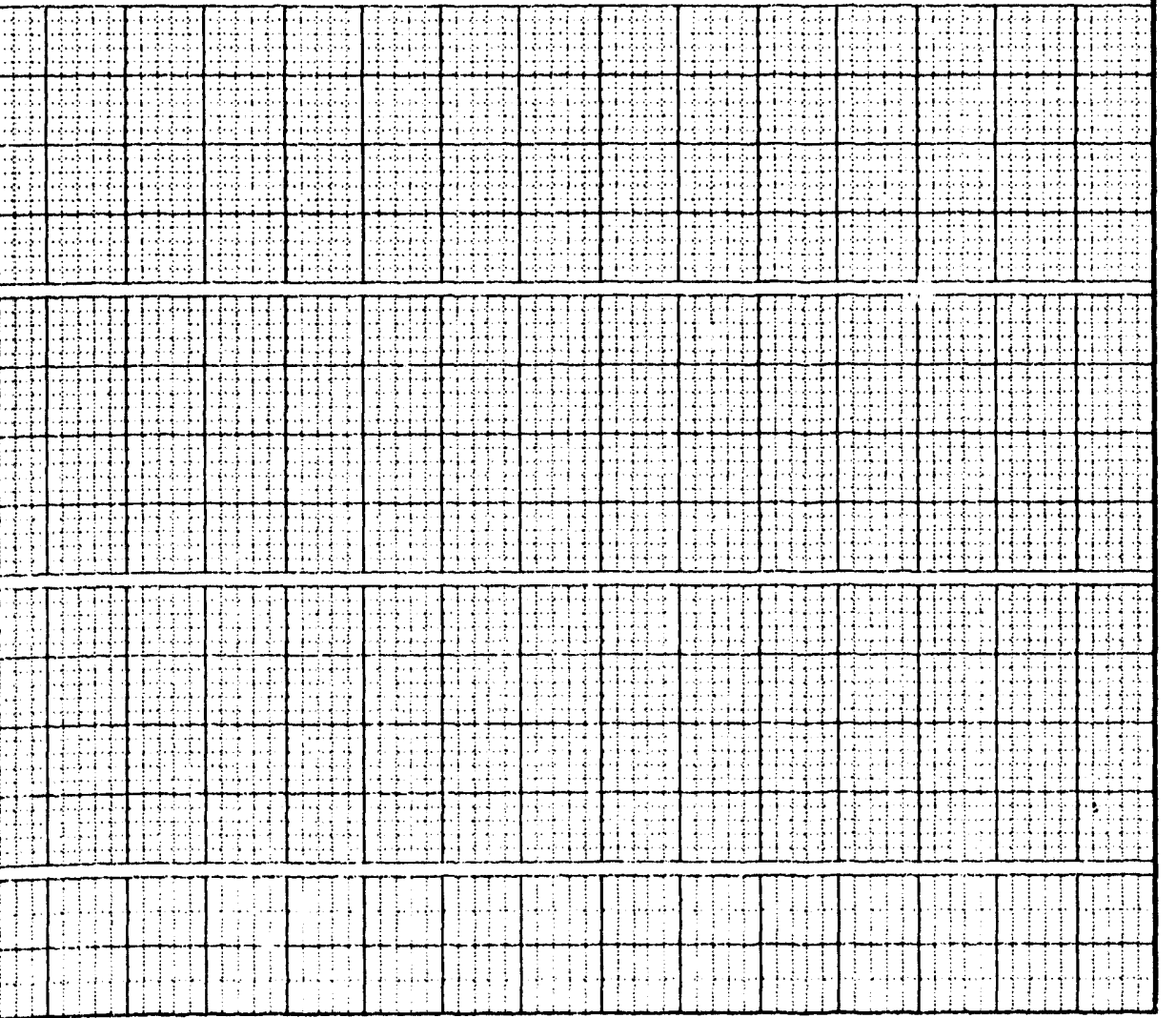




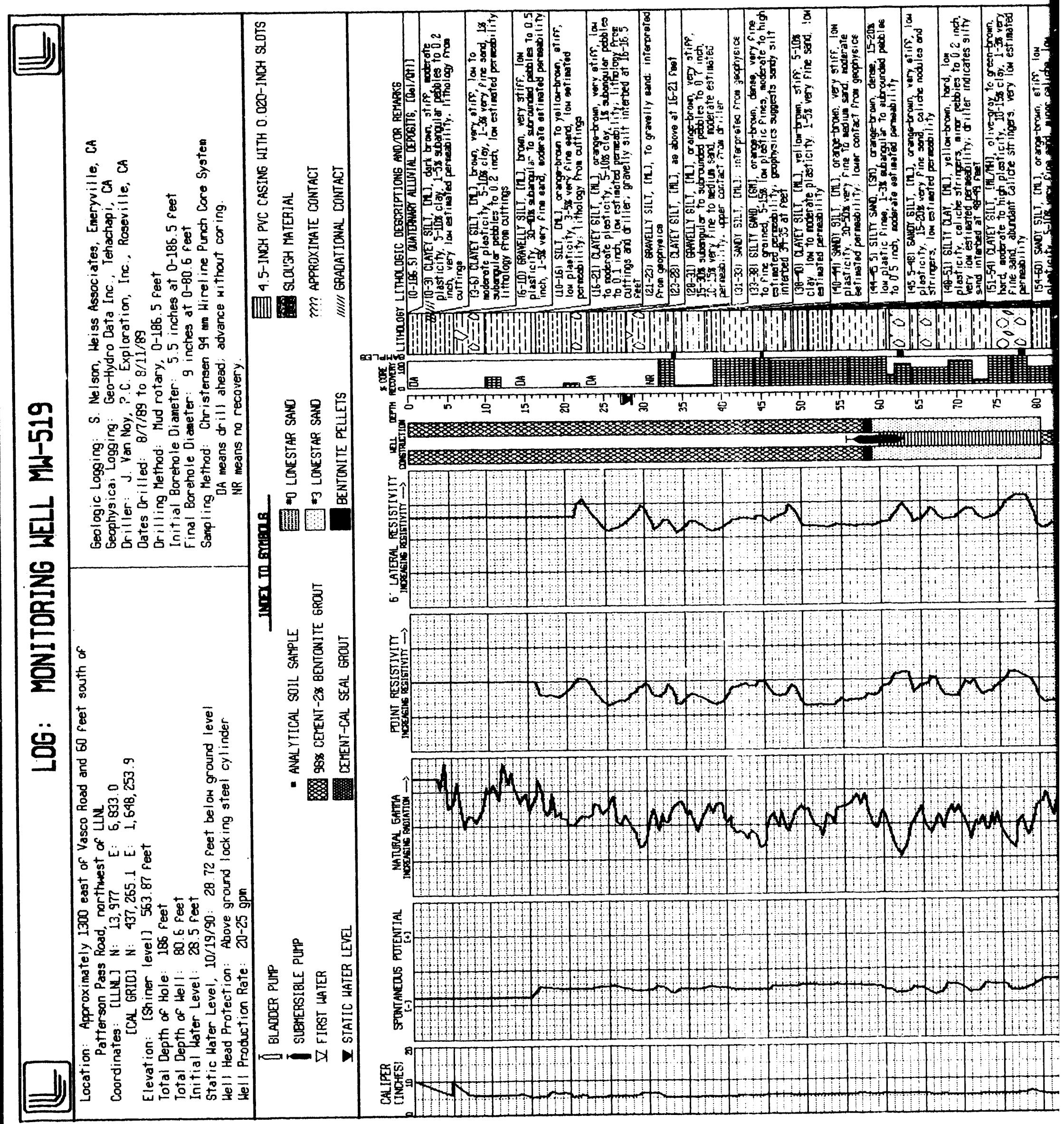




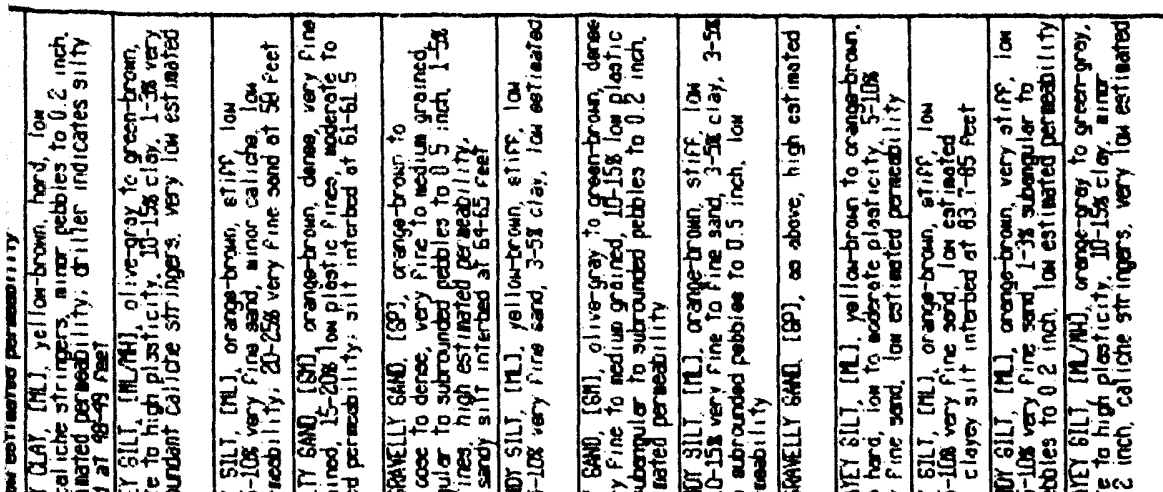

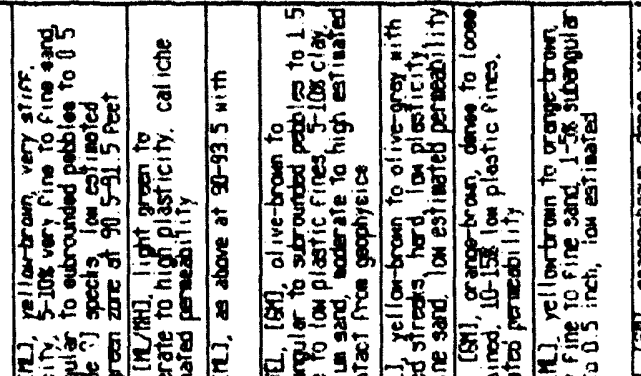

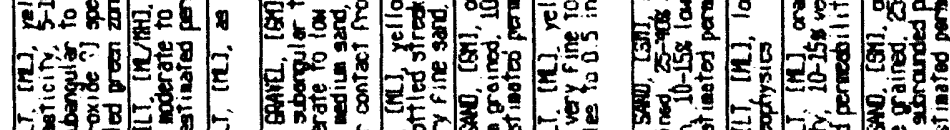

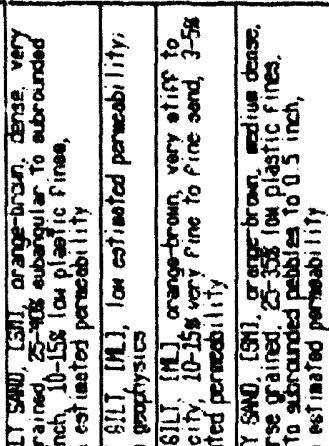

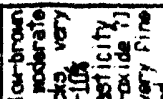
急 35.

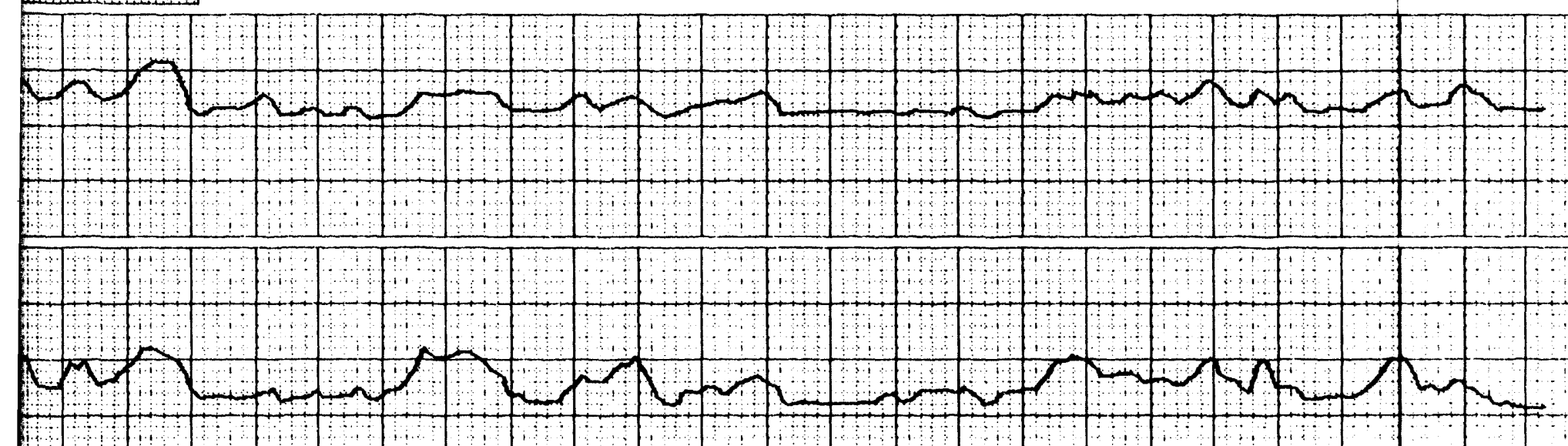

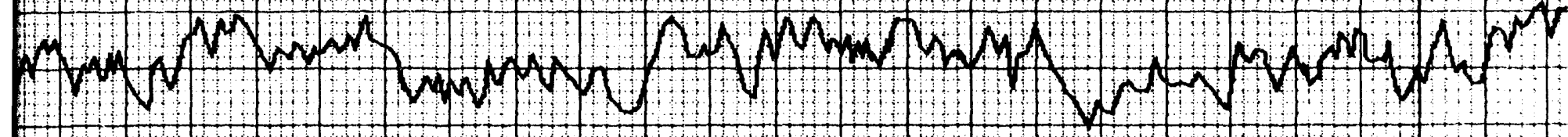




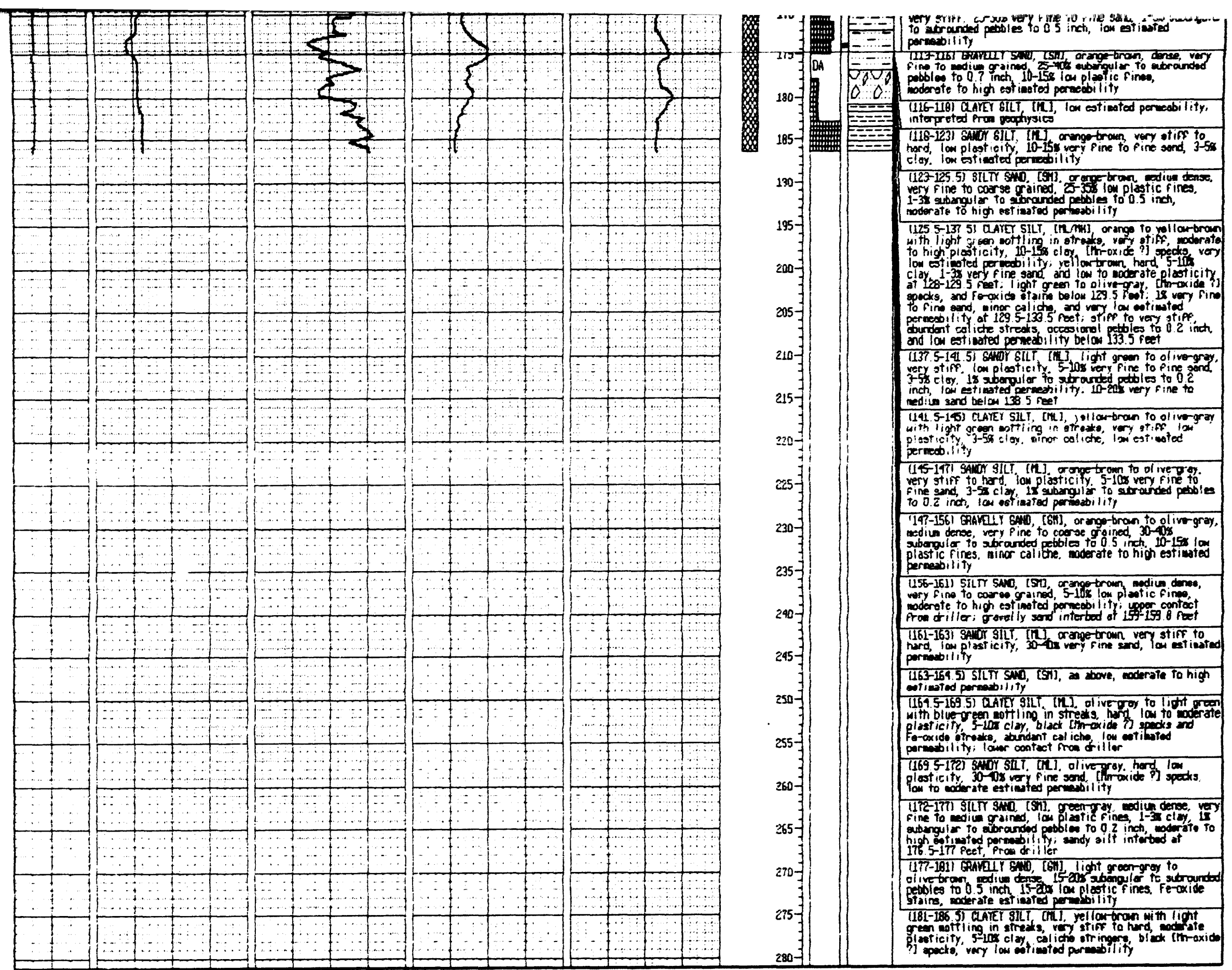




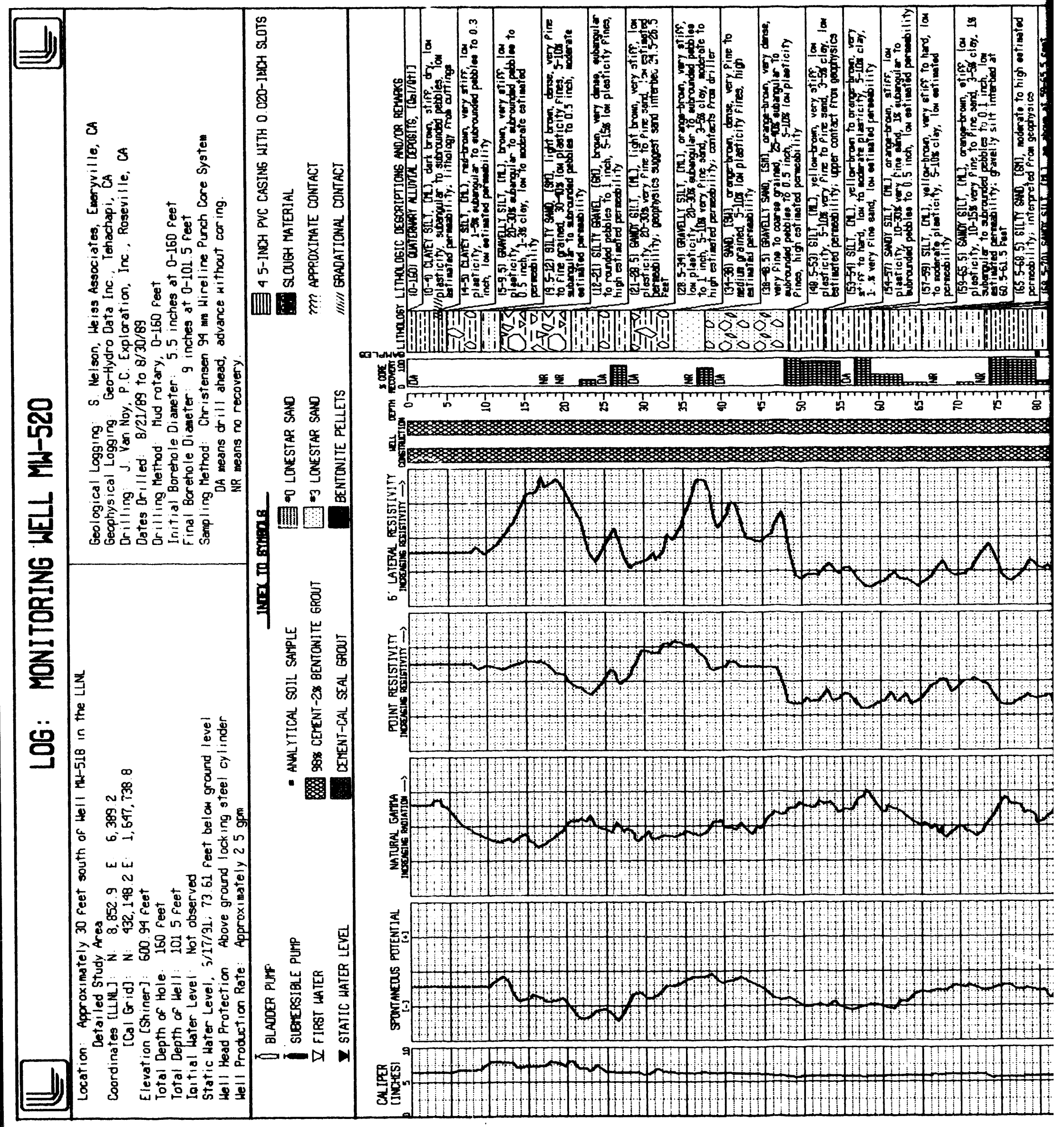




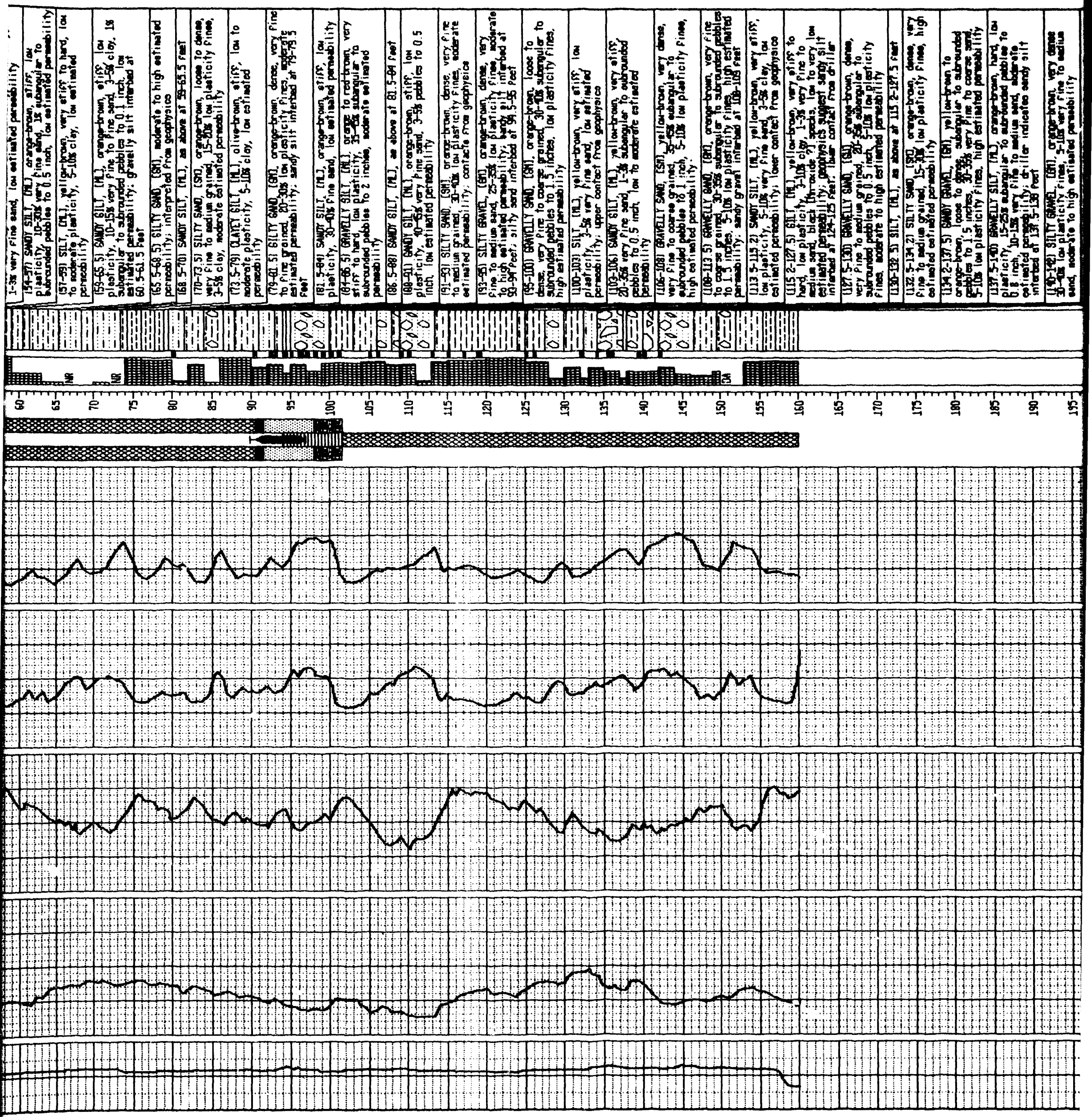




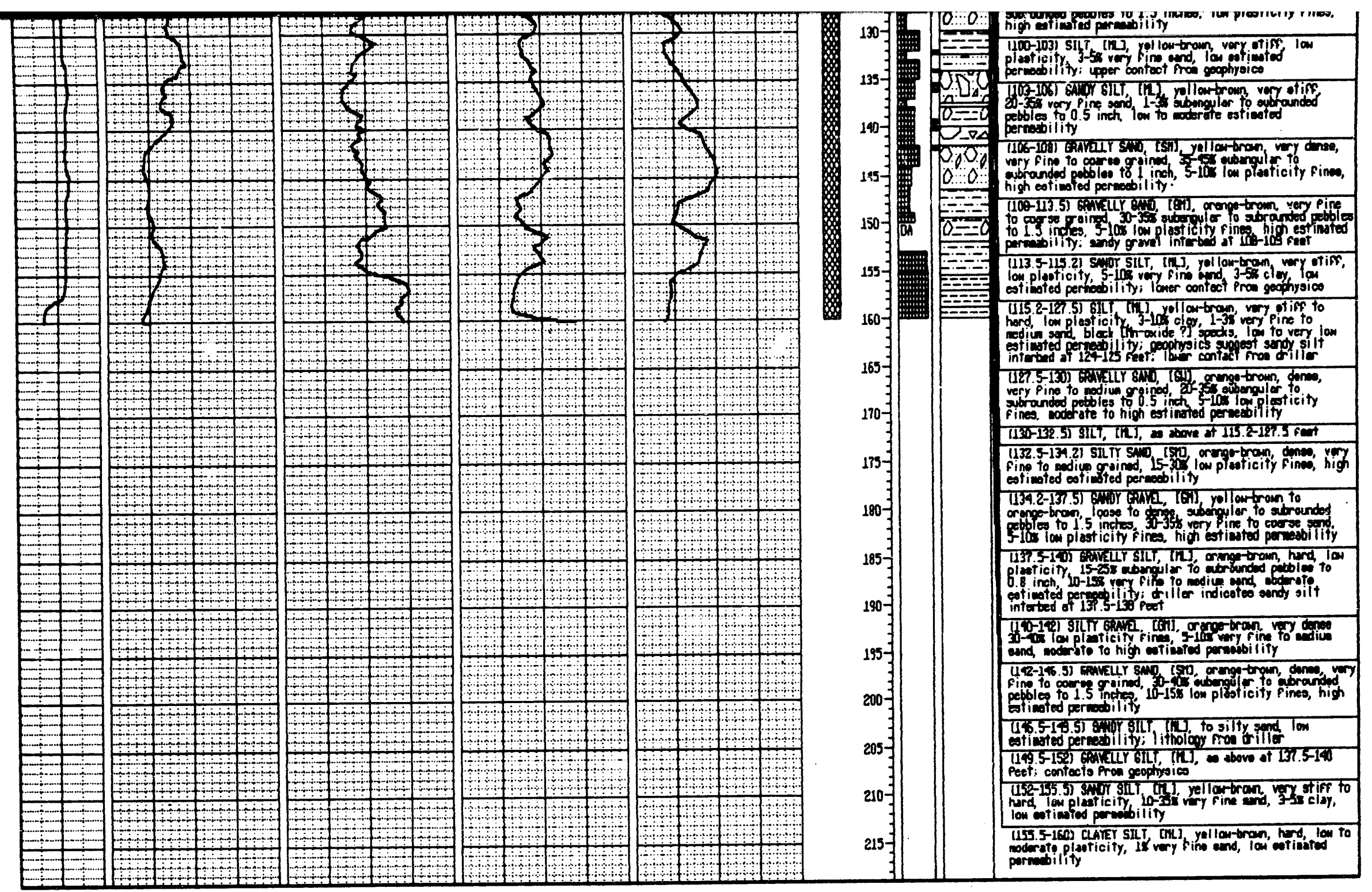




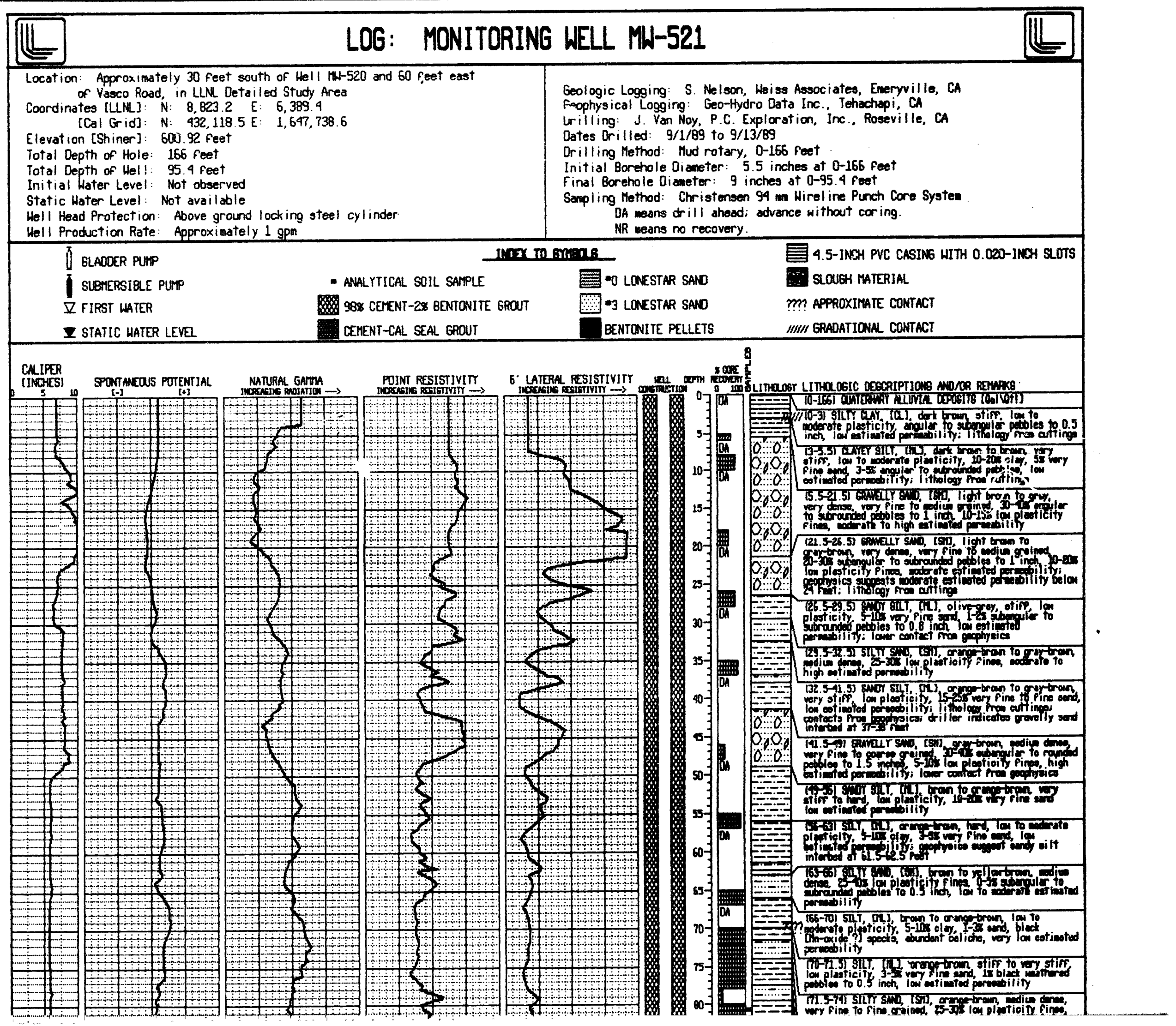



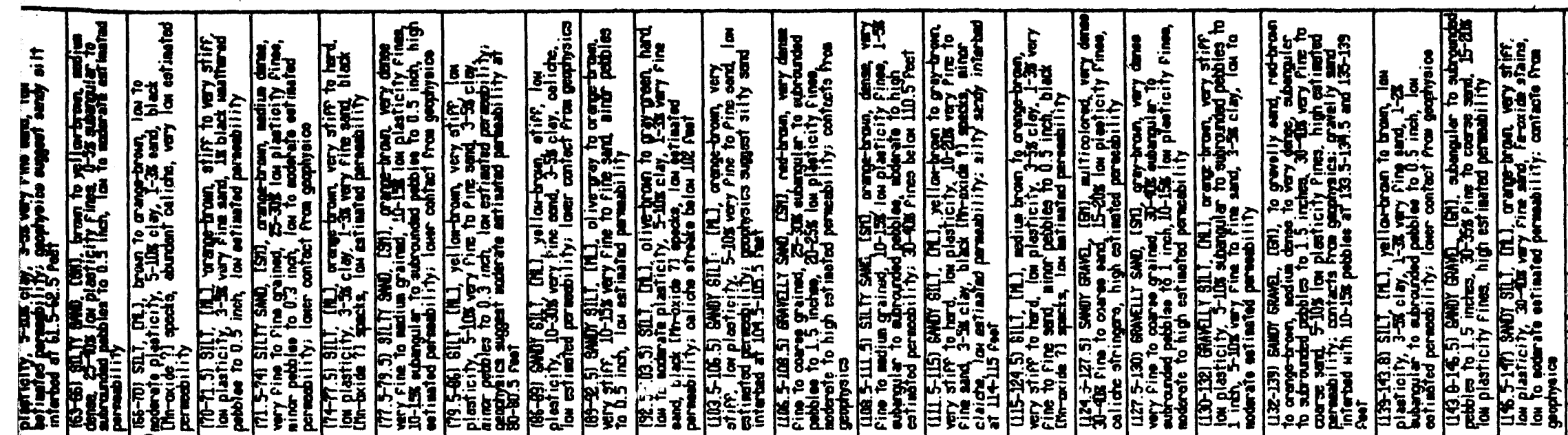
iाi! !

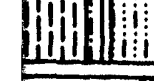

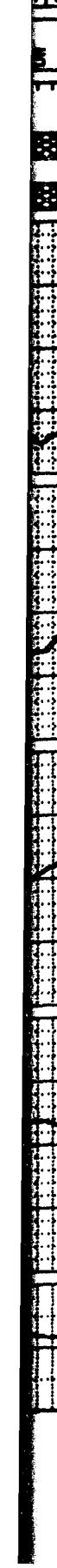

का

,

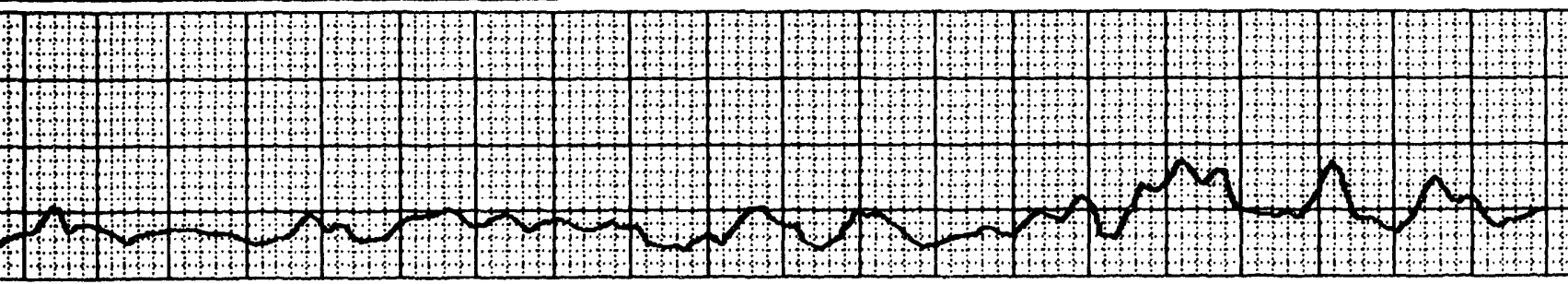

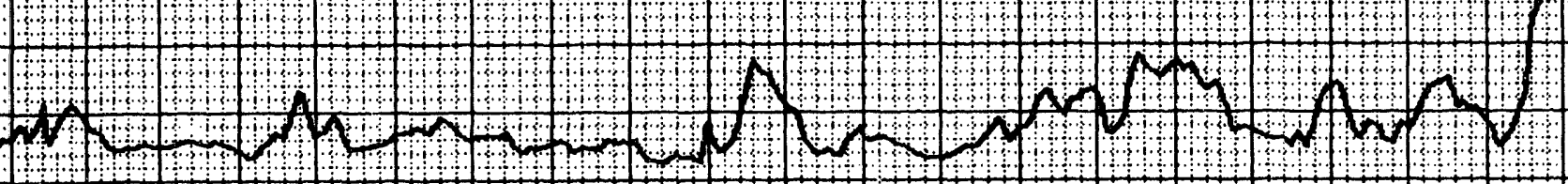

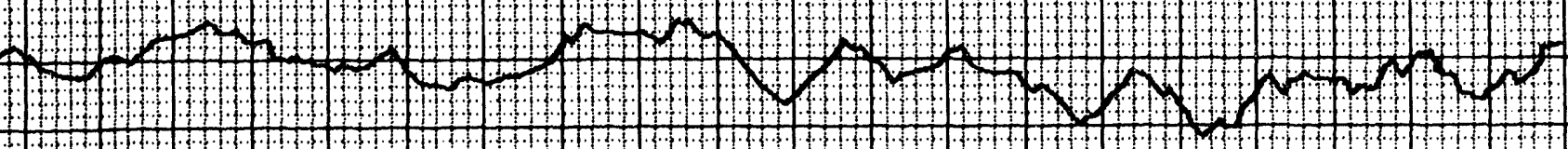




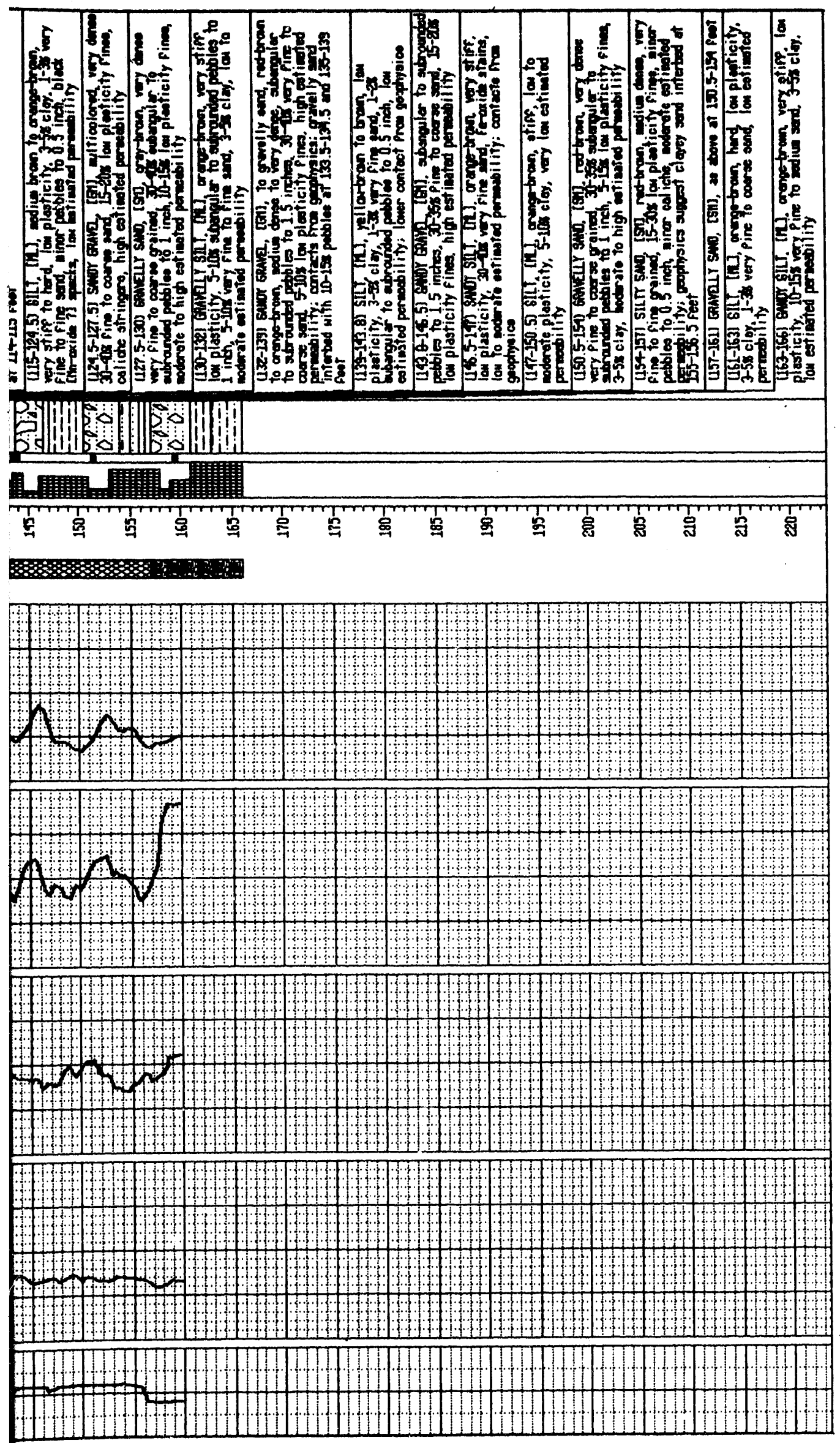




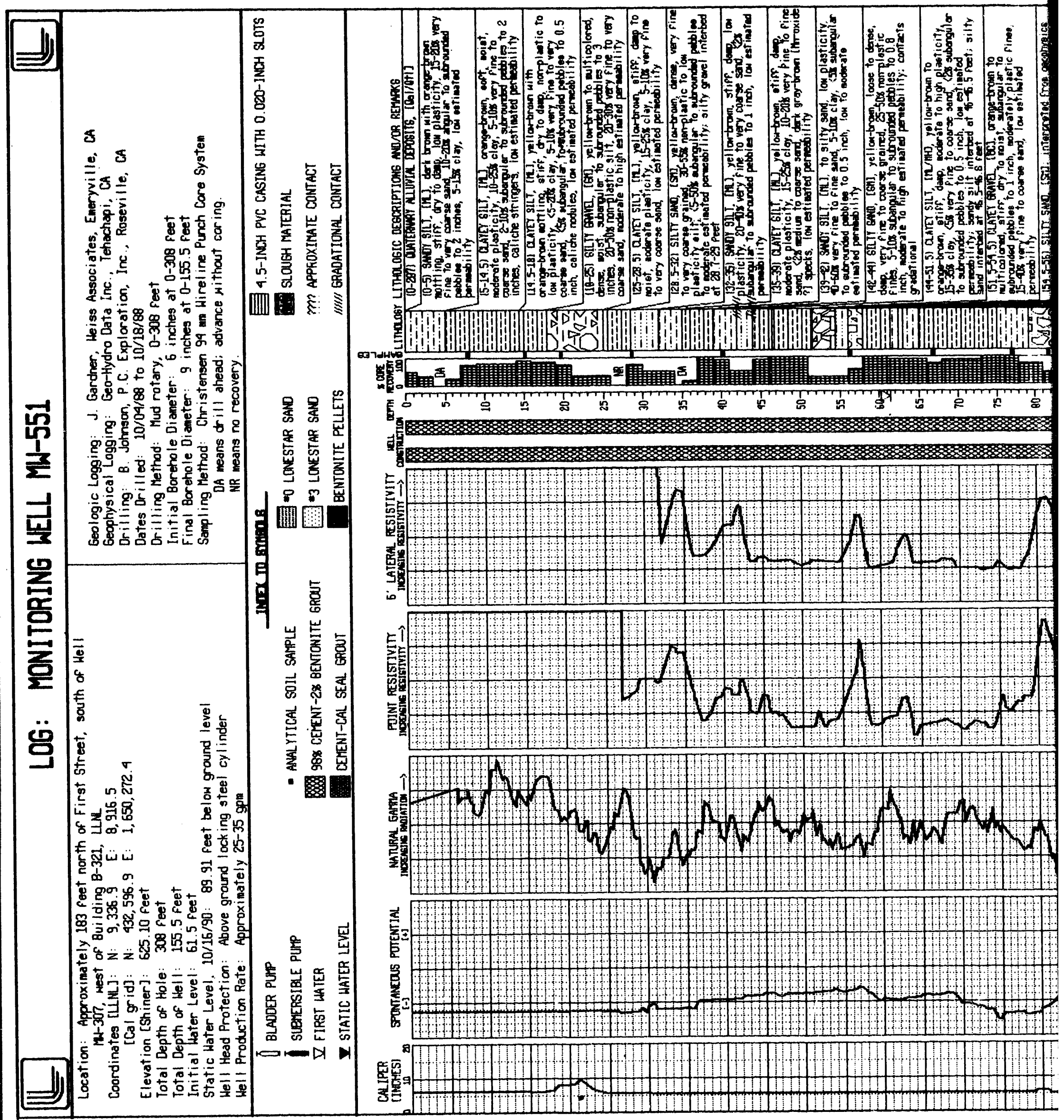



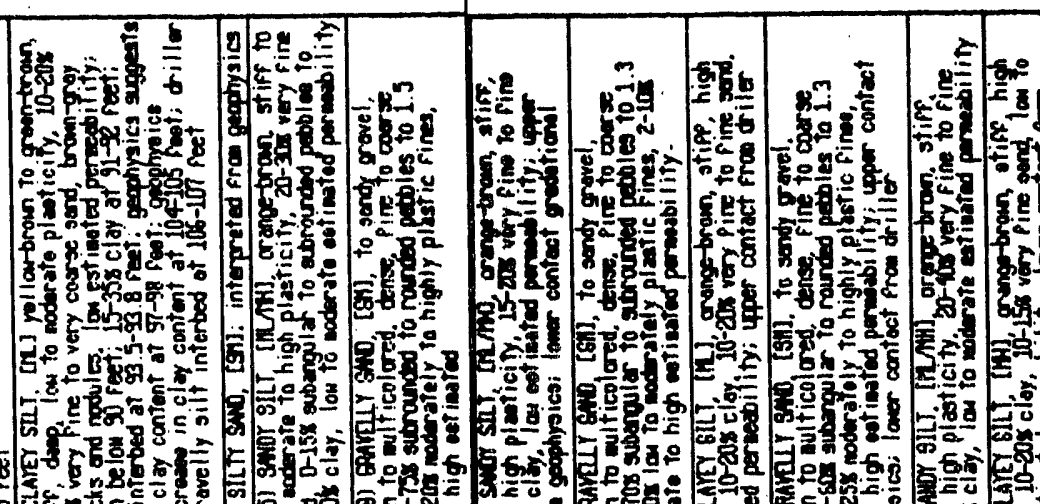

8 Fon

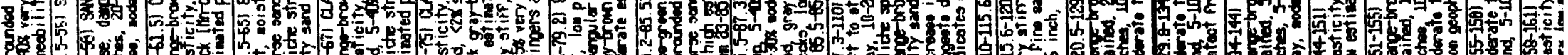

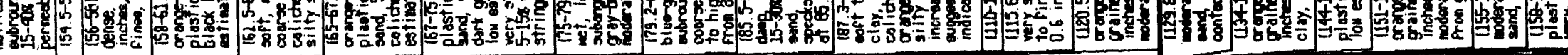

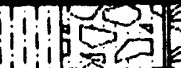
i 00000

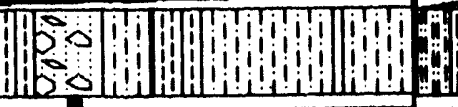

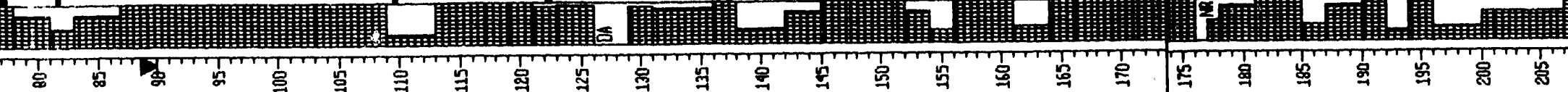
8

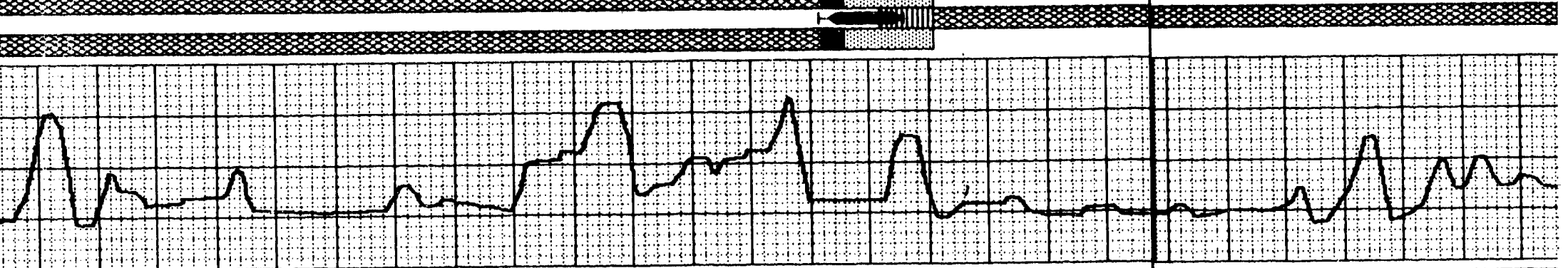

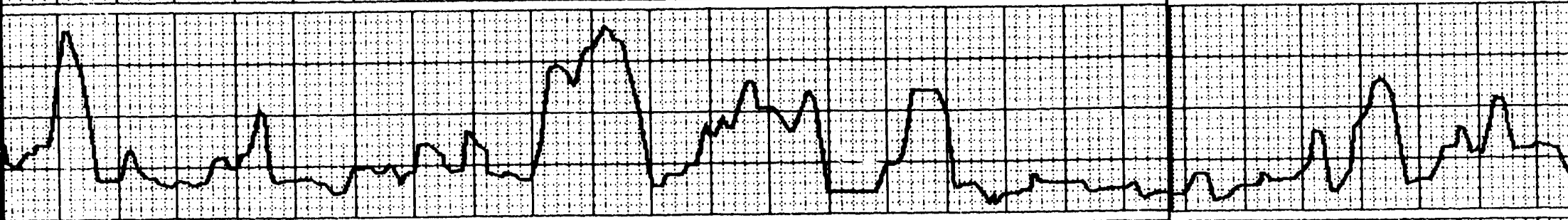

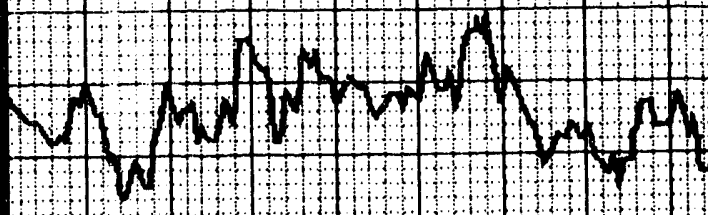

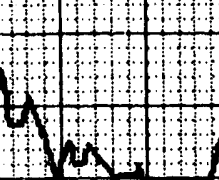

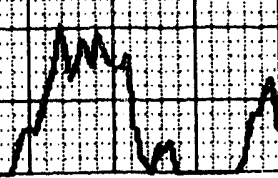
$(n)$

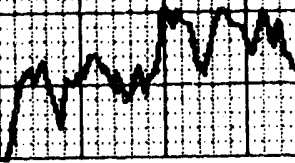
$\ln$ 


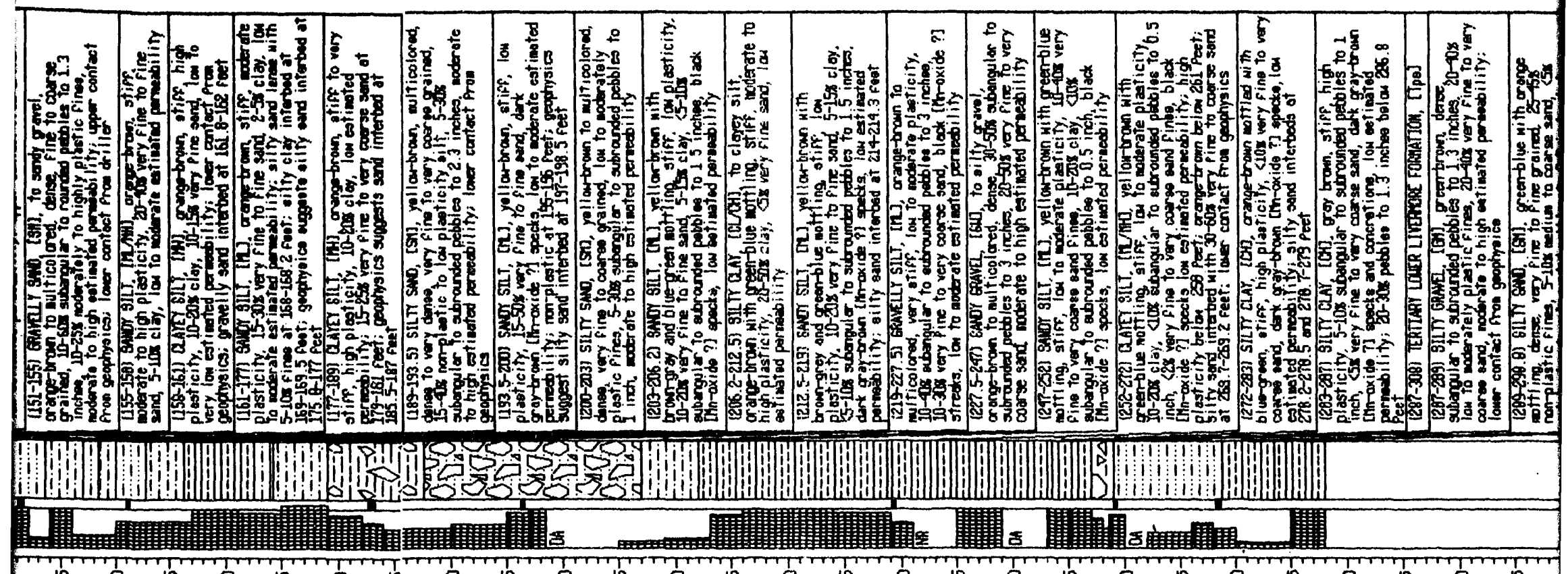

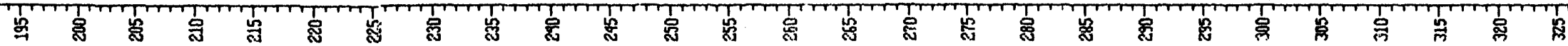
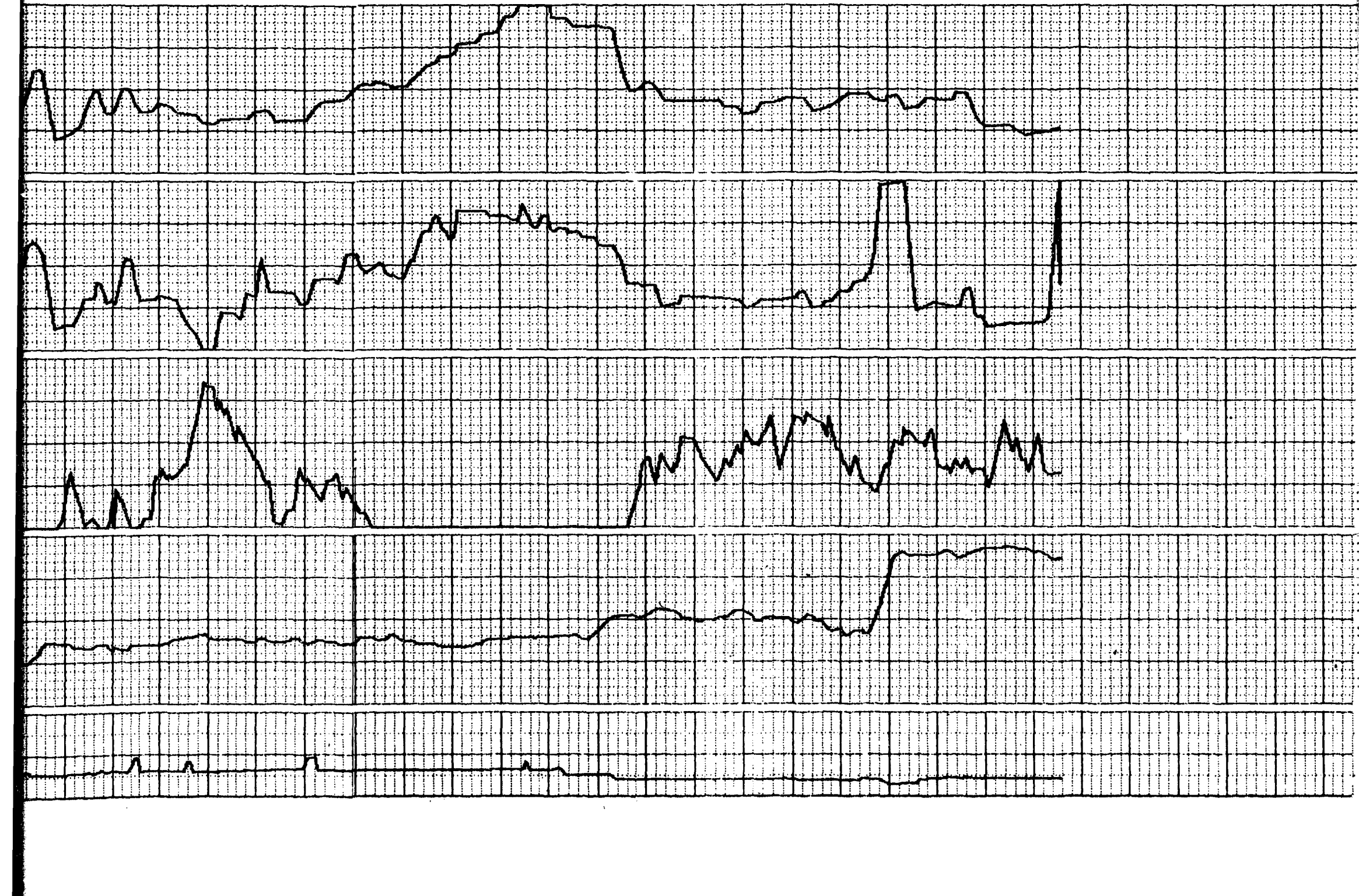


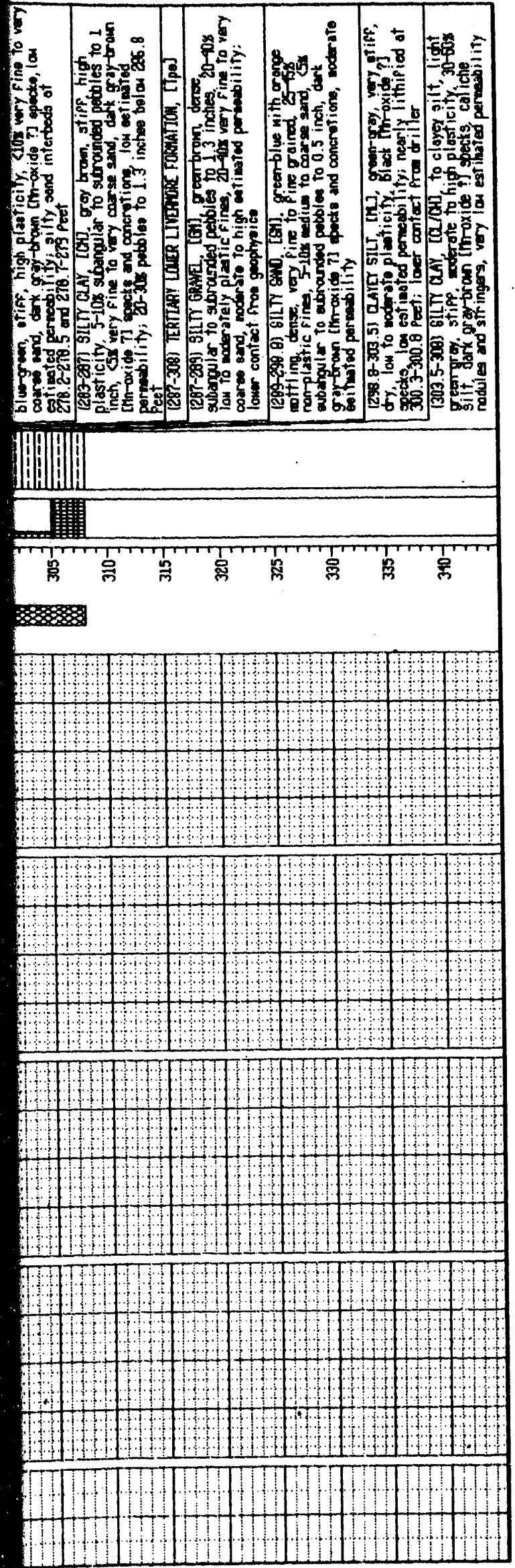




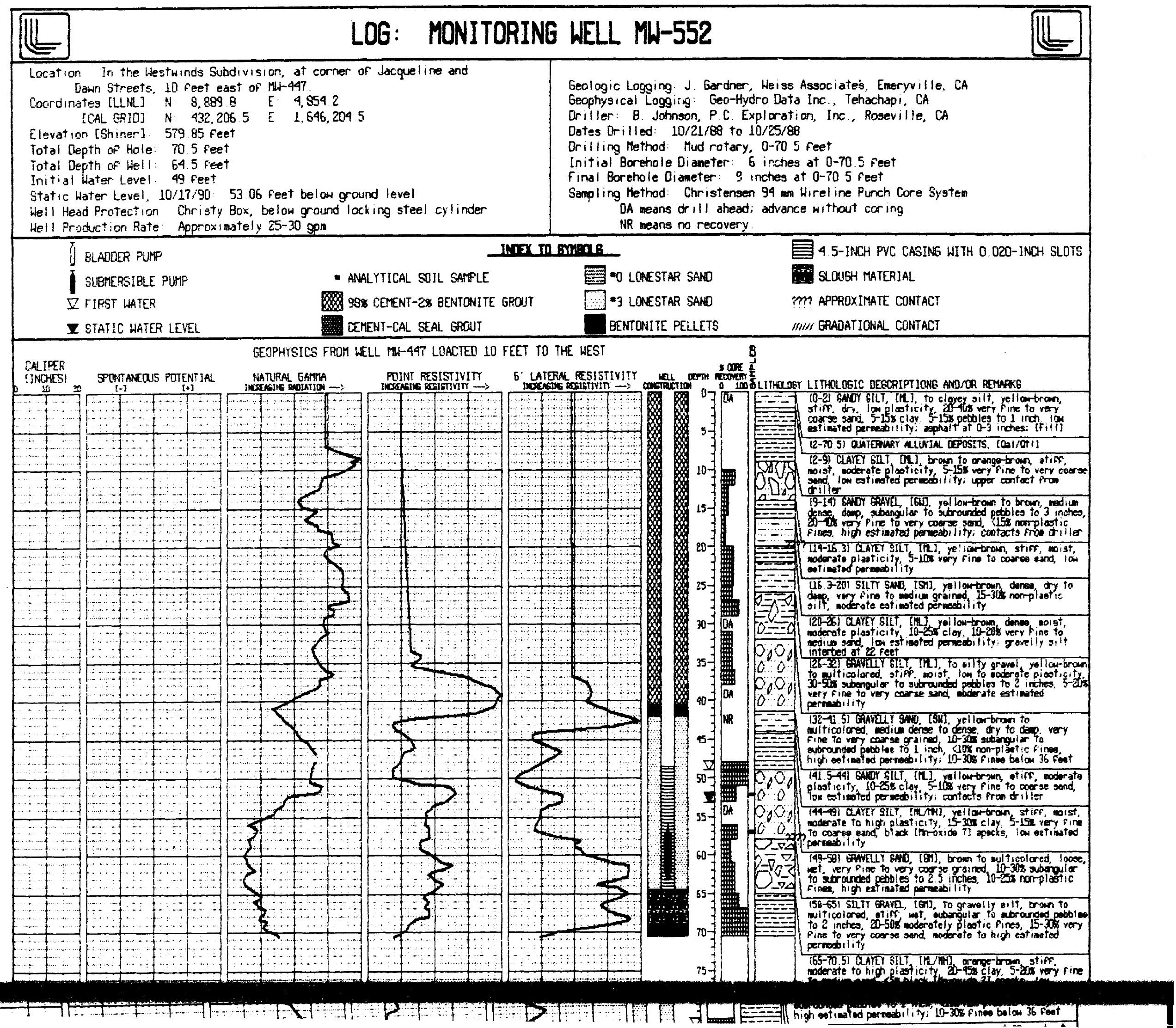




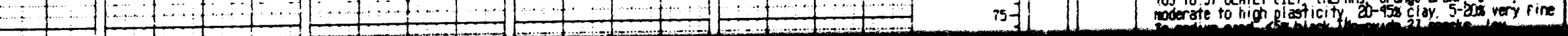

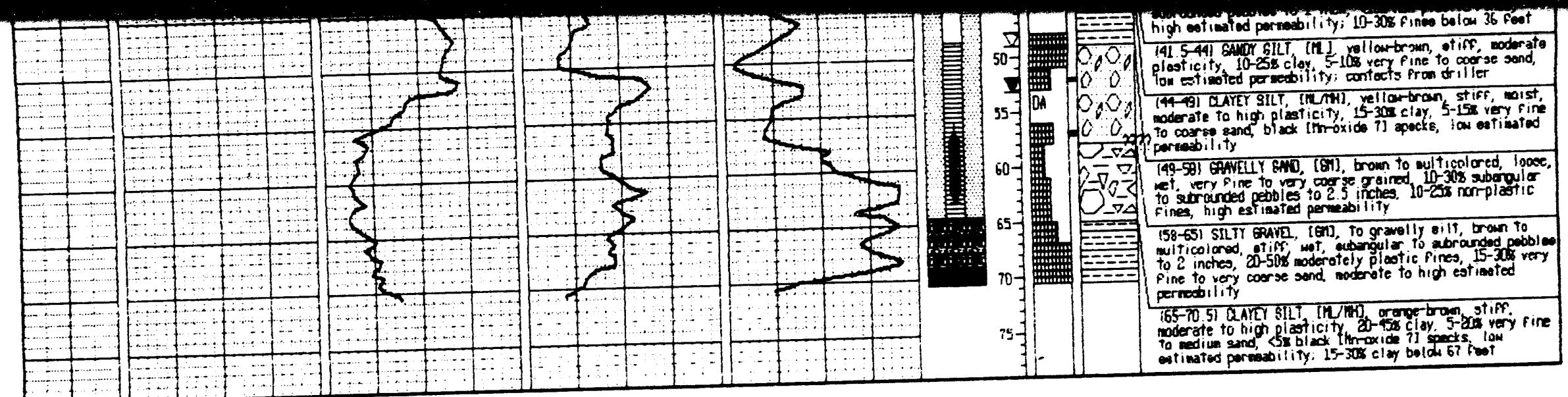




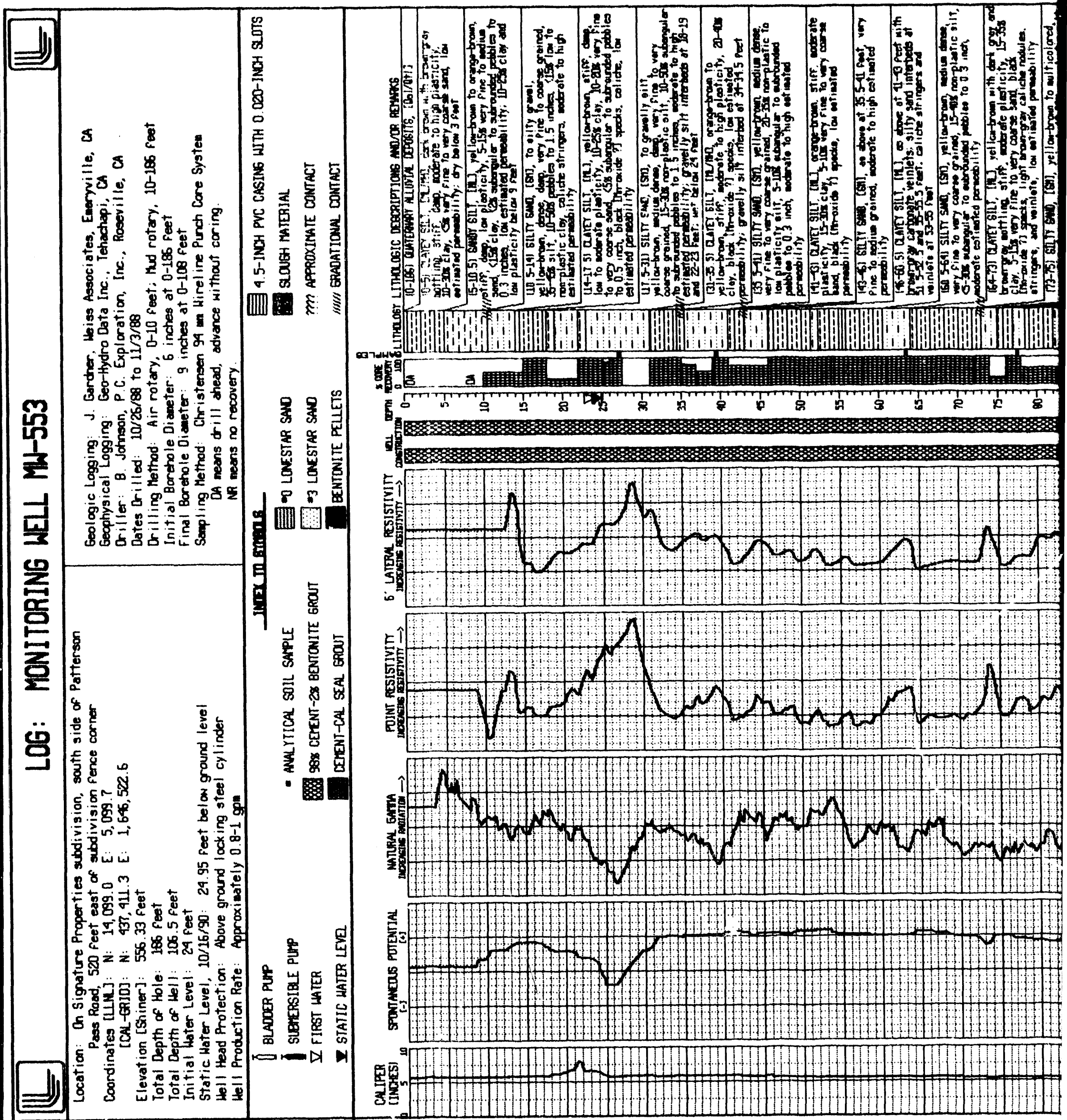




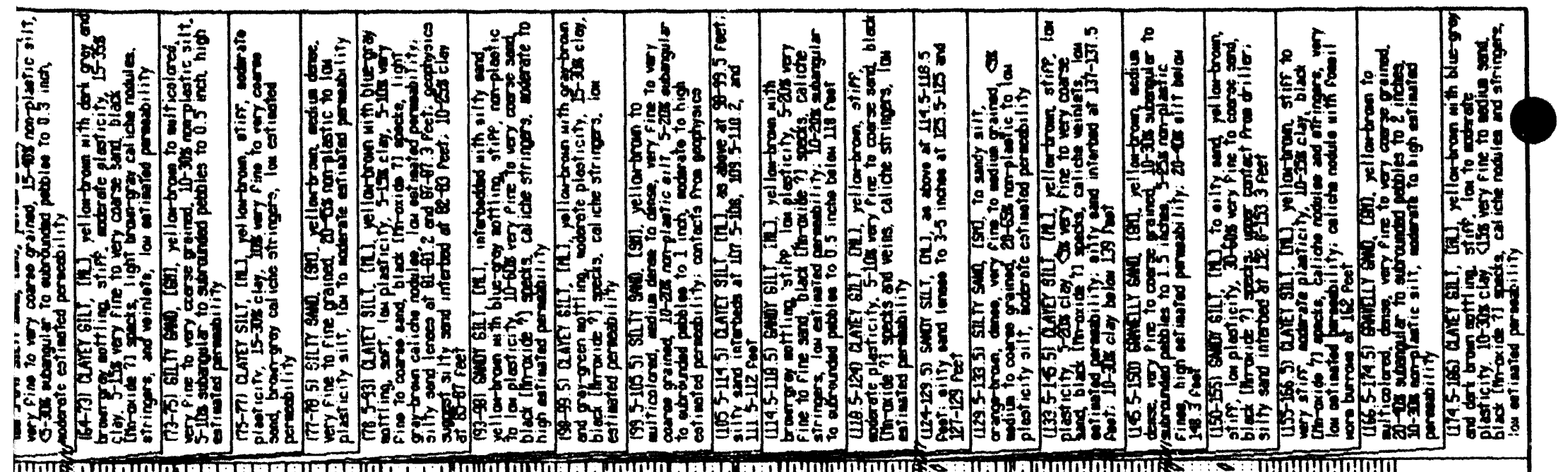

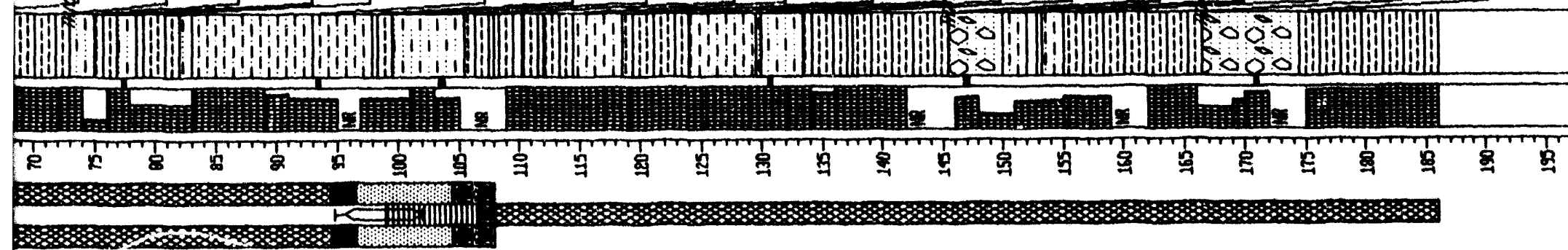

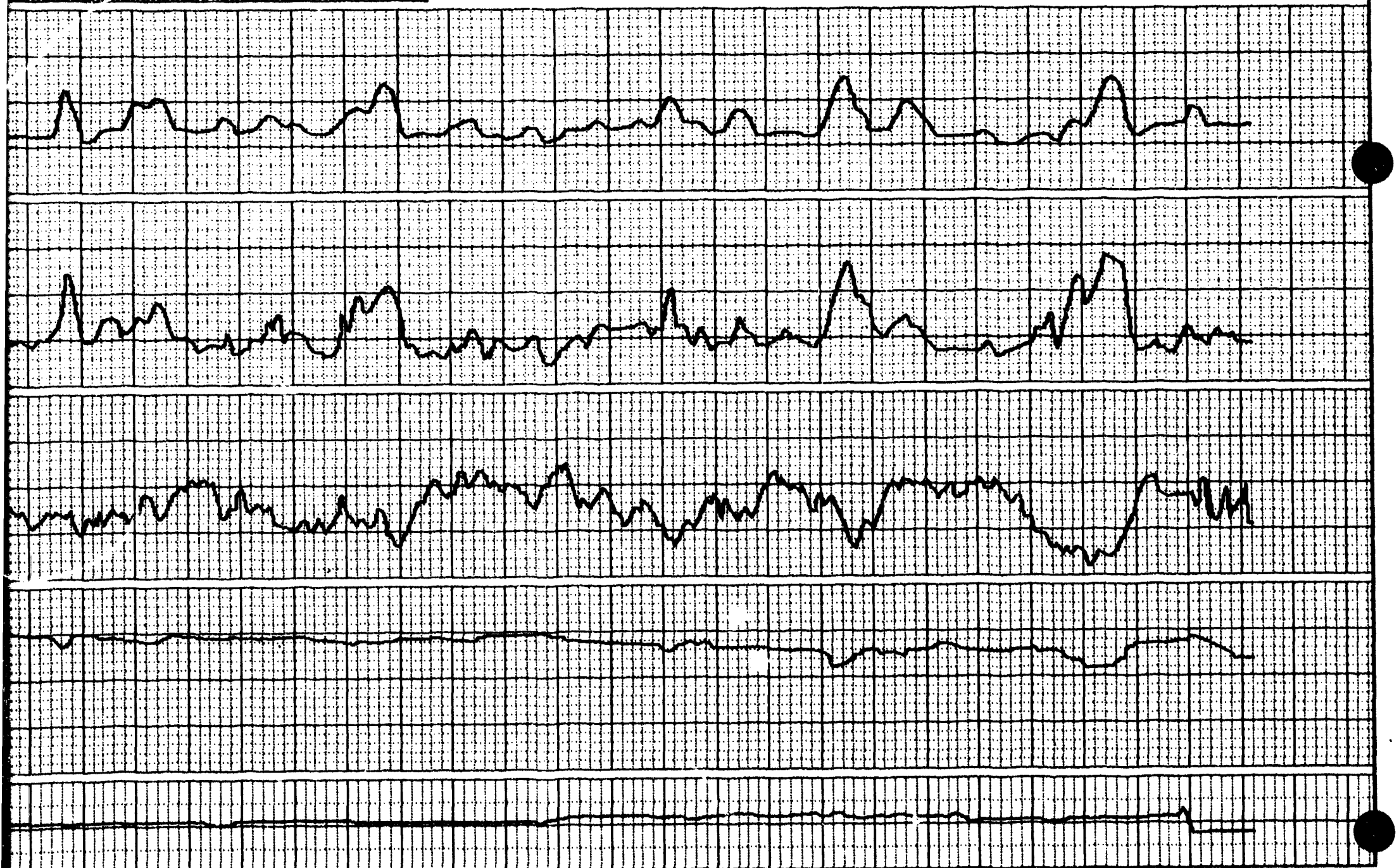




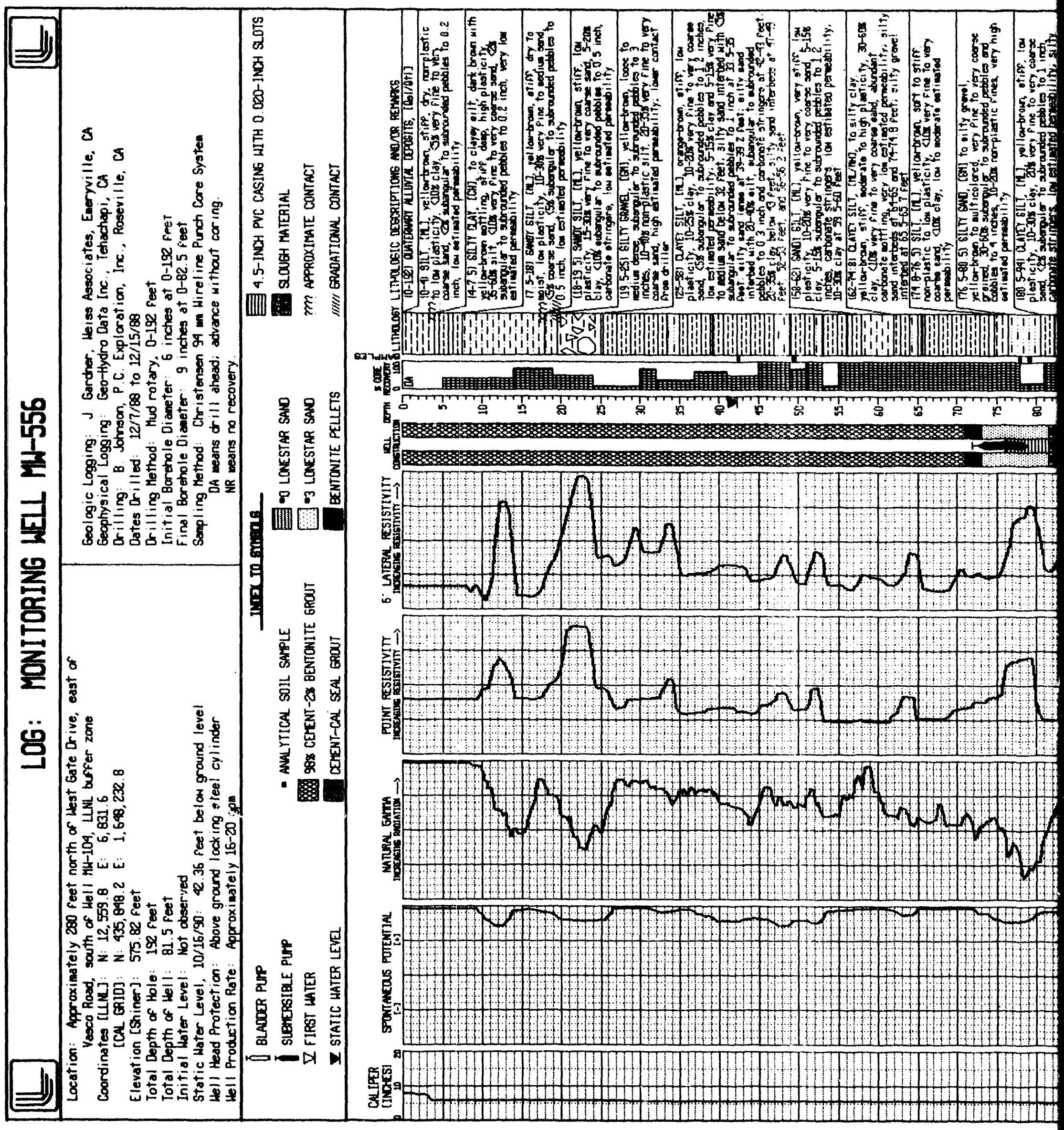




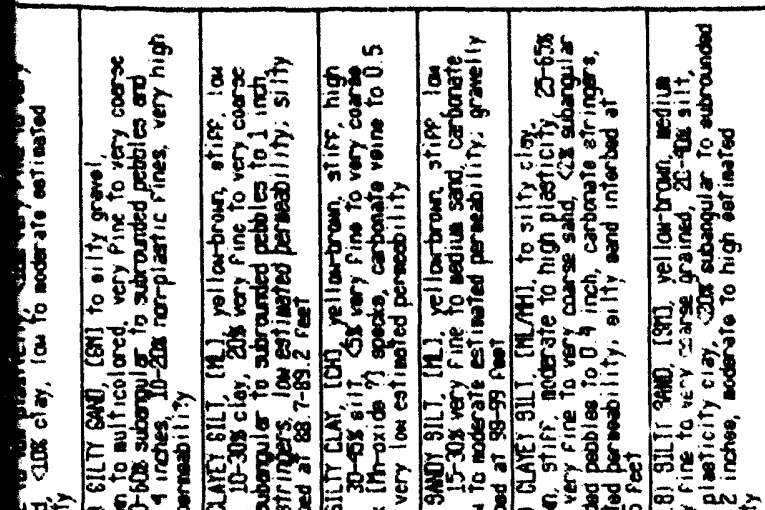

等

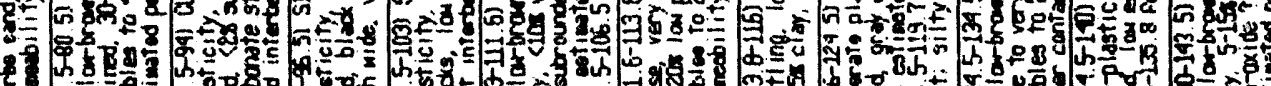

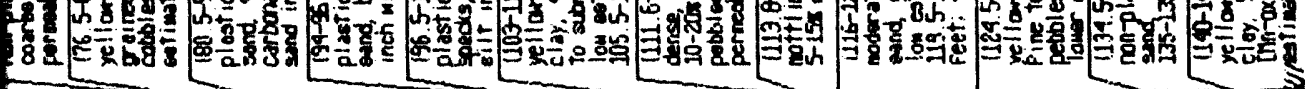
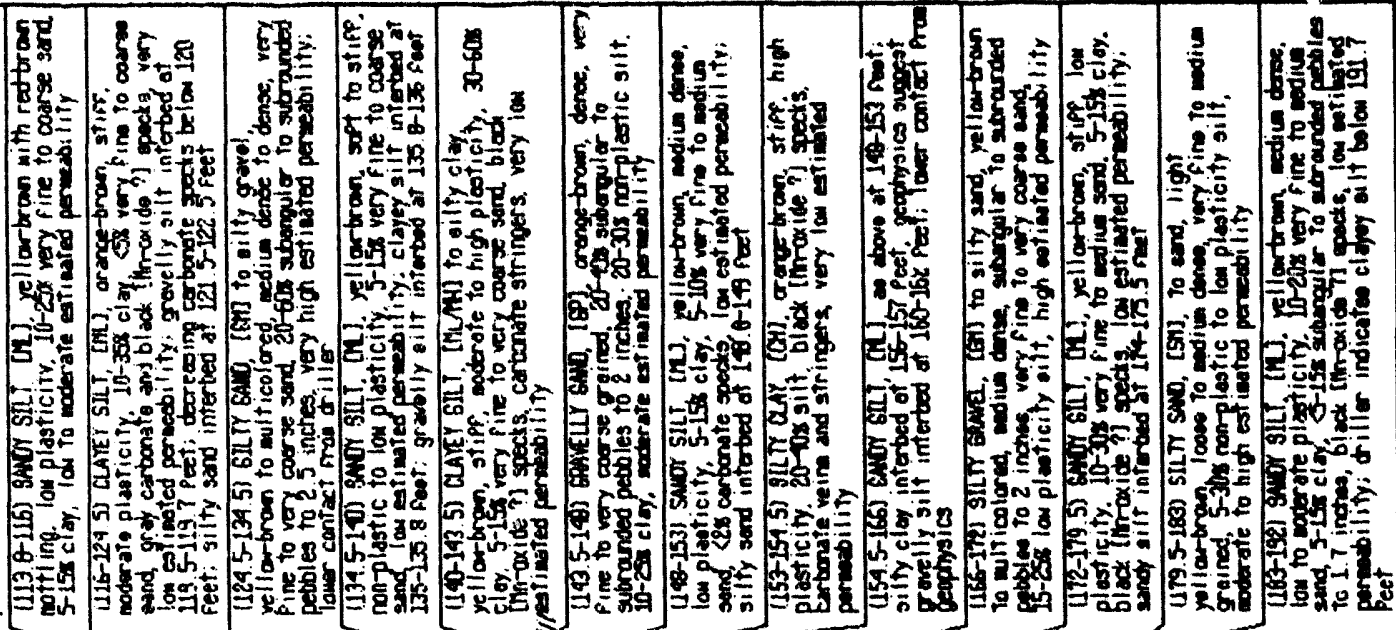

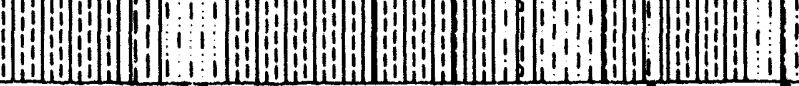
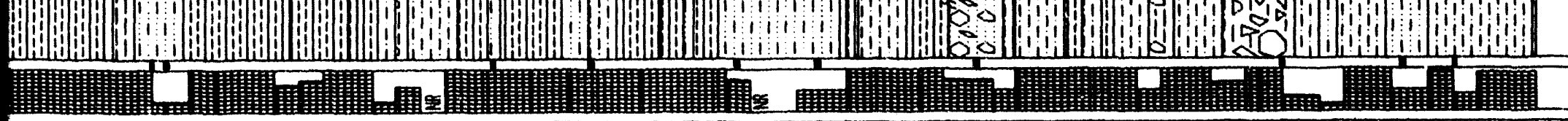

So

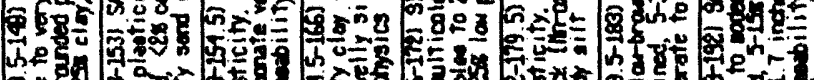

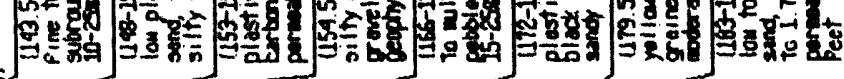
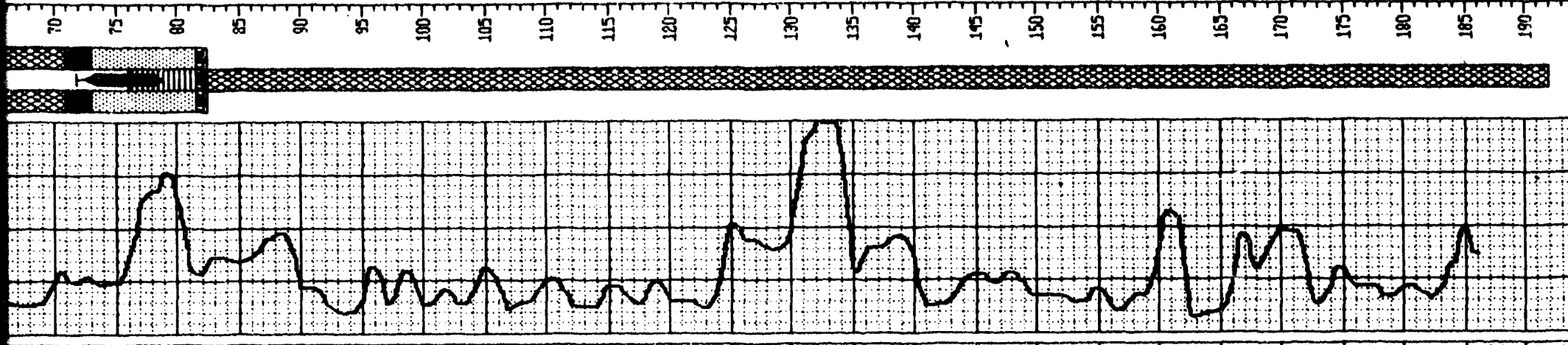

.

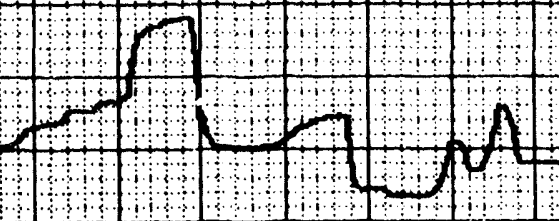

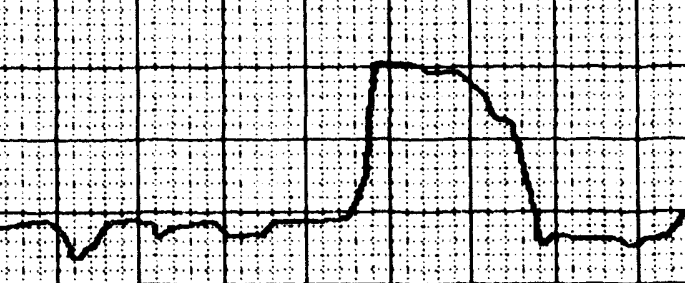

(1)

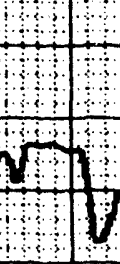

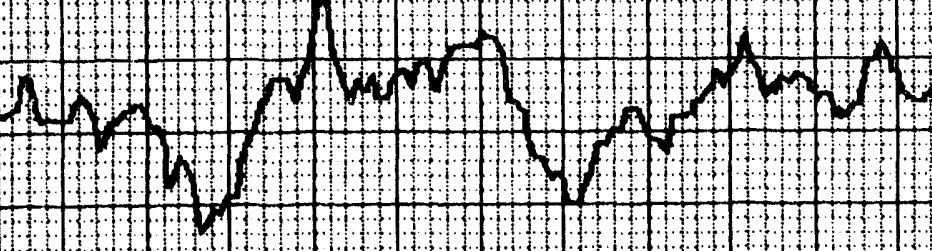

$+1$

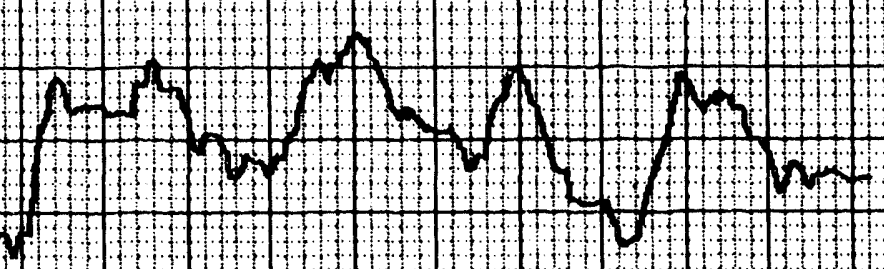

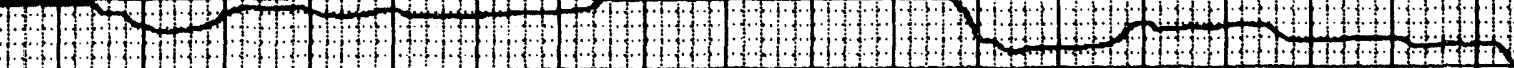




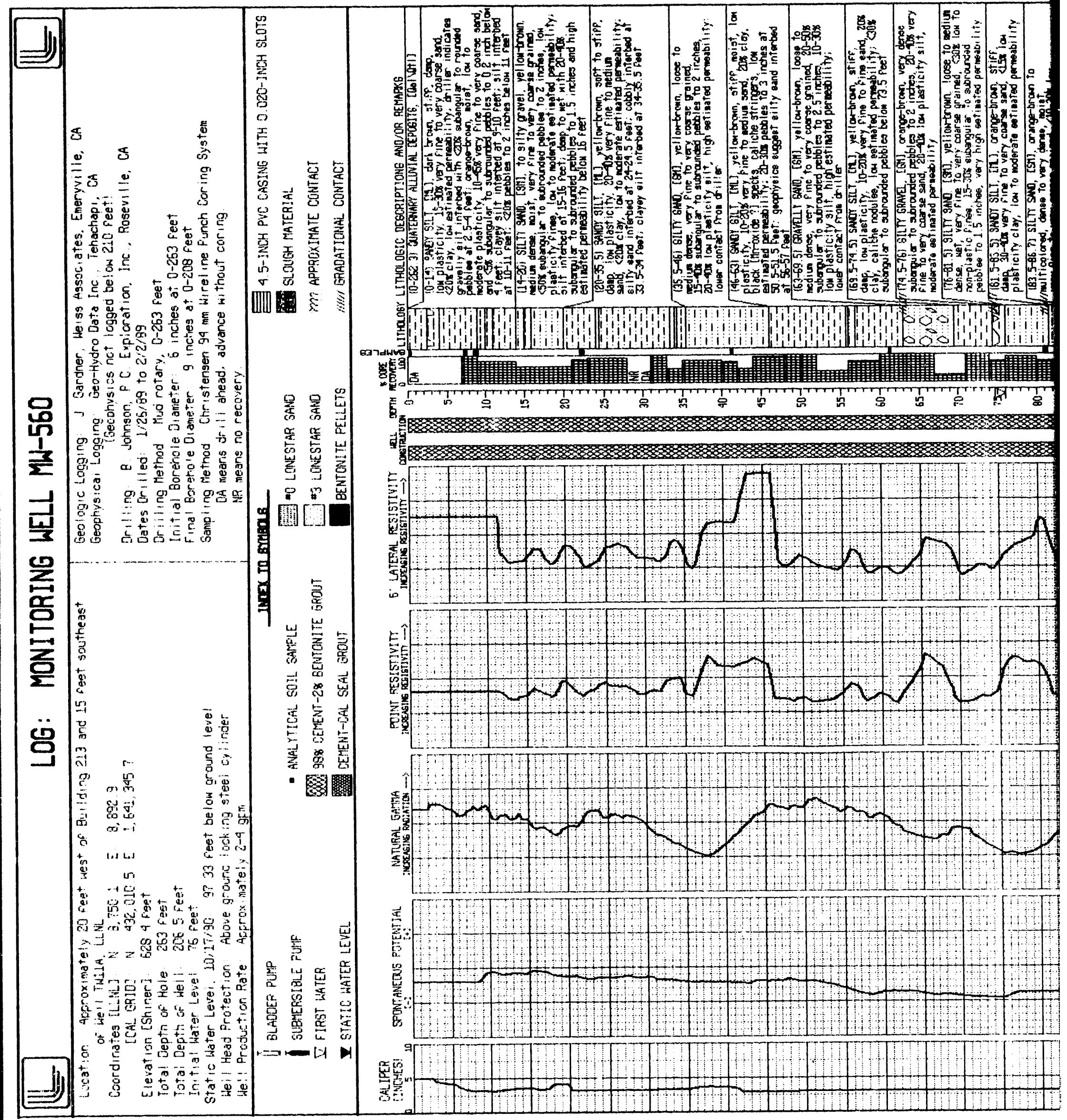




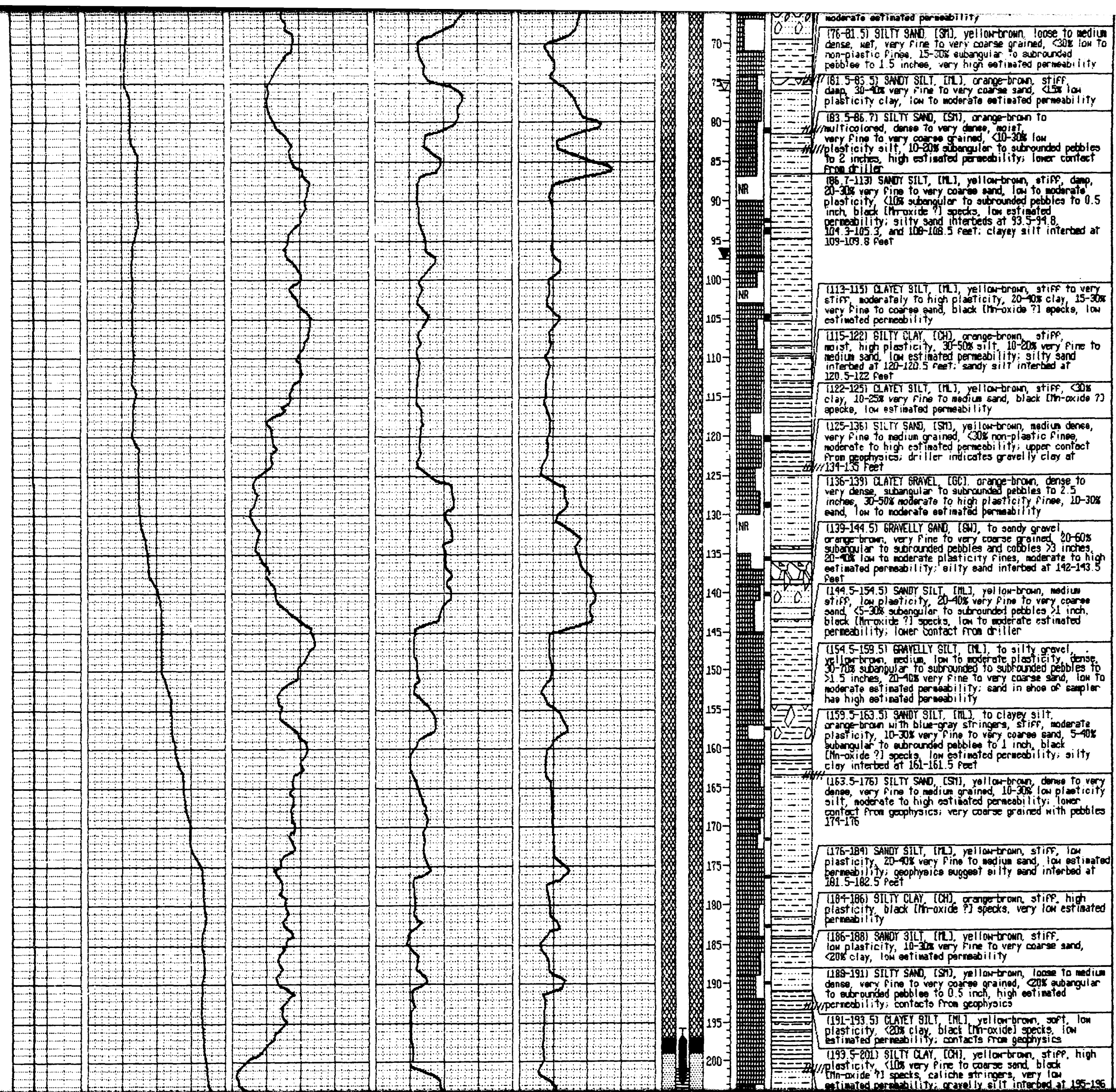




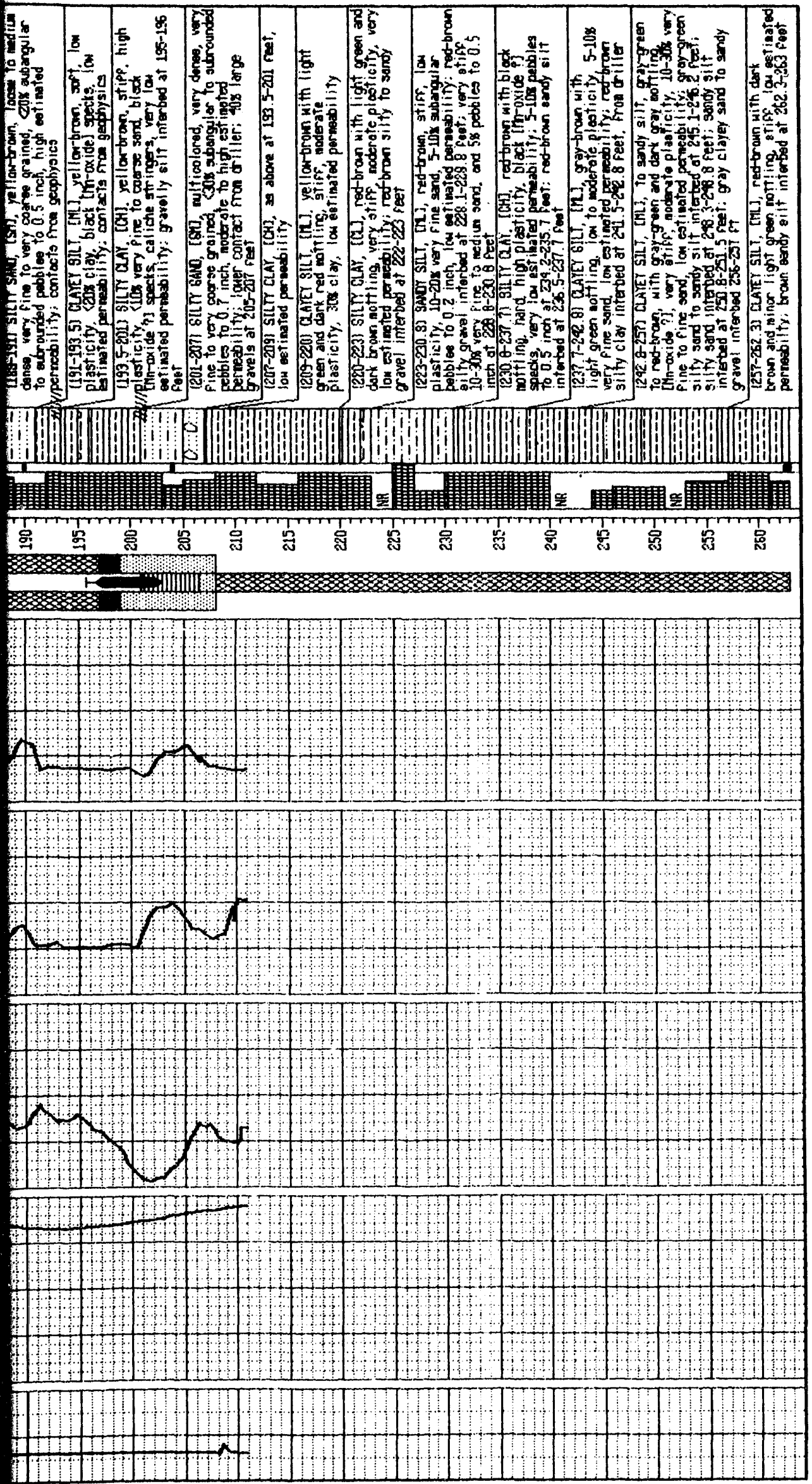




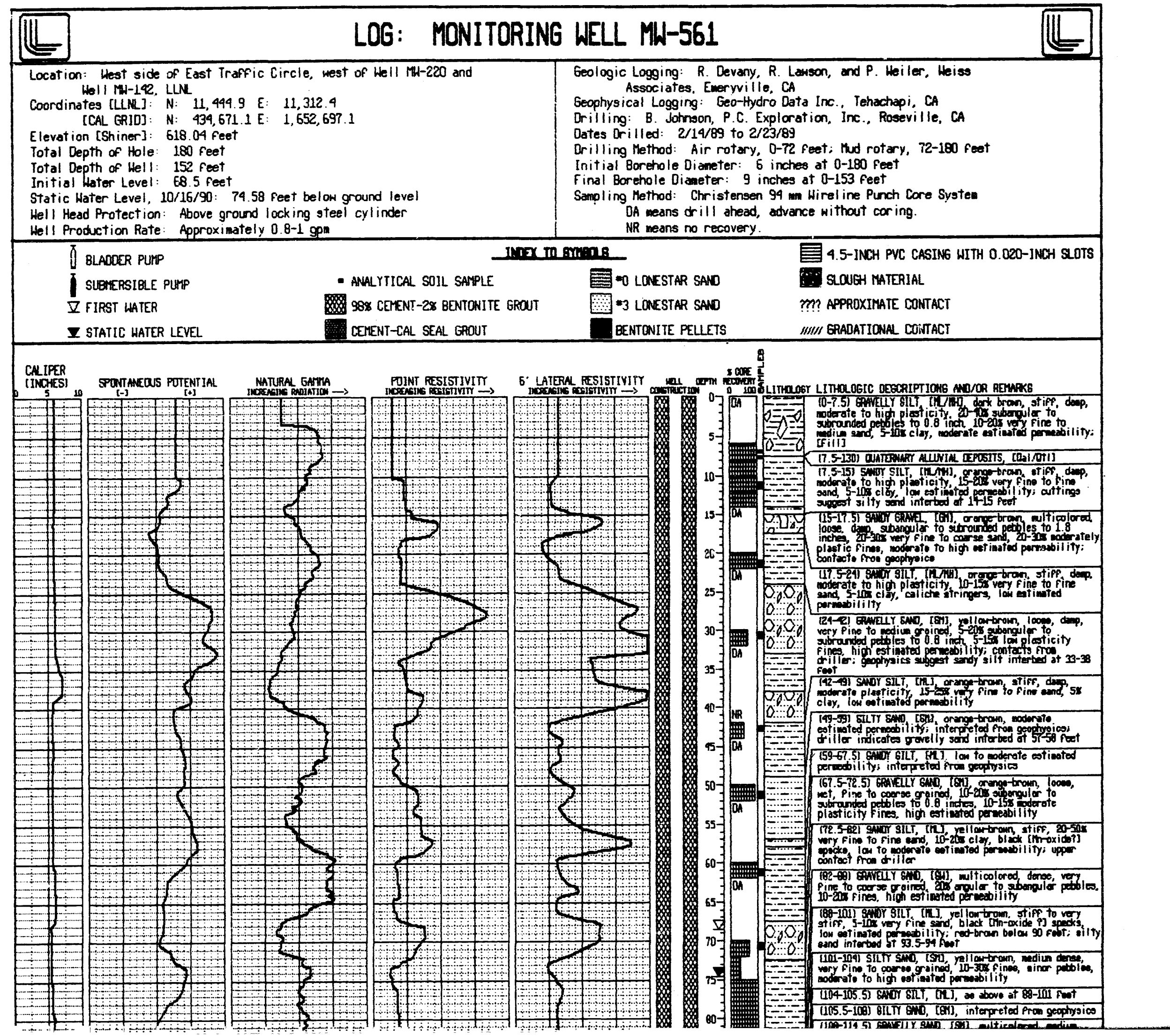




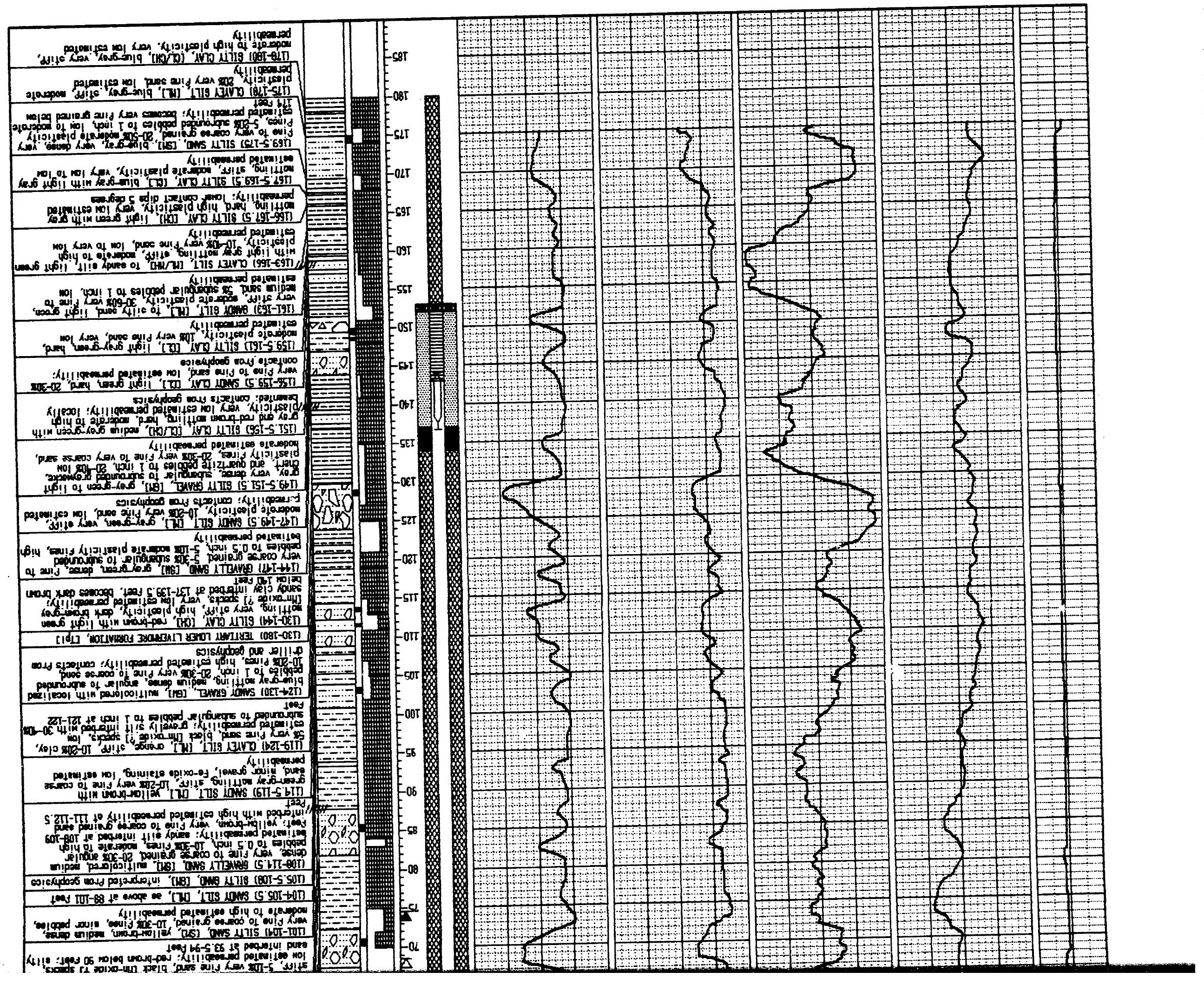




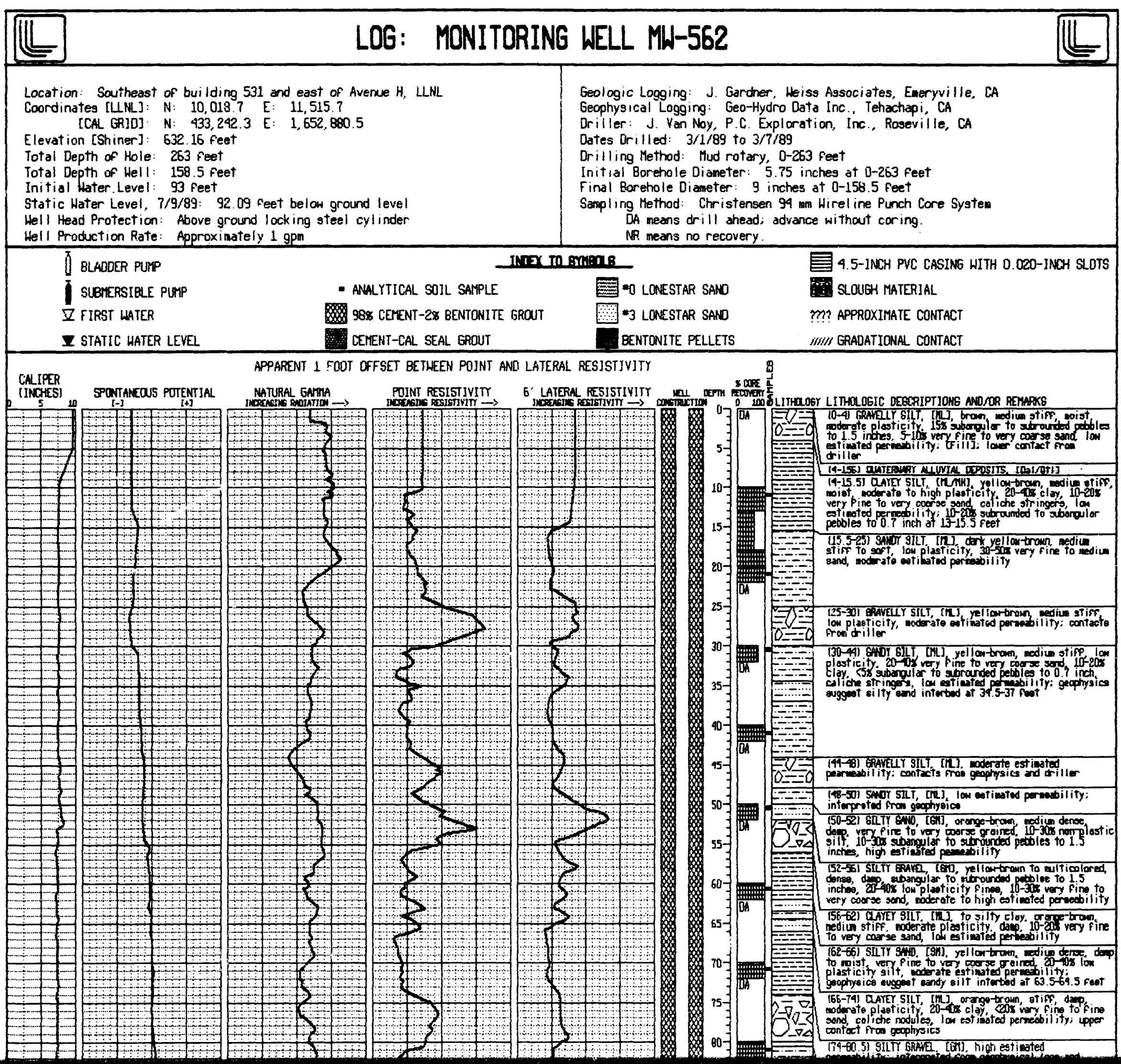




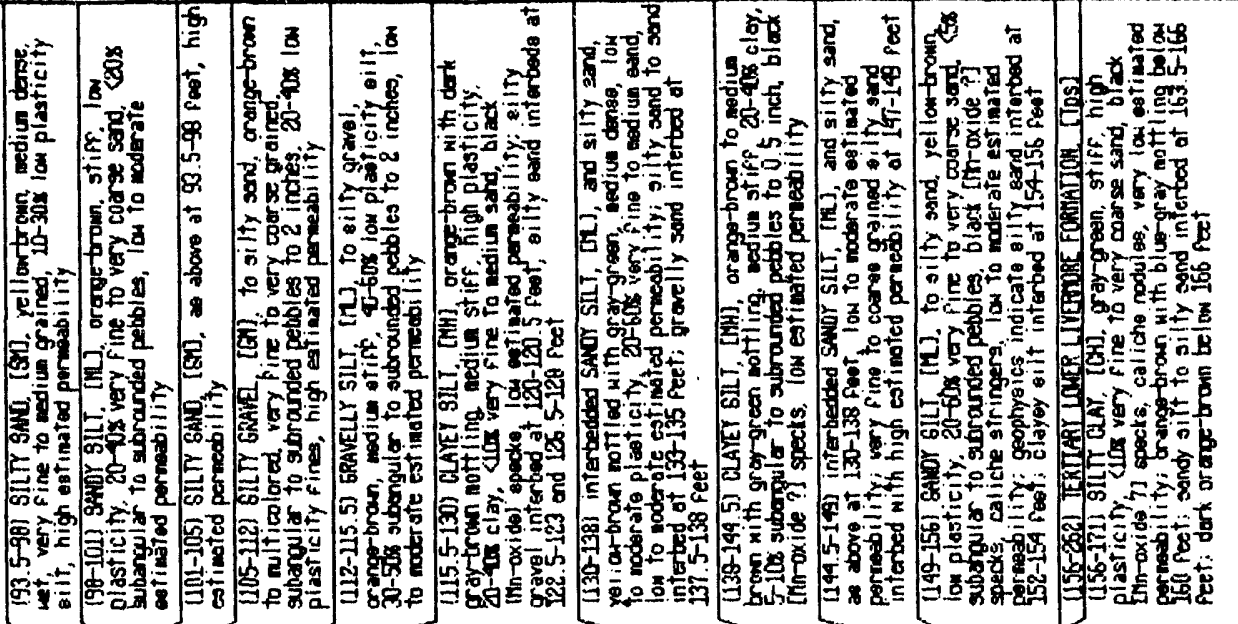

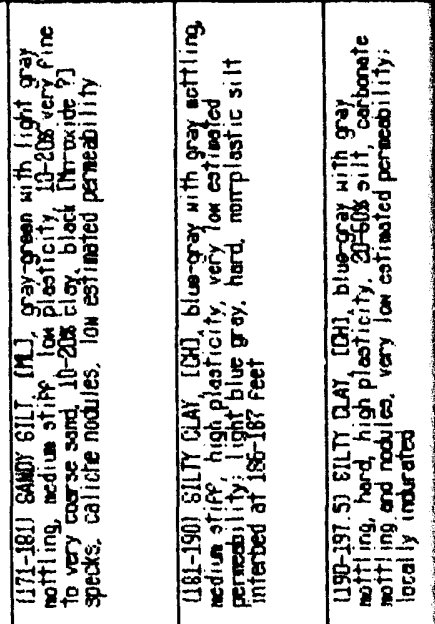
(n)

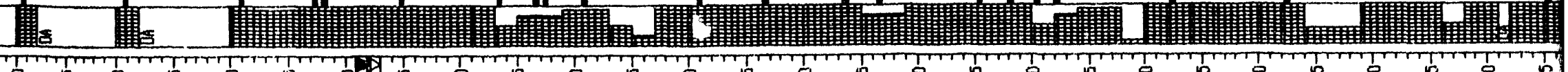
它

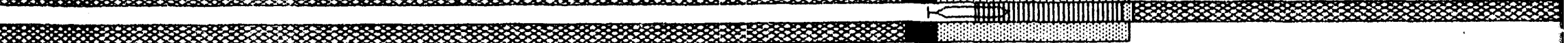
\begin{tabular}{llll}
\hline \\
\hline
\end{tabular}

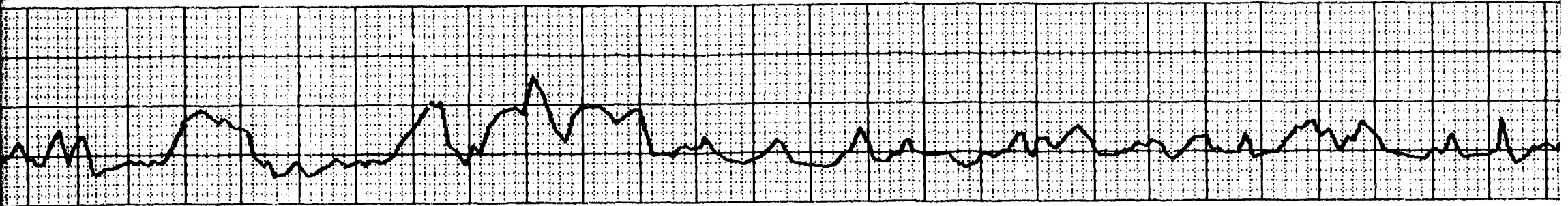

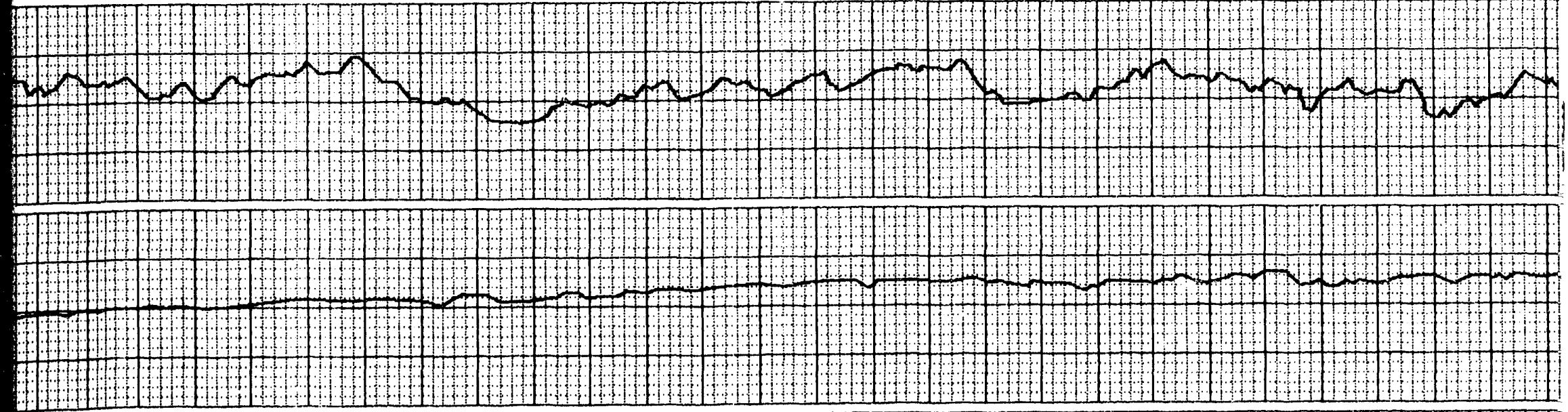




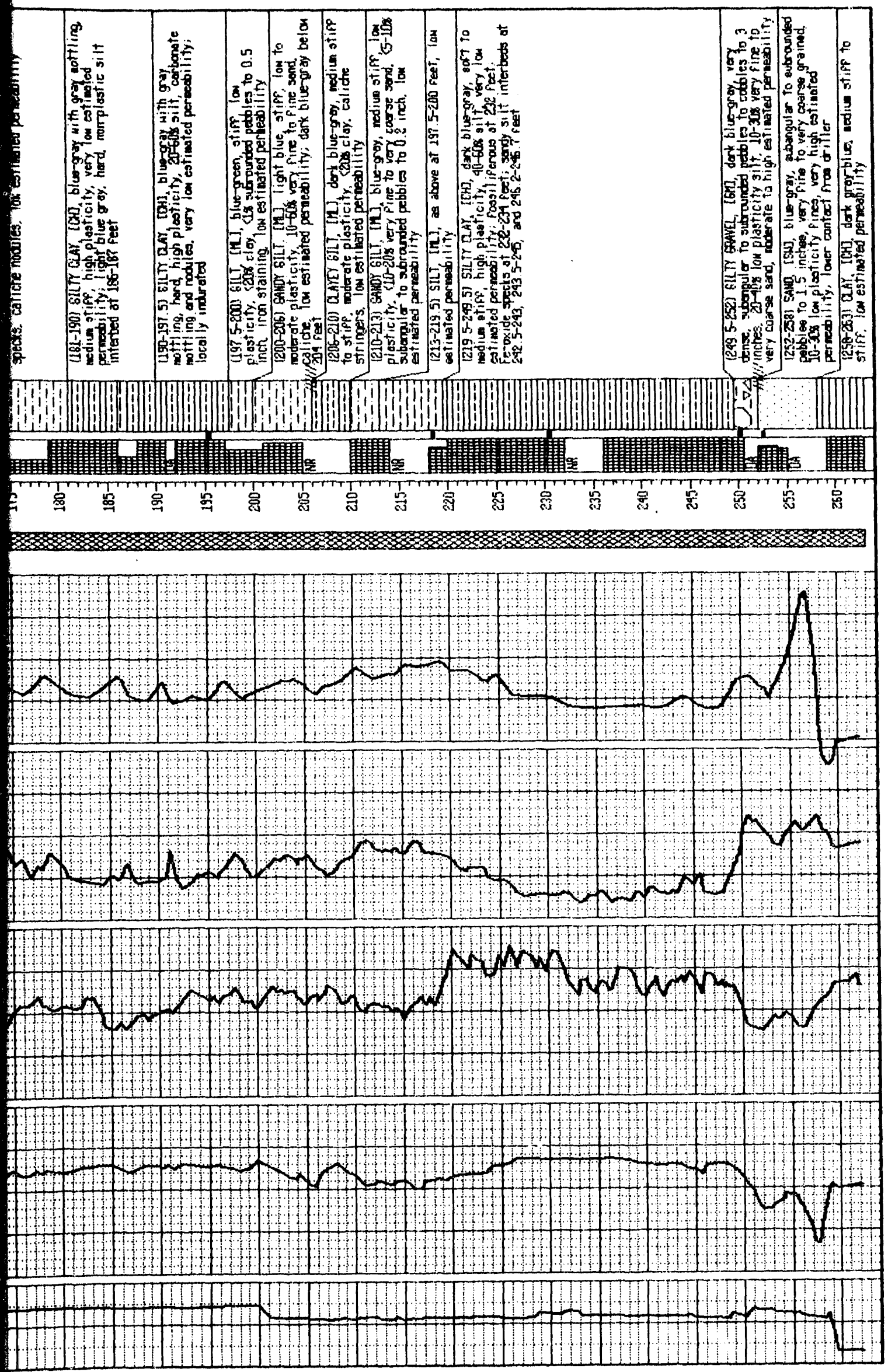




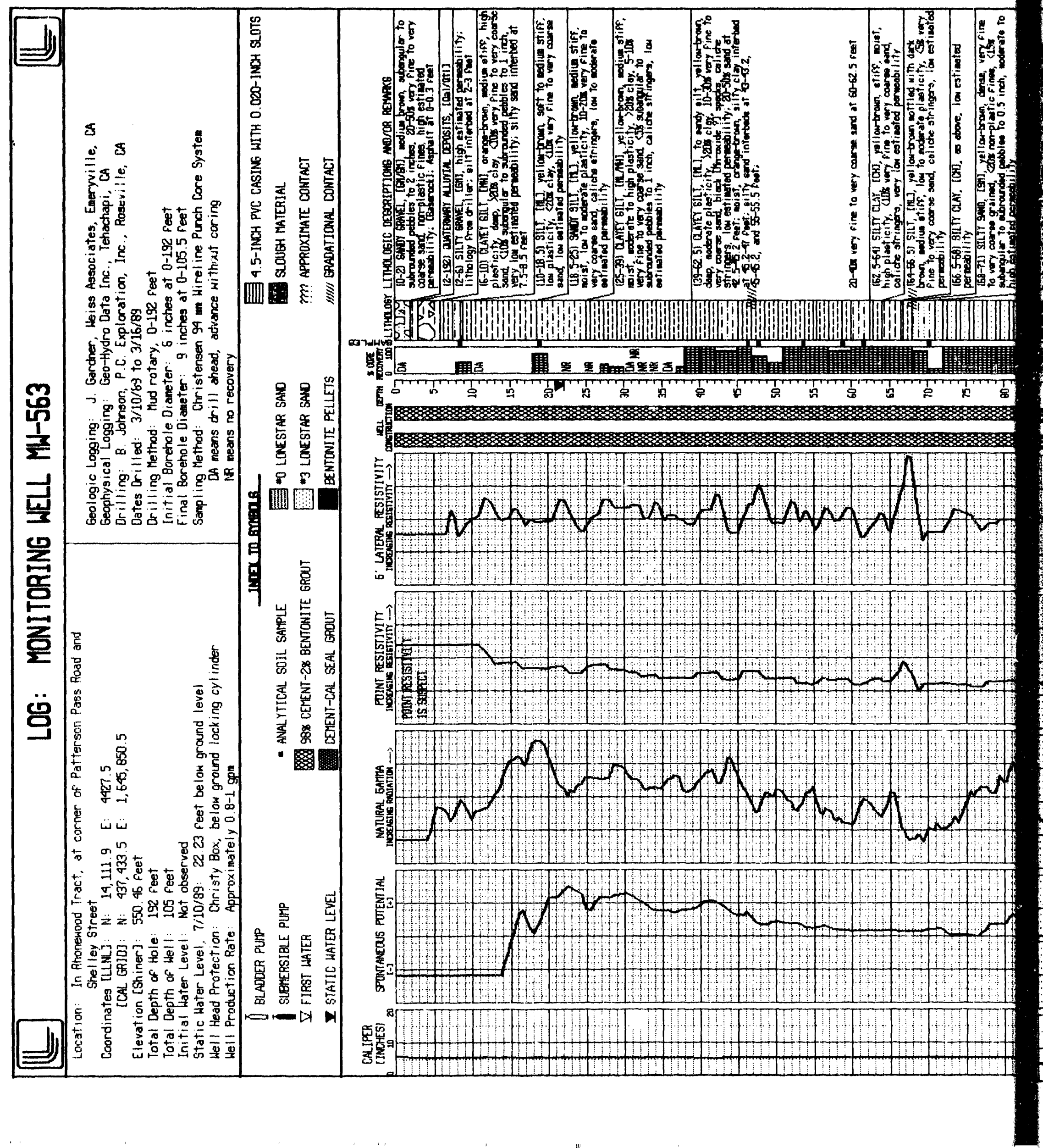




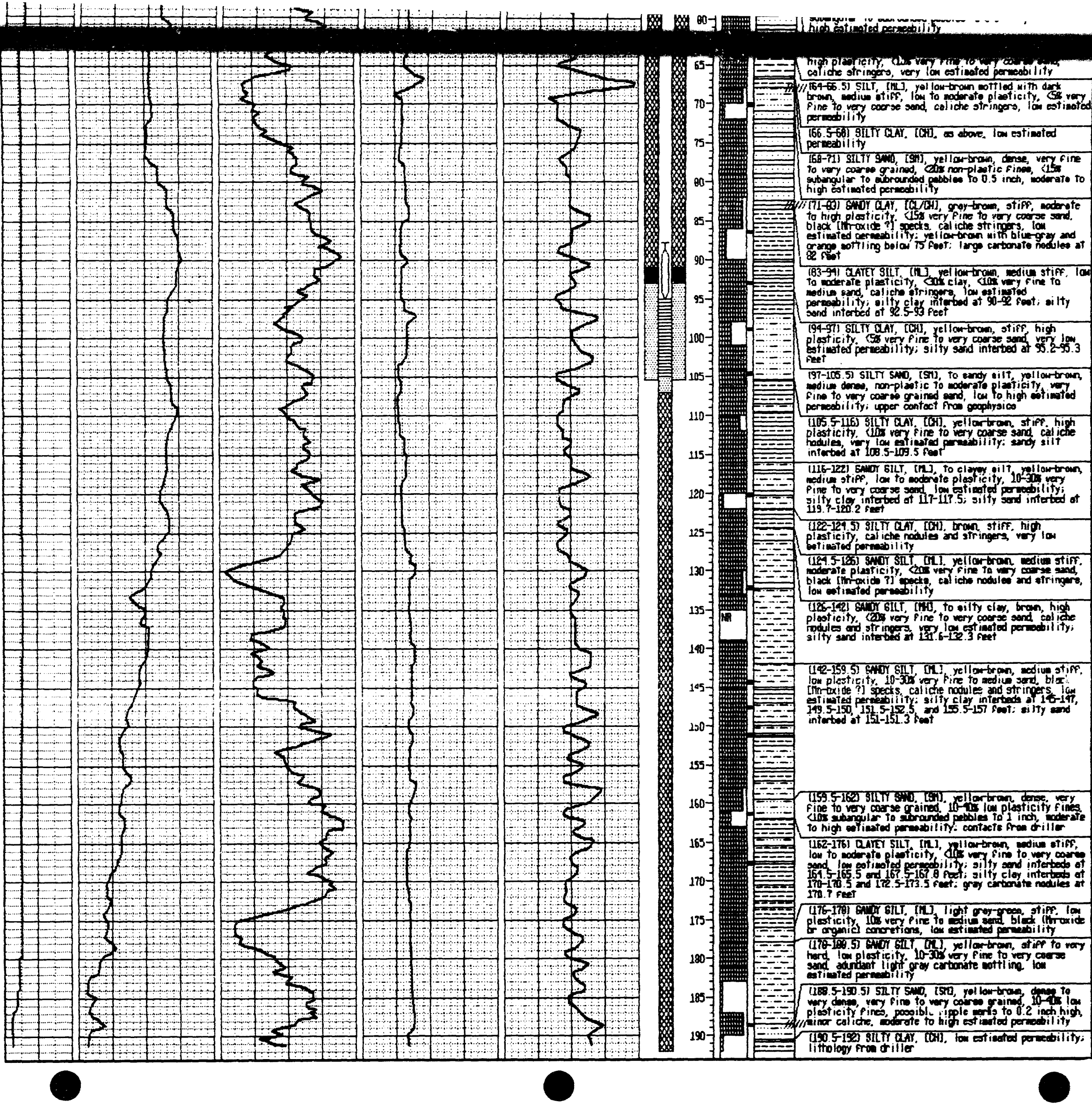




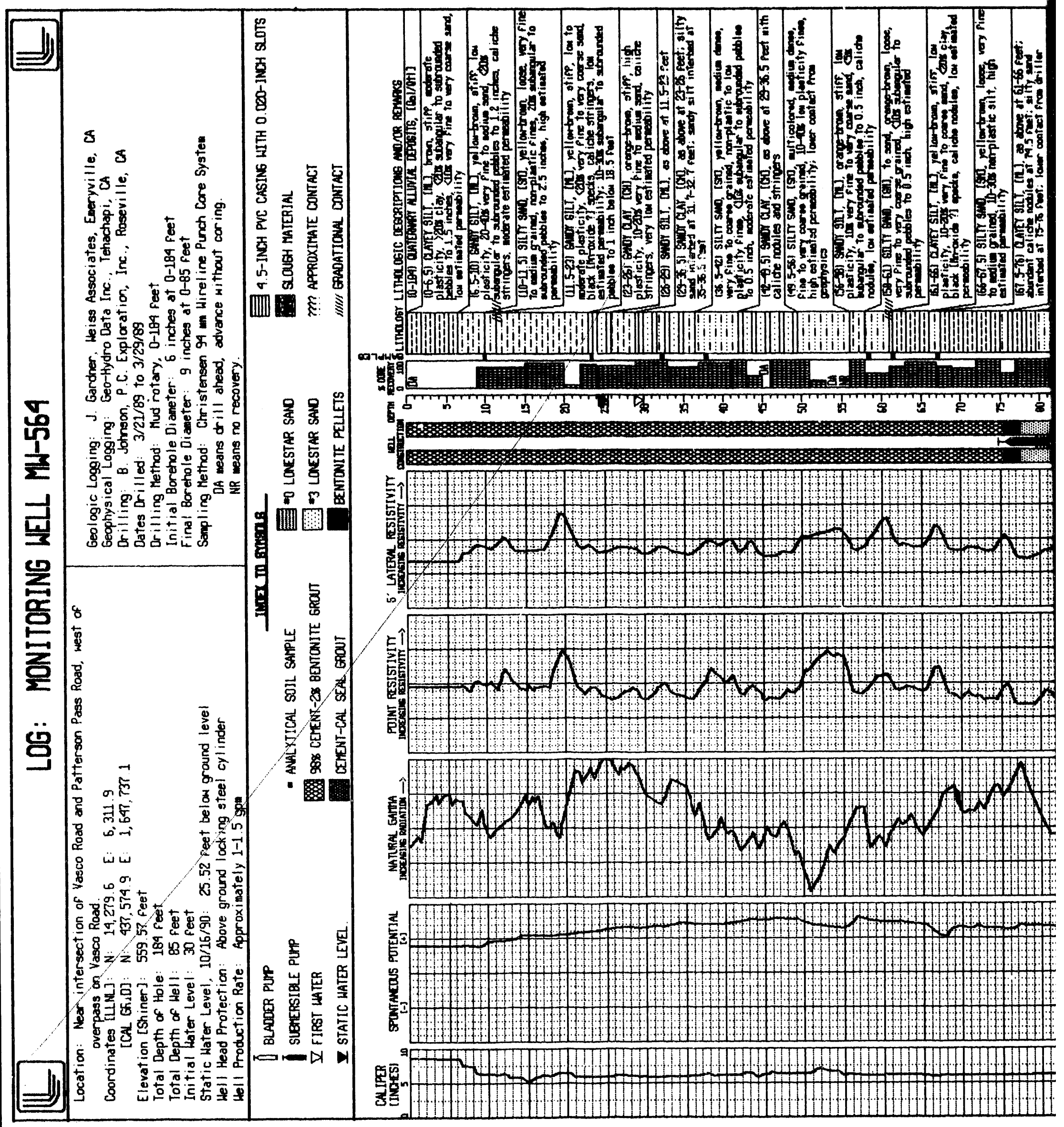



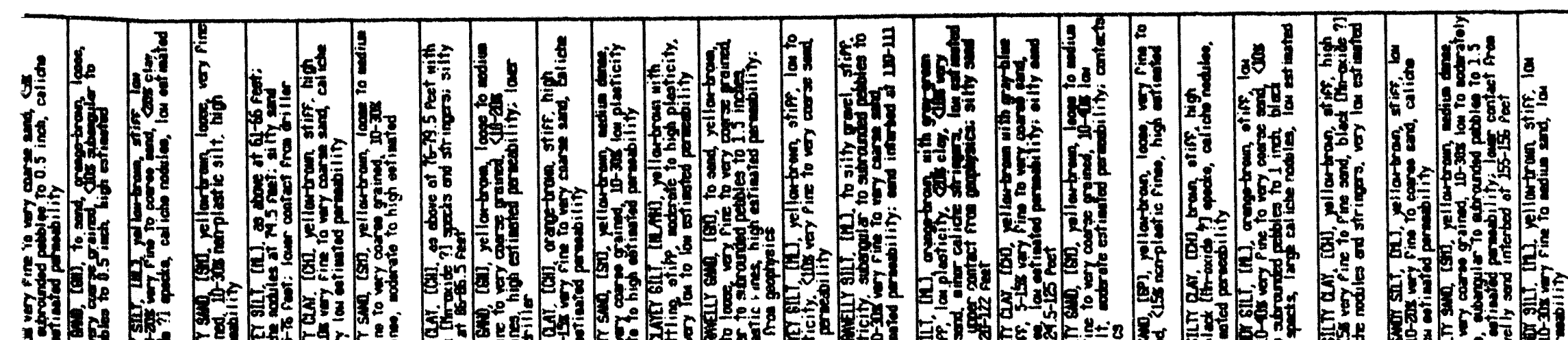

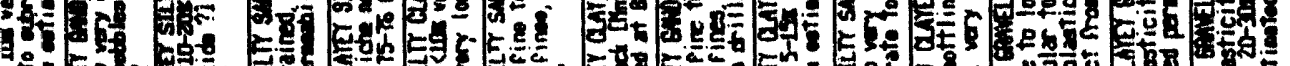

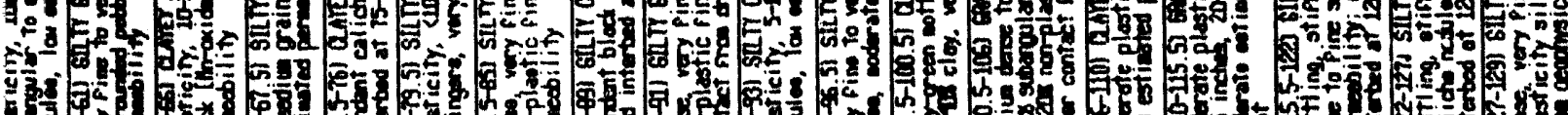

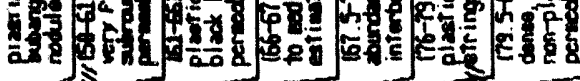
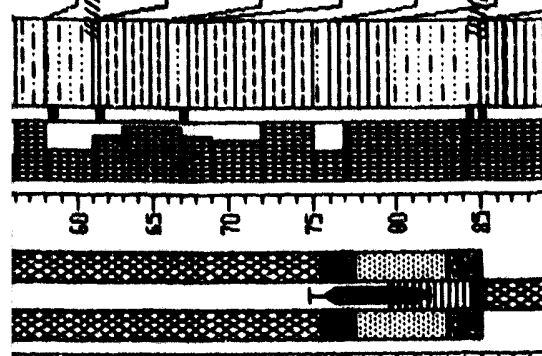

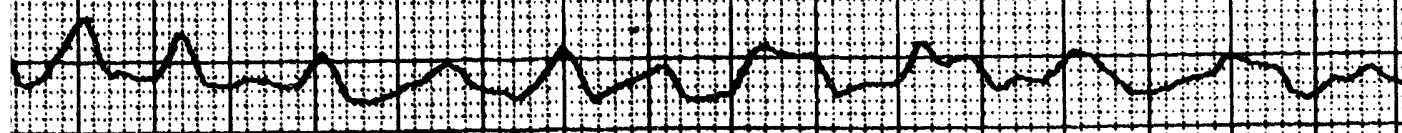

ations

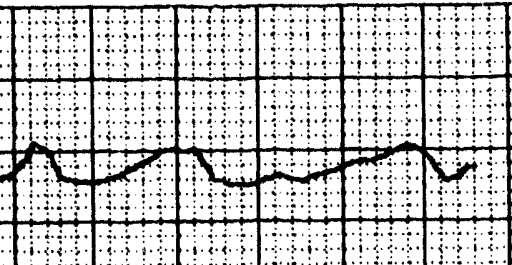
. ind

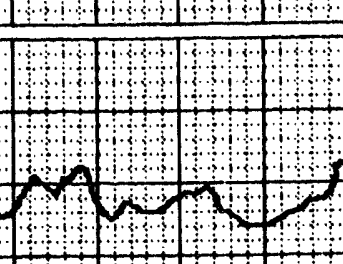
m. $+1$

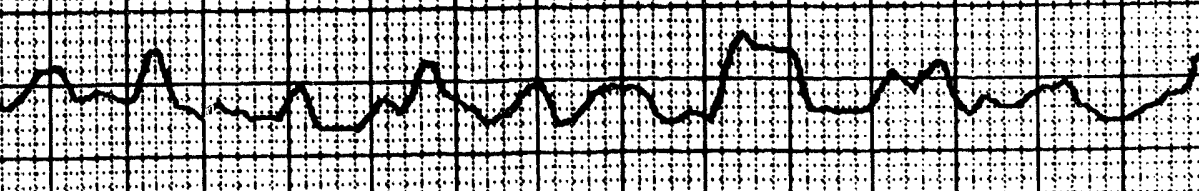

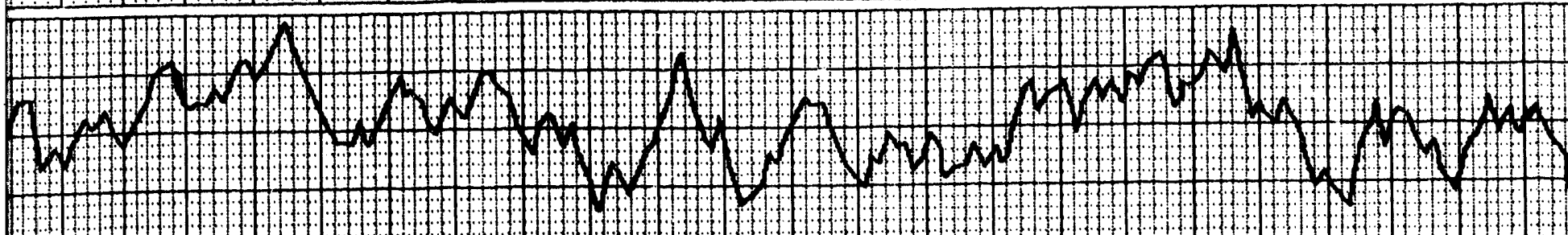




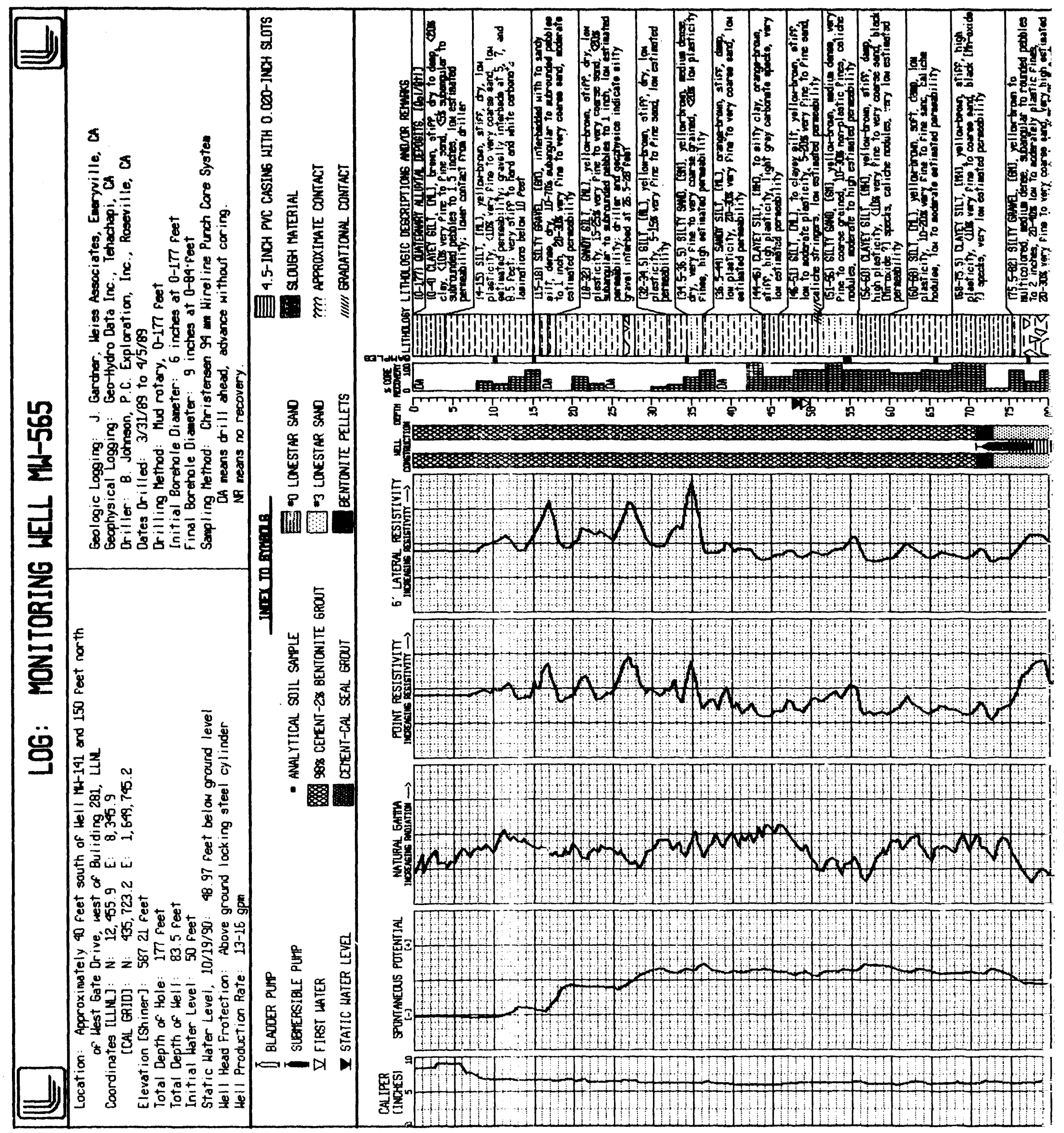




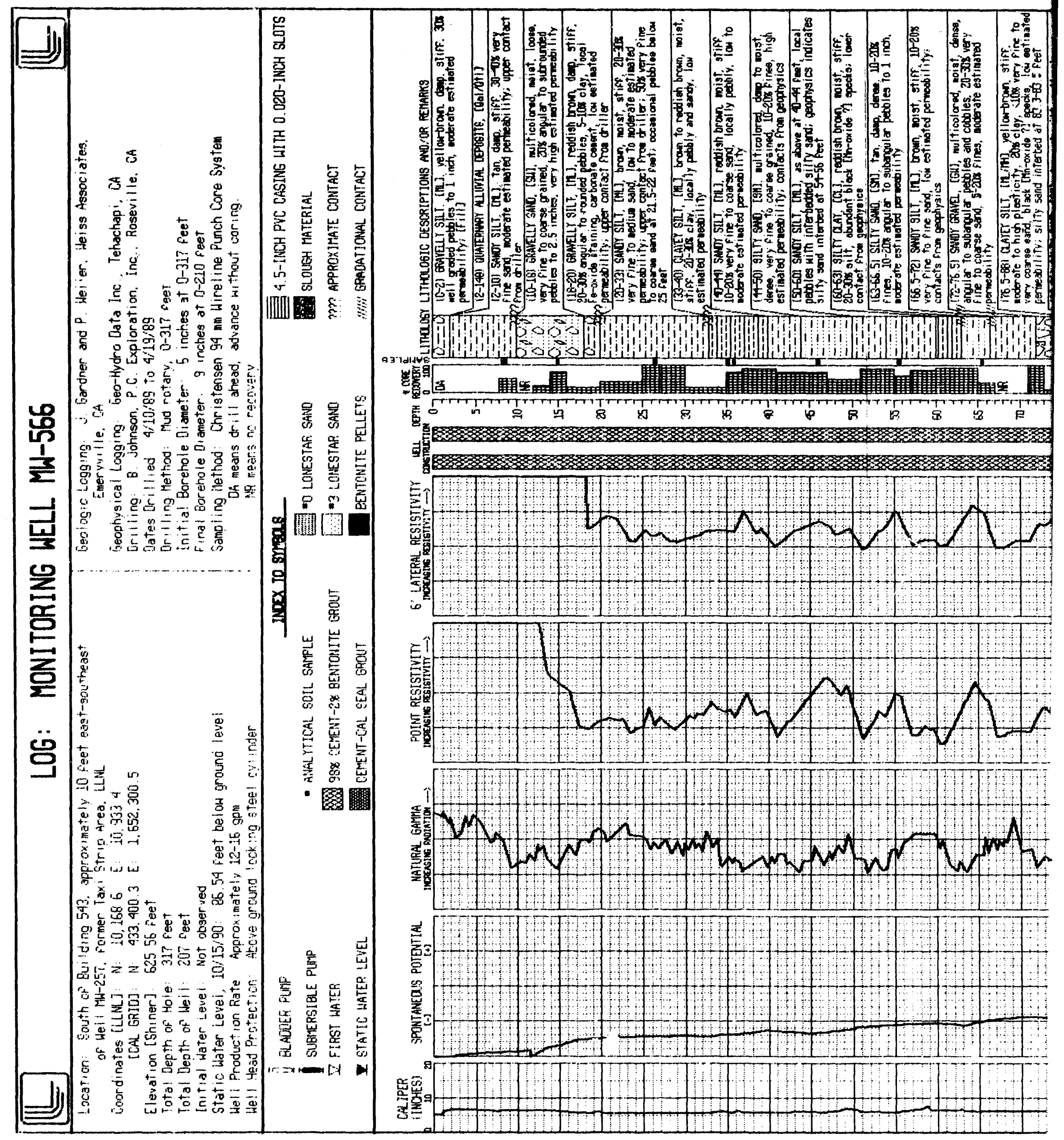




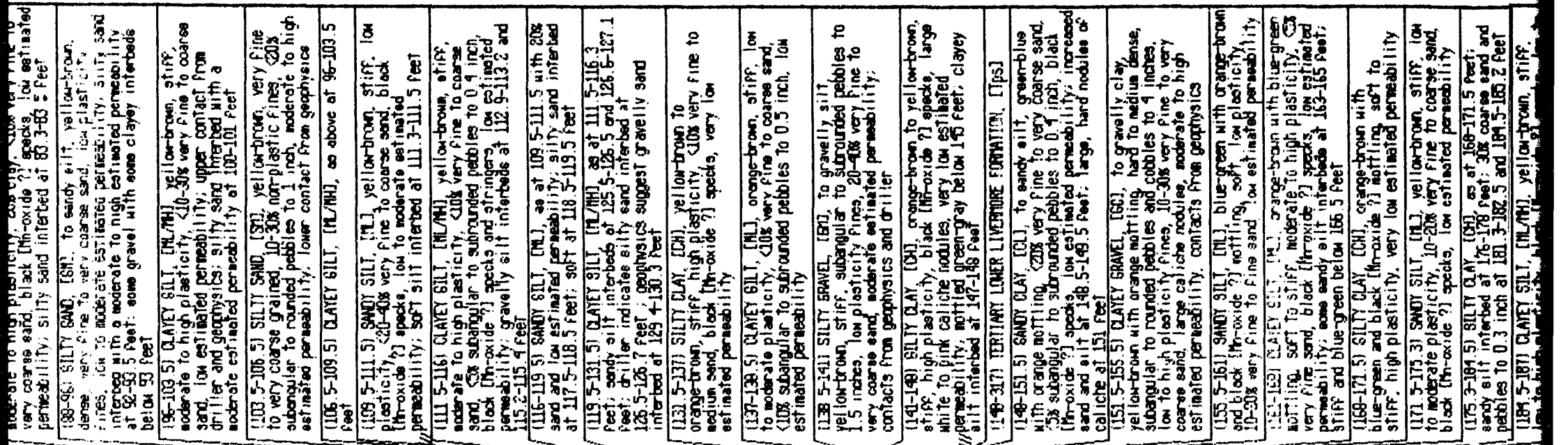

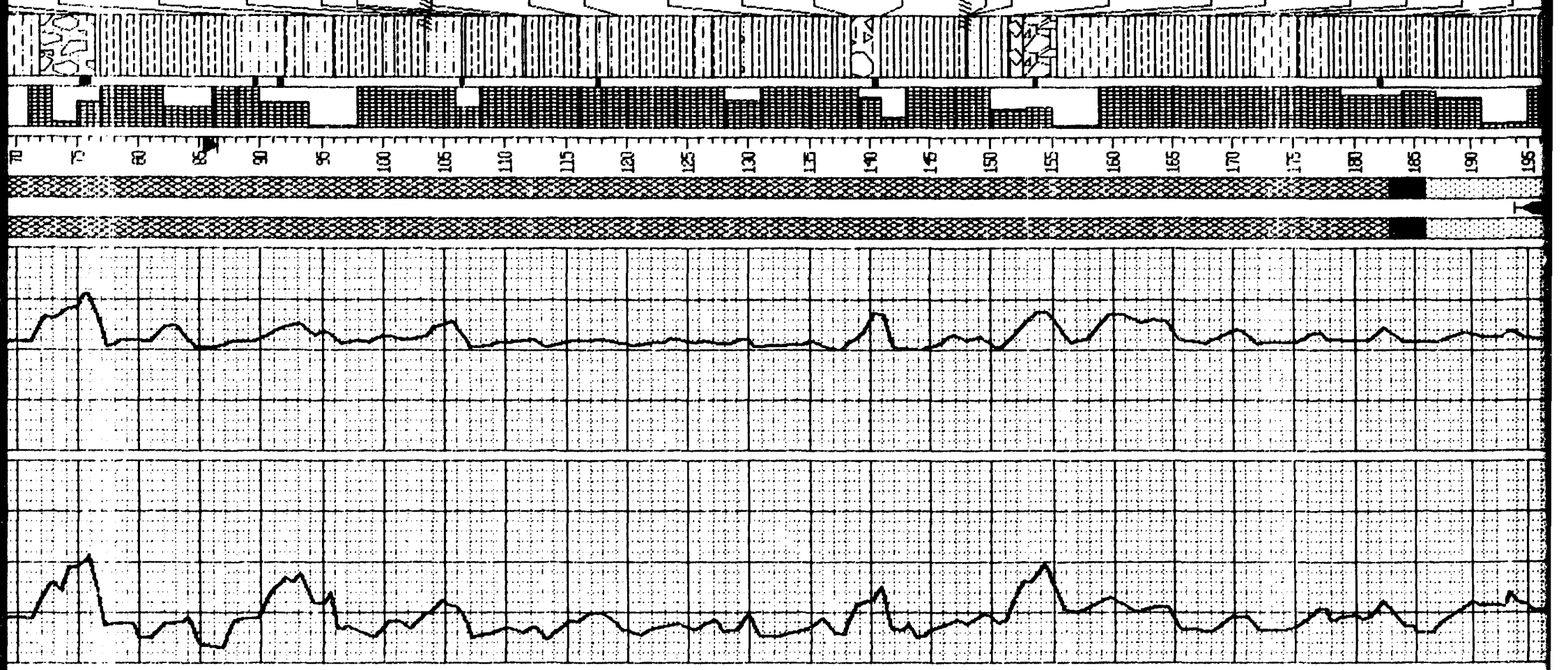

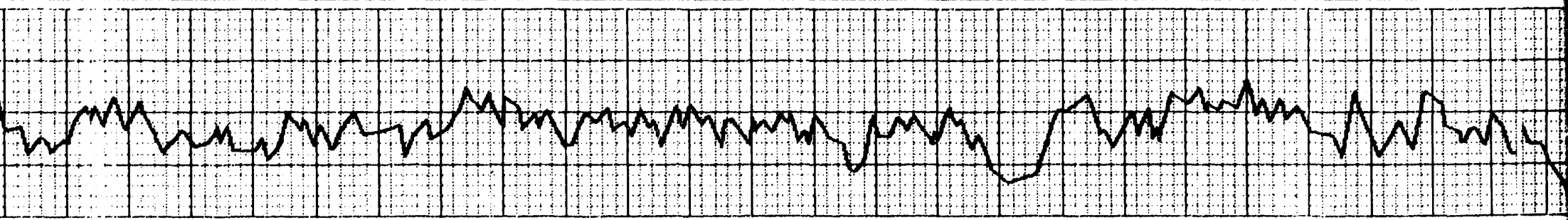




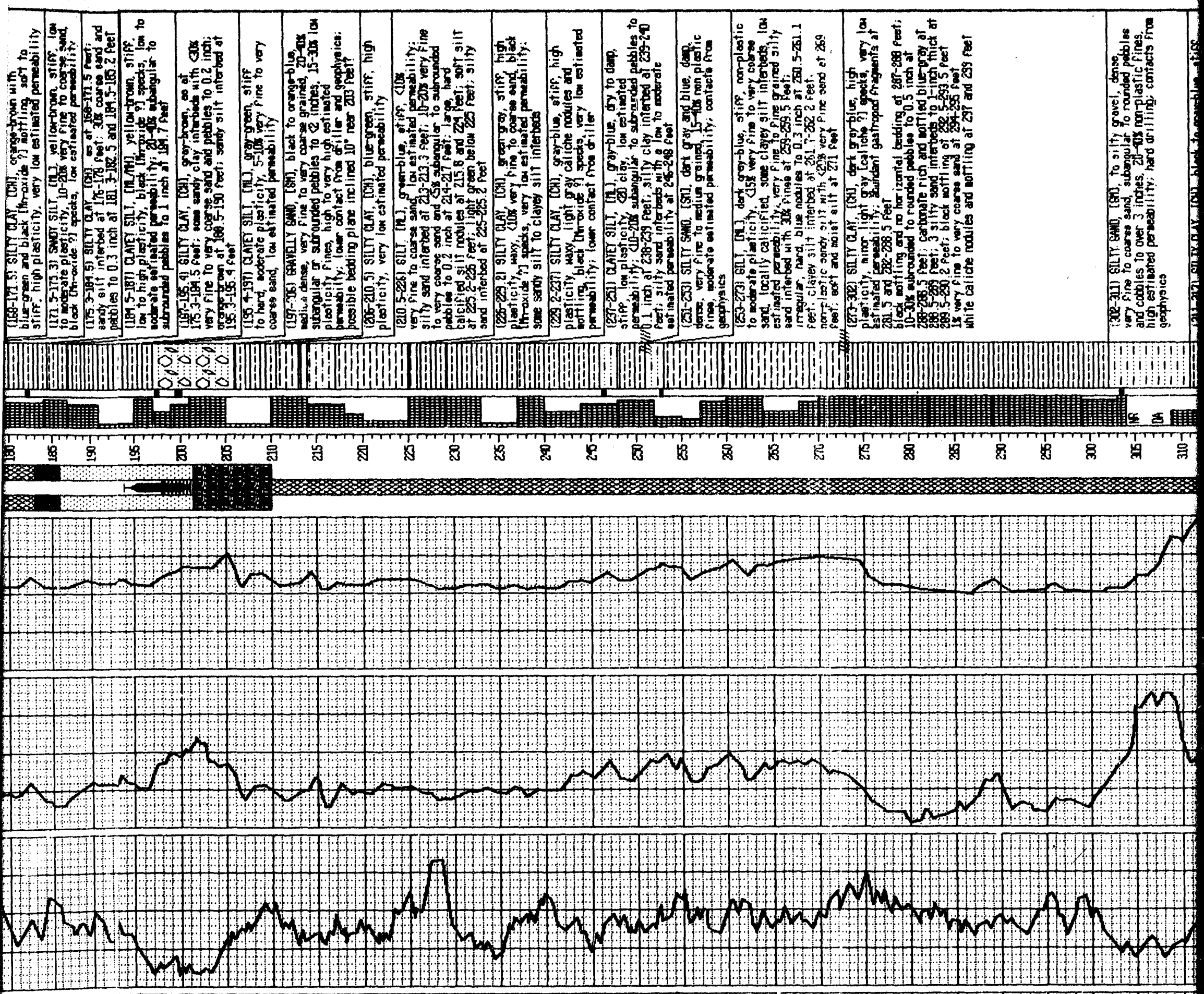




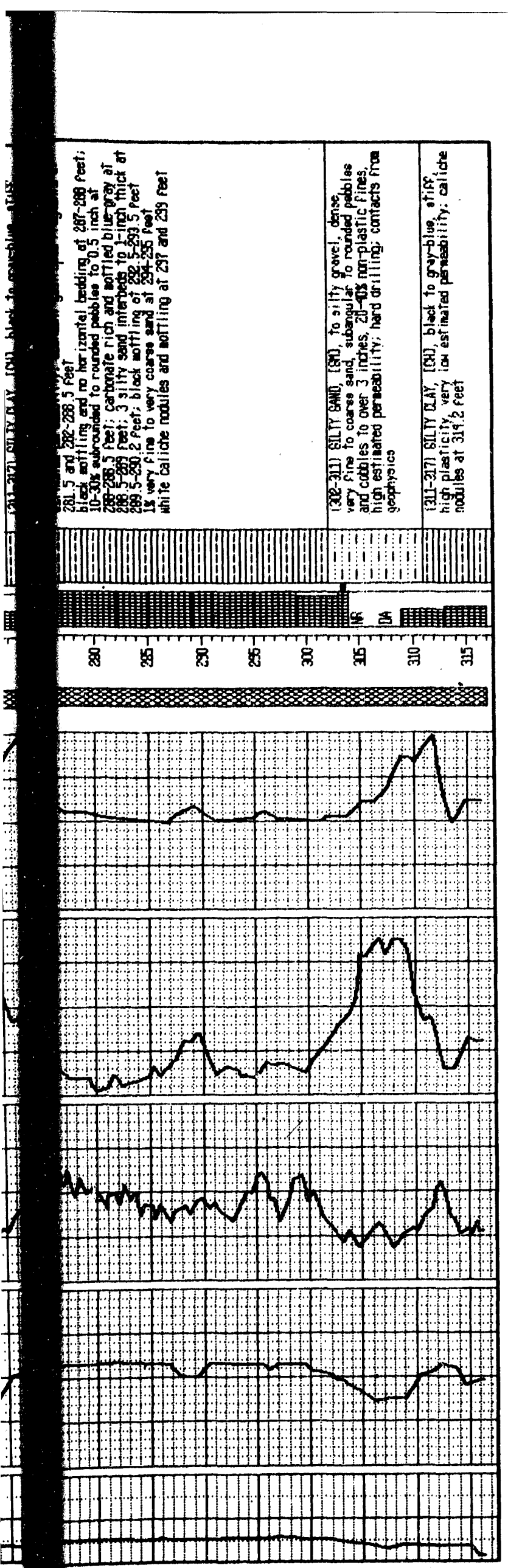




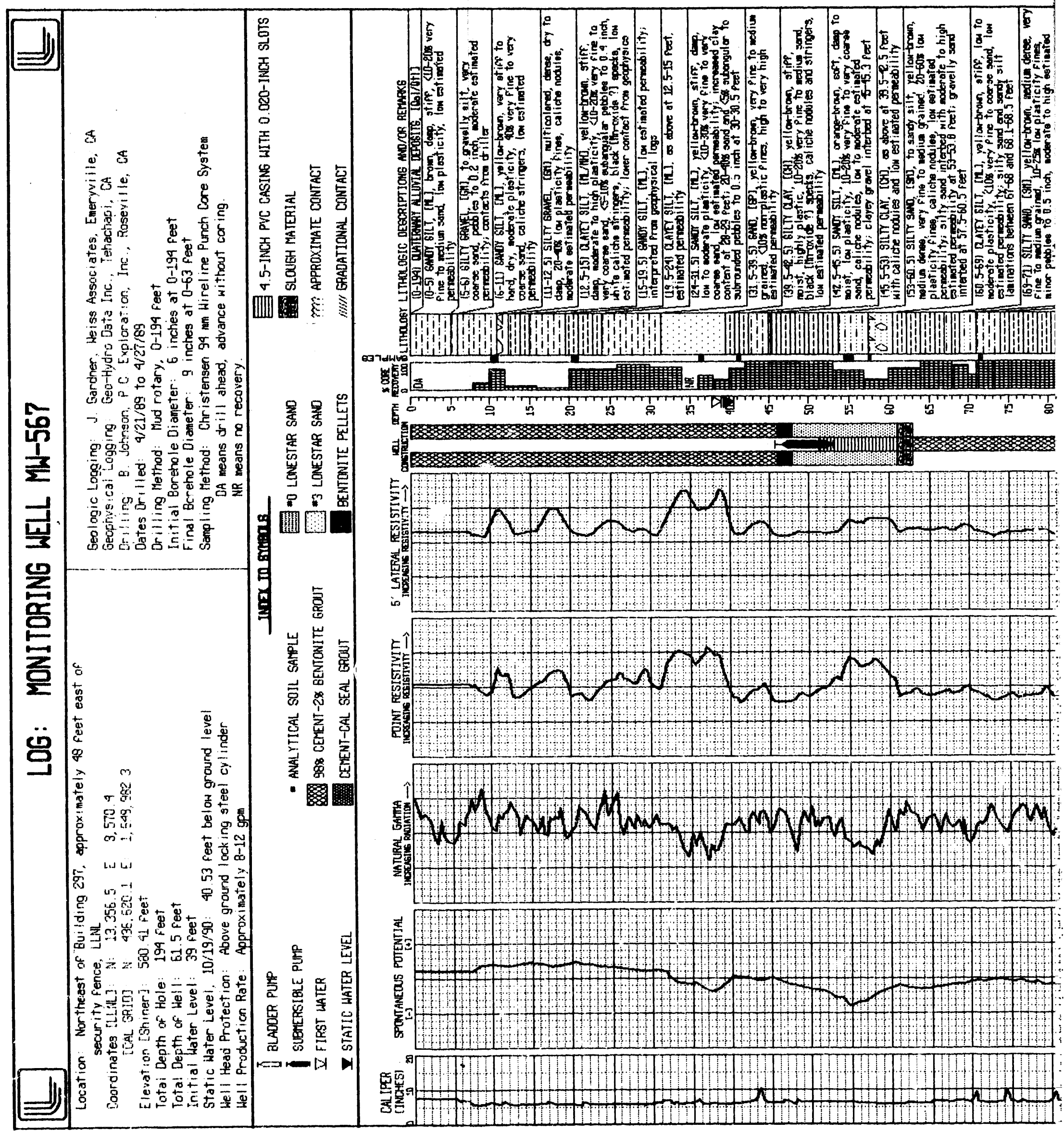



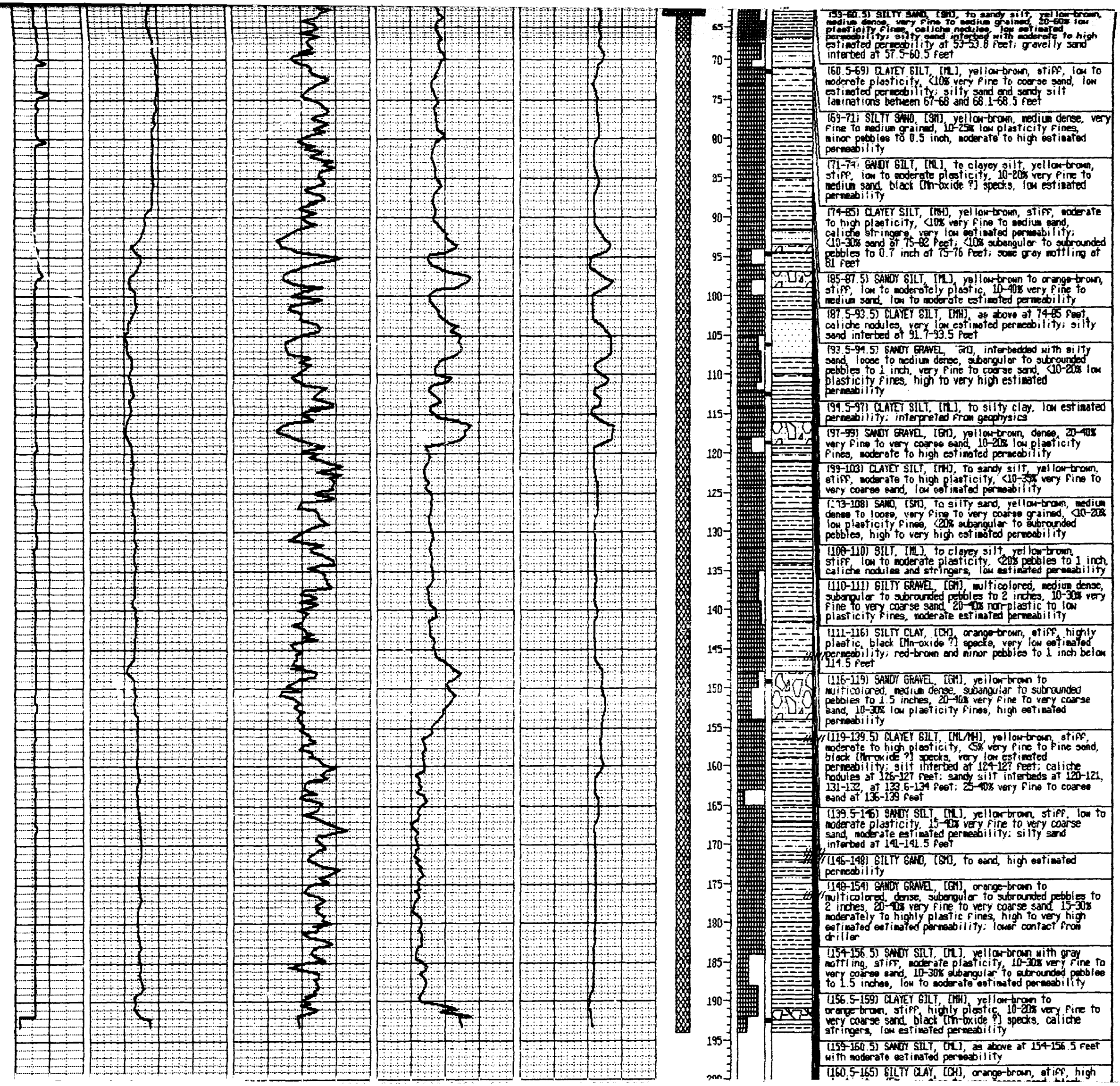


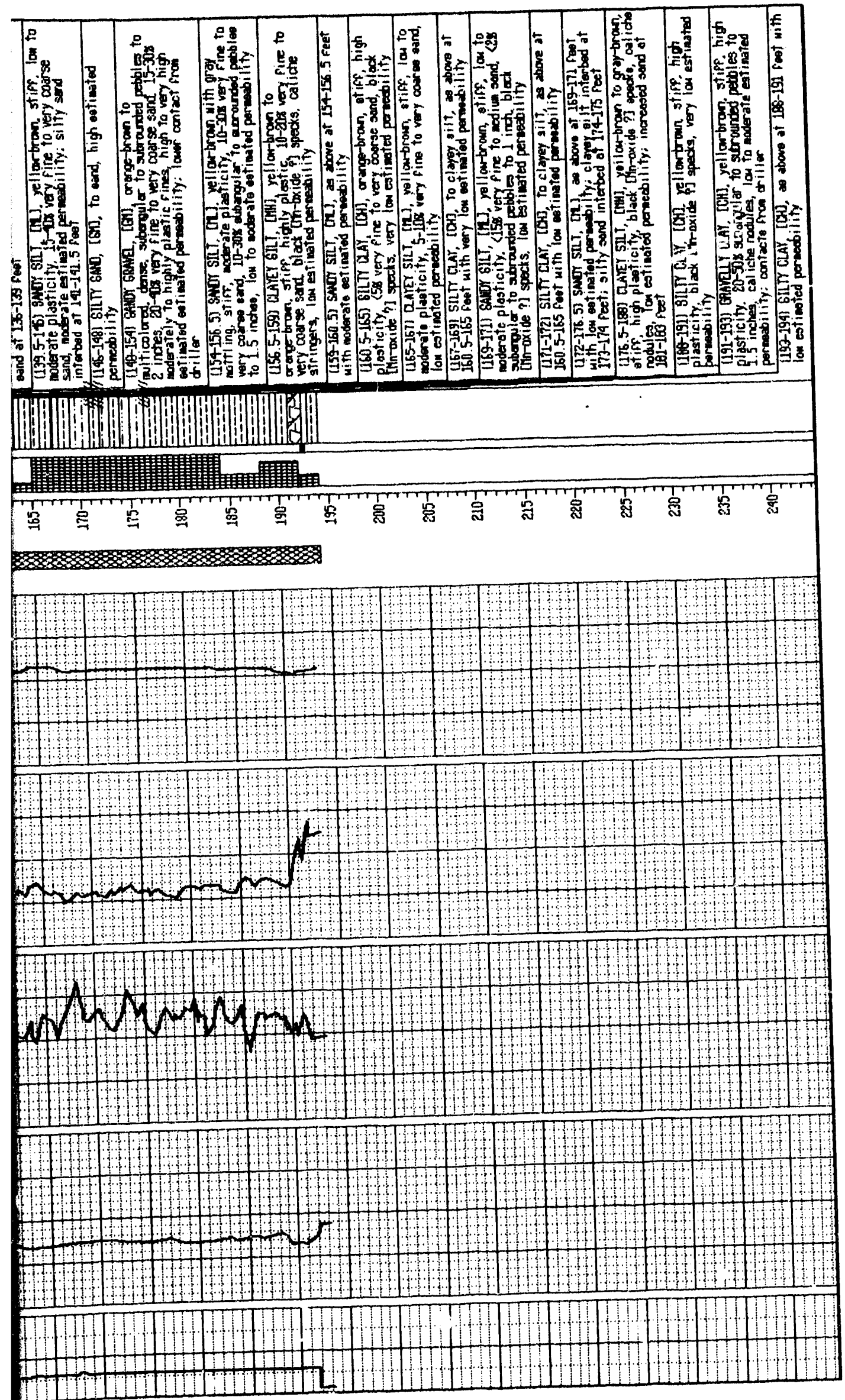




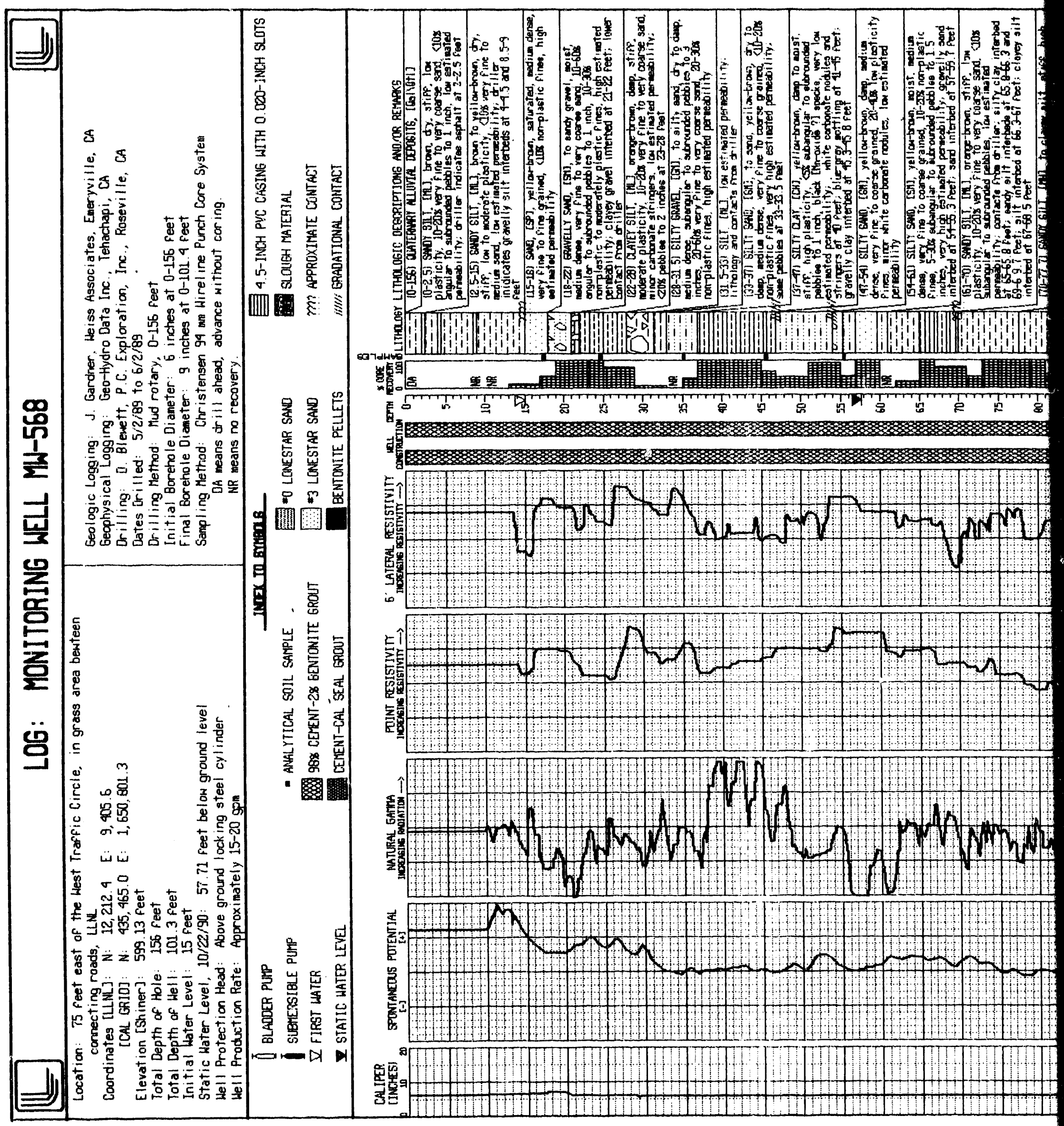




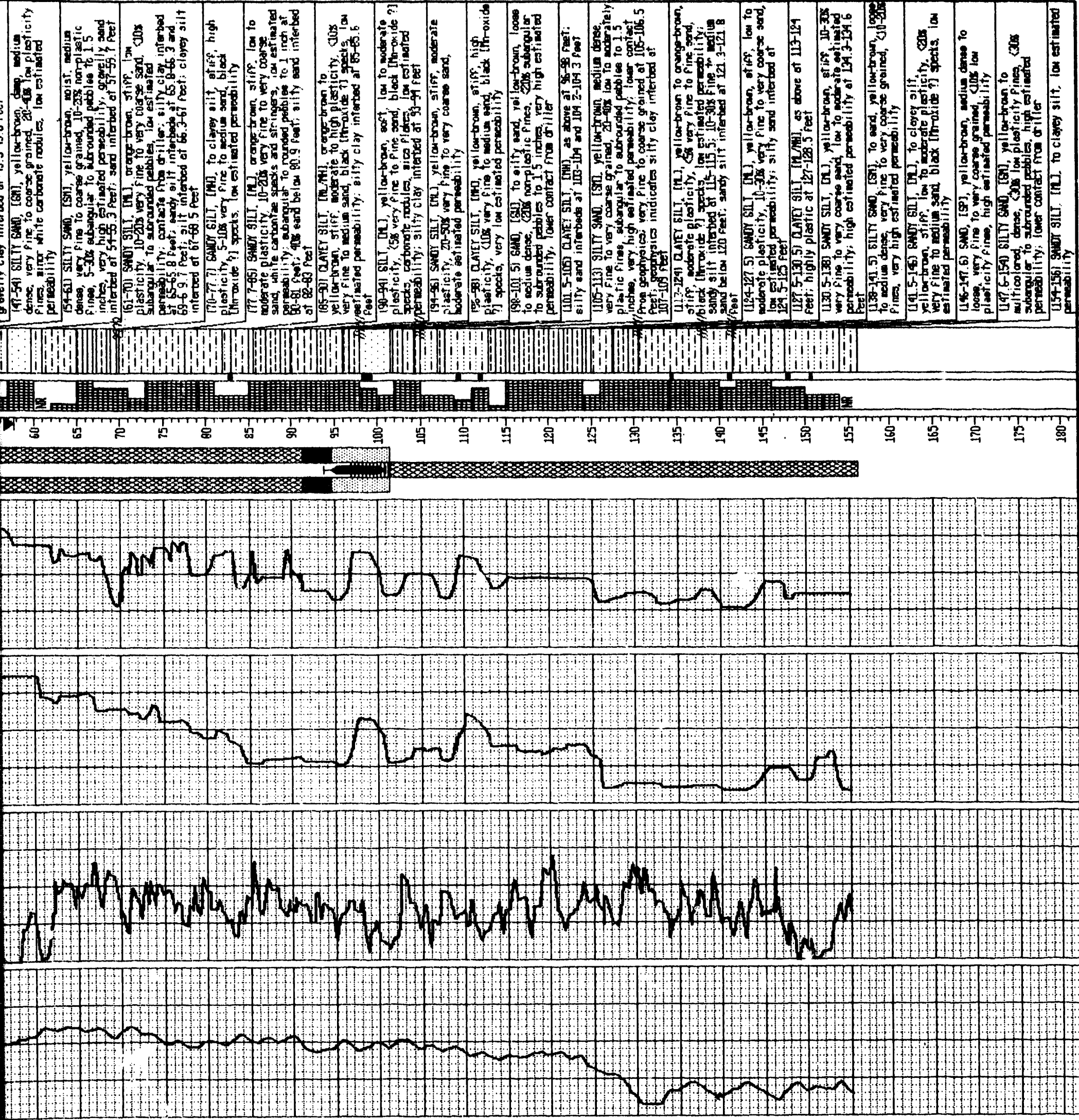




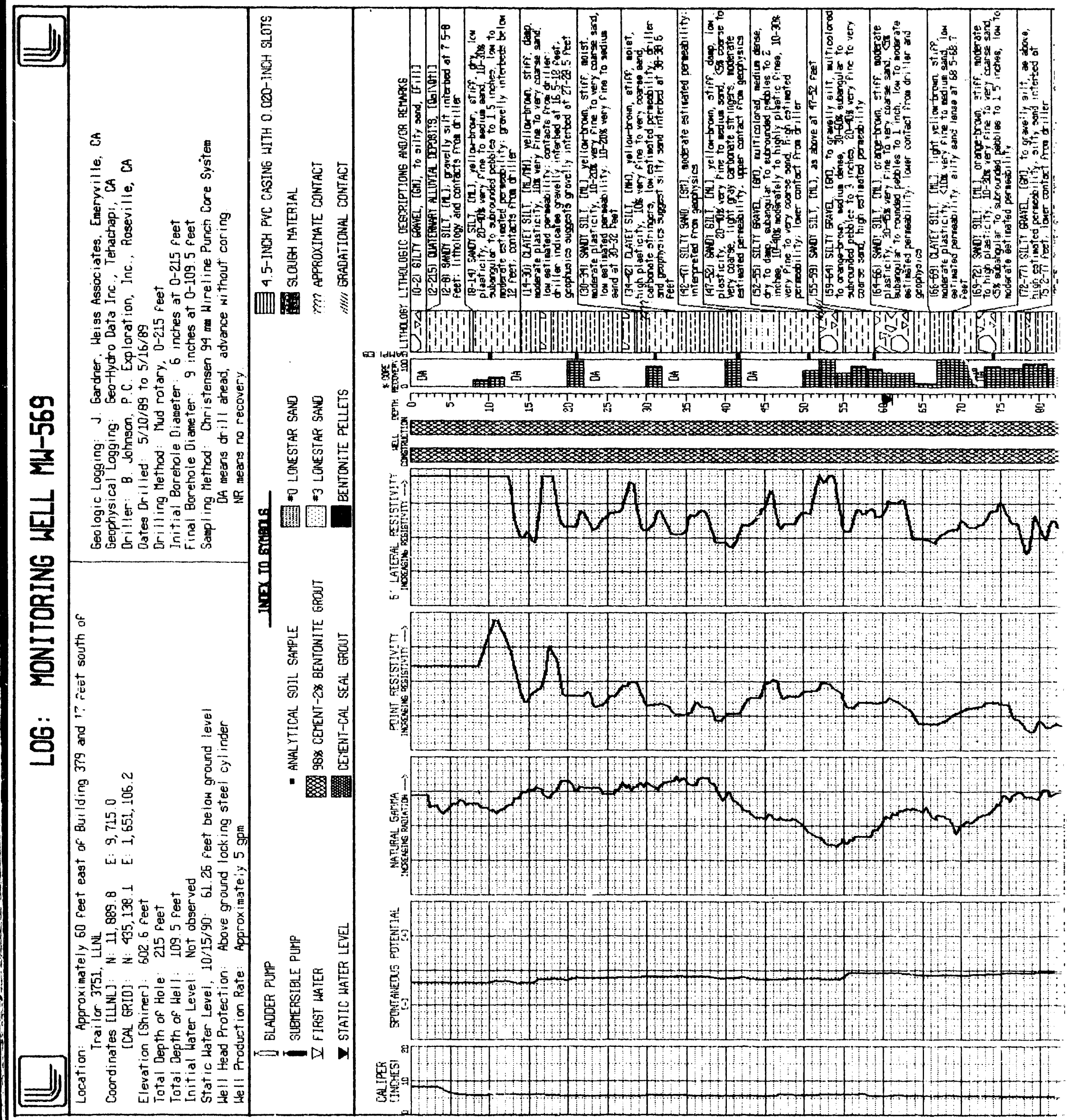



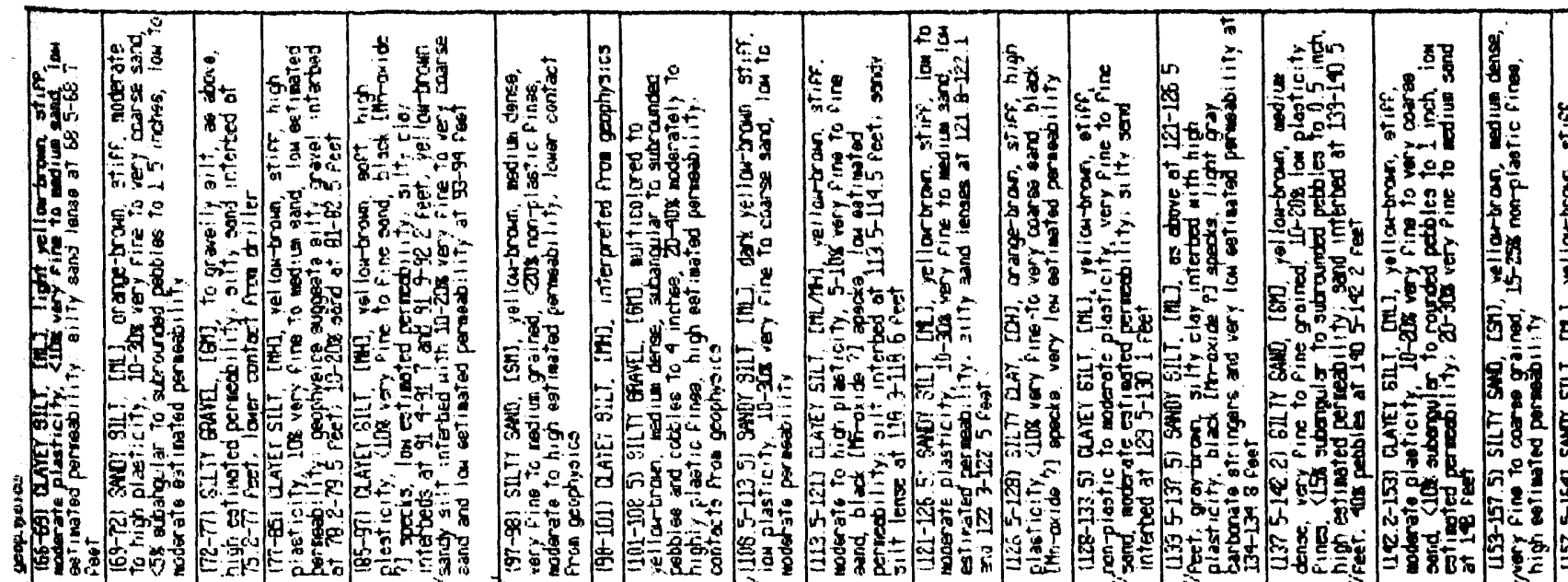

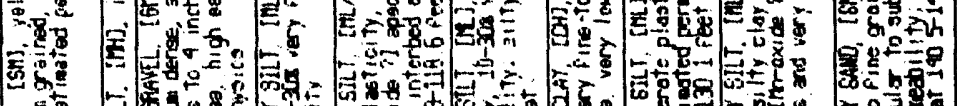

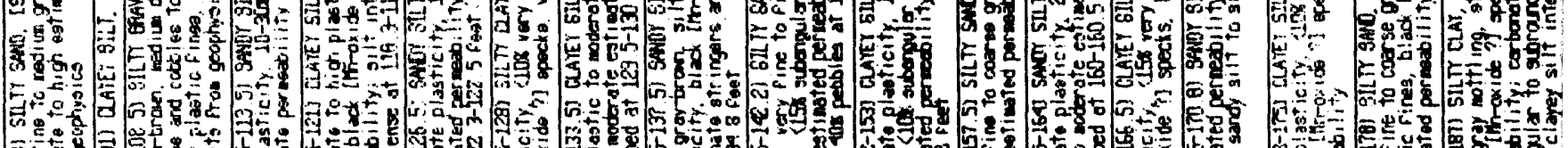

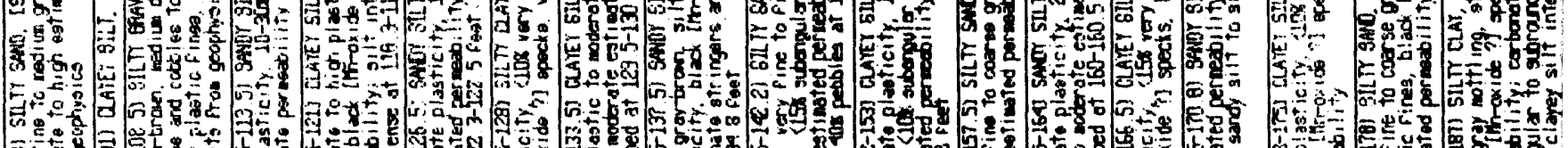

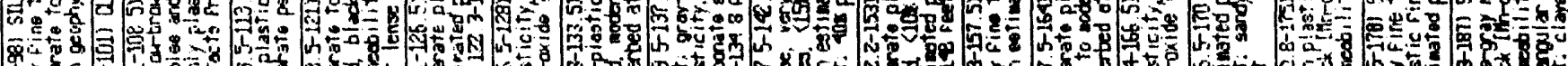

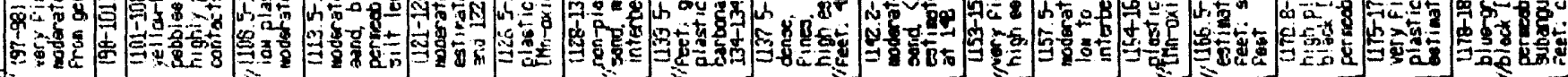
(1) 渄

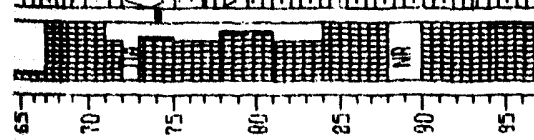

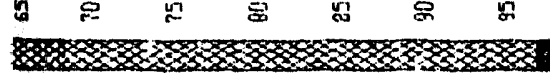

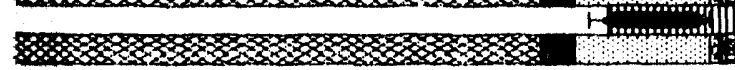

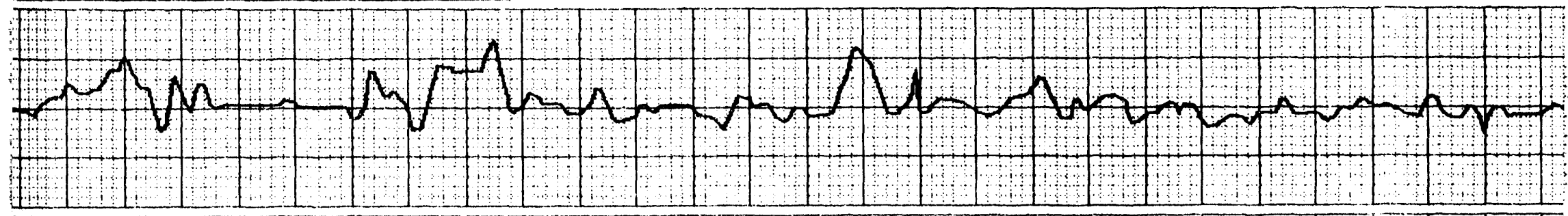

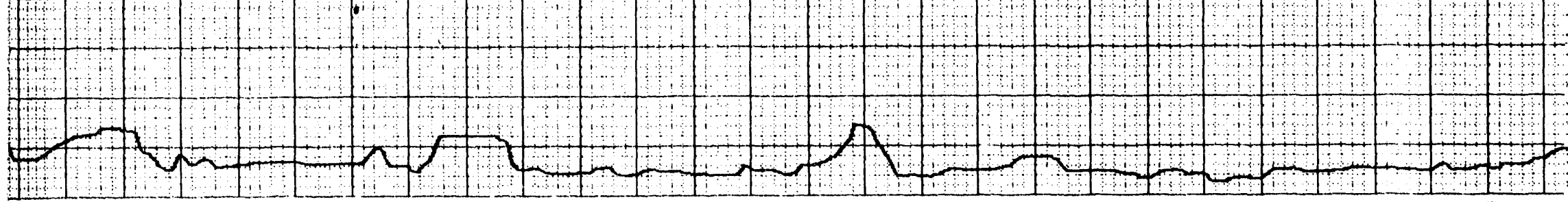

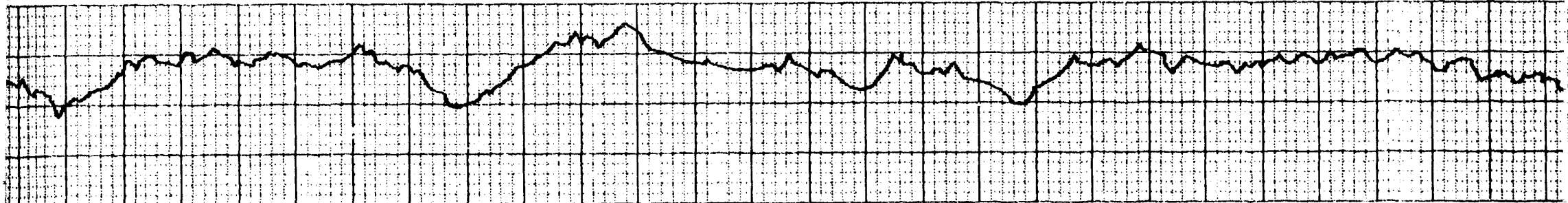




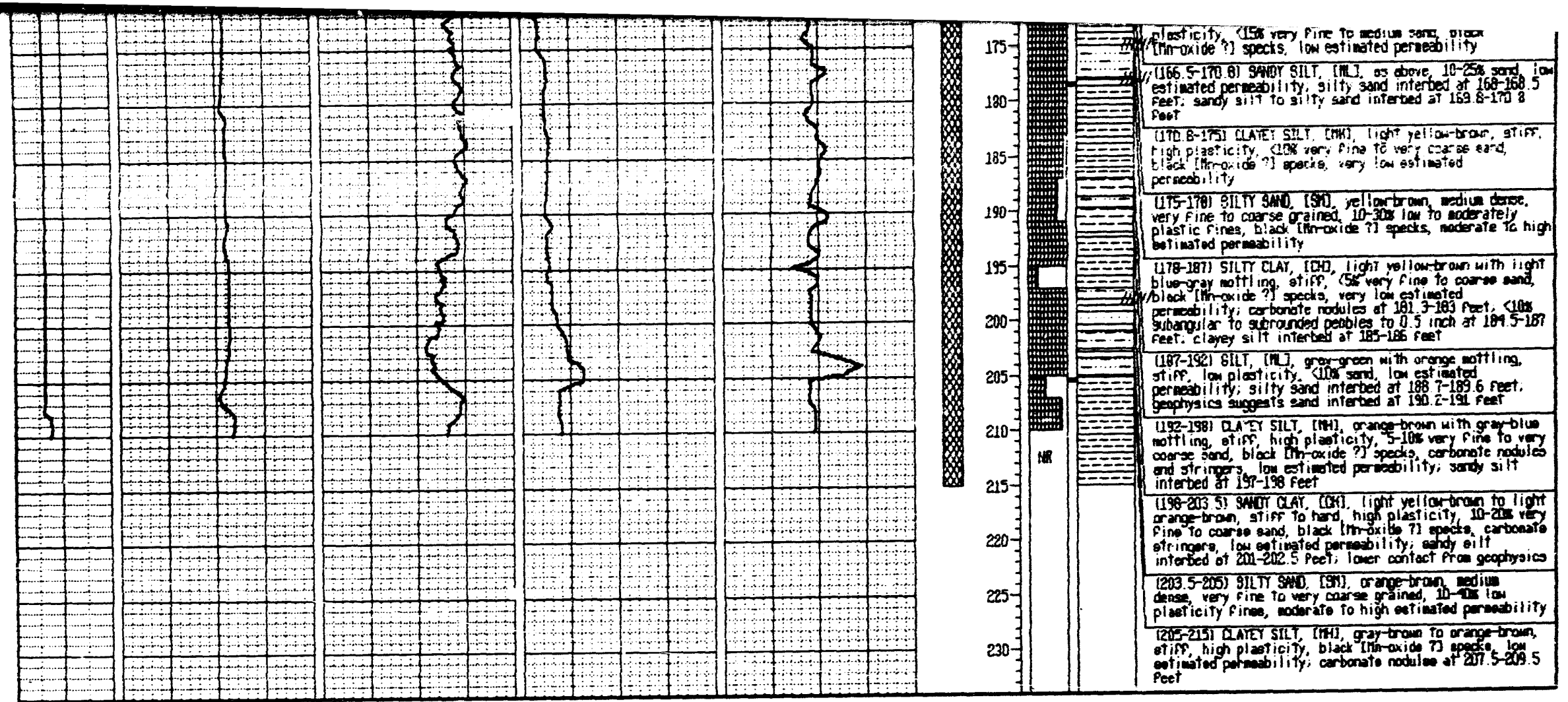




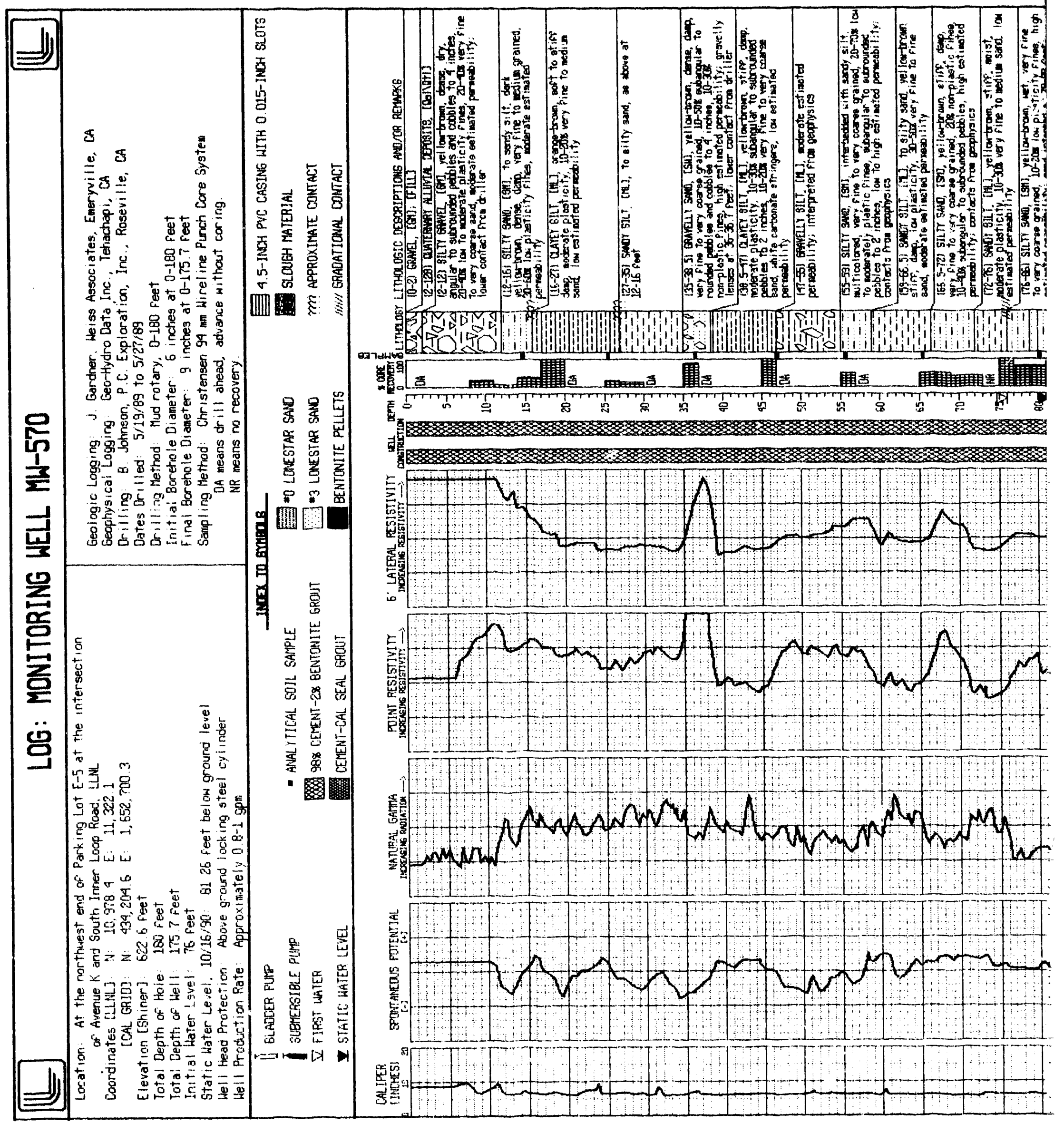




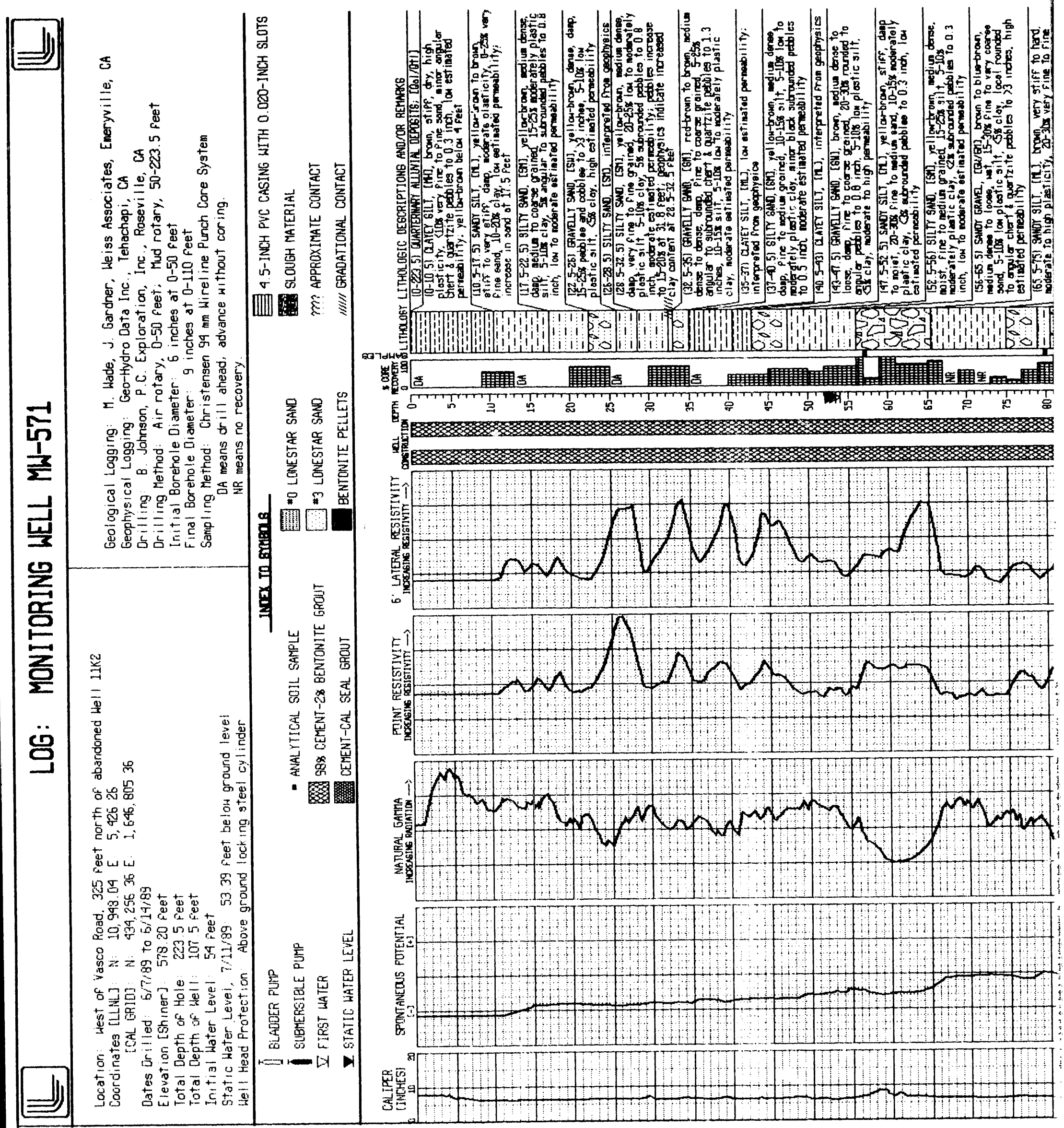



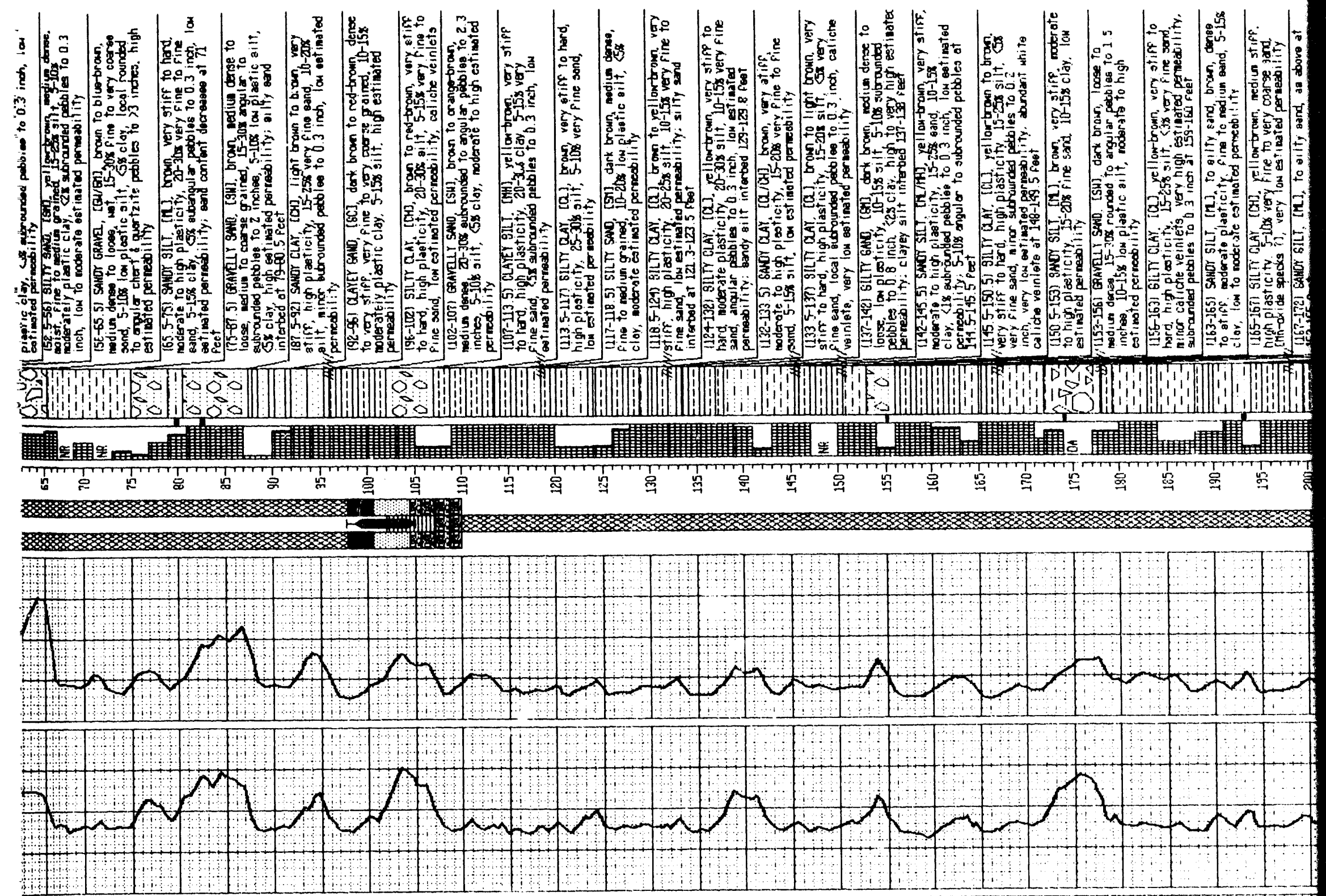

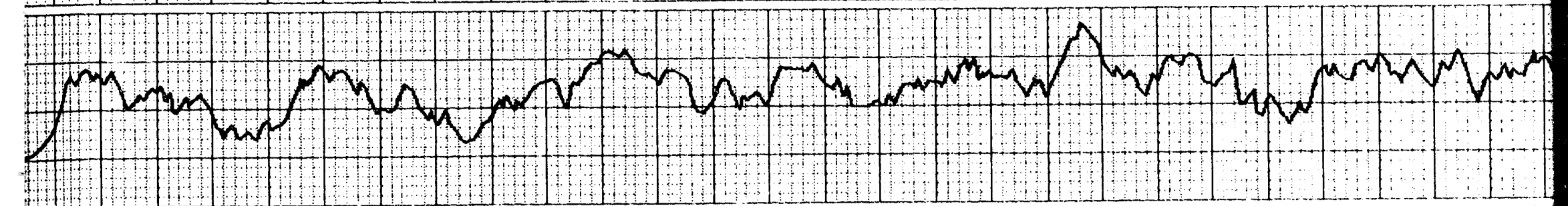




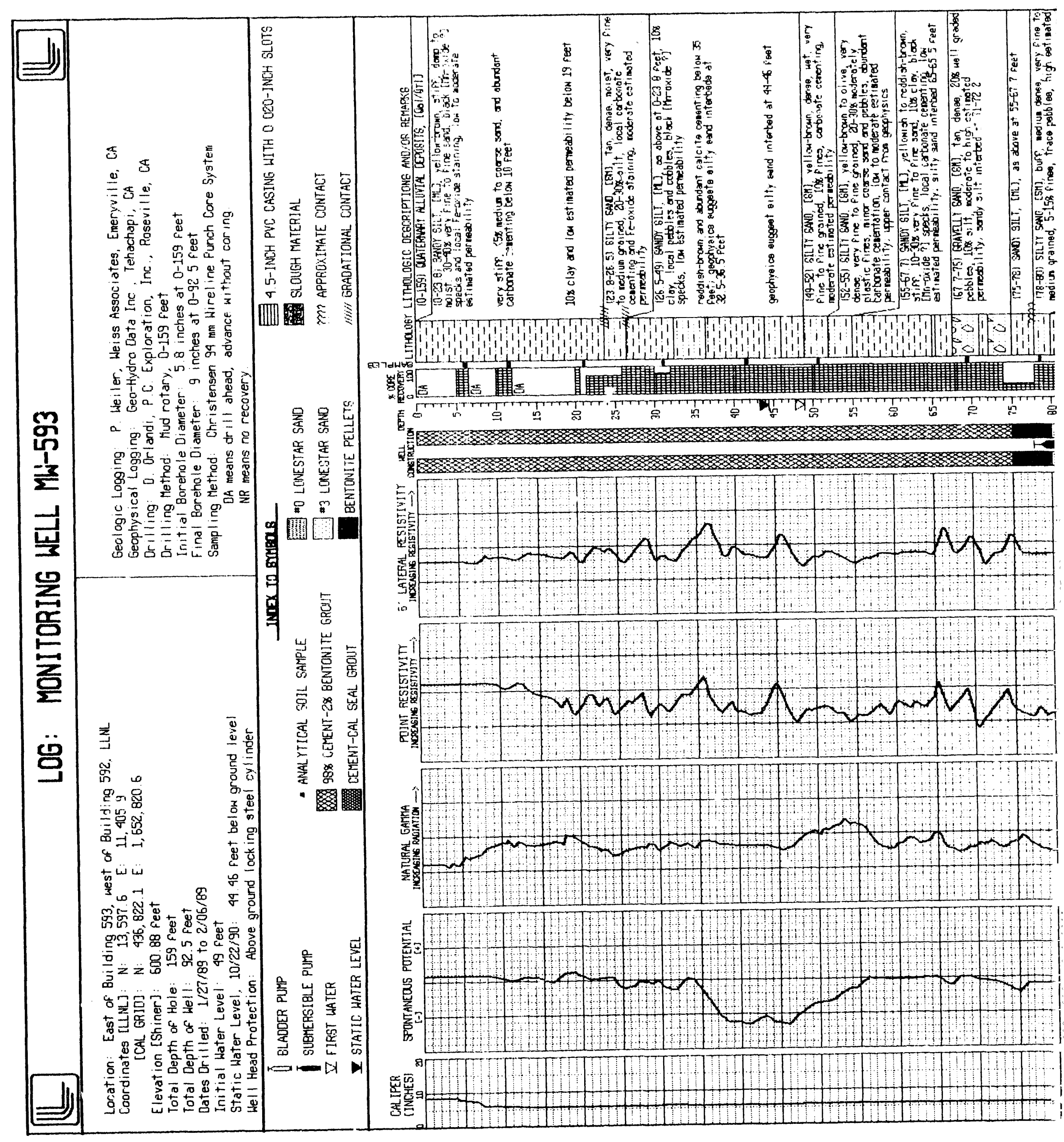




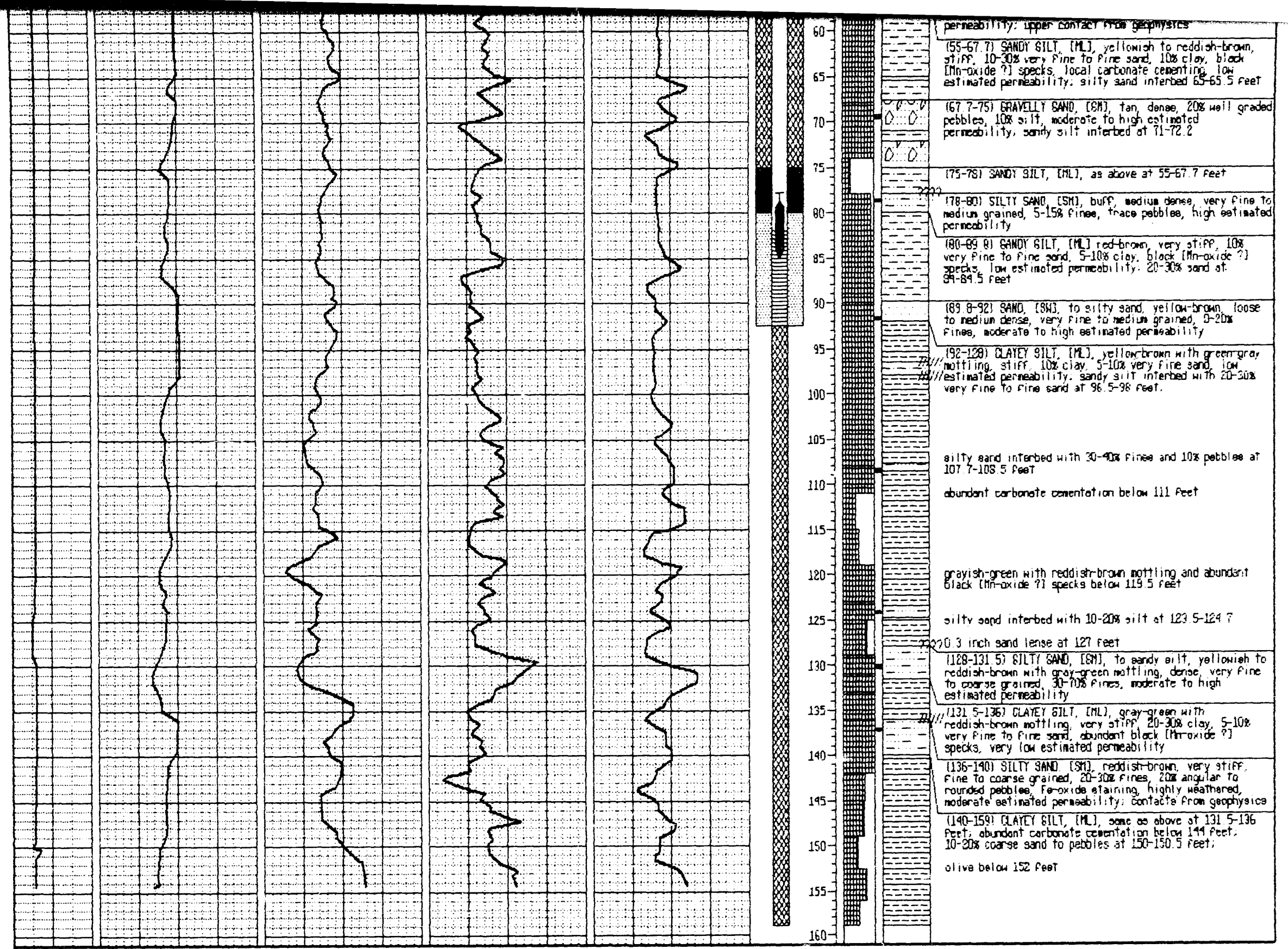




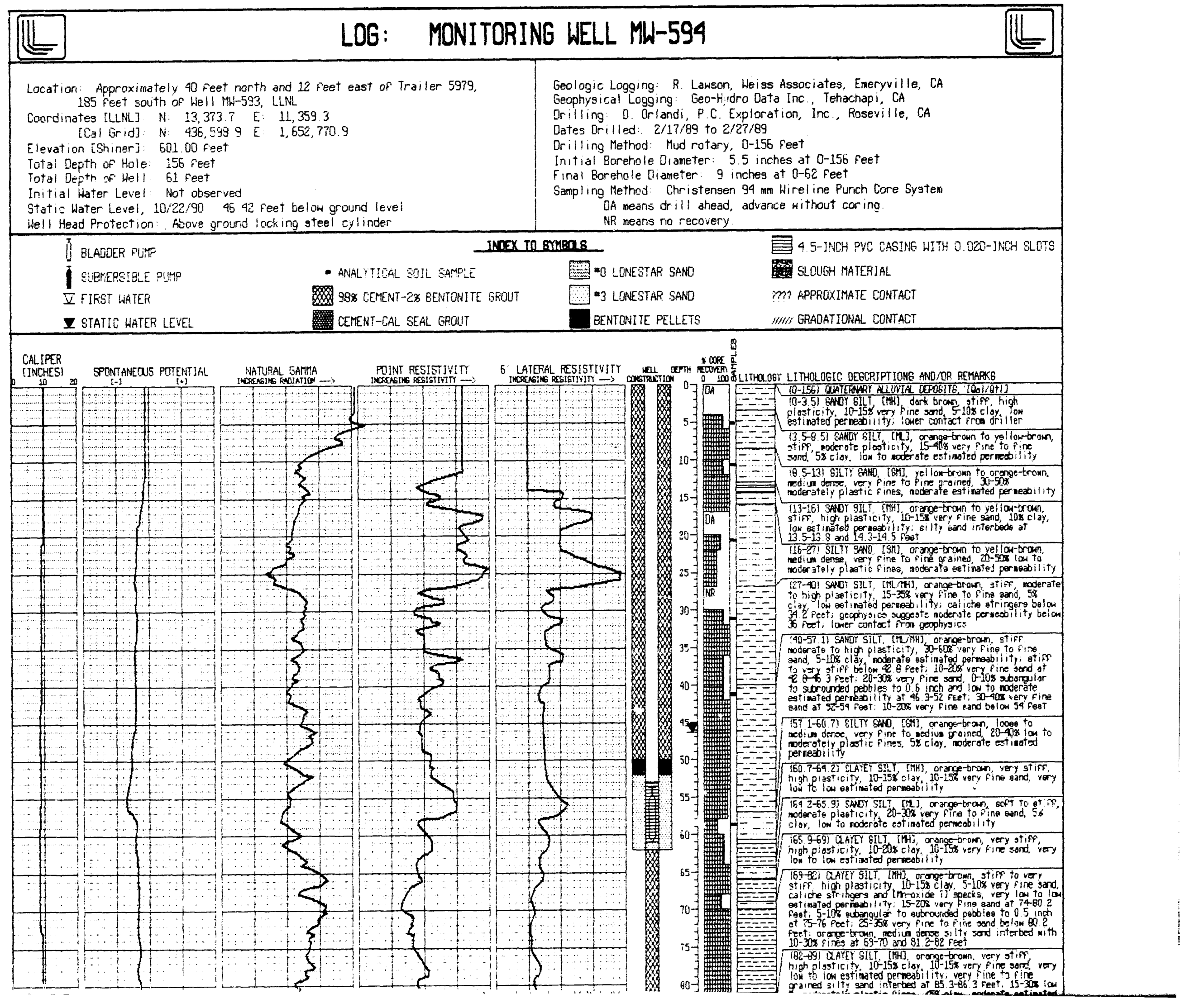




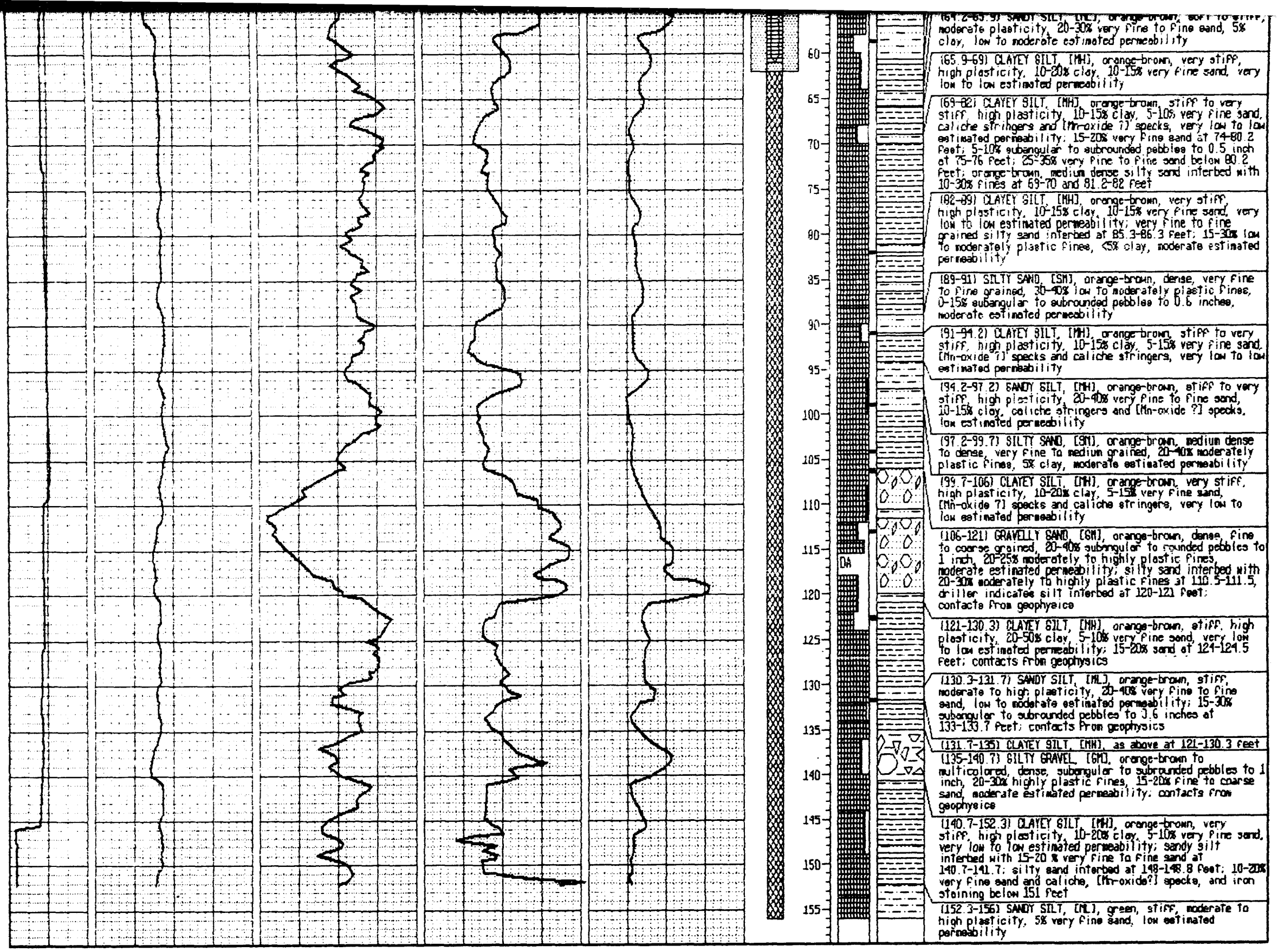




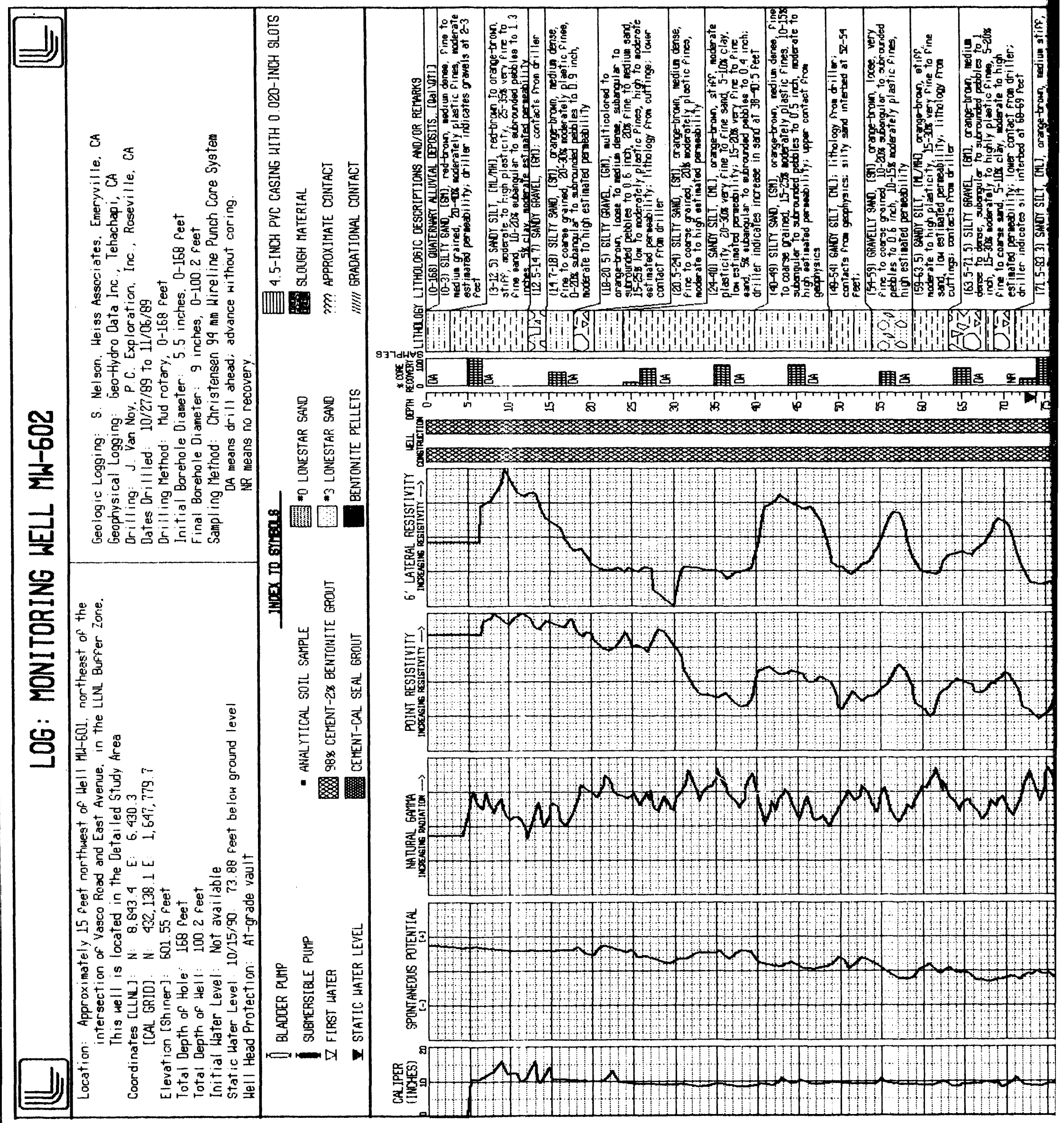




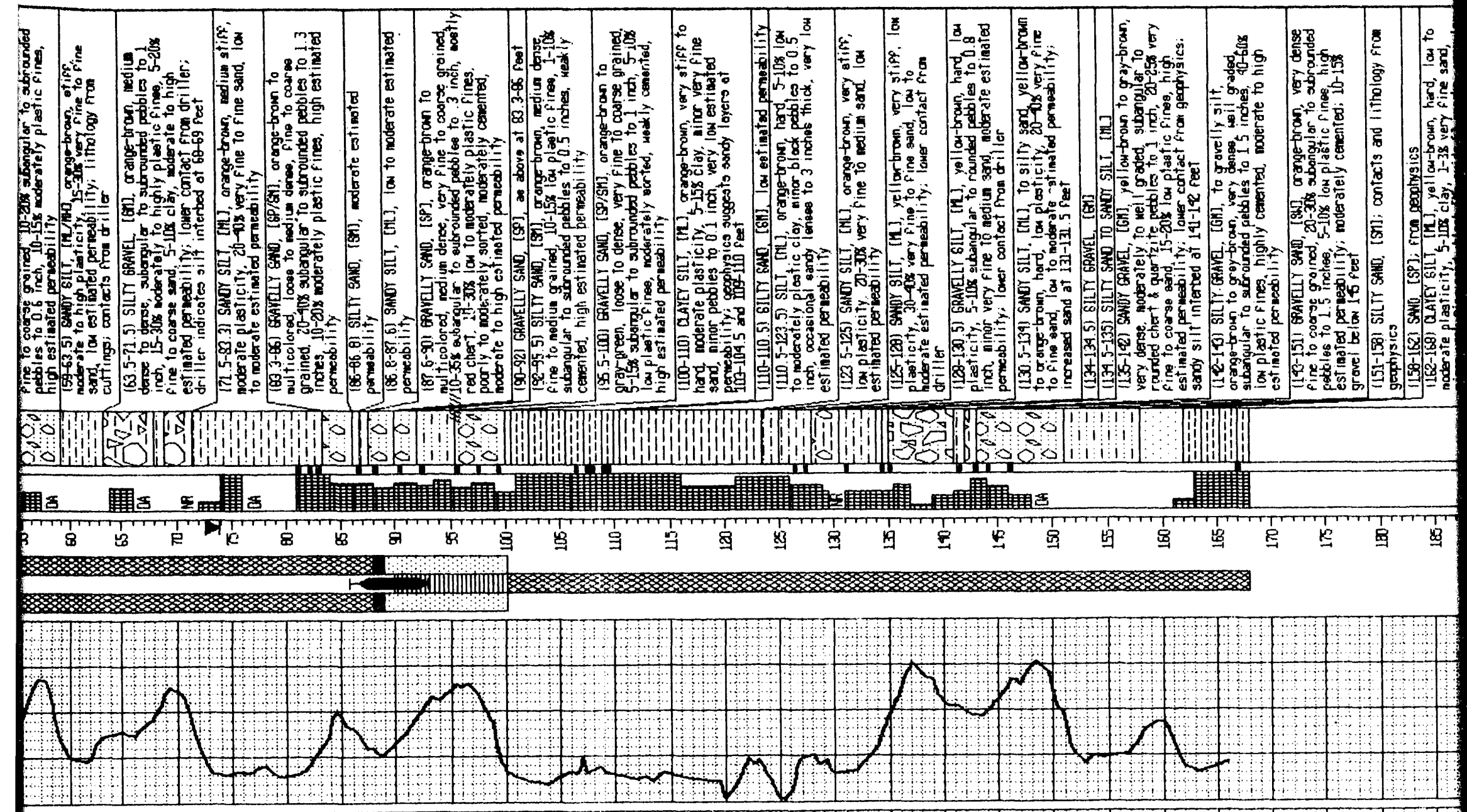

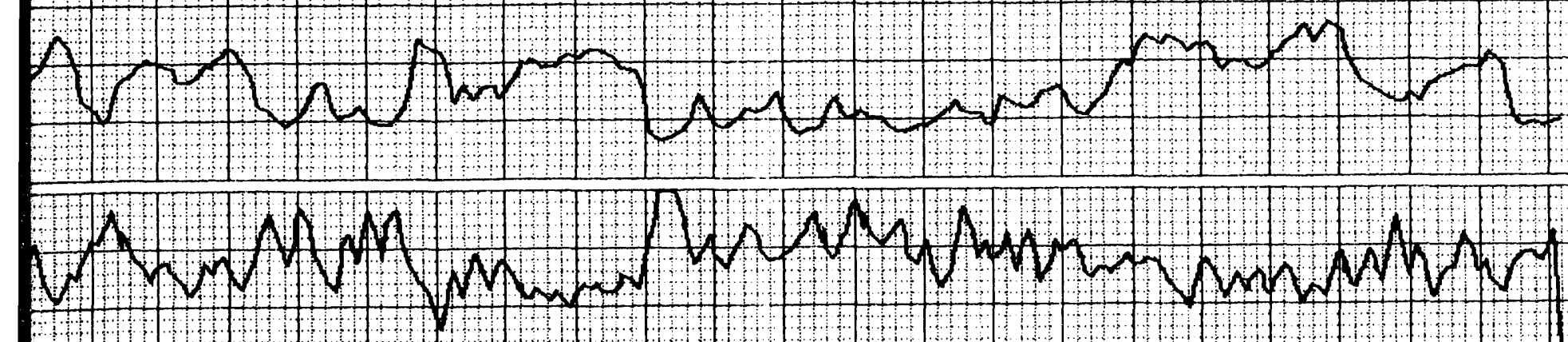

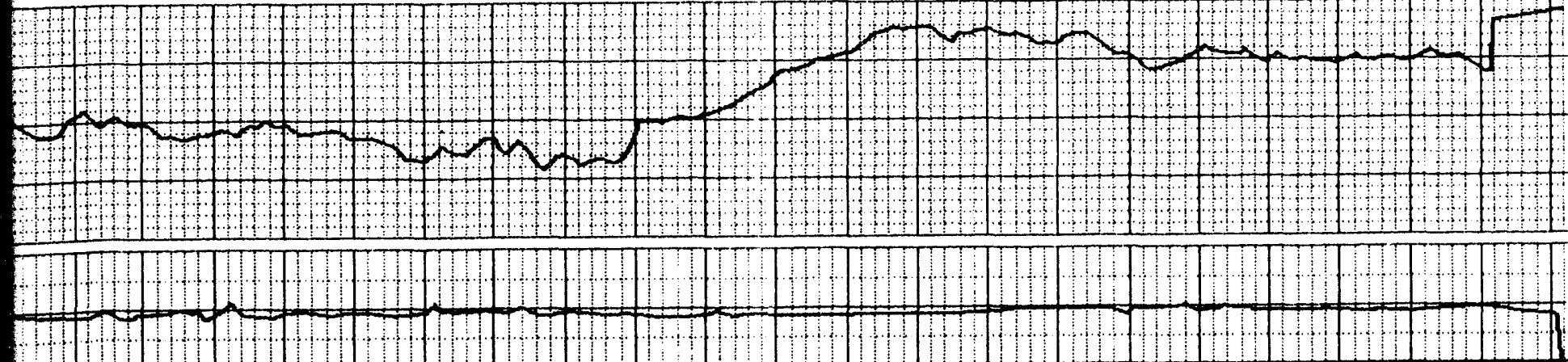




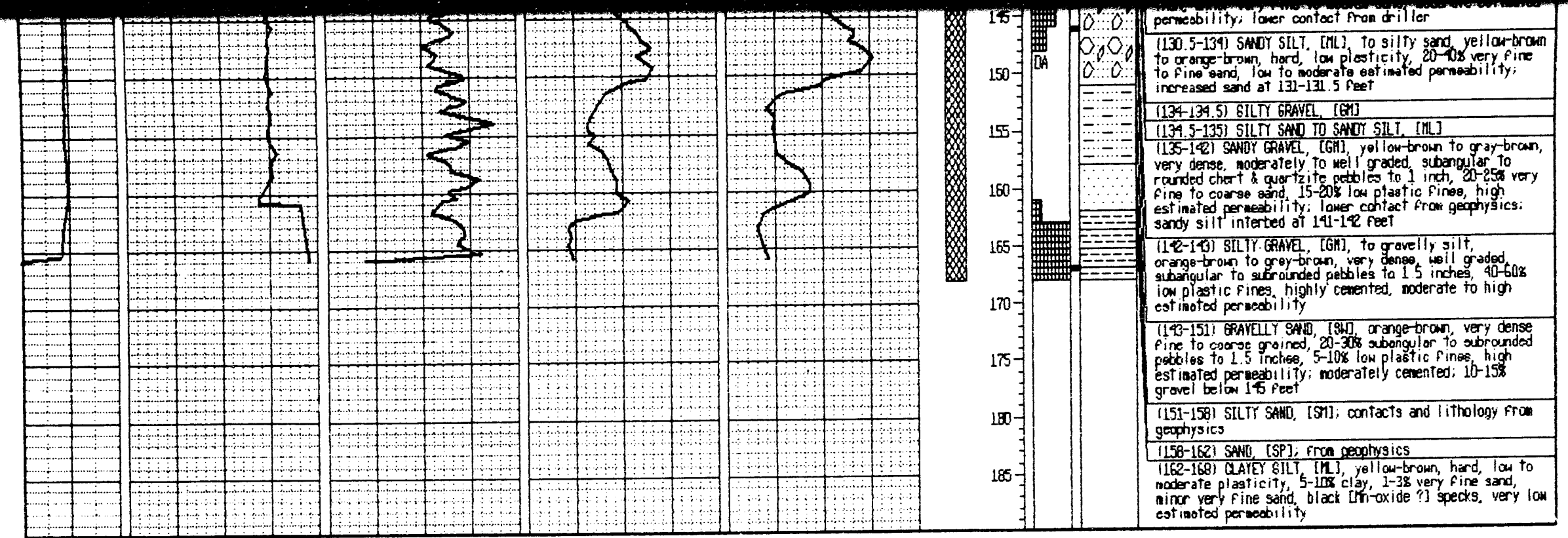




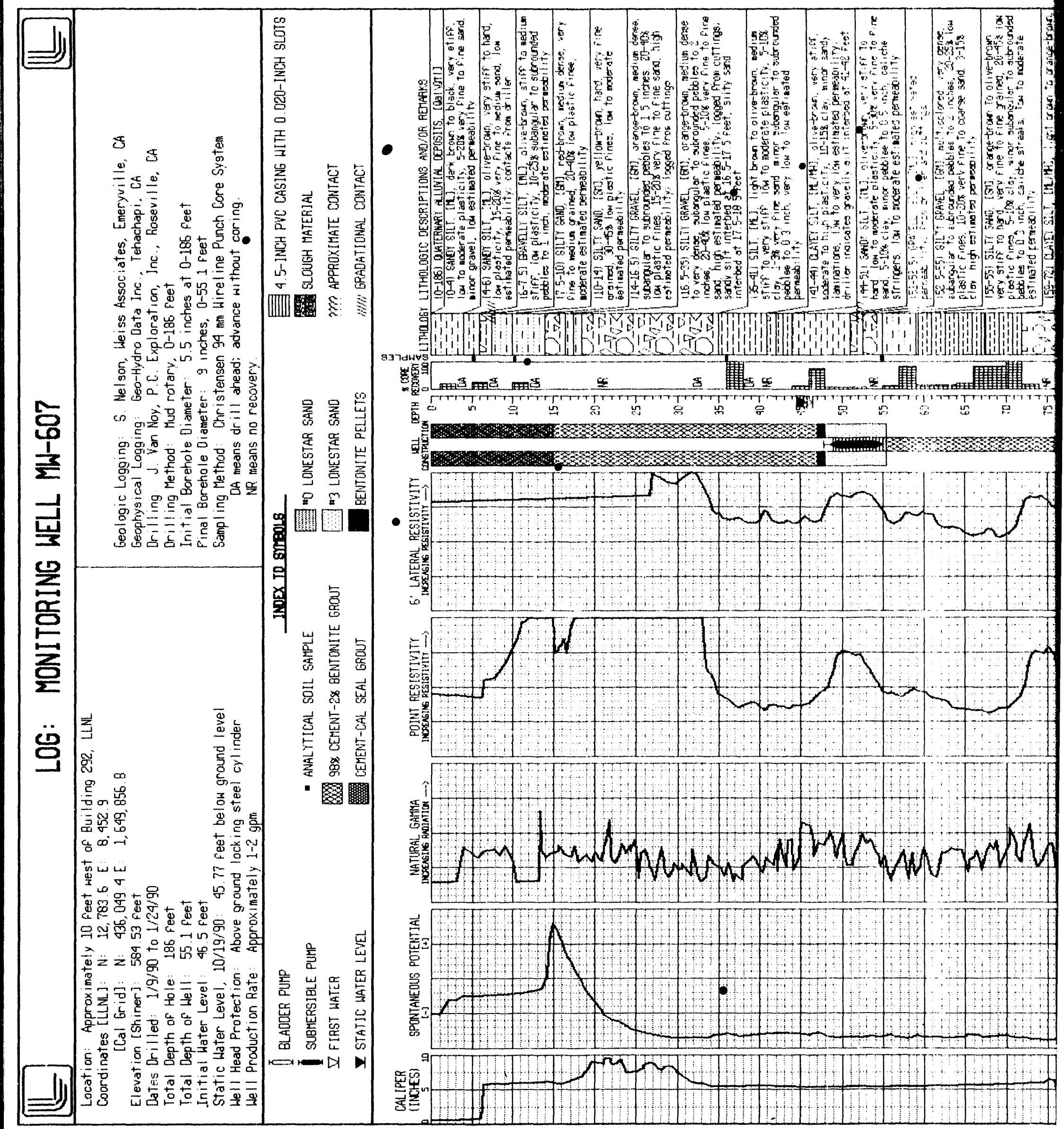




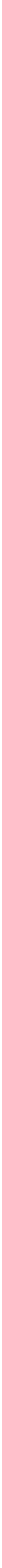




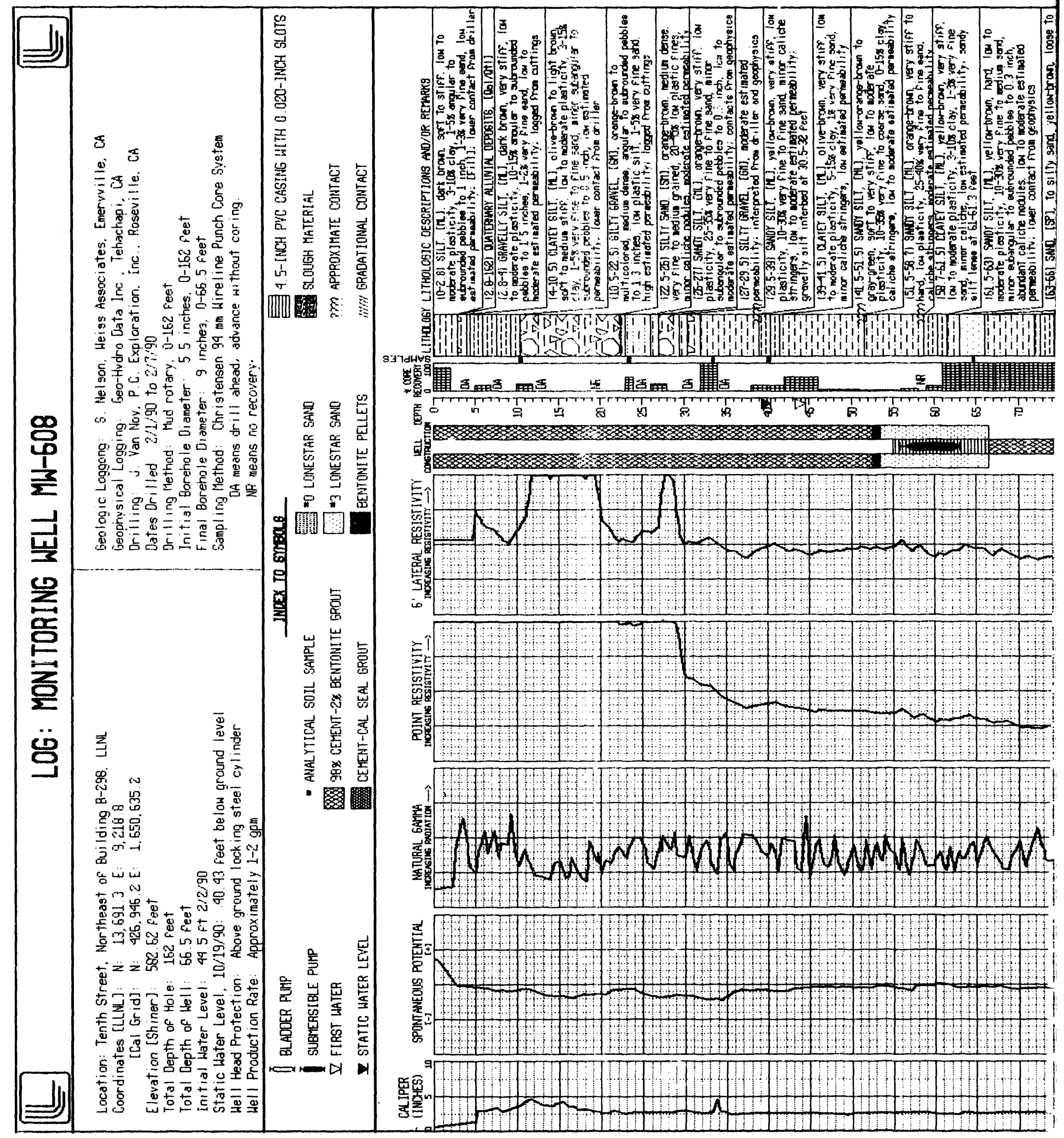




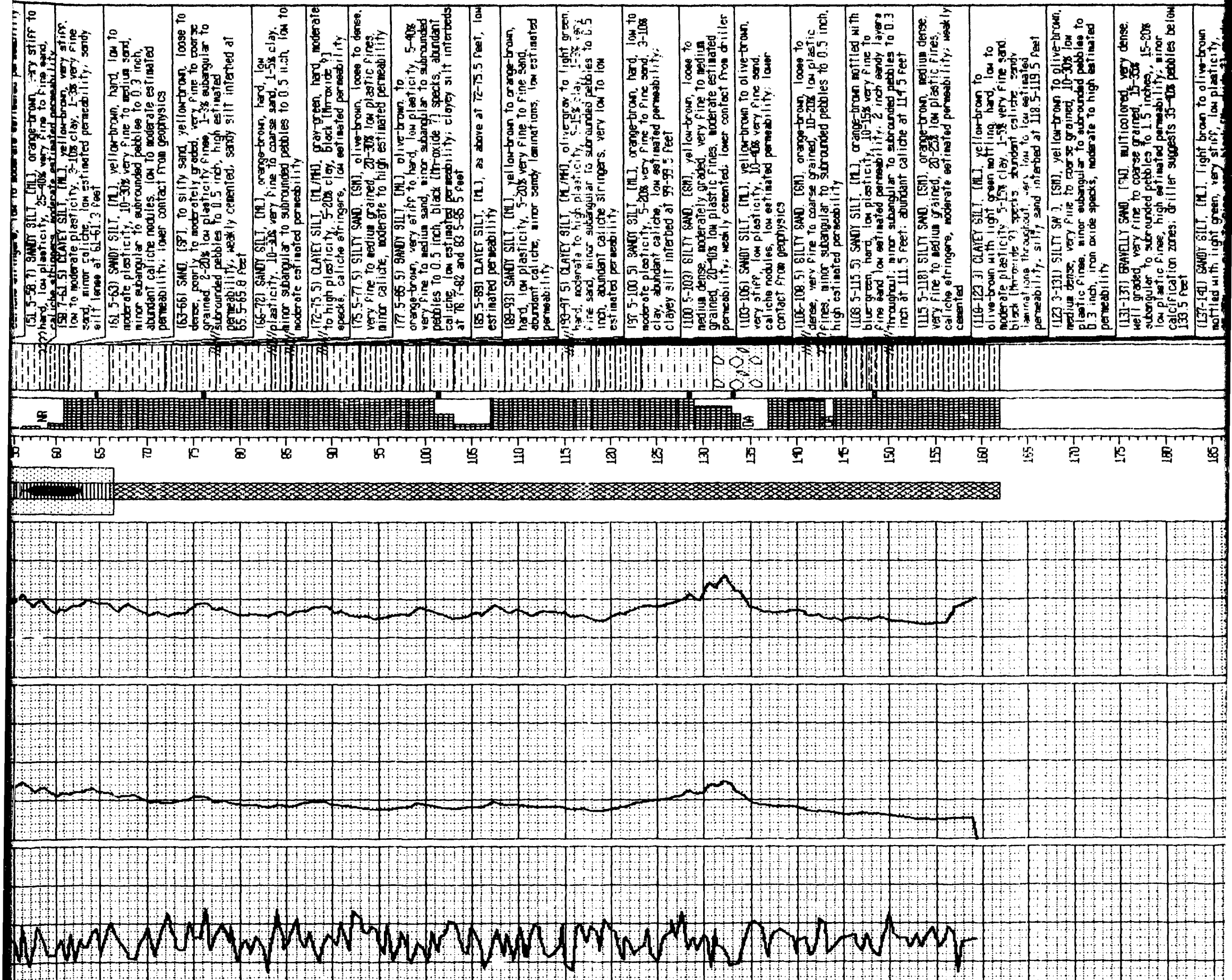




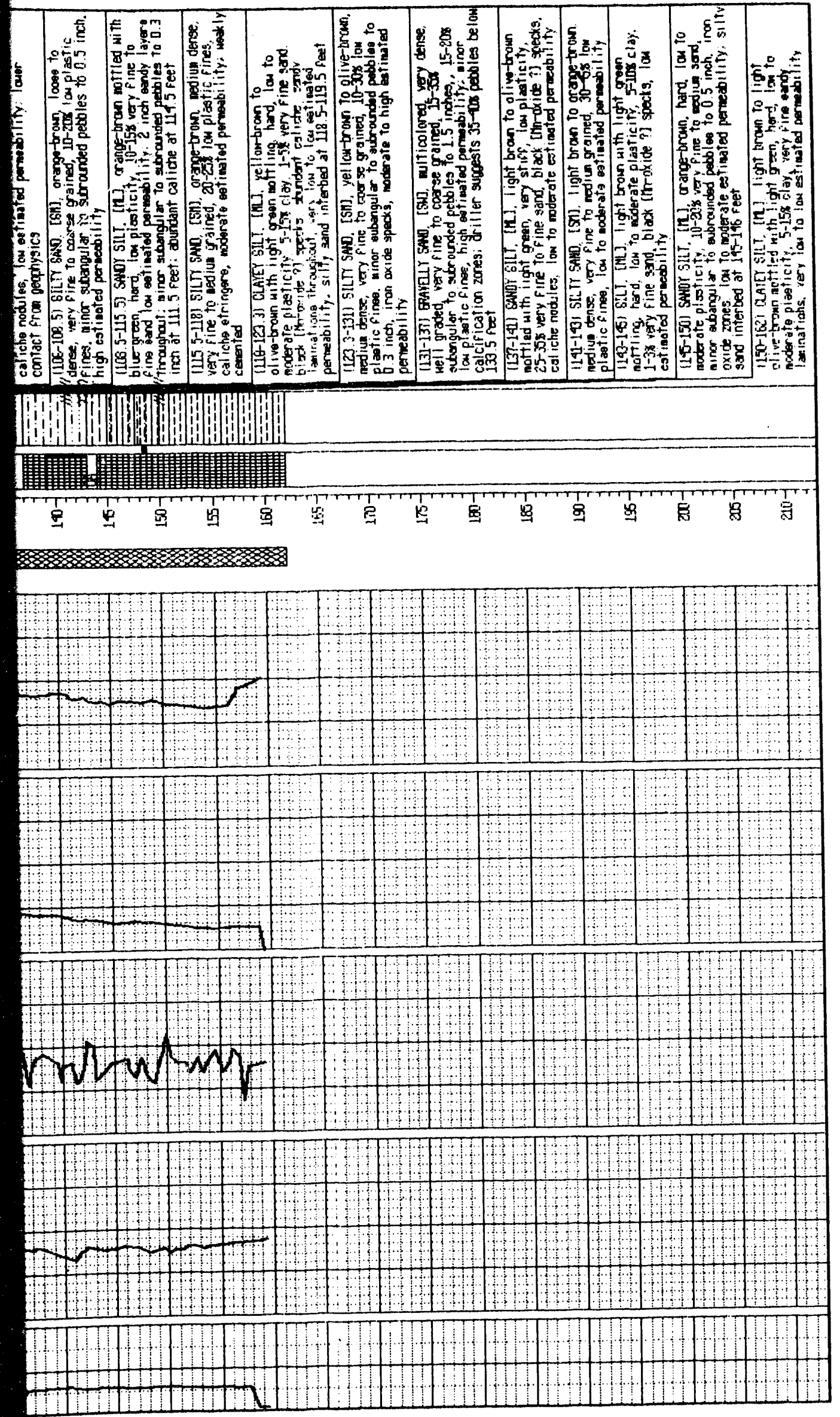




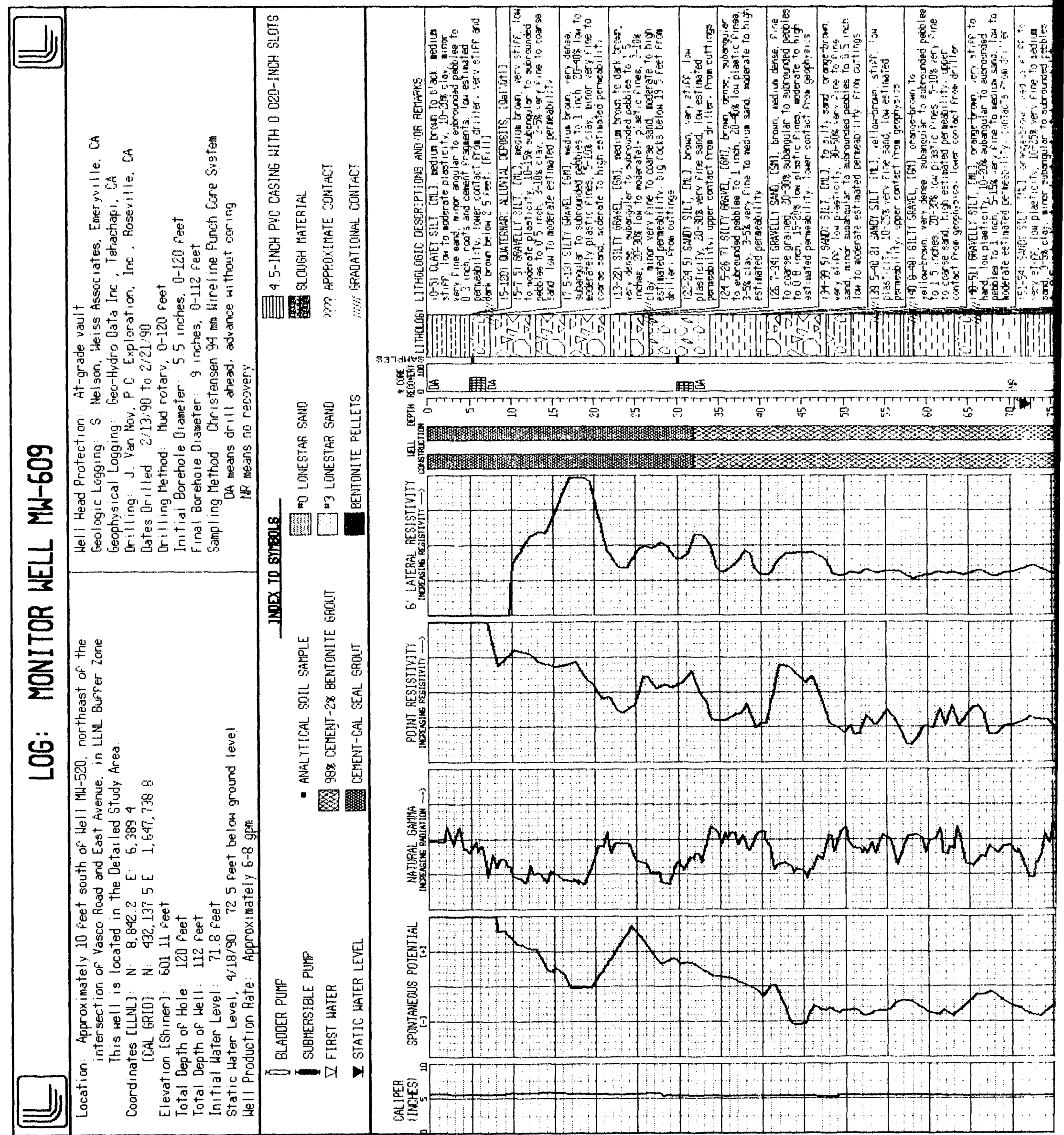




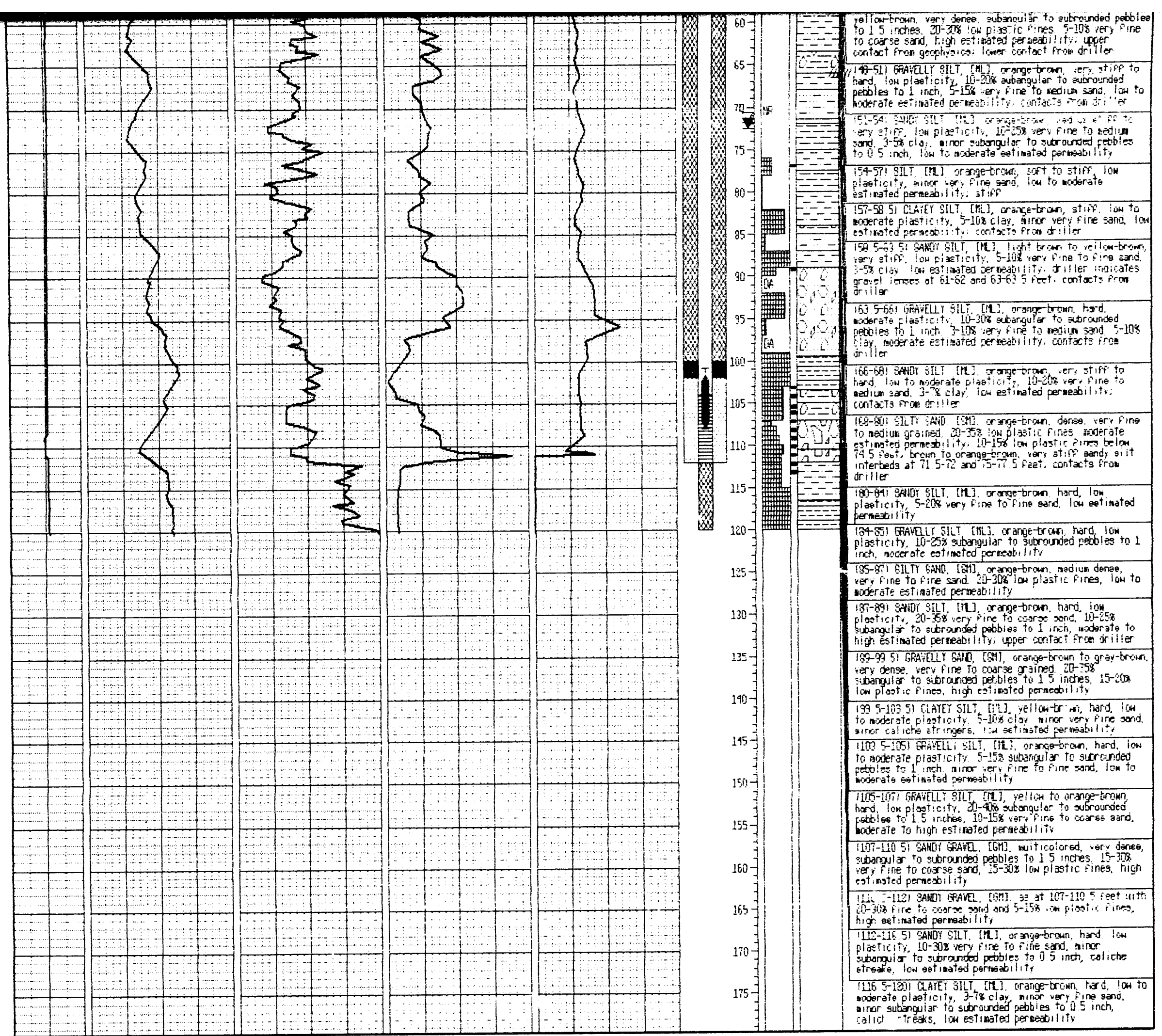




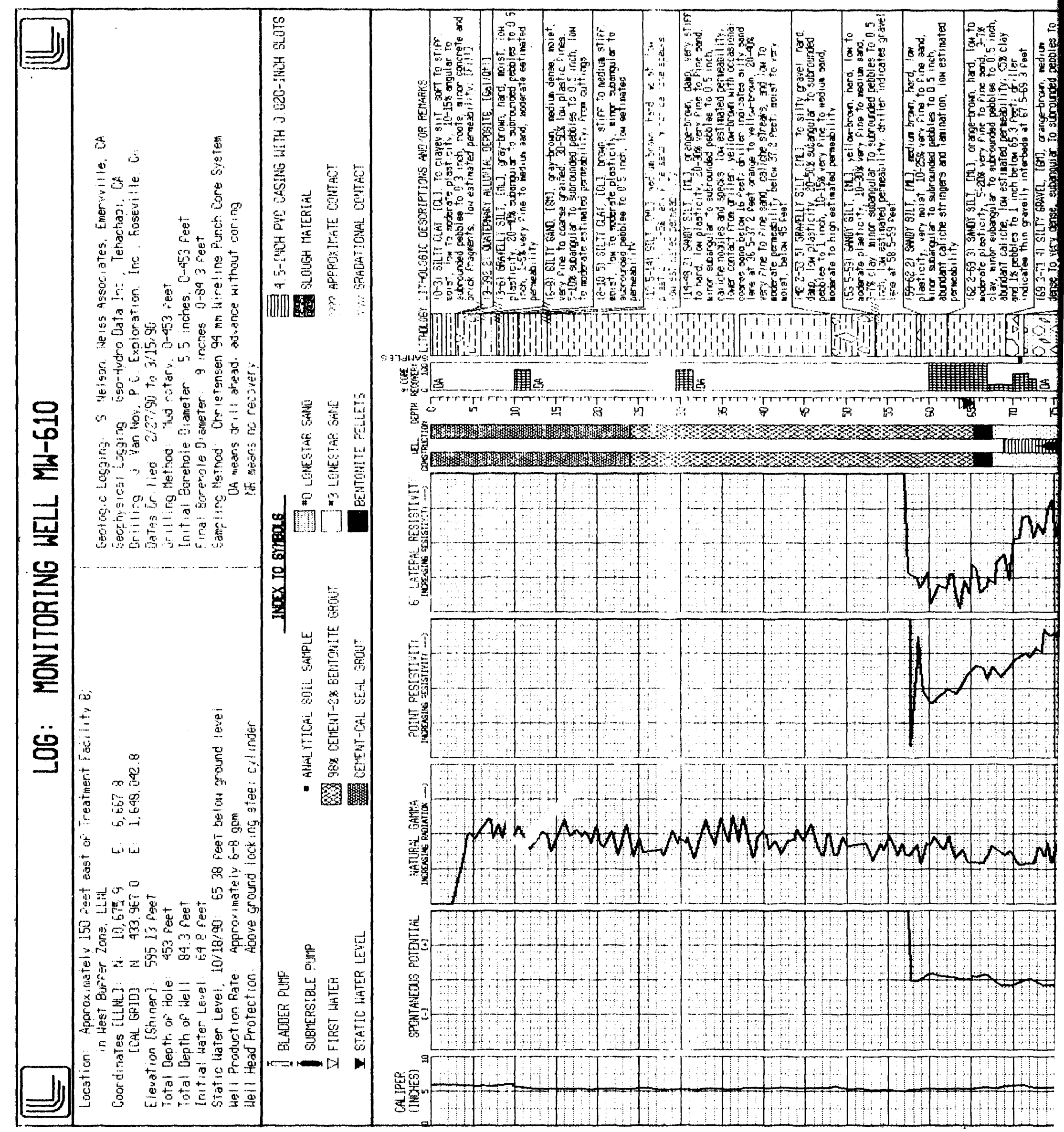




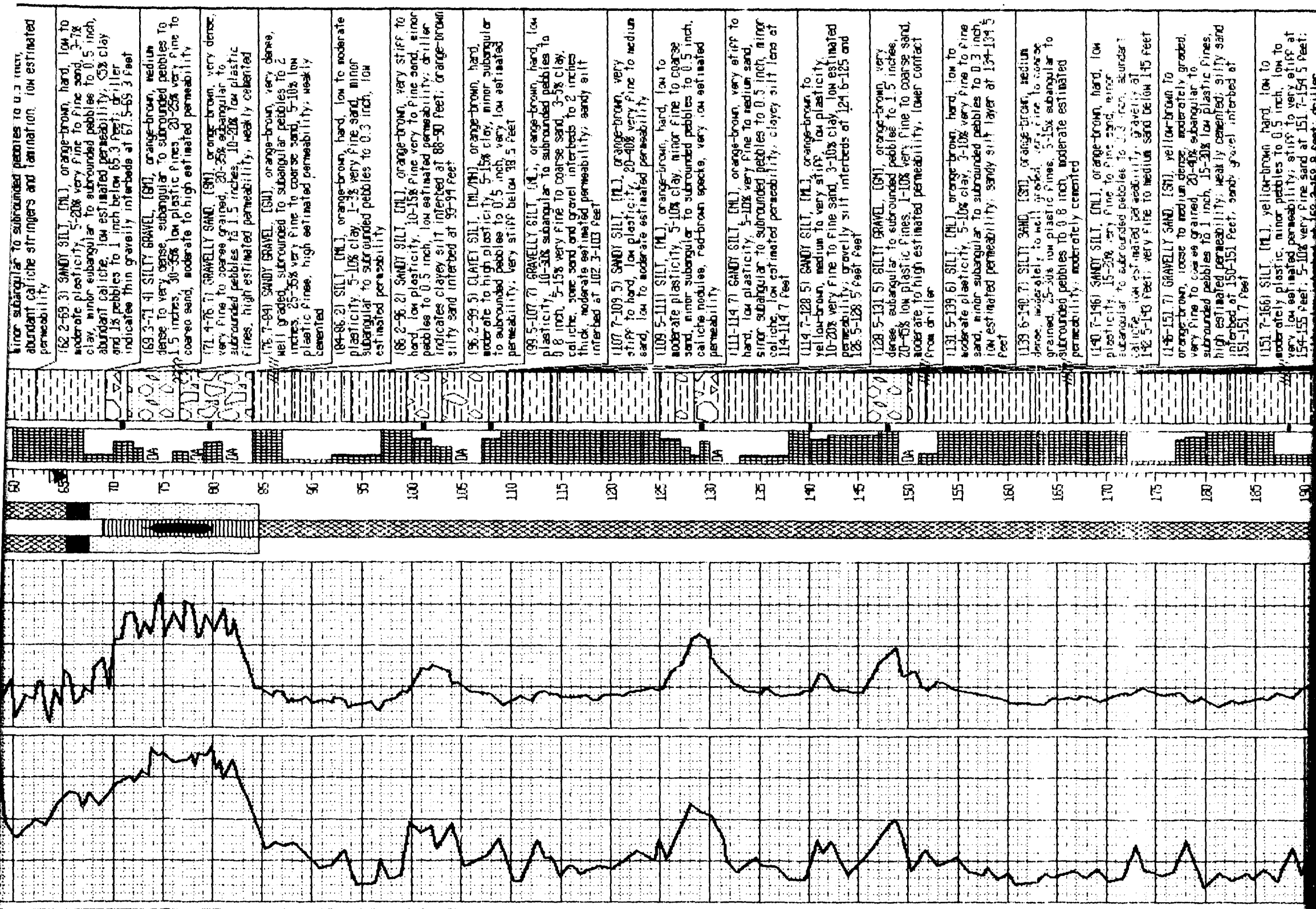

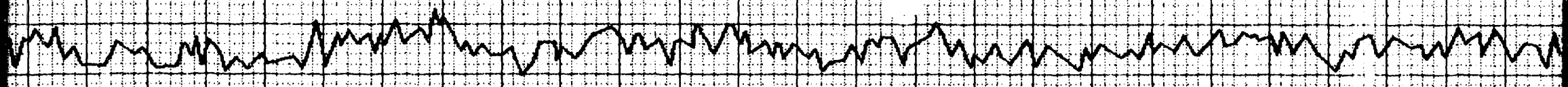




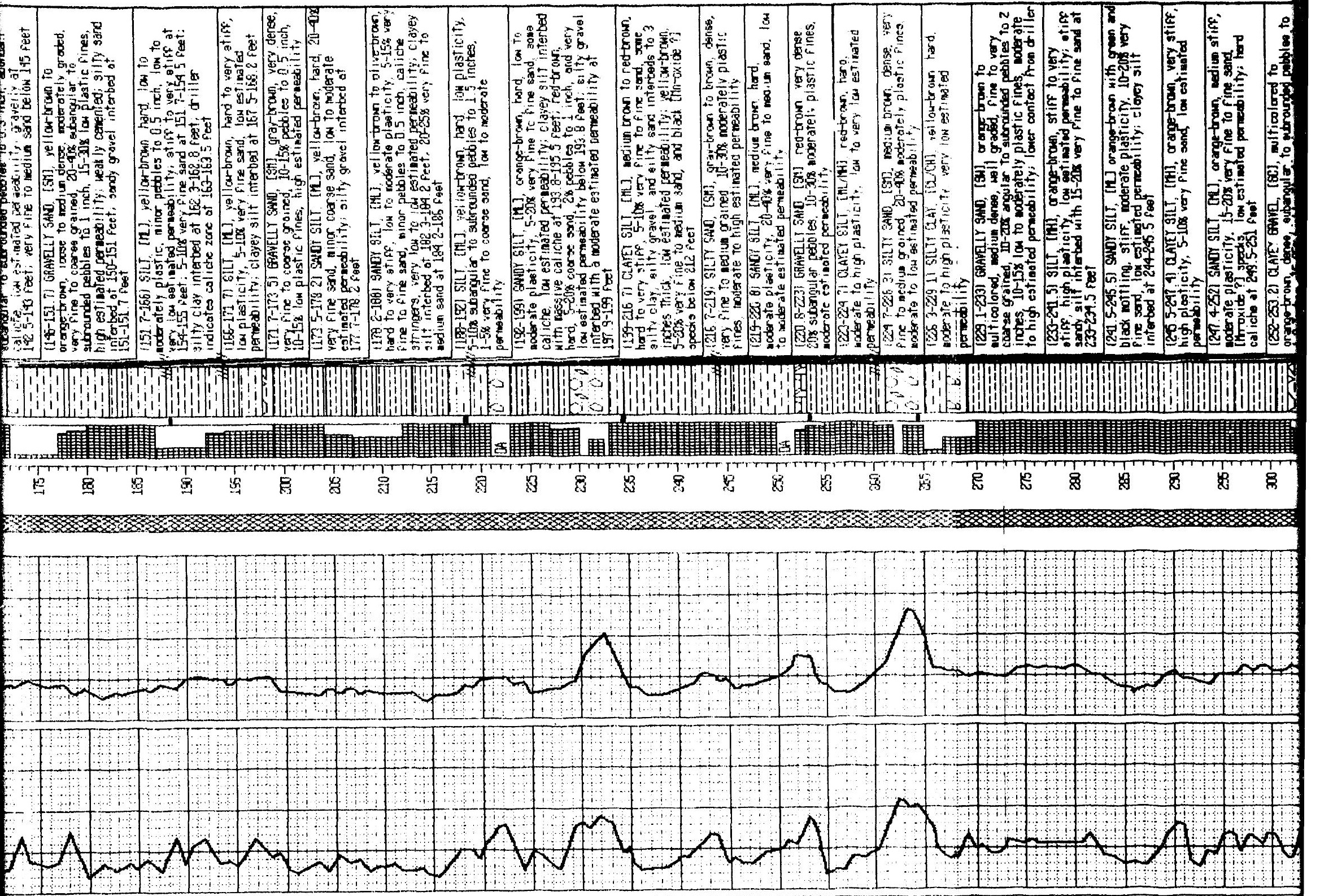

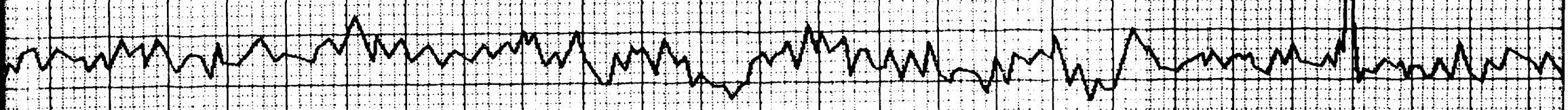



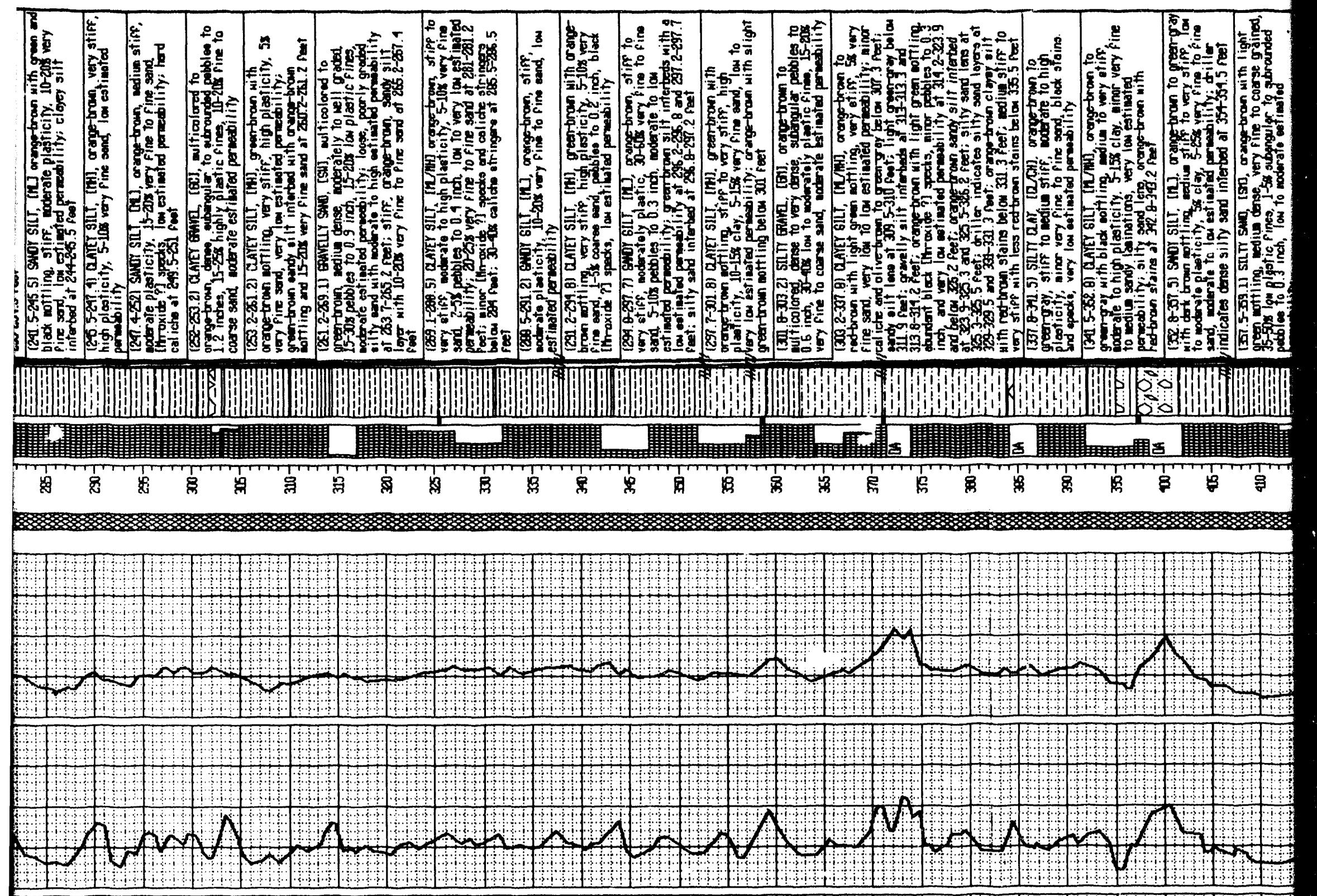

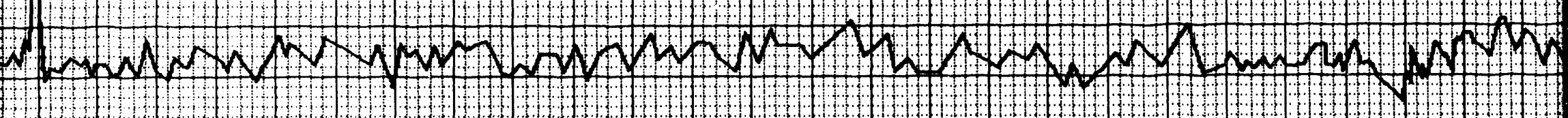




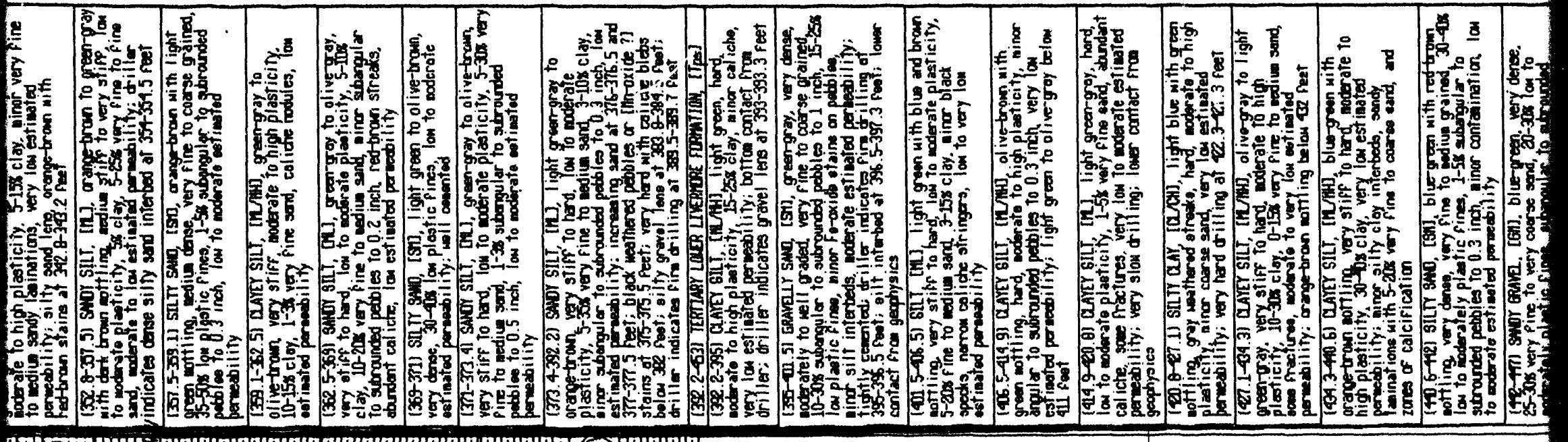

ISO

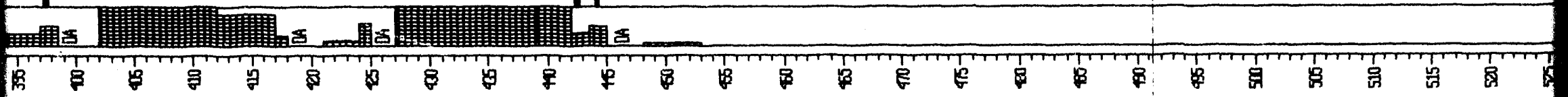
\$
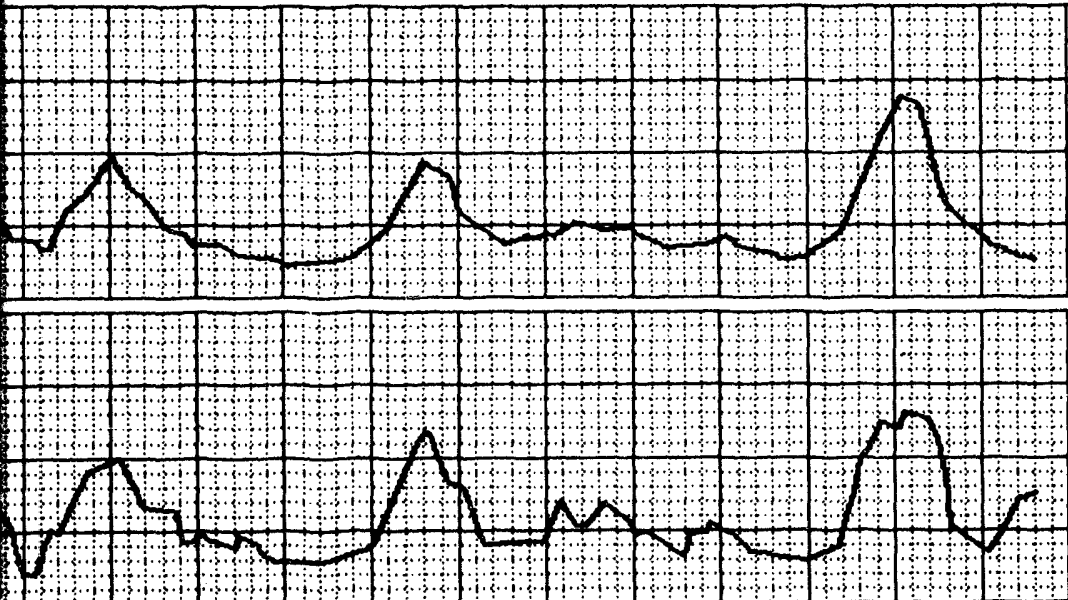

wiminiming

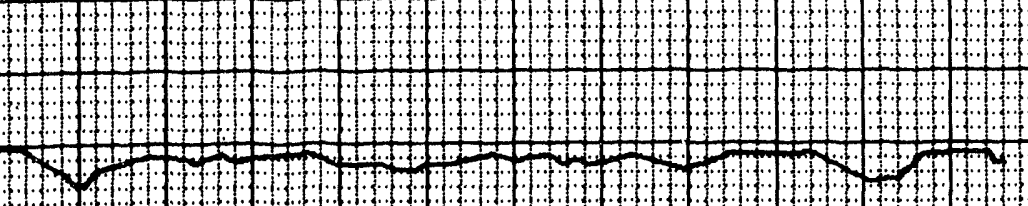




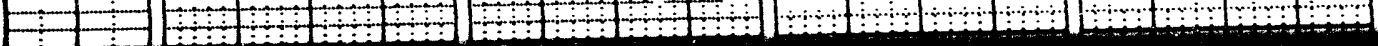
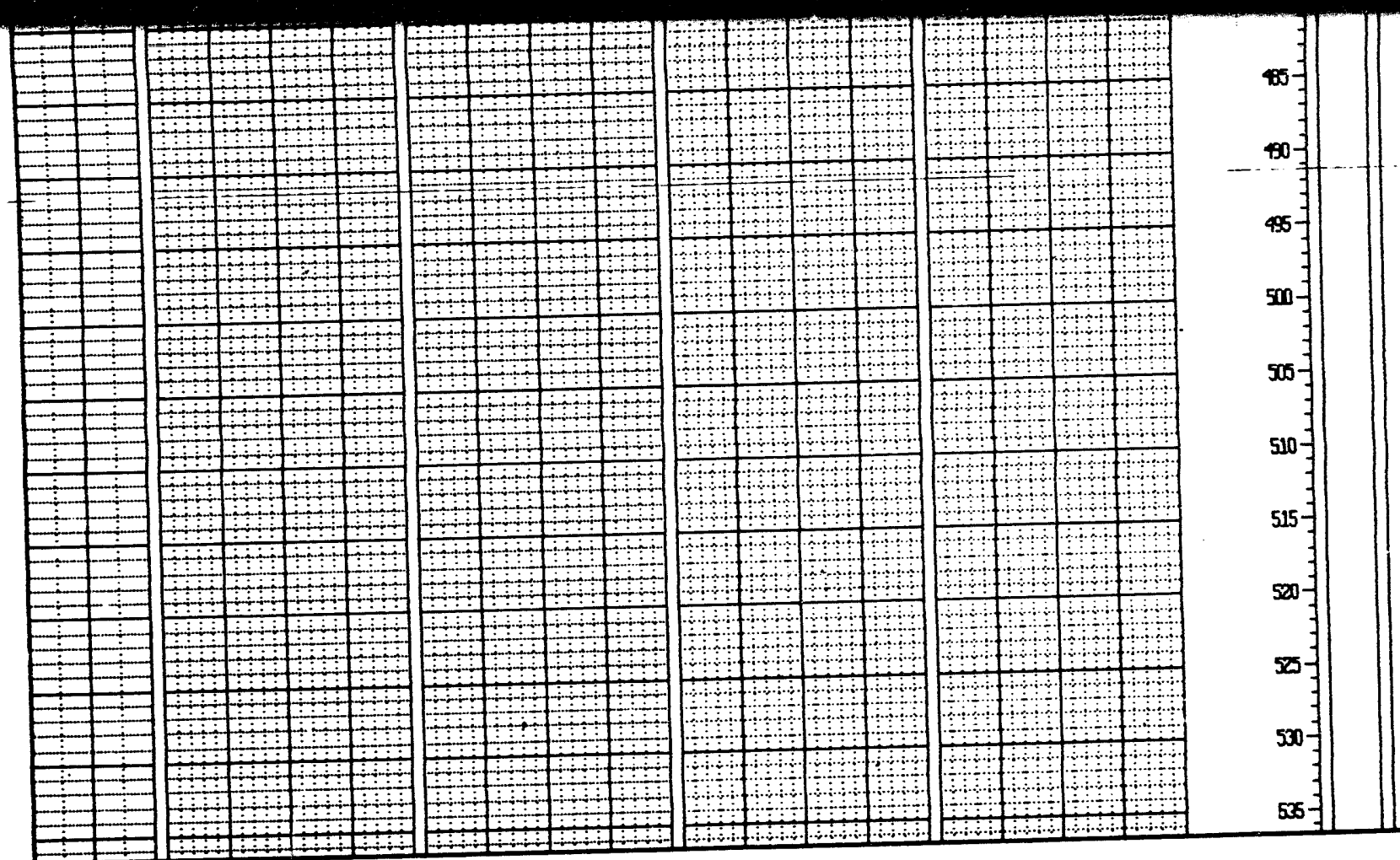

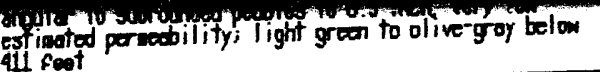

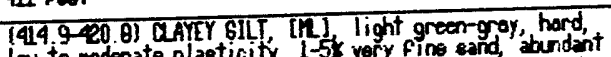
calione

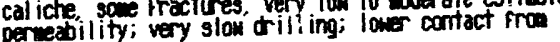
soophyics

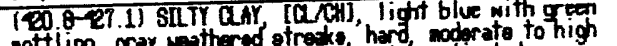

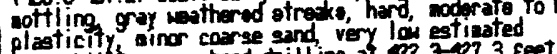

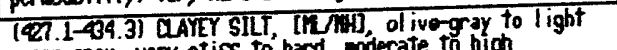

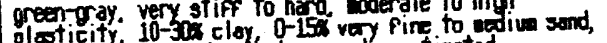

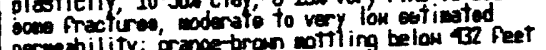

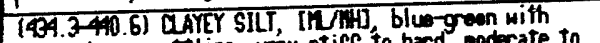
oranoetroun wortl lin very stiff to hare noderate to higi platificity. 30 Pox clay, very low est iated

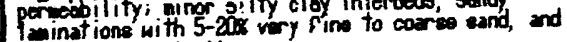
zores of calcification

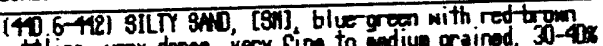

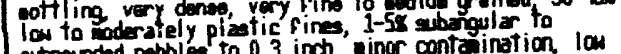
sutrounded pebbles to 0.3 inch, in

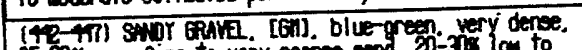

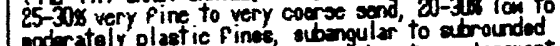

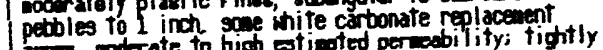
comented

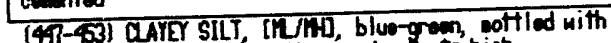

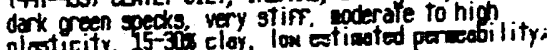

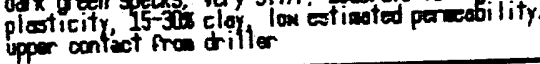




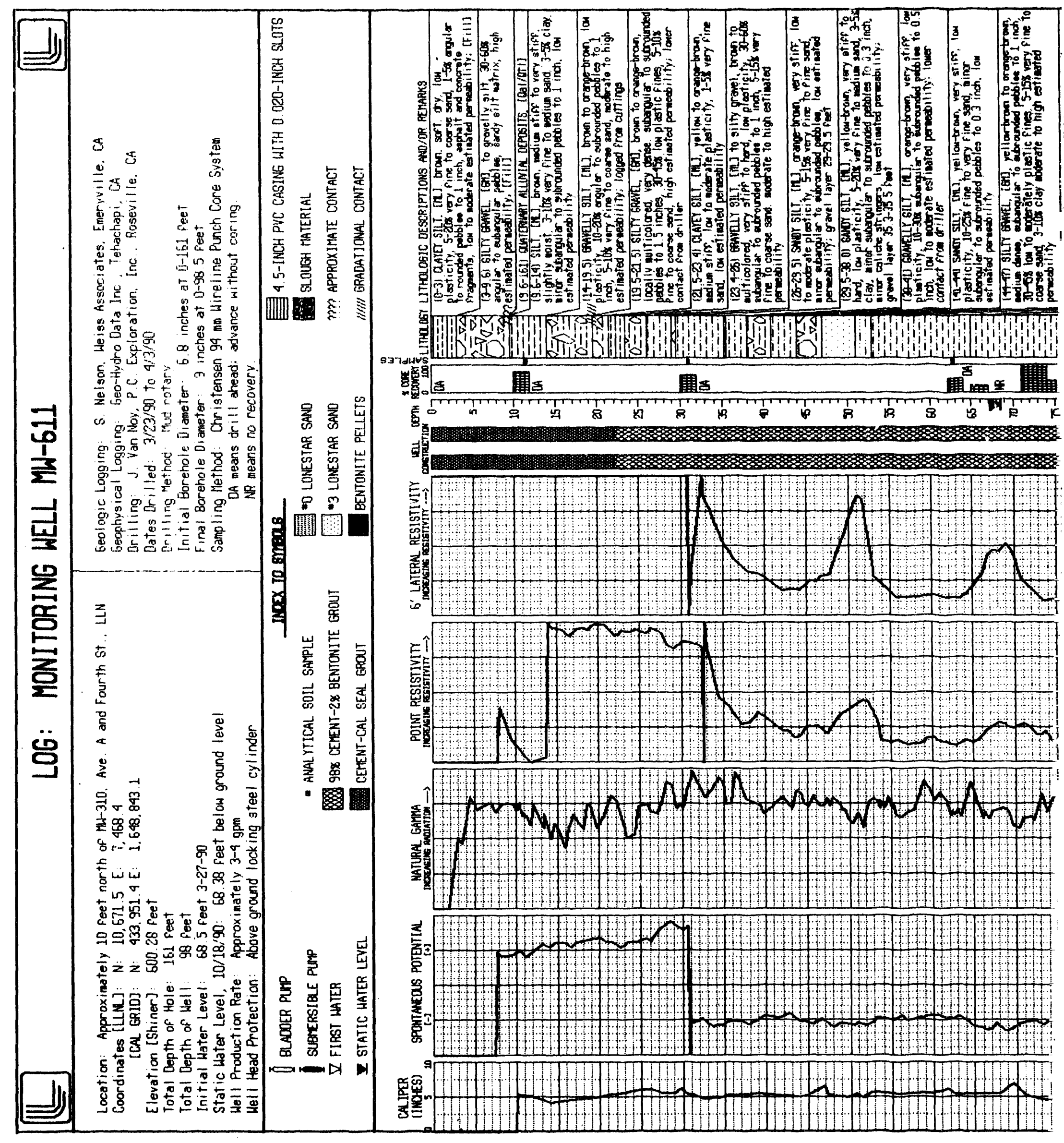




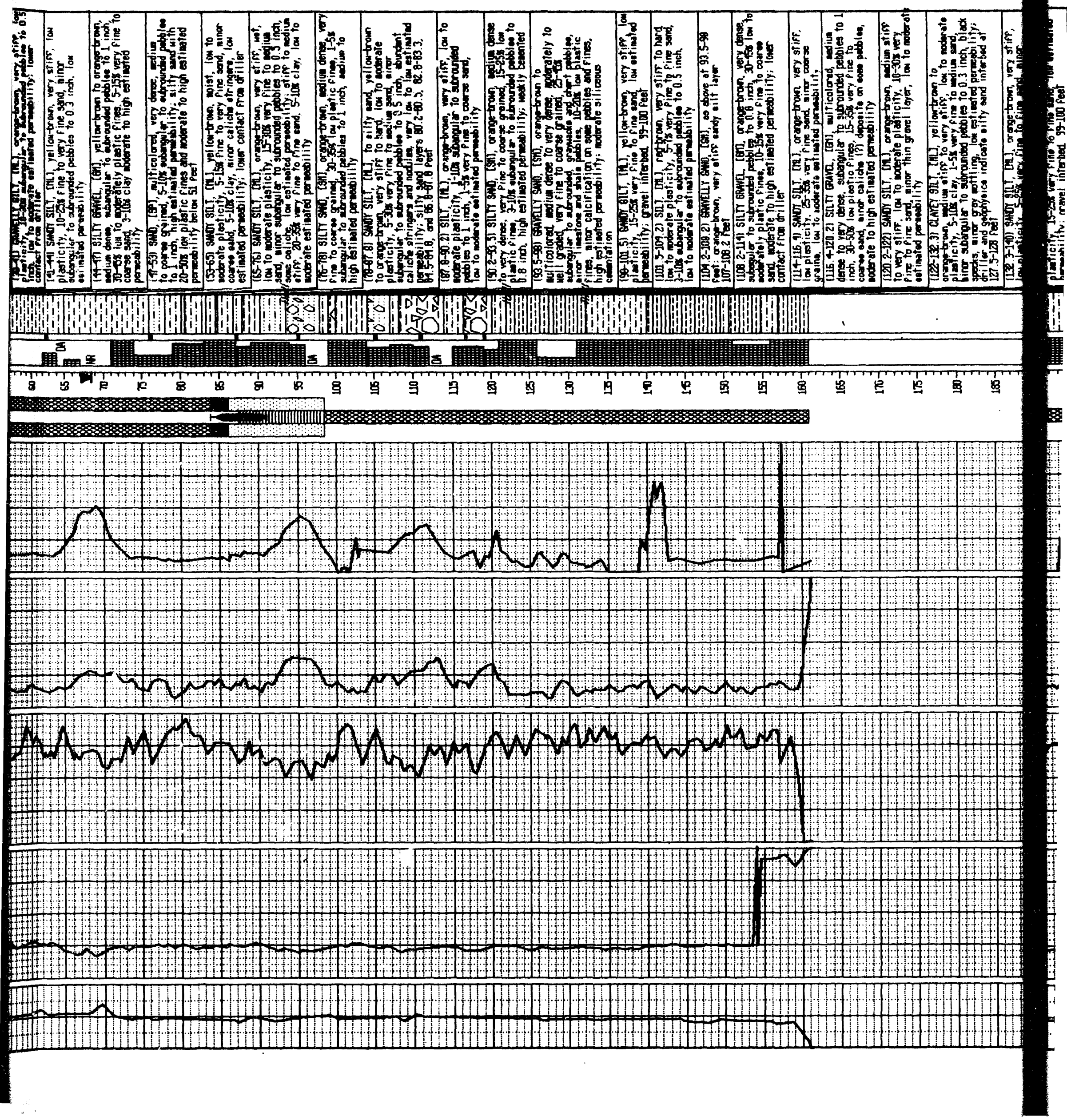




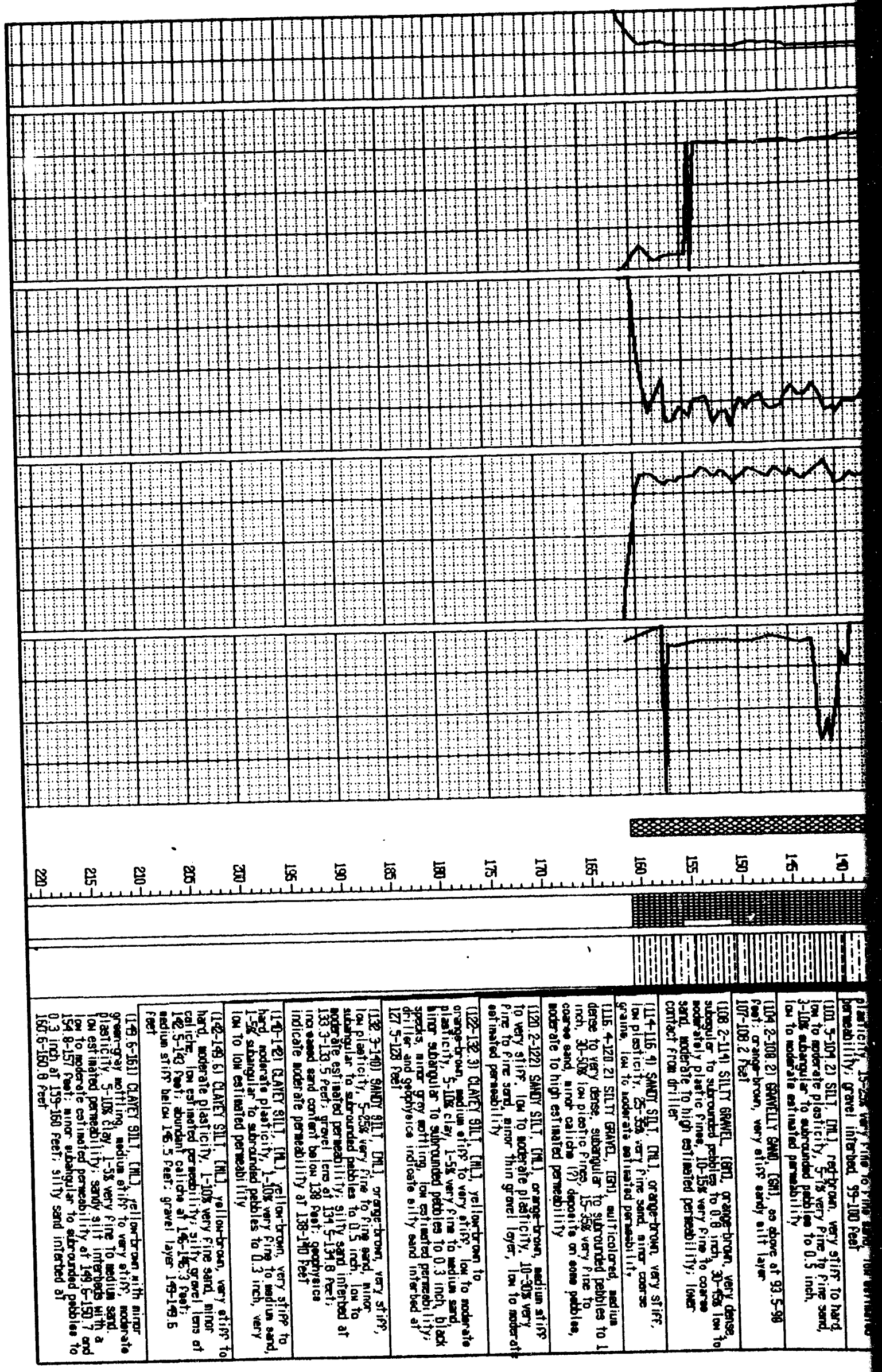




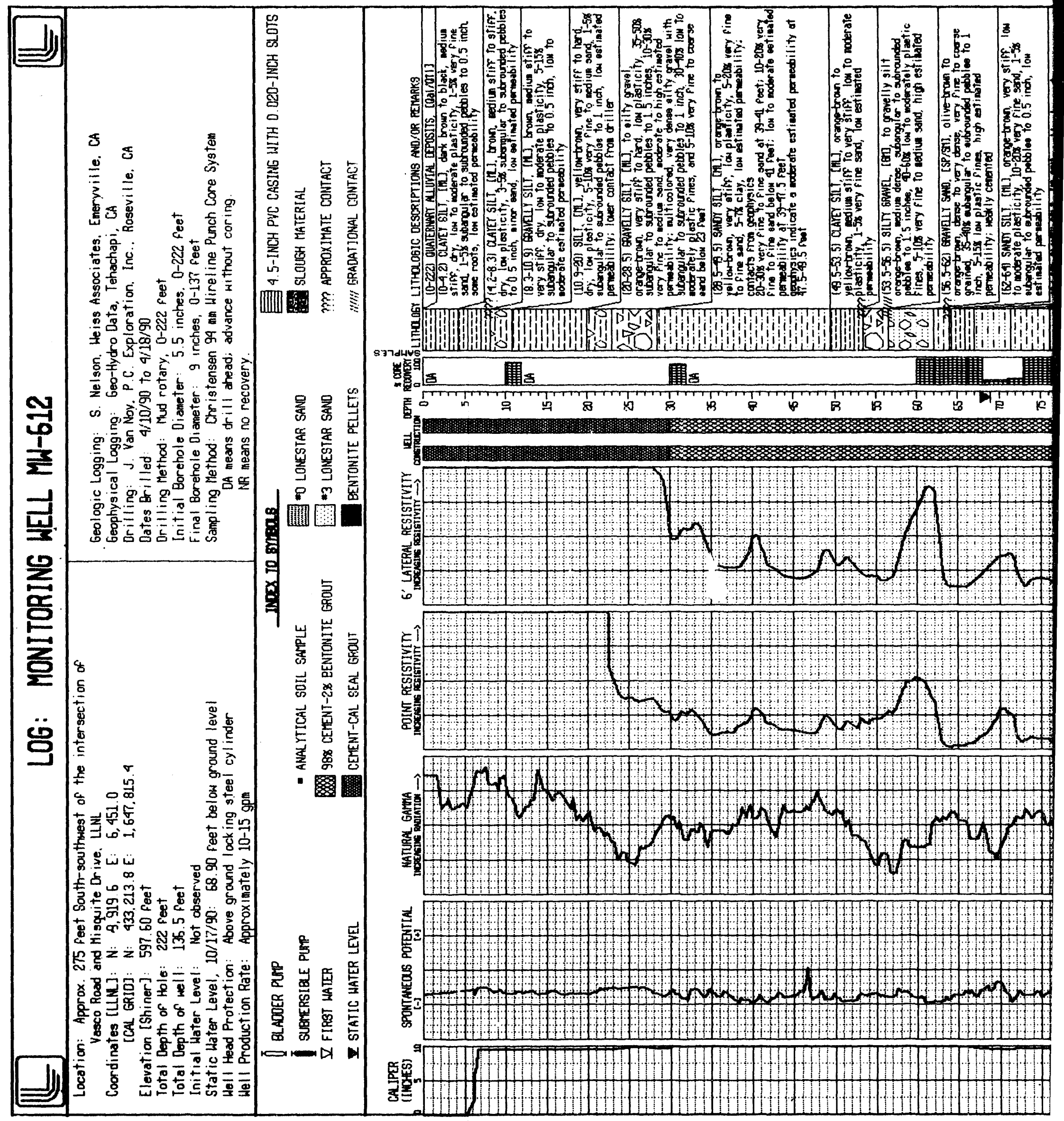



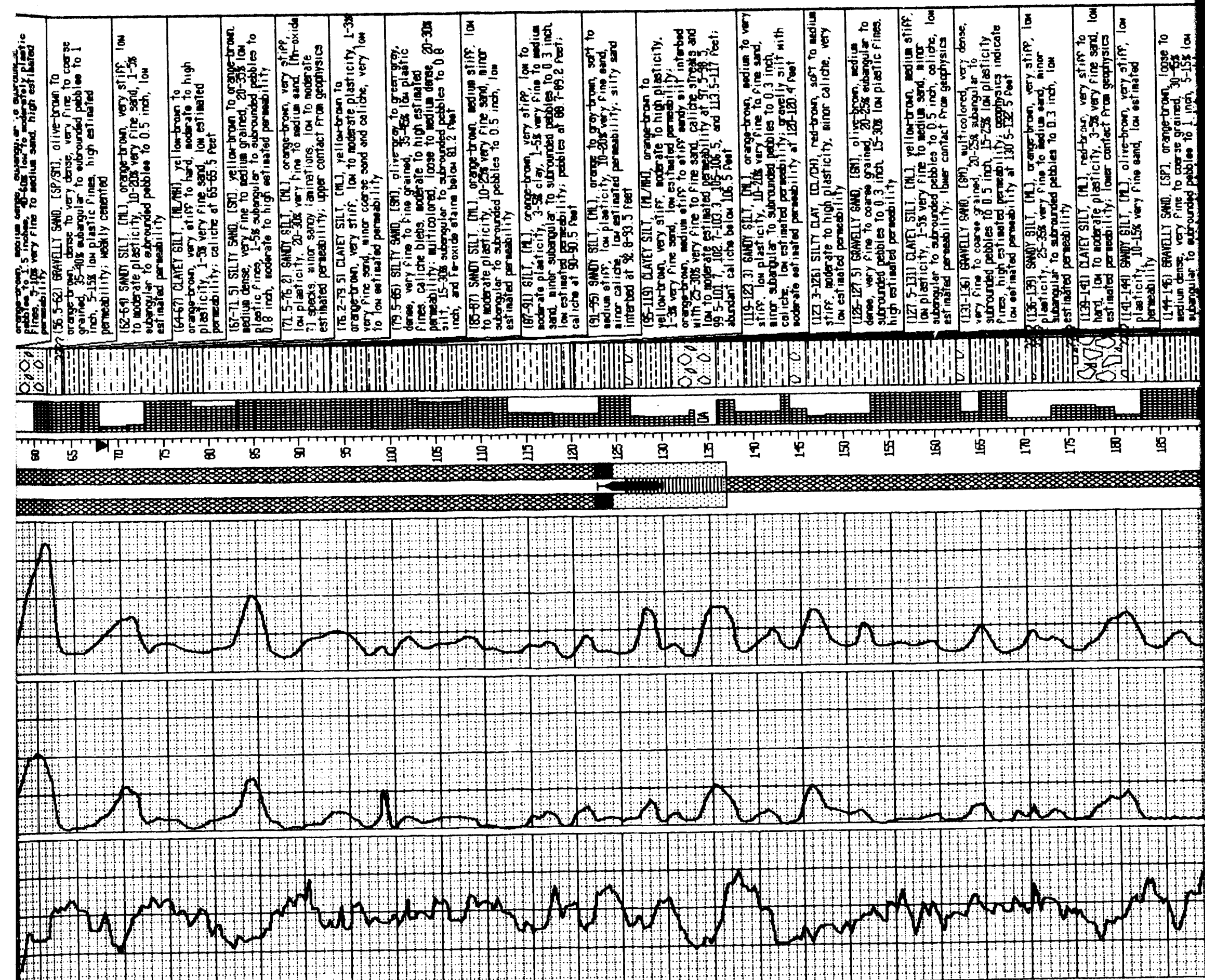

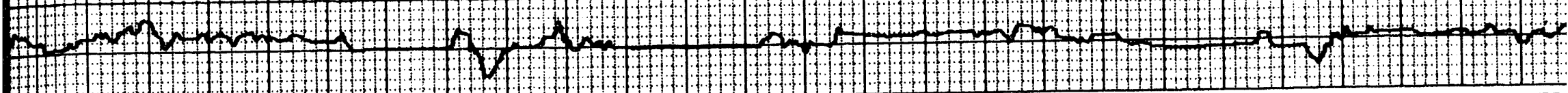




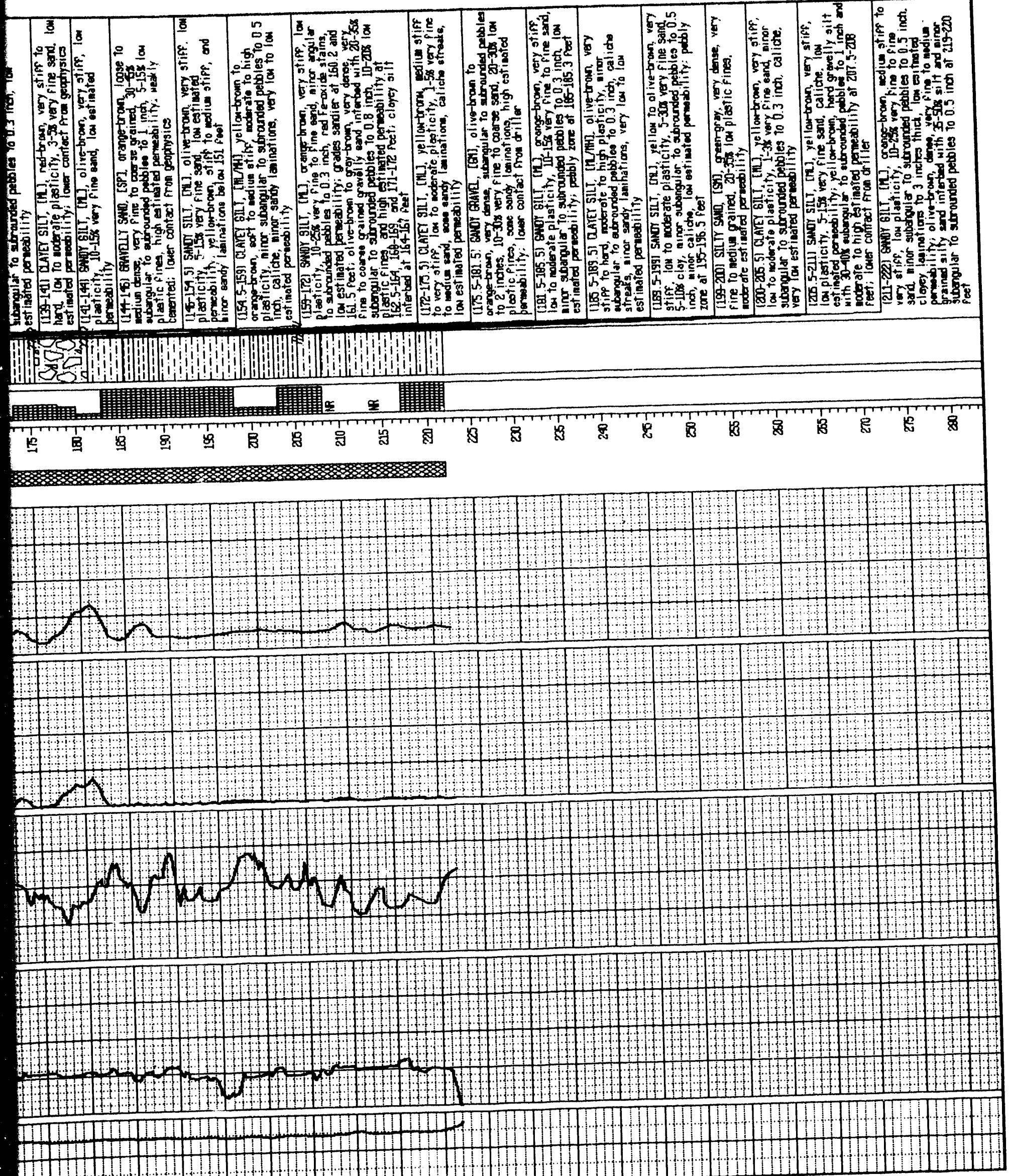




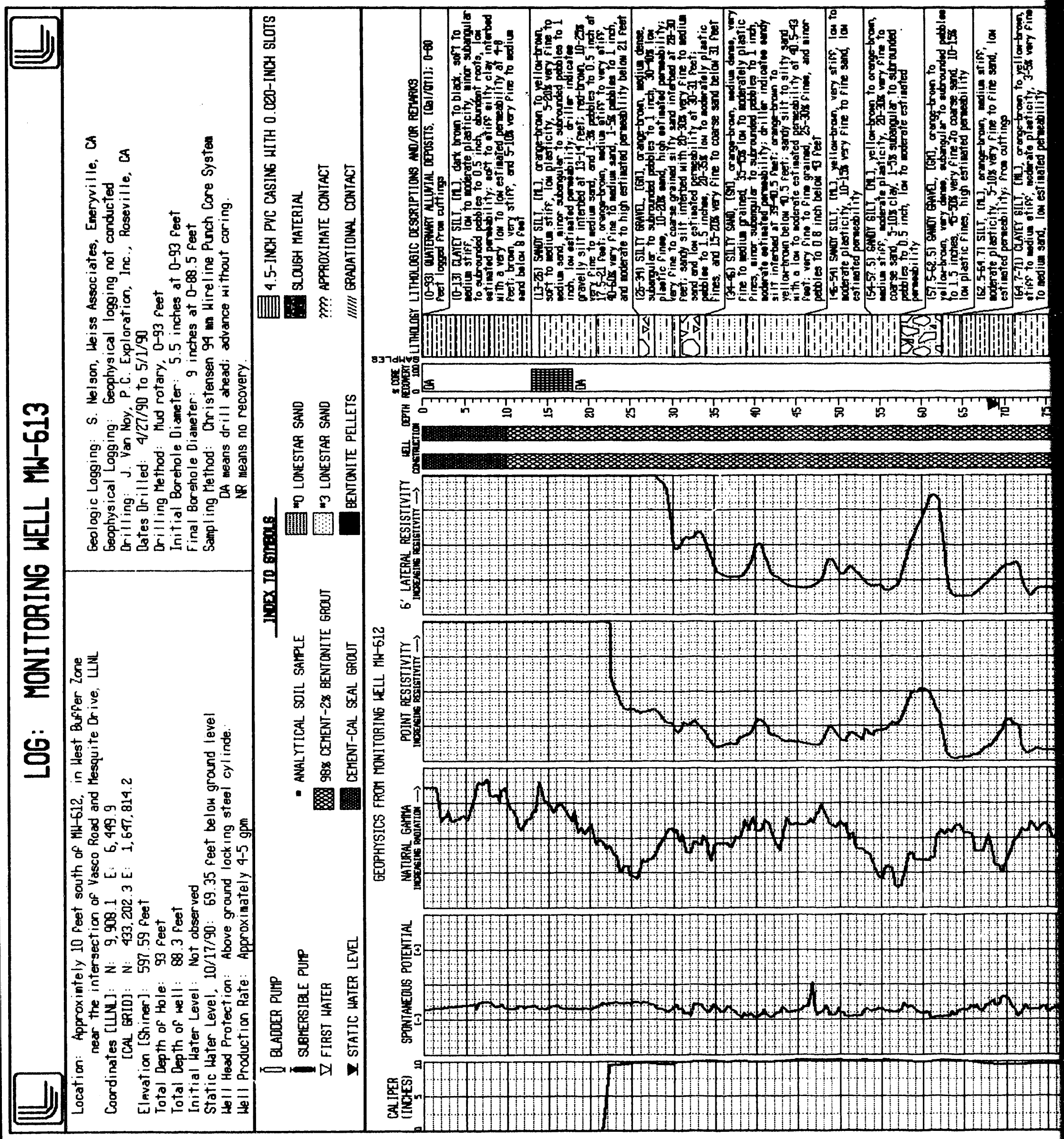



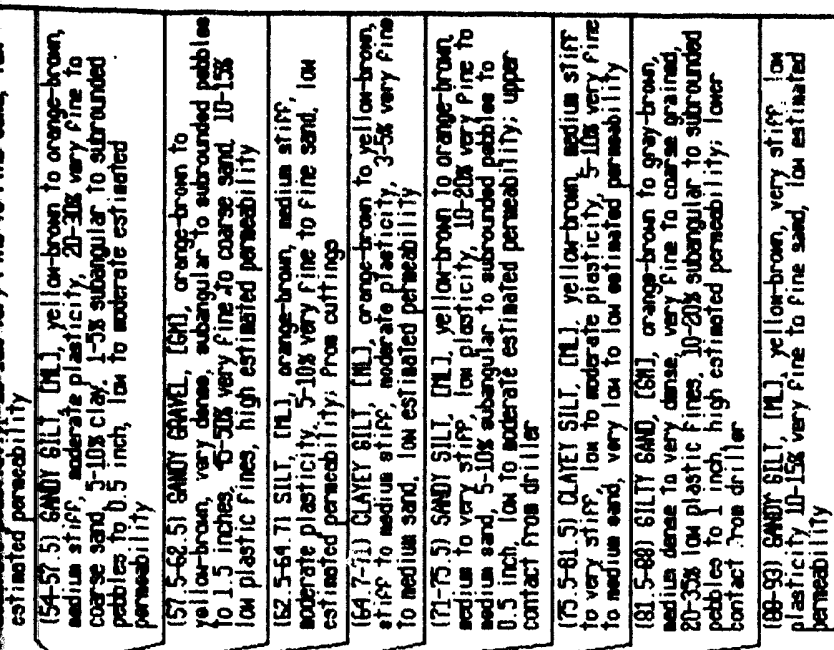

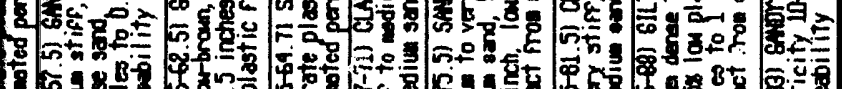

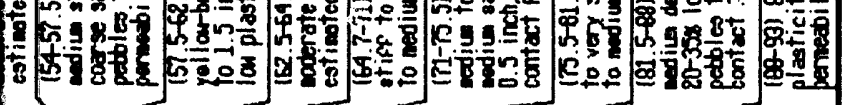

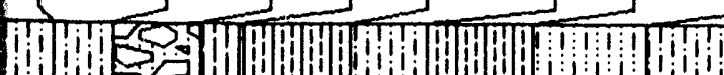
啊

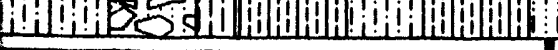
$1 ! 1: 1,1 ! j !$
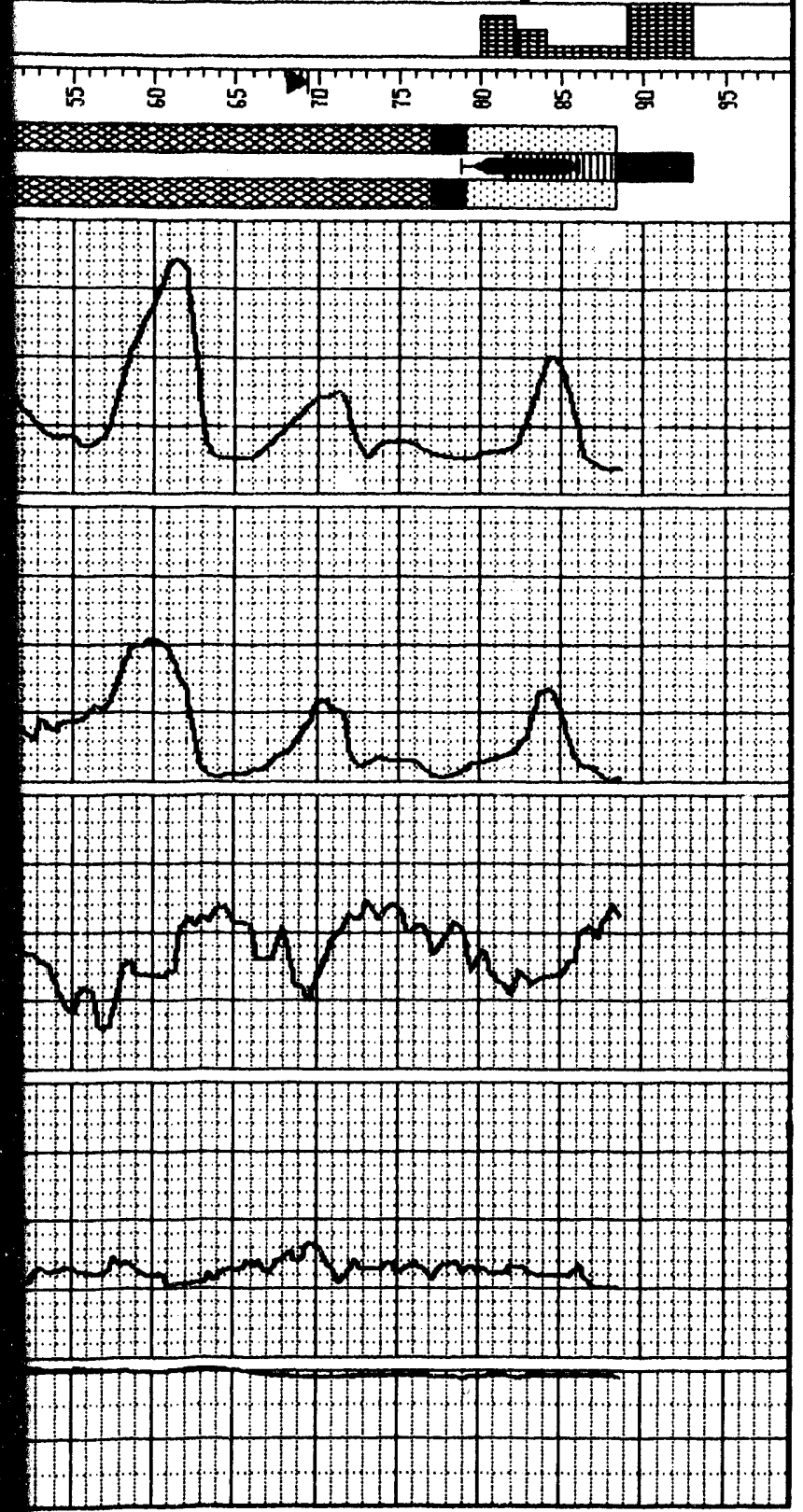


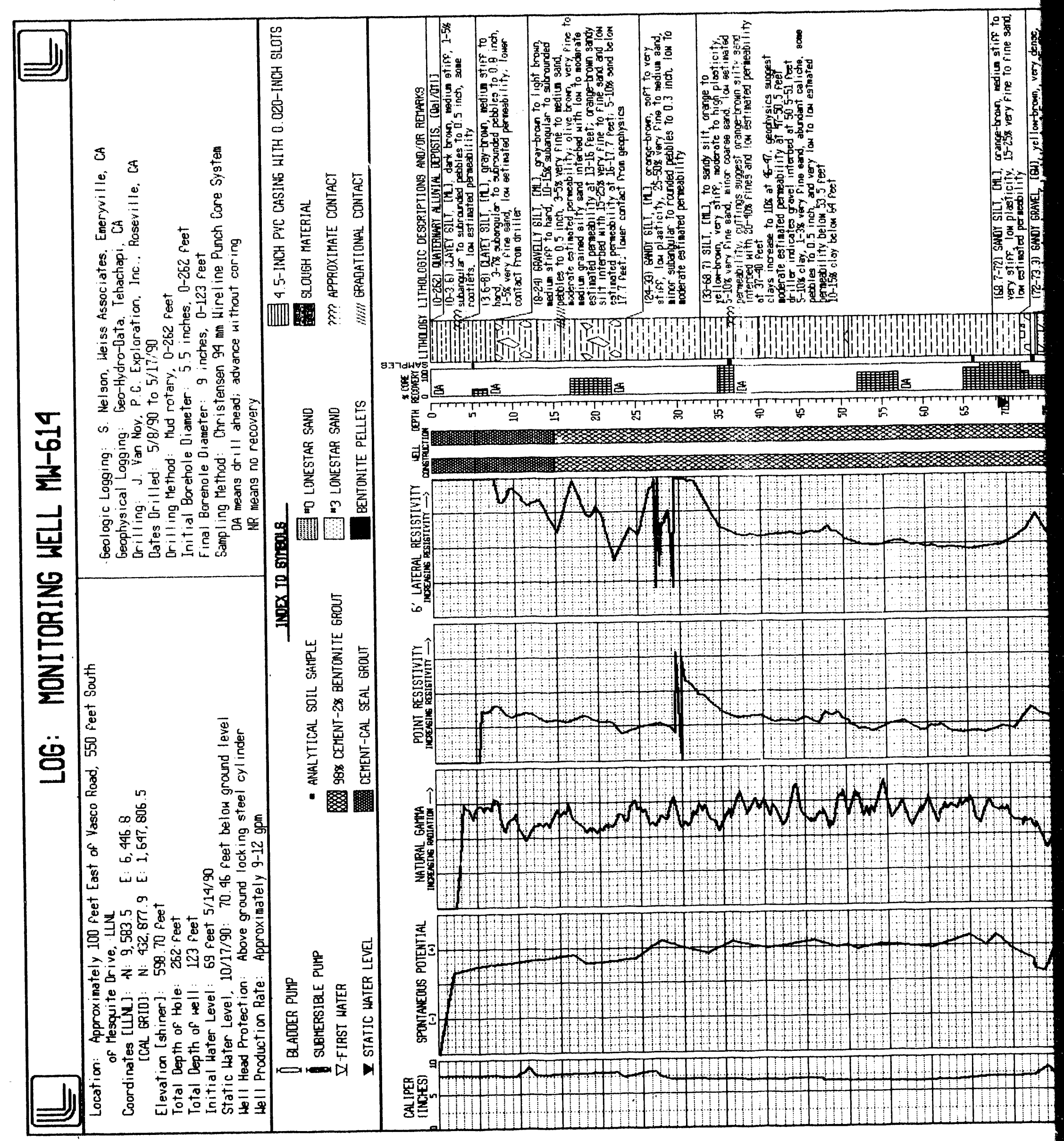




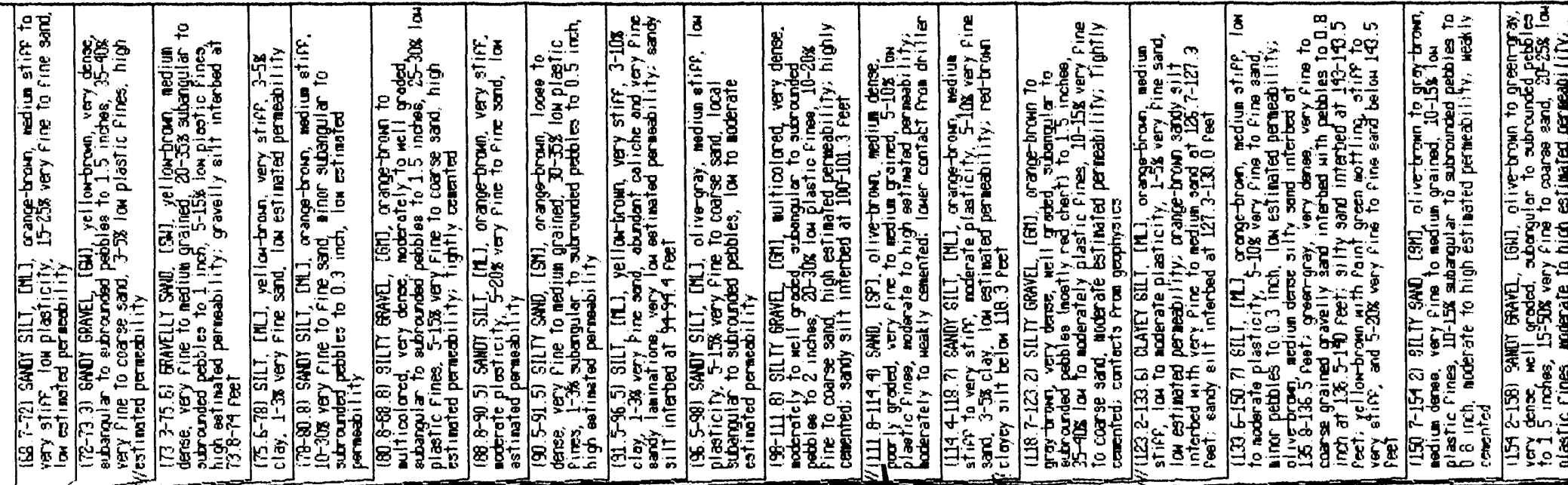

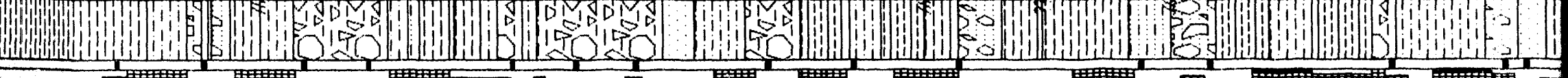

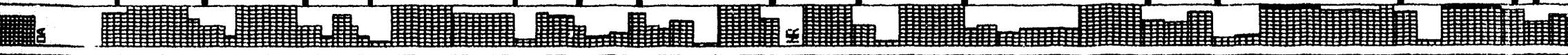

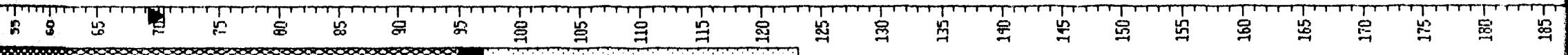

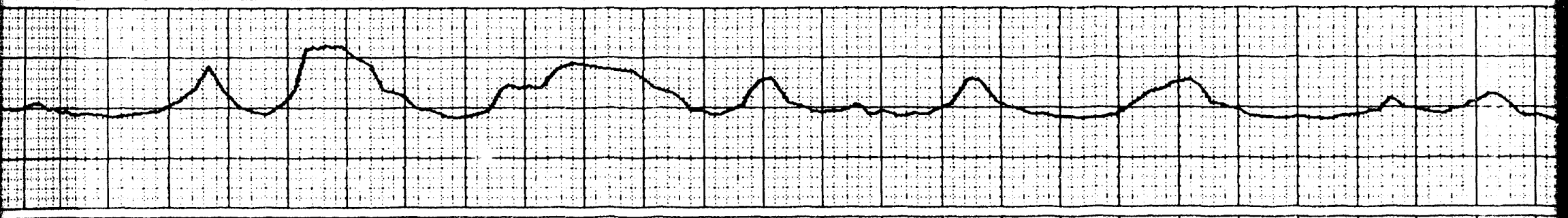

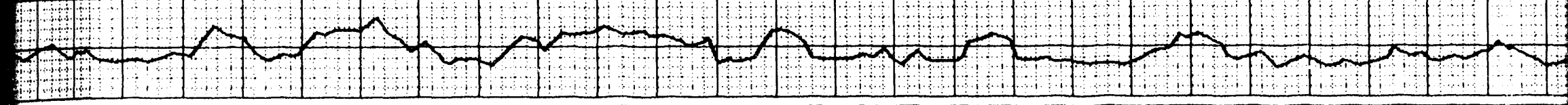

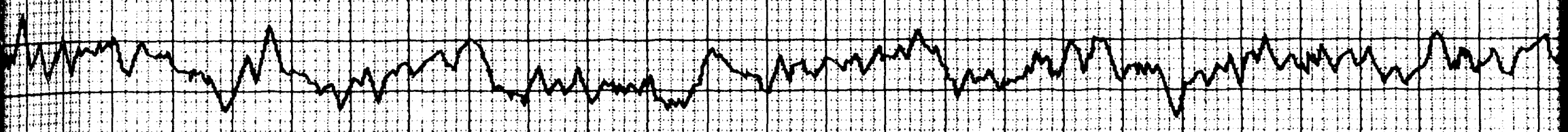
- 


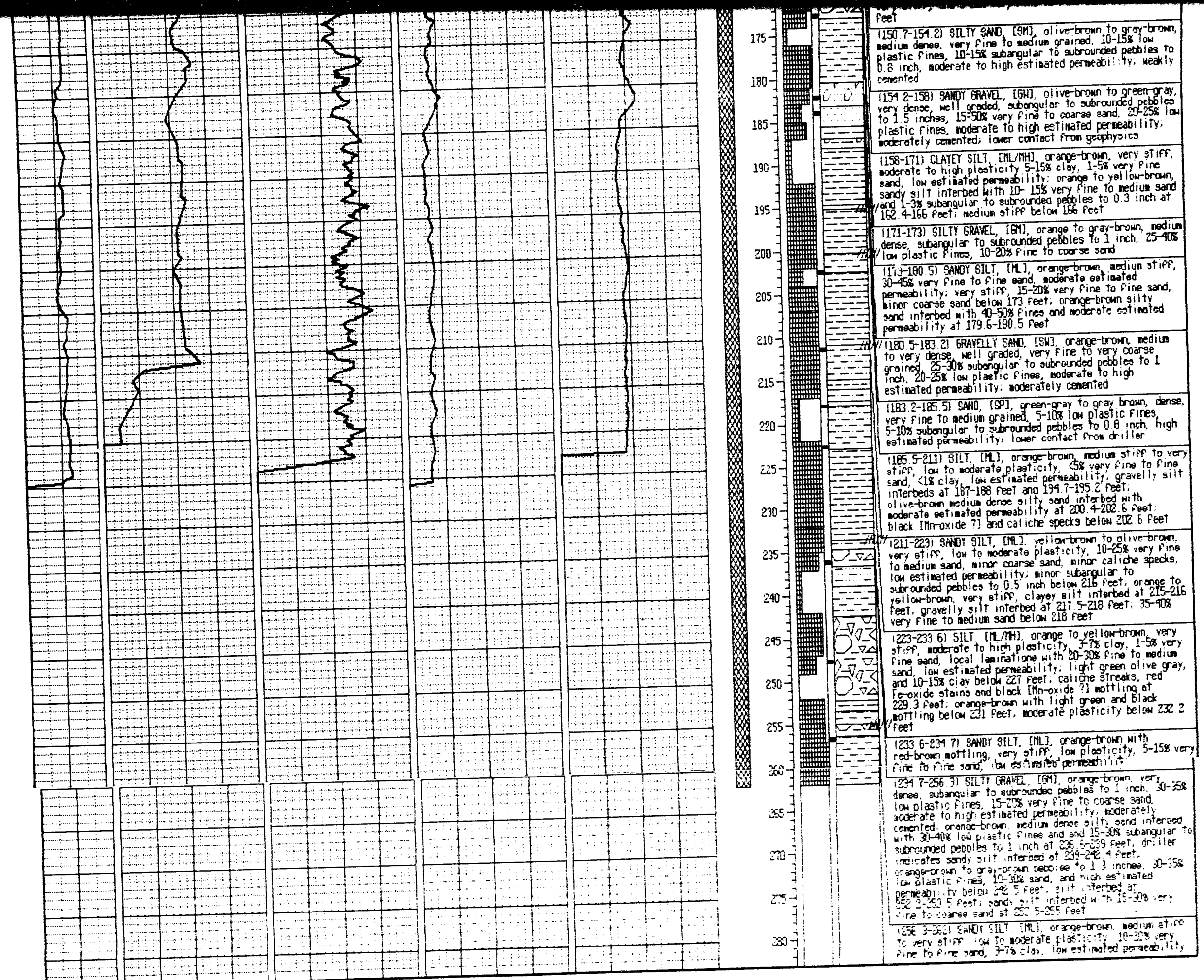




\section{LOG: MONITORING WELL MW-615}

Location: Averwe A, about 200 feet southeast of Well MH-357, LLNL

Coordinates [UNL]: N $10,491.8$ E: 7.4878

[CAL GRID]: $N: 433,7715$ E. 1.698 .860 .0

Elevation [shiner] 601.16 feet

Total Depth of Hole: 121 feet

Total Depth of well: 99.3 feet

Initial Hater Level: Not atserved

Static Water Level, 6/1/90: 684 feet below ground level

Hell Head Protection: Above ground lock ing steel cyl inder

Well Production Rate: Approximately 1-2 gpm

\section{BLADDER PUMP \\ 1 SUBMERSIBLE PUMP \\ Z FIRST WATER}

I STATIC HATER LEVEL
- analytiral soll sample

98X CEMENT-2X BENTONITE GROUT CEMENT-CAL SEAL GROUT
Gealogic Lagging: 5 Helson, Weiss Associates. Emerywille, CA Geophysical Logging Geo-Hydro-[lata, Tehachapi. CA Irilling J.Var Noy. P. C Exploration. Inc. Roseville. CA Llates Urilled: 5/23/90 to 5/24/90

[rilling Method Mud rotary, 0-121 feet

Int ial Borchole Diameter. 55 inches, 0-121 feet

Final Borehole Diameter. 9 inches, $0-993$ feet

Sampling Methad Christensen 94 in Wirel ine Punch Core System OA means drill ahead. advance without coring

WP means no recovery

WNEX I0 8MP018

=

"3 LONESTAR SAND

BENTONITE PELLETS
4.5-INCH PIC CASING HITH O. O20-INCH SLOTS

SLOUGH MATERIAL

M APPROXIMATE CONTACT

IIIII GRADATIONAL CONTACT

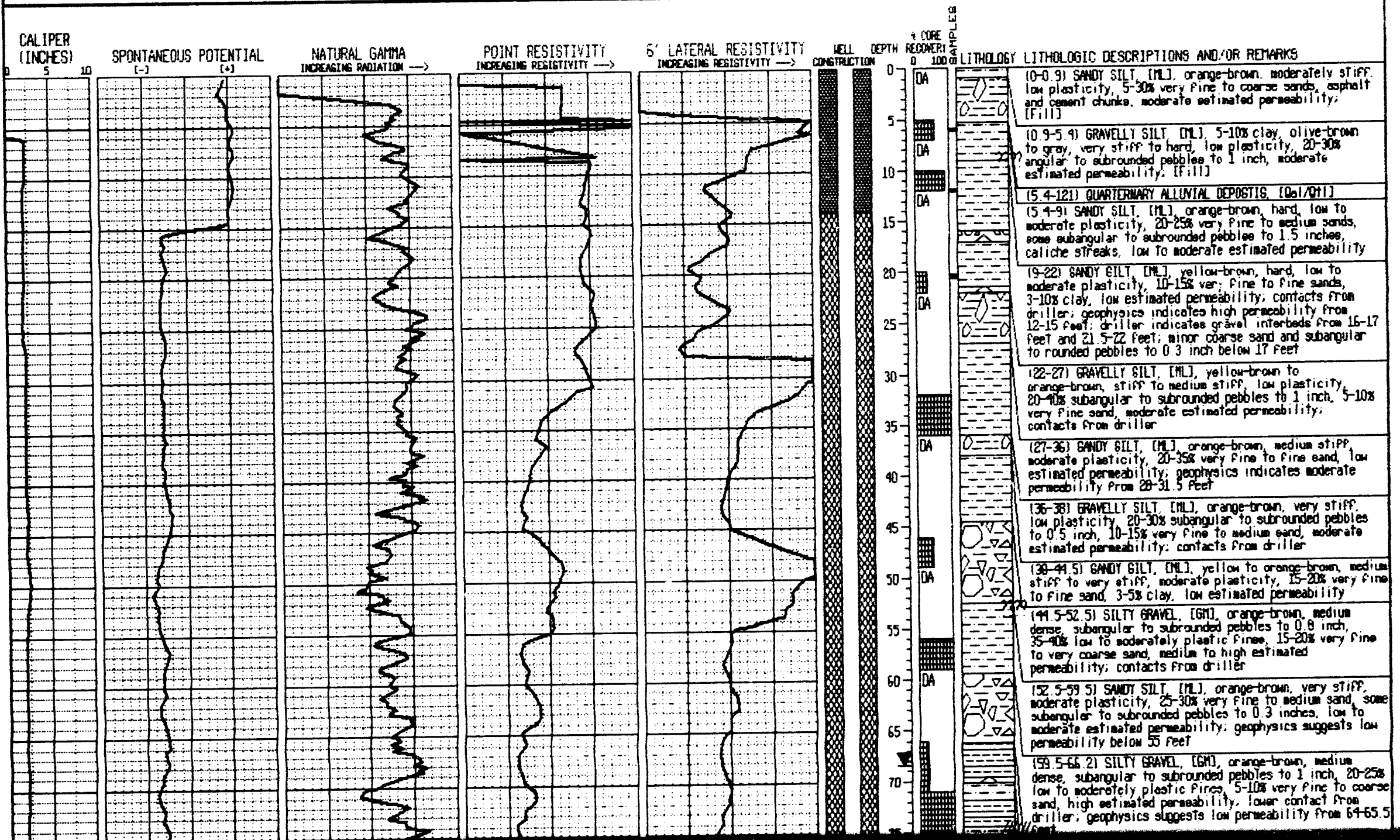



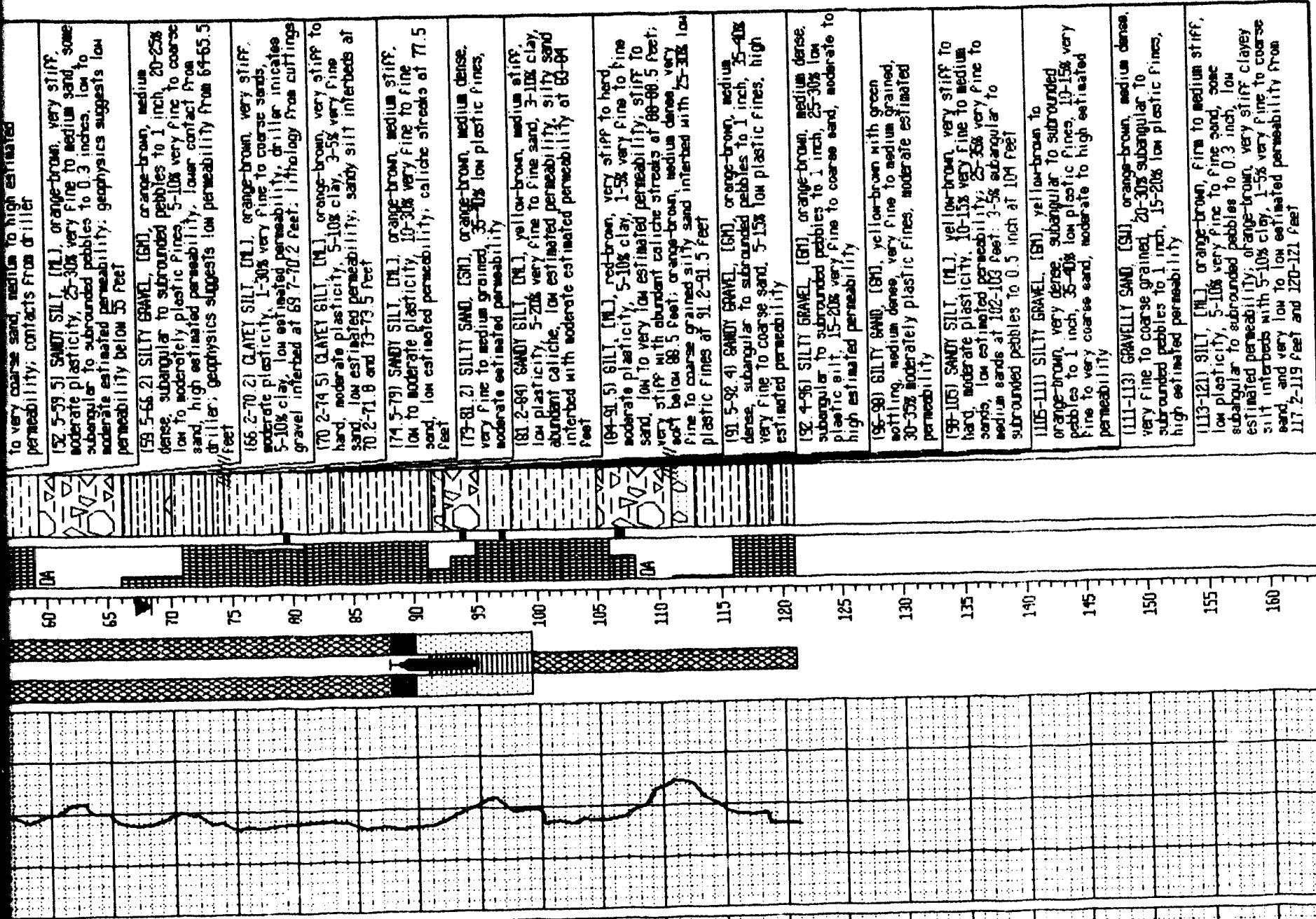

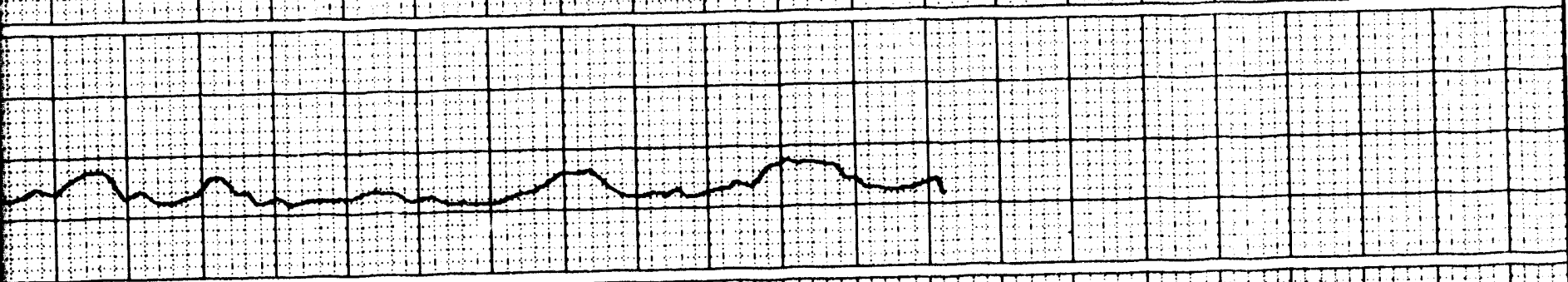

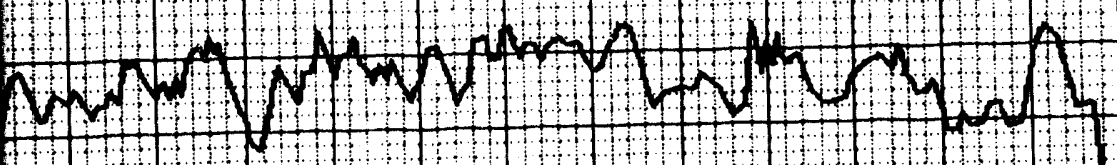

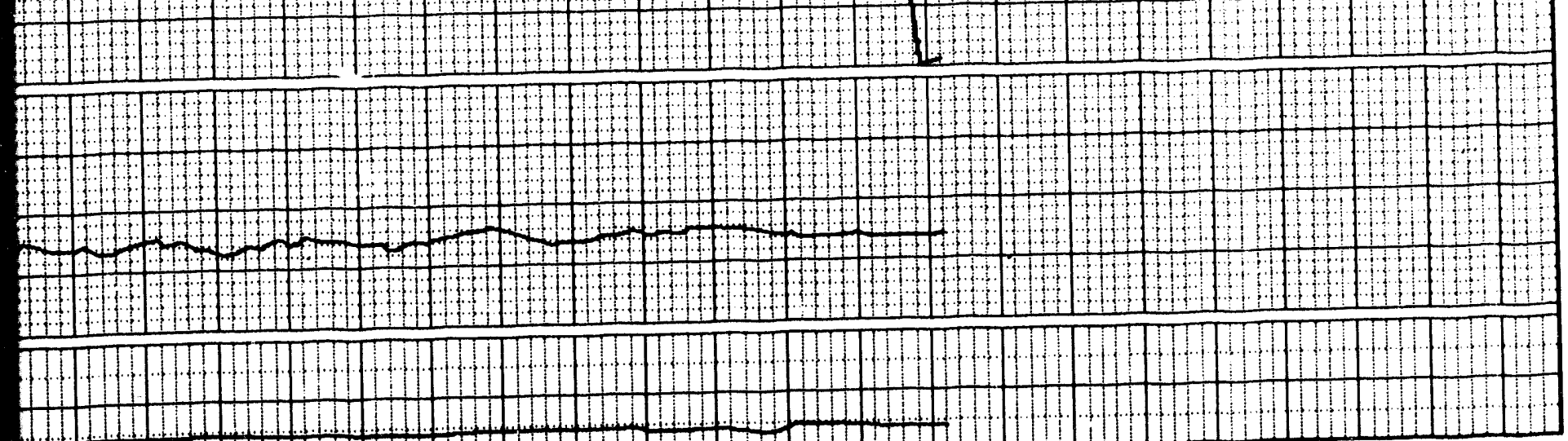




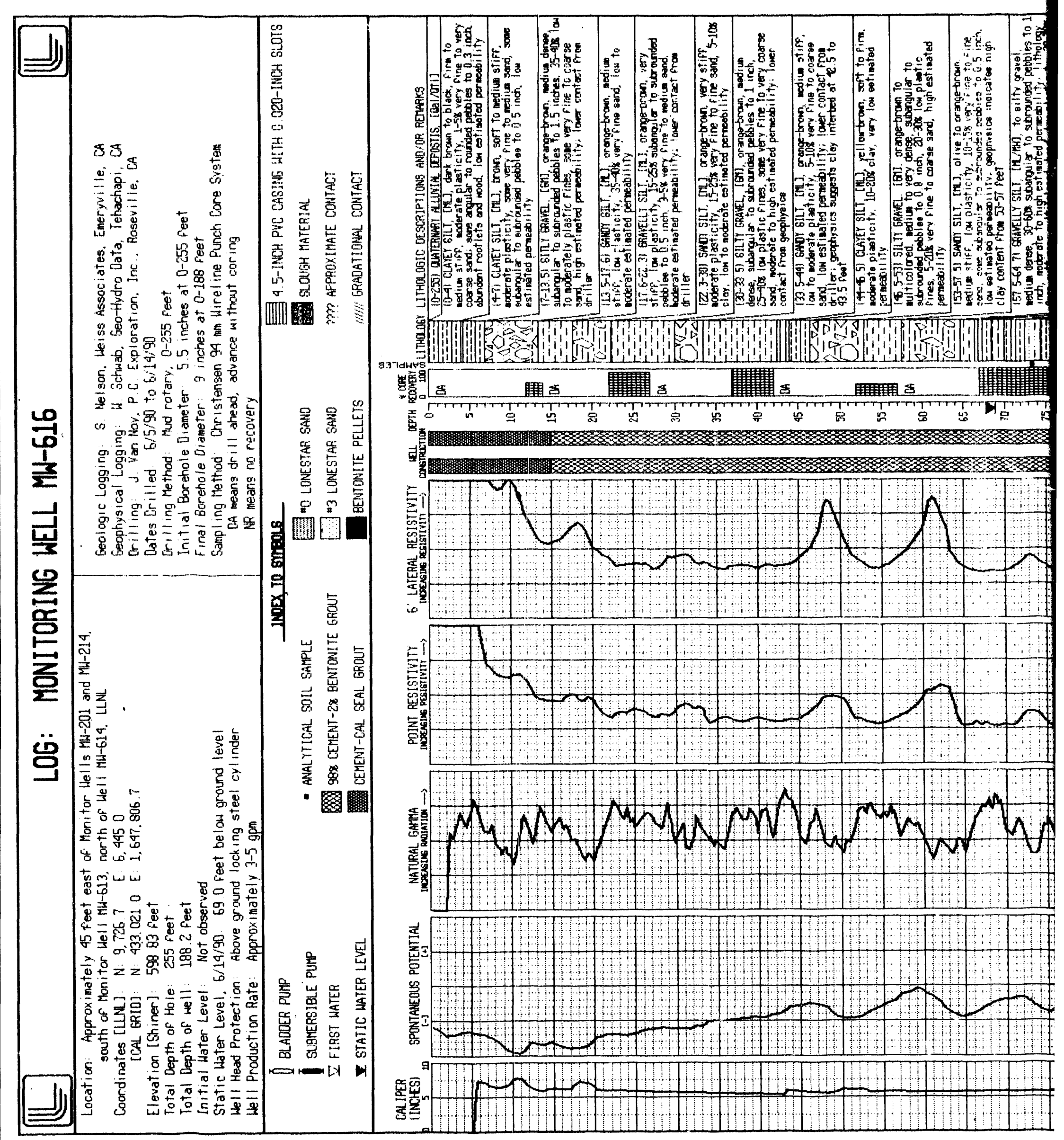




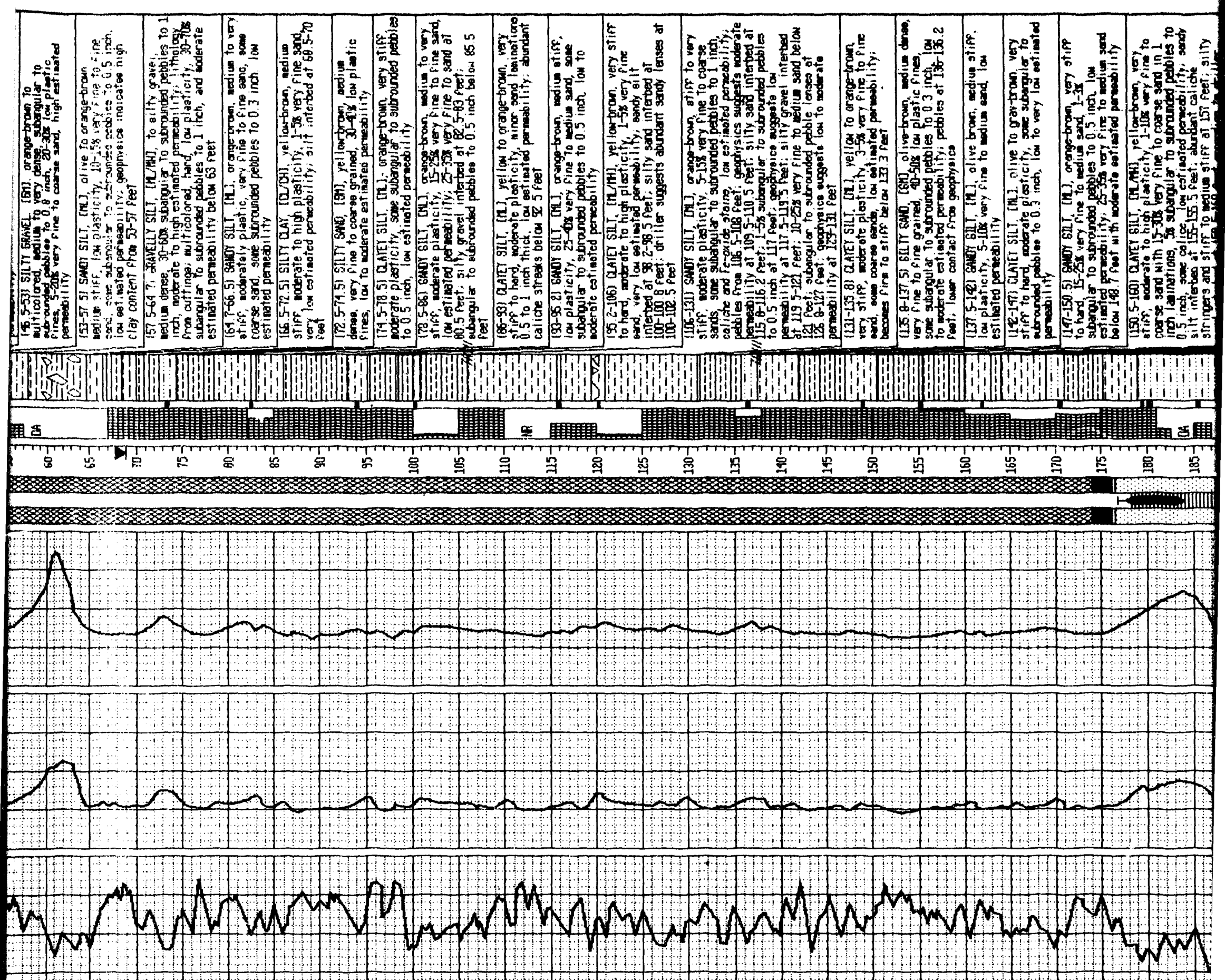

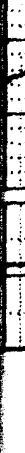




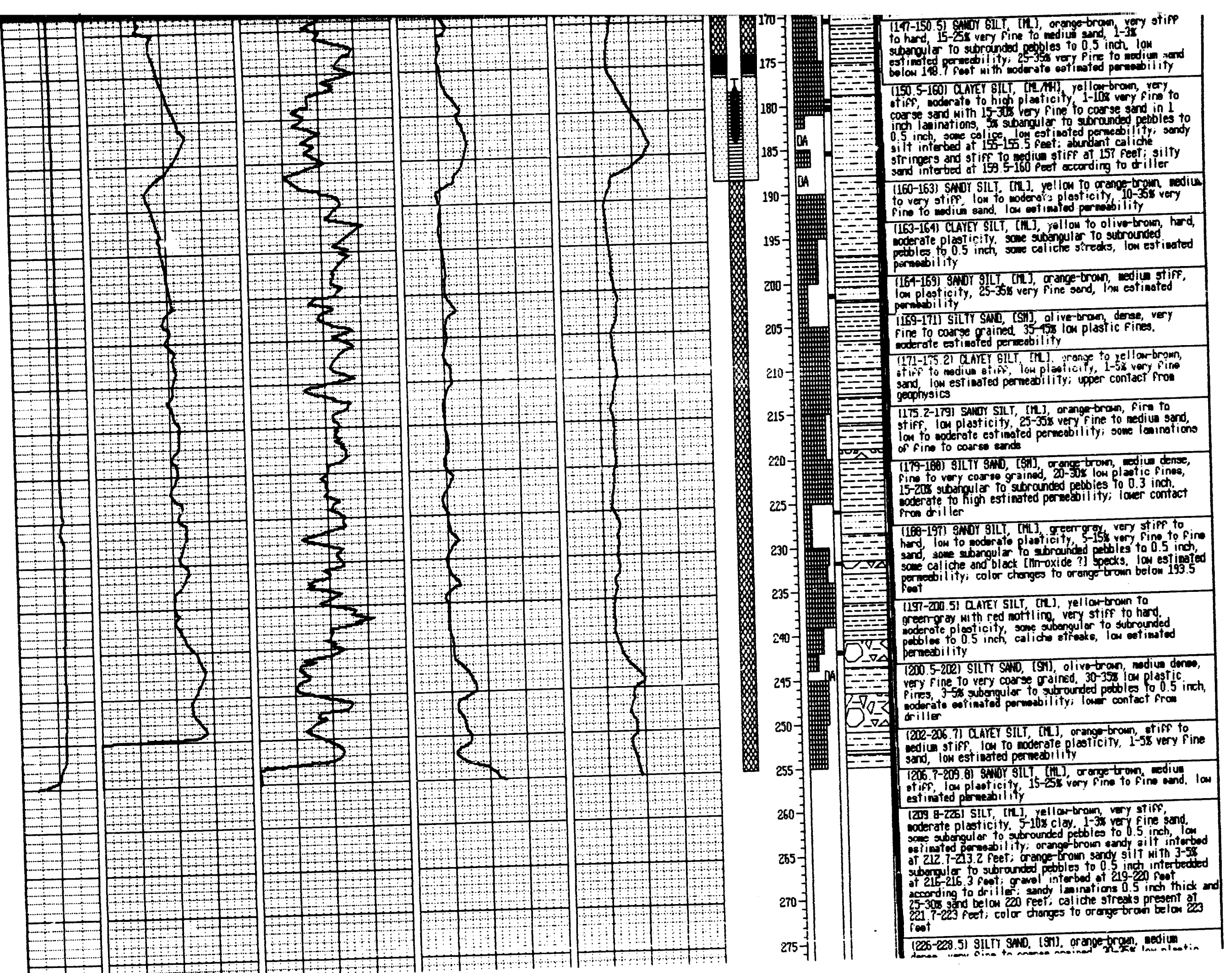




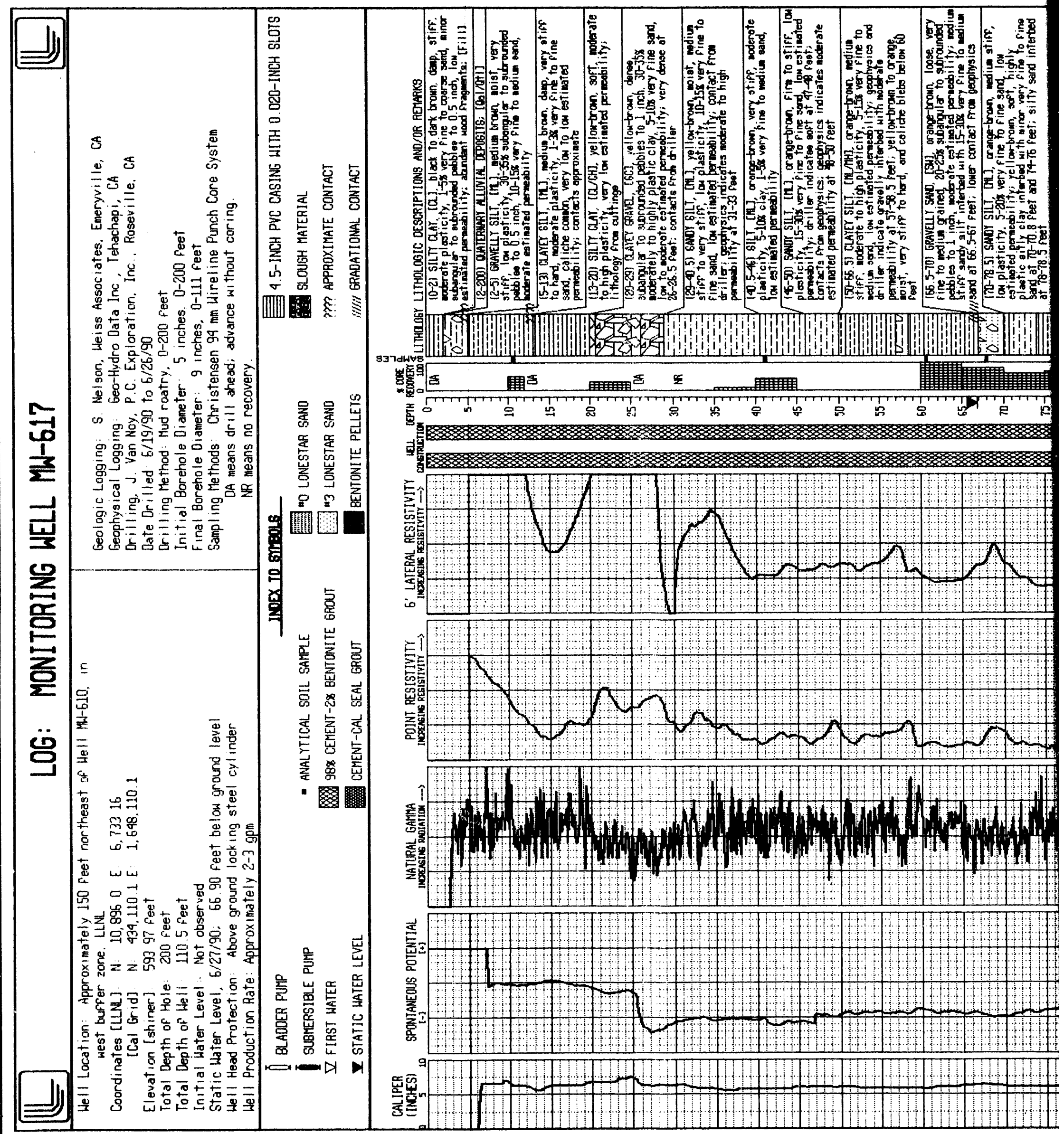



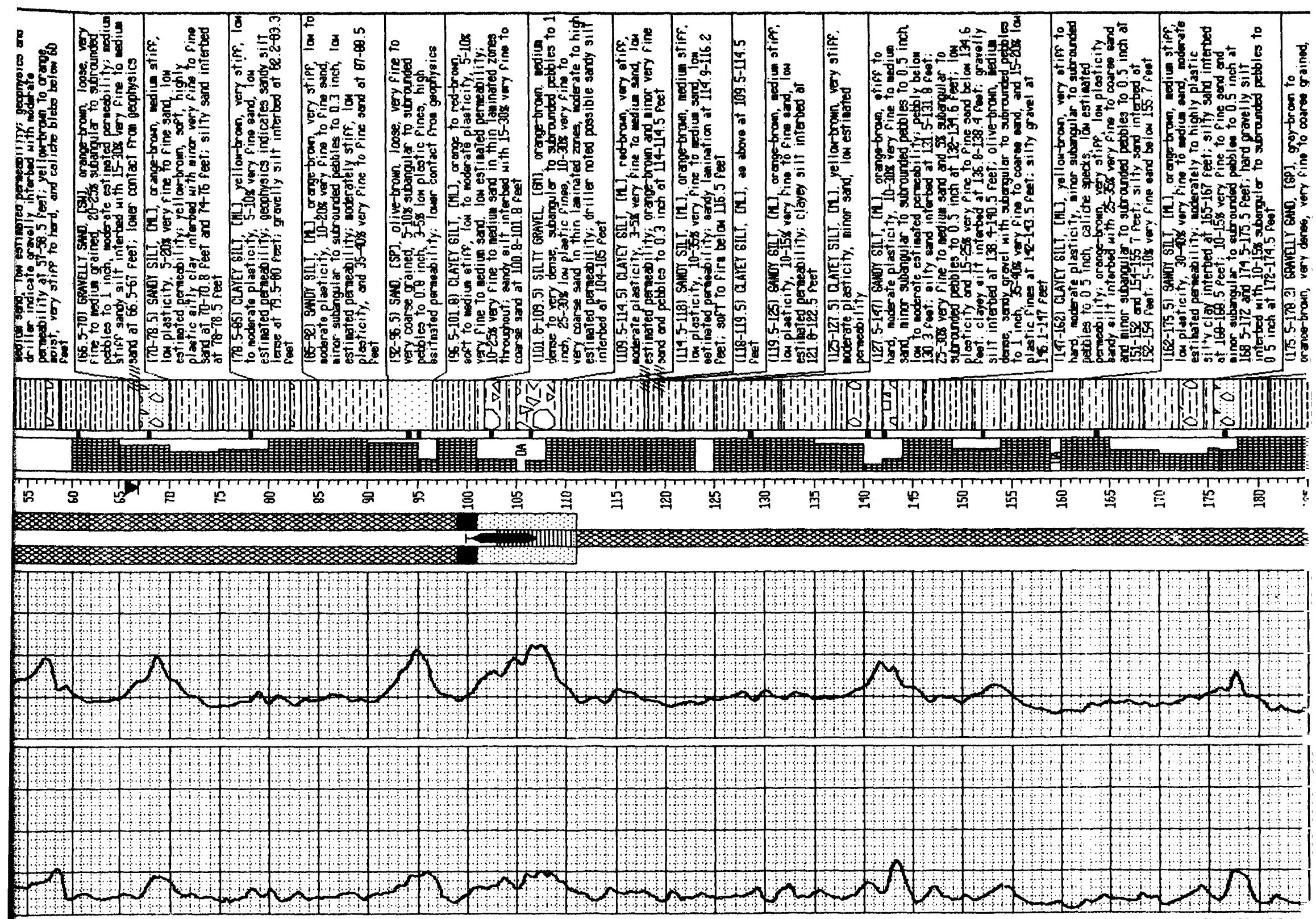

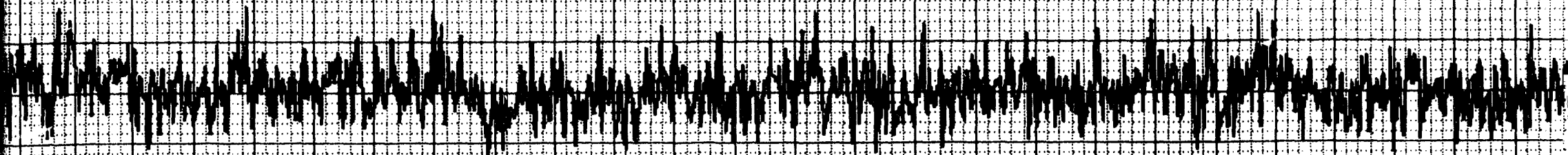
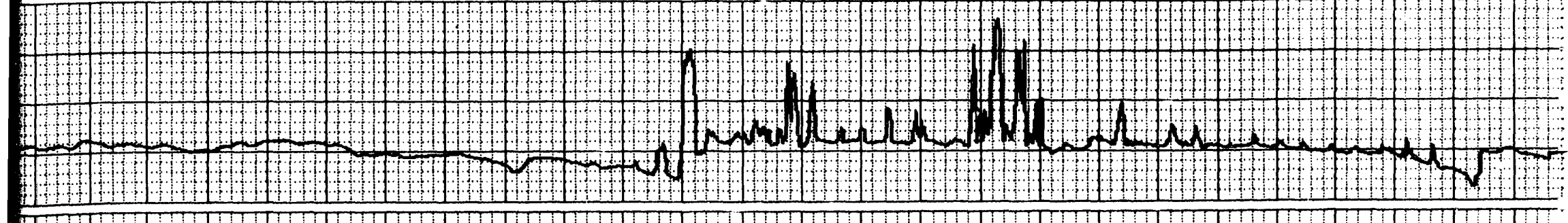



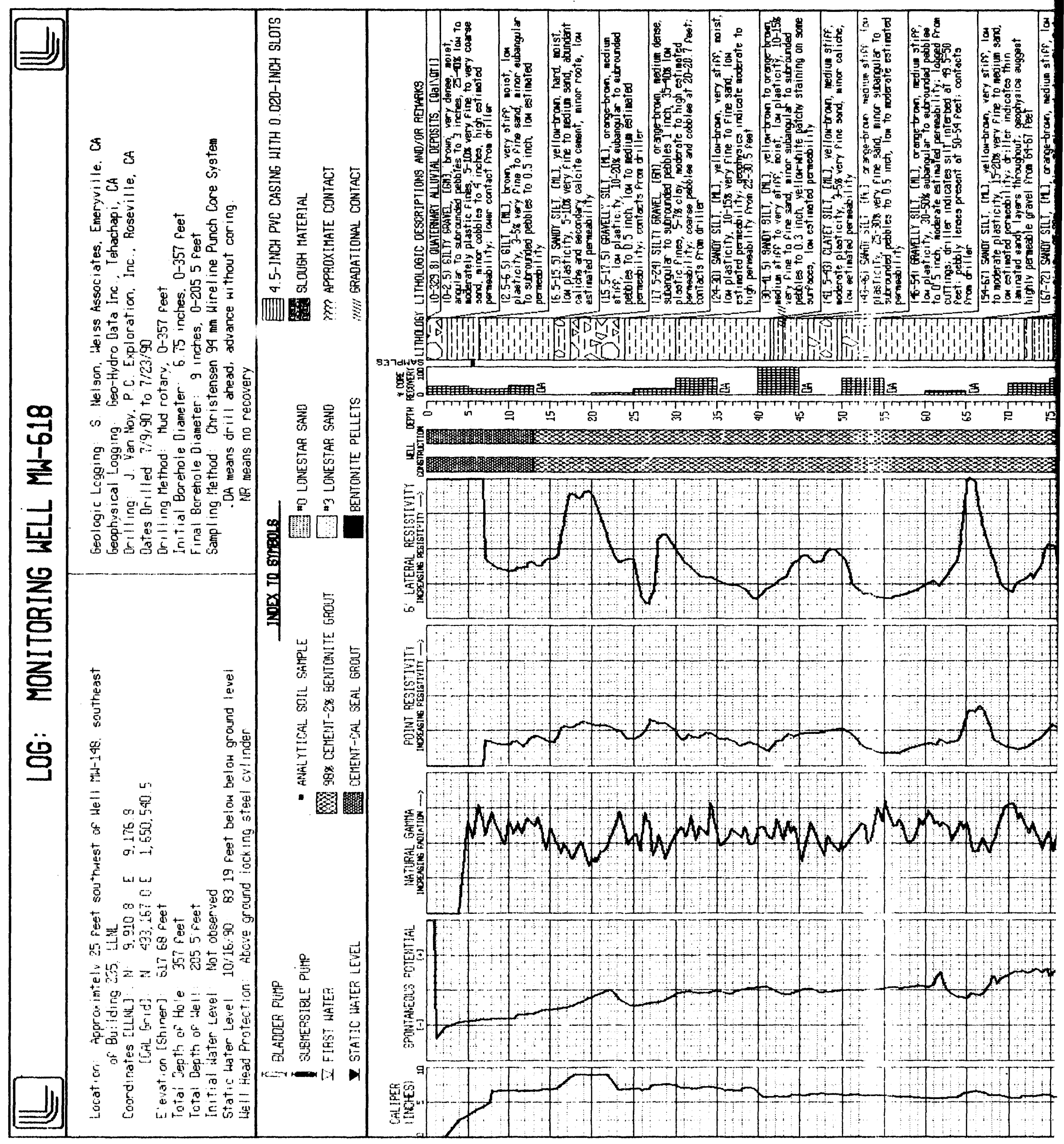




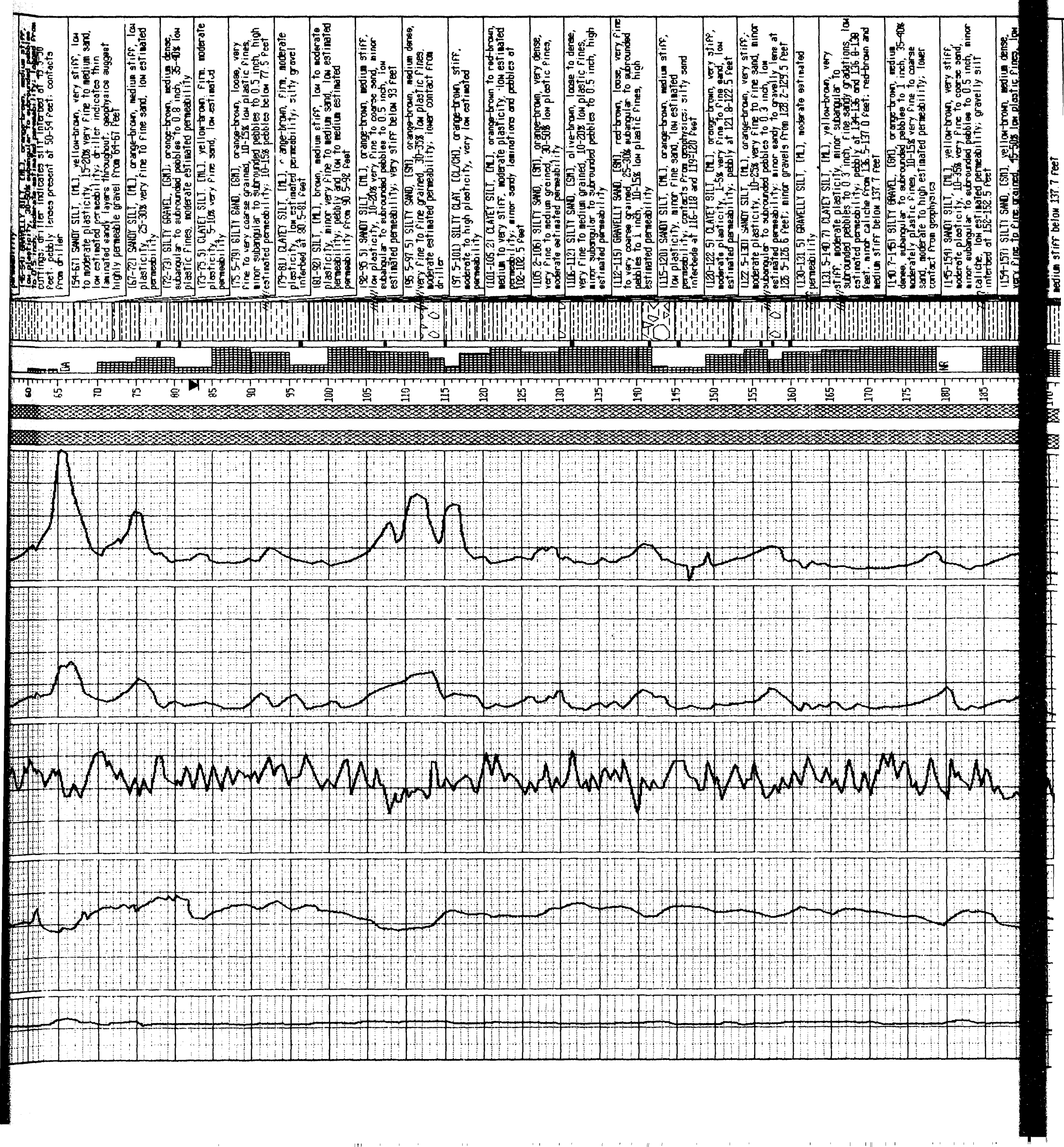




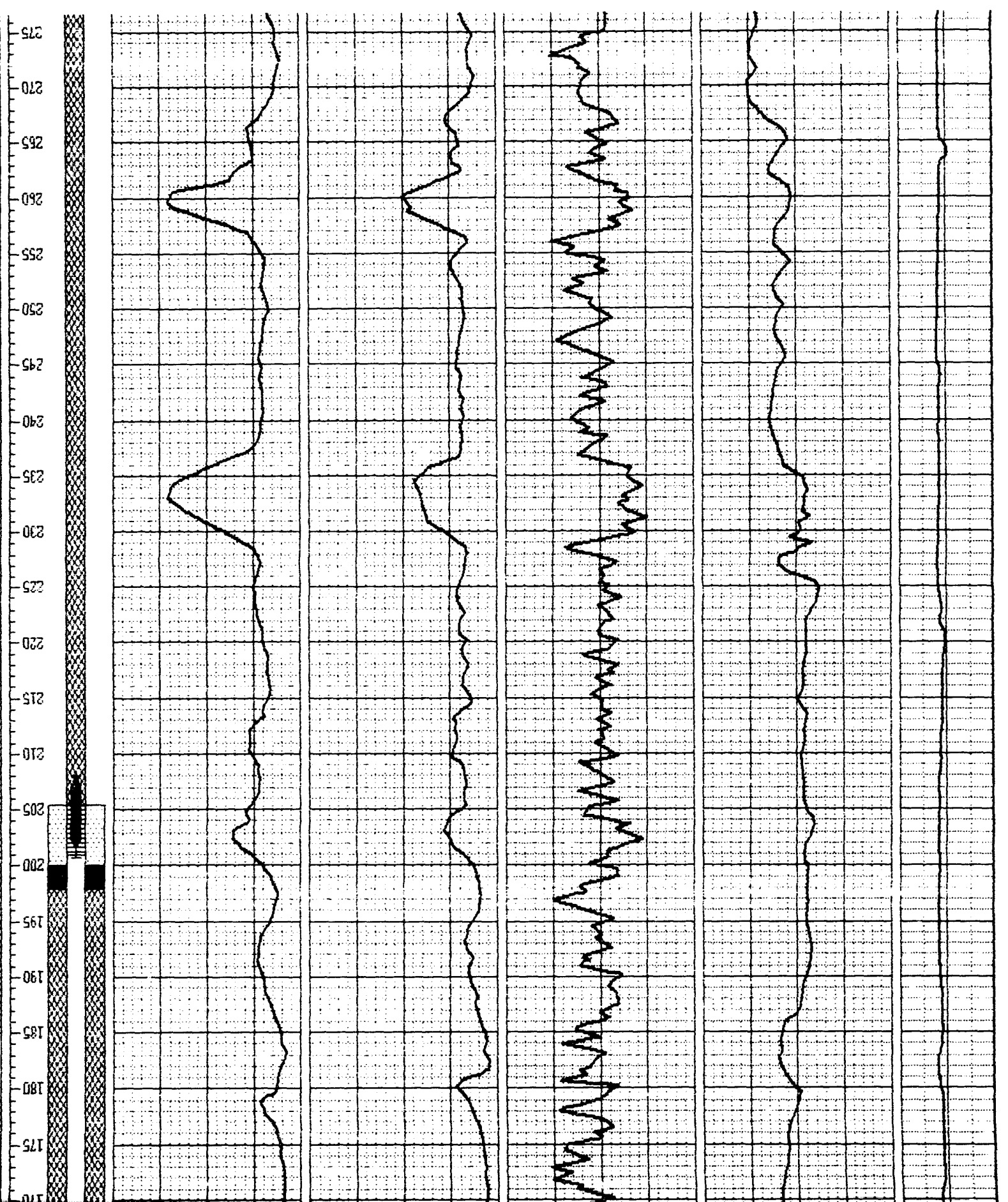




\section{LOG: MONITORING WELL MW-619}

Location: Approximately 20 feet southeast of ML-EO1, nor theast of

intersection: Vasco Foad and East Ayenue. this well is

located in the letailed Study frea, LLHL

Coordinates [LLN]: $N: 8.823 .9 \quad E: \quad 6.469 .2$

[Cal Grid]. H: $432.118 .1 \mathrm{E}$ 1.547.818.3

Elevation [Shiner]: 601.85 feet

Total Depth of Hole: 330 feet

Total Depth of Well: 252 feet

Initial Water Level: Not otserved

Static Hater Level, 10/15/96: 74.23 feet below below ground leve!

Well Production Rate: Approximately $5-10 \mathrm{gem}$

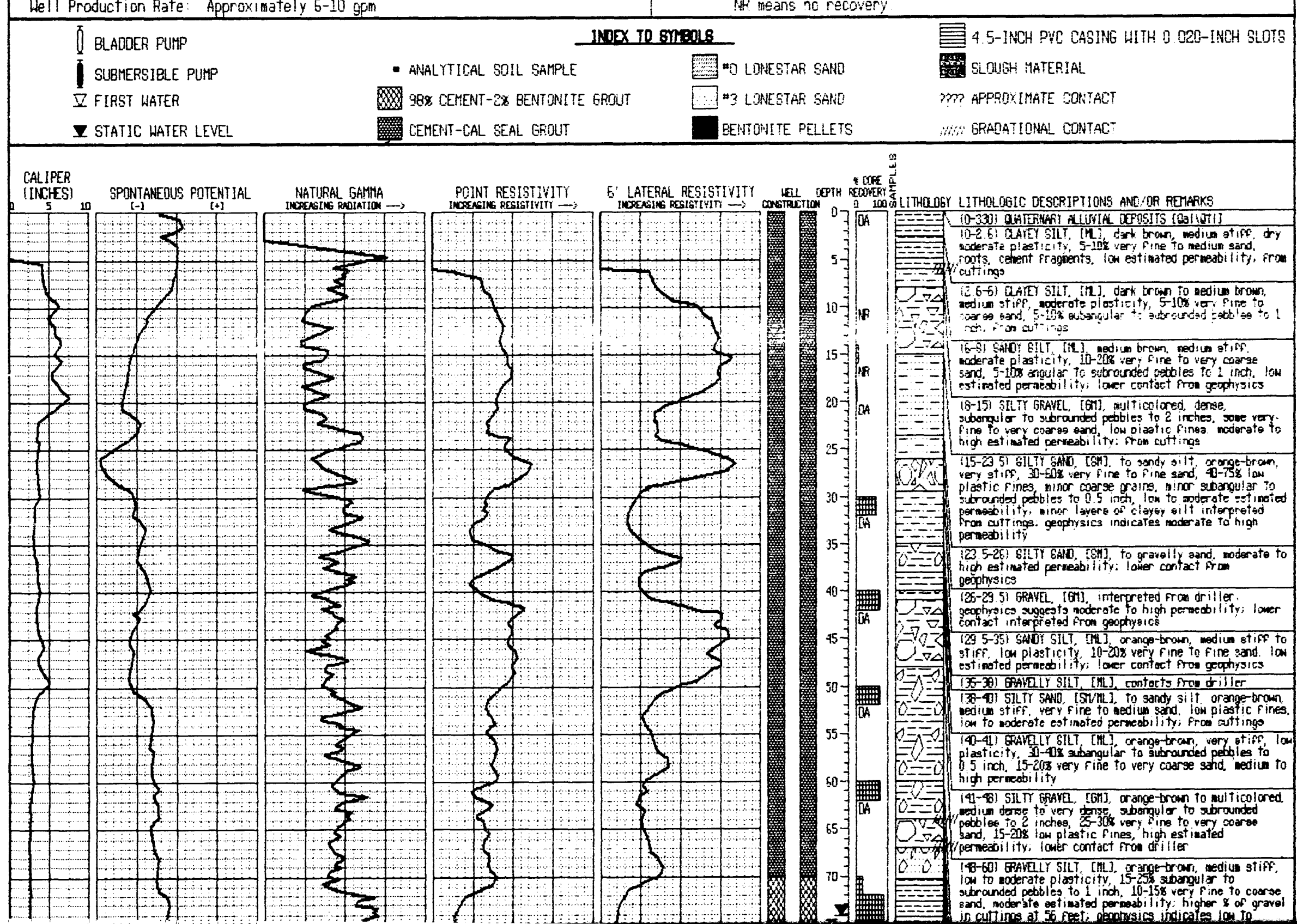

Weli Head Frotection Above around sault

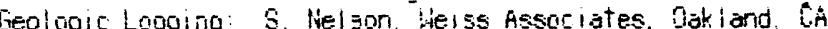

Geophysical Logging. Geo-Hidro Data inc. Tehachap! CA

Erilling I. Van Noy. P E Exploration. Inc. Foseville. CA

Dates Drilled. T/26/90 to 8/1499?

irilling Method: Mud ratary o-30 feet

Initial Eorehole Diameter .58 inches, 01-330 feet

Firia! Borehole Diameter: 9 inches, 0-253 feet

Sampling Method Christensen 94 min Wirelire Funch Core Syctem

OA means drill ahead. advance without coring. 


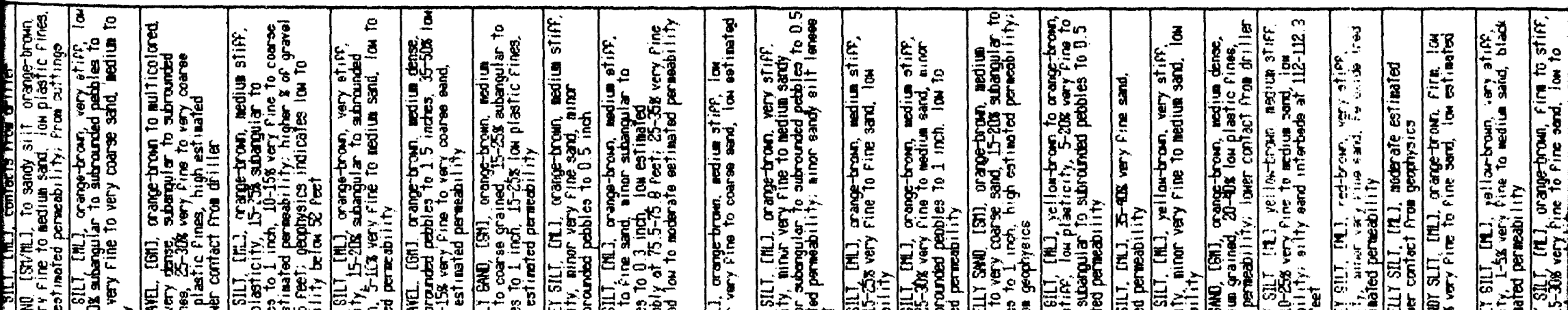
(c)

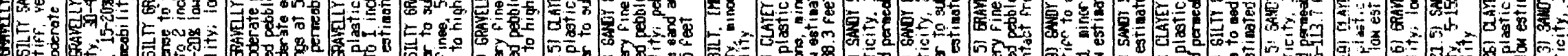

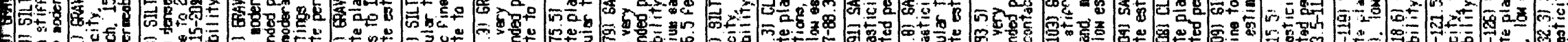

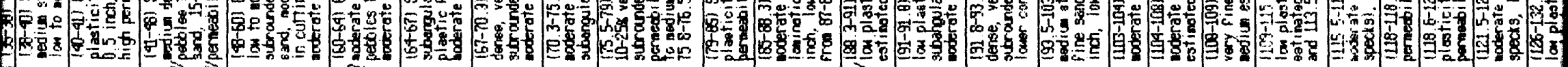

Nons

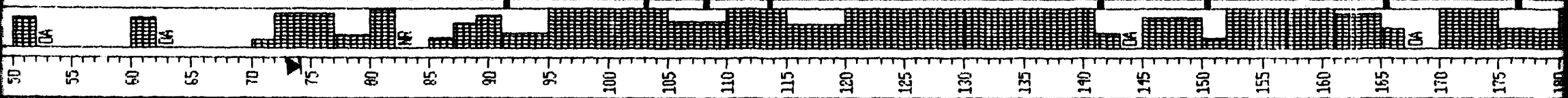

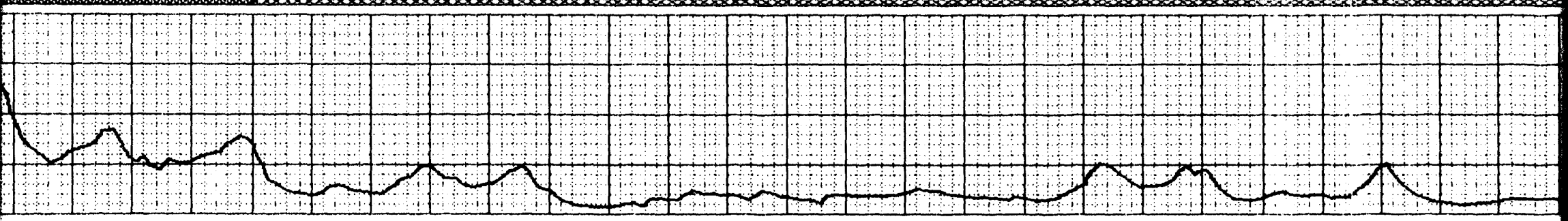

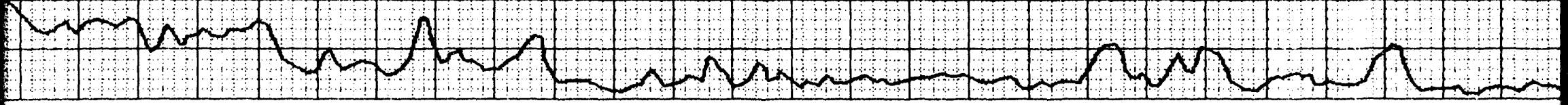

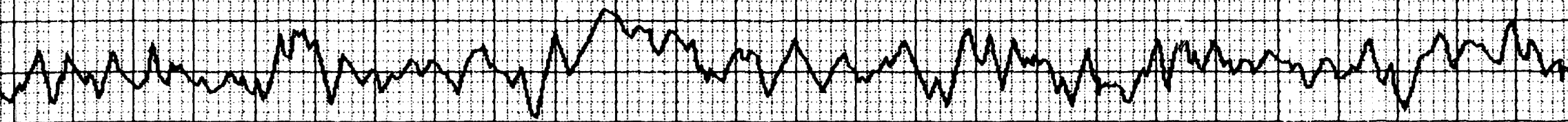




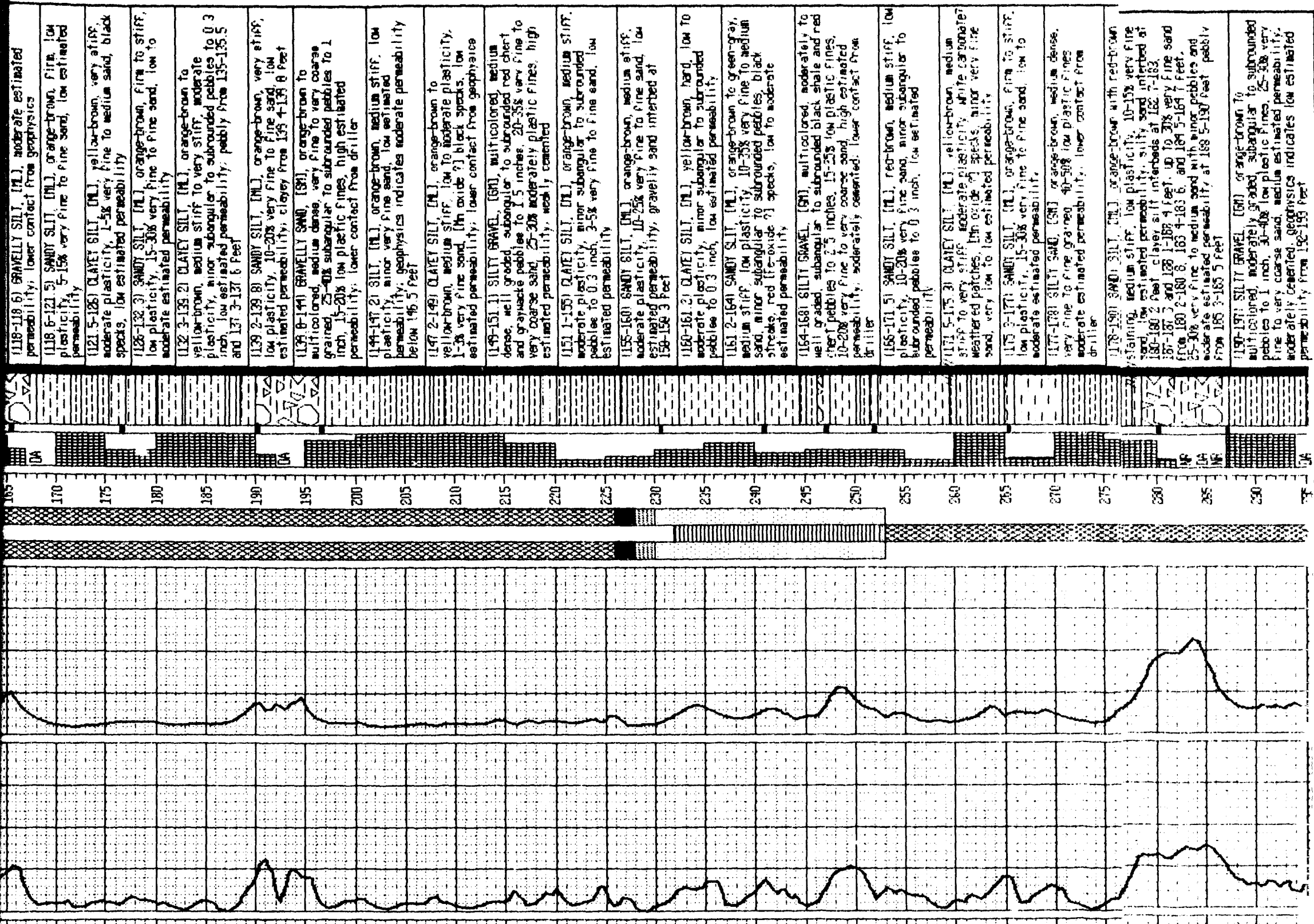

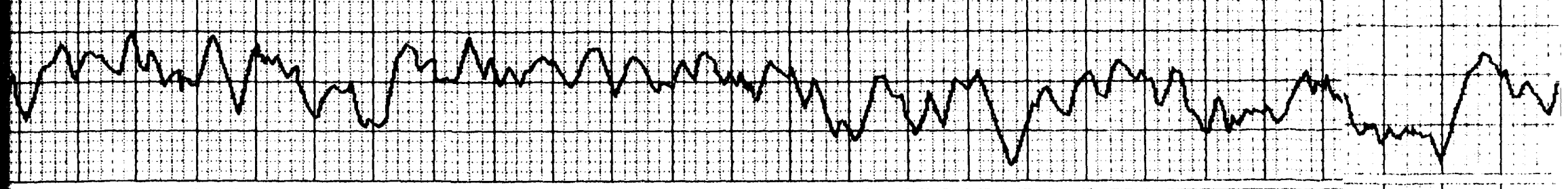
. 


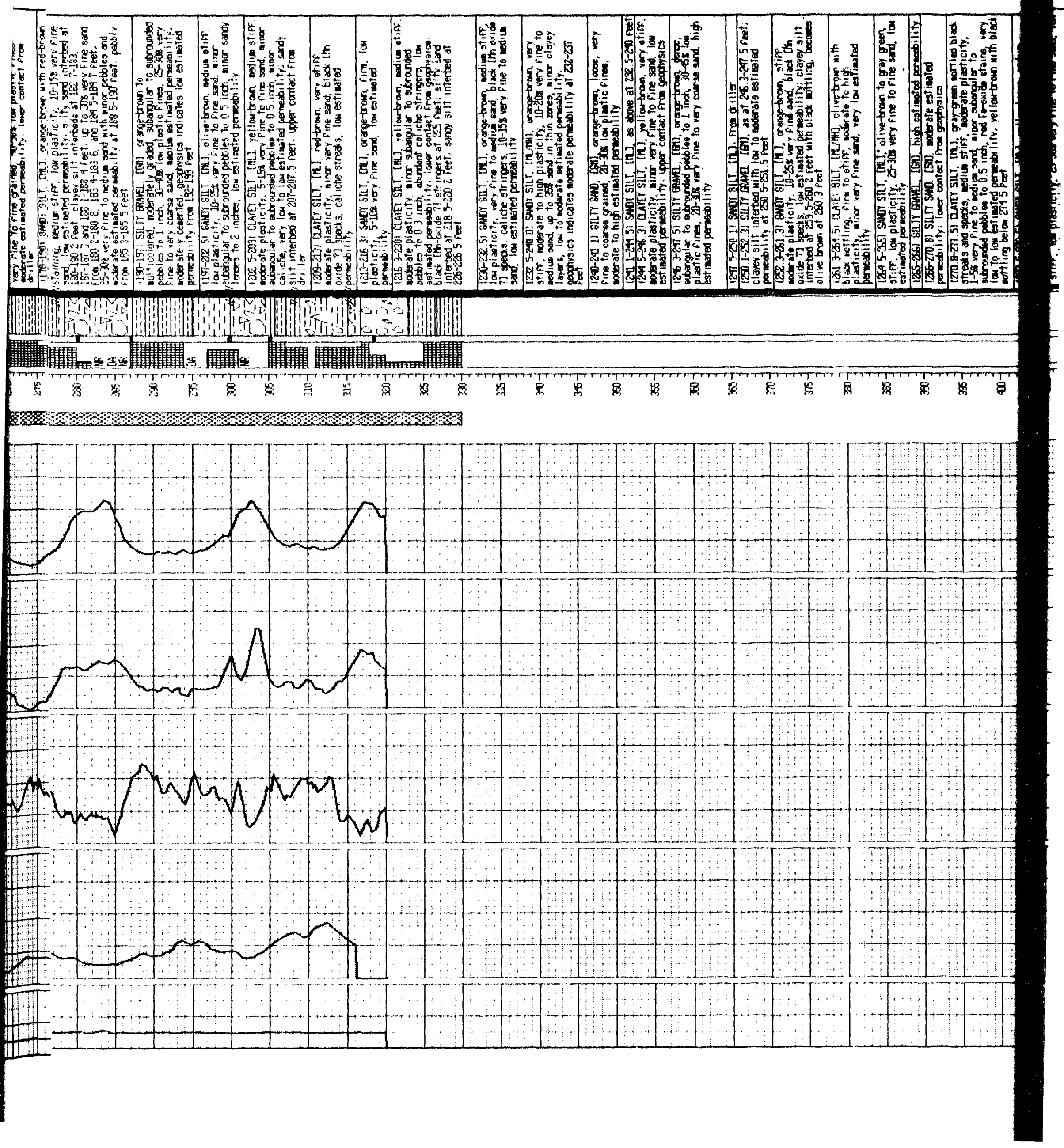




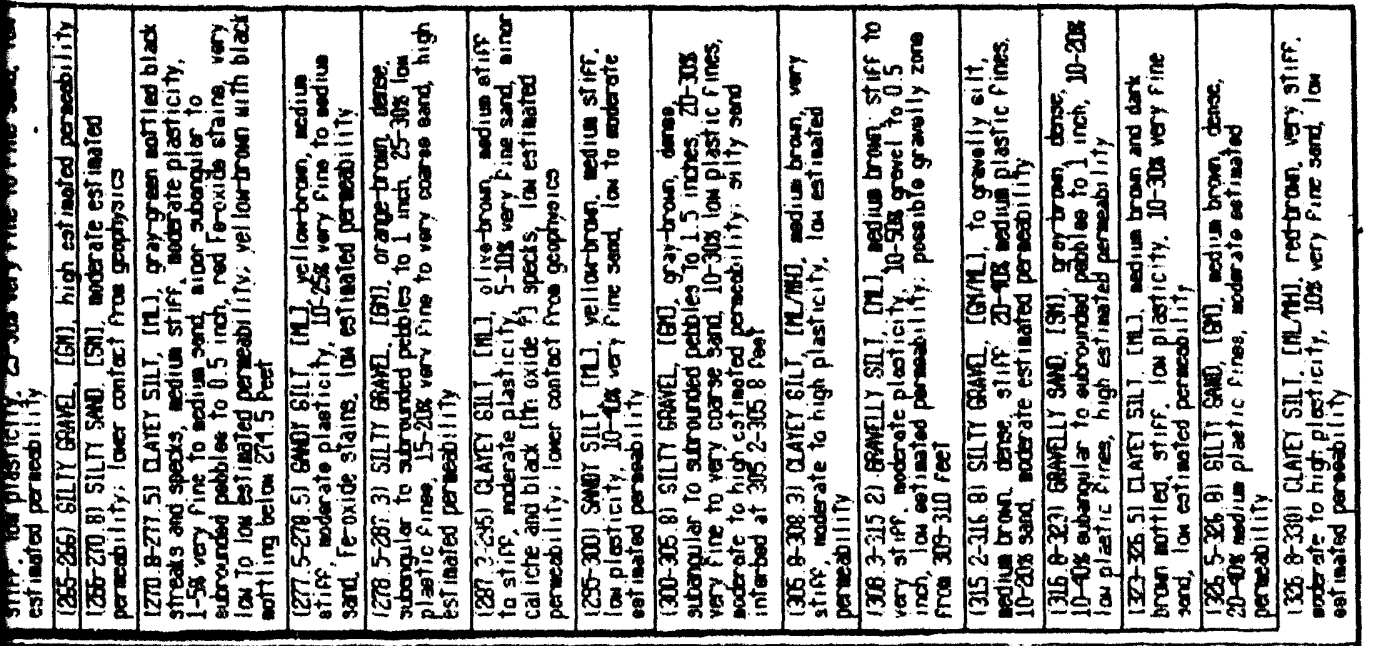

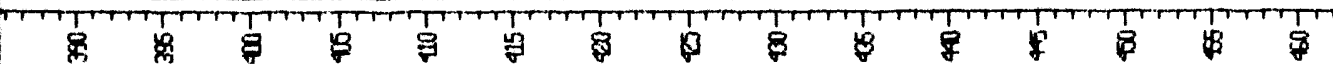
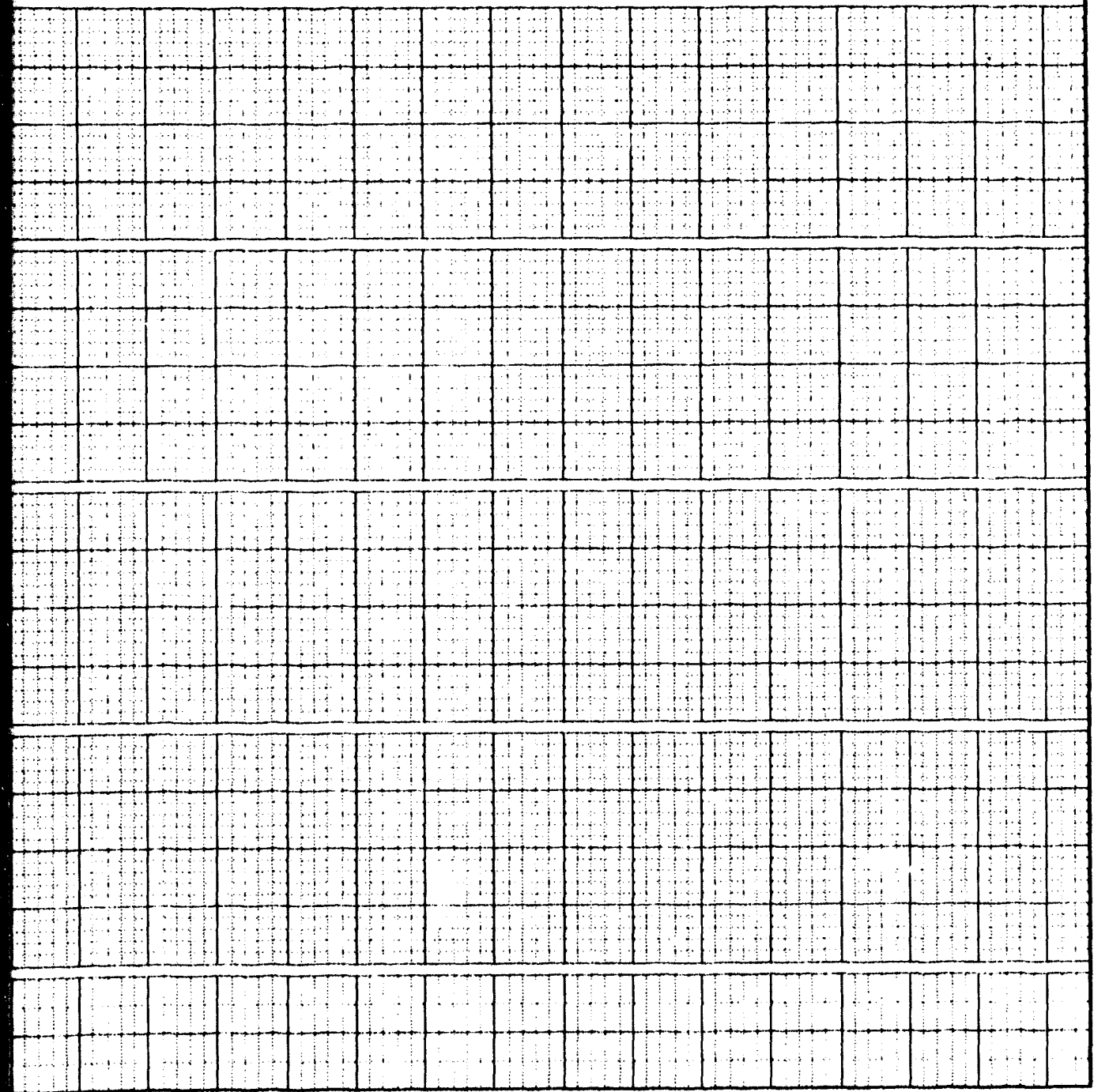


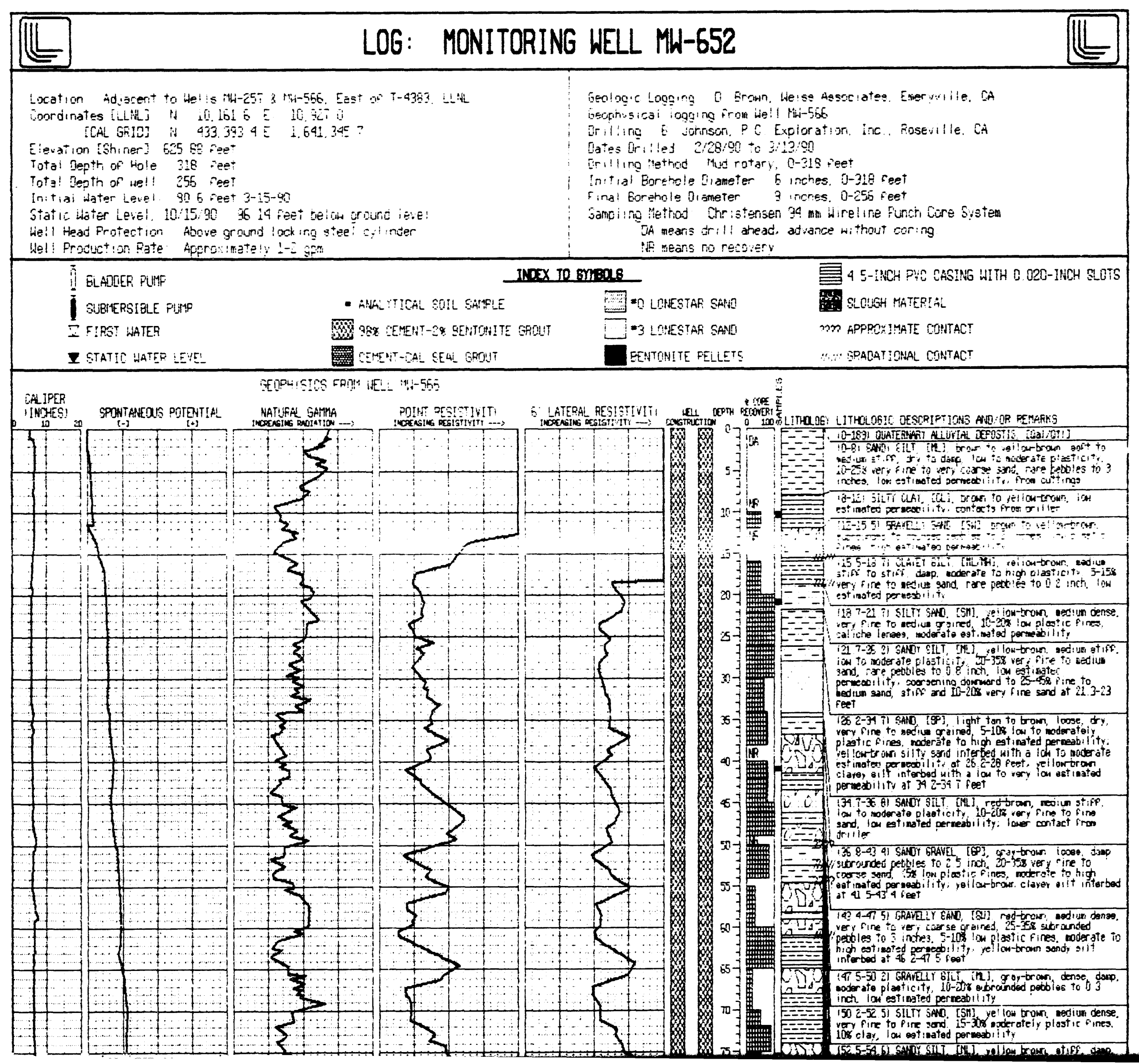




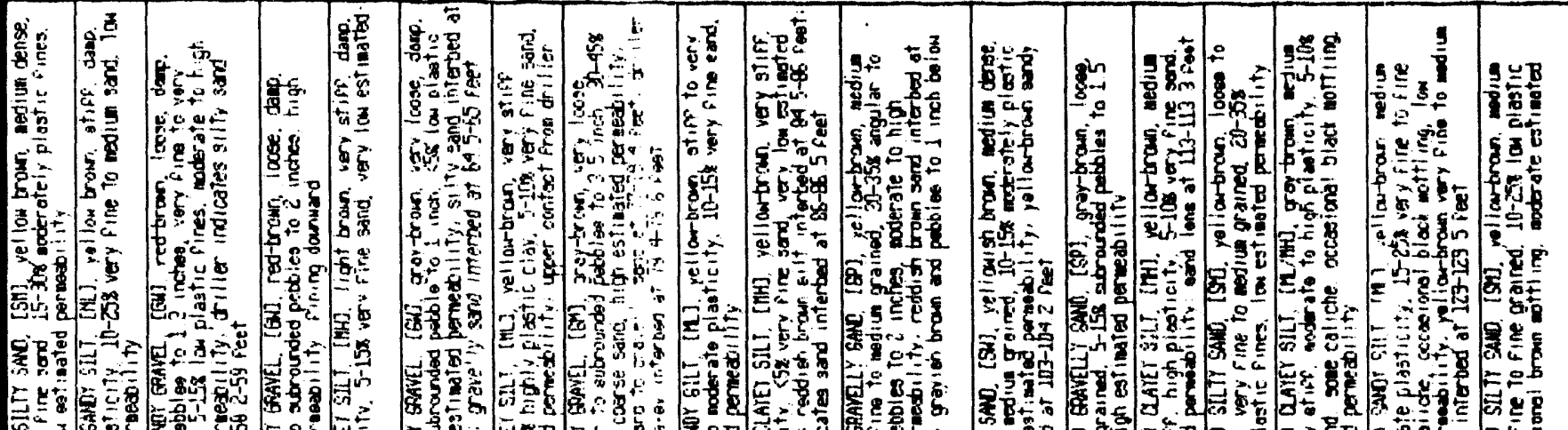

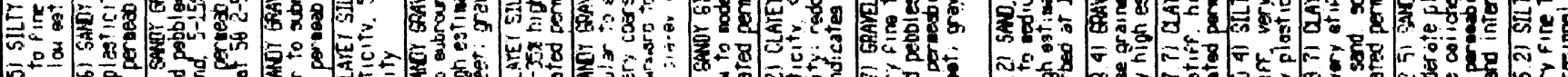

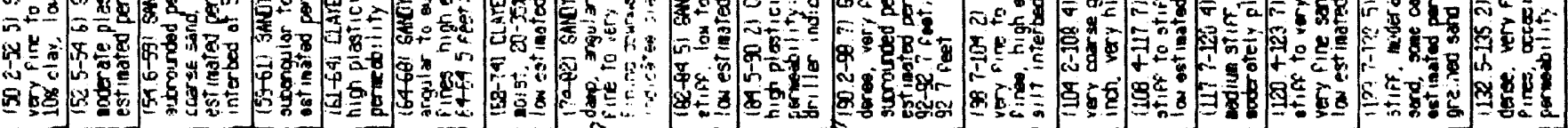

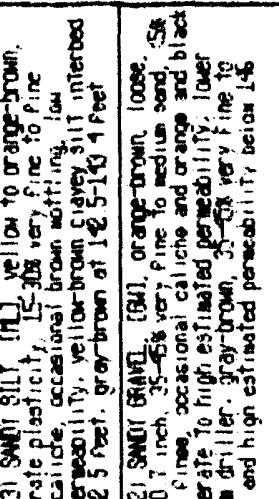

胥.

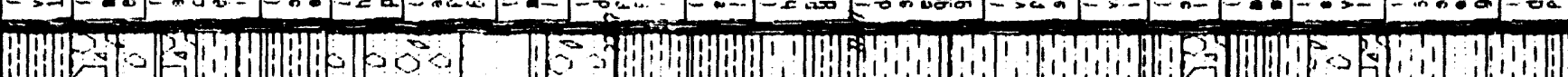

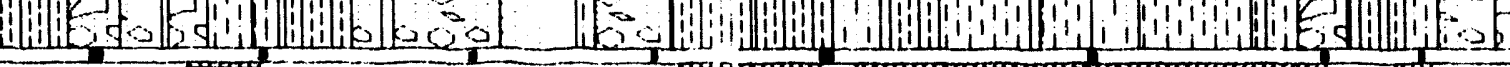

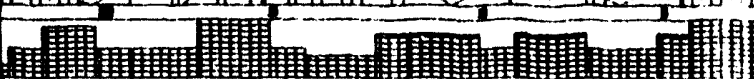

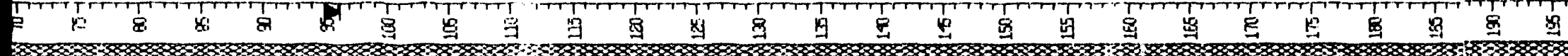
N

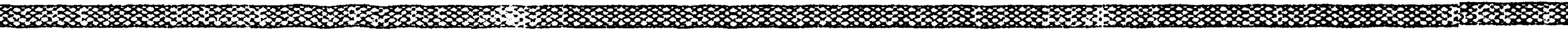
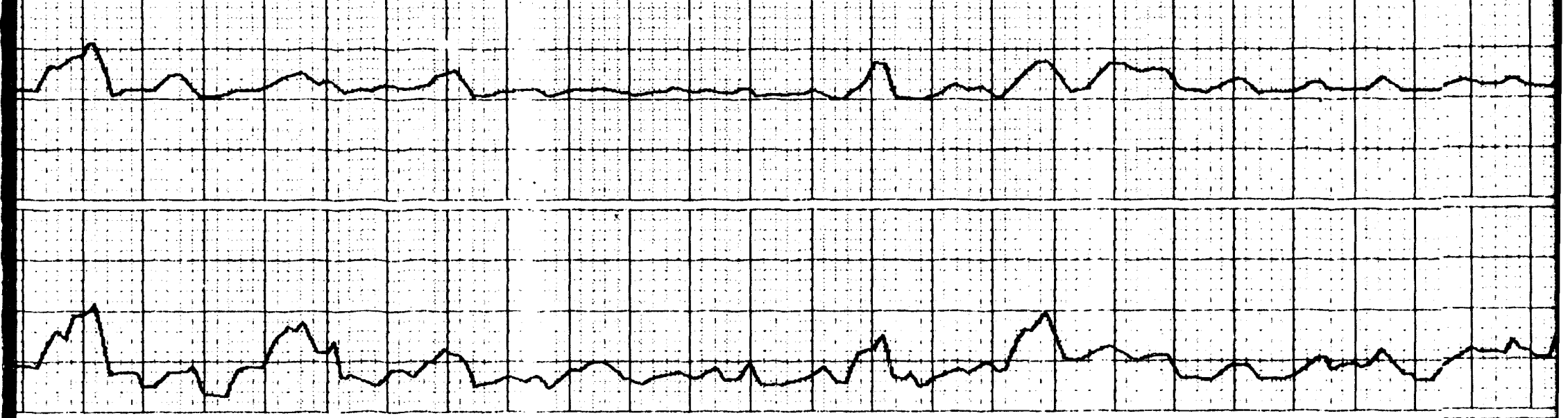

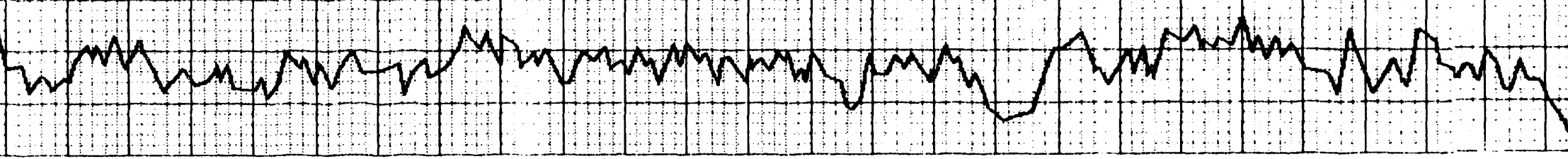



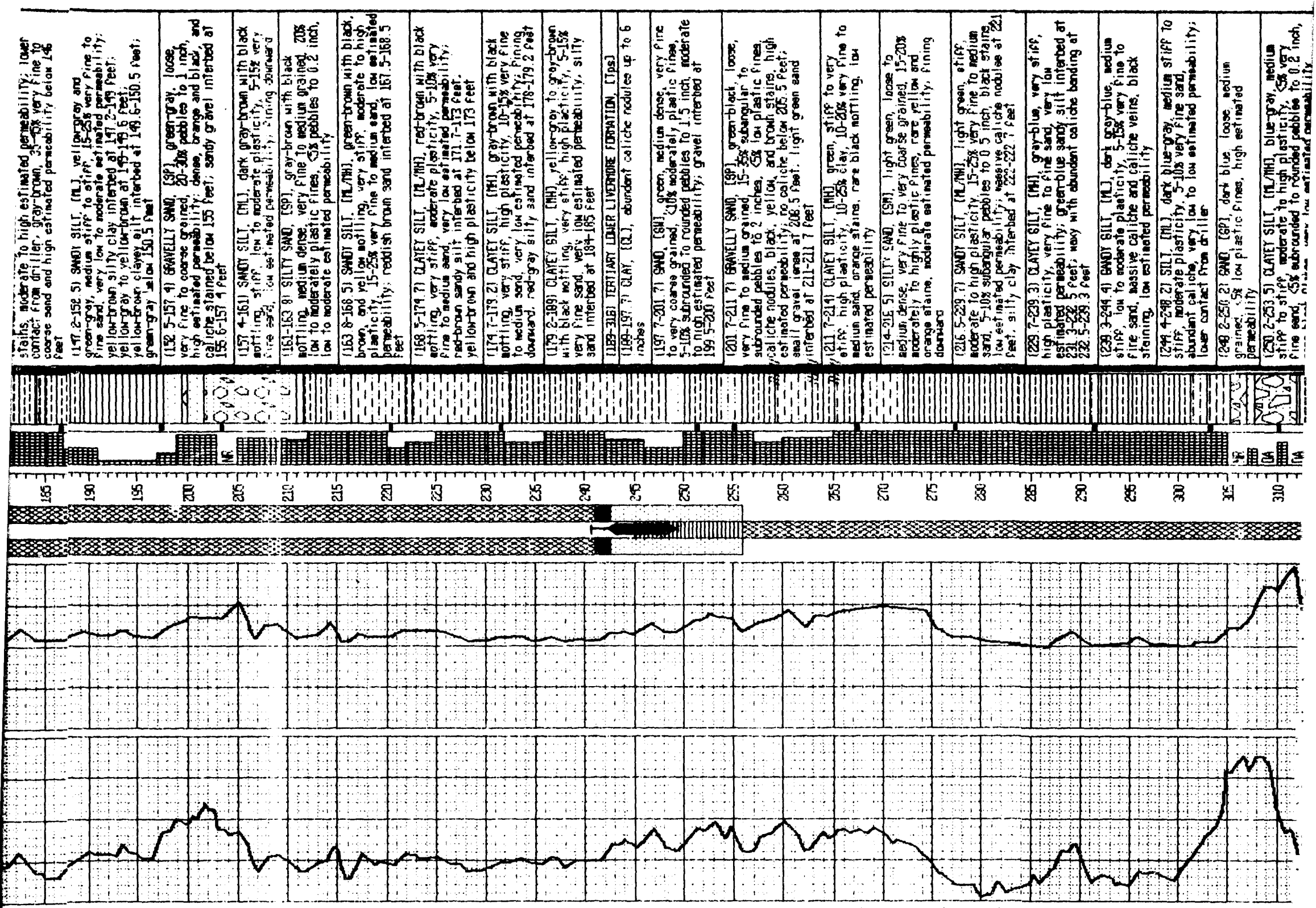

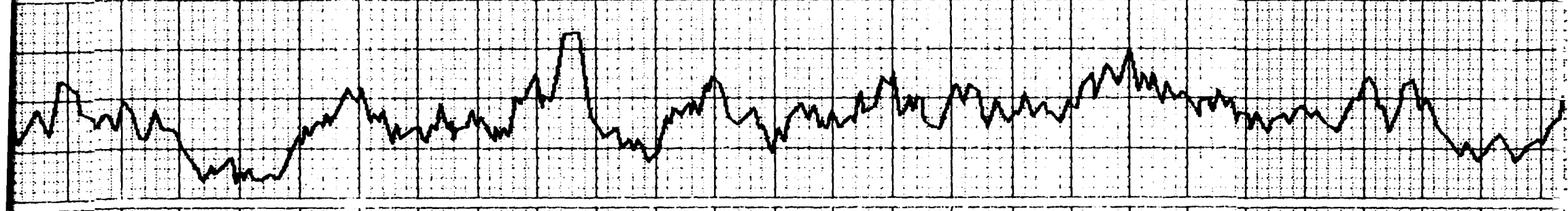




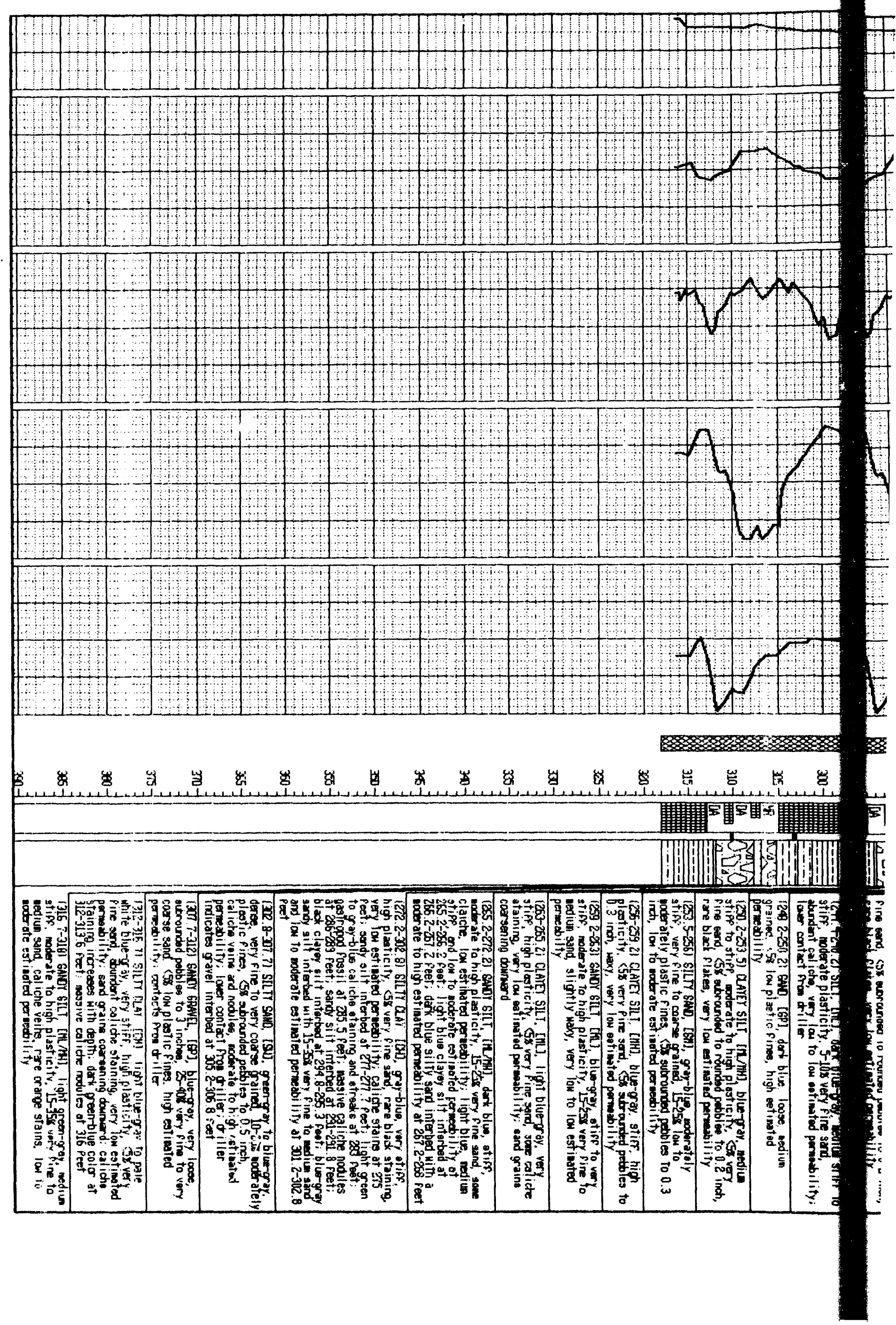




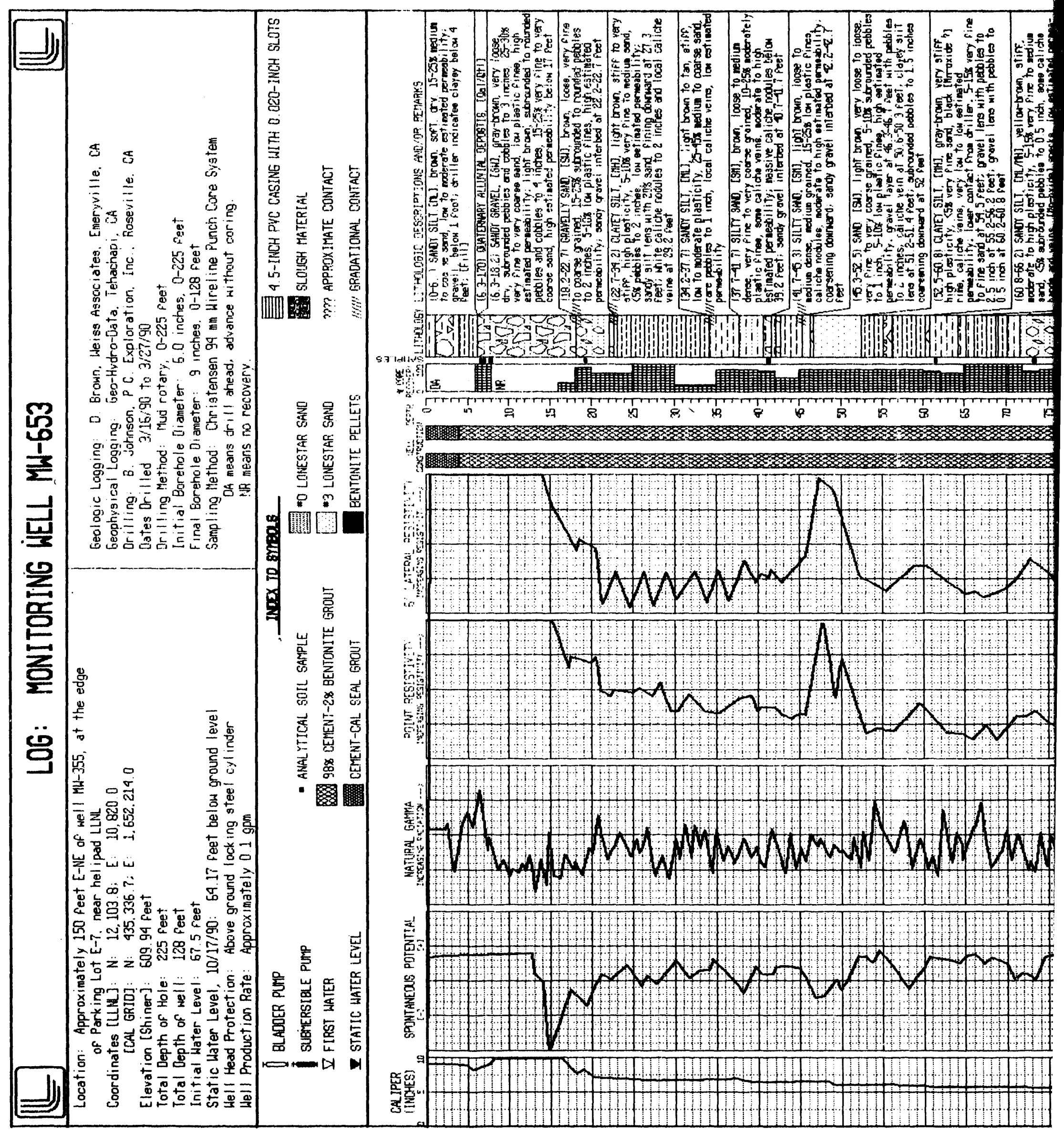




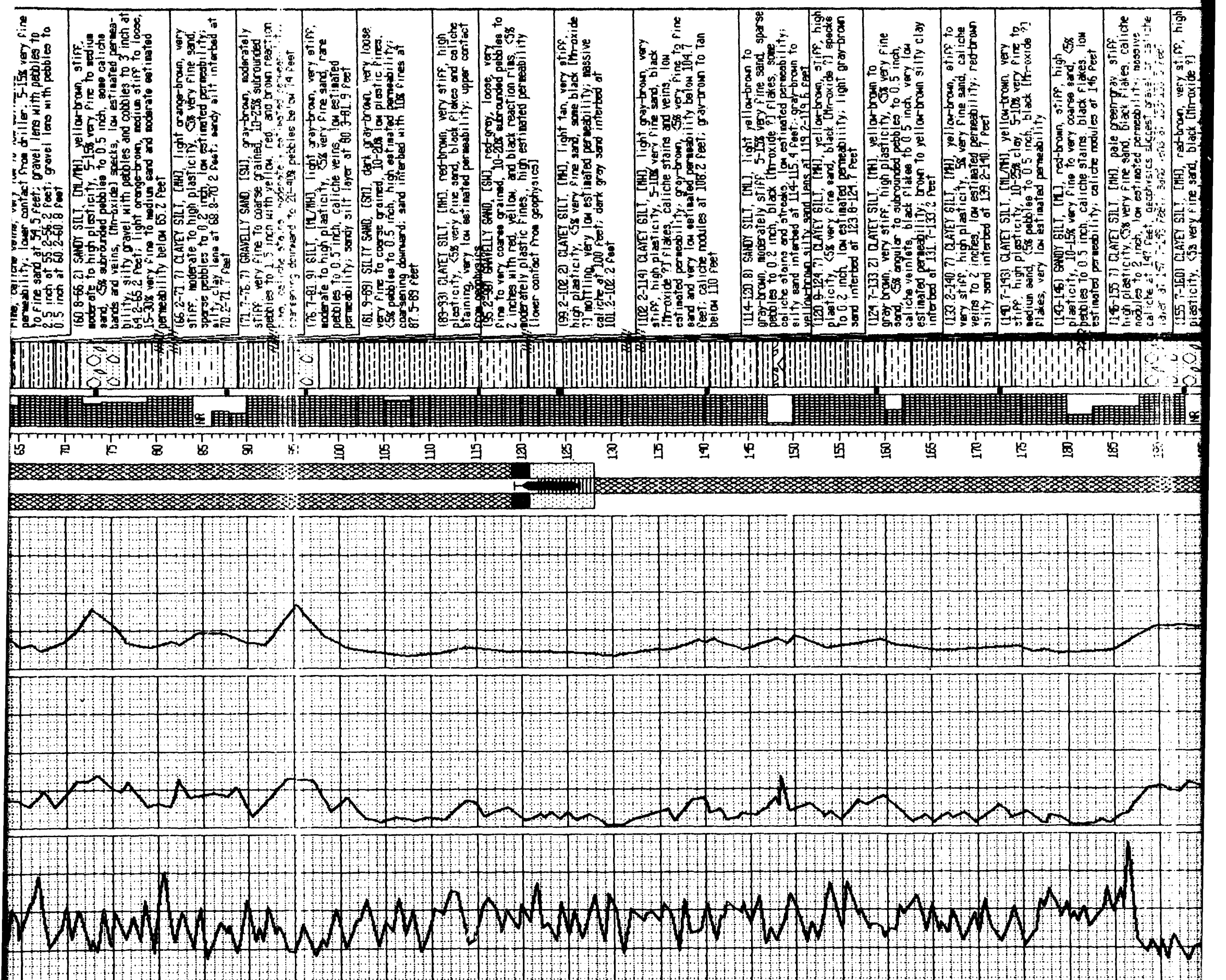

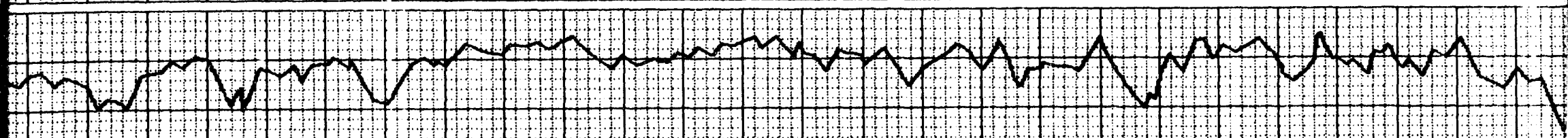




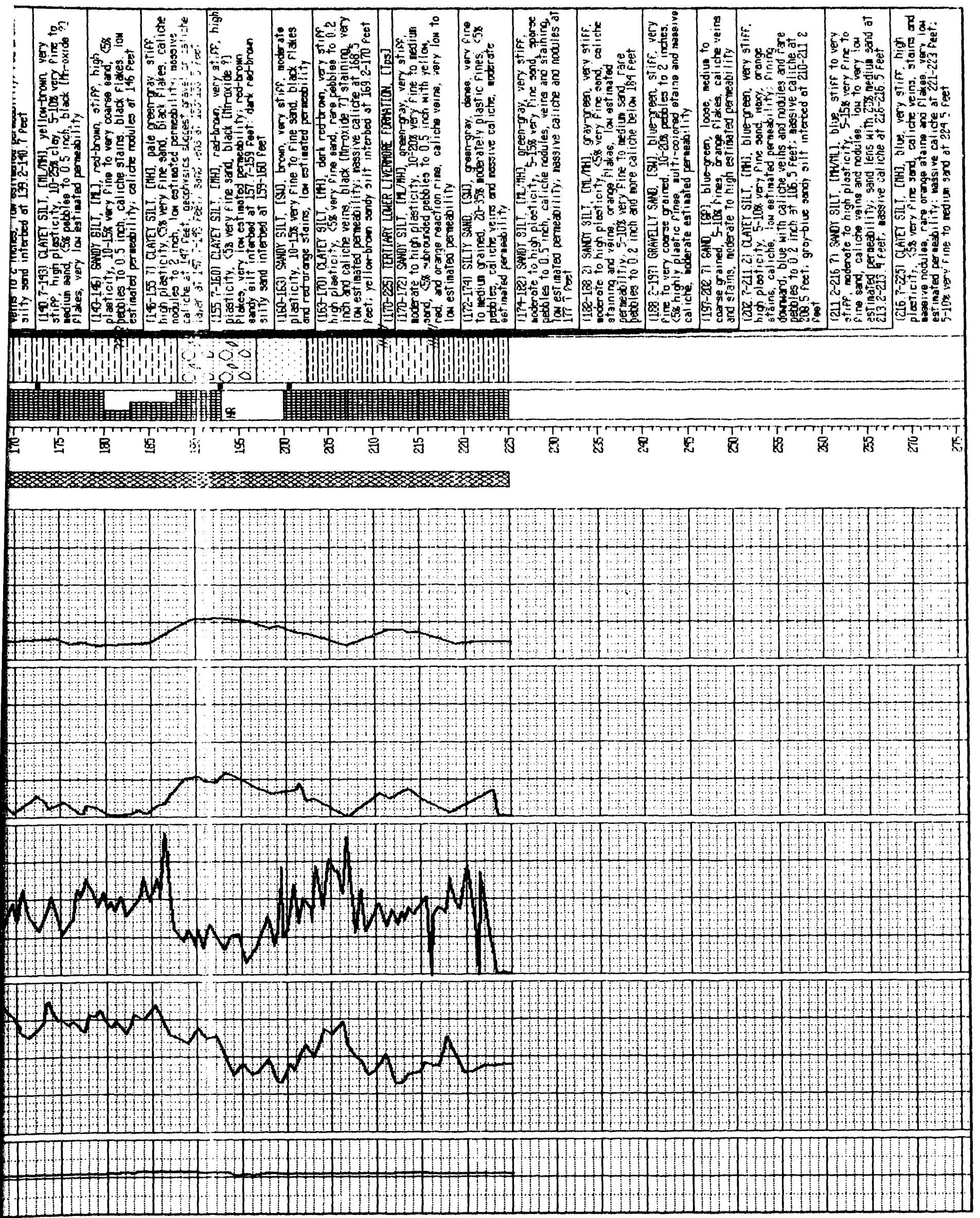




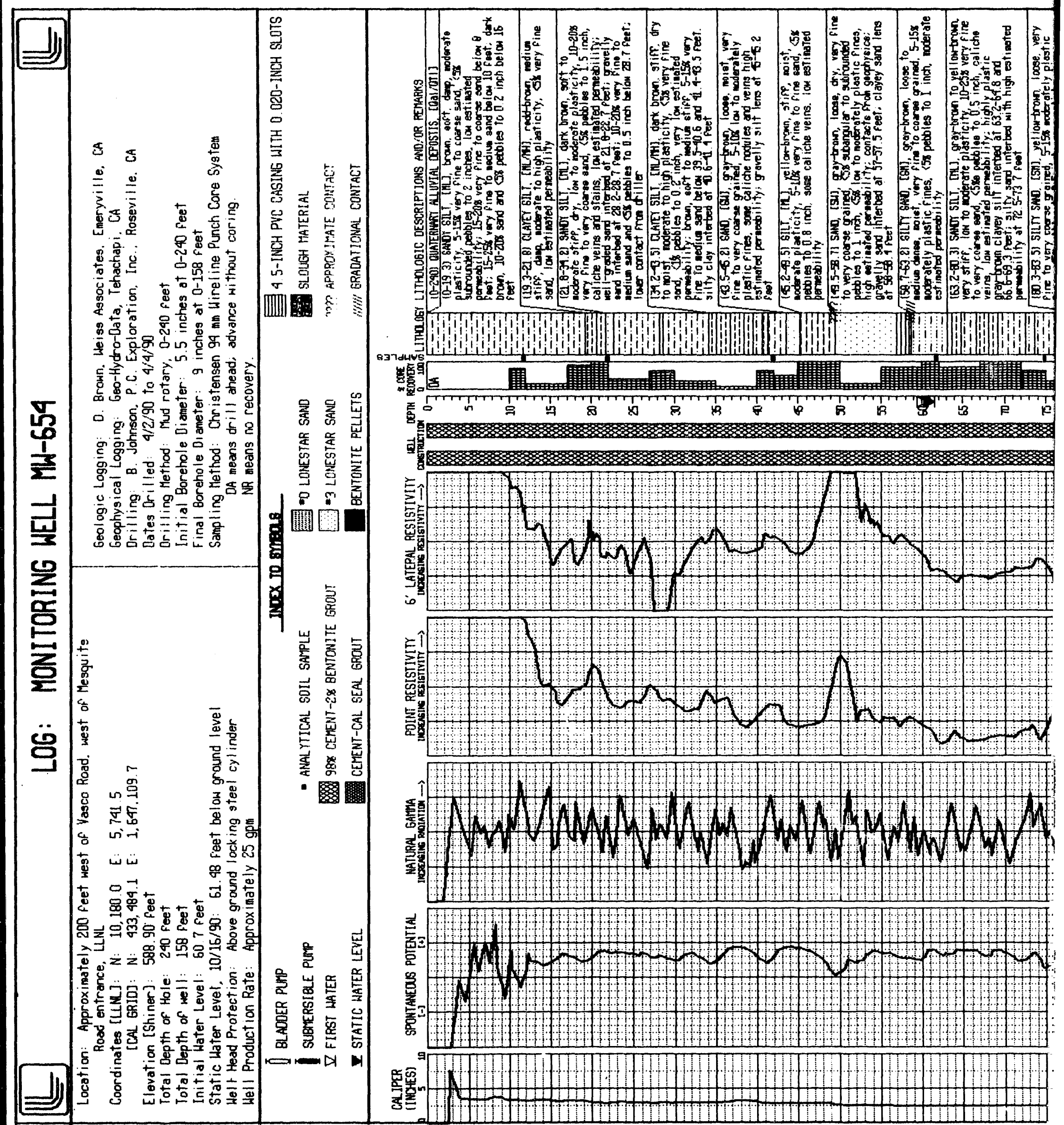




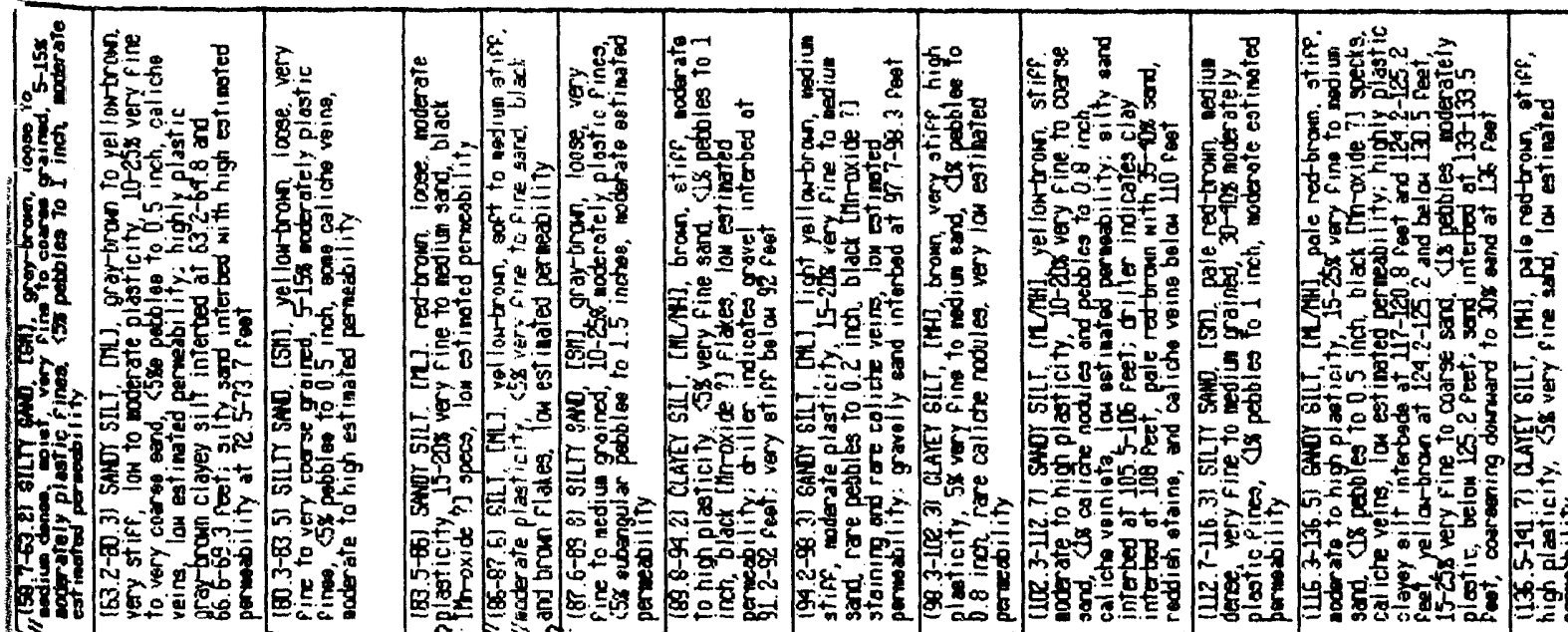

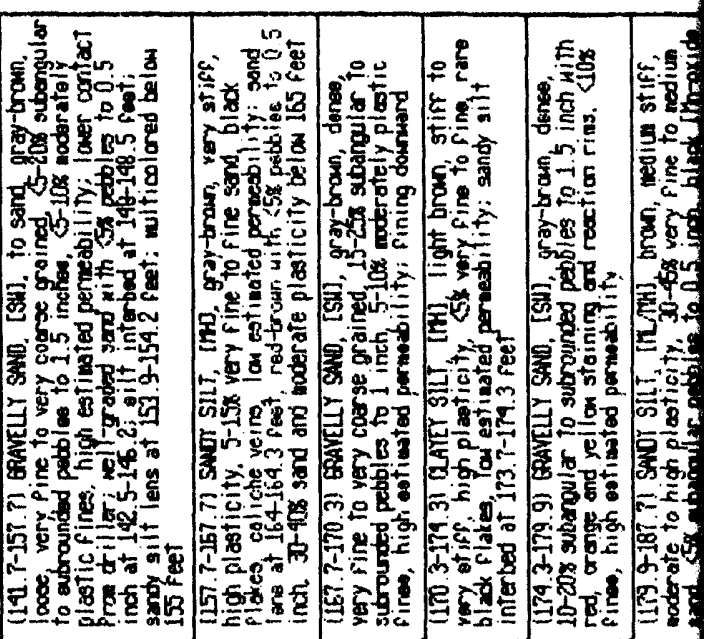

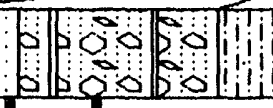

ذ1

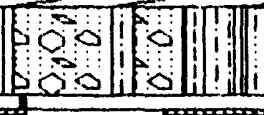

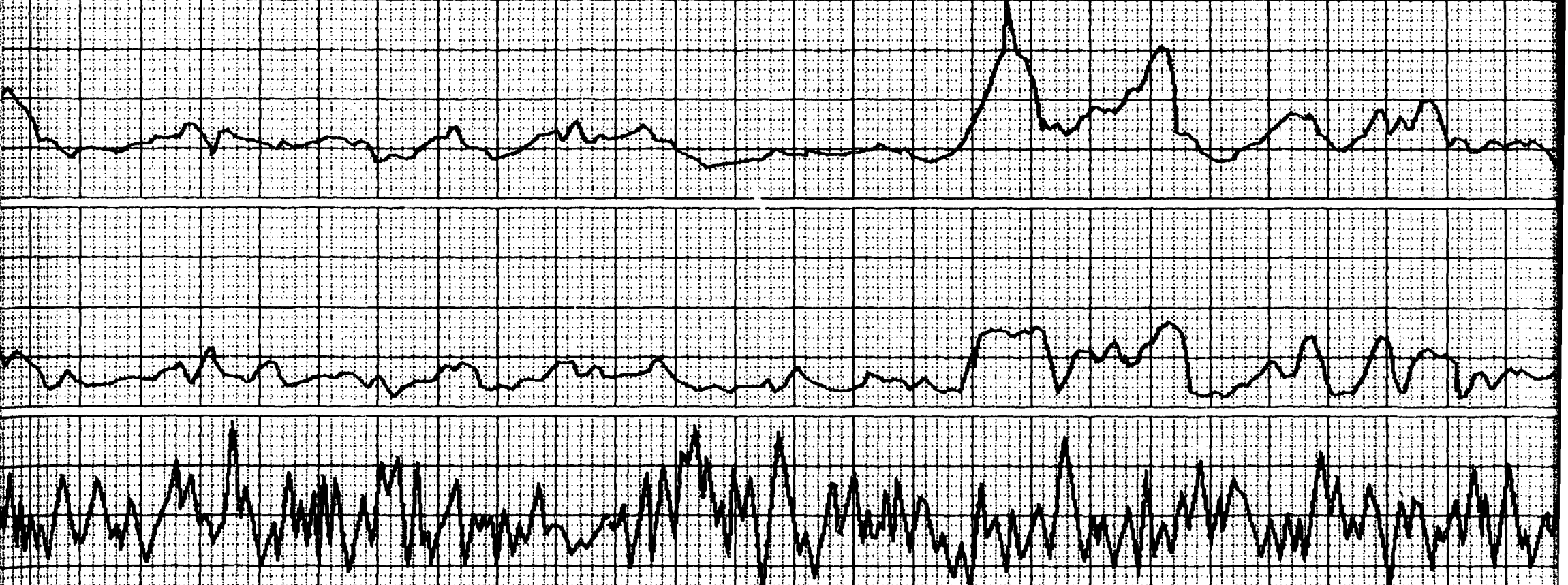




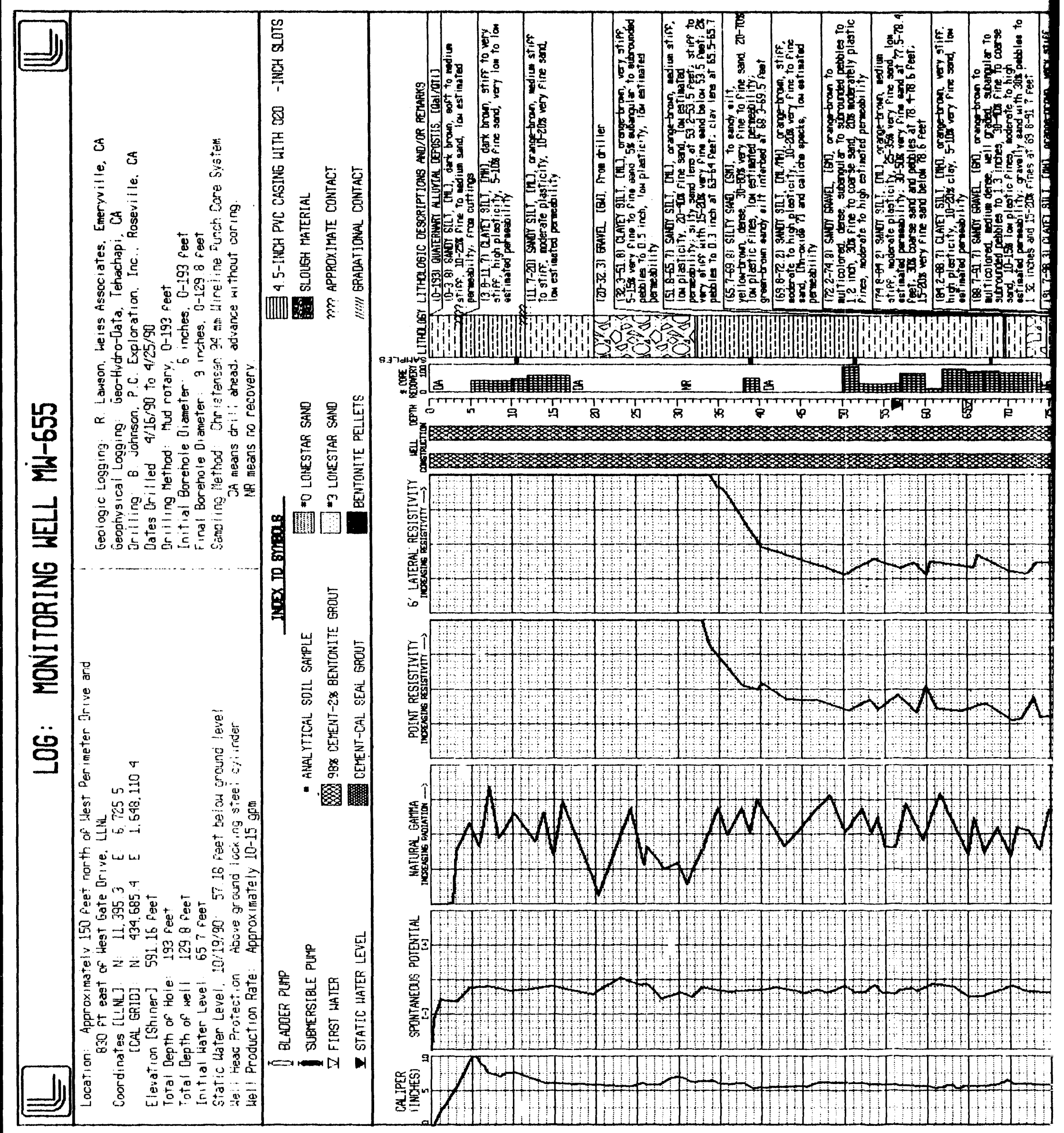




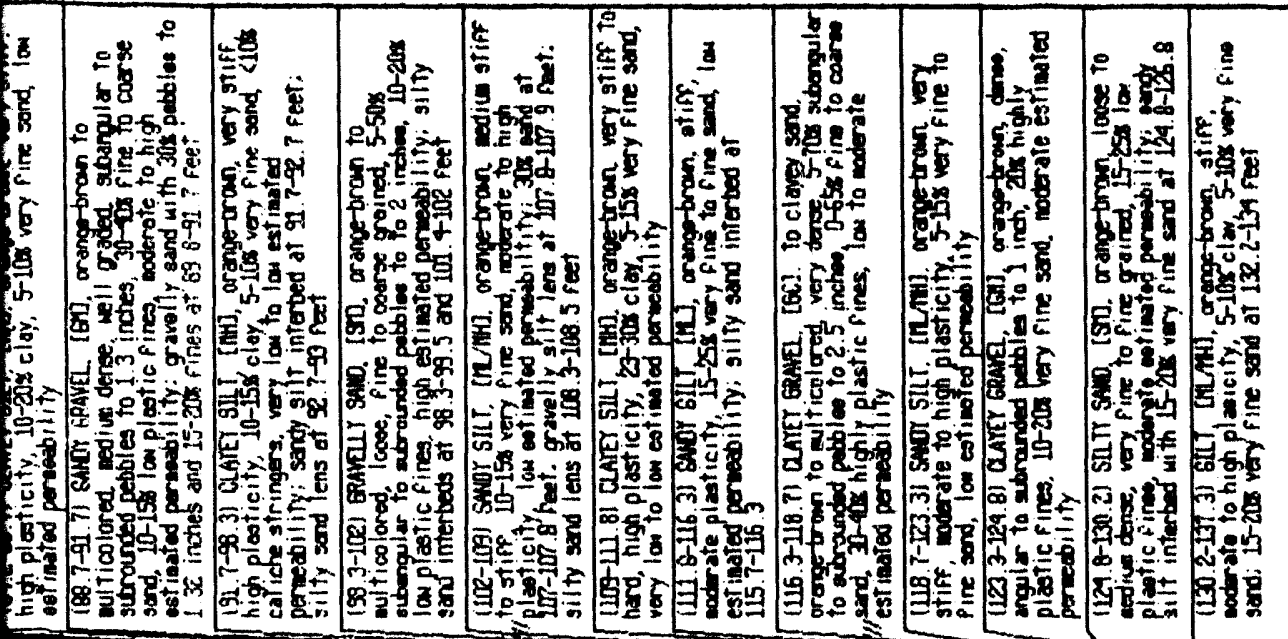

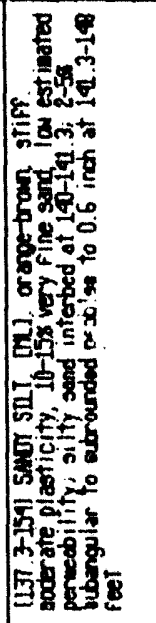

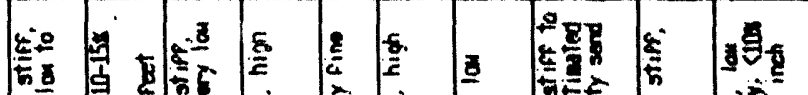

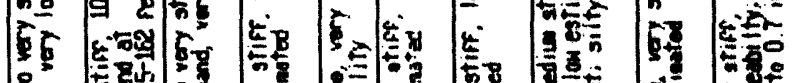

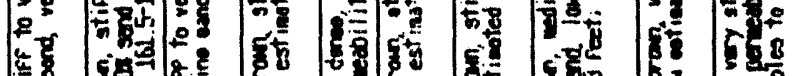

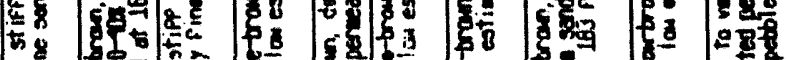

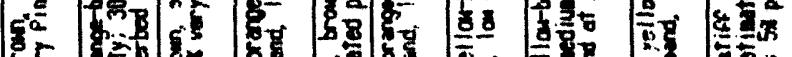

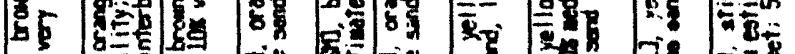

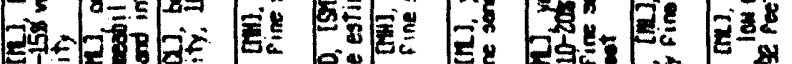

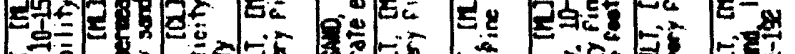
En tre

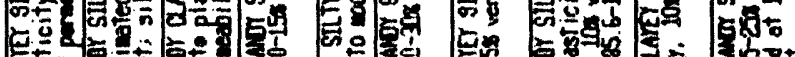

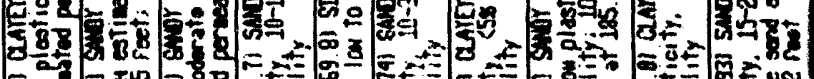
50

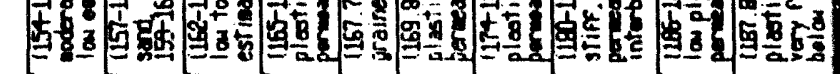

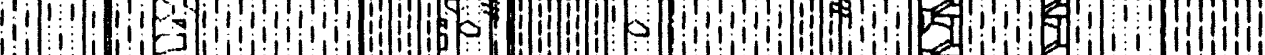

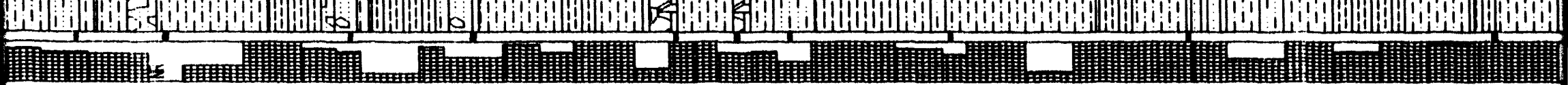

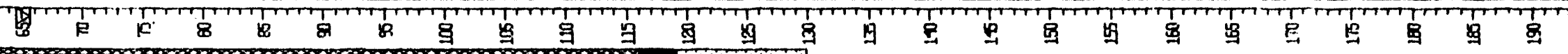

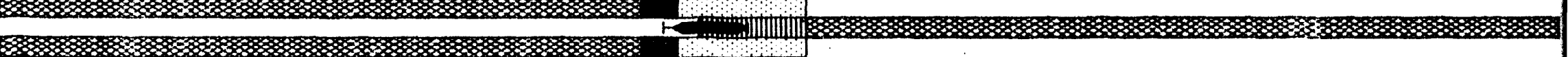

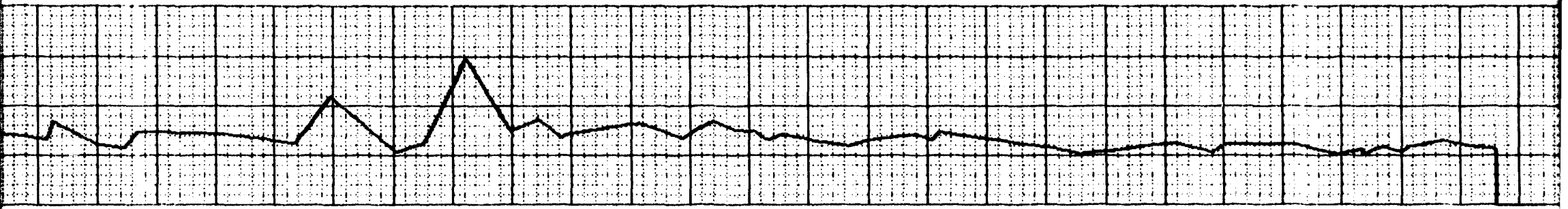

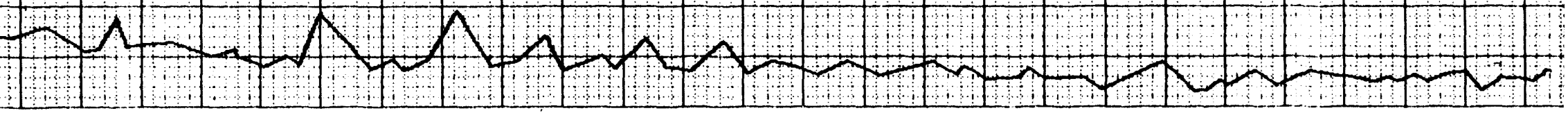

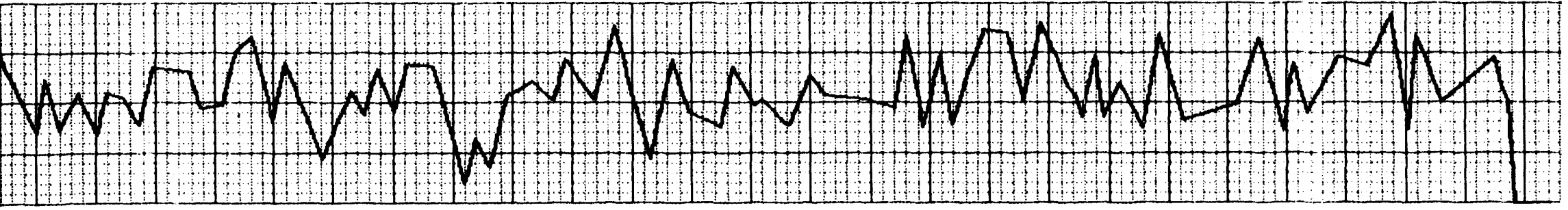

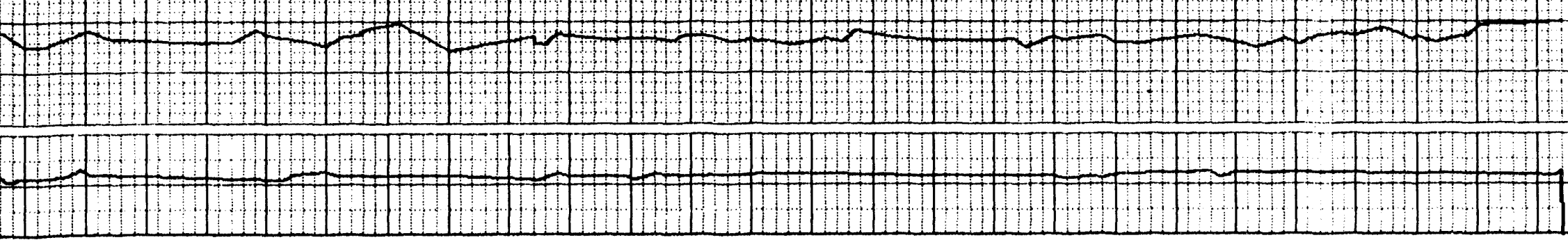




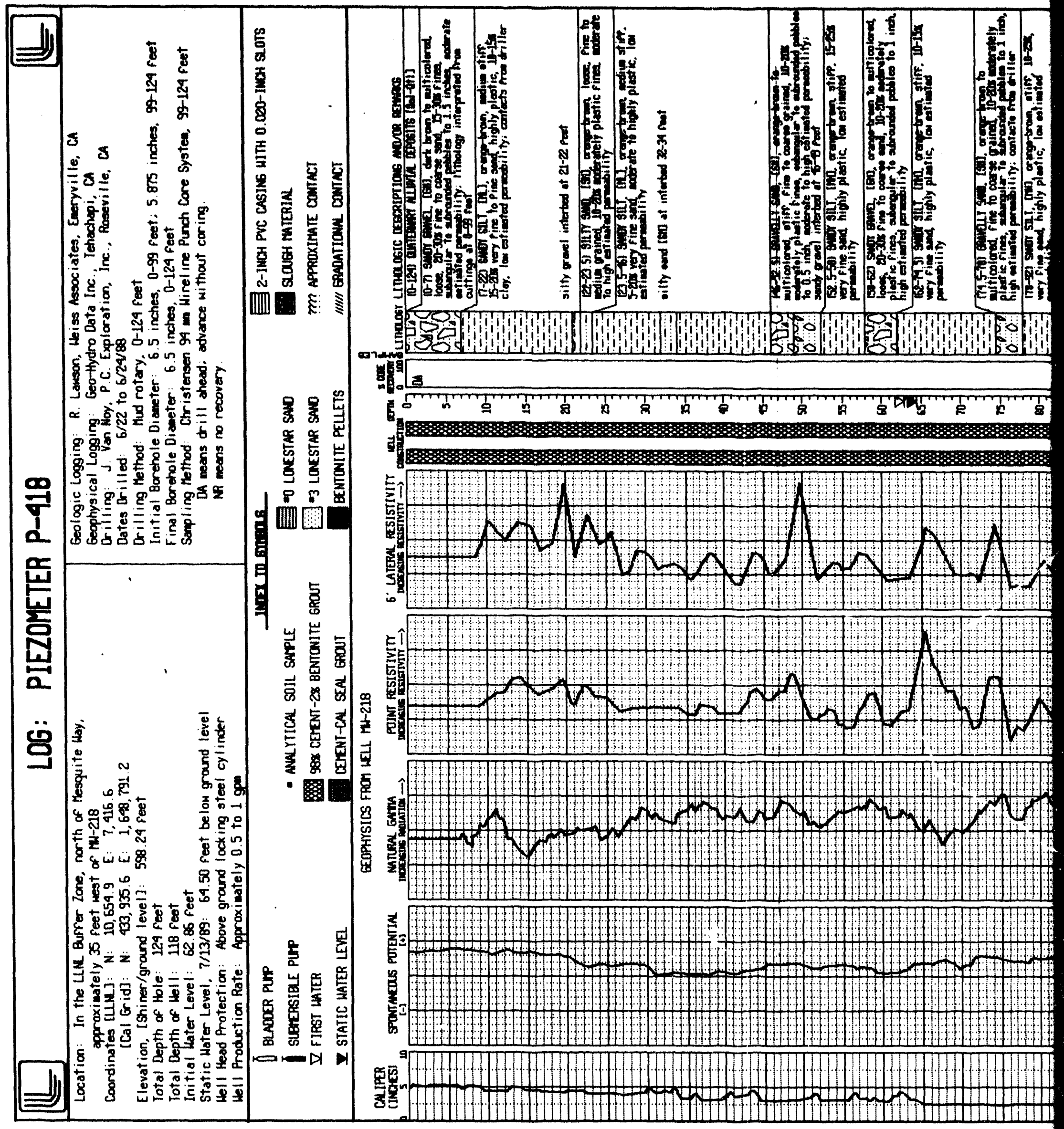




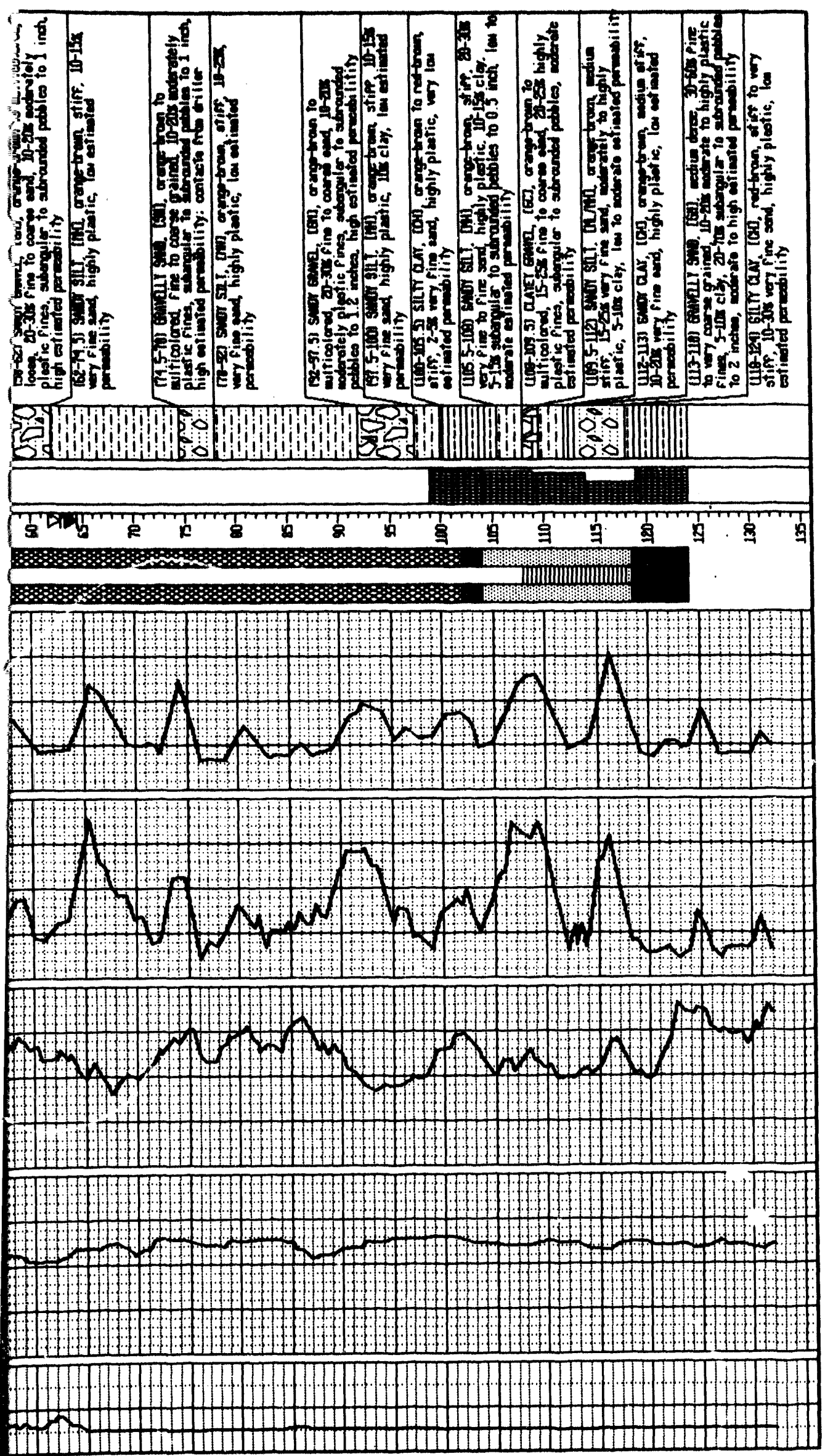




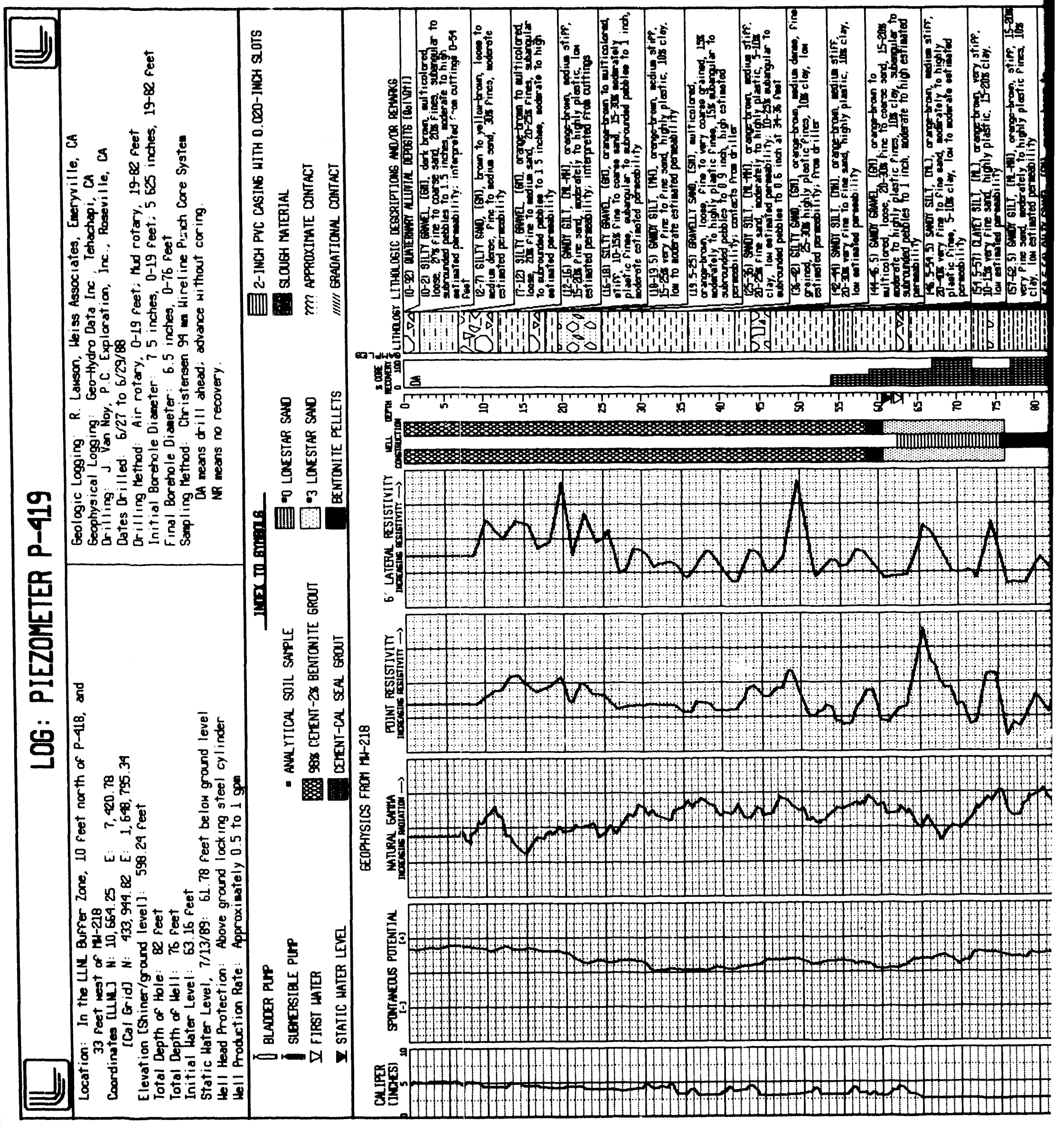




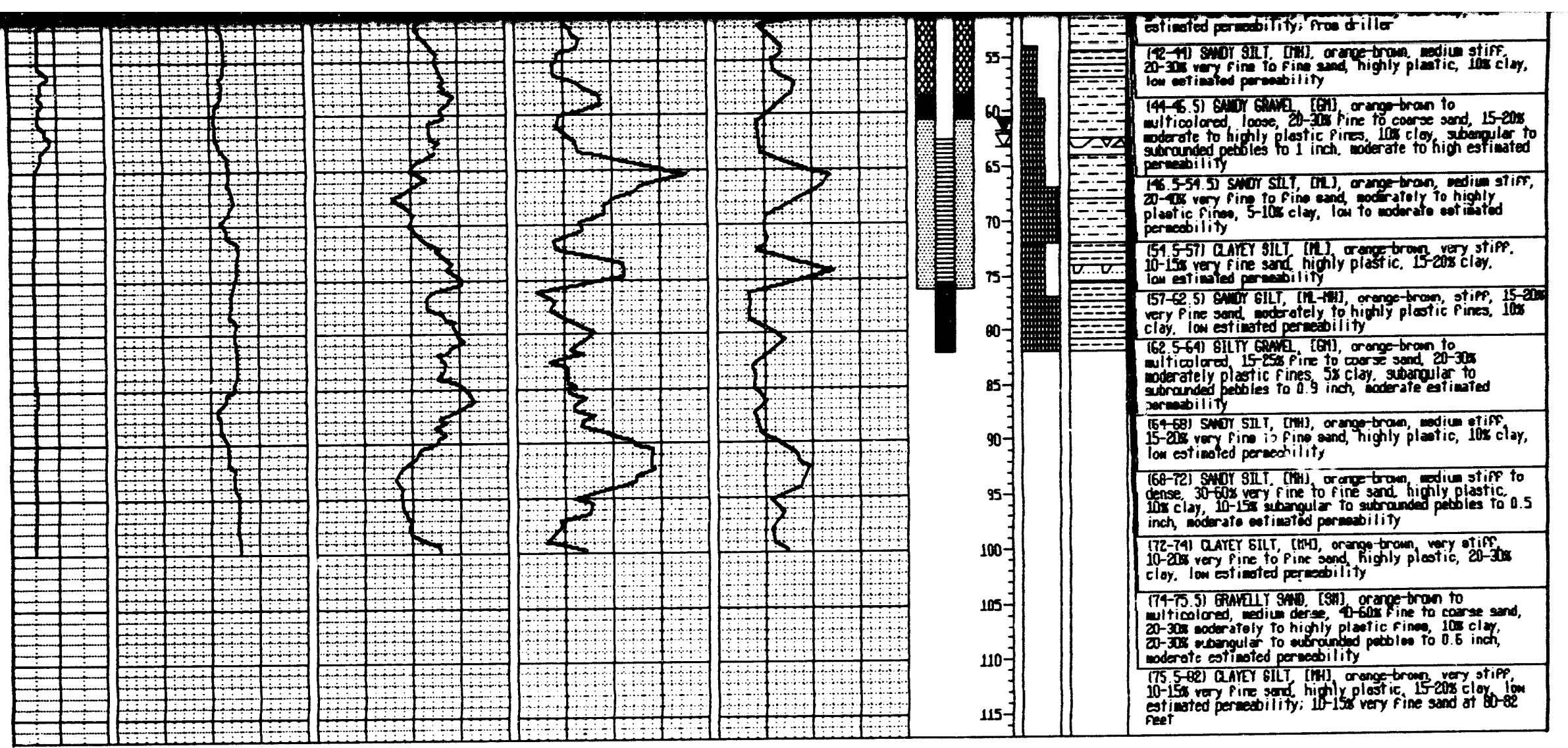




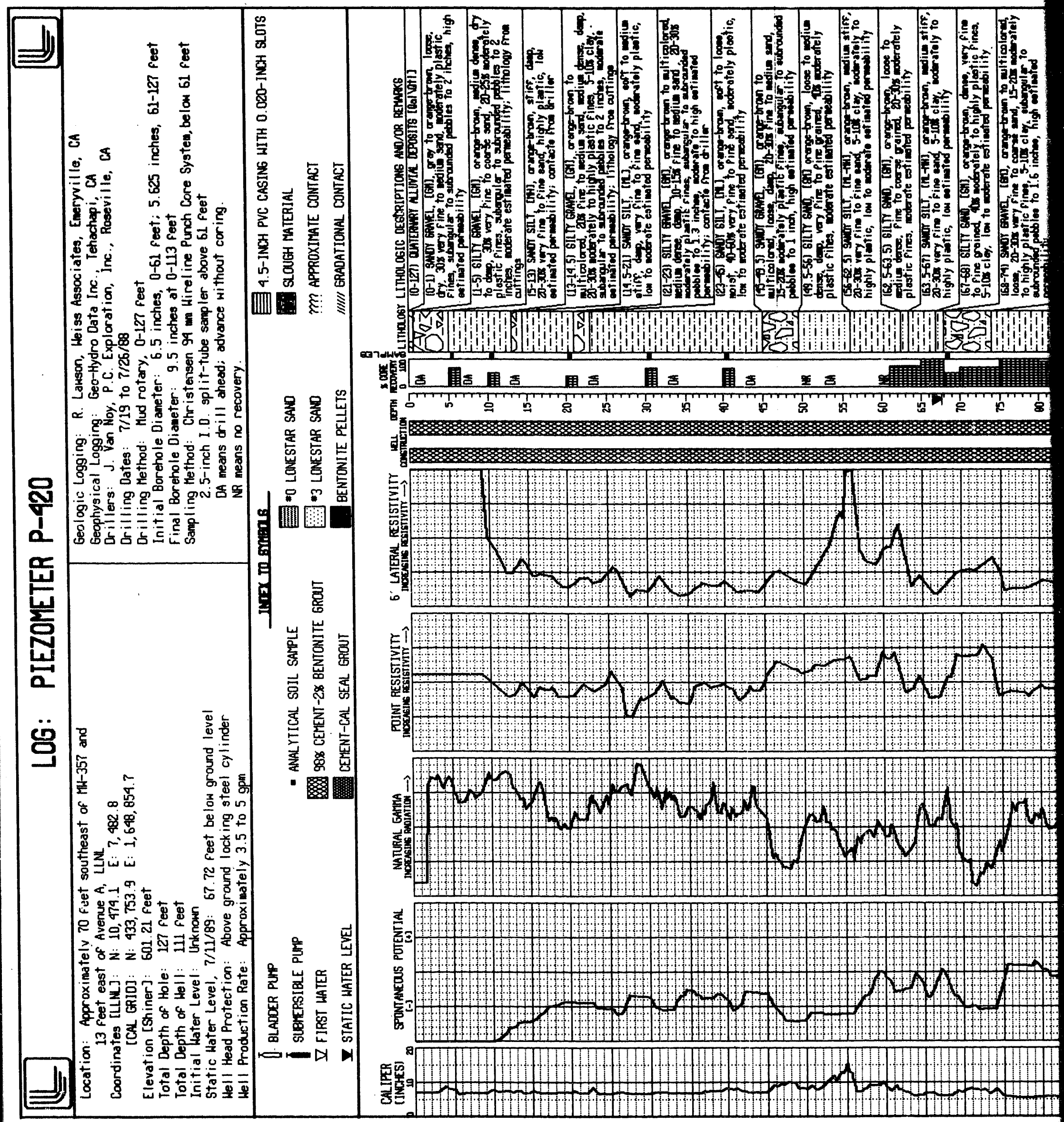




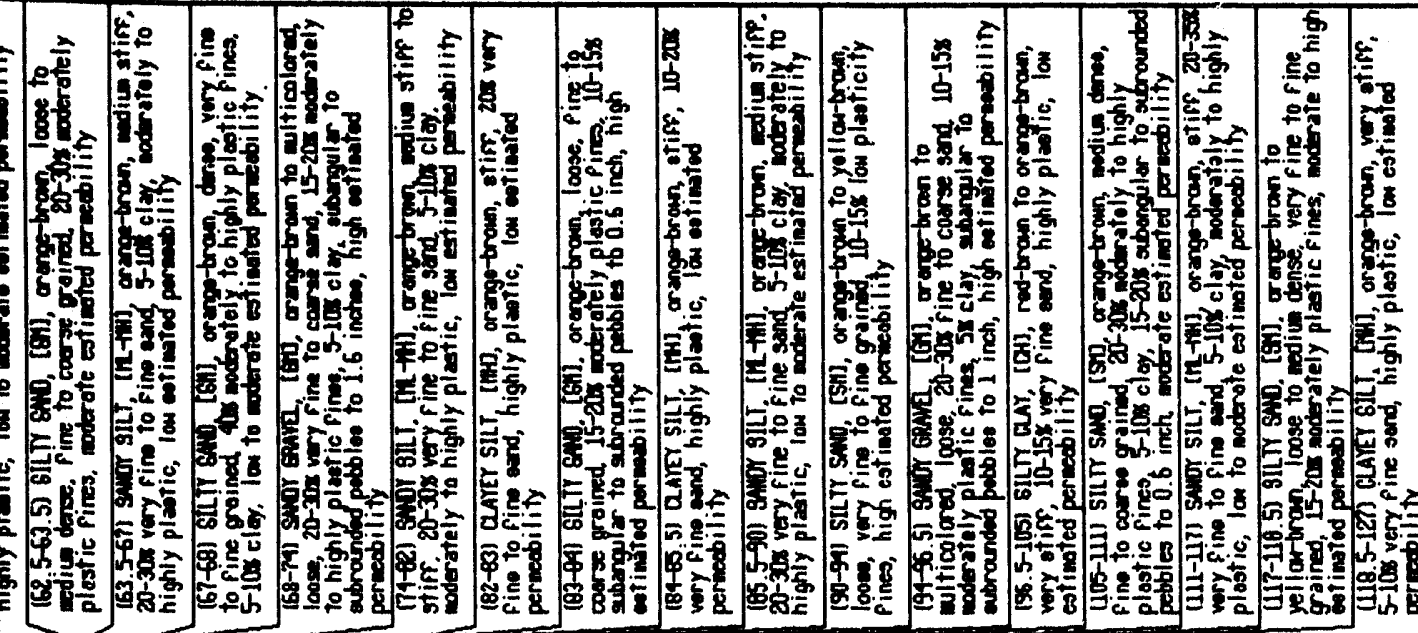

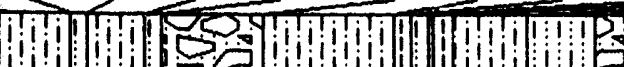

!

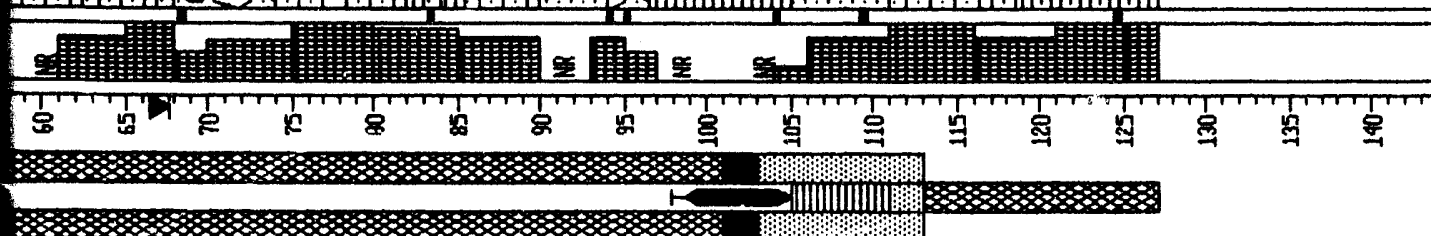

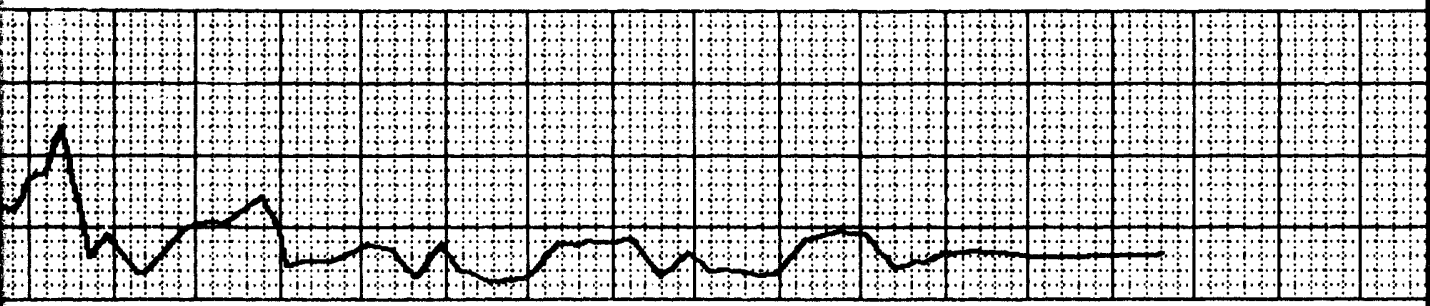

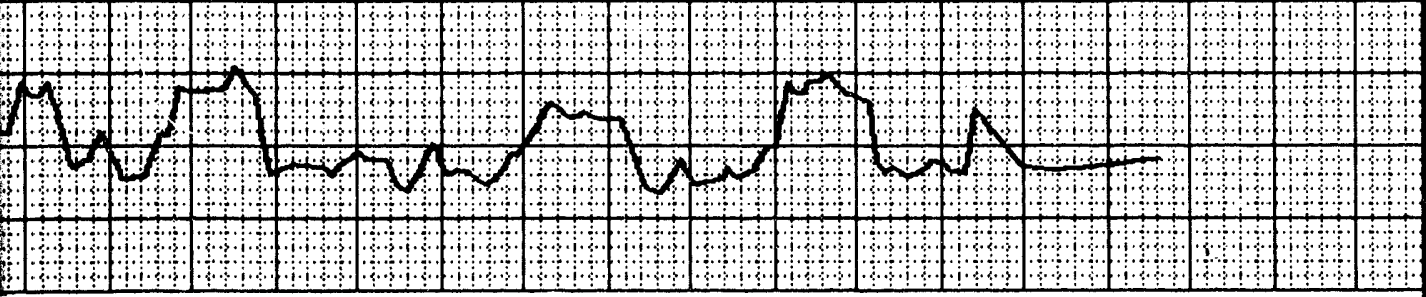

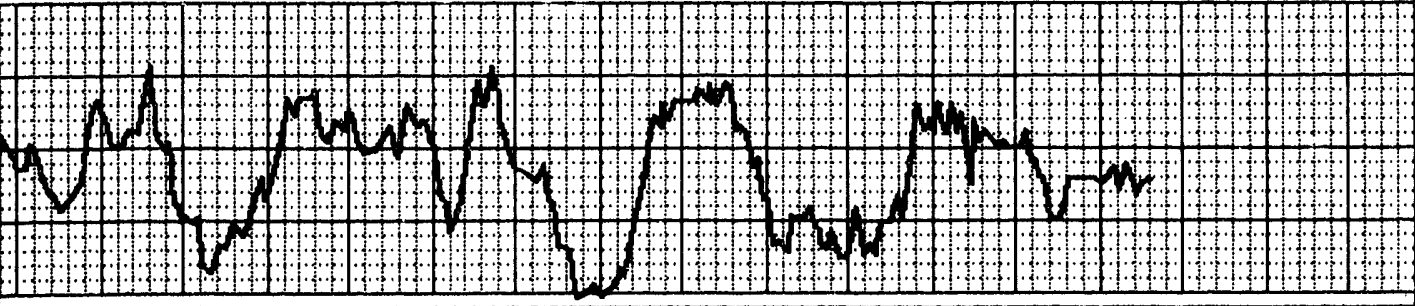

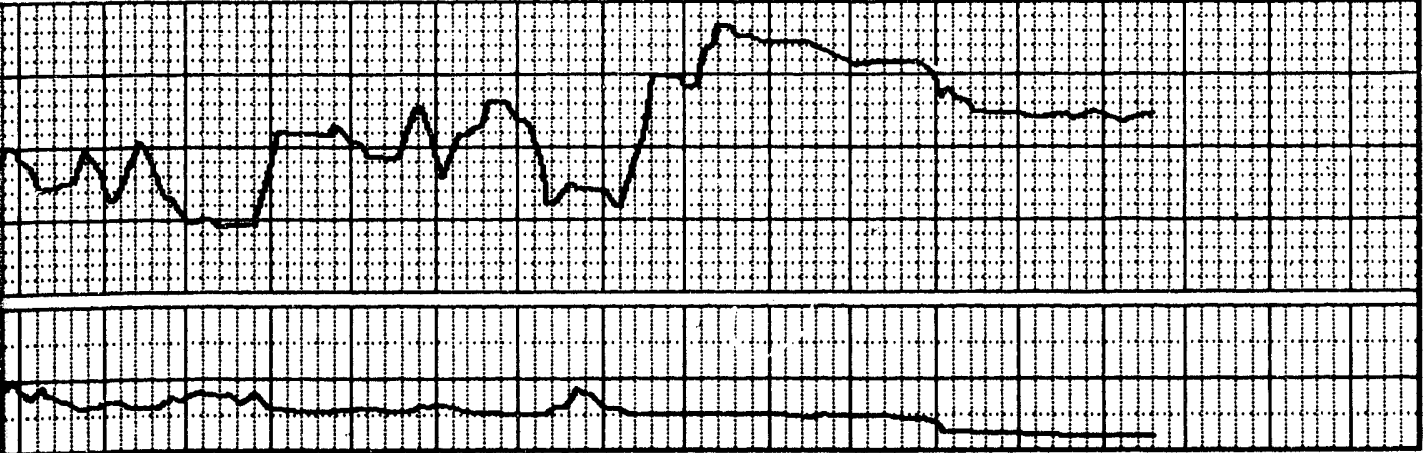




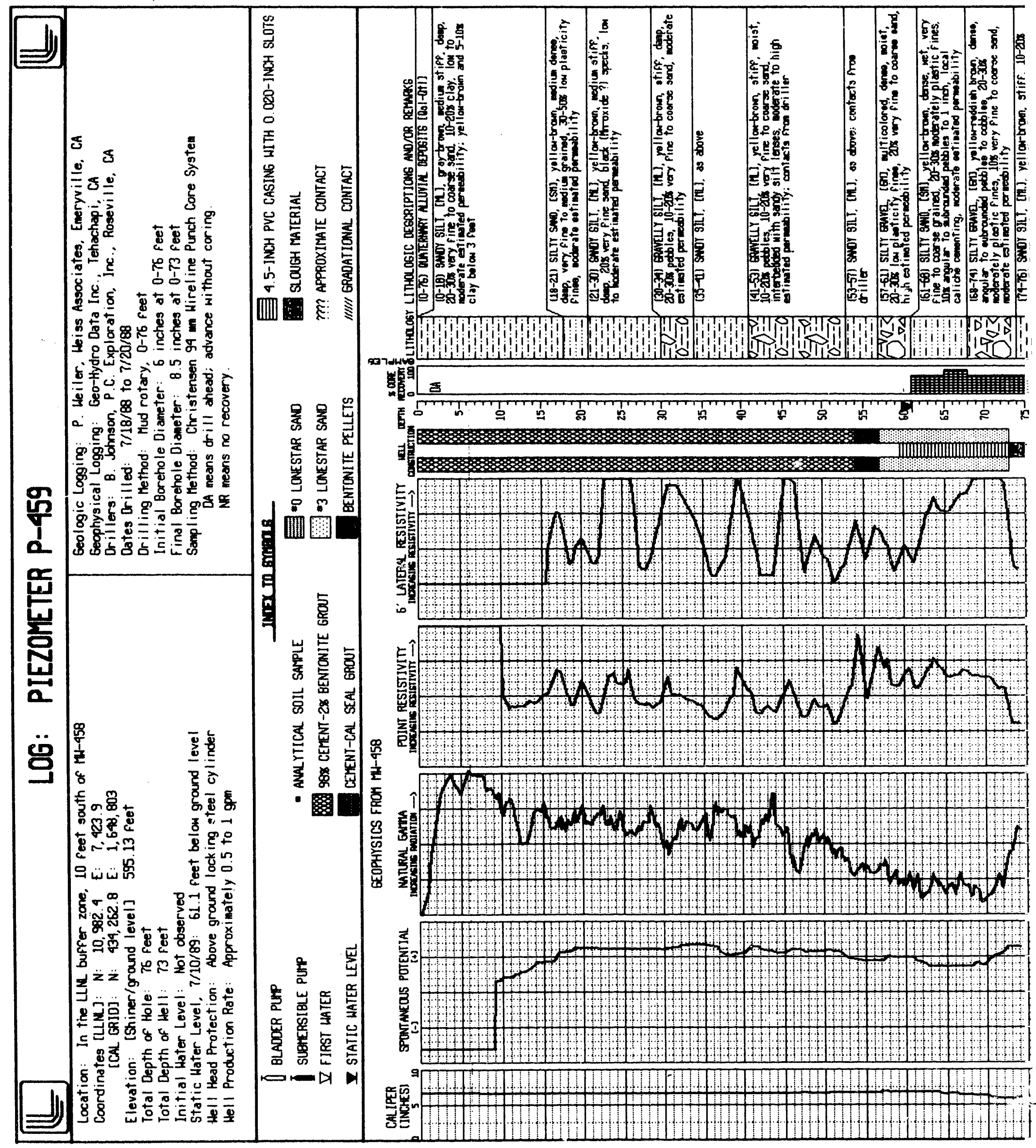




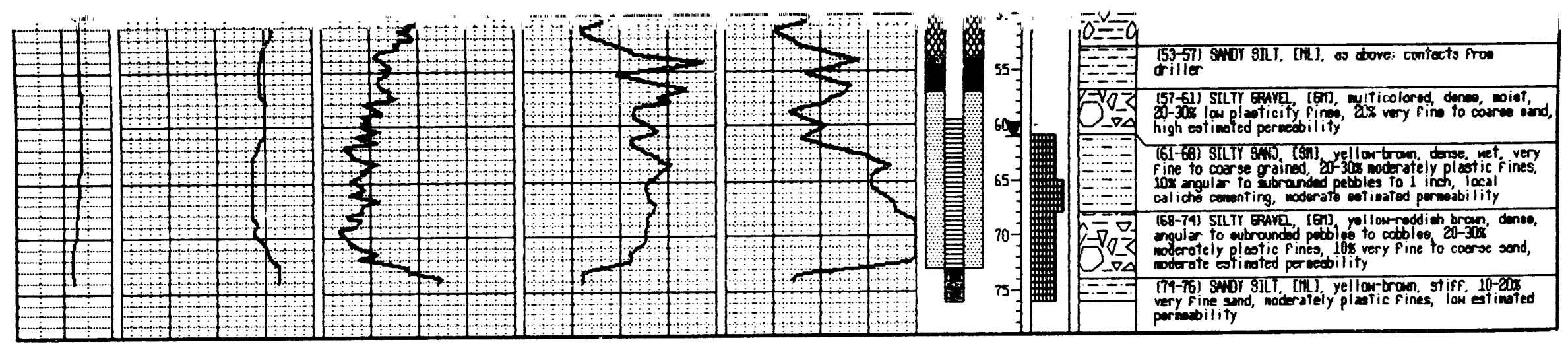




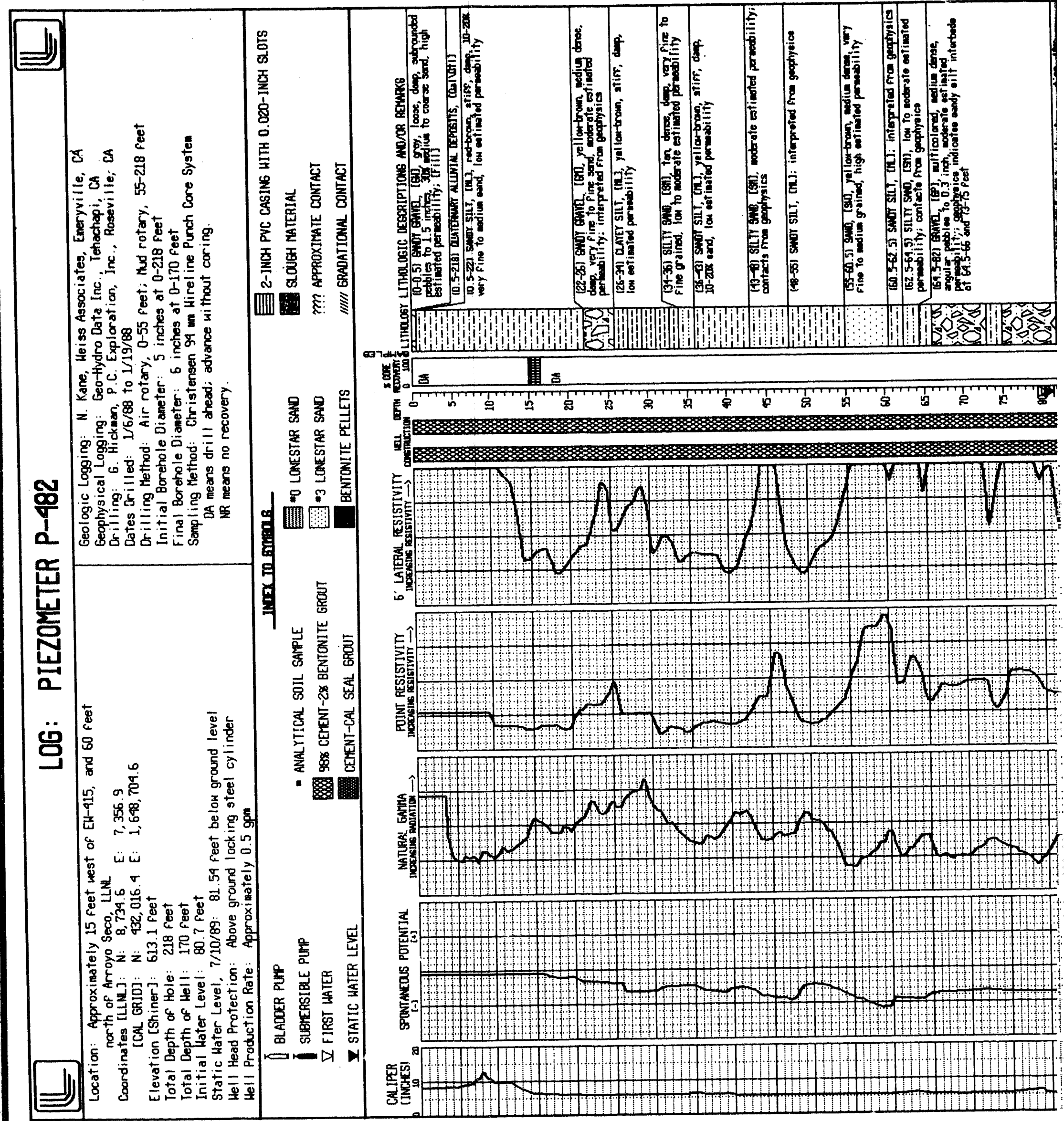




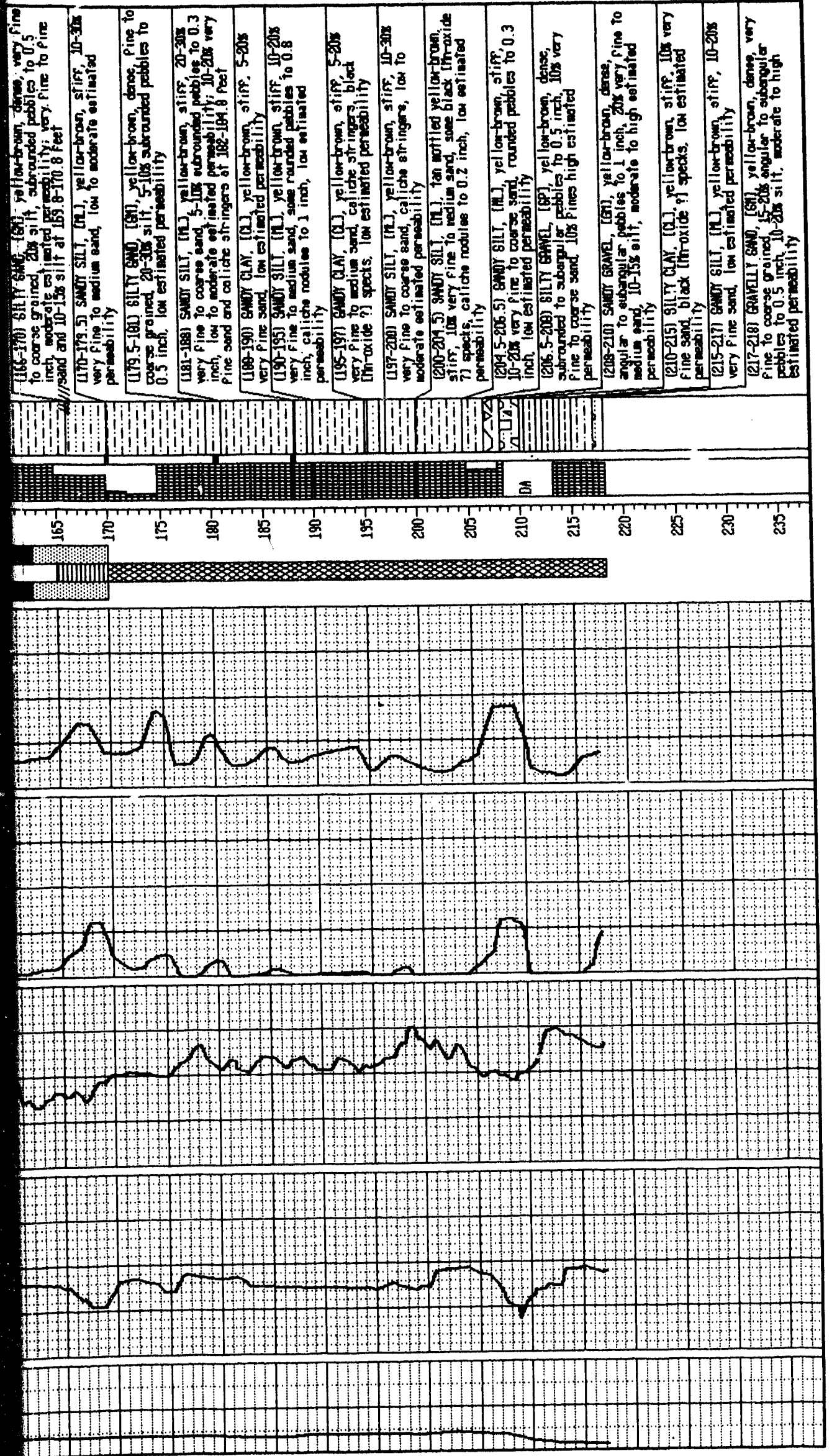




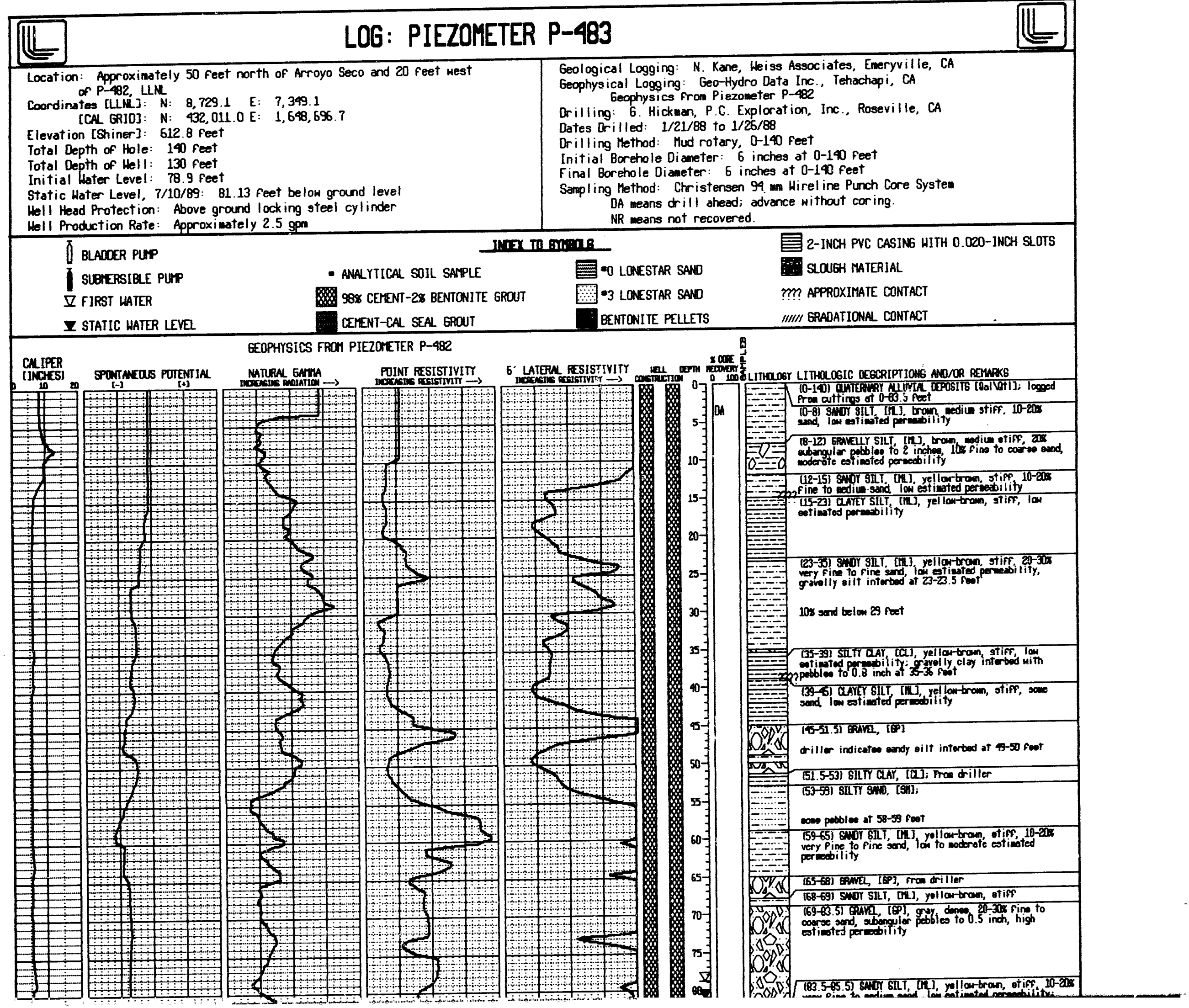




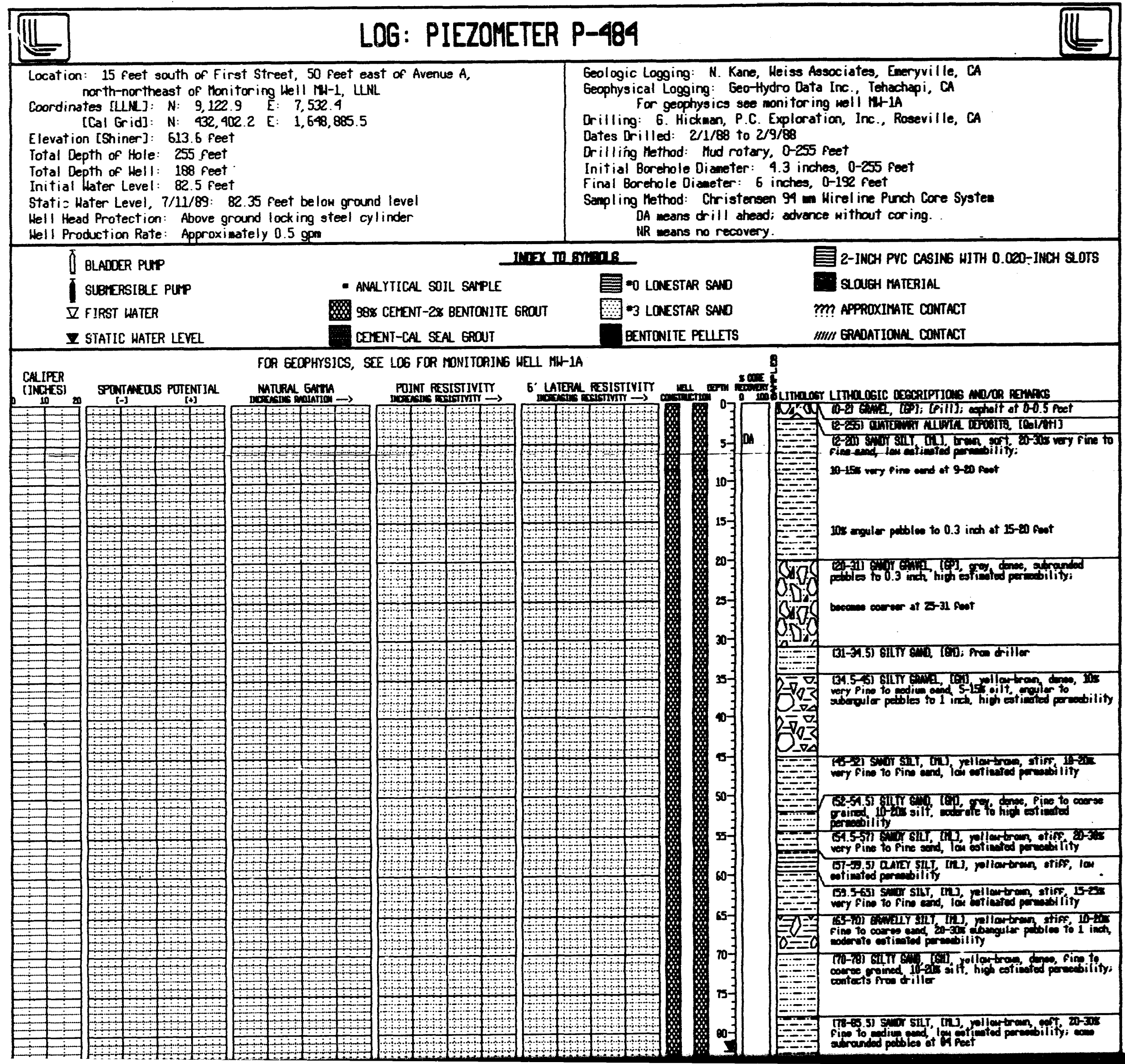



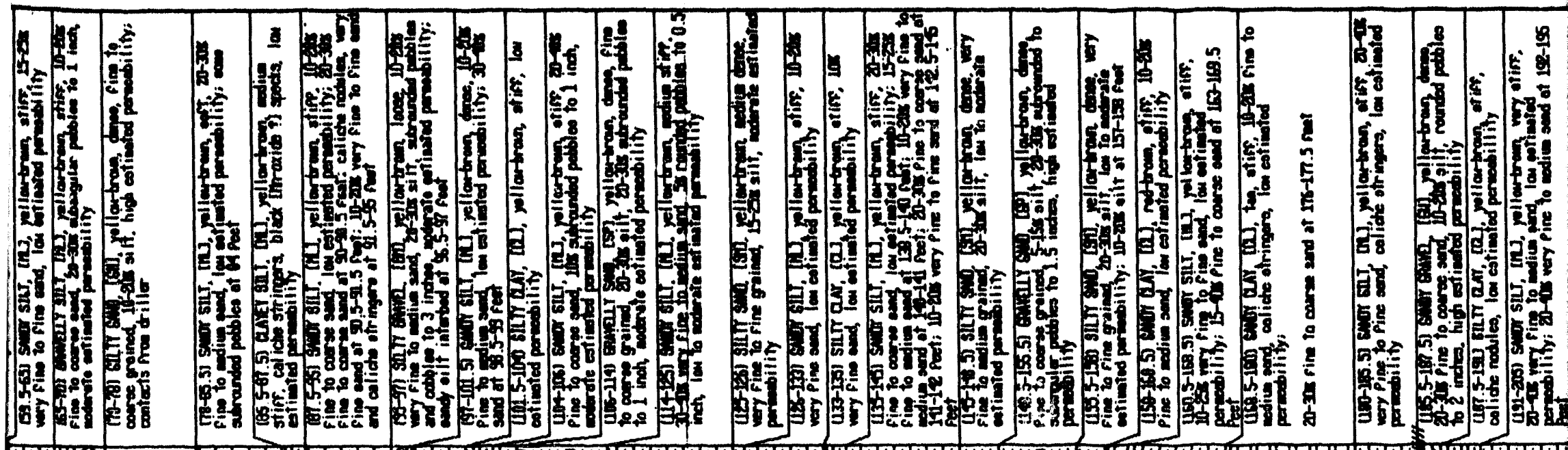

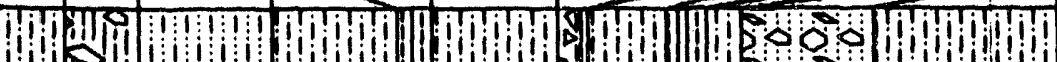

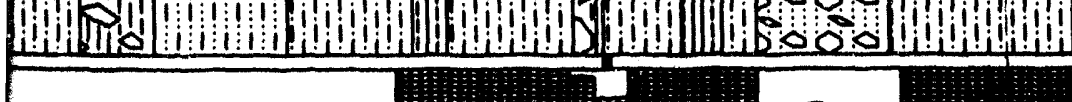
(1)

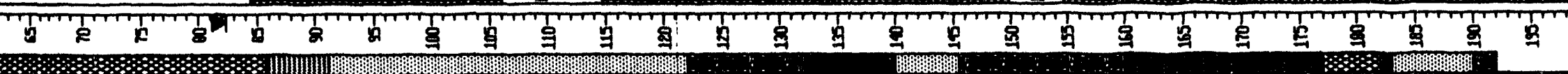

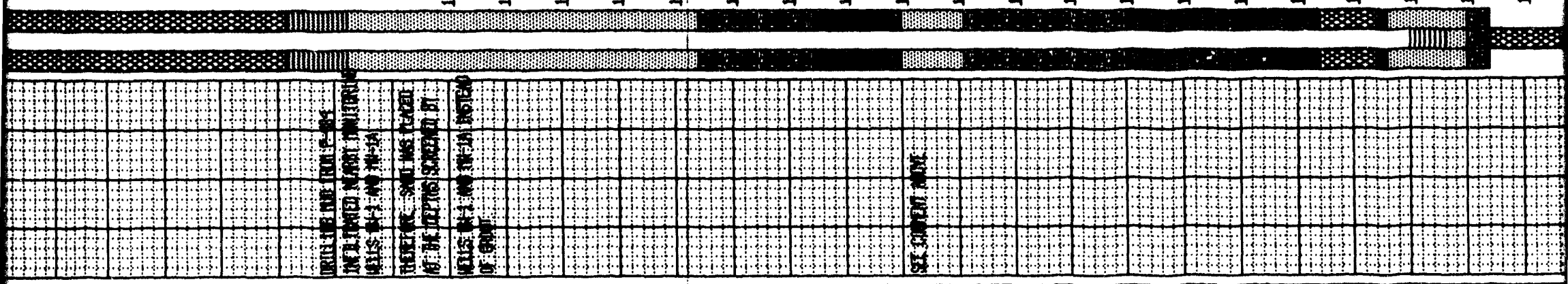

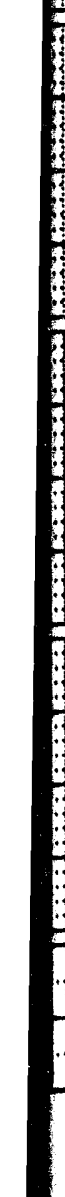



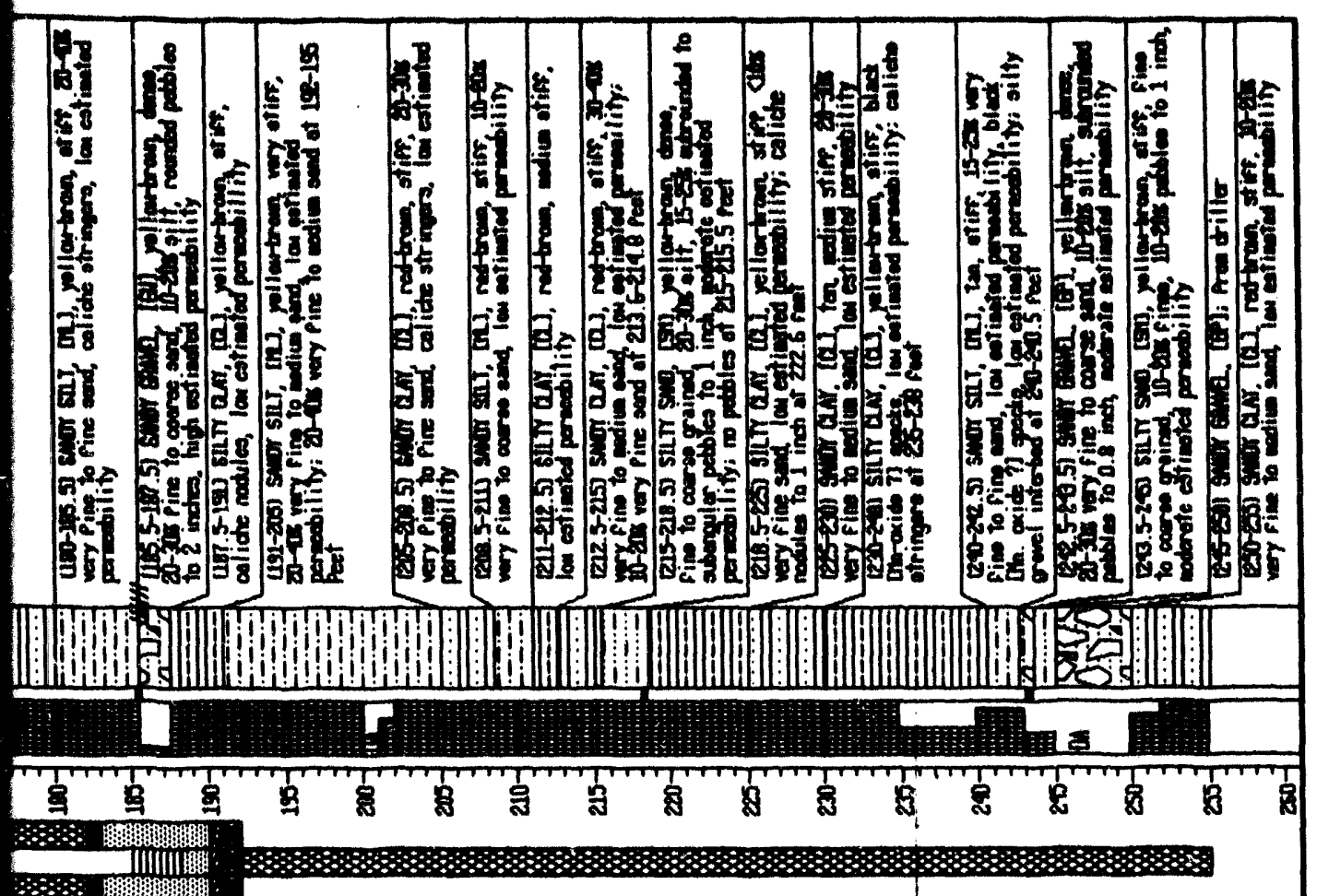

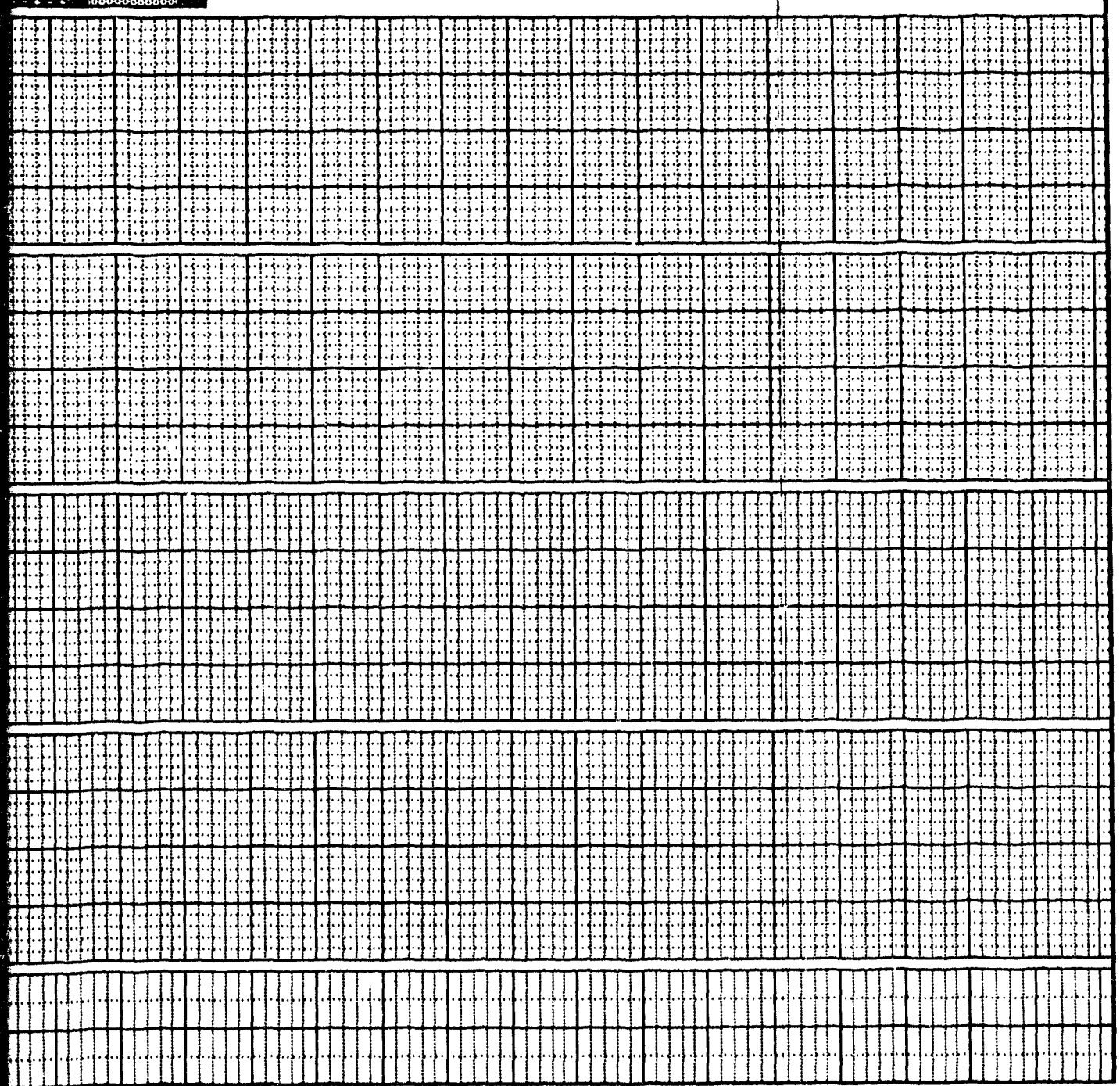




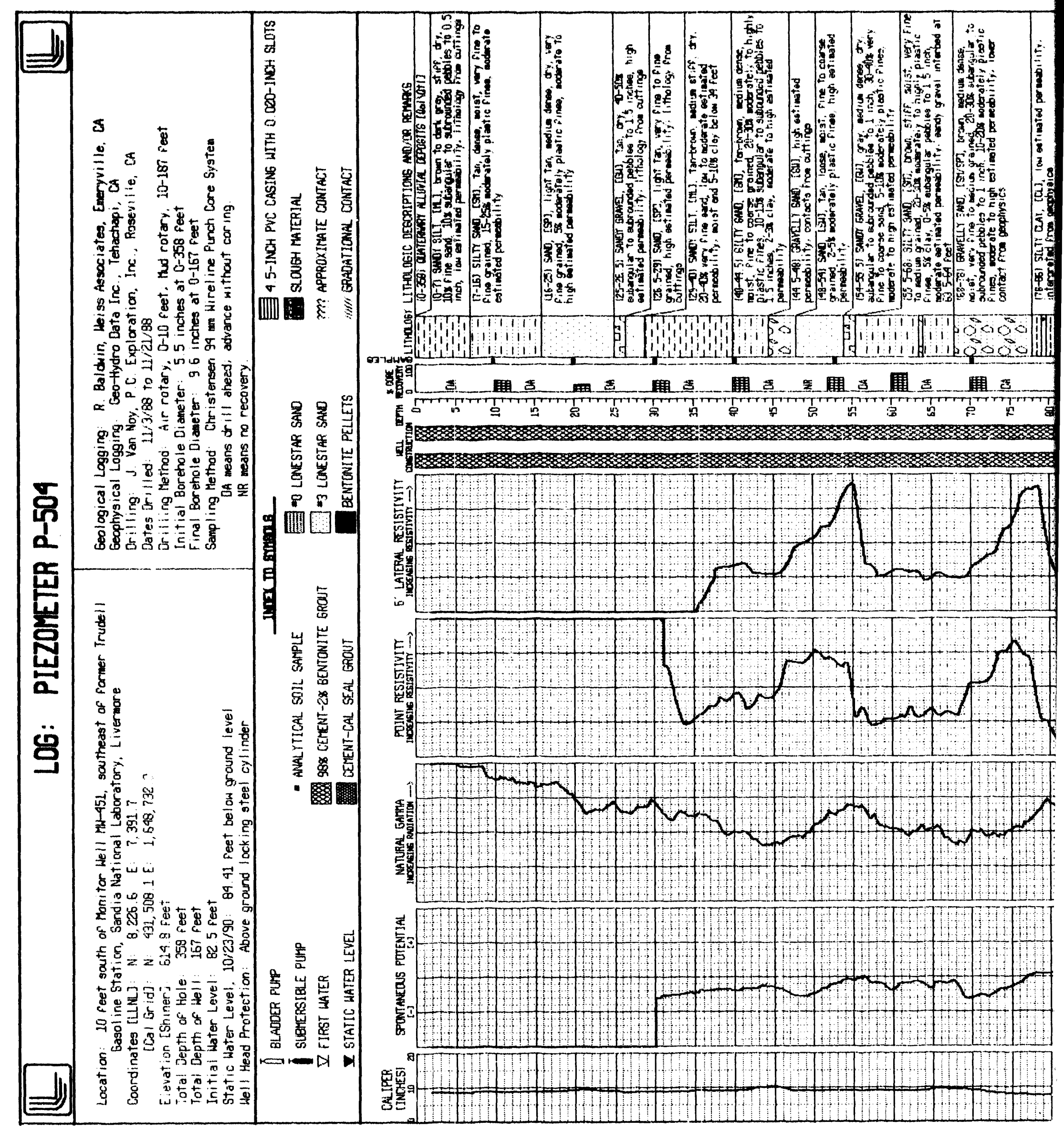



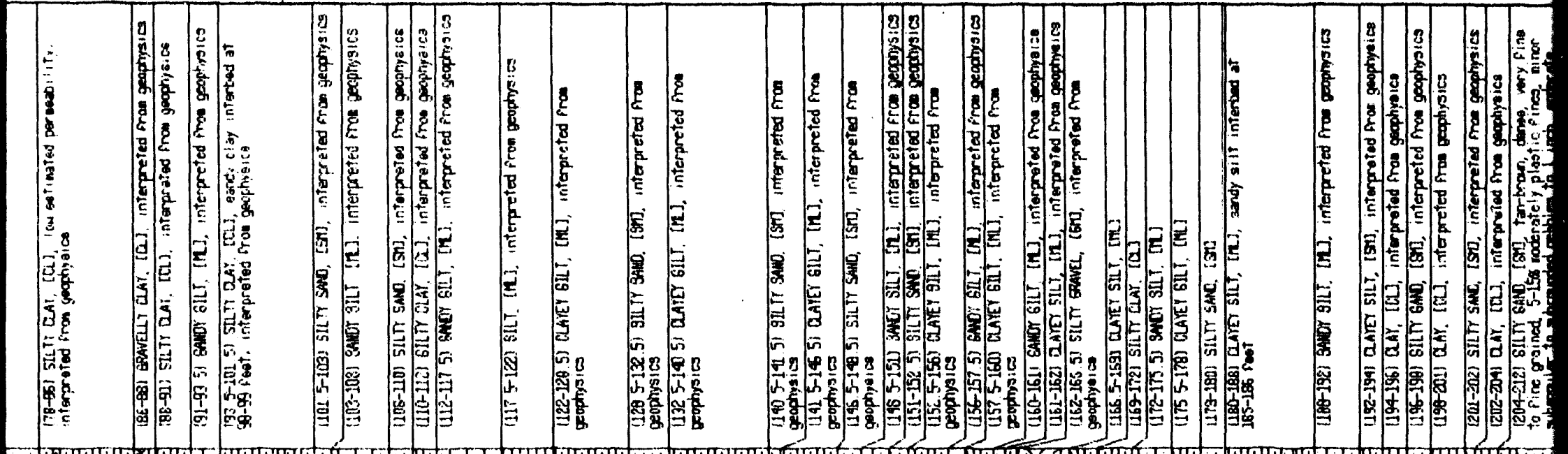

$\therefore$ ini i i i

(1)

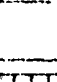

尘

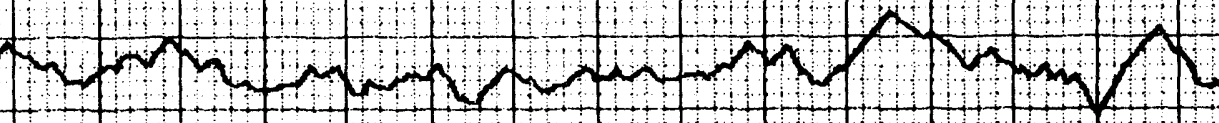

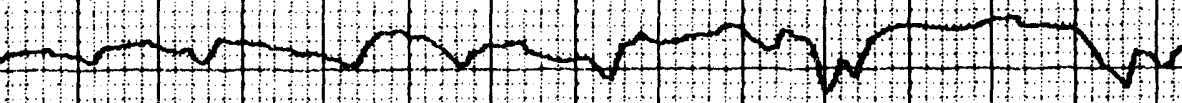




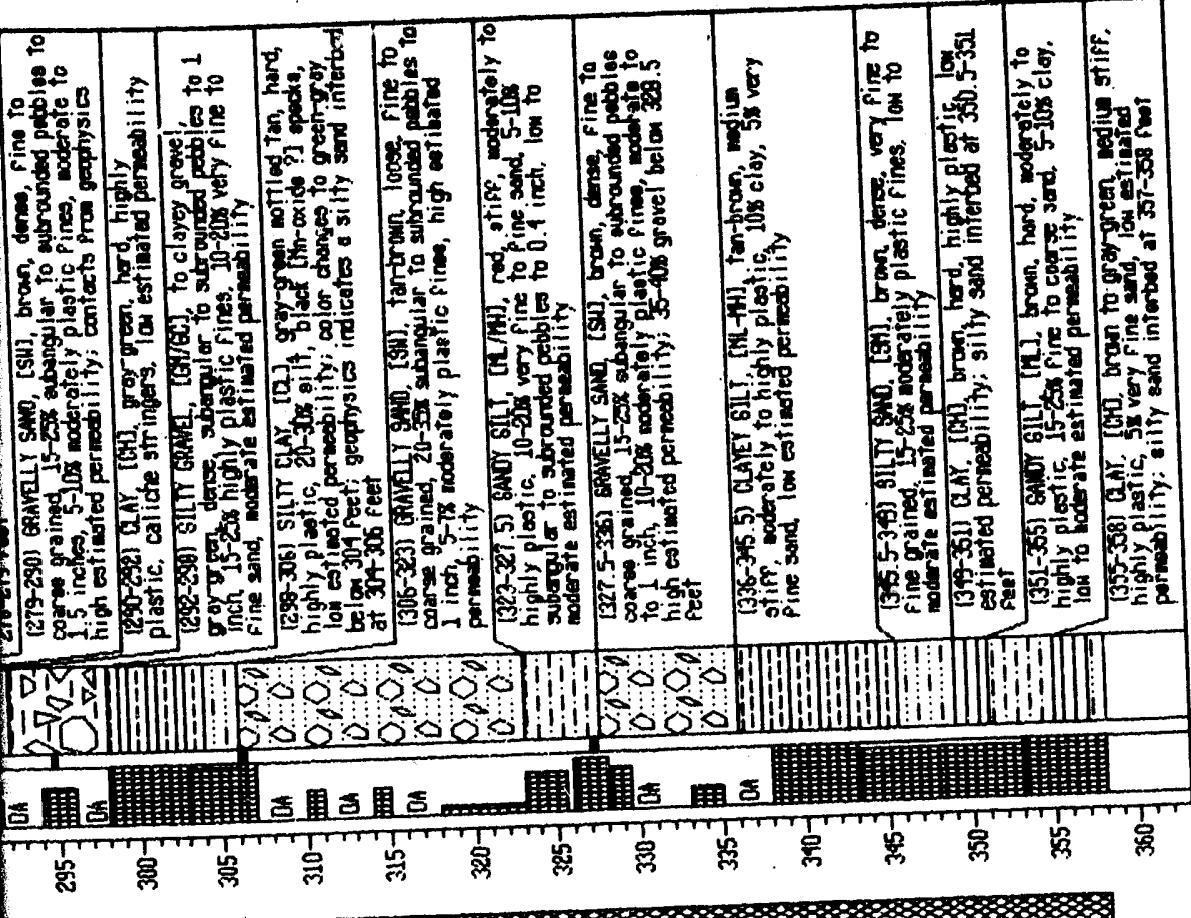

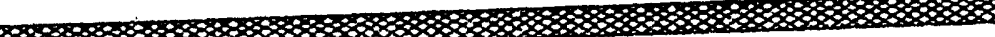

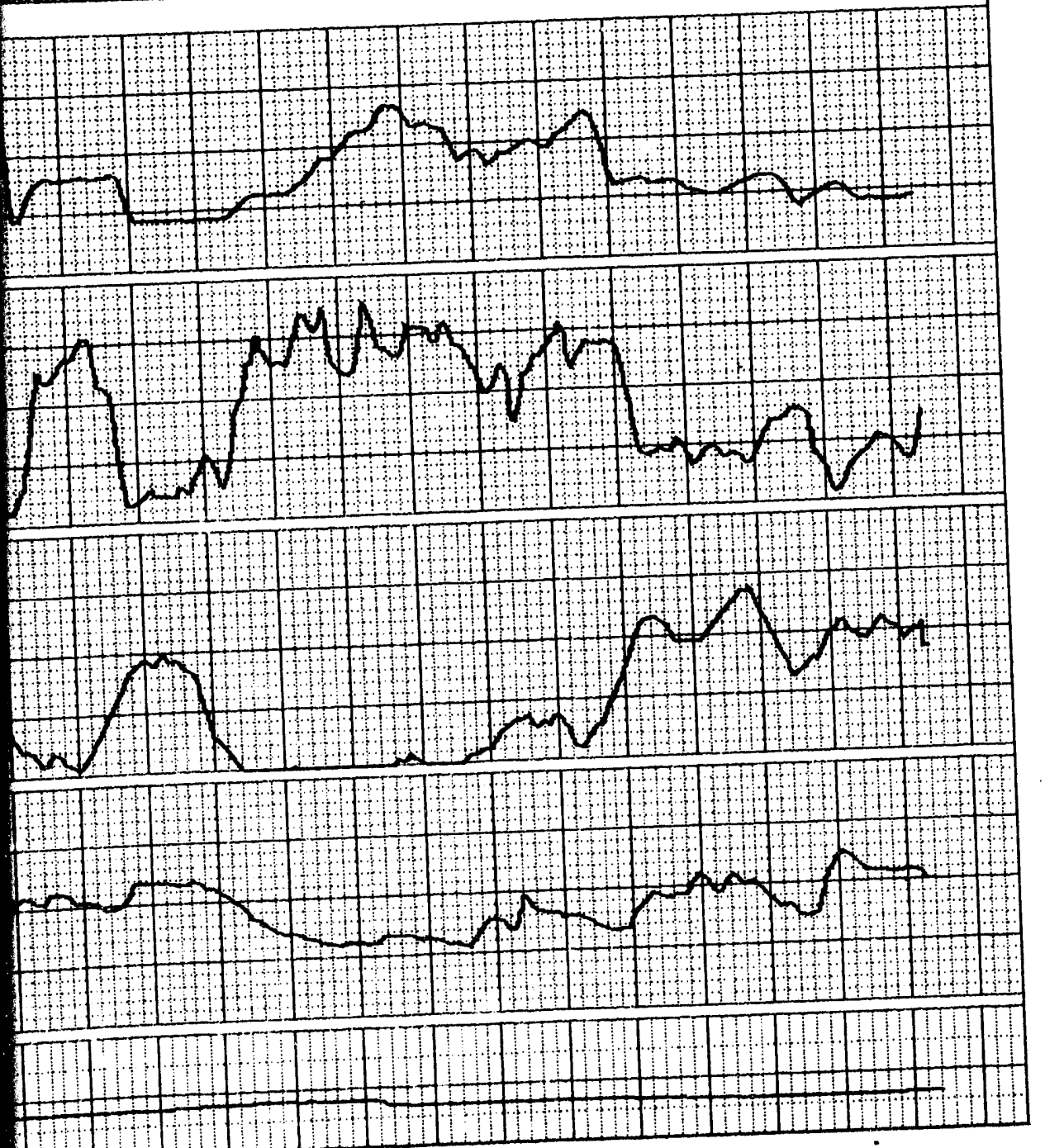




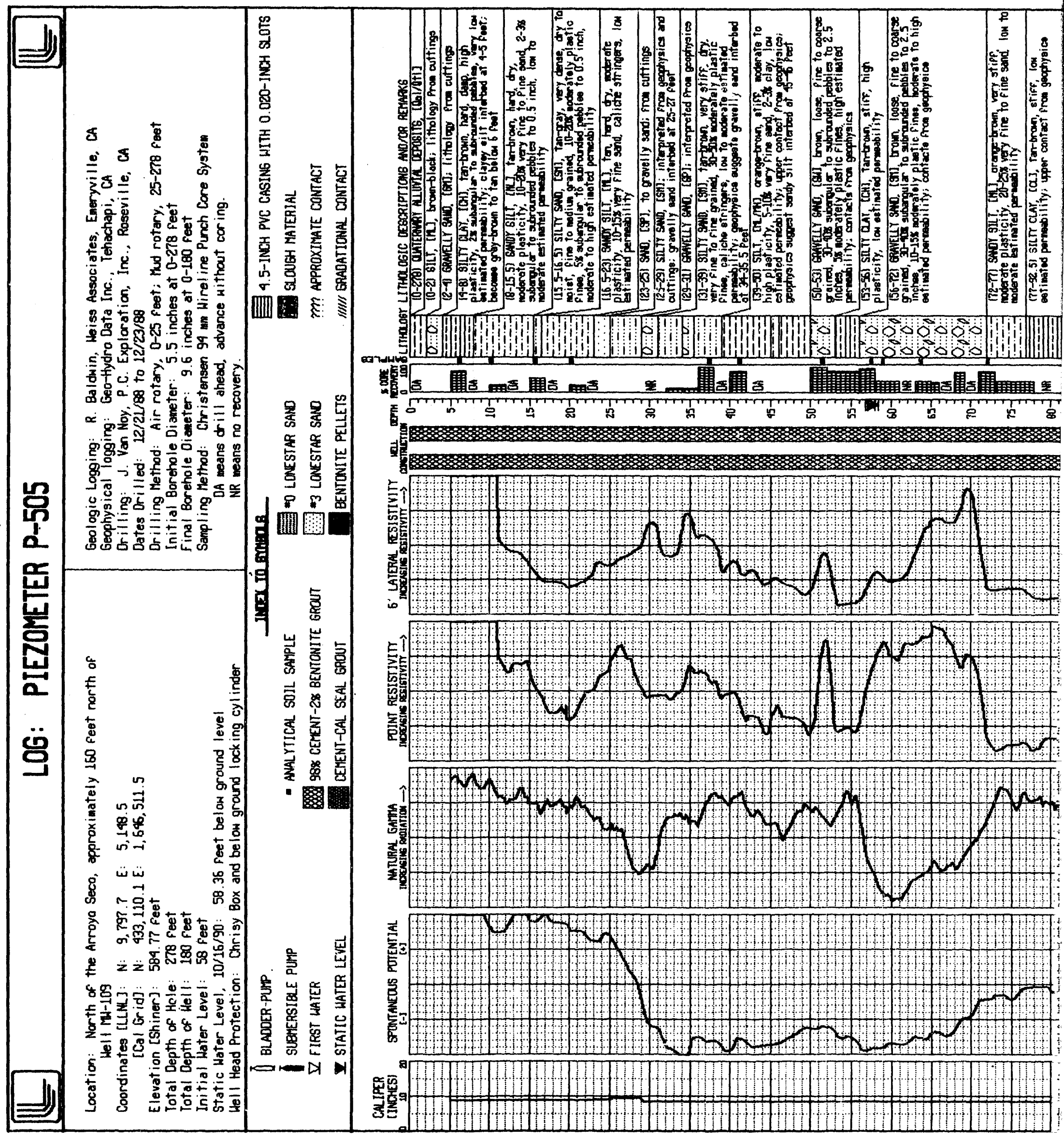




\begin{tabular}{|c|c|}
\hline 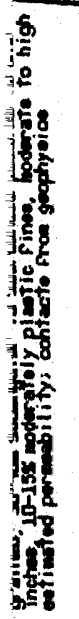 & 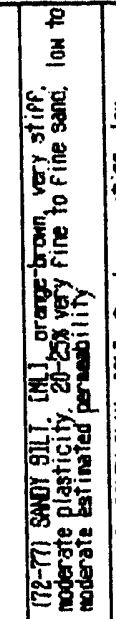 \\
\hline
\end{tabular}

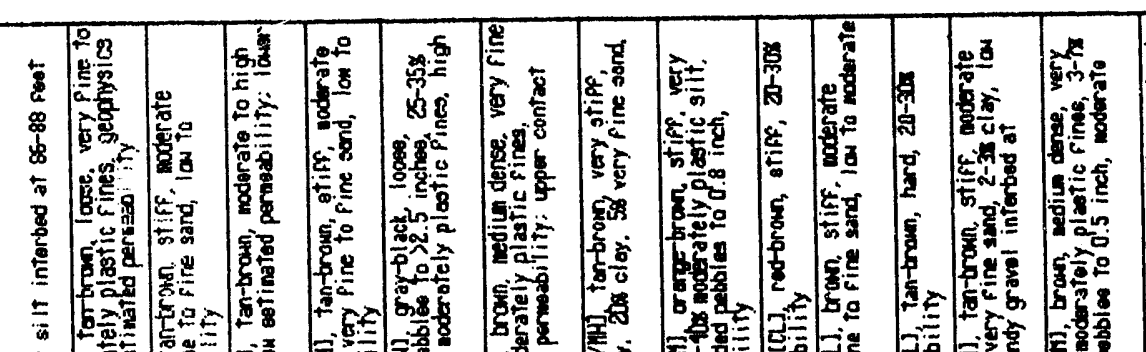

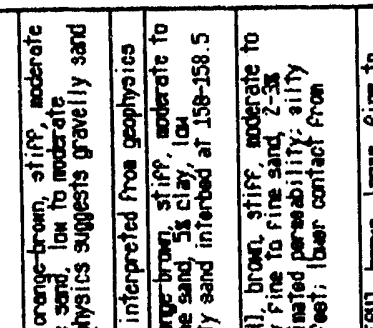

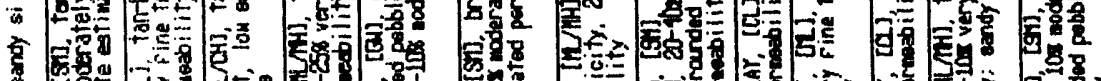

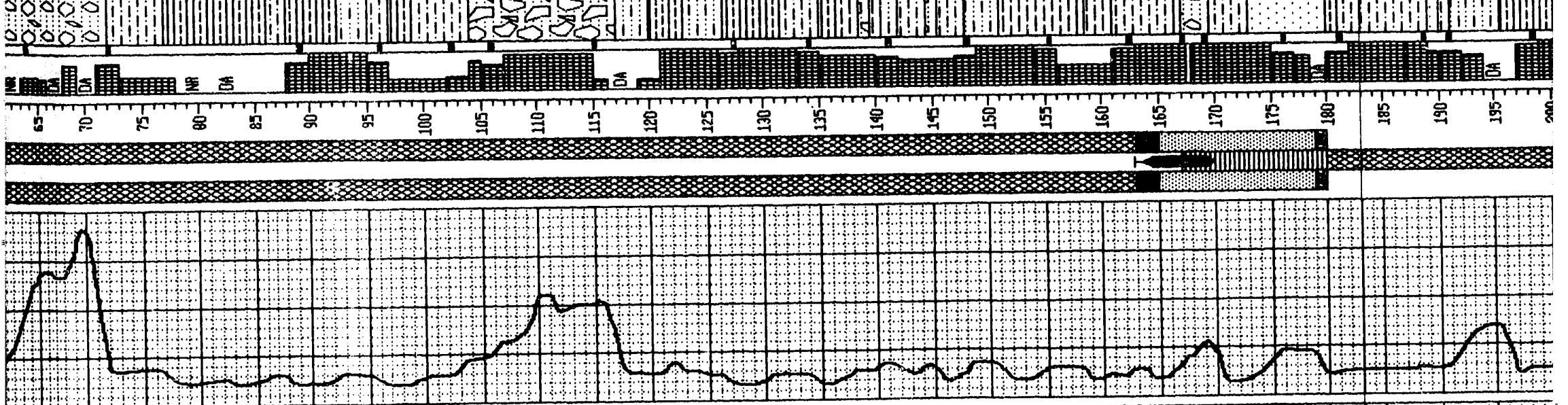

ब1।

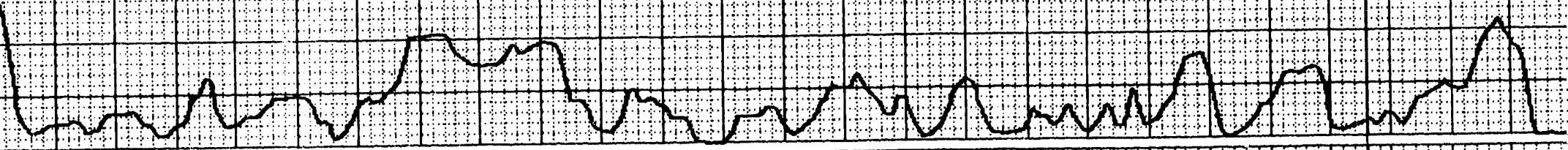

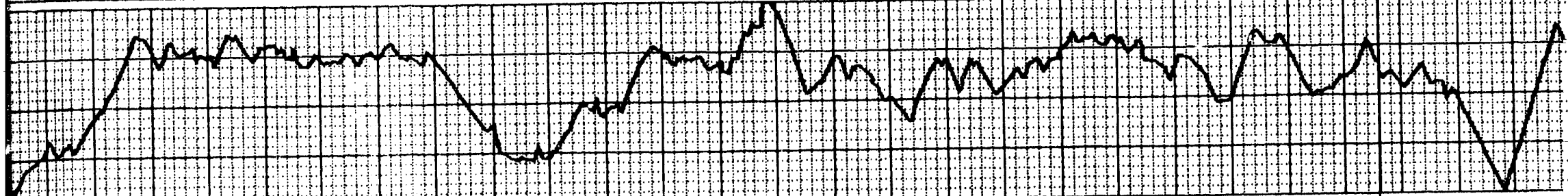




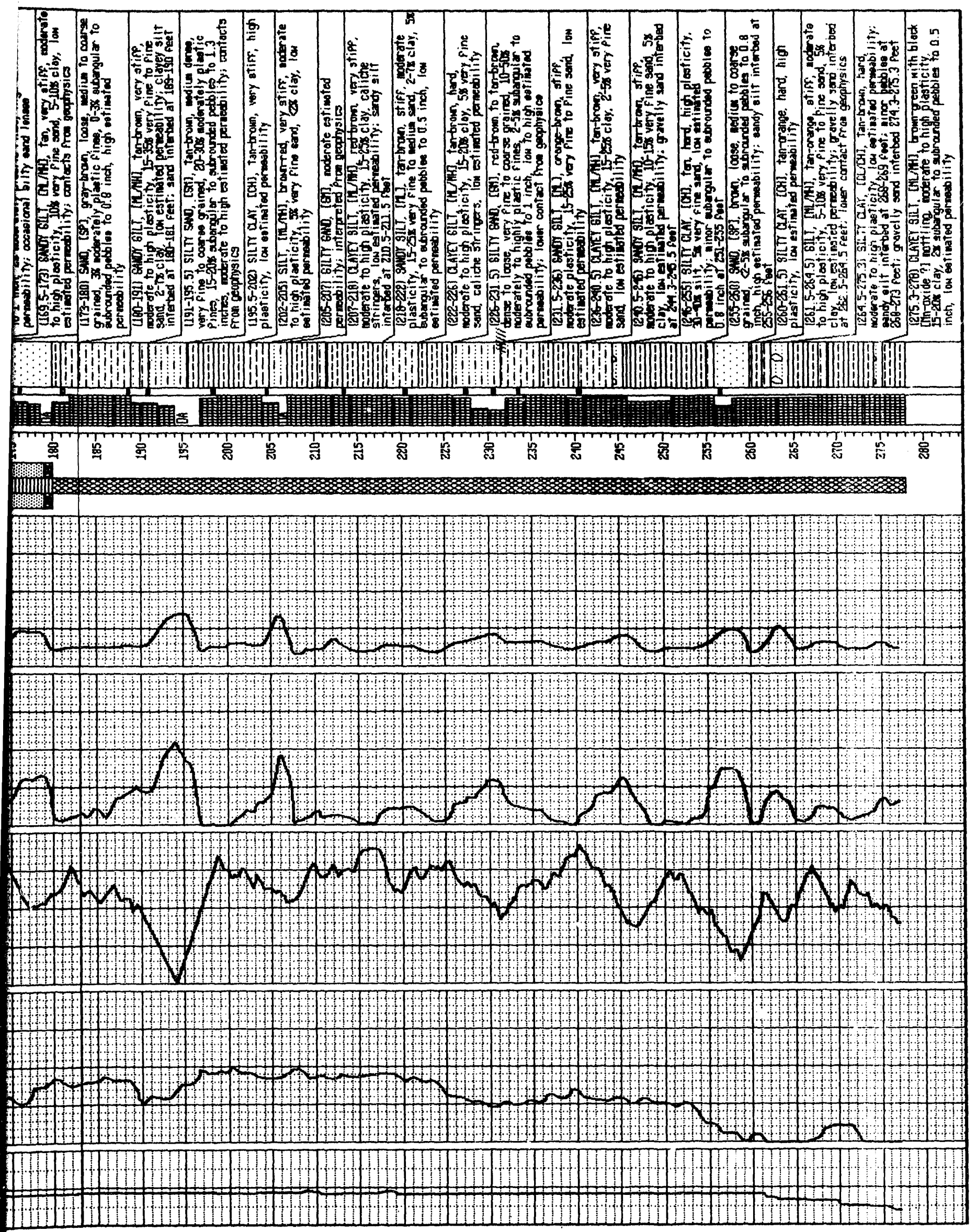




\section{LOG: PIEZOMETER P-506}

Lacation: North of Arroyo Seco, approximately 10 feet east of Piezometer P-505 and Welly Mu-108 and Hu-109, West of LLN

Coordinates rLLin] : $N: 9.800 .5 \quad E: 5,138.9$

[Ca| Grid]: N: 433,113.2 E: 1,646,501.9

Elevation [Shiner]: 584.76 Feet

Total Depth of Hole: 120 feet

Total Depth of Hell: 115 feet

Initial Water Level: Not observed

Static Water Level. 10/16/90: 58.1 feet below ground level

Hell Head Protestion: Above ground locking steel cylincier

Feologic Logging: R. Baldwin, Heiss Assaciates, Emeryville, CA

Geophysical Logoing: Geophysics from Piezometer P-505

Orilling: J. Von Noy, P.C. Exploration, Inc., Roseville, CA

Dates Drilled: 12/16/88 to 12/22/88

Drilling Method: Mud rotary, 0-120 feet

Initial Borehole Diameter: 5.5 inches at 0-120 feet

Final Borehole Diameter: 9.6 inches at 0-115 feet

Sampling Methrod: Christensen 99 Hirel ine Punch Core System

DA meane drill ahead; advance without coring.

\begin{tabular}{|c|c|c|c|}
\hline If BLADDER PUMP & \multicolumn{2}{|c|}{ Inx m m mms } & 4.5-INCH PVC CASING WITH 0.020-INCH SLOTS \\
\hline SLBMERSIBLE PUMP & - ANALYTICAL SOIL SAMPLE & =I LONESTAR SAND & SLOUGH MATERIAL \\
\hline$\square$ FIFST WATER & 988 CEMENT-2\% BENTONITE GRDUTT & * 3 LDNESTAR SAND & m? APPROXIMATE CONTACT \\
\hline I STATIC HATER LEVEL & CEMENT-LAL SEAL GROUT & BENTONITE PELLETS & MIIII GRADATIONAL CONTACT \\
\hline
\end{tabular}

GEOPHYSICS FROM PIEZOMETER P-505

CALIPEB
ITNCHES STINTANETS POTENTAL

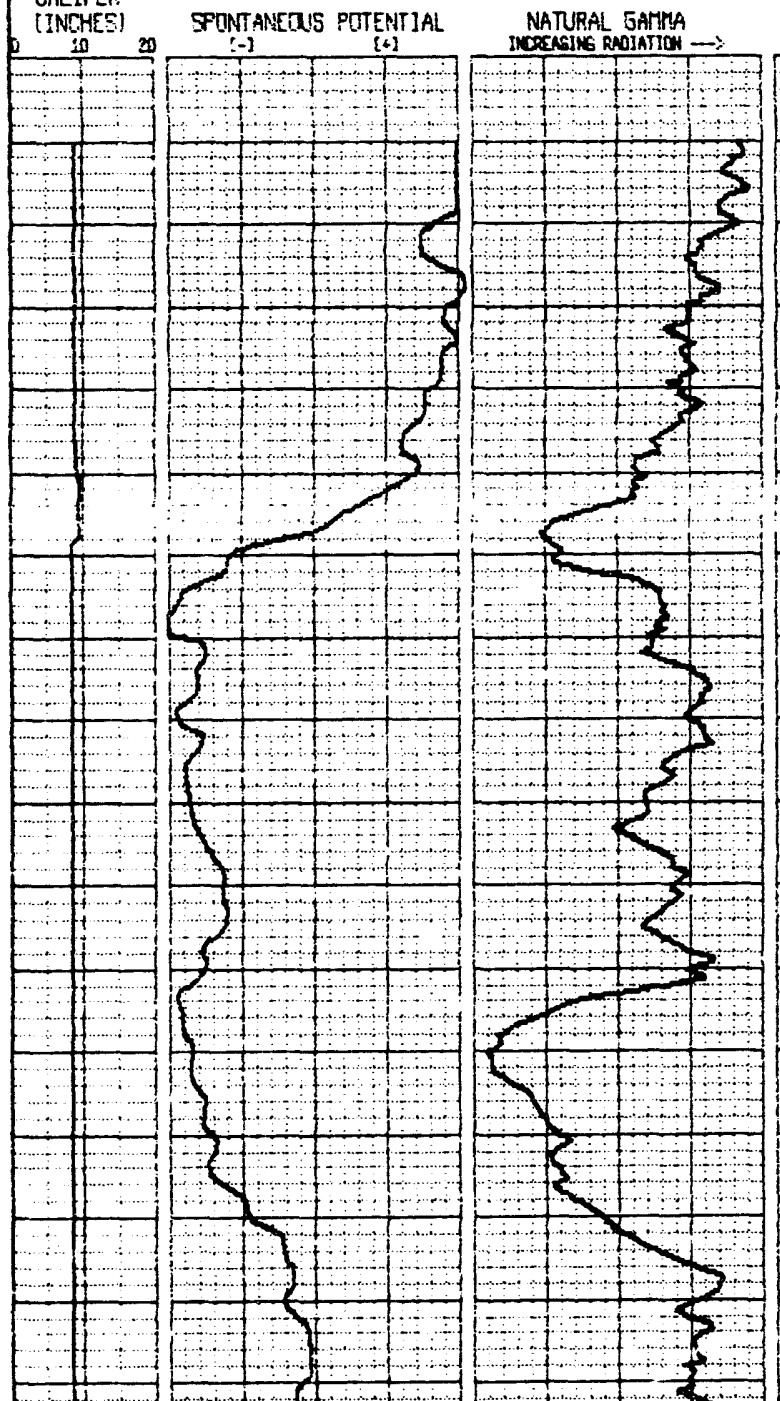

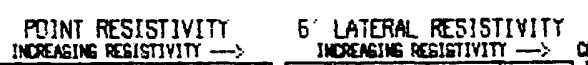

\section{通}

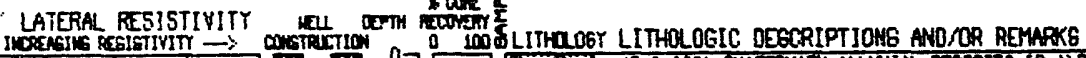

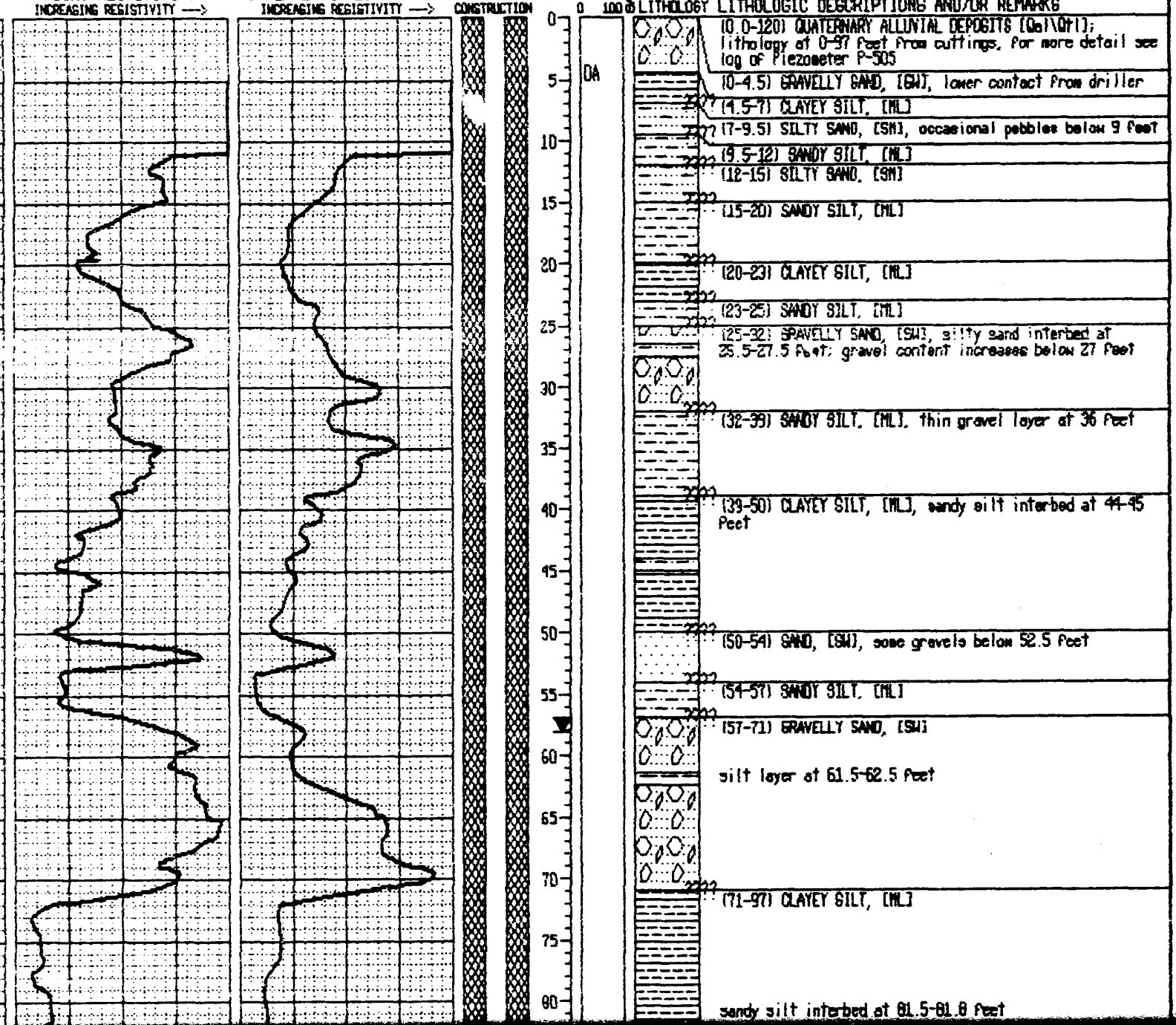




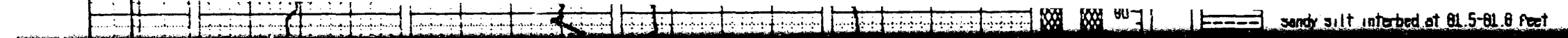

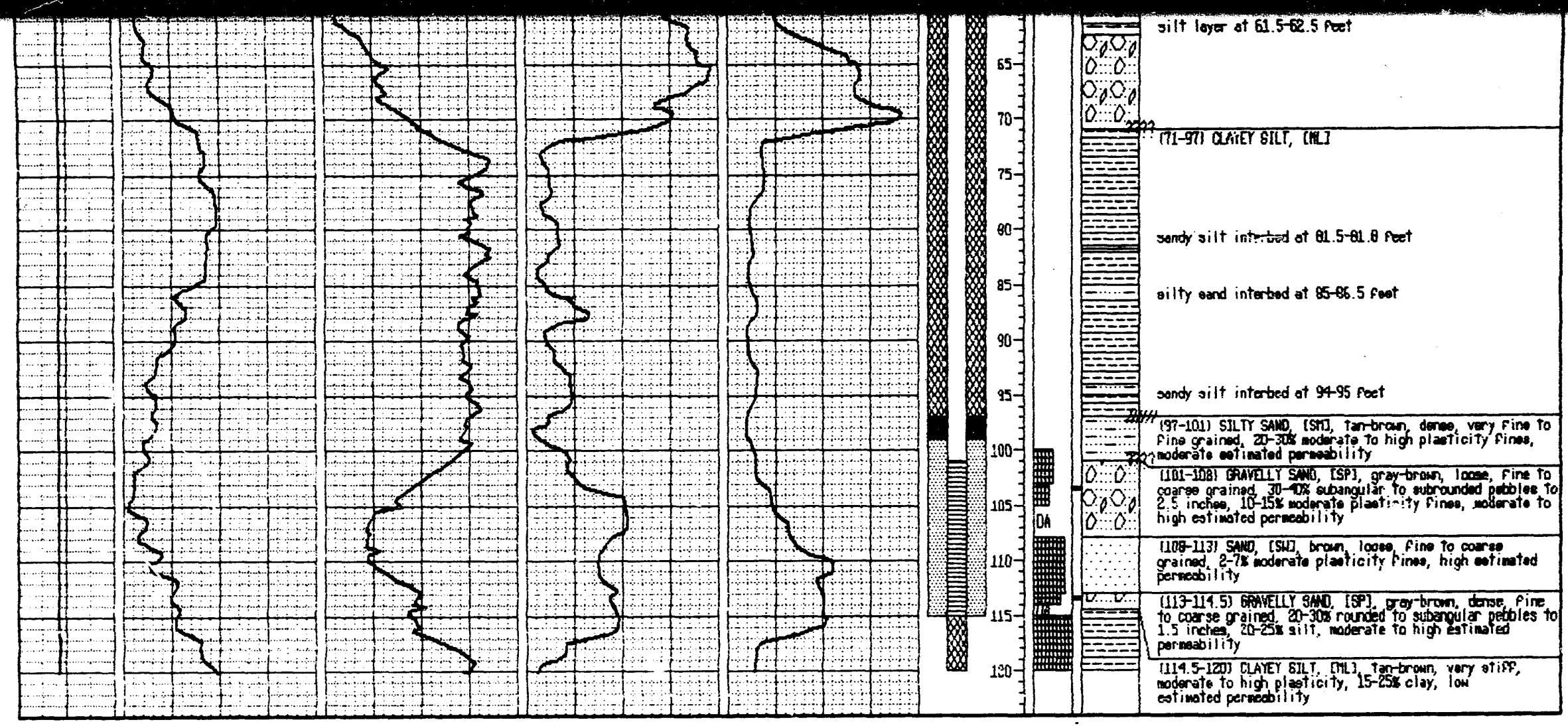




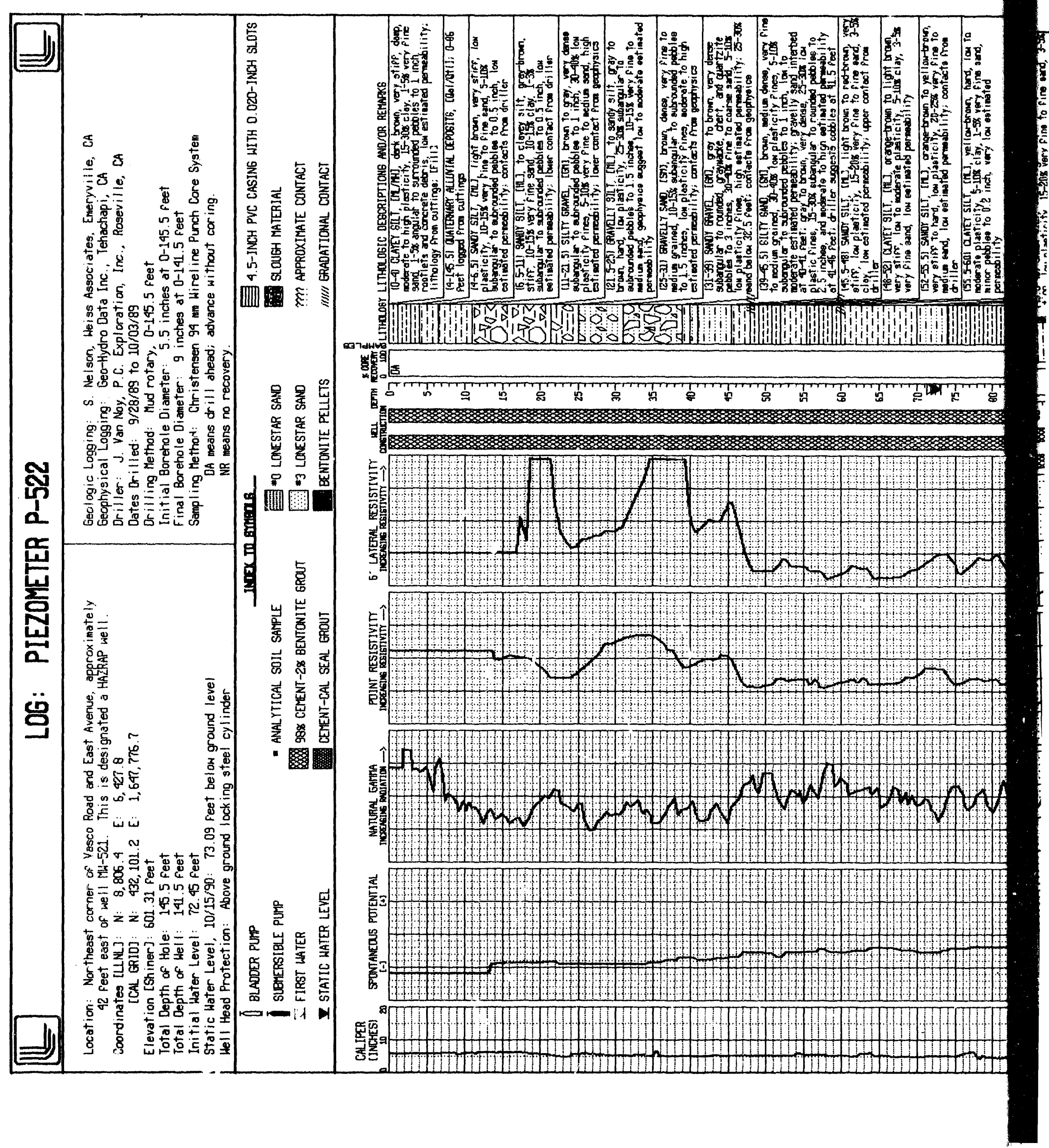




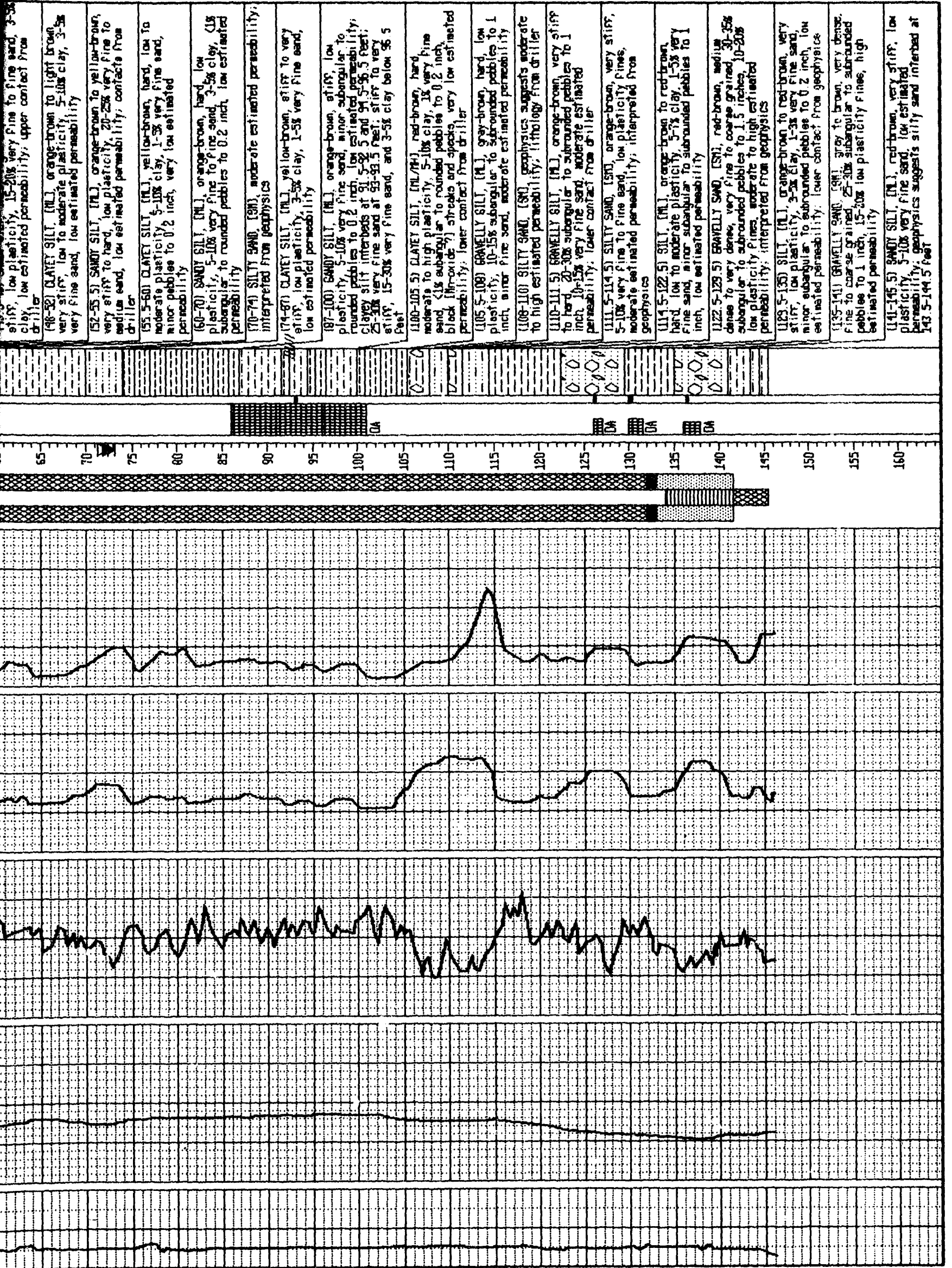




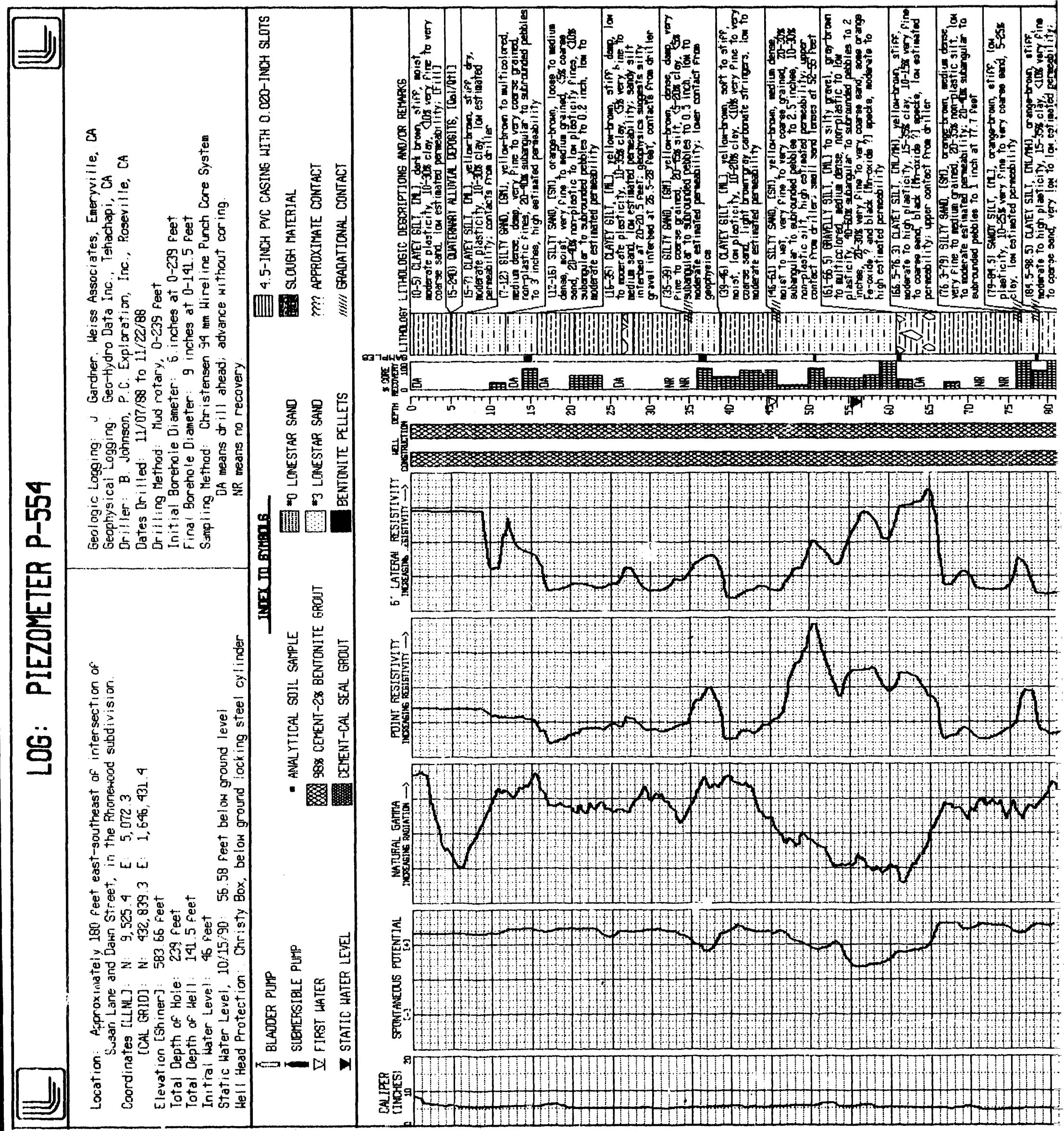




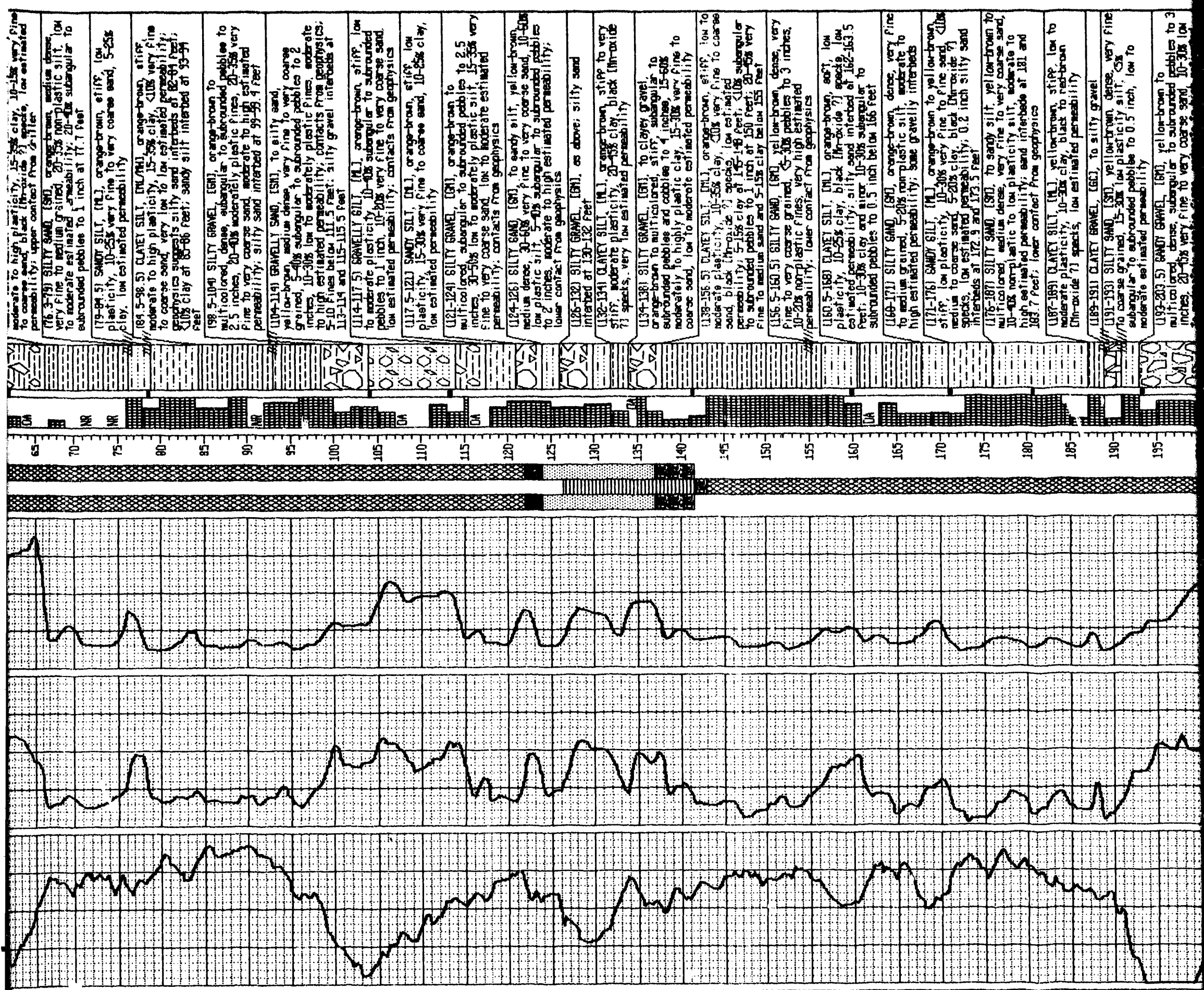




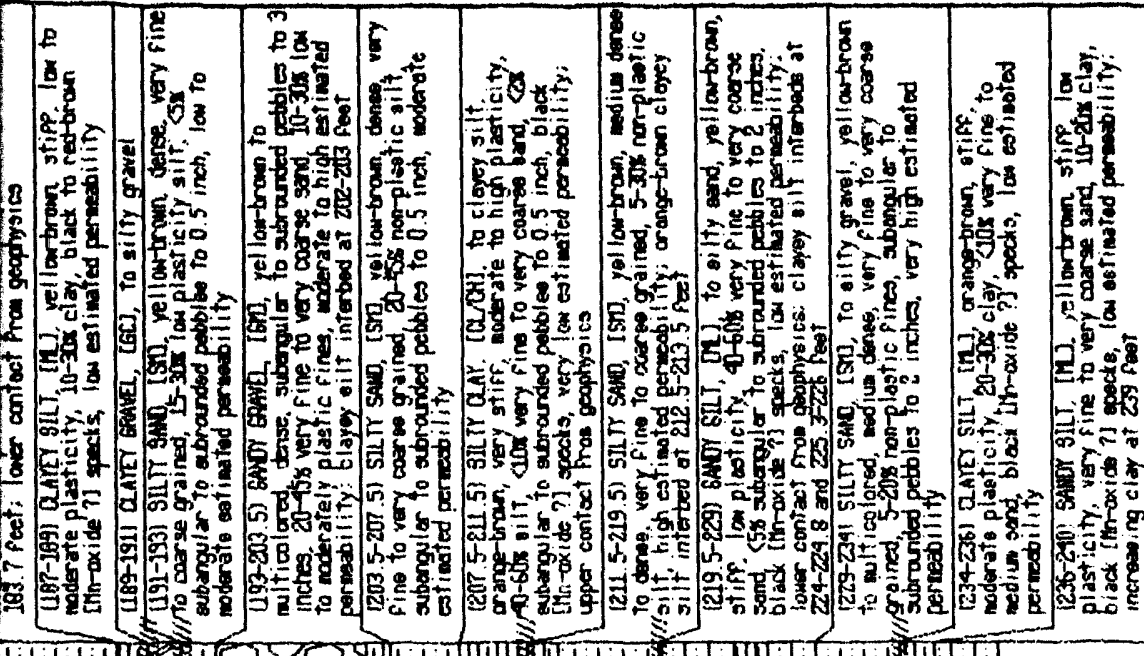

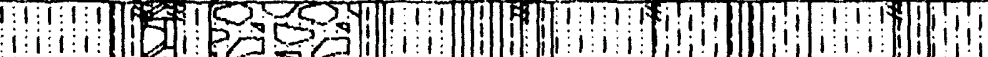

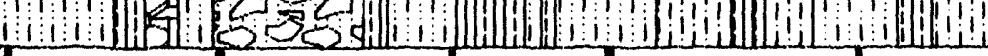

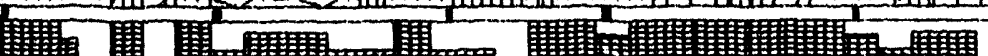

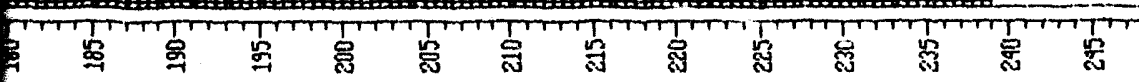

(1)
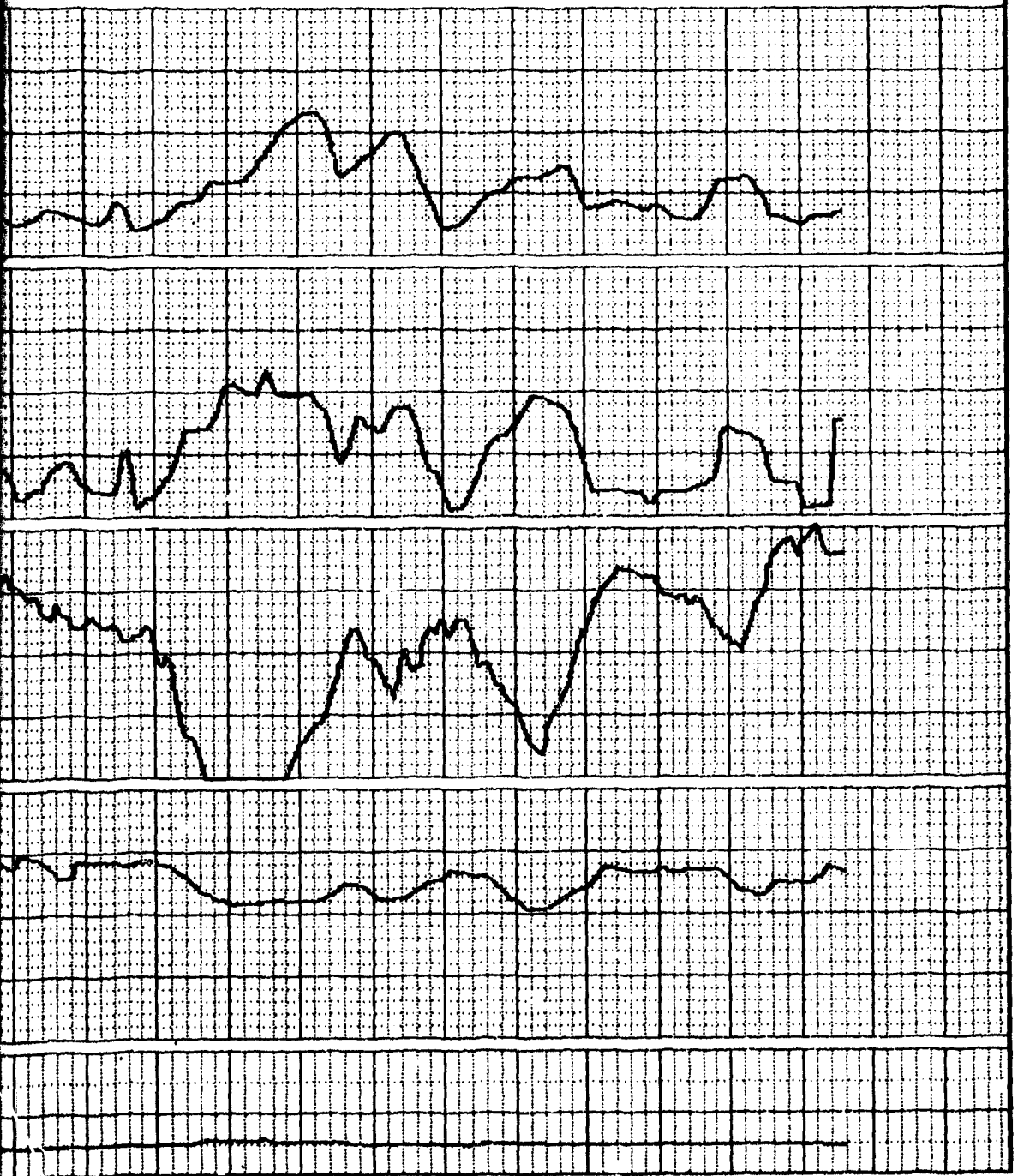


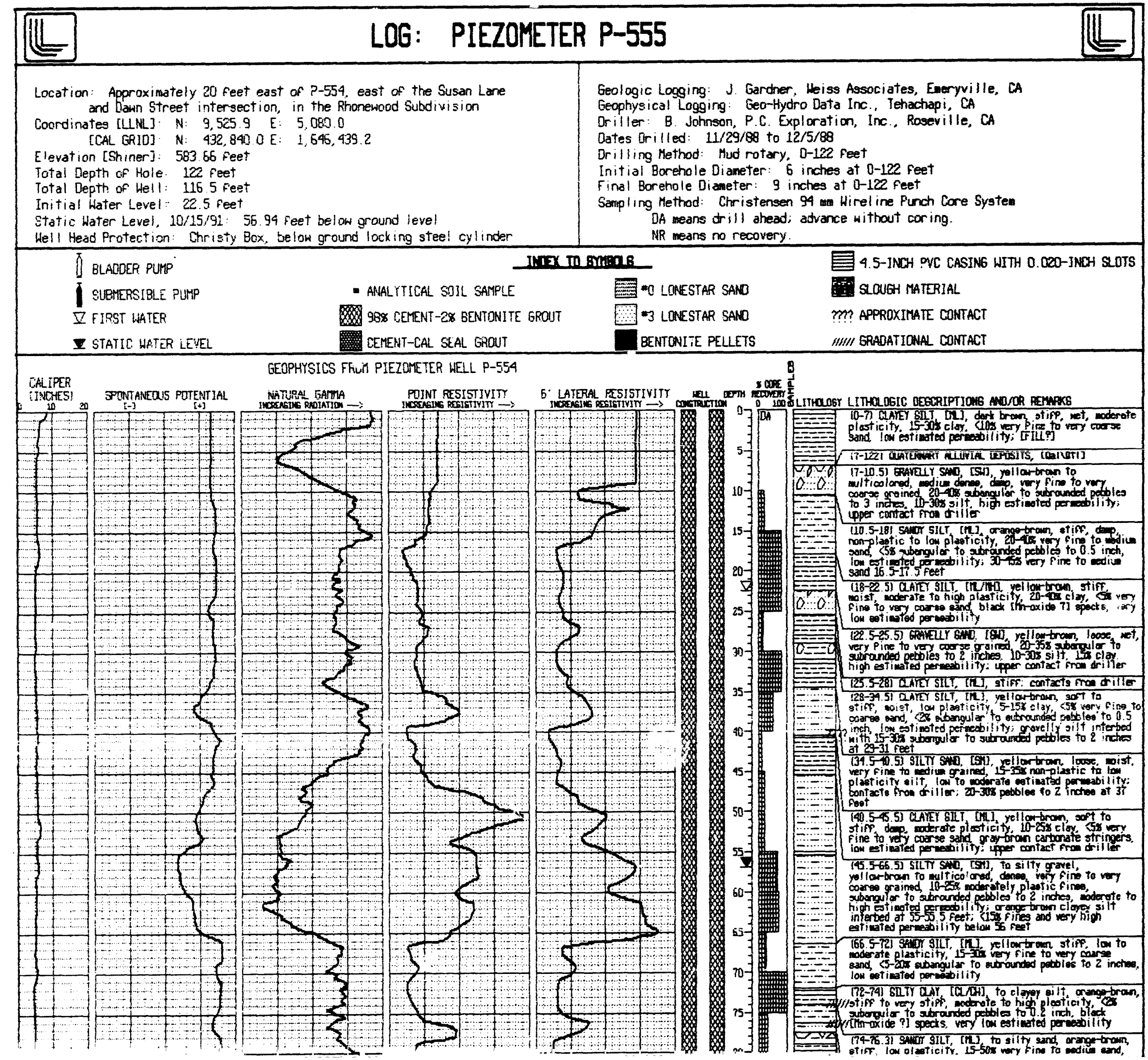




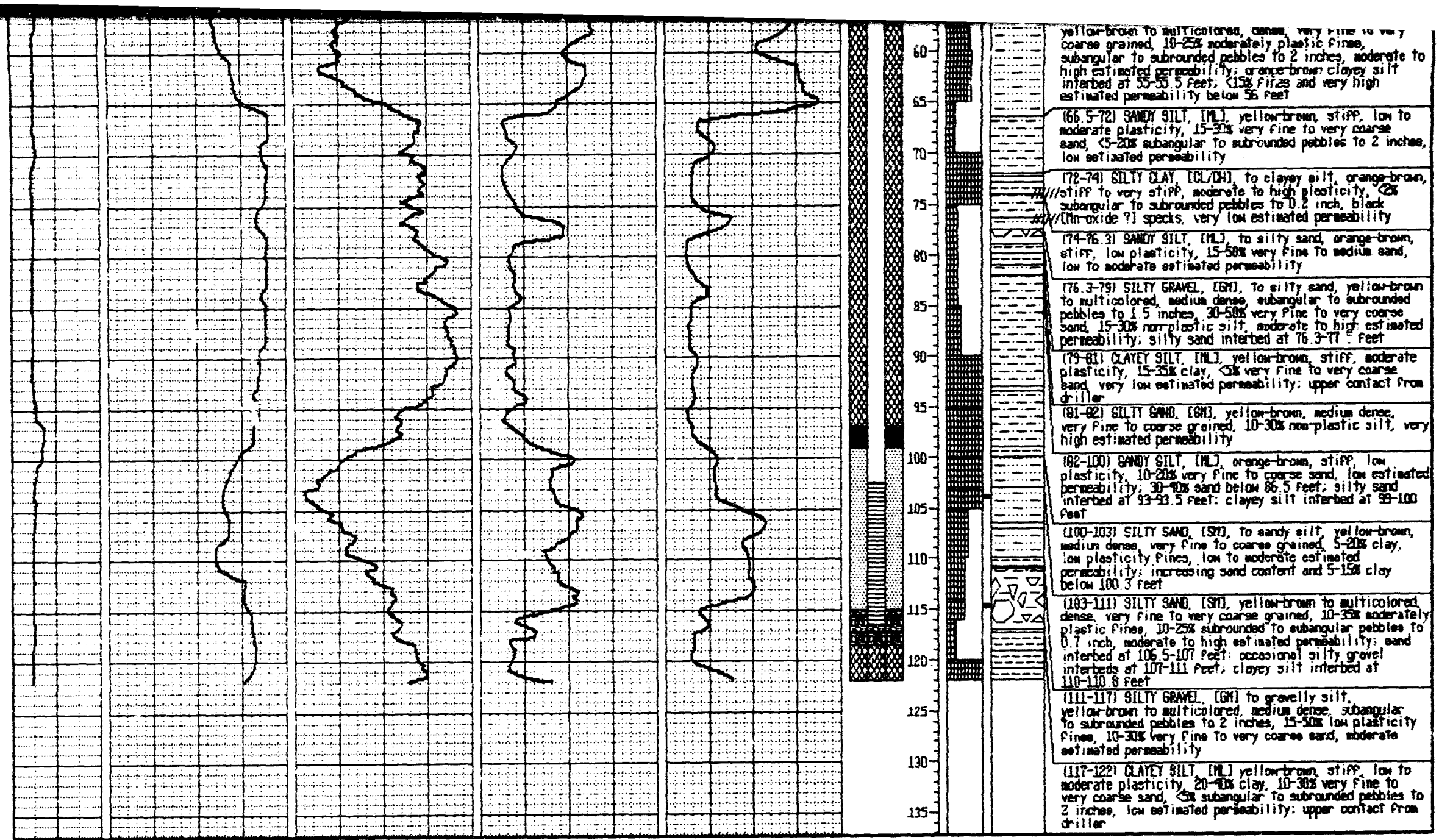




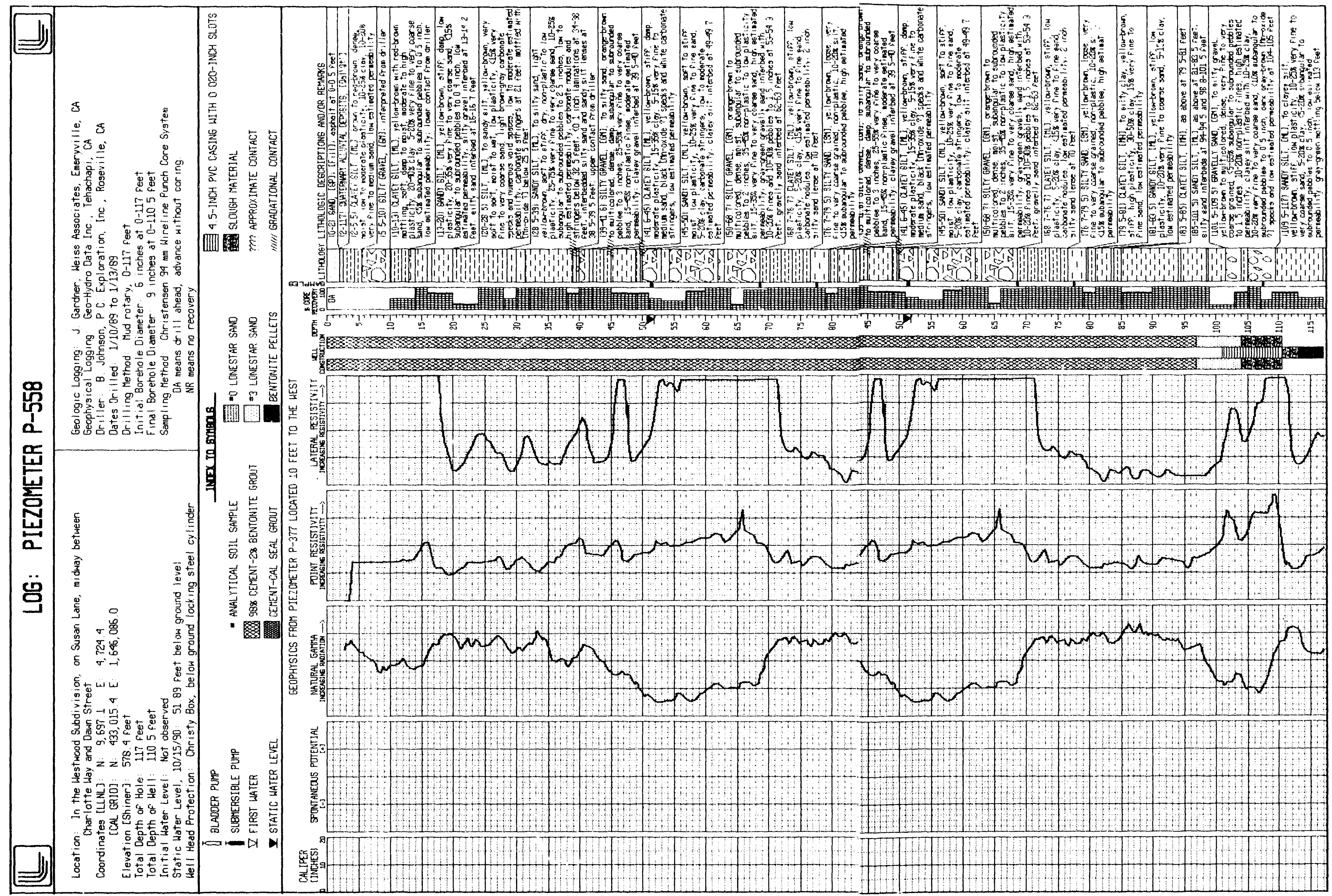




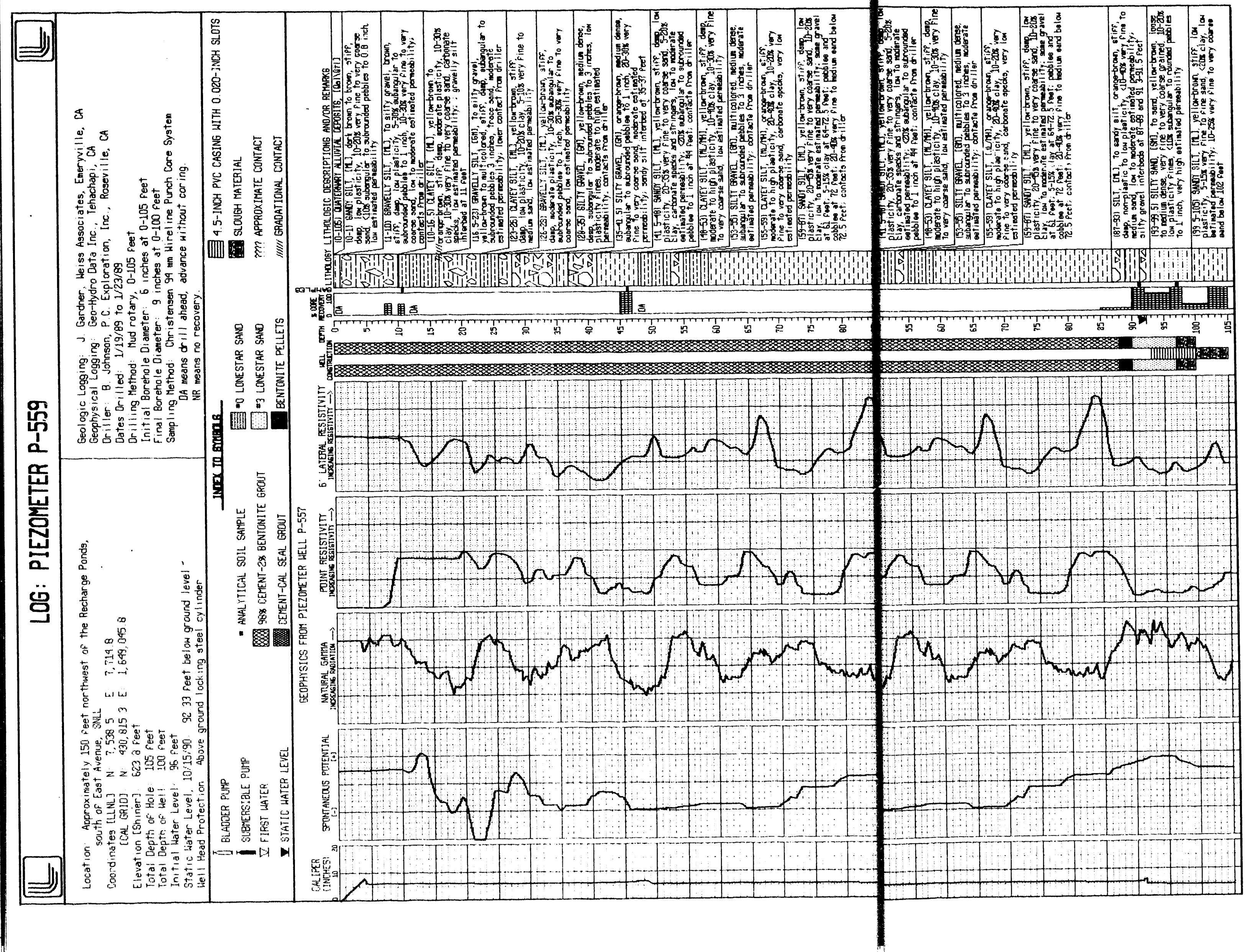




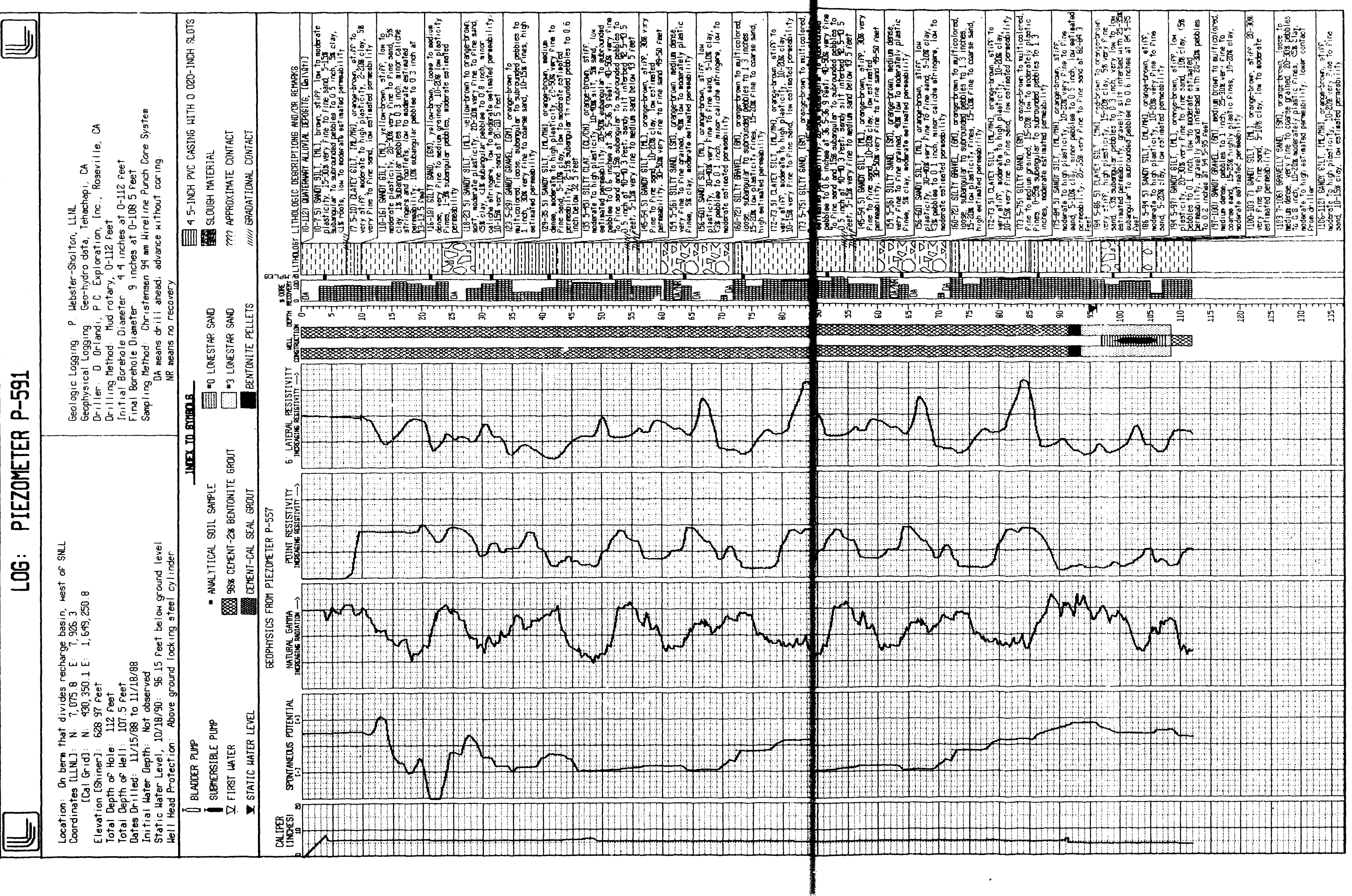




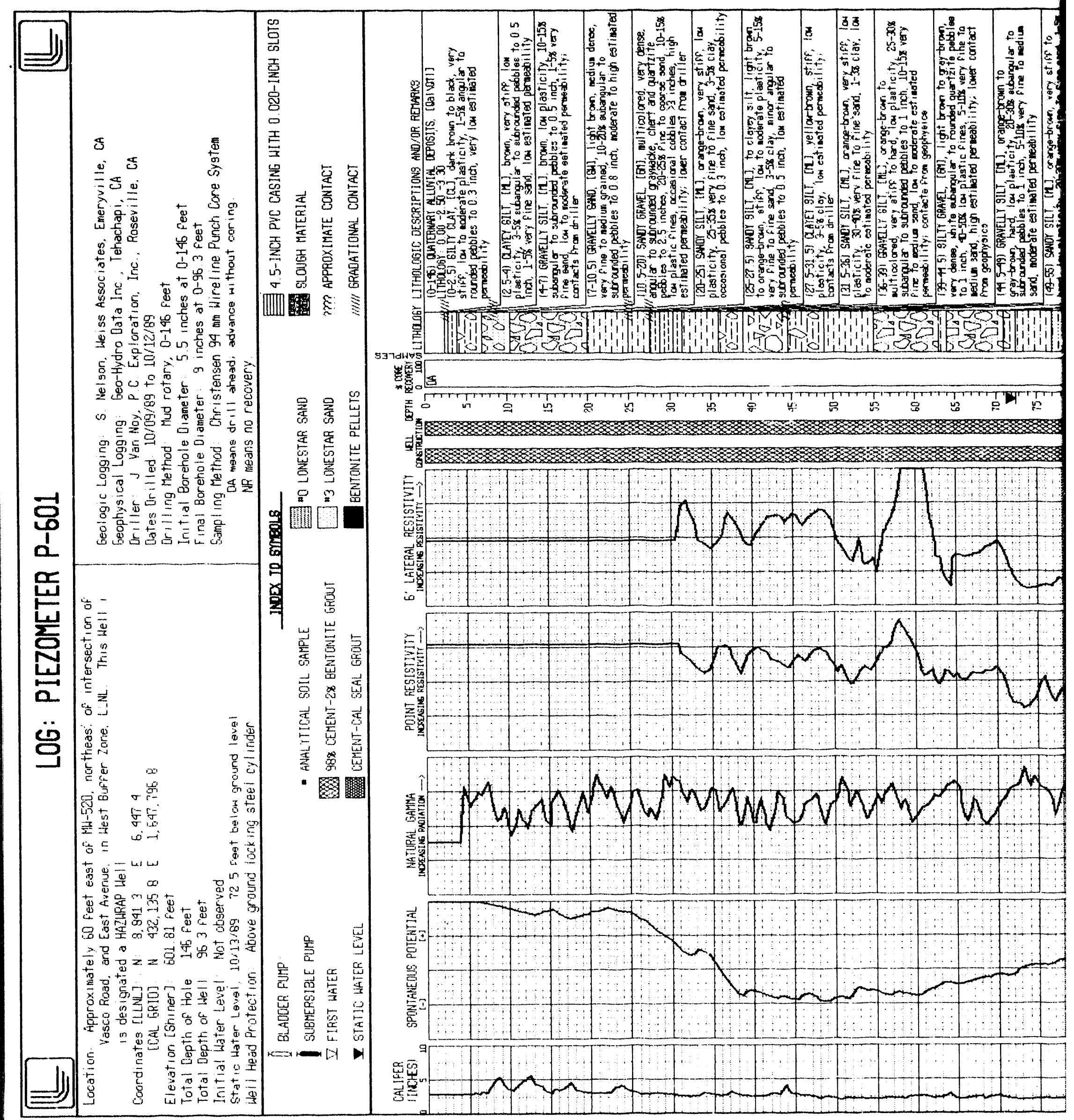




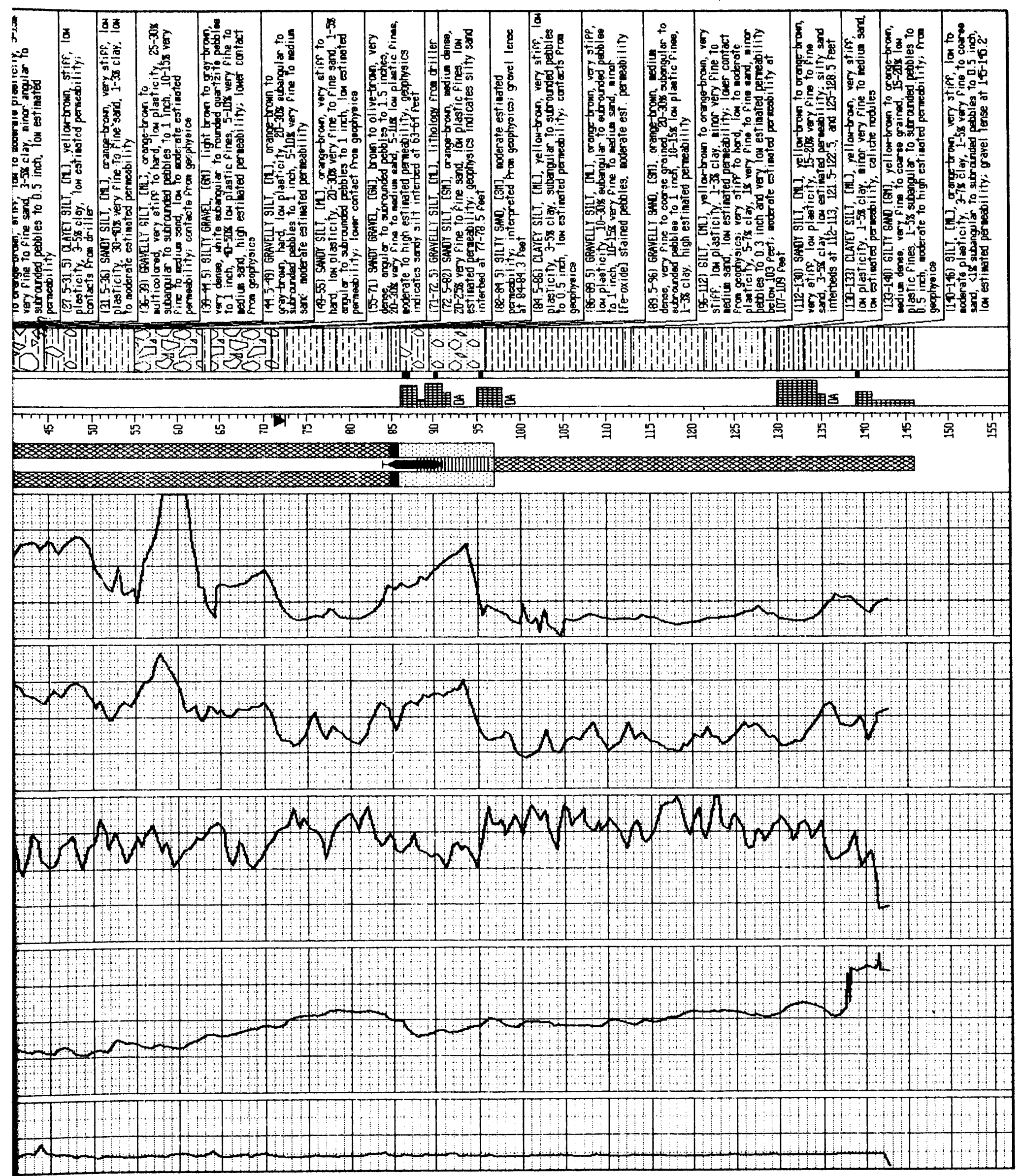




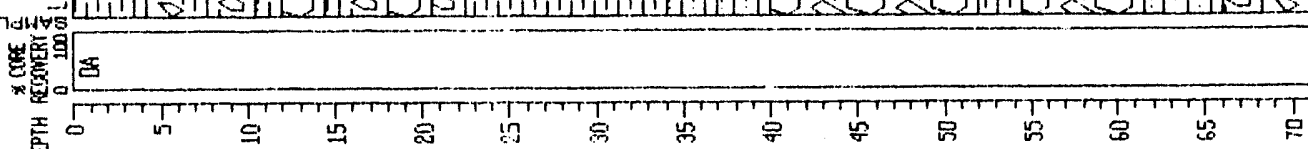

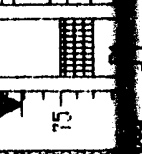

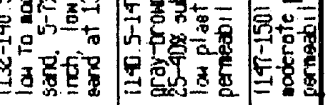
政

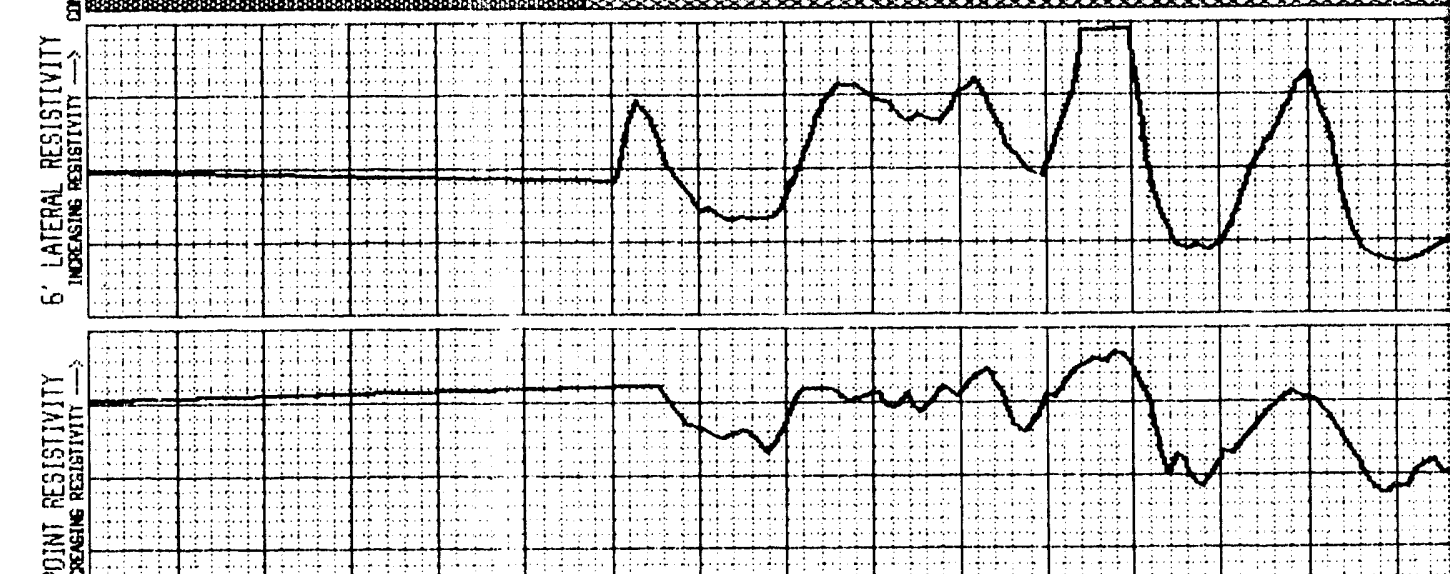

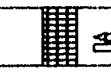
80 in -

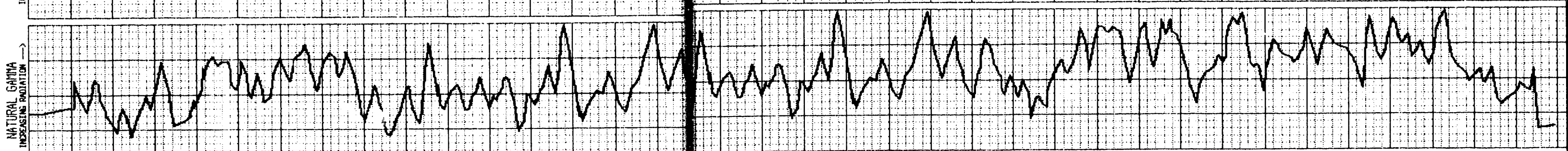
2

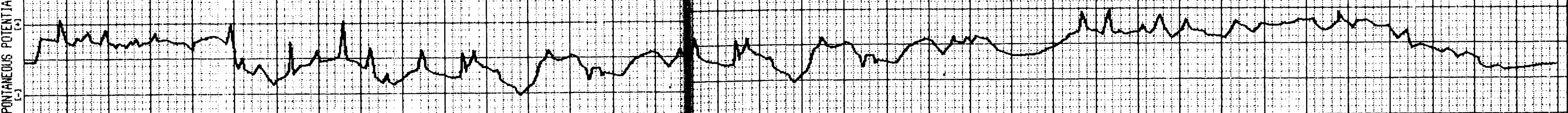

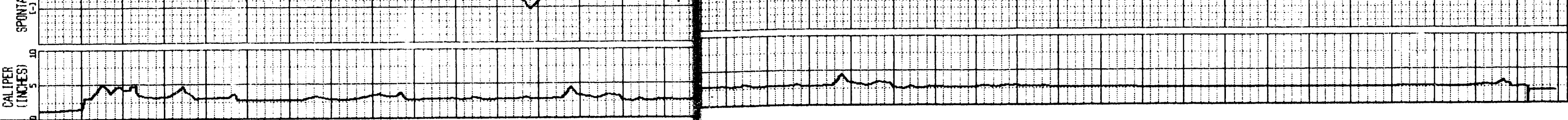
(a) 


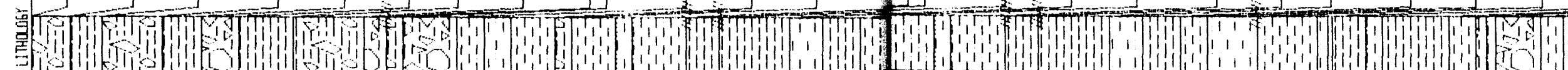
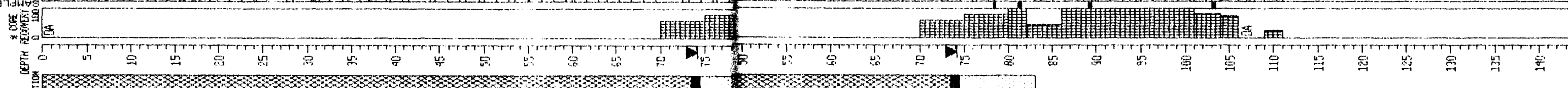

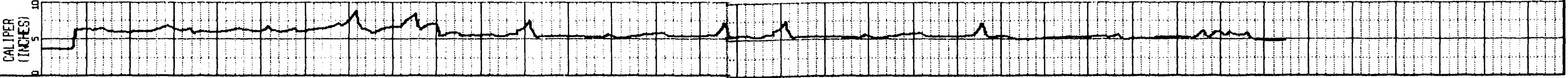




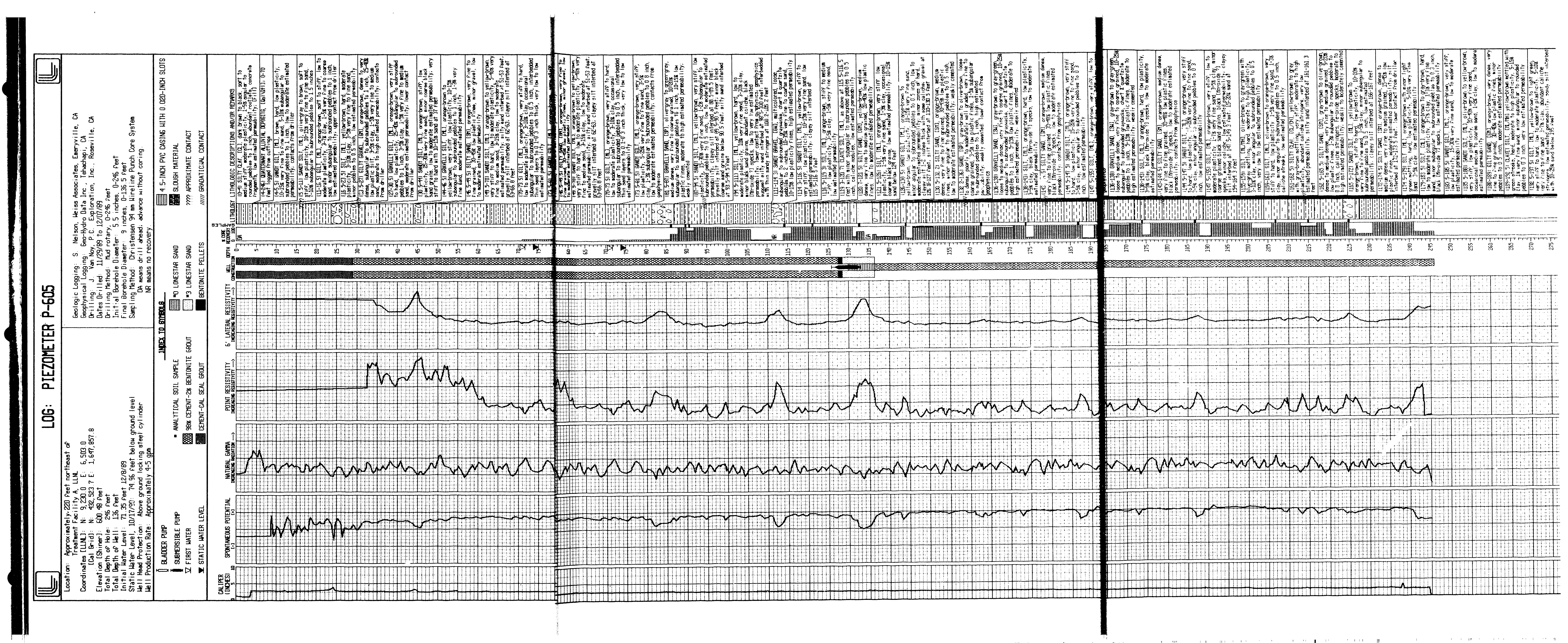




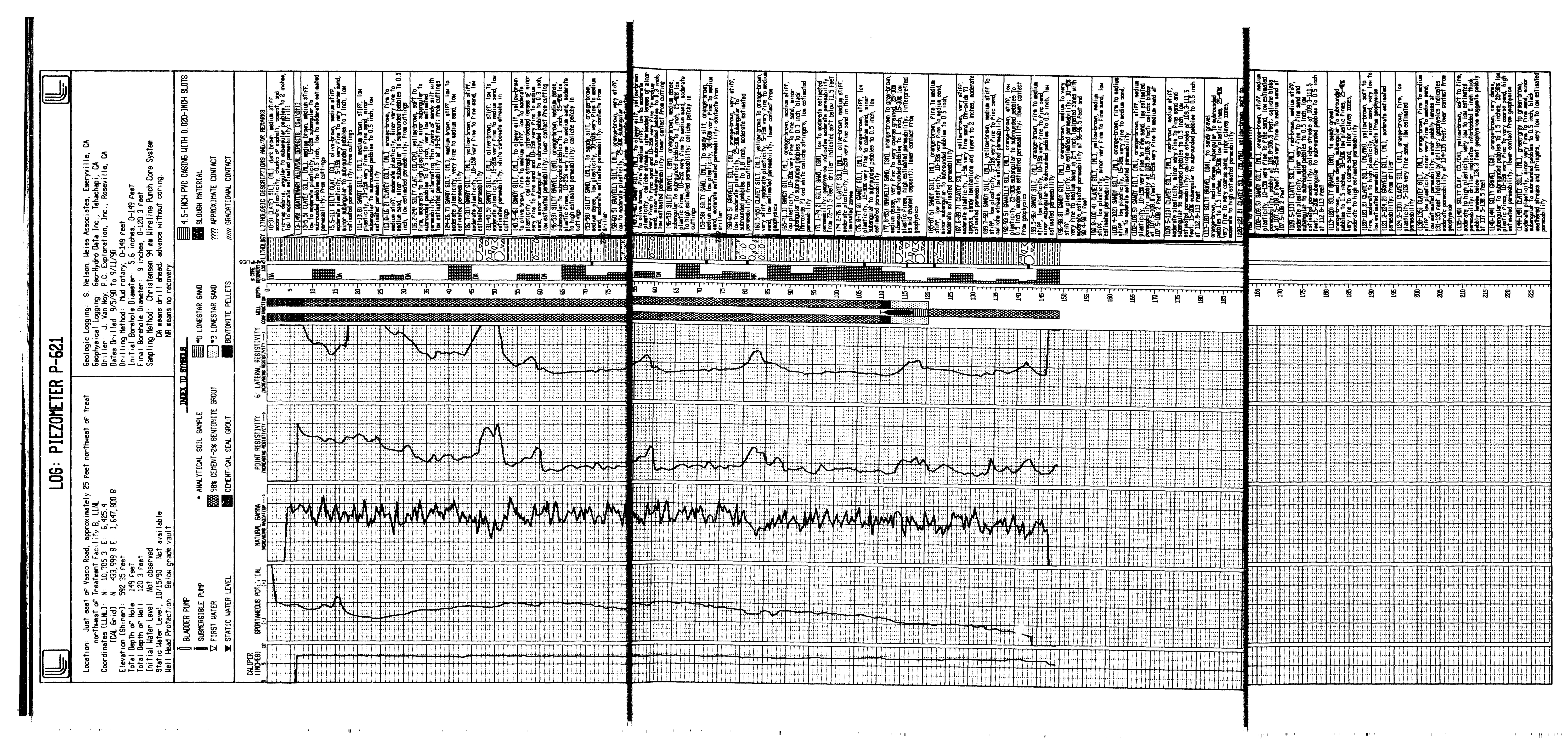




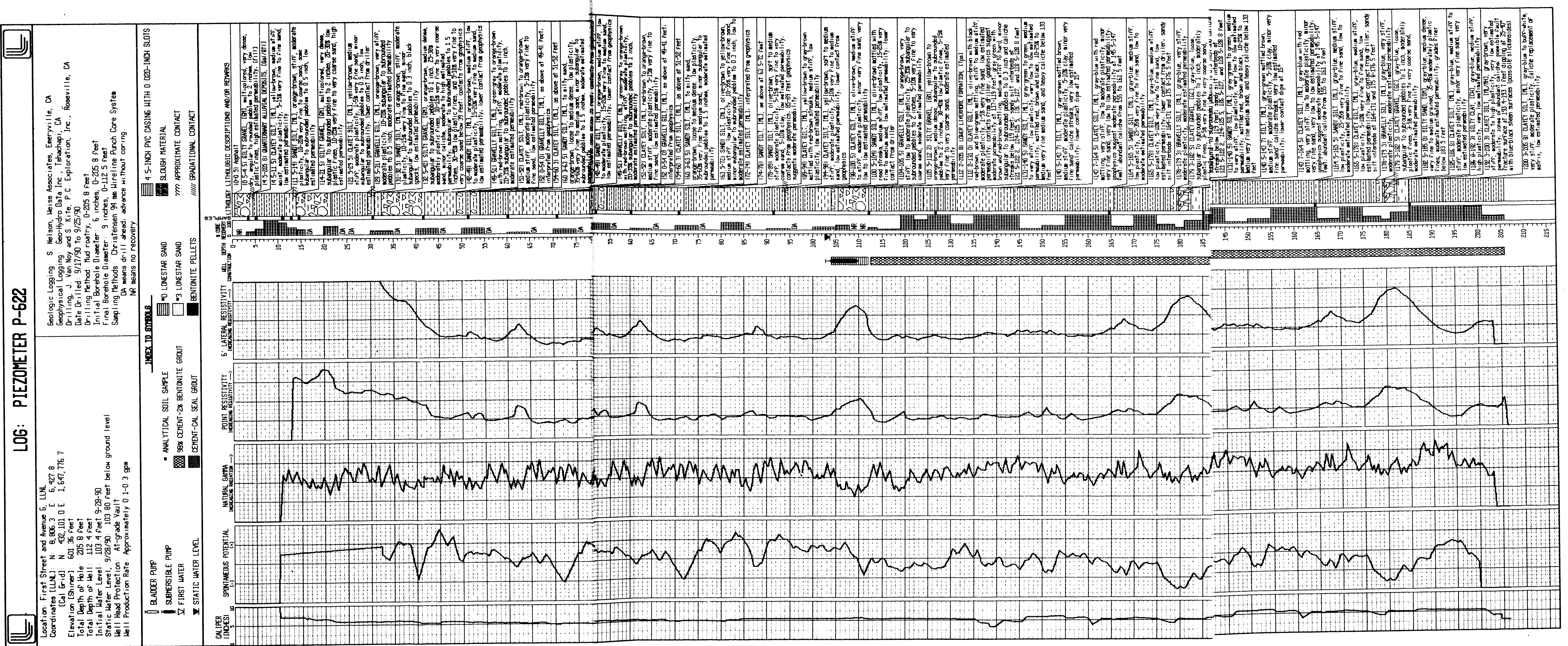




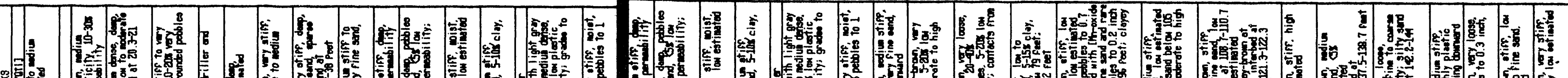

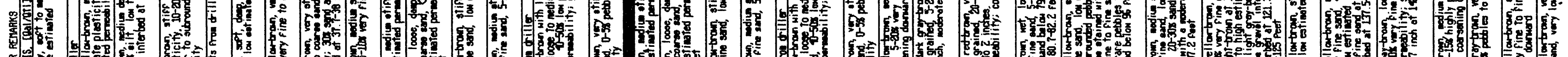

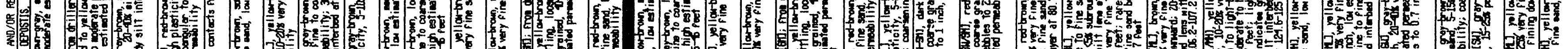

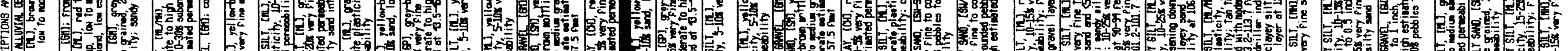

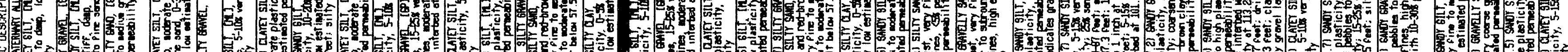

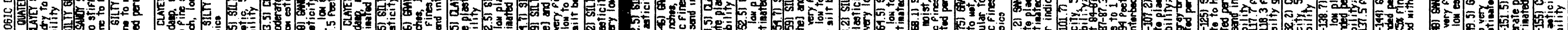

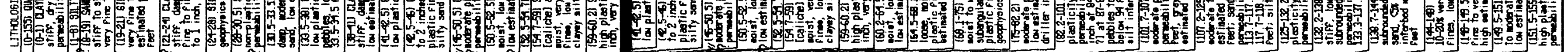

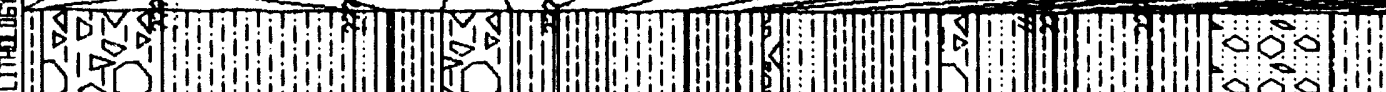

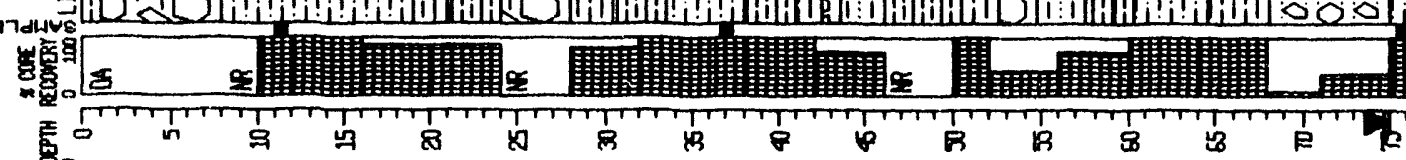
HW

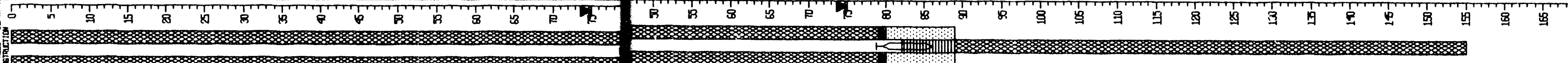

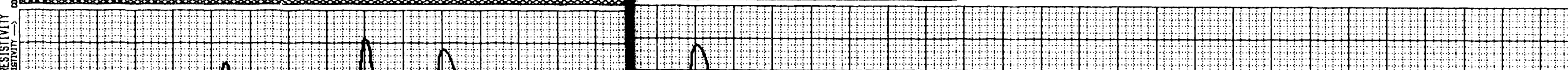

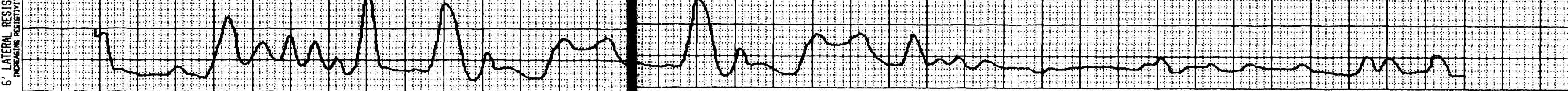

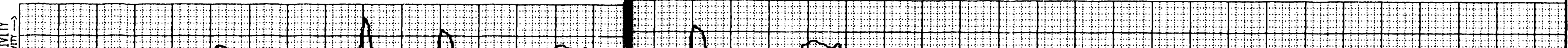

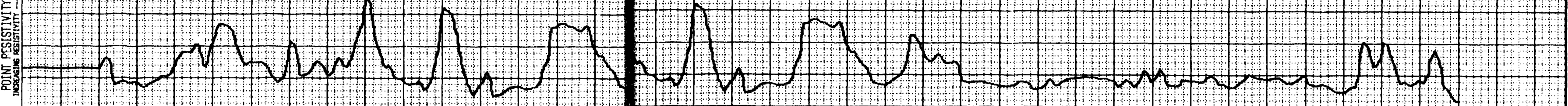

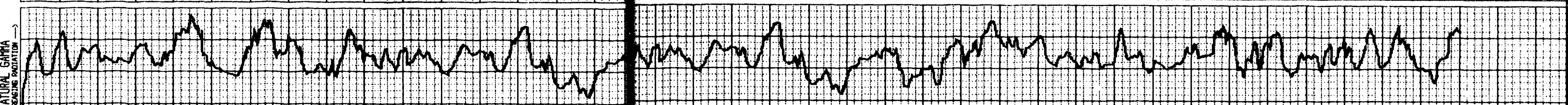
2. z: ( (

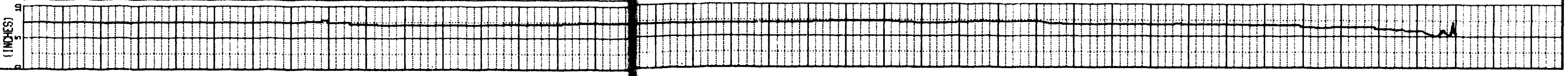



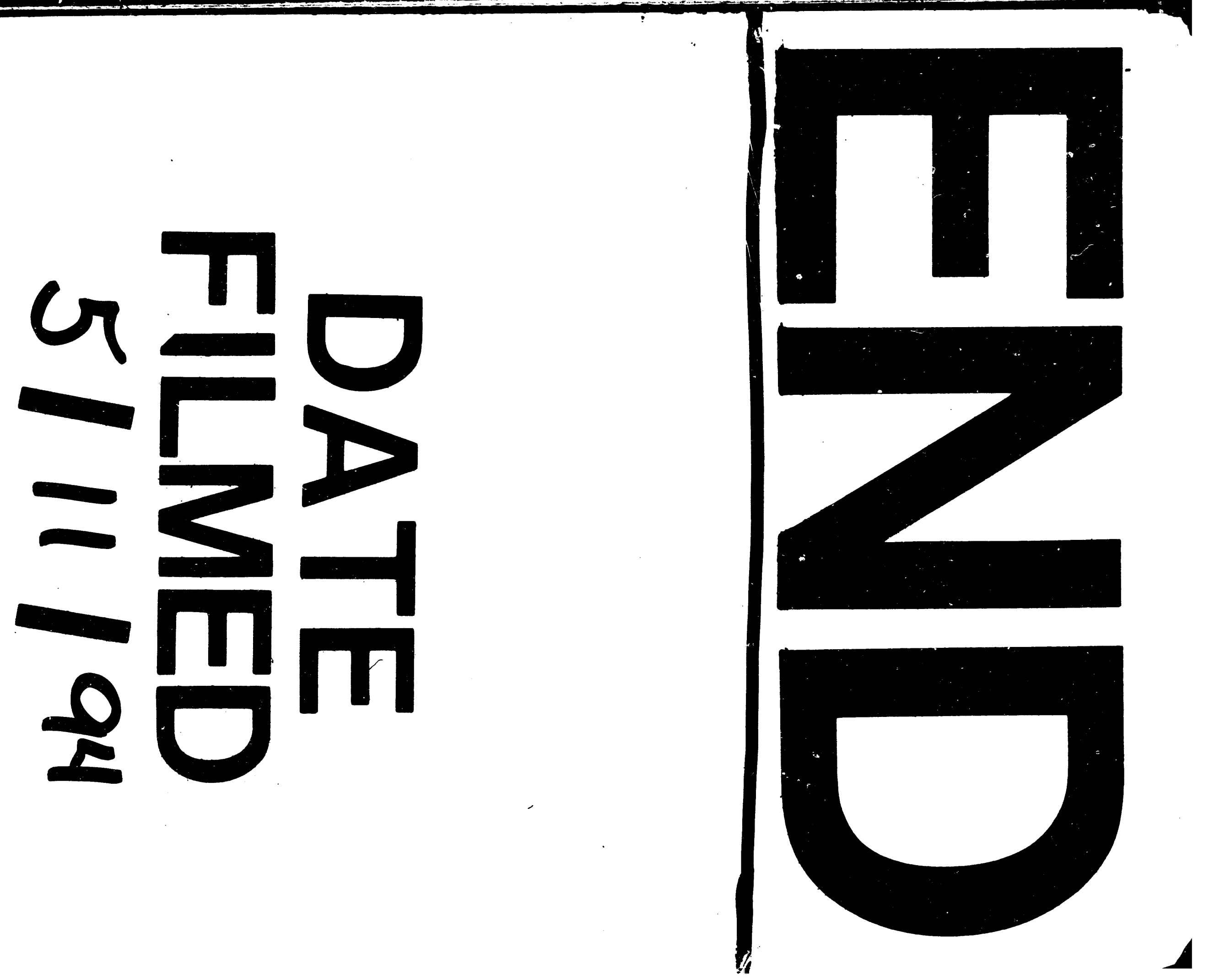
